\title{
Divergent, C-C Bond Forming Macrocyclizations Using Modular Sulfonylhydrazone and Derived Substrates
}

Wenqing Xu, Lauren E. Brown,* and John A. Porco, Jr.*

Department of Chemistry, Center for Molecular Discovery (BU-CMD), Boston University, Boston, Massachusetts 02215, United States

*porco@bu.edu

*brownle@bu.edu 


\section{TABLE OF CONTENTS}

I. Compound Characterization Using Characteristic NMR signals …............................................... 3

A. Table S1: Characteristic NMR signals for the assignment of pyrazole macrocycles. ................ S3

B. Table S2: Characteristic NMR signals for the assignment of dienyl macrocycles.................... S4

II. Compound Characterization Using 2D NMR and DFT Optimized Structures .................................. S4

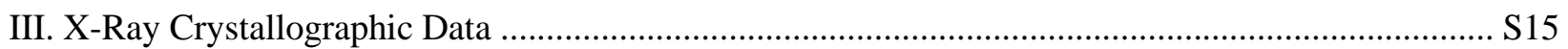

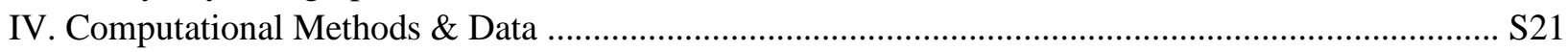

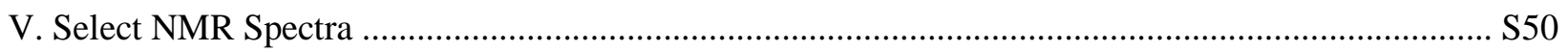




\section{Compound Characterization Using Characteristic NMR signals}

A. Table S1: Characteristic NMR signals for the assignment of pyrazole macrocycles (MCs). ${ }^{\mathrm{a}}$

Key diagnostic shifts used for head-to-tail (MC-htt) vs. head-to-head (MC-hth) assignments are highlighted in red. Head-to-tail structure of macrocycle $20 \mathrm{c}$ was confirmed by X-ray crystallography.

\begin{tabular}{|c|c|c|c|c|c|c|c|}
\hline \multirow[b]{2}{*}{ ID } & \multirow[b]{2}{*}{$X$} & \multicolumn{3}{|c|}{ MC-htt } & \multicolumn{3}{|c|}{ MC-hth } \\
\hline & & $\mathrm{H}_{\mathrm{b}}(\mathrm{ppm})$ & $\mathrm{C}_{\mathrm{b}}(\mathrm{ppm})$ & $\begin{array}{c}\mathrm{nOe} \\
\left(\mathrm{H}_{\mathrm{a}}, \mathrm{H}_{\mathrm{b}}\right)\end{array}$ & $\mathrm{H}_{\mathrm{b}}(\mathrm{ppm})$ & $\mathrm{C}_{\mathrm{b}}(\mathrm{ppm})$ & $\begin{array}{c}\mathrm{nOe} \\
\left(\mathrm{H}_{\mathrm{a}}, \mathrm{H}_{\mathrm{c}}\right)\end{array}$ \\
\hline 17 & $\mathrm{CH}$ & 7.02 & 107.03 & Yes & & & \\
\hline $20 a$ & $\mathrm{CH}$ & 6.81 & 100.77 & Yes & & & \\
\hline $21 a$ & $\mathrm{CH}$ & & & & 7.48 & 139.17 & Yes \\
\hline $20 b$ & $\mathrm{CH}$ & 7.05 & 102.18 & Yes & & & \\
\hline $21 b$ & $\mathrm{CH}$ & & & & $7.76(\mathrm{br})$ & n.o. ${ }^{b}$ & Yes \\
\hline $20 c$ & $\mathrm{CH}$ & 7.20 & 103.07 & Yes & & & \\
\hline $\begin{array}{l}20 d \\
21 d\end{array}$ & $\begin{array}{l}\mathrm{CH} \\
\mathrm{CH}\end{array}$ & 7.30 & 105.35 & Yes & 7.77 (br) & n.o. ${ }^{b}$ & Yes \\
\hline $20 \mathrm{e}$ & $\mathrm{CH}$ & 7.45 & 105.52 & Yes & & & \\
\hline $21 e$ & $\mathrm{CH}$ & & & & $7.76(\mathrm{br})$ & n.o. ${ }^{b}$ & Yes \\
\hline $20 f$ & $\mathrm{CH}$ & n.a. ${ }^{c}$ & $\begin{array}{c}115.40,114.35 \\
\text { (rotamers) }\end{array}$ & n.a. ${ }^{c}$ & & & \\
\hline $21 f$ & $\mathrm{CH}$ & & & & n.a. ${ }^{c}$ & $\begin{array}{c}147.55,144.03 \\
\text { (rotamers) }\end{array}$ & Yes \\
\hline $23 a$ & $\mathrm{CH}$ & 6.94 & 106.89 & Yes & & & \\
\hline $23 b$ & $\mathrm{CH}$ & 6.59 & 107.29 & Yes & & & \\
\hline $24 b$ & $\mathrm{CH}$ & & & & 7.56 & n.o. ${ }^{b}$ & Yes \\
\hline $24 c$ & $\mathrm{CH}$ & & & & 7.52 (br) & n.o. ${ }^{b}$ & Yes \\
\hline 23d & $\mathrm{CH}$ & 6.07 & 106.24 & n.o. ${ }^{b}$ & & & \\
\hline 24d & $\mathrm{CH}$ & & & & n.o. ${ }^{b}$ & n.o. ${ }^{b}$ & n.o. \\
\hline 29 & $\mathrm{~N}$ & 7.01 & 106.95 & n.a. ${ }^{d}$ & & & \\
\hline 30 & $\mathrm{~N}$ & & & & 7.50 & 131.79 & n.a. ${ }^{d}$ \\
\hline 49 & $\mathrm{~N}$ & 7.27 & 107.28 & n.a. ${ }^{d}$ & & & \\
\hline 50 & $\mathrm{~N}$ & & & & n.o. ${ }^{b}$ & n.o. ${ }^{b}$ & n.a. ${ }^{d}$ \\
\hline
\end{tabular}

(a) n.a. = not available; n.o. = not observed. (b) Not observed due to overlap with other signals, broadening, or presence of complex rotamers. (c) Not available due to absence of a diagnostic $\mathrm{H}_{b}$ atom. (d) Not available due to absence of a diagnostic $\mathrm{H}_{a}$ atom $(X=N)$. 
B. Table S2: Characteristic NMR signals for the assignment of dienyl macrocycles

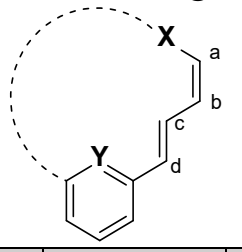

\begin{tabular}{|c|c|c|c|c|c|c|c|c|}
\hline $\mathrm{ID}$ & $\mathbf{X}$ & $\mathbf{Y}$ & $\mathrm{H}_{\mathrm{a}}(\mathrm{ppm})$ & $\mathrm{H}_{\mathrm{b}}(\mathrm{ppm})$ & $\mathrm{H}_{\mathrm{c}}(\mathrm{ppm})$ & $\mathrm{H}_{\mathrm{d}}(\mathrm{ppm})$ & $J_{\mathrm{a}, \mathrm{b}}(\mathrm{Hz})$ & $J_{\mathrm{c}, \mathrm{d}}(\mathrm{Hz})$ \\
\hline $\mathbf{3 2}$ & $\mathrm{C}$ & $\mathrm{N}$ & 5.57 & 6.19 & 8.29 & 6.51 & $11.6($ cis $)$ & 14.7 (trans) \\
\hline $\mathbf{5 1}$ & $\mathrm{O}$ & $\mathrm{N}$ & 6.35 & 5.75 & 7.92 & 6.67 & $5.2($ cis $)$ & 15.7 (trans) \\
\hline $\mathbf{5 2}$ & $\mathrm{C}$ & $\mathrm{CH}$ & 5.58 & 6.21 & 7.39 & 6.58 & $10.9($ cis $)$ & 15.5 (trans) \\
\hline $\mathbf{5 3}$ & $\mathrm{O}$ & $\mathrm{CH}$ & 6.69 & 5.84 & 7.91 & 6.58 & $5.5($ cis $)$ & 16.0 (trans) \\
\hline
\end{tabular}

\section{Compound Characterization Using 2D NMR and DFT Optimized Structures}

Figure S1. DFT-optimized structure consistent with key 2D NMR interactions for structural assignment of 11:
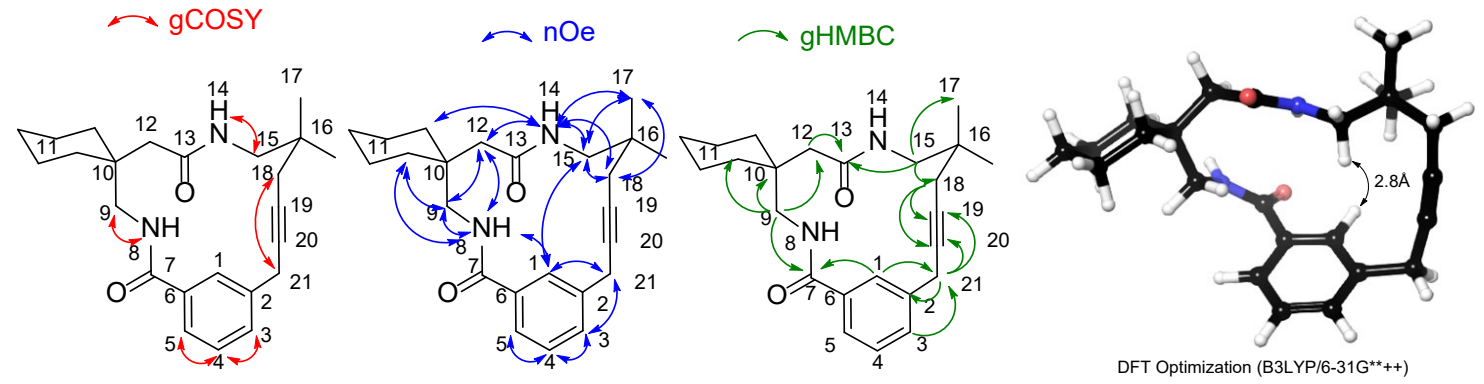

Figure S2. DFT-optimized structure consistent with key 2D NMR interactions for structural assignment of 17:
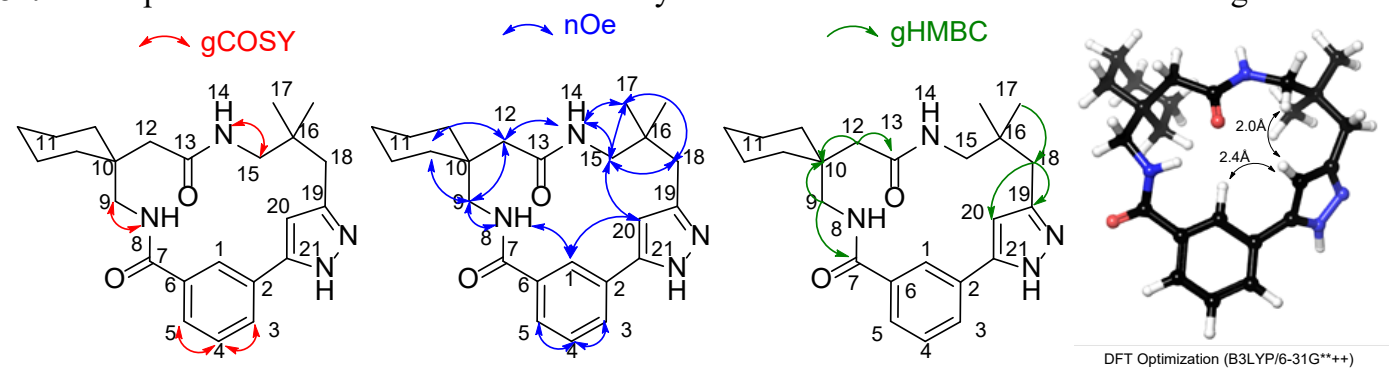

Figure S3. DFT-optimized structure consistent with key 2D NMR interactions for the structural assignment of 20a:
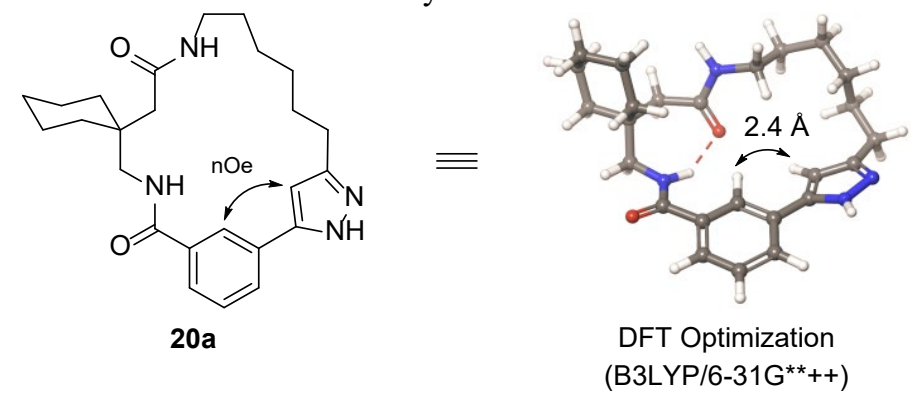

Figure S4. DFT-optimized structure consistent with key 2D NMR interactions for structural assignment of 21a: 


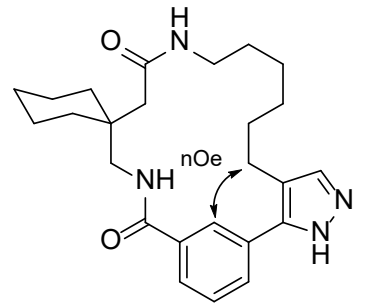

21a

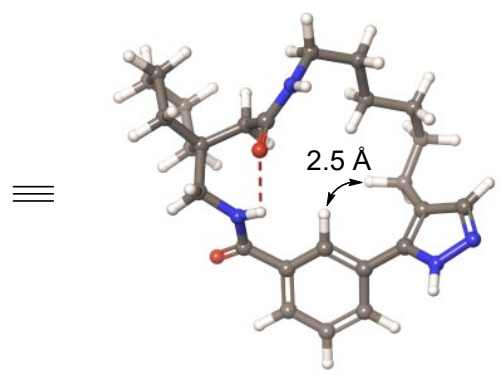

DFT Optimization (B3LYP/6-31G**++)

Figure S5. X-ray crystal structure and key 2D NMR interactions for structural assignment of 20c:

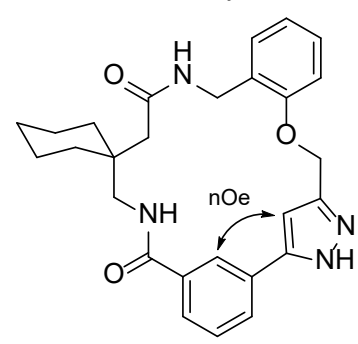

20c

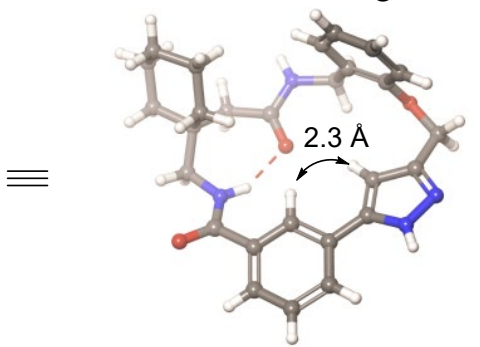

X-ray Crystallography

Figure S6. Key 2D NMR interactions for structural assignment of 20c':

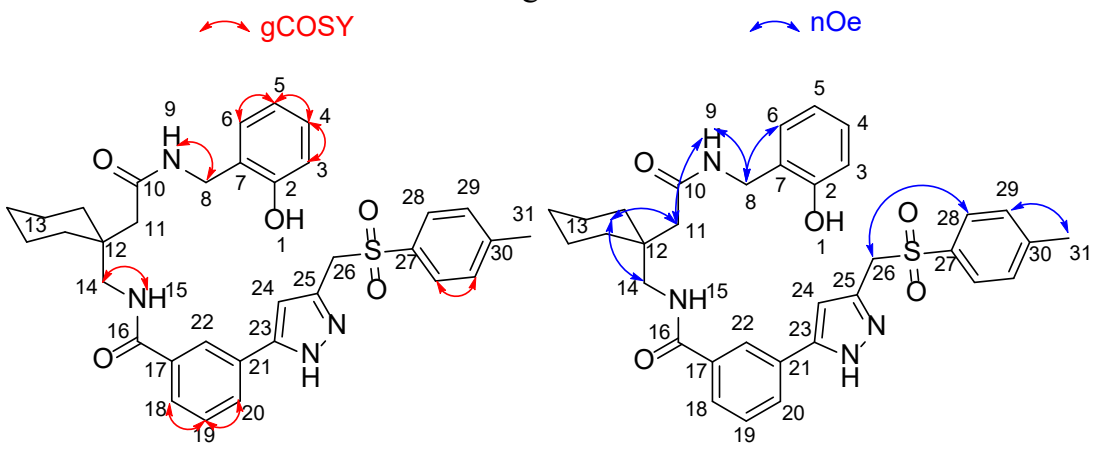


Figure S7. Variable temperature ${ }^{1} \mathrm{H}$ NMR spectra for $20 f$

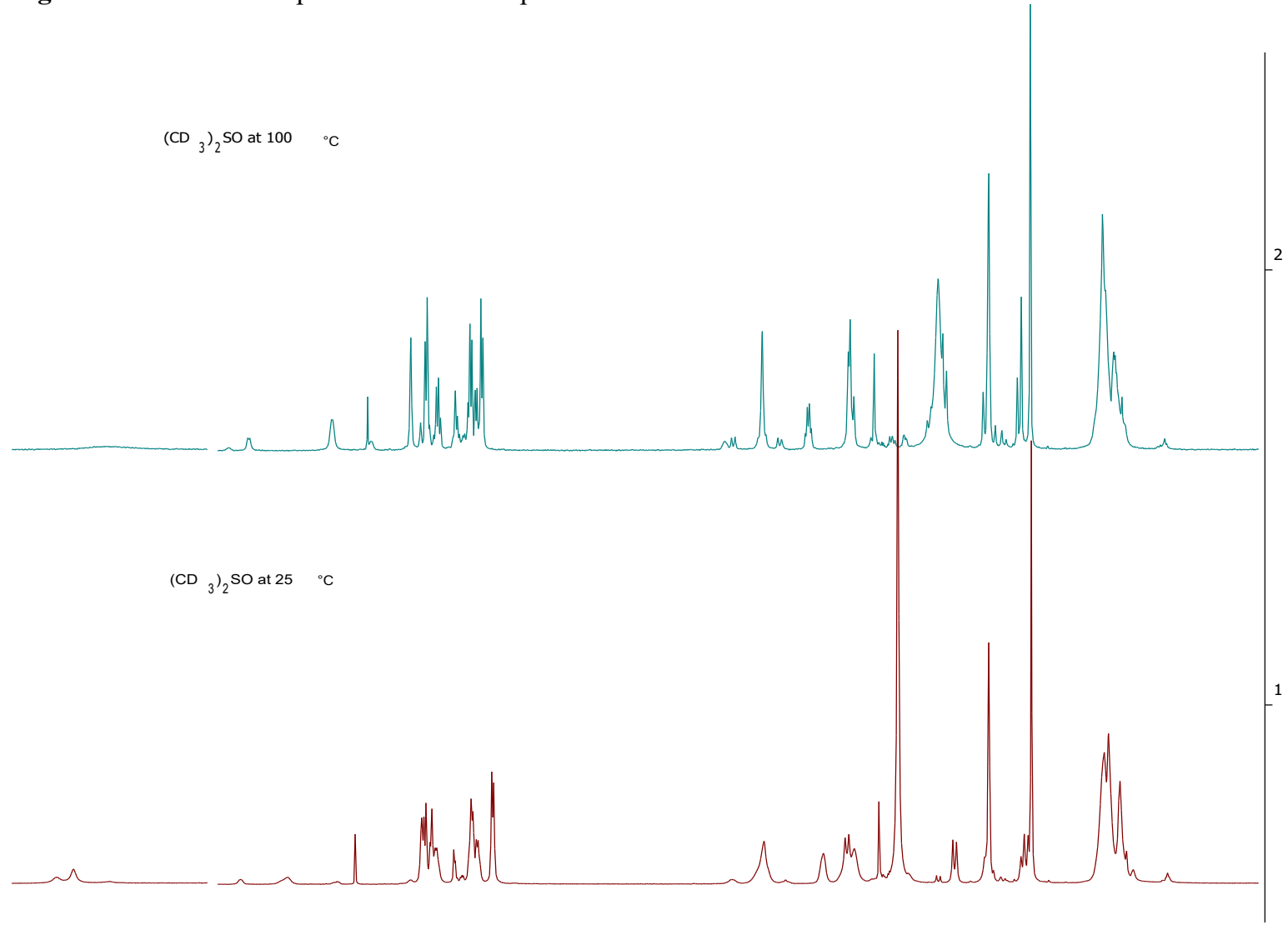

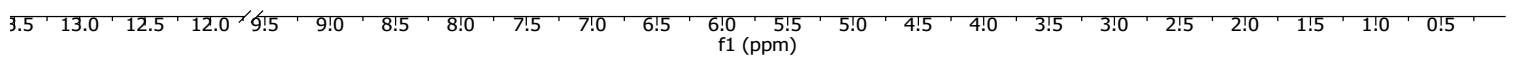

To rationalize the peak broadening and multiple sets of signals observed during variable temperature NMR analysis of 20f, we postulate that "ring flip" conformers 20f-1/20f-4 and 20f-2/20f-3 (Figure S8) interconvert slowly relative to the NMR timescale at $25^{\circ} \mathrm{C}$, resulting in the observed broadening in the ${ }^{1} \mathrm{H}$ NMR spectra. Upon heating, we postulate that the ring flip interconversion occurs more rapidly, resulting in the observed peak sharpening. However, due to the rotational barrier that would be imparted by the pyrazole methyl group, we hypothesize that the interconversion of $\mathbf{2 0 f - 1}$ to $\mathbf{2 0 f - 2}$ and the interconversion of $20 \mathrm{f}-3$ to $\mathbf{2 0 f}-\mathbf{4}$ are still slow relative to the NMR timescale, even with high heating. 
Figure S8. Proposed conformational equilibria for 20f. Variable rates of interconversion of $20 f$ conformers lead to observed broadening and multiple sets of peaks during variable-temperature NMR analysis.

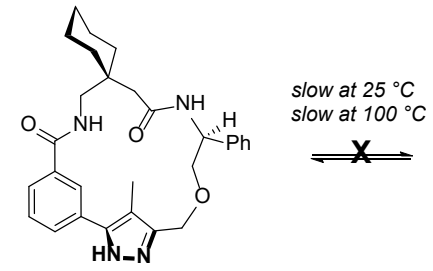

Conf. 20f-1
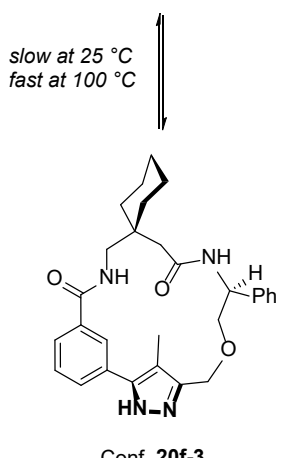
slow at $100^{\circ} \mathrm{C}$

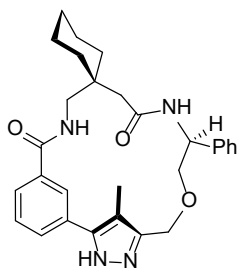

Conf. 20f-2 slow at $25^{\circ} \mathrm{C}$
fast at $100^{\circ} \mathrm{C}$

slow at $25^{\circ} \mathrm{C}$

$\stackrel{\mathbf{X}}{\rightleftharpoons}$

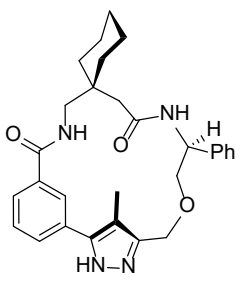

Conf. 20f-4

Figure S9. DFT-optimized structure consistent with key 2D NMR interactions for structural assignment of $\mathbf{2 0 g}$ :
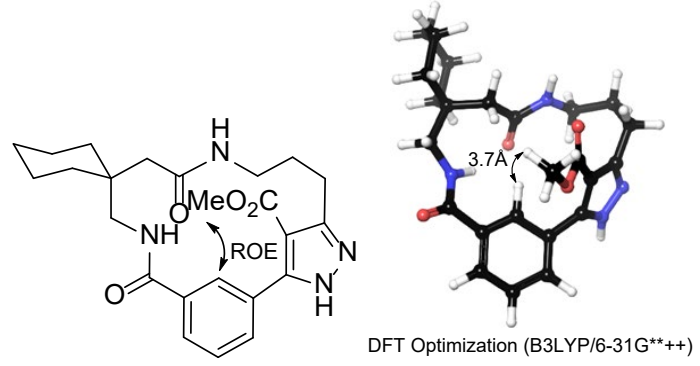

Figure S10. DFT-optimized structure consistent with key 2D NMR interactions for structural assignment of 20h:

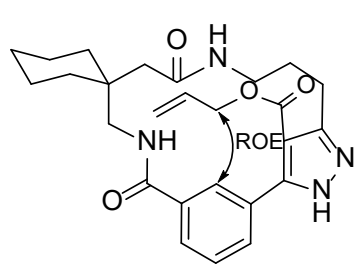

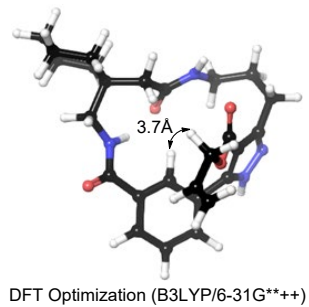

Figure S11. Key ${ }^{13} \mathrm{C}$ NMR shift used in structural assignment of regioisomers $\mathbf{2 3 d}$ and 24d

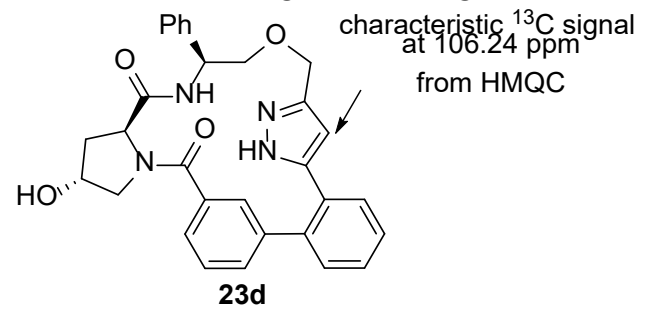

Figure S12. DFT-optimized structure consistent with key 2D NMR interactions for the structural assignment of 29: 


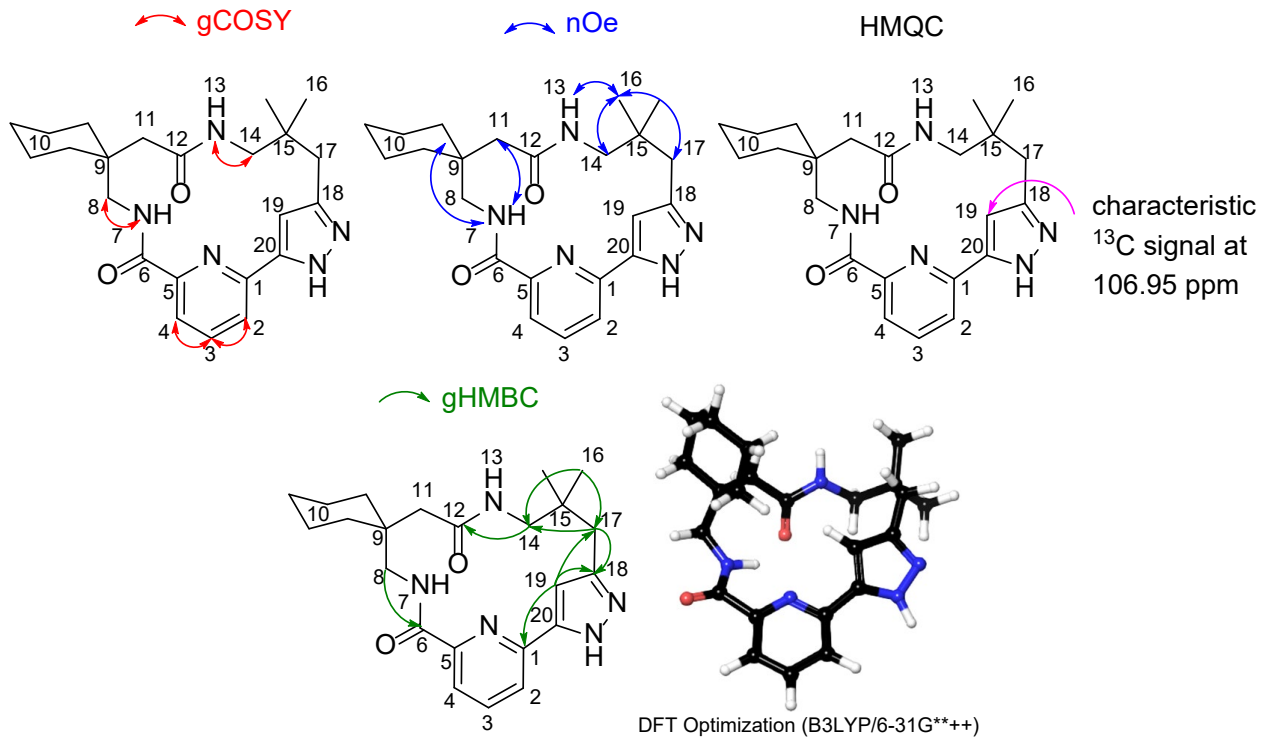

Figure S13. DFT-optimized structure consistent with key 2D NMR interactions for the structural assignment of 30:
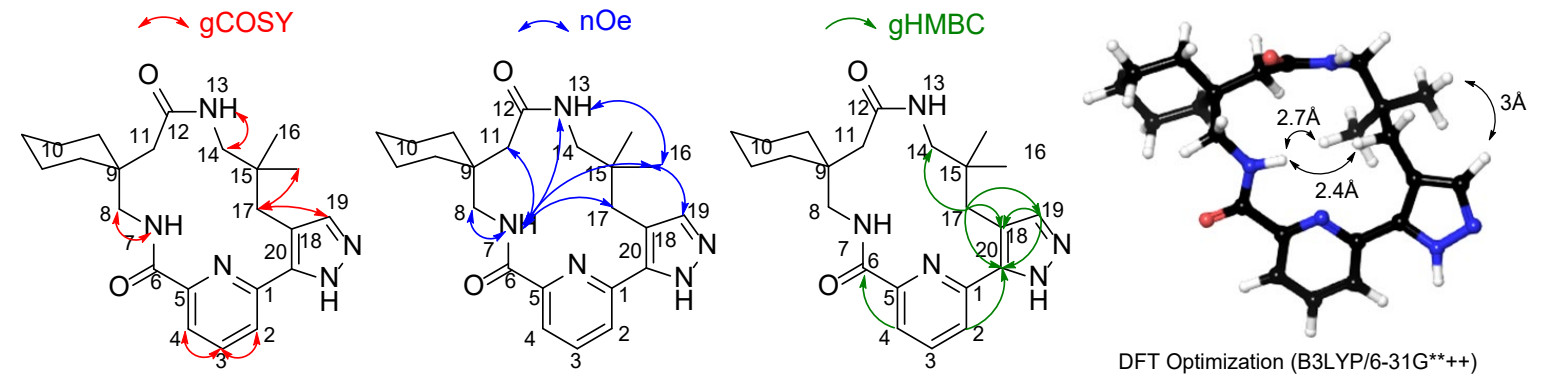

Figure S14. DFT-optimized structure consistent with key 2D NMR interactions for the structural assignment of 31:
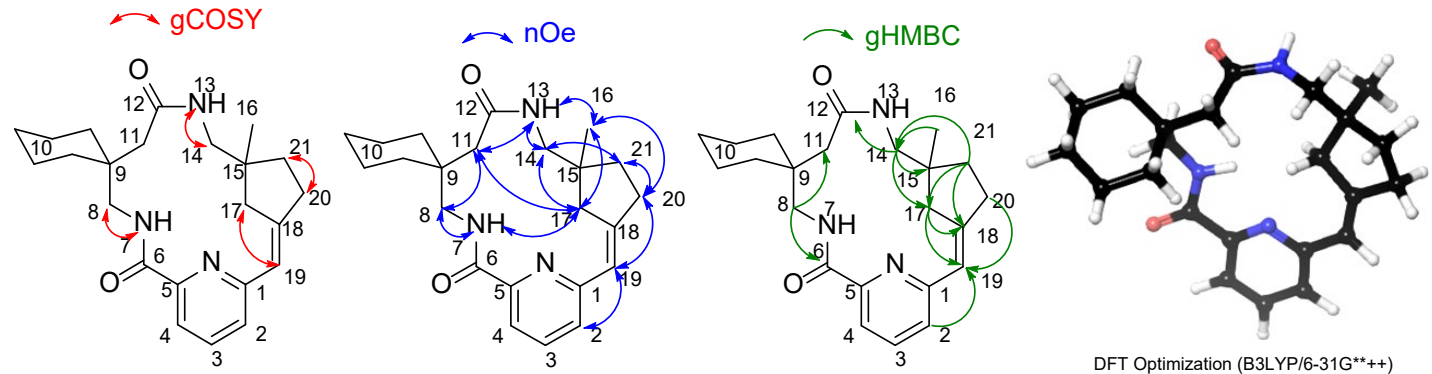

Figure S15. DFT-optimized structure consistent with key 2D NMR interactions for structural assignment of 32:

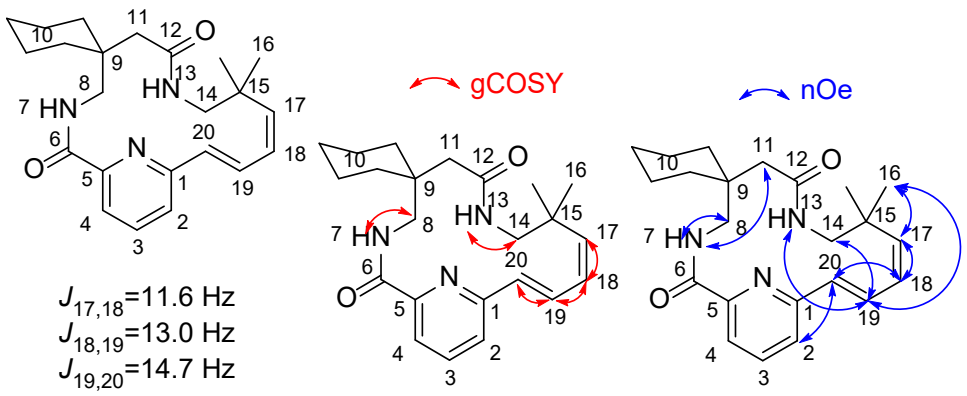




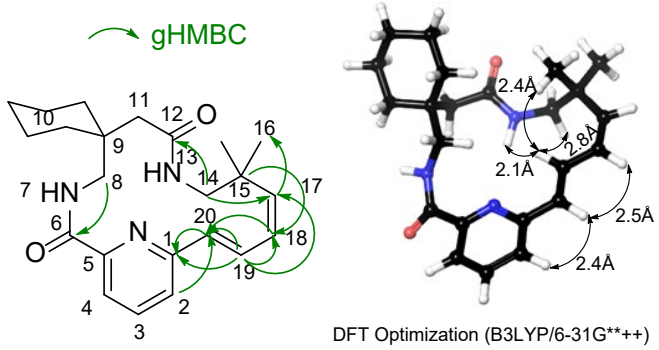

Figure S16. DFT-optimized structure consistent with key 2D NMR interactions for structural assignment of 39:
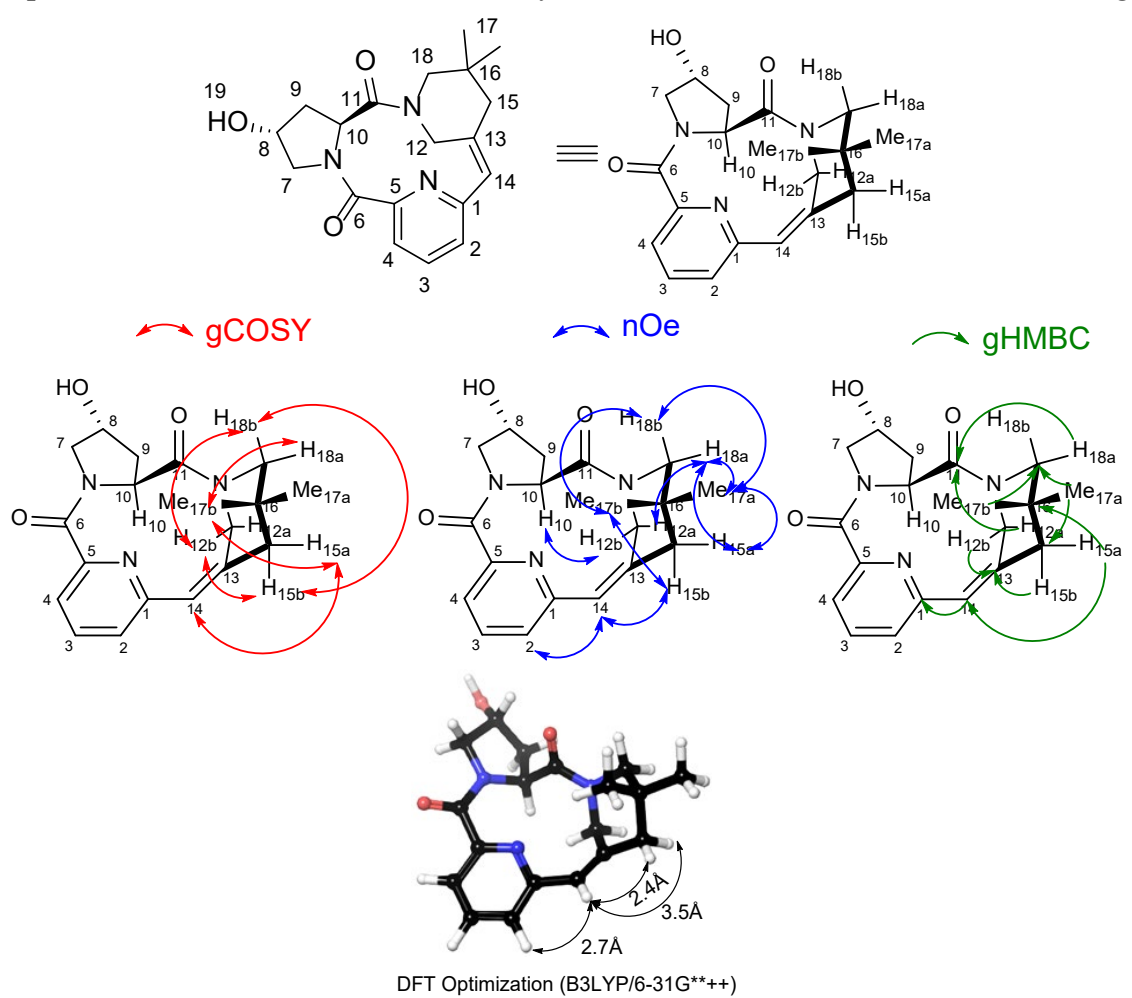

Figure S17. DFT-optimized structure consistent with key 2D NMR interactions for structural assignment of 40:

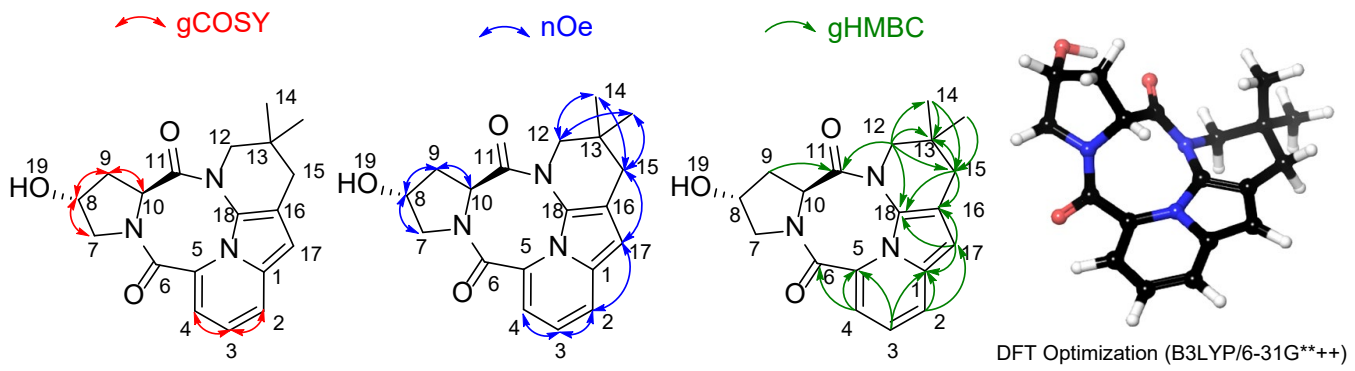

Figure S18. DFT-optimized structure consistent with key 2D NMR interactions for structural assignment of 49: 


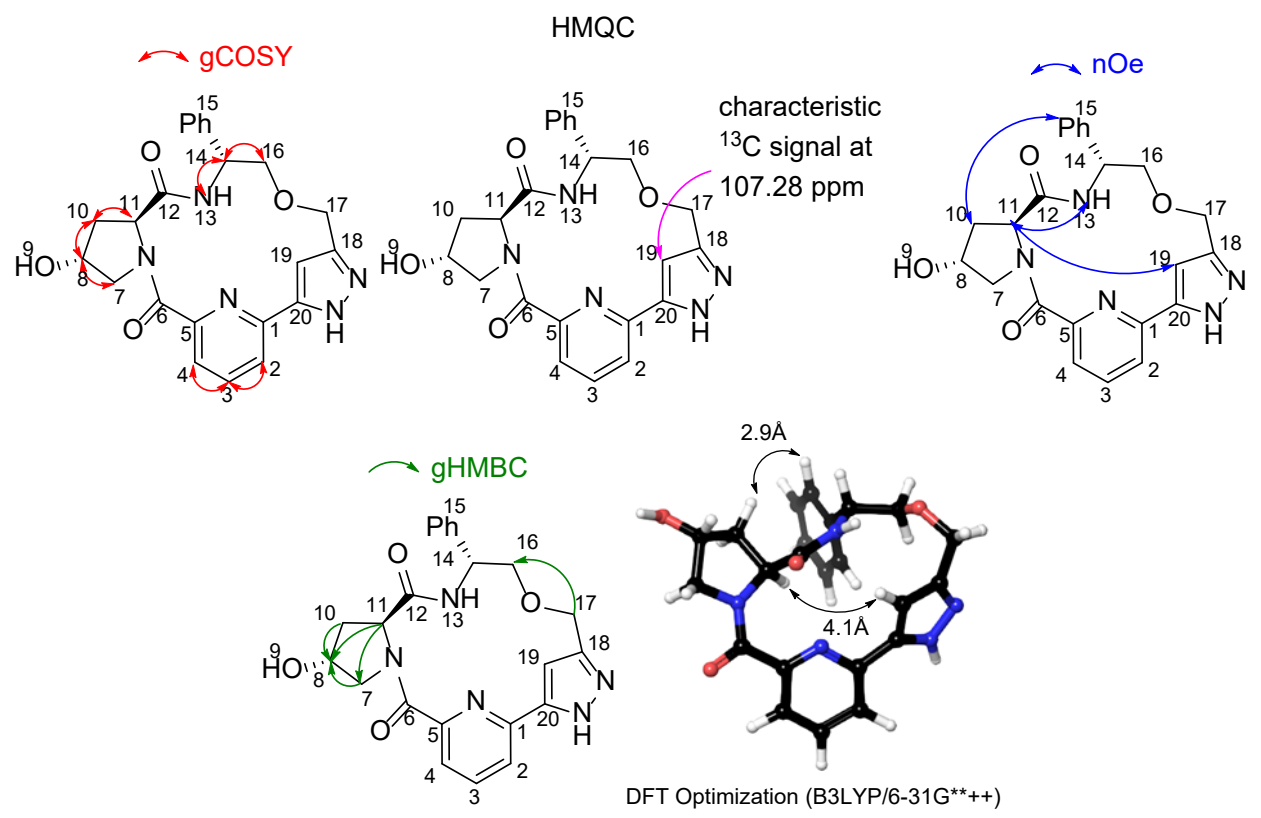

Figure S19. DFT-optimized (B3LYP/6-31 $\mathrm{g}^{* *++}$ ) lowest-energy conformations of two proposed atropisomers of $\mathbf{5 0 .}$

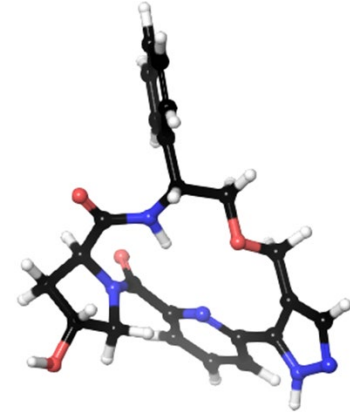

Conf. 50-cis

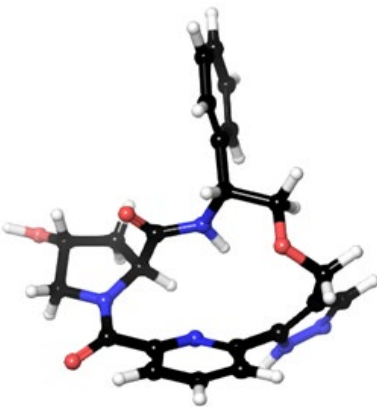

Conf. 50-trans

Figure S20. Variable temperature ${ }^{1} \mathrm{H}$ NMR spectra for $\mathbf{5 0}$ showing discrete proton signals at $25{ }^{\circ} \mathrm{C}$ (bottom) which coalesce upon heating to $90^{\circ} \mathrm{C}$ (top). 


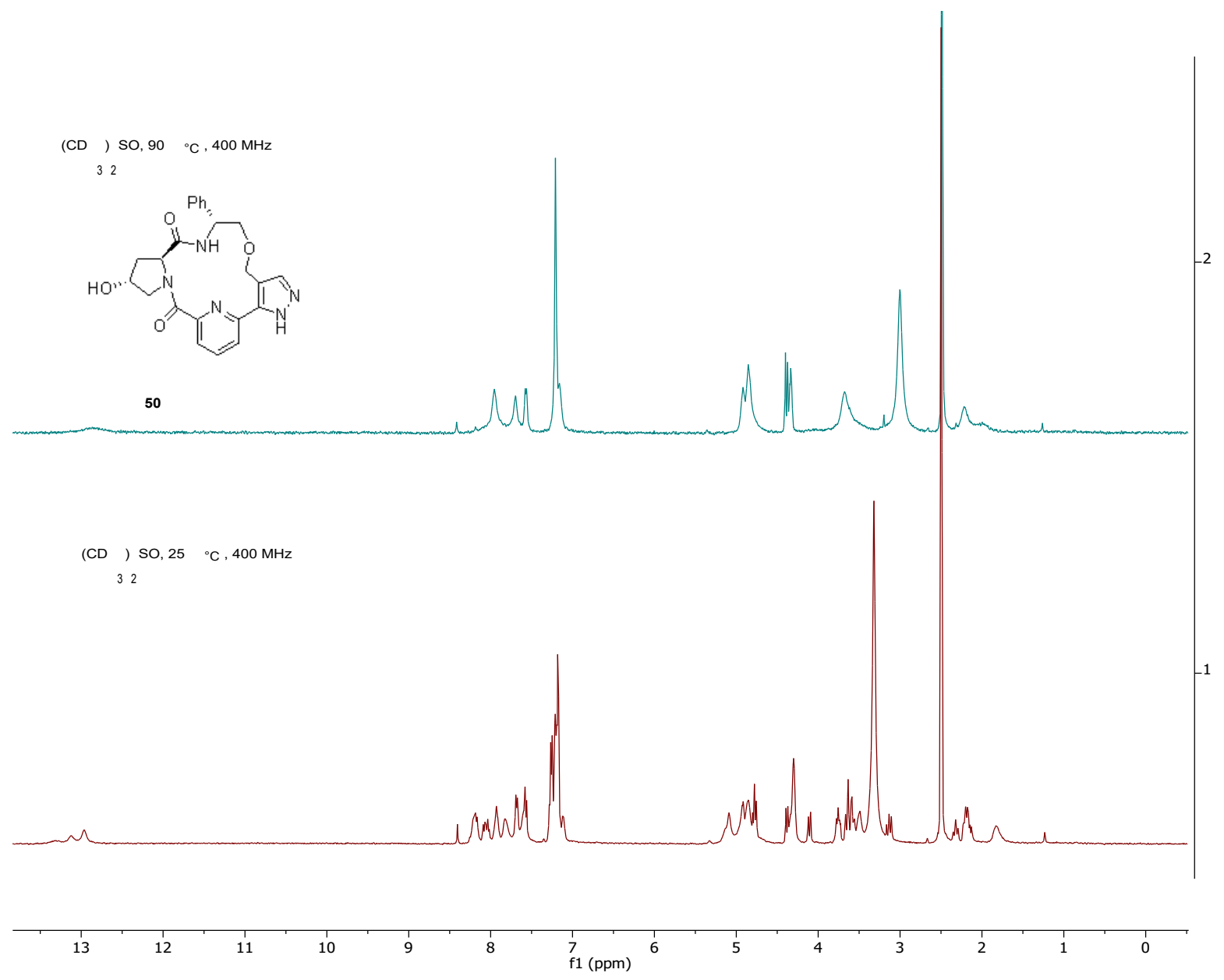

Figure S21. DFT-optimized structure, key 2D NMR interactions, and coupling constants used for structural assignment of 51:

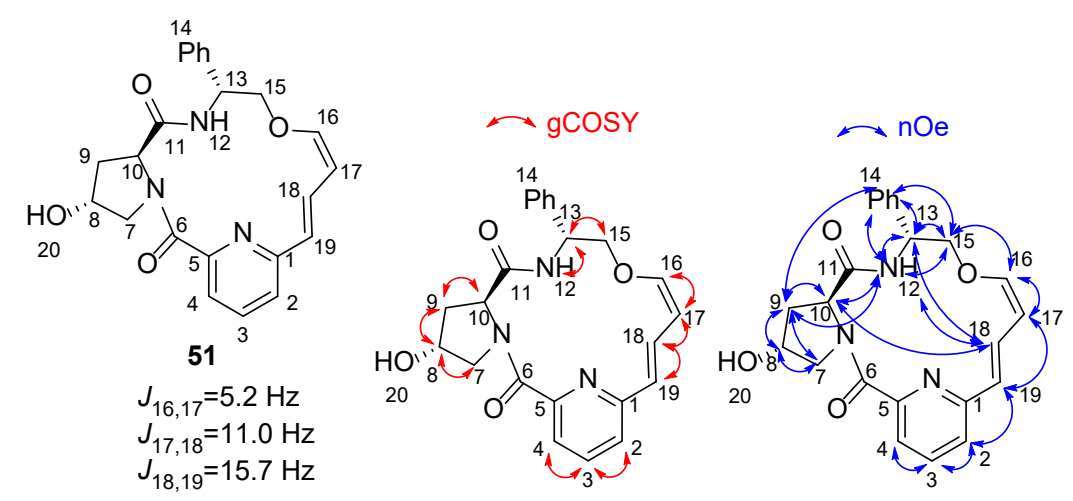



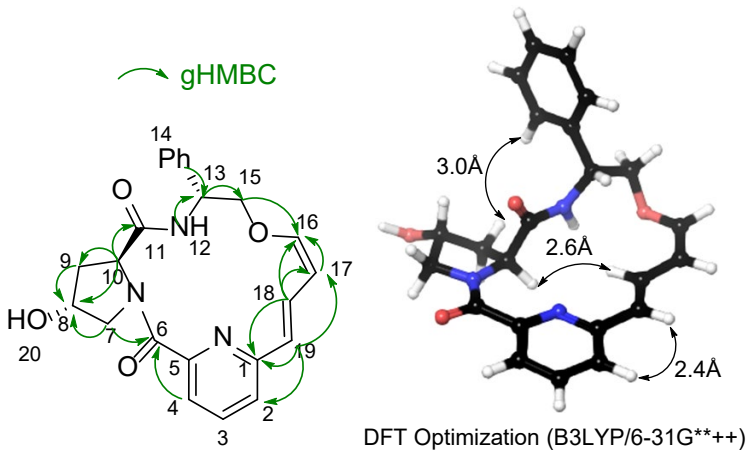

Figure S22. Calculated relative gas phase energies of DFT-optimized s-trans-51 and s-cis-51 (B3LYP/6-31G**++):
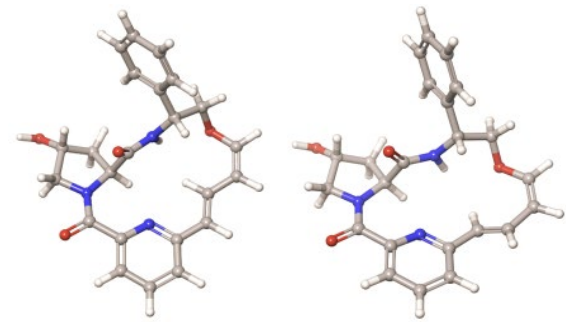

s-trans-51: $0.0 \mathrm{kcal} / \mathrm{mol}$

s-cis-51: $+4.3 \mathrm{kcal} / \mathrm{mol}$

Figure S23. DFT-optimized structure, key 2D NMR interactions and coupling constants used for structural assignment of 52:
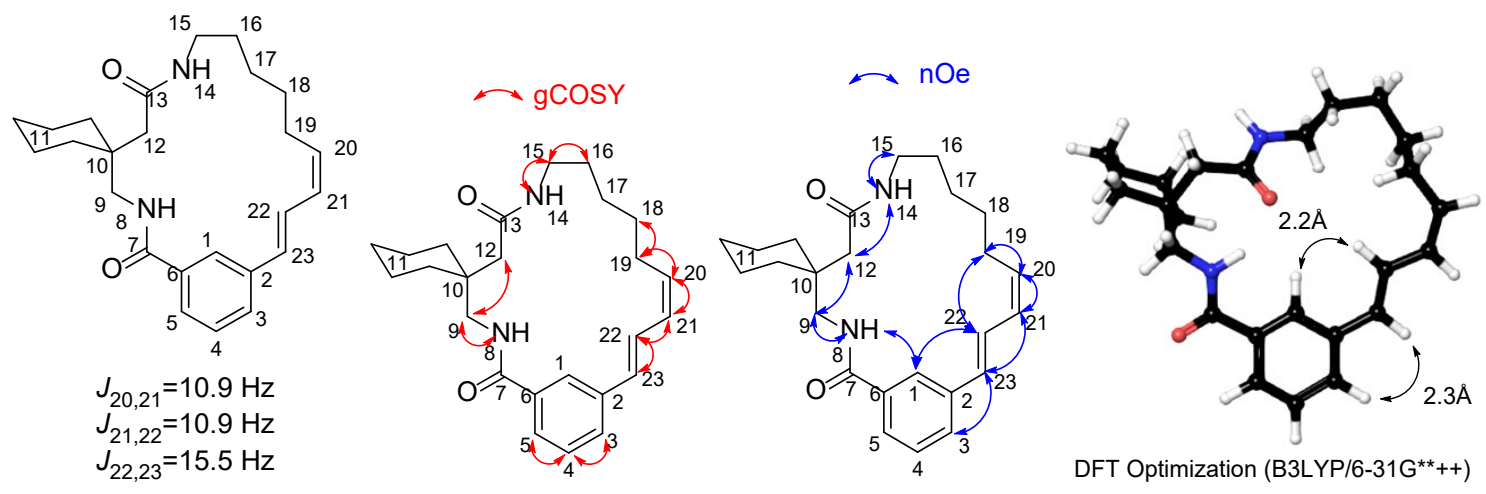

Figure S24. DFT-optimized structure, key 2D NMR interactions and coupling constants used for structural assignment of 53:
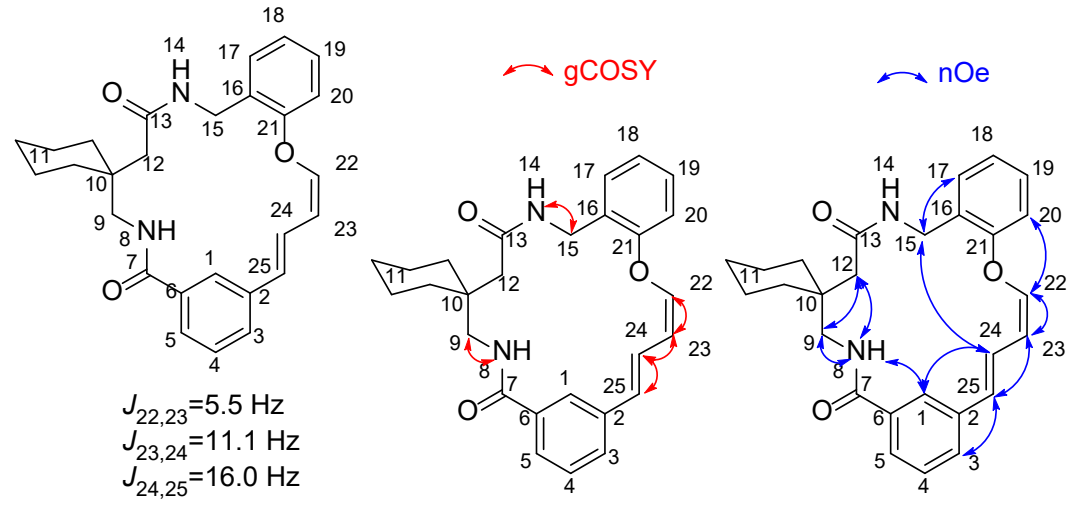


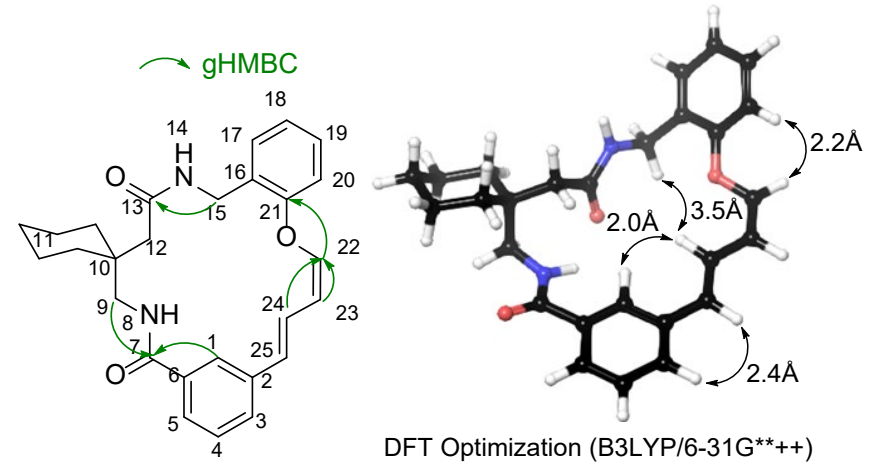

Figure S25. Key 2D NMR interactions and coupling constants used for structural assignment of 56:
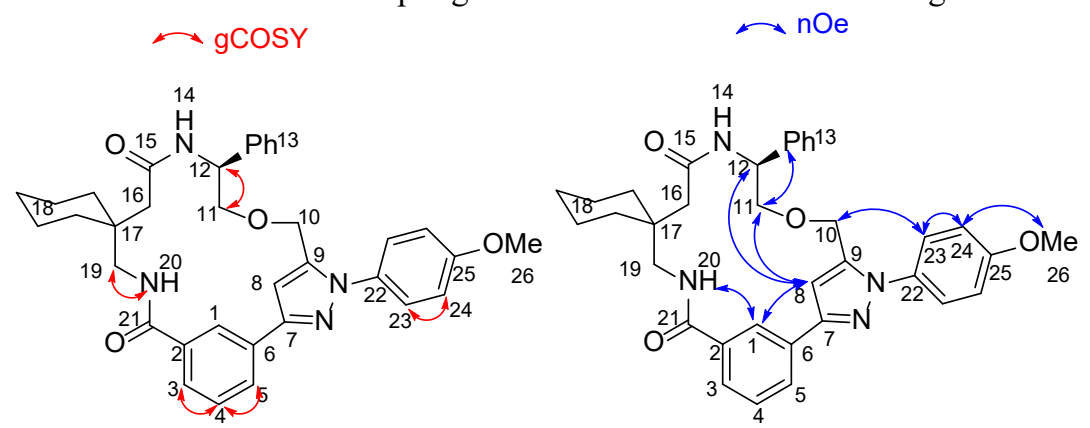

Figure S26. DFT-optimized structure consistent with key 2D NMR interactions used for the structural assignment of 58:
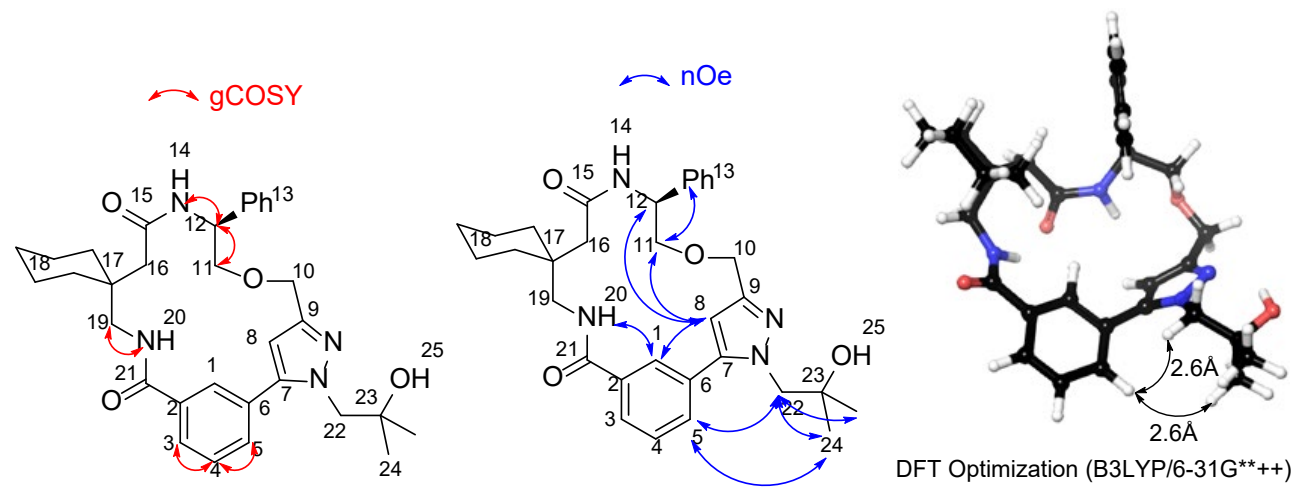

Figure S27. DFT-optimized structure consistent with key 2D NMR interactions used for the structural assignment of 59:
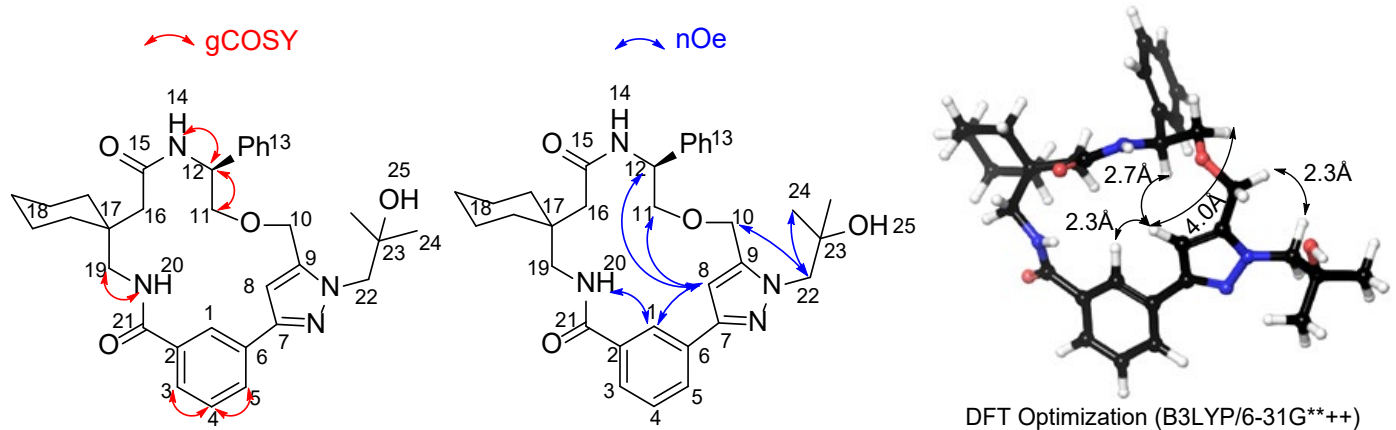
Figure S28. DFT-optimized structure consistent with key 2D NMR interactions used for the structural assignment of 61:

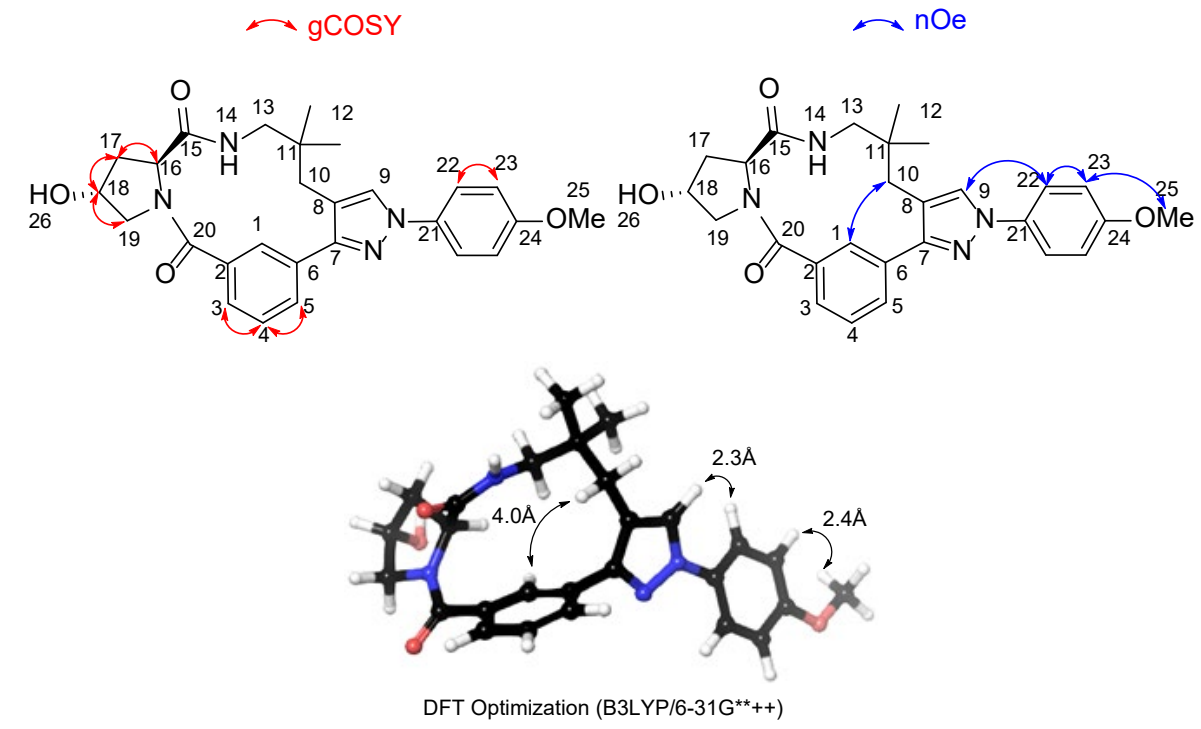

Figure S29. DFT-optimized structure, key 2D NMR interactions and coupling constants used for the structural assignment of pyridazine $\mathbf{6 3}$ :
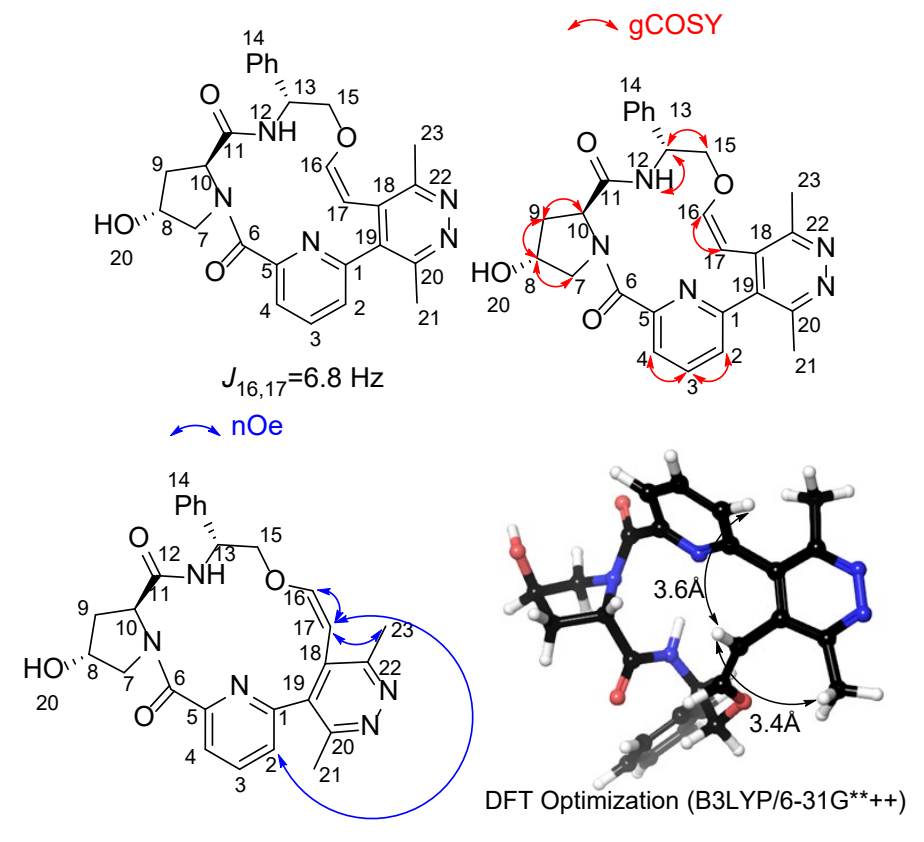


\section{X-Ray Crystallographic Data}

Crystals of compound 20c (containing two molecules of $\mathrm{CD}_{3} \mathrm{OD}$ ) suitable for X-ray analysis were obtained by slow evaporation from $\mathrm{CD}_{3} \mathrm{OD}$. Crystallographic data has been deposited with the Cambridge Crystallographic Data Center (CCDC\#2091562). Copies of the data can be obtained free of charge through application to the CCDC, 12 Union Road, Cambridge CB21EZ, UK (fax: +440-1223-336-033; email: deposit@ccdc.cam.ac.uk)

\section{a. X-ray crystallographic data for compound 20c}

Figure S30. ORTEP drawing of 20c showing thermal ellipsoids at the $50 \%$ probability level:

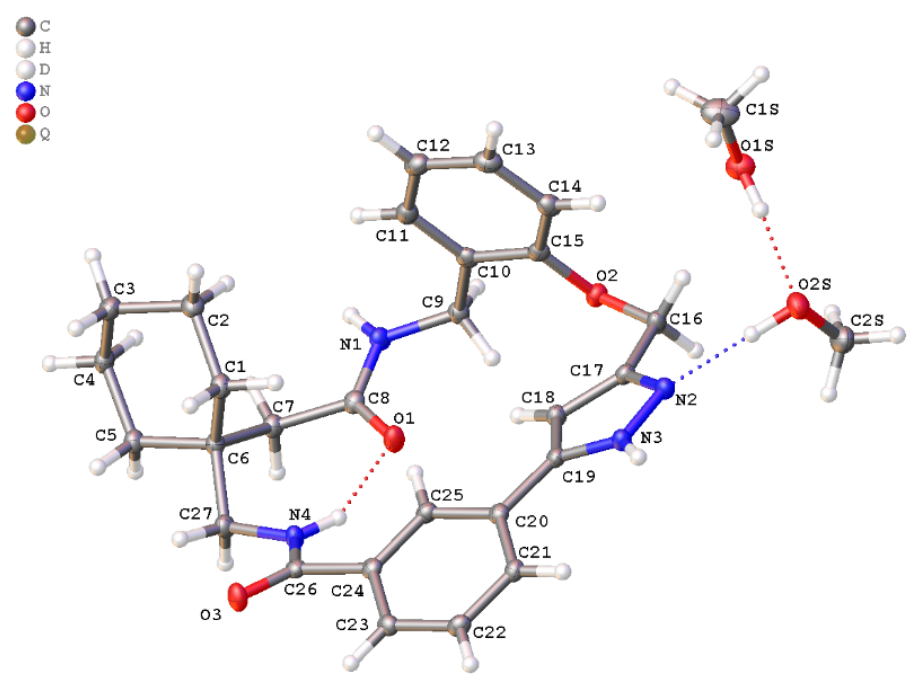

\section{Computing details}

Data collection: APEX3 (Bruker, 2016); cell refinement: SAINT V8.38A (Bruker, 2016); data reduction: SAINT V8.38A (Bruker, 2016); program(s) used to solve structure: ShelXT (Sheldrick, 2015); program(s) used to refine structure: SHELXL (Sheldrick, 2015); molecular graphics: Olex2 (Dolomanov et al., 2009); software used to prepare material for publication: Olex2 (Dolomanov et al., 2009).

\section{References}

Bruker (2006). SAINT. Bruker Analytical X-ray Instruments Inc., Madison, Wisconsin, USA. Bruker (2016). APEX3. Bruker Analytical X-ray Instruments Inc., Madison, Wisconsin, USA. Sheldrick, G. M. A Short History of SHELX. Acta Cryst. A. 2008, 64, 112-122.

Sheldrick, G. M. Crystal Structure Refinement with SHELXL. Acta Cryst.. C Struct. Chem. 2015, 71, 3-8. Krause, L.; Herbst-Irmer, R.; Sheldrick, G. M.; Stalke, D. Comparison of Silver and Molybdenum Microfocus X-ray Sources for Single-Crystal Structure Determination. J. Appl. Cryst. 2015, 48, 3-10.

Dolmanov, O. V.; Bourhis, L. J.; Gildea, R. J.; Howard, J. A. K.; Puschmann, H. OLEX2: A complete structure solution, refinement and analysis program. J. Appl. Cryst. 2009, 42, 339-341.

Table S3. Crystal data for compound 20c

\begin{tabular}{|l|l|}
\hline $\mathrm{C}_{27} \mathrm{H}_{30} \mathrm{~N}_{4} \mathrm{O}_{3} \cdot 2\left(\mathrm{CD}_{4} \mathrm{O}\right)$ & $F(000)=1120$ \\
\hline$M_{r}=530.68$ & $D_{\mathrm{x}}=1.311 \mathrm{Mg} \mathrm{m}^{-3}$ \\
\hline Monoclinic, $P 2_{1} / n$ & $\mathrm{Cu}$ Ka radiation, $\mathrm{l}=1.54178 \AA$ \\
\hline$a=13.5783(4) \AA$ & Cell parameters from 9068 reflections \\
\hline$b=13.6534(4) \AA$ & $\mathrm{q}=3.2-66.6^{\circ}$ \\
\hline$c=15.4288(4) \AA$ & $\mathrm{m}=0.72 \mathrm{~mm}^{-1}$ \\
\hline $\mathrm{b}=109.974(1)^{\circ}$ & $T=100 \mathrm{~K}$ \\
\hline$V=2688.29(13) \AA^{3}$ & Prism, colorless \\
\hline
\end{tabular}




\begin{tabular}{|l|l|}
\hline$Z=4$ & $0.31 \times 0.24 \times 0.19 \mathrm{~mm}$ \\
\hline
\end{tabular}

Table S4. Data collection

\begin{tabular}{|l|l|}
\hline Bruker X8 Proteum-R diffractometer & 4662 independent reflections \\
\hline Radiation source: rotating anode & 4483 reflections with $I>2 \square(I)$ \\
\hline Montel & $R_{\text {int }}=0.038$ \\
\hline$\omega$ and $\phi$ scans & $\theta_{\max }=66.6^{\circ}, \theta_{\min }=3.8^{\circ}$ \\
\hline $\begin{array}{l}\text { Absorption correction: multi-scan } \\
S A D A B S 2016 / 2 \text { (Bruker,2016/2) was used for } \\
\text { absorption correction. wR2(int) was } 0.1259 \\
\text { before and } 0.0521 \text { after correction. The Ratio of } \\
\text { minimum to maximum transmission is } 0.8953 . \\
\text { The } 1 / 2 \text { correction factor is Not present. }\end{array}$ & $h=-15 \rightarrow 16$ \\
\hline$T_{\min }=0.674, T_{\max }=0.753$ & $k=-16 \rightarrow 16$ \\
\hline 68910 measured reflections & $l=-18 \rightarrow 18$ \\
\hline
\end{tabular}

Table S5. Refinement

\begin{tabular}{|l|l|}
\hline Refinement on $F^{2}$ & Primary atom site location: dual \\
\hline Least-squares matrix: full & Hydrogen site location: mixed \\
\hline$R\left[F^{2}>2 \mathrm{~s}\left(F^{2}\right)\right]=0.032$ & $\begin{array}{l}\text { H atoms treated by a mixture of independent } \\
\text { and constrained refinement }\end{array}$ \\
\hline$w R\left(F^{2}\right)=0.078$ & $\begin{array}{l}w=1 /\left[\sigma^{2}\left(F_{\mathrm{o}}^{2}\right)+(0.033 P)^{2}+1.378 P\right] \\
\text { where } P=\left(F_{\mathrm{o}}^{2}+2 F_{\mathrm{c}}^{2}\right) / 3\end{array}$ \\
\hline$S=1.04$ & $(\Delta / \sigma)_{\max }=0.001$ \\
\hline 4662 reflections & $\Delta\rangle_{\max }=0.23 \mathrm{e} \AA^{-3}$ \\
\hline 361 parameters & $\Delta\rangle_{\min }=-0.25 \mathrm{e} \AA^{-3}$ \\
\hline 0 restraints & \\
\hline
\end{tabular}

Special details

Geometry. All esds (except the esd in the dihedral angle between two 1.s. planes) are estimated using the full covariance matrix. The cell esds are taken into account individually in the estimation of esds in distances, angles and torsion angles; correlations between esds in cell parameters are only used when they are defined by crystal symmetry. An approximate (isotropic) treatment of cell esds is used for estimating esds involving 1.s. planes.

Table S6. Fractional atomic coordinates and isotropic or equivalent isotropic displacement parameters $\left(\AA^{2}\right)$

\begin{tabular}{|l|l|l|l|l|}
\hline & $x$ & $y$ & $z$ & $U_{\text {iso }} / U_{\text {eq }}$ \\
\hline O2 & $0.22351(6)$ & $0.37503(6)$ & $0.32250(5)$ & $0.01665(18)$ \\
\hline O1 & $0.05049(7)$ & $0.59518(6)$ & $0.41302(5)$ & $0.01980(19)$ \\
\hline O3 & $0.05647(7)$ & $0.76902(6)$ & $0.69453(5)$ & $0.01991(19)$ \\
\hline O1S & $0.52238(7)$ & $0.23819(7)$ & $0.39896(6)$ & $0.0238(2)$ \\
\hline D1S & $0.5115(13)$ & $0.2048(12)$ & $0.4460(12)$ & $0.036^{*}$ \\
\hline O2S & $0.46185(7)$ & $0.13733(6)$ & $0.52640(6)$ & $0.0237(2)$ \\
\hline N3 & $0.32348(8)$ & $0.32841(7)$ & $0.63103(7)$ & $0.0151(2)$ \\
\hline H3 & $0.3608(11)$ & $0.3077(10)$ & $0.6895(10)$ & $0.018^{*}$ \\
\hline N4 & $0.01874(8)$ & $0.70293(7)$ & $0.55223(7)$ & $0.0161(2)$ \\
\hline H4 & $0.0260(11)$ & $0.6515(11)$ & $0.5190(10)$ & $0.019^{*}$ \\
\hline N1 & $0.05134(8)$ & $0.62888(7)$ & $0.27042(7)$ & $0.0165(2)$ \\
\hline H1 & $0.0300(11)$ & $0.6680(11)$ & $0.2221(10)$ & $0.020^{*}$ \\
\hline N2 & $0.33794(8)$ & $0.28334(7)$ & $0.55780(6)$ & $0.0160(2)$ \\
\hline C15 & $0.28128(9)$ & $0.46035(8)$ & $0.33381(7)$ & $0.0156(2)$ \\
\hline C24 & $0.12313(9)$ & $0.60868(8)$ & $0.68339(8)$ & $0.0142(2)$ \\
\hline
\end{tabular}




\begin{tabular}{|c|c|c|c|c|}
\hline $\mathrm{C} 17$ & $0.27489(9)$ & $0.33071(8)$ & $0.48362(8)$ & $0.0152(2)$ \\
\hline $\mathrm{C} 8$ & $0.02632(9)$ & $0.65007(8)$ & $0.34500(8)$ & $0.0156(2)$ \\
\hline $\mathrm{C} 10$ & $0.22248(9)$ & $0.54533(8)$ & $0.30178(7)$ & $0.0156(2)$ \\
\hline $\mathrm{C} 23$ & $0.13329(9)$ & $0.57966(8)$ & $0.77286(8)$ & $0.0155(2)$ \\
\hline $\mathrm{H} 23$ & 0.1041 & 0.6185 & 0.8091 & $0.019^{*}$ \\
\hline C19 & $0.25432(9)$ & $0.40357(8)$ & $0.60411(8)$ & $0.0145(2)$ \\
\hline $\mathrm{C} 20$ & $0.21932(9)$ & $0.46424(8)$ & $0.66664(8)$ & $0.0143(2)$ \\
\hline $\mathrm{C} 21$ & $0.22940(9)$ & $0.43597(8)$ & $0.75635(8)$ & $0.0150(2)$ \\
\hline $\mathrm{H} 21$ & 0.2656 & 0.3774 & 0.7816 & $0.018^{*}$ \\
\hline $\mathrm{C} 25$ & $0.16726(9)$ & $0.55153(8)$ & $0.63169(8)$ & $0.0148(2)$ \\
\hline $\mathrm{H} 25$ & 0.1619 & 0.5722 & 0.5715 & $0.018^{*}$ \\
\hline $\mathrm{C} 26$ & $0.06333(9)$ & $0.70046(8)$ & $0.64388(8)$ & $0.0144(2)$ \\
\hline C6 & $0.00264(9)$ & $0.81756(8)$ & $0.42053(8)$ & $0.0147(2)$ \\
\hline $\mathrm{C} 22$ & $0.18632(9)$ & $0.49367(8)$ & $0.80846(8)$ & $0.0166(2)$ \\
\hline $\mathrm{H} 22$ & 0.1932 & 0.4740 & 0.8693 & $0.020^{*}$ \\
\hline C14 & $0.38991(10)$ & $0.46387(9)$ & $0.37303(8)$ & $0.0188(2)$ \\
\hline H14 & 0.4287 & 0.4060 & 0.3964 & $0.023^{*}$ \\
\hline C18 & $0.22127(9)$ & $0.40670(8)$ & $0.50885(8)$ & $0.0158(2)$ \\
\hline $\mathrm{H} 18$ & 0.1726 & 0.4510 & 0.4691 & $0.019^{*}$ \\
\hline $\mathrm{C} 1$ & $0.12242(9)$ & $0.82960(8)$ & $0.45591(8)$ & $0.0173(2)$ \\
\hline H1A & 0.1437 & 0.8671 & 0.5143 & $0.021 *$ \\
\hline H1B & 0.1551 & 0.7640 & 0.4696 & $0.021^{*}$ \\
\hline $\mathrm{C} 27$ & $-0.03408(9)$ & $0.78893(8)$ & $0.50151(8)$ & $0.0173(2)$ \\
\hline $\mathrm{H} 27 \mathrm{~A}$ & -0.0219 & 0.8449 & 0.5447 & $0.021 *$ \\
\hline H27B & -0.1104 & 0.7762 & 0.4771 & $0.021 *$ \\
\hline C5 & $-0.05125(9)$ & $0.91634(8)$ & $0.38370(8)$ & $0.0167(2)$ \\
\hline $\mathrm{H} 5 \mathrm{~A}$ & -0.1266 & 0.9041 & 0.3502 & $0.020^{*}$ \\
\hline $\mathrm{H} 5 \mathrm{~B}$ & -0.0460 & 0.9591 & 0.4369 & $0.020^{*}$ \\
\hline C11 & $0.27573(10)$ & $0.63331(9)$ & $0.30684(8)$ & $0.0183(2)$ \\
\hline H11 & 0.2371 & 0.6916 & 0.2847 & $0.022^{*}$ \\
\hline C7 & $-0.03579(9)$ & $0.74369(8)$ & $0.33905(8)$ & $0.0161(2)$ \\
\hline H7A & -0.1087 & 0.7252 & 0.3316 & $0.019^{*}$ \\
\hline H7B & -0.0379 & 0.7785 & 0.2822 & $0.019^{*}$ \\
\hline $\mathrm{C} 16$ & $0.26450(10)$ & $0.29811(8)$ & $0.38798(8)$ & $0.0175(2)$ \\
\hline H16A & 0.3340 & 0.2780 & 0.3867 & $0.021^{*}$ \\
\hline H16B & 0.2173 & 0.2407 & 0.3708 & $0.021 *$ \\
\hline C9 & $0.10396(9)$ & $0.53772(8)$ & $0.26424(8)$ & $0.0167(2)$ \\
\hline H9A & 0.0817 & 0.5171 & 0.1988 & $0.020^{*}$ \\
\hline H9B & 0.0816 & 0.4863 & 0.2988 & $0.020^{*}$ \\
\hline $\mathrm{C} 2$ & $0.16439(9)$ & $0.88174(9)$ & $0.38798(9)$ & $0.0199(3)$ \\
\hline $\mathrm{H} 2 \mathrm{~A}$ & 0.2413 & 0.8897 & 0.4161 & $0.024 *$ \\
\hline $\mathrm{H} 2 \mathrm{~B}$ & 0.1498 & 0.8415 & 0.3316 & $0.024 *$ \\
\hline C4 & $-0.00561(9)$ & $0.97100(8)$ & $0.31946(8)$ & $0.0187(2)$ \\
\hline $\mathrm{H} 4 \mathrm{~A}$ & -0.0227 & 0.9347 & 0.2606 & $0.022 *$ \\
\hline $\mathrm{H} 4 \mathrm{~B}$ & -0.0382 & 1.0366 & 0.3057 & $0.022 *$ \\
\hline C13 & $0.44113(10)$ & $0.55299(9)$ & $0.37770(8)$ & $0.0211(3)$ \\
\hline $\mathrm{H} 13$ & 0.5153 & 0.5559 & 0.4043 & $0.025^{*}$ \\
\hline $\mathrm{C} 3$ & $0.11299(10)$ & $0.98218(9)$ & $0.36248(8)$ & $0.0200(3)$ \\
\hline H3A & 0.1302 & 1.0235 & 0.4184 & $0.024 *$ \\
\hline H3B & 0.1405 & 1.0149 & 0.3182 & $0.024 *$ \\
\hline $\mathrm{C} 12$ & $0.38467(10)$ & $0.63756(9)$ & $0.34378(8)$ & $0.0209(3)$ \\
\hline $\mathrm{H} 12$ & 0.4200 & 0.6980 & 0.3457 & $0.025^{*}$ \\
\hline
\end{tabular}




\begin{tabular}{|l|l|l|l|l|}
\hline C2S & $0.38103(11)$ & $0.06888(10)$ & $0.48156(10)$ & $0.0287(3)$ \\
\hline D2SA & 0.3521 & 0.0841 & 0.4156 & $0.043^{*}$ \\
\hline D2SB & 0.3254 & 0.0731 & 0.5084 & $0.043^{*}$ \\
\hline D2SC & 0.4101 & 0.0025 & 0.4899 & $0.043^{*}$ \\
\hline C1S & $0.62365(10)$ & $0.28065(12)$ & $0.42857(10)$ & $0.0327(3)$ \\
\hline D1SA & 0.6202 & 0.3456 & 0.4006 & $0.049^{*}$ \\
\hline D1SB & 0.6714 & 0.2388 & 0.4097 & $0.049^{*}$ \\
\hline D1SC & 0.6494 & 0.2869 & 0.4959 & $0.049^{*}$ \\
\hline D2S & $0.4289(14)$ & $0.1889(14)$ & $0.5469(12)$ & $0.044(5)^{*}$ \\
\hline
\end{tabular}

Table S7. Atomic displacement parameters $\left(\AA^{2}\right)$

\begin{tabular}{|c|c|c|c|c|c|c|}
\hline & $U^{11}$ & $U^{22}$ & $U^{33}$ & $U^{12}$ & $U^{13}$ & $U^{23}$ \\
\hline $\mathrm{O} 2$ & $0.0203(4)$ & $0.0144(4)$ & $0.0146(4)$ & $-0.0004(3)$ & $0.0051(3)$ & $0.0012(3)$ \\
\hline $\mathrm{O} 1$ & $0.0286(5)$ & $0.0158(4)$ & $0.0148(4)$ & 0.0039 (3) & $0.0072(3)$ & $0.0014(3)$ \\
\hline $\mathrm{O} 3$ & $0.0266(5)$ & $0.0149(4)$ & $0.0167(4)$ & $0.0031(3)$ & $0.0055(3)$ & $-0.0034(3)$ \\
\hline $\mathrm{O} 1 \mathrm{~S}$ & $0.0227(5)$ & $0.0285(5)$ & $0.0191(4)$ & $-0.0032(4)$ & $0.0056(4)$ & $-0.0029(4)$ \\
\hline $\mathrm{O} 2 \mathrm{~S}$ & $0.0248(5)$ & $0.0192(4)$ & $0.0285(5)$ & $0.0029(4)$ & $0.0109(4)$ & $-0.0025(4)$ \\
\hline N3 & $0.0197(5)$ & $0.0135(5)$ & $0.0128(5)$ & $0.0017(4)$ & $0.0063(4)$ & $0.0001(4)$ \\
\hline $\mathrm{N} 4$ & $0.0214(5)$ & $0.0128(5)$ & $0.0142(5)$ & $0.0029(4)$ & $0.0061(4)$ & $-0.0001(4)$ \\
\hline $\mathrm{N} 1$ & $0.0186(5)$ & $0.0162(5)$ & $0.0144(5)$ & $0.0030(4)$ & $0.0053(4)$ & $0.0024(4)$ \\
\hline $\mathrm{N} 2$ & $0.0200(5)$ & $0.0140(5)$ & $0.0154(5)$ & 0.0007 (4) & $0.0079(4)$ & $-0.0010(4)$ \\
\hline $\mathrm{C} 15$ & $0.0203(6)$ & $0.0167(6)$ & $0.0117(5)$ & $-0.0011(5)$ & $0.0080(5)$ & $-0.0010(4)$ \\
\hline $\mathrm{C} 24$ & $0.0141(5)$ & $0.0128(5)$ & $0.0149(5)$ & $-0.0033(4)$ & $0.0039(4)$ & $-0.0017(4)$ \\
\hline $\mathrm{C} 17$ & $0.0185(6)$ & $0.0128(5)$ & $0.0153(6)$ & $-0.0014(4)$ & $0.0070(5)$ & $0.0008(4)$ \\
\hline $\mathrm{C} 8$ & $0.0154(6)$ & $0.0148(5)$ & $0.0148(5)$ & $-0.0023(4)$ & $0.0027(4)$ & $-0.0014(4)$ \\
\hline $\mathrm{C} 10$ & $0.0187(6)$ & $0.0184(6)$ & $0.0110(5)$ & $0.0006(5)$ & $0.0066(4)$ & $-0.0007(4)$ \\
\hline $\mathrm{C} 23$ & $0.0167(6)$ & $0.0151(5)$ & $0.0152(5)$ & $-0.0023(4)$ & $0.0060(4)$ & $-0.0037(4)$ \\
\hline C19 & $0.0161(6)$ & $0.0116(5)$ & $0.0166(5)$ & $-0.0012(4)$ & $0.0067(4)$ & $0.0002(4)$ \\
\hline $\mathrm{C} 20$ & $0.0140(5)$ & $0.0137(5)$ & $0.0152(5)$ & $-0.0029(4)$ & $0.0048(4)$ & $-0.0018(4)$ \\
\hline $\mathrm{C} 21$ & $0.0157(6)$ & $0.0127(5)$ & $0.0153(5)$ & $-0.0009(4)$ & $0.0036(4)$ & $0.0003(4)$ \\
\hline $\mathrm{C} 25$ & $0.0169(6)$ & $0.0145(5)$ & $0.0129(5)$ & $-0.0020(4)$ & $0.0049(4)$ & $0.0000(4)$ \\
\hline $\mathrm{C} 26$ & $0.0143(5)$ & $0.0137(5)$ & $0.0163(6)$ & $-0.0024(4)$ & $0.0066(4)$ & $-0.0011(4)$ \\
\hline C6 & $0.0156(6)$ & $0.0128(5)$ & $0.0147(5)$ & $0.0010(4)$ & $0.0039(4)$ & $0.0001(4)$ \\
\hline $\mathrm{C} 22$ & $0.0199(6)$ & $0.0170(6)$ & $0.0125(5)$ & $-0.0027(5)$ & $0.0052(5)$ & $-0.0001(4)$ \\
\hline C14 & $0.0198(6)$ & $0.0218(6)$ & $0.0156(6)$ & $0.0031(5)$ & $0.0070(5)$ & $0.0014(5)$ \\
\hline $\mathrm{C} 18$ & $0.0184(6)$ & $0.0140(5)$ & $0.0153(5)$ & $0.0012(4)$ & $0.0062(5)$ & $0.0011(4)$ \\
\hline $\mathrm{C} 1$ & $0.0159(6)$ & $0.0153(5)$ & $0.0183(6)$ & $0.0009(4)$ & $0.0028(5)$ & $-0.0006(4)$ \\
\hline $\mathrm{C} 27$ & $0.0191(6)$ & $0.0153(6)$ & $0.0174(6)$ & $0.0035(5)$ & $0.0063(5)$ & $0.0019(5)$ \\
\hline $\mathrm{C} 5$ & $0.0166(6)$ & $0.0144(6)$ & $0.0176(6)$ & $0.0020(5)$ & $0.0040(5)$ & $-0.0001(5)$ \\
\hline C11 & $0.0236(6)$ & $0.0170(6)$ & $0.0161(6)$ & $0.0008(5)$ & $0.0090(5)$ & $0.0001(4)$ \\
\hline $\mathrm{C} 7$ & $0.0164(6)$ & $0.0152(6)$ & $0.0149(5)$ & $0.0008(5)$ & $0.0032(4)$ & $0.0004(4)$ \\
\hline $\mathrm{C} 16$ & $0.0237(6)$ & $0.0135(5)$ & $0.0161(6)$ & $0.0024(5)$ & $0.0080(5)$ & $0.0012(4)$ \\
\hline C9 & $0.0189(6)$ & $0.0167(6)$ & $0.0145(5)$ & $0.0012(5)$ & $0.0059(5)$ & $-0.0007(4)$ \\
\hline $\mathrm{C} 2$ & $0.0168(6)$ & $0.0185(6)$ & $0.0246(6)$ & $-0.0007(5)$ & $0.0073(5)$ & $-0.0010(5)$ \\
\hline $\mathrm{C} 4$ & $0.0220(6)$ & $0.0139(5)$ & $0.0190(6)$ & $0.0019(5)$ & $0.0055(5)$ & $0.0017(5)$ \\
\hline $\mathrm{C} 13$ & $0.0170(6)$ & $0.0283(7)$ & $0.0182(6)$ & $-0.0026(5)$ & $0.0064(5)$ & $-0.0023(5)$ \\
\hline $\mathrm{C} 3$ & $0.0220(6)$ & $0.0164(6)$ & $0.0224(6)$ & $-0.0019(5)$ & $0.0088(5)$ & $0.0007(5)$ \\
\hline C12 & $0.0239(6)$ & $0.0206(6)$ & $0.0206(6)$ & $-0.0054(5)$ & $0.0104(5)$ & $-0.0030(5)$ \\
\hline $\mathrm{C} 2 \mathrm{~S}$ & $0.0357(8)$ & $0.0197(6)$ & $0.0353(7)$ & $-0.0035(6)$ & $0.0183(6)$ & $-0.0048(6)$ \\
\hline $\mathrm{C} 1 \mathrm{~S}$ & $0.0194(7)$ & $0.0506(9)$ & $0.0251(7)$ & $-0.0024(6)$ & $0.0037(5)$ & $-0.0003(6)$ \\
\hline
\end{tabular}

Table S8. Geometric parameters $\left(\AA,{ }^{\circ}\right)$ 


\begin{tabular}{|c|c|c|c|}
\hline $\mathrm{O} 2-\mathrm{C} 15$ & $1.3817(14)$ & C6-C5 & $1.5470(15)$ \\
\hline $\mathrm{O} 2-\mathrm{C} 16$ & $1.4316(14)$ & C6-C7 & $1.5561(15)$ \\
\hline $\mathrm{O} 1-\mathrm{C} 8$ & $1.2391(14)$ & $\mathrm{C} 22-\mathrm{H} 22$ & 0.9500 \\
\hline $\mathrm{O} 3-\mathrm{C} 26$ & $1.2431(14)$ & $\mathrm{C} 14-\mathrm{H} 14$ & 0.9500 \\
\hline $\mathrm{O} 1 \mathrm{~S}-\mathrm{D} 1 \mathrm{~S}$ & $0.911(18)$ & $\mathrm{C} 14-\mathrm{C} 13$ & $1.3911(17)$ \\
\hline $\mathrm{O} 1 \mathrm{~S}-\mathrm{C} 1 \mathrm{~S}$ & $1.4166(16)$ & C18-H18 & 0.9500 \\
\hline $\mathrm{O} 2 \mathrm{~S}-\mathrm{C} 2 \mathrm{~S}$ & $1.4274(16)$ & $\mathrm{C} 1-\mathrm{H} 1 \mathrm{~A}$ & 0.9900 \\
\hline $\mathrm{O} 2 \mathrm{~S}-\mathrm{D} 2 \mathrm{~S}$ & $0.945(19)$ & $\mathrm{C} 1-\mathrm{H} 1 \mathrm{~B}$ & 0.9900 \\
\hline N3-H3 & $0.916(15)$ & $\mathrm{C} 1-\mathrm{C} 2$ & $1.5291(16)$ \\
\hline $\mathrm{N} 3-\mathrm{N} 2$ & $1.3584(13)$ & $\mathrm{C} 27-\mathrm{H} 27 \mathrm{~A}$ & 0.9900 \\
\hline $\mathrm{N} 3-\mathrm{C} 19$ & $1.3567(15)$ & $\mathrm{C} 27-\mathrm{H} 27 \mathrm{~B}$ & 0.9900 \\
\hline $\mathrm{N} 4-\mathrm{H} 4$ & $0.895(15)$ & C5-H5A & 0.9900 \\
\hline $\mathrm{N} 4-\mathrm{C} 26$ & $1.3350(15)$ & C5-H5B & 0.9900 \\
\hline $\mathrm{N} 4-\mathrm{C} 27$ & $1.4567(14)$ & $\mathrm{C} 5-\mathrm{C} 4$ & $1.5307(16)$ \\
\hline $\mathrm{N} 1-\mathrm{H} 1$ & $0.881(15)$ & C11-H11 & 0.9500 \\
\hline $\mathrm{N} 1-\mathrm{C} 8$ & $1.3380(15)$ & $\mathrm{C} 11-\mathrm{C} 12$ & $1.3929(18)$ \\
\hline $\mathrm{N} 1-\mathrm{C} 9$ & $1.4544(15)$ & C7-H7A & 0.9900 \\
\hline $\mathrm{N} 2-\mathrm{C} 17$ & $1.3386(15)$ & C7-H7B & 0.9900 \\
\hline $\mathrm{C} 15-\mathrm{C} 10$ & $1.3994(16)$ & $\mathrm{C} 16-\mathrm{H} 16 \mathrm{~A}$ & 0.9900 \\
\hline $\mathrm{C} 15-\mathrm{C} 14$ & $1.3909(17)$ & C16-H16B & 0.9900 \\
\hline $\mathrm{C} 24-\mathrm{C} 23$ & $1.3968(16)$ & C9-H9A & 0.9900 \\
\hline $\mathrm{C} 24-\mathrm{C} 25$ & $1.3895(16)$ & C9-H9B & 0.9900 \\
\hline $\mathrm{C} 24-\mathrm{C} 26$ & $1.5043(15)$ & $\mathrm{C} 2-\mathrm{H} 2 \mathrm{~A}$ & 0.9900 \\
\hline $\mathrm{C} 17-\mathrm{C} 18$ & $1.3970(16)$ & $\mathrm{C} 2-\mathrm{H} 2 \mathrm{~B}$ & 0.9900 \\
\hline $\mathrm{C} 17-\mathrm{C} 16$ & $1.5010(15)$ & $\mathrm{C} 2-\mathrm{C} 3$ & $1.5280(16)$ \\
\hline $\mathrm{C} 8-\mathrm{C} 7$ & $1.5168(16)$ & $\mathrm{C} 4-\mathrm{H} 4 \mathrm{~A}$ & 0.9900 \\
\hline $\mathrm{C} 10-\mathrm{C} 11$ & $1.3904(17)$ & C4-H4B & 0.9900 \\
\hline $\mathrm{C} 10-\mathrm{C} 9$ & $1.5163(16)$ & $\mathrm{C} 4-\mathrm{C} 3$ & $1.5254(17)$ \\
\hline $\mathrm{C} 23-\mathrm{H} 23$ & 0.9500 & C13-H13 & 0.9500 \\
\hline $\mathrm{C} 23-\mathrm{C} 22$ & $1.3886(16)$ & $\mathrm{C} 13-\mathrm{C} 12$ & $1.3859(18)$ \\
\hline $\mathrm{C} 19-\mathrm{C} 20$ & $1.4684(15)$ & $\mathrm{C} 3-\mathrm{H} 3 \mathrm{~A}$ & 0.9900 \\
\hline $\mathrm{C} 19-\mathrm{C} 18$ & $1.3830(16)$ & $\mathrm{C} 3-\mathrm{H} 3 \mathrm{~B}$ & 0.9900 \\
\hline $\mathrm{C} 20-\mathrm{C} 21$ & $1.3979(16)$ & $\mathrm{C} 12-\mathrm{H} 12$ & 0.9500 \\
\hline $\mathrm{C} 20-\mathrm{C} 25$ & $1.3966(16)$ & $\mathrm{C} 2 \mathrm{~S}-\mathrm{D} 2 \mathrm{SA}$ & 0.9800 \\
\hline $\mathrm{C} 21-\mathrm{H} 21$ & 0.9500 & $\mathrm{C} 2 \mathrm{~S}-\mathrm{D} 2 \mathrm{SB}$ & 0.9800 \\
\hline $\mathrm{C} 21-\mathrm{C} 22$ & $1.3894(16)$ & $\mathrm{C} 2 \mathrm{~S}-\mathrm{D} 2 \mathrm{SC}$ & 0.9800 \\
\hline $\mathrm{C} 25-\mathrm{H} 25$ & 0.9500 & C1S-D1SA & 0.9800 \\
\hline $\mathrm{C} 6-\mathrm{C} 1$ & $1.5374(16)$ & $\mathrm{C} 1 \mathrm{~S}-\mathrm{D} 1 \mathrm{SB}$ & 0.9800 \\
\hline $\mathrm{C} 6-\mathrm{C} 27$ & $1.5453(15)$ & $\mathrm{C} 1 \mathrm{~S}-\mathrm{D} 1 \mathrm{SC}$ & 0.9800 \\
\hline $\mathrm{C} 15-\mathrm{O} 2-\mathrm{C} 16$ & $117.54(9)$ & N4-C27-H27A & 108.8 \\
\hline $\mathrm{C} 1 \mathrm{~S}-\mathrm{O} 1 \mathrm{~S}-\mathrm{D} 1 \mathrm{~S}$ & $110.6(10)$ & $\mathrm{N} 4-\mathrm{C} 27-\mathrm{H} 27 \mathrm{~B}$ & 108.8 \\
\hline $\mathrm{C} 2 \mathrm{~S}-\mathrm{O} 2 \mathrm{~S}-\mathrm{D} 2 \mathrm{~S}$ & $106.0(11)$ & $\mathrm{C} 6-\mathrm{C} 27-\mathrm{H} 27 \mathrm{~A}$ & 108.8 \\
\hline $\mathrm{N} 2-\mathrm{N} 3-\mathrm{H} 3$ & $119.4(9)$ & $\mathrm{C} 6-\mathrm{C} 27-\mathrm{H} 27 \mathrm{~B}$ & 108.8 \\
\hline $\mathrm{C} 19-\mathrm{N} 3-\mathrm{H} 3$ & $128.9(9)$ & $\mathrm{H} 27 \mathrm{~A}-\mathrm{C} 27-\mathrm{H} 27 \mathrm{~B}$ & 107.7 \\
\hline $\mathrm{C} 19-\mathrm{N} 3-\mathrm{N} 2$ & $111.69(9)$ & $\mathrm{C} 6-\mathrm{C} 5-\mathrm{H} 5 \mathrm{~A}$ & 108.6 \\
\hline $\mathrm{C} 26-\mathrm{N} 4-\mathrm{H} 4$ & $119.2(9)$ & C6- $55-\mathrm{H} 5 \mathrm{~B}$ & 108.6 \\
\hline $\mathrm{C} 26-\mathrm{N} 4-\mathrm{C} 27$ & $123.45(10)$ & $\mathrm{H} 5 \mathrm{~A}-\mathrm{C} 5-\mathrm{H} 5 \mathrm{~B}$ & 107.6 \\
\hline $\mathrm{C} 27-\mathrm{N} 4-\mathrm{H} 4$ & $117.1(9)$ & $\mathrm{C} 4-\mathrm{C} 5-\mathrm{C} 6$ & $114.68(9)$ \\
\hline $\mathrm{C} 8-\mathrm{N} 1-\mathrm{H} 1$ & $119.3(9)$ & $\mathrm{C} 4-\mathrm{C} 5-\mathrm{H} 5 \mathrm{~A}$ & 108.6 \\
\hline $\mathrm{C} 8-\mathrm{N} 1-\mathrm{C} 9$ & $121.07(10)$ & $\mathrm{C} 4-\mathrm{C} 5-\mathrm{H} 5 \mathrm{~B}$ & 108.6 \\
\hline $\mathrm{C} 9-\mathrm{N} 1-\mathrm{H} 1$ & $119.4(9)$ & $\mathrm{C} 10-\mathrm{C} 11-\mathrm{H} 11$ & 119.4 \\
\hline
\end{tabular}




\begin{tabular}{|c|c|c|c|}
\hline $\mathrm{C} 17-\mathrm{N} 2-\mathrm{N} 3$ & $105.09(9)$ & $\mathrm{C} 10-\mathrm{C} 11-\mathrm{C} 12$ & $121.21(11)$ \\
\hline $\mathrm{O} 2-\mathrm{C} 15-\mathrm{C} 10$ & $115.06(10)$ & $\mathrm{C} 12-\mathrm{C} 11-\mathrm{H} 11$ & 119.4 \\
\hline $\mathrm{O} 2-\mathrm{C} 15-\mathrm{C} 14$ & $123.78(10)$ & $\mathrm{C} 8-\mathrm{C} 7-\mathrm{C} 6$ & $118.12(9)$ \\
\hline $\mathrm{C} 14-\mathrm{C} 15-\mathrm{C} 10$ & $121.15(11)$ & $\mathrm{C} 8-\mathrm{C} 7-\mathrm{H} 7 \mathrm{~A}$ & 107.8 \\
\hline $\mathrm{C} 23-\mathrm{C} 24-\mathrm{C} 26$ & $119.62(10)$ & $\mathrm{C} 8-\mathrm{C} 7-\mathrm{H} 7 \mathrm{~B}$ & 107.8 \\
\hline $\mathrm{C} 25-\mathrm{C} 24-\mathrm{C} 23$ & $119.44(10)$ & C6- $-\mathrm{C} 7-\mathrm{H} 7 \mathrm{~A}$ & 107.8 \\
\hline $\mathrm{C} 25-\mathrm{C} 24-\mathrm{C} 26$ & $120.93(10)$ & C6- $-\mathrm{C} 7-\mathrm{H} 7 \mathrm{~B}$ & 107.8 \\
\hline $\mathrm{N} 2-\mathrm{C} 17-\mathrm{C} 18$ & $111.25(10)$ & $\mathrm{H} 7 \mathrm{~A}-\mathrm{C} 7-\mathrm{H} 7 \mathrm{~B}$ & 107.1 \\
\hline $\mathrm{N} 2-\mathrm{C} 17-\mathrm{C} 16$ & $121.01(10)$ & $\mathrm{O} 2-\mathrm{C} 16-\mathrm{C} 17$ & $111.07(9)$ \\
\hline $\mathrm{C} 18-\mathrm{C} 17-\mathrm{C} 16$ & $127.68(10)$ & $\mathrm{O} 2-\mathrm{C} 16-\mathrm{H} 16 \mathrm{~A}$ & 109.4 \\
\hline $\mathrm{O} 1-\mathrm{C} 8-\mathrm{N} 1$ & $121.74(11)$ & $\mathrm{O} 2-\mathrm{C} 16-\mathrm{H} 16 \mathrm{~B}$ & 109.4 \\
\hline $\mathrm{O} 1-\mathrm{C} 8-\mathrm{C} 7$ & $122.81(10)$ & $\mathrm{C} 17-\mathrm{C} 16-\mathrm{H} 16 \mathrm{~A}$ & 109.4 \\
\hline $\mathrm{N} 1-\mathrm{C} 8-\mathrm{C} 7$ & $115.43(10)$ & $\mathrm{C} 17-\mathrm{C} 16-\mathrm{H} 16 \mathrm{~B}$ & 109.4 \\
\hline $\mathrm{C} 15-\mathrm{C} 10-\mathrm{C} 9$ & $118.76(10)$ & $\mathrm{H} 16 \mathrm{~A}-\mathrm{C} 16-\mathrm{H} 16 \mathrm{~B}$ & 108.0 \\
\hline $\mathrm{C} 11-\mathrm{C} 10-\mathrm{C} 15$ & $118.26(11)$ & $\mathrm{N} 1-\mathrm{C} 9-\mathrm{C} 10$ & $113.41(9)$ \\
\hline $\mathrm{C} 11-\mathrm{C} 10-\mathrm{C} 9$ & $122.98(10)$ & $\mathrm{N} 1-\mathrm{C} 9-\mathrm{H} 9 \mathrm{~A}$ & 108.9 \\
\hline $\mathrm{C} 24-\mathrm{C} 23-\mathrm{H} 23$ & 120.2 & N1-C9-H9B & 108.9 \\
\hline $\mathrm{C} 22-\mathrm{C} 23-\mathrm{C} 24$ & $119.58(10)$ & $\mathrm{C} 10-\mathrm{C} 9-\mathrm{H} 9 \mathrm{~A}$ & 108.9 \\
\hline $\mathrm{C} 22-\mathrm{C} 23-\mathrm{H} 23$ & 120.2 & $\mathrm{C} 10-\mathrm{C} 9-\mathrm{H} 9 \mathrm{~B}$ & 108.9 \\
\hline $\mathrm{N} 3-\mathrm{C} 19-\mathrm{C} 20$ & $124.83(10)$ & H9A-C9-H9B & 107.7 \\
\hline $\mathrm{N} 3-\mathrm{C} 19-\mathrm{C} 18$ & $106.74(10)$ & $\mathrm{C} 1-\mathrm{C} 2-\mathrm{H} 2 \mathrm{~A}$ & 109.6 \\
\hline $\mathrm{C} 18-\mathrm{C} 19-\mathrm{C} 20$ & $128.35(10)$ & $\mathrm{C} 1-\mathrm{C} 2-\mathrm{H} 2 \mathrm{~B}$ & 109.6 \\
\hline $\mathrm{C} 21-\mathrm{C} 20-\mathrm{C} 19$ & $123.41(10)$ & $\mathrm{H} 2 \mathrm{~A}-\mathrm{C} 2-\mathrm{H} 2 \mathrm{~B}$ & 108.1 \\
\hline $\mathrm{C} 25-\mathrm{C} 20-\mathrm{C} 19$ & $117.55(10)$ & $\mathrm{C} 3-\mathrm{C} 2-\mathrm{C} 1$ & $110.48(10)$ \\
\hline $\mathrm{C} 25-\mathrm{C} 20-\mathrm{C} 21$ & $118.91(10)$ & $\mathrm{C} 3-\mathrm{C} 2-\mathrm{H} 2 \mathrm{~A}$ & 109.6 \\
\hline $\mathrm{C} 20-\mathrm{C} 21-\mathrm{H} 21$ & 120.1 & $\mathrm{C} 3-\mathrm{C} 2-\mathrm{H} 2 \mathrm{~B}$ & 109.6 \\
\hline $\mathrm{C} 22-\mathrm{C} 21-\mathrm{C} 20$ & $119.88(11)$ & $\mathrm{C} 5-\mathrm{C} 4-\mathrm{H} 4 \mathrm{~A}$ & 109.3 \\
\hline $\mathrm{C} 22-\mathrm{C} 21-\mathrm{H} 21$ & 120.1 & $\mathrm{C} 5-\mathrm{C} 4-\mathrm{H} 4 \mathrm{~B}$ & 109.3 \\
\hline $\mathrm{C} 24-\mathrm{C} 25-\mathrm{C} 20$ & $121.21(10)$ & $\mathrm{H} 4 \mathrm{~A}-\mathrm{C} 4-\mathrm{H} 4 \mathrm{~B}$ & 108.0 \\
\hline $\mathrm{C} 24-\mathrm{C} 25-\mathrm{H} 25$ & 119.4 & $\mathrm{C} 3-\mathrm{C} 4-\mathrm{C} 5$ & $111.58(10)$ \\
\hline $\mathrm{C} 20-\mathrm{C} 25-\mathrm{H} 25$ & 119.4 & $\mathrm{C} 3-\mathrm{C} 4-\mathrm{H} 4 \mathrm{~A}$ & 109.3 \\
\hline $\mathrm{O} 3-\mathrm{C} 26-\mathrm{N} 4$ & $122.86(10)$ & $\mathrm{C} 3-\mathrm{C} 4-\mathrm{H} 4 \mathrm{~B}$ & 109.3 \\
\hline $\mathrm{O} 3-\mathrm{C} 26-\mathrm{C} 24$ & $121.23(10)$ & $\mathrm{C} 14-\mathrm{C} 13-\mathrm{H} 13$ & 119.8 \\
\hline $\mathrm{N} 4-\mathrm{C} 26-\mathrm{C} 24$ & $115.91(10)$ & $\mathrm{C} 12-\mathrm{C} 13-\mathrm{C} 14$ & $120.46(11)$ \\
\hline $\mathrm{C} 1-\mathrm{C} 6-\mathrm{C} 27$ & $109.53(9)$ & $\mathrm{C} 12-\mathrm{C} 13-\mathrm{H} 13$ & 119.8 \\
\hline $\mathrm{C} 1-\mathrm{C} 6-\mathrm{C} 5$ & $110.33(9)$ & $\mathrm{C} 2-\mathrm{C} 3-\mathrm{H} 3 \mathrm{~A}$ & 109.6 \\
\hline $\mathrm{C} 1-\mathrm{C} 6-\mathrm{C} 7$ & $112.22(9)$ & $\mathrm{C} 2-\mathrm{C} 3-\mathrm{H} 3 \mathrm{~B}$ & 109.6 \\
\hline $\mathrm{C} 27-\mathrm{C} 6-\mathrm{C} 5$ & $105.95(9)$ & $\mathrm{C} 4-\mathrm{C} 3-\mathrm{C} 2$ & $110.10(10)$ \\
\hline $\mathrm{C} 27-\mathrm{C} 6-\mathrm{C} 7$ & $112.18(9)$ & $\mathrm{C} 4-\mathrm{C} 3-\mathrm{H} 3 \mathrm{~A}$ & 109.6 \\
\hline $\mathrm{C} 5-\mathrm{C} 6-\mathrm{C} 7$ & $106.40(9)$ & $\mathrm{C} 4-\mathrm{C} 3-\mathrm{H} 3 \mathrm{~B}$ & 109.6 \\
\hline $\mathrm{C} 23-\mathrm{C} 22-\mathrm{C} 21$ & $120.96(10)$ & $\mathrm{H} 3 \mathrm{~A}-\mathrm{C} 3-\mathrm{H} 3 \mathrm{~B}$ & 108.2 \\
\hline $\mathrm{C} 23-\mathrm{C} 22-\mathrm{H} 22$ & 119.5 & $\mathrm{C} 11-\mathrm{C} 12-\mathrm{H} 12$ & 120.2 \\
\hline $\mathrm{C} 21-\mathrm{C} 22-\mathrm{H} 22$ & 119.5 & $\mathrm{C} 13-\mathrm{C} 12-\mathrm{C} 11$ & $119.53(11)$ \\
\hline C15-C14-H14 & 120.3 & $\mathrm{C} 13-\mathrm{C} 12-\mathrm{H} 12$ & 120.2 \\
\hline $\mathrm{C} 13-\mathrm{C} 14-\mathrm{C} 15$ & $119.33(11)$ & $\mathrm{O} 2 \mathrm{~S}-\mathrm{C} 2 \mathrm{~S}-\mathrm{D} 2 \mathrm{SA}$ & 109.5 \\
\hline $\mathrm{C} 13-\mathrm{C} 14-\mathrm{H} 14$ & 120.3 & $\mathrm{O} 2 \mathrm{~S}-\mathrm{C} 2 \mathrm{~S}-\mathrm{D} 2 \mathrm{SB}$ & 109.5 \\
\hline $\mathrm{C} 17-\mathrm{C} 18-\mathrm{H} 18$ & 127.4 & $\mathrm{O} 2 \mathrm{~S}-\mathrm{C} 2 \mathrm{~S}-\mathrm{D} 2 \mathrm{SC}$ & 109.5 \\
\hline $\mathrm{C} 19-\mathrm{C} 18-\mathrm{C} 17$ & $105.21(10)$ & $\mathrm{D} 2 \mathrm{SA}-\mathrm{C} 2 \mathrm{~S}-\mathrm{D} 2 \mathrm{SB}$ & 109.5 \\
\hline $\mathrm{C} 19-\mathrm{C} 18-\mathrm{H} 18$ & 127.4 & $\mathrm{D} 2 \mathrm{SA}-\mathrm{C} 2 \mathrm{~S}-\mathrm{D} 2 \mathrm{SC}$ & 109.5 \\
\hline
\end{tabular}




\begin{tabular}{|l|l|l|l|}
\hline $\mathrm{C} 6-\mathrm{C} 1-\mathrm{H} 1 \mathrm{~A}$ & 108.8 & $\mathrm{D} 2 \mathrm{SB}-\mathrm{C} 2 \mathrm{~S}-\mathrm{D} 2 \mathrm{SC}$ & 109.5 \\
\hline $\mathrm{C} 6-\mathrm{C} 1-\mathrm{H} 1 \mathrm{~B}$ & 108.8 & $\mathrm{O} 1 \mathrm{~S}-\mathrm{C} 1 \mathrm{~S}-\mathrm{D} 1 \mathrm{SA}$ & 109.5 \\
\hline $\mathrm{H} 1 \mathrm{~A}-\mathrm{C} 1-\mathrm{H} 1 \mathrm{~B}$ & 107.7 & $\mathrm{O} 1 \mathrm{~S}-\mathrm{C} 1 \mathrm{~S}-\mathrm{D} 1 \mathrm{SB}$ & 109.5 \\
\hline $\mathrm{C} 2-\mathrm{C} 1-\mathrm{C} 6$ & $113.78(9)$ & $\mathrm{O} 1 \mathrm{~S}-\mathrm{C} 1 \mathrm{~S}-\mathrm{D} 1 \mathrm{SC}$ & 109.5 \\
\hline $\mathrm{C} 2-\mathrm{C} 1-\mathrm{H} 1 \mathrm{~A}$ & 108.8 & D1SA-C1S-D1SB & 109.5 \\
\hline $\mathrm{C} 2-\mathrm{C} 1-\mathrm{H} 1 \mathrm{~B}$ & 108.8 & D1SA-C1S-D1SC & 109.5 \\
\hline $\mathrm{N} 4-\mathrm{C} 27-\mathrm{C} 6$ & $113.74(9)$ & $\mathrm{D} 1 \mathrm{SB}-\mathrm{C} 1 \mathrm{~S}-\mathrm{D} 1 \mathrm{SC}$ & 109.5 \\
\hline
\end{tabular}

\section{Computational Methods \& Data}

All DFT-optimized structures to aid interpretation of nOe data were obtained using Maestro software (Version 10.5 Schrodinger, LLC) according to the following workflow: First, conformers were generated using a Macromodel conformational search (force field: OPLS3, mixed torsional/low-mode sampling, $5 \mathrm{kcal} / \mathrm{mol} \mathrm{MM}$ energy window, no solvent). Next, all unique conformers within the $5 \mathrm{kcal} / \mathrm{mol}$ energy window were subjected to gas-phase DFT optimization (B3LYP/6-31 G**++) using Jaguar. For each macrocycle, we depict the lowestenergy optimized conformation that is consistent with the observed nOe interactions. In the case of compound 51, single point energy calculations with vibrational frequencies computed were performed to directly compare the gas phase energies of the two product isomers; otherwise vibrational frequency calculations were not included in the DFT optimizations.

Computational data for DFT-optimized 11

\begin{tabular}{|c|c|c|c|}
\hline \multicolumn{4}{|c|}{$\begin{array}{l}\text { Number of imagi } \\
\text { Total energy: } \\
\text { Final geometry: }\end{array}$} \\
\hline atom & $\mathrm{x}$ & $\mathrm{Y}$ & z \\
\hline $\mathrm{C} 1$ & -4.9940364019 & 8.3057320765 & 0.4271539075 \\
\hline $\mathrm{C} 2$ & -4.3507739162 & 8.2449047468 & -0.9820222126 \\
\hline C3 & -6.9706541180 & 6.9974165370 & -0.4746189263 \\
\hline $\mathrm{C} 4$ & -6.5452670279 & 8.2092458358 & 0.2349028649 \\
\hline N5 & -2.9026962826 & 8.0516054110 & -0.9875638129 \\
\hline C6 & -2.0860410387 & 8.6792115990 & -1.8889955948 \\
\hline 07 & -2.4737096915 & 9.6005647777 & -2.6083014529 \\
\hline $\mathrm{C} 8$ & -0.6452386338 & 8.1707486390 & -1.9302221631 \\
\hline $\mathrm{C} 9$ & -0.2138880596 & 7.4721957406 & -3.2569410921 \\
\hline $\mathrm{C} 10$ & -1.1700634660 & 6.2942895623 & -3.5986986226 \\
\hline $\mathrm{N} 11$ & -1.2170861965 & 5.2619665135 & -2.5589272739 \\
\hline C12 & -2.2842170939 & 4.5604546663 & -2.0617737420 \\
\hline C13 & -3.5665958103 & 4.5305558774 & -2.8479918825 \\
\hline 014 & -2.1928915858 & 3.9639865673 & -0.9842649584 \\
\hline C15 & -3.6142021244 & 4.3702500558 & -4.2410945054 \\
\hline C16 & -4.8485891379 & 4.3072845502 & -4.8874868592 \\
\hline C17 & -6.0364883739 & 4.4403025072 & -4.1606313644 \\
\hline C18 & -6.0042762878 & 4.6018336307 & -2.7710439655 \\
\hline C19 & -4.7622247500 & 4.6138480001 & -2.1272455211 \\
\hline $\mathrm{C} 20$ & -7.1978370328 & 5.9957730582 & -1.1188559139 \\
\hline $\mathrm{C} 21$ & -7.2861911911 & 4.7955795700 & -1.9610189410 \\
\hline
\end{tabular}




$\begin{array}{lrrr}\text { C22 } & 1.2510376853 & 6.9868540421 & -3.0659751999 \\ \text { C23 } & 1.9161830012 & 6.4581044797 & -4.3474249795 \\ \text { C24 } & 1.8726229040 & 7.5041645274 & -5.4709907602 \\ \text { C25 } & 0.4361106823 & 7.9826374294 & -5.7248019295 \\ \text { C26 } & -0.2387995846 & 8.4860498055 & -4.4363046865 \\ \text { C27 } & -4.6671438657 & 9.6456564541 & 1.1075985390 \\ \text { C28 } & -4.5167279332 & 7.1451807445 & 1.3208445636 \\ \text { H29 } & -4.8147557793 & 7.4322189558 & -1.5503062817 \\ \text { H30 } & -4.5400717734 & 9.1749623717 & -1.5234189144 \\ \text { H31 } & -7.0218208604 & 8.2583319846 & 1.2224432655 \\ \text { H32 } & -6.8922034465 & 9.0913095829 & -0.3205866728 \\ \text { H33 } & -2.5449116600 & 7.2126303656 & -0.5517067655 \\ \text { H34 } & -0.0002615797 & 9.0442824678 & -1.7859538801 \\ \text { H35 } & -0.4587551472 & 7.4947053951 & -1.0871721435 \\ \text { H36 } & -0.8627792811 & 5.8506930276 & -4.5515099344 \\ \text { H37 } & -2.1794913590 & 6.6878040997 & -3.7436096971 \\ \text { H38 } & -0.3864668057 & 5.1307968325 & -1.9948286847 \\ \text { H39 } & -2.6985097945 & 4.2740329340 & -4.8151976887 \\ \text { H40 } & -4.8883107479 & 4.1678391829 & -5.9638194995 \\ \text { H41 } & -6.9907237182 & 4.4244617464 & -4.6811837126 \\ \text { H42 } & -4.7102255387 & 4.7049107150 & -1.0476329945 \\ \text { H43 } & -8.1426959366 & 4.8691024508 & -2.6413313074 \\ \text { H44 } & -7.4674989682 & 3.9123887651 & -1.3329266371 \\ \text { H45 } & 1.8364002855 & 7.8416247103 & -2.6994768260 \\ \text { H46 } & 1.3141386572 & 6.2314080159 & -2.2705578127 \\ \text { H47 } & 2.9536489855 & 6.1799405152 & -4.1257659530 \\ \text { H48 } & 1.4192327145 & 5.5372642422 & -4.6823545865 \\ \text { H49 } & 2.4937320547 & 8.3648541175 & -5.1830677893 \\ \text { H50 } & 2.3098386207 & 7.0953656452 & -6.3900697322 \\ \text { H51 } & 0.4326944306 & 8.7932770479 & -6.4635011460 \\ \text { H52 } & -0.1466365325 & 7.1665626592 & -6.1732169633 \\ \text { H53 } & -1.2671818512 & 8.7999266338 & -4.6346632361 \\ \text { H54 } & 0.2849050567 & 9.3918369814 & -4.1025161130 \\ \text { H55 } & -5.0521855948 & 10.4923896195 & 0.5281013922 \\ \text { H56 } & -5.1031981071 & 9.6941090925 & 2.1121610247 \\ \text { H57 } & -3.5841588452 & 9.7738014985 & 1.2051455291 \\ \text { H58 } & -3.4493786852 & 7.2312693221 & 1.5505441710 \\ \text { H59 } & -4.6918710979 & 6.1707896469 & 0.8554159541 \\ \text { H60 } & -5.0518779921 & 7.1541583381 & 2.2769696858 \\ & & & \end{array}$

\section{Computational data for DFT-optimized 17}

$\begin{array}{lccc}\begin{array}{l}\text { Number of imaginary frequencies: } \\ \text { Total energy: }\end{array} & \begin{array}{l}0 \\ \text { Final geometry: }\end{array} & & \\ \text { Fi303.40645066532 hartrees } & \\ \text { atom } & \mathrm{x} & \text { angstroms } & \mathrm{z} \\ \text { N1 } & -4.2351602992 & 2.3006139150 & 4.4888438271 \\ \text { C2 } & -5.3323734414 & 2.8573165465 & 3.7198096602 \\ \text { C3 } & -5.4843621679 & 2.2944460402 & 2.2671724104 \\ \text { C4 } & -4.4246346745 & 2.8875705998 & 1.2737542488 \\ \text { C5 } & -2.9642138116 & 2.4525995550 & 1.3921148192 \\ \text { N6 } & -2.3866404895 & 1.9459845482 & 0.2649055948 \\ \text { C7 } & -0.9695127344 & 1.5817670299 & 0.1916549012 \\ \text { C8 } & -0.6381101631 & 0.1003034948 & -0.2101406684 \\ \text { C9 } & 0.6719078859 & -0.3423142772 & 0.5390222872 \\ \text { C10 } & 0.4605955841 & -0.7127949460 & 1.9825043465\end{array}$




\begin{tabular}{|c|c|c|c|}
\hline 011 & -2.3171432073 & 2.6001498630 & 2.4387016475 \\
\hline C12 & -1.7914372027 & -0.8641018926 & 0.1195789281 \\
\hline C13 & -0.3623269954 & 0.0445081058 & -1.7271539678 \\
\hline C14 & -3.1685285148 & 0.6998706868 & 5.9642812841 \\
\hline C15 & -2.2261231563 & 0.3660712350 & 4.9937684123 \\
\hline C16 & -0.2841463386 & -0.7538794553 & 4.0920336240 \\
\hline C17 & -4.3991689552 & 1.4849935522 & 5.5714572334 \\
\hline 018 & -5.4695866311 & 1.3421964107 & 6.1685488291 \\
\hline N19 & 0.1086821233 & -1.9880420746 & 3.6615400660 \\
\hline $\mathrm{C} 20$ & -3.0102323262 & 0.1862087662 & 7.2592292062 \\
\hline $\mathrm{C} 21$ & -1.9102777374 & -0.6245812214 & 7.5525574086 \\
\hline C22 & -0.9687451250 & -0.9564685502 & 6.5681202262 \\
\hline C23 & -1.1256268868 & -0.4617656550 & 5.2663609270 \\
\hline C24 & -0.0184758683 & 0.1017449952 & 3.0337813031 \\
\hline N25 & 0.5480136583 & -1.9902645470 & 2.3787941149 \\
\hline C26 & -6.8856659634 & 2.7693354052 & 1.7760749098 \\
\hline C27 & -7.3830859068 & 2.1004894811 & 0.4833821404 \\
\hline C28 & -7.3443900076 & 0.5695919418 & 0.5944674769 \\
\hline C29 & -5.9439396025 & 0.0828772170 & 0.9929135217 \\
\hline C30 & -5.4590698668 & 0.7452900794 & 2.2936055871 \\
\hline H31 & -3.3167101854 & 2.4202583444 & 4.0736850249 \\
\hline H32 & -6.2440577630 & 2.6359615569 & 4.2798094824 \\
\hline H33 & -5.2328067207 & 3.9503255623 & 3.6701082275 \\
\hline H34 & -4.4157834599 & 3.9776924651 & 1.4063206940 \\
\hline H35 & -4.7637609236 & 2.7082990103 & 0.2491510101 \\
\hline H36 & -2.9501424755 & 1.8912454278 & -0.5709034240 \\
\hline H37 & -0.4707747055 & 2.2569205633 & -0.5158517557 \\
\hline H38 & -0.5765320202 & 1.8120204389 & 1.1777603071 \\
\hline H39 & 1.0852163557 & -1.2186851625 & 0.0286458662 \\
\hline H4 0 & 1.4160812668 & 0.4603300451 & 0.4458342061 \\
\hline H4 1 & -1.4728272081 & -1.8981988812 & -0.0470144595 \\
\hline H4 2 & -2.1124152059 & -0.7804923933 & 1.1604561129 \\
\hline H4 3 & -2.6616766876 & -0.6756076903 & -0.5181206636 \\
\hline H4 4 & -1.2159691784 & 0.4262385377 & -2.3018946366 \\
\hline H4 5 & 0.5150000207 & 0.6447842911 & -1.9961797195 \\
\hline H4 6 & -0.1788912434 & -0.9842602535 & -2.0560459151 \\
\hline H4 7 & -2.3550638257 & 0.7057928023 & 3.9755625665 \\
\hline H4 8 & 0.0185583056 & -2.8679556057 & 4.1460771943 \\
\hline H4 9 & -3.7551900898 & 0.4140608065 & 8.0146053883 \\
\hline H5O & -1.7826497627 & -1.0086069806 & 8.5604354218 \\
\hline H51 & -0.1180246645 & -1.5835436426 & 6.8218098528 \\
\hline H52 & -0.2201298141 & 1.1605687119 & 3.0320741717 \\
\hline H53 & -7.6100657324 & 2.5434159779 & 2.5697322949 \\
\hline H5 4 & -6.8862095039 & 3.8619975985 & 1.6624359030 \\
\hline H5 5 & -8.4044398982 & 2.4420833469 & 0.2758003399 \\
\hline H5 6 & -6.7798137842 & 2.4194968996 & -0.3779711214 \\
\hline H57 & -8.0677226598 & 0.2443801751 & 1.3559667456 \\
\hline H5 8 & -7.6561312197 & 0.1098378700 & -0.3514017828 \\
\hline H59 & -5.9432739768 & -1.0059147790 & 1.1232469462 \\
\hline H60 & -5.2398070405 & 0.2892828259 & 0.1743047958 \\
\hline H61 & -4.4529702292 & 0.3902110140 & 2.5410130621 \\
\hline H62 & -6.1055975016 & 0.4130783811 & 3.116929566 \\
\hline
\end{tabular}

\section{Computational data for DFT-optimized 20a}

Number of imaginary frequencies: 0

Total energy: $\quad-1342.74347736378$ hartrees 
Final geometry:

\begin{tabular}{|c|c|c|c|}
\hline atom & $x$ & y & z \\
\hline N1 & -3.2787798077 & -0.4835694185 & 0.3893826994 \\
\hline $\mathrm{C} 2$ & -3.5858366824 & 0.7622118121 & 1.0667637643 \\
\hline $\mathrm{C} 3$ & -3.1398349024 & 2.0580811568 & 0.3291481098 \\
\hline $\mathrm{C} 4$ & -3.9959415978 & 2.3072607372 & -0.9607120061 \\
\hline $\mathrm{C} 5$ & -3.7364037879 & 1.4143412417 & -2.1727718446 \\
\hline N6 & -3.0768641639 & 1.9967854241 & -3.2071445531 \\
\hline C7 & -2.7111431962 & 1.3005933454 & -4.4404422357 \\
\hline $\mathrm{C} 8$ & -4.3230350637 & -1.3336711301 & -6.7996084594 \\
\hline $\mathrm{Cg}$ & -4.1154118526 & -2.8611979466 & -6.7837611790 \\
\hline 010 & -4.1139715657 & 0.2338375417 & -2.2254550551 \\
\hline $\mathrm{C} 11$ & -2.1579120764 & -2.6185418424 & 0.0216323674 \\
\hline $\mathrm{C} 12$ & -2.4538112217 & -2.6489653799 & -1.3446942662 \\
\hline $\mathrm{C} 13$ & -2.4168075512 & -1.4105416030 & 0.8919347348 \\
\hline 014 & -1.8706010066 & -1.2961484306 & 1.9935634995 \\
\hline C15 & -1.5618149347 & -3.7383676717 & 0.6189097705 \\
\hline C16 & -1.2929906922 & -4.8739186647 & -0.1443477657 \\
\hline $\mathrm{C} 17$ & -1.6045810839 & -4.9056352248 & -1.5056015948 \\
\hline C18 & -2.1831824632 & -3.7856718399 & -2.1251617749 \\
\hline C19 & -3.6730143452 & 1.5846900950 & -5.6041732292 \\
\hline $\mathrm{C} 20$ & -3.2012423895 & 0.9943499544 & -6.9464044397 \\
\hline $\mathrm{C} 21$ & -3.0256815656 & -0.5352814403 & -6.9889696685 \\
\hline $\mathrm{C} 22$ & -3.4663951147 & -3.0026422169 & -4.2412049777 \\
\hline $\mathrm{C} 23$ & -2.5190724591 & -3.7520031230 & -3.5539727562 \\
\hline N24 & -1.8878048318 & -4.4929985610 & -4.5105145706 \\
\hline N25 & -2.3483957119 & -4.2604378011 & -5.7625906207 \\
\hline $\mathrm{C} 26$ & -3.3222511393 & -3.3576650700 & -5.6054304619 \\
\hline $\mathrm{C} 27$ & -1.6196678010 & 2.0018291740 & 0.0332482606 \\
\hline $\mathrm{C} 28$ & -1.0037109314 & 3.3509402112 & -0.3749947190 \\
\hline $\mathrm{C} 29$ & -1.2913081664 & 4.4440588410 & 0.6642061926 \\
\hline $\mathrm{C} 30$ & -2.7997930010 & 4.5763275514 & 0.9176788258 \\
\hline C31 & -3.4237338169 & 3.2284988617 & 1.3160981547 \\
\hline H32 & -3.7112628056 & -0.6173073703 & -0.5184723466 \\
\hline H33 & -4.6684043495 & 0.8165902685 & 1.2502233528 \\
\hline H34 & -3.0871001970 & 0.7164977949 & 2.0375960764 \\
\hline H35 & -5.0518117062 & 2.1703898550 & -0.6992970502 \\
\hline H36 & -3.8828475996 & 3.3523356347 & -1.2651220107 \\
\hline H37 & -2.8250512830 & 2.9704847876 & -3.1200134577 \\
\hline H38 & -1.6924783279 & 1.6063956643 & -4.7094076153 \\
\hline H39 & -2.6890384589 & 0.2351443880 & -4.2056793847 \\
\hline $\mathrm{H} 4 \mathrm{O}$ & -5.0211846551 & -1.0832840317 & -7.6100414621 \\
\hline $\mathrm{H} 41$ & -4.8196929612 & -1.0345830063 & -5.8680242063 \\
\hline $\mathrm{H} 42$ & -5.1007688895 & -3.3470291879 & -6.7821833720 \\
\hline $\mathrm{H} 43$ & -3.6078542386 & -3.1876744228 & -7.6986376178 \\
\hline $\mathrm{H} 44$ & -2.8728643499 & -1.7810098197 & -1.8409370234 \\
\hline H4 5 & -1.3174944254 & -3.6948237882 & 1.6746284131 \\
\hline H4 6 & -0.8462934051 & -5.7459958077 & 0.3241706906 \\
\hline H 47 & -1.4180958892 & -5.8117831824 & -2.0755001515 \\
\hline $\mathrm{H} 48$ & -4.6663634364 & 1.2052457393 & -5.3377007467 \\
\hline H4 9 & -3.7778184617 & 2.6722190931 & -5.7189637515 \\
\hline $\mathrm{H} 50$ & -3.9158152597 & 1.2958382444 & -7.7243377625 \\
\hline H51 & -2.2445536082 & 1.4633973016 & -7.2168398579 \\
\hline H52 & -2.2914129920 & -0.8491460294 & -6.2366743975 \\
\hline H53 & -2.5876118891 & -0.8041710060 & -7.9596151690 \\
\hline H5 4 & -4.1709014827 & -2.3094220622 & -3.8059378274 \\
\hline
\end{tabular}




$\begin{array}{lrrr}\text { H55 } & -1.0946496237 & -5.1037859835 & -4.3917250221 \\ \text { H56 } & -1.1155977034 & 1.6525089411 & 0.9439884650 \\ \text { H57 } & -1.4129847517 & 1.2442655666 & -0.7308683849 \\ \text { H58 } & 0.0778061210 & 3.2270939257 & -0.5079821481 \\ \text { H59 } & -1.3929058279 & 3.6732341465 & -1.3519278970 \\ \text { H60 } & -0.7874592947 & 4.1851365915 & 1.6064933254 \\ \text { H61 } & -0.8730970233 & 5.4041539600 & 0.3377890085 \\ \text { H62 } & -2.9902489606 & 5.3073587857 & 1.7128384122 \\ \text { H63 } & -3.2870381369 & 4.9777644007 & 0.0179958590 \\ \text { H64 } & -4.5077704500 & 3.3393183546 & 1.4545494827 \\ \text { H65 } & -3.0167981433 & 2.9444696513 & 2.2961306438\end{array}$

\section{Computational data for DFT-optimized 21a}

Number of imaginary frequencies: 0 Total energy: $\quad-1342.72793458194$ hartrees Final geometry:

\begin{tabular}{|c|c|c|c|}
\hline & & & \\
\hline atom & $\mathrm{x}$ & $\mathrm{y}$ & $\mathrm{z}$ \\
\hline $\mathrm{N} 1$ & -0.6702358692 & -1.1240418533 & 5.2832756200 \\
\hline $\mathrm{C} 2$ & -0.1173141138 & -0.6662342794 & 4.0174174215 \\
\hline C3 & 1.1148275721 & 0.2841115425 & 4.1388295558 \\
\hline C4 & 1.0182134928 & 1.2086614173 & 5.3857813735 \\
\hline C5 & -0.2340295690 & 2.0682884160 & 5.5180278214 \\
\hline N6 & -0.1247808155 & 3.3096160706 & 6.0797903400 \\
\hline $\mathrm{C} 7$ & 0.9728010469 & 3.9499649369 & 6.8025345826 \\
\hline $\mathrm{C} 8$ & 0.9328270630 & 1.3473447107 & 11.2792846638 \\
\hline C9 & 0.2607423036 & 0.0336484592 & 10.8354188234 \\
\hline $\mathrm{C} 10$ & -2.0476699160 & 0.6815936243 & 11.9300340224 \\
\hline 011 & -1.3492081017 & 1.6663925102 & 5.1606129901 \\
\hline C12 & -1.1483843058 & -2.6266989959 & 7.1314803821 \\
\hline C13 & -1.3472516079 & -1.6275566415 & 8.0926292467 \\
\hline C14 & -2.1347167487 & -0.8724061744 & 10.3511400223 \\
\hline C15 & -0.4733037918 & -2.3635475282 & 5.8064755221 \\
\hline 016 & 0.2061684512 & -3.2342506375 & 5.2570254414 \\
\hline N17 & -3.3539787129 & -0.6365464141 & 10.9220079497 \\
\hline C18 & -1.5630005738 & -3.9368979700 & 7.4053419496 \\
\hline C19 & -2.1967513711 & -4.2308773878 & 8.6114459463 \\
\hline C20 & -2.3900720790 & -3.2321570744 & 9.5688117898 \\
\hline C21 & -1.9556898789 & -1.9181048564 & 9.3247118175 \\
\hline C22 & -1.2391905839 & -0.0179624743 & 10.9973764015 \\
\hline N23 & -3.3291111002 & 0.3150549852 & 11.8738614163 \\
\hline C24 & 0.7757328872 & 3.9525996828 & 8.3287365827 \\
\hline C25 & 0.8934001734 & 2.5759004492 & 8.9970022178 \\
\hline C26 & 0.5549709138 & 2.6161349214 & 10.4954514084 \\
\hline C27 & 2.4073891427 & -0.5702881272 & 4.2710622088 \\
\hline C28 & 3.7146267664 & 0.2241285655 & 4.1097034519 \\
\hline C29 & 3.7314074323 & 1.0098464487 & 2.7896447617 \\
\hline C30 & 2.4923231006 & 1.9084214182 & 2.6566138283 \\
\hline C31 & 1.1889690763 & 1.1103007385 & 2.8285247364 \\
\hline H32 & -1.3401782684 & -0.4943289730 & 5.7056498020 \\
\hline H33 & 0.1686596550 & -1.5495968797 & 3.4409406531 \\
\hline H34 & -0.9179771416 & -0.1456948974 & 3.4888675615 \\
\hline H35 & 1.0514710079 & 0.5753566118 & 6.2821817064 \\
\hline H36 & 1.9091420947 & 1.8350104364 & 5.4314029323 \\
\hline H37 & -1.0304709522 & 3.7605991660 & 6.1598573254 \\
\hline 0 & 1.9103045140 & 3.4528644420 & 6.54471545 \\
\hline
\end{tabular}




$\begin{array}{lrrr}\text { H39 } & 1.0543724781 & 4.9830622642 & 6.4437036123 \\ \text { H40 } & 0.7211930289 & 1.5164053404 & 12.3429956007 \\ \text { H41 } & 2.0196459867 & 1.2031944531 & 11.2114346944 \\ \text { H42 } & 0.5281637194 & -0.1845324350 & 9.7961303571 \\ \text { H43 } & 0.7072278526 & -0.7802518624 & 11.4243626024 \\ \text { H44 } & -1.7472863985 & 1.4362723737 & 12.6439338955 \\ \text { H45 } & -1.0243859973 & -0.6093240929 & 7.8993975123 \\ \text { H46 } & -4.2383915478 & -1.0379447008 & 10.6478845527 \\ \text { H47 } & -1.3777929067 & -4.7067344601 & 6.6637976886 \\ \text { H48 } & -2.5274002980 & -5.2447684108 & 8.8168560008 \\ \text { H49 } & -2.8497548087 & -3.4757074093 & 10.5226429087 \\ \text { H50 } & 1.5216817239 & 4.6325975209 & 8.7625106497 \\ \text { H51 } & -0.2057922111 & 4.3923290481 & 8.5561552927 \\ \text { H52 } & 1.9177303738 & 2.1980768886 & 8.8604071447 \\ \text { H53 } & 0.2276564097 & 1.8664107738 & 8.4901903912 \\ \text { H54 } & -0.5162796606 & 2.8238182785 & 10.6128431591 \\ \text { H55 } & 1.0832940079 & 3.4630677864 & 10.9552680949 \\ \text { H56 } & 2.3985665995 & -1.1132240006 & 5.2238525059 \\ \text { H57 } & 2.3781660520 & -1.3413859985 & 3.4902835562 \\ \text { H58 } & 3.8558702238 & 0.9174615345 & 4.9508623367 \\ \text { H59 } & 4.5633748535 & -0.4695997860 & 4.1473232607 \\ \text { H60 } & 4.6471480486 & 1.6088489887 & 2.7112324160 \\ \text { H61 } & 3.7490204039 & 0.2986121287 & 1.9510119489 \\ \text { H62 } & 2.5434485588 & 2.7142788718 & 3.4025127145 \\ \text { H63 } & 2.4870815114 & 2.4040771787 & 1.6780675495 \\ \text { H64 } & 1.0973868490 & 0.4103293697 & 1.9850998970 \\ \text { H65 } & 0.3206142023 & 1.7778886313 & 2.7665783088 \\ \text { C61960 }\end{array}$

\section{Computational data for DFT-optimized $\mathbf{2 0 g}$}

Number of imaginary frequencies: 0 Total energy: $\quad-1452.66001568986$ hartrees Final geometry:

\begin{tabular}{lrrr} 
atom & $\mathrm{x}$ & \multicolumn{1}{c}{$\mathrm{y}$} & \multicolumn{1}{c}{$\mathrm{z}$} \\
N1 & -7.9423455764 & 4.8600314945 & 2.7017225391 \\
C2 & -7.6430789066 & 3.5253476057 & 2.1990354011 \\
C3 & -6.4226403601 & 3.4726620202 & 1.2302605578 \\
C4 & -6.4780239607 & 4.6251348440 & 0.1629536458 \\
C5 & -5.7873451444 & 5.9500679988 & 0.4993097407 \\
N6 & -5.2117120470 & 6.6130604991 & -0.5365905014 \\
C7 & -4.5074631425 & 7.8862206386 & -0.3575905377 \\
C8 & -4.5703086828 & 8.8503729150 & -1.5510990084 \\
C9 & -5.9614239474 & 9.4114328456 & -1.9637760427 \\
C10 & -6.8495843793 & 9.6929596554 & -0.7862423878 \\
O11 & -5.7741054676 & 6.4176514615 & 1.6490566280 \\
C12 & -9.4575195042 & 6.7034330216 & 3.1177380605 \\
C13 & -8.8525281625 & 7.4742362896 & 2.1314717832 \\
C14 & -8.3318823116 & 9.4718379646 & 0.8932965528 \\
C15 & -9.1885707524 & 5.2168359937 & 3.1232041177 \\
O16 & -10.0732501287 & 4.4032274001 & 3.4010746916 \\
N17 & -7.4609029779 & 10.4880167152 & 1.0967063304 \\
C18 & -10.3704973854 & 7.3213401060 & 3.9837106658 \\
C19 & -10.6248275749 & 8.6902081203 & 3.8560150947 \\
C20 & -10.0079521750 & 9.4576285819 & 2.8562068542 \\
C21 & -9.1159244295 & 8.8399092548 & 1.9721565133 \\
C22 & -7.9948072591 & 8.9533814235 & -0.3662669148 \\
N23 & -6.5499091517 & 10.6409557077 & 0.0999757910
\end{tabular}




$\begin{array}{lrrr}\text { C24 } & -6.5492952025 & 2.1163418735 & 0.4743273141 \\ \text { C25 } & -5.3126152400 & 1.7178531644 & -0.3490855148 \\ \text { C26 } & -4.0249193092 & 1.7551660935 & 0.4860805894 \\ \text { C27 } & -3.8618492973 & 3.1114693830 & 1.1863366133 \\ \text { C28 } & -5.0960039356 & 3.4494624144 & 2.0381679356 \\ \text { C29 } & -8.6832250818 & 7.9046259063 & -1.1348579763 \\ \text { O30 } & -8.1748730879 & 7.2236616296 & -2.0160092488 \\ \text { O31 } & -9.9817755843 & 7.7813202620 & -0.7809916325 \\ \text { C32 } & -10.7362619226 & 6.7601175571 & -1.4584218982 \\ \text { H33 } & -7.2150524205 & 5.5658591369 & 2.5914696009 \\ \text { H34 } & -7.4627686281 & 2.8262413436 & 3.0262289461 \\ \text { H35 } & -8.5409300636 & 3.1636683801 & 1.6826378519 \\ \text { H36 } & -7.5313199943 & 4.8607325441 & -0.0388170302 \\ \text { H37 } & -6.0749004082 & 4.2630446398 & -0.7865199594 \\ \text { H38 } & -5.3049184759 & 6.2307753887 & -1.4671332796 \\ \text { H39 } & -4.9225703463 & 8.3548795654 & 0.5361651686 \\ \text { H40 } & -3.4499039380 & 7.6799202402 & -0.1429977213 \\ \text { H41 } & -3.9246778664 & 9.6909423118 & -1.2726179050 \\ \text { H42 } & -4.1070561700 & 8.3895513957 & -2.4342053001 \\ \text { H43 } & -5.7976241409 & 10.3293110365 & -2.5390902416 \\ \text { H44 } & -6.4744627551 & 8.7005182493 & -2.6127165034 \\ \text { H45 } & -8.1426322069 & 7.0161740999 & 1.4616194046 \\ \text { H46 } & -7.3460323319 & 11.0216390101 & 1.9463278879 \\ \text { H47 } & -10.8737310191 & 6.7297329632 & 4.7419481116 \\ \text { H48 } & -11.3256152810 & 9.1686355808 & 4.5339471139 \\ \text { H49 } & -10.2473482924 & 10.5129431907 & 2.7566802620 \\ \text { H50 } & -6.7365547304 & 1.3327636827 & 1.2220834263 \\ \text { H51 } & -7.4366825141 & 2.1410023273 & -0.1723033621 \\ \text { H52 } & -5.4687929876 & 0.7155835221 & -0.7664682074 \\ \text { H53 } & -5.1997604042 & 2.3872108080 & -1.2132877028 \\ \text { H54 } & -4.0633250814 & 0.9625206085 & 1.2474350740 \\ \text { H55 } & -3.1560962983 & 1.5390879938 & -0.1478396178 \\ \text { H56 } & -2.9711343600 & 3.1041445439 & 1.8261947577 \\ \text { H57 } & -3.6903133639 & 3.8960043181 & 0.4355139228 \\ \text { H58 } & -4.9501294145 & 4.3981838252 & 2.5598704794 \\ \text { H59 } & -5.1992792935 & 2.6753896506 & 2.8116475842 \\ \text { H60 } & -10.2933777004 & 5.7759688242 & -1.2864428643 \\ \text { H61 } & -10.7678215923 & 6.9580213782 & -2.5325529295 \\ \text { H62 } & -11.7353173796 & 6.8087774426 & -1.0265269448 \\ & & & \end{array}$

\section{Computational data for DFT-optimized 20h}

$\begin{array}{lc}\text { Number of imaginary frequenci } \\ \text { Total energy: } & -1530.0596378 \\ \text { Final geometry: } & \text { x } \\ \text { atom } & -2.9461085432 \\ \text { N1 } & -2.9117682005 \\ \text { C2 } & -1.8238809685 \\ \text { C3 } & -1.7129646574 \\ \text { C4 } & -0.7588956512 \\ \text { C5 } & -0.1570192840 \\ \text { N6 } & 0.7607666664 \\ \text { C7 } & 0.8581308587 \\ \text { C8 } & -0.3990504746 \\ \text { C9 } & -1.1912328743 \\ \text { C10 } & \end{array}$

\section{0}

\section{5 hartrees}

angstroms
y
10.8094469924
9.9751384707
8.8515584033
8.1842579543
8.8153152364
7.9626175085
8.4381175833
7.5520788903
7.4233317706
8.6957228458

$$
\begin{array}{r}
0.4743273141 \\
-0.3490855148 \\
0.4860805894 \\
1.1863366133 \\
2.0381679356 \\
-1.1348579763 \\
-2.0160092488 \\
-0.7809916325 \\
-1.4584218982 \\
2.5914696009 \\
3.0262289461 \\
1.6826378519 \\
-0.0388170302 \\
-0.7865199594 \\
-1.4671332796 \\
0.5361651686 \\
-0.1429977213 \\
-1.2726179050 \\
-2.4342053001 \\
-2.5390902416 \\
-2.6127165034 \\
1.4616194046 \\
1.9463278879 \\
4.7419481116 \\
4.5339471139 \\
2.7566802620 \\
1.2220834263 \\
-0.1723033621 \\
-0.7664682074 \\
-1.2132877028 \\
1.2474350740 \\
-0.1478396178 \\
1.8261947577 \\
0.4355139228 \\
2.5598704794 \\
2.8116475842 \\
-1.2864428643 \\
-2.5325529295 \\
-1.0265269448
\end{array}
$$




\begin{tabular}{|c|c|c|c|}
\hline 011 & -0.5526887522 & 10.0383268962 & 0.4516931115 \\
\hline C12 & -4.0899654841 & 11.8541387314 & -0.0787430302 \\
\hline C13 & -3.3887558866 & 11.0776923717 & -0.9942613989 \\
\hline C14 & -2.6171983226 & 10.3716159812 & -3.163468743 \\
\hline C15 & -4.0827353305 & 11.4355120126 & 1.372738670 \\
\hline 016 & -5.0847626735 & 11.5711245307 & 2.080711583 \\
\hline N17 & -1.6058392117 & 10.7606917997 & -3.974662017 \\
\hline C18 & -4.8753916069 & 12.9110702074 & -0.56049982 \\
\hline C19 & -4.9148578144 & 13.1727444201 & -1.93318604 \\
\hline $\mathrm{C} 20$ & -4.2116484675 & 12.3751187648 & -2.84838577 \\
\hline C21 & -3.4454508767 & 11.3037454779 & -2.37504481 \\
\hline $\mathrm{C} 22$ & -2.4088612983 & 8.9989508046 & -2.95860836 \\
\hline N23 & -0.7253751779 & 9.7692572353 & -4.27288944 \\
\hline C24 & -2.2847107194 & 7.7292710628 & 3.92161463 \\
\hline C25 & -2.2914134624 & 8.1467890050 & 5.40060313 \\
\hline C26 & -0.8993063344 & 8.6292703470 & 5.833965538 \\
\hline C27 & -0.3933177200 & 9.7533993159 & 4.918597322 \\
\hline C28 & -0.4374159339 & 9.3668371065 & 3.42900019 \\
\hline C29 & -3.2711640972 & 8.0377975005 & -2.253696517 \\
\hline 030 & -2.8982969857 & 6.9690349677 & -1.785115746 \\
\hline 031 & -4.5565854066 & 8.4489847669 & $-2.19693187 \mathrm{~g}$ \\
\hline C32 & -7.9538406831 & 7.8667641063 & -1.775864346 \\
\hline C33 & -6.7789396591 & 8.3339059966 & -1.347132814 \\
\hline C34 & -5.4888310024 & 7.5884503092 & -1.48952002 \\
\hline H35 & -2.0975514476 & 10.8403179663 & 1.222485298 \\
\hline H36 & -2.7716438648 & 10.5948227617 & 3.873951755 \\
\hline H37 & -3.9054849049 & 9.5239371293 & 3.082568188 \\
\hline H3 8 & -2.7079145941 & 8.1496752944 & 1.066151420 \\
\hline H39 & -1.4146855231 & 7.1387533920 & 1.664971400 \\
\hline H 40 & -0.4521865052 & 6.9960429971 & -0.369969682 \\
\hline H4 1 & 0.4513529729 & 9.4499142892 & -1.67123766 \\
\hline H4 2 & 1.7662178343 & 8.5252611723 & -0.968654026 \\
\hline H4 3 & 1.6683617110 & 7.9892933225 & -3.24555838 \\
\hline H 44 & 1.1928994198 & 6.5432989748 & -2.374731951 \\
\hline H4 5 & -0.0621749496 & 7.1333961723 & -4.563446153 \\
\hline H4 6 & -1.0535316095 & 6.6342029749 & -3.189948122 \\
\hline H4 7 & -2.7732972523 & 10.2660264598 & -0.640129800 \\
\hline H4 8 & -1.3855726355 & 11.7062384276 & -4.252468075 \\
\hline H 49 & -5.4508761745 & 13.5101671339 & 0.137967736 \\
\hline H50 & -5.5175750681 & 13.9971690156 & -2.302710015 \\
\hline H51 & -4.2916711505 & 12.5726310092 & -3.91390761 \\
\hline H52 & -3.2753945480 & 7.3640475185 & 3.620289342 \\
\hline H53 & -1.5940518775 & 6.8803852825 & 3.815780427 \\
\hline H5 4 & -3.0314646385 & 8.9389838339 & 5.57361821 \\
\hline H55 & -2.6066861451 & 7.2939645035 & 6.014242571 \\
\hline H5 6 & -0.9171356514 & 8.9664479964 & 6.877497813 \\
\hline H5 7 & -0.1984176878 & 7.7824510406 & 5.78896669 \\
\hline H5 8 & -0.9925267464 & 10.6574235949 & 5.089801491 \\
\hline H59 & 0.6367227960 & 10.0221298209 & 5.183214087 \\
\hline $\mathrm{H} 60$ & 0.2962435389 & 8.5646130855 & 3.257113483 \\
\hline H61 & -0.1146357269 & 10.2122489103 & 2.816107096 \\
\hline H62 & -8.8746191995 & 8.4200706633 & -1.617846923 \\
\hline H63 & -8.0359635903 & 6.9132489683 & -2.292896164 \\
\hline H6 64 & -6.7215823562 & 9.2931336947 & -0.83531201 \\
\hline H65 & -5.6129351481 & 6.6579855357 & $-2.05135562 \mathrm{~s}$ \\
\hline H6 6 & -5.0556365533 & 7.3411931799 & -0.51396279 \\
\hline
\end{tabular}




\section{Computational data for DFT-optimized 29}

Number of imaginary frequencies: 0 Total energy: $\quad-1319.45113536393$ hartrees Final geometry:

\begin{tabular}{|c|c|c|c|}
\hline & & & \\
\hline atom & $\mathrm{x}$ & Y & z \\
\hline N1 & -3.2980151928 & 0.1433288526 & 3.2728545222 \\
\hline $\mathrm{C} 2$ & -4.6051796231 & 0.3547453270 & 2.6823505961 \\
\hline $\mathrm{C} 3$ & -4.7424731749 & 1.6819145863 & 1.8601133569 \\
\hline $\mathrm{C} 4$ & -4.1346572063 & 1.5764617476 & 0.4197019473 \\
\hline $\mathrm{C} 5$ & -2.6493009246 & 1.2563399237 & 0.2320967718 \\
\hline N6 & -1.9249533882 & 2.1183537737 & -0.5499002896 \\
\hline $\mathrm{C} 7$ & -0.5957828936 & 1.7440354314 & -1.0517720676 \\
\hline $\mathrm{C} 8$ & 0.3647842804 & 2.9054270876 & -1.4146747706 \\
\hline $\mathrm{C} 9$ & 0.7444526920 & 3.8141693223 & -0.1984304656 \\
\hline C10 & 1.1269121992 & 3.0961981632 & 1.0629057967 \\
\hline 011 & -2.1372730576 & 0.2389762104 & 0.7063270188 \\
\hline C12 & 1.6455598386 & 2.2473152032 & -1.9697386237 \\
\hline C13 & -0.2542539714 & 3.8006873625 & -2.5069449585 \\
\hline C14 & -1.5497551098 & 0.3068053984 & 4.9032155449 \\
\hline N15 & -0.8603340856 & 0.9282497768 & 3.9504232743 \\
\hline C16 & 0.9821512396 & 1.9552612367 & 2.9740502809 \\
\hline C17 & -3.0318113518 & 0.1442083509 & 4.6014321395 \\
\hline 018 & -3.8643082599 & 0.0680337253 & 5.5088238321 \\
\hline N19 & 2.2772659094 & 2.1088068279 & 2.5691149731 \\
\hline C20 & -0.9621221102 & -0.1045521082 & 6.1055412313 \\
\hline C21 & 0.4008408669 & 0.1473529221 & 6.2741404901 \\
\hline C22 & 1.1231804733 & 0.8155416418 & 5.2789169237 \\
\hline C23 & 0.4432852305 & 1.2040969301 & 4.1157921797 \\
\hline C24 & 0.2204561802 & 2.6060039328 & 2.0198185229 \\
\hline N25 & 2.3891000318 & 2.7914520305 & 1.4012214080 \\
\hline C26 & -4.1346635350 & 2.8632693574 & 2.6591830008 \\
\hline C27 & -4.4989940631 & 4.2522566549 & 2.1074680548 \\
\hline C28 & -6.0186281014 & 4.4372347512 & 1.9812558901 \\
\hline C29 & -6.6471346269 & 3.3150590852 & 1.1415516888 \\
\hline C30 & -6.2723784688 & 1.9292561123 & 1.6944757143 \\
\hline H31 & -2.5090557080 & 0.1704482828 & 2.6332947514 \\
\hline H32 & -4.8637859322 & -0.4963527113 & 2.0390287374 \\
\hline H33 & -5.3205683969 & 0.3764924268 & 3.5086099602 \\
\hline H34 & -4.6568295734 & 0.7605994547 & -0.0986922703 \\
\hline H35 & -4.3770380273 & 2.4907603528 & -0.1298229104 \\
\hline H36 & -2.4309836193 & 2.8463549669 & -1.0340402417 \\
\hline H37 & -0.1497096663 & 1.1086429484 & -0.2860724013 \\
\hline H38 & -0.7204411765 & 1.1199318735 & -1.9488125433 \\
\hline H39 & 1.5628797128 & 4.4705507170 & -0.5141597961 \\
\hline $\mathrm{H} 4 \mathrm{O}$ & -0.1089530771 & 4.4619981186 & 0.0351050801 \\
\hline H 41 & 2.3566558055 & 3.0123532531 & -2.2996002436 \\
\hline $\mathrm{H} 42$ & 2.1437541876 & 1.6415713320 & -1.2066171869 \\
\hline $\mathrm{H} 43$ & 1.4216464368 & 1.6044384525 & -2.8287385752 \\
\hline H 44 & -1.1598853040 & 4.3139872070 & -2.1595451315 \\
\hline H4 5 & 0.4517055665 & 4.5800642938 & -2.8146254200 \\
\hline H 46 & -0.5137063647 & 3.2170349776 & -3.3980120664 \\
\hline H4 7 & 3.1159930143 & 1.7482432249 & 2.9968930808 \\
\hline $\mathrm{H} 48$ & -1.5602283039 & -0.5949114295 & 6.8650302267 \\
\hline H 49 & 0.9023656887 & -0.1616440991 & 7.1866342850 \\
\hline $\mathrm{H} 50$ & 2.1747969605 & 1.0458238468 & 5.4224425658 \\
\hline
\end{tabular}




$\begin{array}{llll}\text { H51 } & -0.8519455038 & 2.6846022613 & 2.0165789778 \\ \text { H52 } & -4.5013570929 & 2.7918945229 & 3.6923857713 \\ \text { H53 } & -3.0471829933 & 2.7541165478 & 2.7223310643 \\ \text { H54 } & -4.0786672435 & 5.0212333221 & 2.7668834114 \\ \text { H55 } & -4.0301796401 & 4.4088166287 & 1.1254462745 \\ \text { H56 } & -6.4650221727 & 4.4263798488 & 2.9860556204 \\ \text { H57 } & -6.2508857723 & 5.4168377065 & 1.5455310116 \\ \text { H58 } & -7.7396331810 & 3.4132247050 & 1.1337983208 \\ \text { H59 } & -6.3287653553 & 3.4159506214 & 0.0949854734 \\ \text { H60 } & -6.7097162057 & 1.1389639660 & 1.0694450763 \\ \text { H61 } & -6.7347212213 & 1.8285536578 & 2.6855644181\end{array}$

\section{Computational data for DFT-optimized 30}

Number of imaginary frequencies: 0 Total energy: $\quad-1319.44747024160$ hartrees Final geometry:

\begin{tabular}{|c|c|c|c|}
\hline & & & \\
\hline atom & $\mathrm{x}$ & y & $z$ \\
\hline $\mathrm{C} 1$ & 0.9691495934 & -0.8574285286 & 3.9782103465 \\
\hline C2 & 1.1808842512 & -2.2072679076 & 4.3271496532 \\
\hline C3 & 0.1933332000 & -3.1477026208 & 4.0515597170 \\
\hline C4 & -0.9741210995 & -2.7404708253 & 3.4097573405 \\
\hline C5 & -1.0952140532 & -1.3896106488 & 3.0749837217 \\
\hline N6 & -0.1630695793 & -0.4691939252 & 3.3617481056 \\
\hline C7 & -2.3333262732 & -0.9482797685 & 2.3136869914 \\
\hline N8 & -2.3782424133 & 0.3720065464 & 2.0173691746 \\
\hline C9 & -3.4919931690 & 1.0147957217 & 1.3253034750 \\
\hline C10 & -3.1881101668 & 1.4355140169 & -0.1438263456 \\
\hline C11 & -1.8563472536 & 2.2364741032 & -0.2304796332 \\
\hline C12 & -1.6573142911 & 3.3477302318 & 0.7943033990 \\
\hline $\mathrm{C} 13$ & 0.9239191331 & 3.3235071456 & 0.5633358134 \\
\hline C14 & 1.7854894925 & 2.2641921596 & 1.3477086036 \\
\hline 015 & -3.1950699315 & -1.7698640336 & 1.9909887834 \\
\hline 016 & -2.5979171555 & 3.9293746665 & 1.3404007422 \\
\hline C17 & 1.4291786216 & 2.3400214166 & 2.8635790132 \\
\hline C18 & 2.2178608754 & 1.4709378169 & 3.8065033143 \\
\hline C19 & 3.2637795823 & 2.6313449517 & 1.1118990341 \\
\hline C20 & 1.5435065571 & 0.8491998042 & 0.7895834995 \\
\hline N21 & -0.3727689676 & 3.7005188405 & 1.1244295136 \\
\hline C22 & 1.9877489820 & 0.1657699426 & 4.2650307605 \\
\hline N23 & 3.0087192591 & -0.0951595445 & 5.1447161791 \\
\hline N24 & 3.8628173802 & 0.9280831948 & 5.3046372350 \\
\hline C25 & 3.3917872801 & 1.8720713134 & 4.4909609501 \\
\hline C26 & -4.3712549565 & 2.2911264577 & -0.6733430799 \\
\hline C27 & -5.6693569050 & 1.4944974327 & -0.8894547147 \\
\hline C28 & -5.4437776565 & 0.2884999342 & -1.8125527678 \\
\hline C29 & -4.3116433841 & -0.6072441151 & -1.2885230242 \\
\hline C30 & -3.0172147052 & 0.1887337077 & -1.0541348074 \\
\hline H31 & 2.1127263115 & -2.5280812141 & 4.7804986029 \\
\hline H32 & 0.3432198852 & -4.1895699018 & 4.3186641724 \\
\hline H33 & -1.7730036838 & -3.4237016438 & 3.1484023274 \\
\hline H34 & -1.6386587626 & 0.9346770043 & 2.4183105638 \\
\hline H35 & -4.3208920561 & 0.3073100453 & 1.3503025889 \\
\hline H36 & -3.7765657260 & 1.9039261988 & 1.8922414787 \\
\hline H37 & -1.7962315929 & 2.6986204806 & -1.2260605941 \\
\hline H3 8 & -1.0179946383 & 1.5373330502 & 3630 \\
\hline
\end{tabular}




$\begin{array}{lrrr}\text { H39 } & 1.5018913867 & 4.2527208403 & 0.5016805724 \\ \text { H40 } & 0.7864596395 & 2.9818475193 & -0.4667594250 \\ \text { H41 } & 1.5710297517 & 3.3797419563 & 3.1894753976 \\ \text { H42 } & 0.3674224910 & 2.1210608362 & 2.9822165708 \\ \text { H43 } & 3.5082246796 & 3.6116447500 & 1.5389396817 \\ \text { H44 } & 3.9320694756 & 1.8921173554 & 1.5629742770 \\ \text { H45 } & 3.4883478127 & 2.6703192370 & 0.0390529870 \\ \text { H46 } & 0.5120055297 & 0.5199046383 & 0.9309524454 \\ \text { H47 } & 1.7749532675 & 0.8153264311 & -0.2818334079 \\ \text { H48 } & 2.1884435459 & 0.1194617544 & 1.2889230973 \\ \text { H49 } & -0.3678798675 & 4.4588924395 & 1.7961969810 \\ \text { H50 } & 3.1249076007 & -0.9087335709 & 5.7282995616 \\ \text { H51 } & 3.9023380451 & 2.8239117820 & 4.4241985664 \\ \text { H52 } & -4.5386166934 & 3.1367360533 & -0.0000586542 \\ \text { H53 } & -4.0677589621 & 2.7183974764 & -1.6408443972 \\ \text { H54 } & -6.0700060532 & 1.1494160185 & 0.0733002446 \\ \text { H55 } & -6.4312131837 & 2.1607907096 & -1.3130798498 \\ \text { H56 } & -6.3689885030 & -0.2914557988 & -1.9183415696 \\ \text { H57 } & -5.1842904718 & 0.6518062484 & -2.8185321584 \\ \text { H58 } & -4.6191418499 & -1.0942268507 & -0.3553981684 \\ \text { H59 } & -4.1085332508 & -1.4169932154 & -2.0004899263 \\ \text { H60 } & -2.6331783558 & 0.5310215630 & -2.0271371456 \\ \text { H61 } & -2.2480690664 & -0.4704346510 & -0.6323058154\end{array}$

\section{Computational data for DFT-optimized 31}

\begin{tabular}{|c|c|c|c|}
\hline \multicolumn{4}{|c|}{$\begin{array}{l}\text { Number of imagil } \\
\text { Total energy: } \\
\text { Final geometry: }\end{array}$} \\
\hline atom & $x$ & $\mathrm{y}$ & z \\
\hline $\mathrm{C} 1$ & 6.9227631330 & -2.1070839323 & 2.2619334407 \\
\hline C2 & 8.2332410697 & -1.5920627209 & 2.3512614452 \\
\hline C3 & 9.2912007233 & -2.4580815682 & 2.6153738686 \\
\hline C4 & 9.0371134291 & -3.8185224839 & 2.8060721337 \\
\hline C5 & 7.7135588055 & -4.2494290885 & 2.6982134282 \\
\hline N6 & 6.6960404908 & -3.4252746746 & 2.4210963442 \\
\hline C7 & 7.3617545307 & -5.7085705867 & 2.9416790713 \\
\hline N8 & 6.0570004195 & -5.9947813445 & 2.7065373430 \\
\hline C9 & 5.4318930783 & -7.2943188298 & 2.9102118793 \\
\hline C10 & 4.2775169659 & -7.2801472240 & 3.9561520897 \\
\hline C11 & 3.1718197578 & -6.2611900975 & 3.5559879393 \\
\hline C12 & 2.5580212512 & -6.3767433396 & 2.1603162155 \\
\hline C13 & 1.6104025507 & -3.9638780900 & 2.2142923548 \\
\hline C14 & 2.4107497667 & -2.8366460071 & 1.5157276069 \\
\hline 015 & 8.2063101537 & -6.5168334877 & 3.3343238995 \\
\hline 016 & 2.6381060495 & -7.3985619095 & 1.4760979740 \\
\hline C17 & 3.9318553929 & -2.9710523548 & 1.7786688670 \\
\hline C18 & 4.4883261898 & -1.5642526030 & 1.8715021681 \\
\hline C19 & 2.1130579333 & -2.8294629526 & 0.0033742459 \\
\hline C20 & 2.0910437996 & -1.4476491742 & 2.1308833551 \\
\hline N21 & 1.8539529673 & -5.3007336036 & 1.6737866983 \\
\hline C22 & 5.7769413118 & -1.2103069444 & 2.0561777912 \\
\hline C23 & 3.3280881299 & -0.5845303916 & 1.8010069649 \\
\hline C24 & 4.7996466949 & -6.8335484397 & 5.3498389359 \\
\hline C25 & 5.7401710785 & -7.8439525519 & 6.0258423341 \\
\hline C26 & 5.0892632372 & -9.2305421928 & 6.1321763836 \\
\hline
\end{tabular}




$\begin{array}{lrrr}\text { C27 } & 4.6143911497 & -9.7238413379 & 4.7581471458 \\ \text { C28 } & 3.6806369512 & -8.7095137054 & 4.0751412634 \\ \text { H29 } & 8.4067627545 & -0.5281861671 & 2.2201011091 \\ \text { H30 } & 10.3053079664 & -2.0748628623 & 2.6839024802 \\ \text { H31 } & 9.8160511401 & -4.5352033505 & 3.0380874522 \\ \text { H32 } & 5.5133995519 & -5.2149613332 & 2.3605709421 \\ \text { H33 } & 5.0361806467 & -7.6604909900 & 1.9579614942 \\ \text { H34 } & 6.2277339705 & -7.9689484609 & 3.2263504914 \\ \text { H35 } & 3.5564517327 & -5.2475575950 & 3.7067654473 \\ \text { H36 } & 2.3414757493 & -6.3608913285 & 4.2700553955 \\ \text { H37 } & 1.8357897493 & -3.9656424087 & 3.2836340636 \\ \text { H38 } & 0.5363397636 & -3.7532961219 & 2.1213904023 \\ \text { H39 } & 4.1139968724 & -3.4731075548 & 2.7348296690 \\ \text { H40 } & 4.4461545060 & -3.5690404146 & 1.0205161666 \\ \text { H41 } & 2.6478406665 & -2.0213139904 & -0.5054784719 \\ \text { H42 } & 1.0420771745 & -2.6894052091 & -0.1883682238 \\ \text { H43 } & 2.4279927837 & -3.7654198344 & -0.4695701698 \\ \text { H44 } & 1.1575843498 & -1.0195774195 & 1.7499284576 \\ \text { H45 } & 1.9906552081 & -1.5389157498 & 3.2211168555 \\ \text { H46 } & 1.4450161082 & -5.5092515317 & 0.7706561827 \\ \text { H47 } & 6.0119067191 & -0.1477844856 & 2.1058937321 \\ \text { H48 } & 3.4545439889 & 0.2646866068 & 2.4801104233 \\ \text { H49 } & 3.2398828015 & -0.1680858312 & 0.7882031405 \\ \text { H50 } & 5.2986699251 & -5.8605247168 & 5.2596699257 \\ \text { H51 } & 3.9285196868 & -6.6766016327 & 6.0037145415 \\ \text { H52 } & 6.6795968200 & -7.9146943353 & 5.4639082059 \\ \text { H53 } & 6.0075983658 & -7.4721874283 & 7.0229337633 \\ \text { H54 } & 5.7908300185 & -9.9481915390 & 6.5750353956 \\ \text { H55 } & 4.2257757046 & -9.1740525206 & 6.8123965495 \\ \text { H56 } & 5.4866130493 & -9.9257414563 & 4.1222381092 \\ \text { H57 } & 4.0861394925 & -10.6801414982 & 4.8592040412 \\ \text { H58 } & 2.7579647027 & -8.6368743919 & 4.6705947308 \\ \text { H59 } & 3.3812058340 & -9.0632103174 & 3.0850549168\end{array}$

\section{Computational data for DFT-optimized 32}

Number of imaginary frequencies: 0 Total energy: $\quad-1209.91587462178$ hartrees Final geometry:

$\begin{array}{lc}\text { atom } & x \\ \text { C1 } & -0.9219413954 \\ \text { C2 } & -0.6174452205 \\ \text { C3 } & -1.2587158434 \\ \text { C4 } & -2.1747562487 \\ \text { C5 } & -2.4312492054 \\ \text { N6 } & -1.8354283480 \\ \text { C7 } & -3.3629753819 \\ \text { N8 } & -4.1012361401 \\ \text { C9 } & -4.5687530303 \\ \text { C10 } & -5.3110483439 \\ \text { C11 } & -4.3570164609 \\ \text { C12 } & -3.4405084468 \\ \text { C13 } & -1.1960357391 \\ \text { C14 } & -1.1549525757 \\ \text { 015 } & -3.4193525420 \\ \text { O16 } & -3.7526882711\end{array}$

angstroms Y

$-3.8527189949$

$-4.8078594434$

$-4.7437449579$

$-3.7210289603$

$-2.8163980010$

$-2.8790982677$

$-1.6637132547$

$-1.0791195599$

$-1.6474082471$

$-0.5976725836$

0.5949968829

0.4459556900

$-0.1548995181$

$-1.2829933818$

$-1.2359515879$

0.9123461497
4. 7581471458

4.0751412634

2. 2201011091

2. 3605709421

1. 9579614942

3.2263504914

3. 7067654473

.2700553955

3.2836340636

2.1213904023

2.7348296690

1.0205161666

$-0.1883682238$

$-0.4695701698$

1. 7499284576

3. 2211168555

0.7706561827

.4801104233

0.7882031405

5.2596699257

6.0037145415

5.4639082059

6.5750353956

6.8123965495

4.1222381092

3. 0850549168
1.0437205947
0.0556262193
$-1.1783772059$
$-1.4102817087$
$-0.3690320363$
0.8258171208
$-0.6729572415$
0.3199595689
1. 5912025245
2. 4622869265
2.8114488124
4.0250121038
4.8567234423
5.9648391016
$-1.8285747153$
5.1222286752 


\begin{tabular}{|c|c|c|c|}
\hline $\mathrm{C} 17$ & 0.1798073379 & -3.1038139551 & 4.6446528670 \\
\hline C18 & -0.5440750894 & -3.1068333321 & 3.3916316899 \\
\hline C19 & -0.8199463750 & -0.5676834426 & 7.2950987781 \\
\hline C20 & -2.5195834198 & -1.9924363314 & 6.1271290196 \\
\hline N21 & -2.2475330088 & -0.2018454340 & 3.8380612977 \\
\hline C22 & -0.2279742132 & -3.8855623812 & 2.3329996418 \\
\hline C23 & -0.0642620052 & -2.3268565533 & 5.7235508519 \\
\hline C24 & -5.8316972881 & -1.3439569954 & 3.7191051332 \\
\hline C25 & -6.8070829764 & -0.5285726724 & 4.5825028529 \\
\hline C26 & -8.0073824779 & -0.0472568129 & 3.7571837576 \\
\hline C27 & -7.5391143961 & 0.7519453638 & 2.5344412088 \\
\hline C28 & -6.5363244686 & -0.0409274638 & 1.6775853768 \\
\hline H29 & 0.1140621984 & -5.5826755401 & 0.2637072134 \\
\hline $\mathrm{H} 30$ & -1.0371100314 & -5.4720932940 & -1.9528563456 \\
\hline H31 & -2.6720583701 & -3.5906014588 & -2.3636154887 \\
\hline H32 & -4.7075464634 & -0.3766433456 & -0.0888316225 \\
\hline H33 & -5.2618866590 & -2.4791591822 & 1.3979498101 \\
\hline H34 & -3.7115987150 & -2.0551486135 & 2.1281797971 \\
\hline H35 & -4.9531509623 & 1.4806147787 & 3.0366668051 \\
\hline H36 & -3.7594775180 & 0.8246645985 & 1.9212936816 \\
\hline H37 & -1.3249102020 & 0.8003924339 & 5.3715939467 \\
\hline H38 & -0.2361573663 & -0.1294633532 & 4.3351674250 \\
\hline H39 & 1.0156568008 & -3.8002465344 & 4.7112301493 \\
\hline H4 0 & -1.3966605538 & -2.4465185196 & 3.2893616478 \\
\hline H4 1 & -1.6046722385 & 0.1504402565 & 7.5511508562 \\
\hline H4 2 & -0.7342577628 & -1.2889961899 & 8.1153697709 \\
\hline H4 3 & 0.1311773548 & -0.0256899075 & 7.2257439168 \\
\hline H4 4 & -2.8041985630 & -2.5316920440 & 5.2201787301 \\
\hline H4 5 & -3.3006781716 & -1.2644505579 & 6.3623422553 \\
\hline H4 6 & -2.4630813462 & -2.7217100274 & 6.9421332227 \\
\hline H4 7 & -1.9968091809 & -0.4666272963 & 2.8948582738 \\
\hline H4 8 & 0.6003440888 & -4.5880352721 & 2.4121830999 \\
\hline H4 9 & 0.5985888529 & -2.4835088060 & 6.5743345830 \\
\hline H5O & -6.3486650468 & -2.2529606856 & 3.3777102823 \\
\hline H51 & -4.9854028096 & -1.6822144327 & 4.3260790873 \\
\hline H52 & -7.1472215760 & -1.1507592404 & 5.4193890373 \\
\hline H53 & -6.2836562333 & 0.3281125838 & 5.0222398618 \\
\hline H5 4 & -8.5942982688 & -0.9154747404 & 3.4217349494 \\
\hline H5 5 & -8.6765863799 & 0.5644564513 & 4.3741170488 \\
\hline H5 6 & -8.3938777724 & 1.0341491988 & 1.9077074204 \\
\hline H5 7 & -7.0859761860 & 1.6932563428 & 2.872233084 \\
\hline H5 8 & -6.2059055305 & 0.5993501980 & 0.8483256378 \\
\hline H5 9 & -7.0633253033 & -0.8929070452 & 1.2240668866 \\
\hline
\end{tabular}

\section{Computational data for DFT-optimized 39}

Number of imaginary frequencies: 0 Total energy: $\quad-1127.87422709055$ hartrees Final geometry:

$\begin{array}{lc}\text { atom } & x \\ \text { C1 } & -7.5387126991 \\ \text { C2 } & -6.8001688789 \\ \text { O3 } & -7.0935889355 \\ \text { C4 } & -6.8050731503 \\ \text { N5 } & -5.8043533813 \\ \text { C6 } & -5.6058493994\end{array}$

angstroms
y
-0.4259808695
0.5960990811
1.7894333780
-3.7615988753
0.1842592175
-1.1667684505

4.6446528670

3.3916316899

7.2950987781

2.3329996418

5.7235508519

3.7191051332

4.5825028529

.7571837576

.6775853768

0.2637072134

$-1.9528563456$

$-2.3636154887$

1. 3979498101

2.1281797971

3.0366668051

1.9212936816

.3715939467

4.3351674250

4.7112301493

3.2893616478

7.5511508562

.1153697709

5.2201787301

6.3623422553

6.9421332227

. 8948582738

.5743345830

3.3777102823

4.3260790873

5.4193890373

5.0222398618

4. 3741170488

1. 9077074204

0.8483256378

1. 2240668866 


\begin{tabular}{|c|c|c|c|}
\hline $\mathrm{C} 7$ & -4.4524775354 & -1.9321377698 & 0.5494423179 \\
\hline 08 & -3.3762095785 & -1.3571689454 & 0.7141335262 \\
\hline $\mathrm{C} 9$ & -5.1774685669 & 1.1807457726 & -0.4446413491 \\
\hline C10 & -5.4142080611 & 0.6006026961 & -1.8393814237 \\
\hline C11 & -5.1854099528 & -0.8961179995 & -1.6219070039 \\
\hline 012 & -6.7727545412 & 0.7584867386 & -2.2641977552 \\
\hline C13 & -5.9773230352 & -4.3486817803 & 2.4763310067 \\
\hline C14 & -5.8805434204 & -3.9625629641 & 1.0134123138 \\
\hline C15 & -2.3975115079 & -5.7033766001 & 2.7855639594 \\
\hline C16 & -3.5013590999 & -4.6448669310 & 2.6233083152 \\
\hline C17 & -4.8976738219 & -5.3176463451 & 2.8668024440 \\
\hline C18 & -3.4461215995 & -4.0984161977 & 1.1695004460 \\
\hline N19 & -4.6031916208 & -3.2775507438 & 0.7871179748 \\
\hline $\mathrm{C} 20$ & -3.2729331524 & -3.5009534166 & 3.6286285189 \\
\hline C21 & -8.7456030945 & -0.0536106071 & 2.7260180059 \\
\hline $\mathrm{C} 22$ & -9.3704170115 & -0.9814040867 & 3.5583549140 \\
\hline C23 & -8.7742230602 & -2.2245553193 & 3.7736050216 \\
\hline C24 & -7.5638365371 & -2.5175831518 & 3.1249482436 \\
\hline N25 & -6.9732076247 & -1.6183726795 & 2.3123808075 \\
\hline H2 6 & -6.8412220399 & -4.1544806784 & 4.3782049817 \\
\hline H2 7 & -6.5468077592 & -1.7045153136 & -0.1106680320 \\
\hline H2 8 & -5.6355300305 & 2.1564893576 & -0.2771682897 \\
\hline H29 & -4.1040831608 & 1.2399286595 & -0.2305547919 \\
\hline H3O & -4.7280144139 & 1.0225397335 & -2.5857015735 \\
\hline H31 & -4.1276832272 & -1.1293282298 & -1.7646909157 \\
\hline H32 & -5.7774779564 & -1.4869053460 & -2.3247910310 \\
\hline H33 & -7.0270433396 & 1.6874983700 & -2.1833906917 \\
\hline H34 & -5.8848757599 & -4.8817919519 & 0.4076957323 \\
\hline H35 & -6.7155694062 & -3.3509631505 & 0.6974944140 \\
\hline H36 & -2.4002788880 & -6.1128121732 & 3.8020622331 \\
\hline H37 & -2.5324034543 & -6.5379508422 & 2.0872179255 \\
\hline H38 & -1.4073137596 & -5.2691665622 & 2.6054910698 \\
\hline H39 & -4.9816900597 & -5.6180411848 & 3.9173386561 \\
\hline H4 0 & -4.9554705499 & -6.2283317537 & 2.2523933523 \\
\hline H4 1 & -2.5489522896 & -3.4949024549 & 1.0260183643 \\
\hline H4 2 & -3.4100611295 & -4.9500373960 & 0.4734558514 \\
\hline H4 3 & -2.2764434313 & -3.0675669922 & 3.4952462561 \\
\hline H4 4 & -4.0007437275 & -2.6953195982 & 3.5052147989 \\
\hline H4 5 & -3.3470913266 & -3.8716308436 & 4.6580306672 \\
\hline H4 6 & -9.1458716130 & 0.9382753499 & 2.5549299834 \\
\hline H4 7 & -10.3090485282 & -0.7343206633 & 4.0457910708 \\
\hline H4 8 & -9.2334690520 & -2.9580554924 & 4.4294278507 \\
\hline
\end{tabular}

\section{Computational data for DFT-optimized $\mathbf{4 0}$}

Number of imaginary frequencies: 0 Total energy: $\quad-1126.67101028546$ hartrees Final geometry:

$\begin{array}{lc}\text { atom } & \text { X } \\ \text { C1 } & -5.7254998586 \\ \text { C2 } & -4.6382543282 \\ \text { C3 } & -3.7834432815 \\ \text { N4 } & -4.0897119647 \\ \text { C5 } & -5.2044306247 \\ \text { C6 } & -5.9785876233 \\ \text { C7 } & -5.6770006896\end{array}$

$$
\text { angstroms }
$$

$$
\text { Y }
$$

$-5.2183597468$

$-5.5520157780$

$-4.5472597279$

$-3.1672792496$

$-2.8355202165$

$-3.8493416309$

$-1.4430071530$
0.5494423179

0.7141335262

$-0.4446413491$

$-1.8393814237$

.6219070039

2.2641977552

2.4763310067

1.0134123138

.7855639594

1.1695004460

0.7871179748

3. 6286285189

3.5583549140

3.7736050216

3.1249482436

2.3123808075

4.3782049817

$-0.2771682897$

$-0.2305547919$

$-2.5857015735$

$-1.7646909157$

2.3247910310

0.4076957323

0.6974944140

3. 8020622331

2.0872179255

3. 9173386561

2.2523933523

1. 0260183643

0.4734558514

3.5052147989

4. 6580306672

2.5549299834

4. 4294278507 


$\begin{array}{lrrr}\text { O8 } & -6.0998594218 & -1.2429725101 & -1.3379675788 \\ \text { C9 } & -2.5850677237 & -4.6180817295 & 1.8917215933 \\ \text { N10 } & -5.7898580397 & -0.4928665574 & 0.7666426061 \\ \text { C11 } & -5.2144494678 & -0.5394038110 & 2.1142923620 \\ \text { C12 } & -3.7534192772 & -0.0882523725 & 1.9813369293 \\ \text { O13 } & -3.4235906401 & 1.0911272855 & 2.1442981223 \\ \text { C14 } & -6.4462015295 & 0.7973220938 & 0.4855765684 \\ \text { C15 } & -6.5175336271 & 1.5269040478 & 1.8539463676 \\ \text { C16 } & -6.0980800228 & 0.4501279380 & 2.8867654219 \\ \text { O17 } & -5.7196326815 & 2.6959499577 & 1.9007882105 \\ \text { C18 } & -2.1380111029 & -3.3055136999 & 2.1133597421 \\ \text { C19 } & -3.0783531138 & -2.4348823718 & 1.5686753262 \\ \text { C20 } & 0.8105688931 & -0.9120423833 & 2.6130724865 \\ \text { C21 } & -0.6733280221 & -1.3032938454 & 2.7236069029 \\ \text { C22 } & -0.8154967724 & -2.8495178374 & 2.6701999048 \\ \text { C23 } & -1.4267982325 & -0.6907215723 & 1.5151027311 \\ \text { N24 } & -2.8647723660 & -1.0362897156 & 1.5420892558 \\ \text { C25 } & -1.2467656326 & -0.7641765511 & 4.0493731675 \\ \text { H26 } & -6.3836820937 & -5.9795540922 & -0.4797272821 \\ \text { H27 } & -4.3884021713 & -6.5842149276 & 0.9129683960 \\ \text { H28 } & -6.8069284105 & -3.5544497075 & -0.9744055082 \\ \text { H29 } & -2.0914676928 & -5.5349823698 & 2.1827678974 \\ \text { H30 } & -5.2718118154 & -1.5503772513 & 2.5243023309 \\ \text { H31 } & -5.8741014141 & 1.3910120876 & -0.2342091834 \\ \text { H32 } & -7.4299224275 & 0.6007474668 & 0.0487224835 \\ \text { H33 } & -7.5372923127 & 1.8694196298 & 2.0471841918 \\ \text { H34 } & -6.9810198825 & -0.0914032545 & 3.2418920516 \\ \text { H35 } & -5.5964545470 & 0.8974398218 & 3.7469618022 \\ \text { H36 } & -4.7887602092 & 2.4104669322 & 1.9688240024 \\ \text { H37 } & 1.2363766900 & -1.2211870844 & 1.6511708889 \\ \text { H38 } & 0.9432345529 & 0.1712603828 & 2.7100858721 \\ \text { H39 } & 1.3955120641 & -1.3918214788 & 3.4058967908 \\ \text { H40 } & -0.6476550541 & -3.2684569858 & 3.6703197313 \\ \text { H41 } & -0.0232350837 & -3.2657853692 & 2.0323573796 \\ \text { H42 } & -1.3535653754 & 0.3965299255 & 1.5204877011 \\ \text { H43 } & -1.0108318384 & -1.0729145639 & 0.5768983495 \\ \text { H44 } & -0.6517023603 & -1.1326282496 & 4.8923045990 \\ \text { H45 } & -1.2340643901 & 0.3299296755 & 4.0740913545 \\ \text { H46 } & -2.2785978097 & -1.0924606719 & 4.2120126350 \\ & & & \end{array}$

\section{Computational data for DFT-optimized 49}

Number of imaginary frequencies: 0 Total energy: $\quad-1465.00897496514$ hartrees Final geometry:

$\begin{array}{lc}\text { atom } & x \\ \text { C1 } & -6.9391728061 \\ \text { C2 } & -5.8734065143 \\ \text { C3 } & -5.3646682309 \\ \text { N4 } & -5.8683879365 \\ \text { C5 } & -6.9061075315 \\ \text { C6 } & -7.4776604543 \\ \text { C7 } & -7.5576823277 \\ \text { O8 } & -8.7317625887 \\ \text { C9 } & -4.2294794629\end{array}$

angstroms

$$
\text { Y }
$$

$-5.3150109931$

$-5.2435780626$

$-3.9807168971$

$-2.8444323646$

$-2.9178955757$

$-4.1400450987$

$-1.6425468791$

$-1.7074882679$

$-3.7554583142$ $\mathrm{z}$

0.7214185886

1.6212305293

1.9499084037

1.4277494513

0.5934069841

0.2042669723

0.0951708795

$-0.2891574671$

2.8583414663 


\begin{tabular}{|c|c|c|c|}
\hline $\mathrm{N} 10$ & -3.0778542084 & -4.4965917792 & 2.8910278702 \\
\hline $\mathrm{N} 11$ & -2.0910467864 & -3.9038036208 & 3.5967096352 \\
\hline $\mathrm{N} 12$ & -6.8884835882 & -0.4566984335 & 0.0797779504 \\
\hline $\mathrm{C} 13$ & -5.4627969818 & -0.1532932748 & 0.2969883632 \\
\hline C14 & -5.1333200103 & 0.0534428081 & 1.7878053541 \\
\hline 015 & -6.0305453967 & 0.2111287798 & 2.6149460265 \\
\hline C16 & -7.6486050346 & 0.7279345235 & -0.3529024796 \\
\hline C17 & -6.6255566831 & 1.8744737317 & -0.3041143273 \\
\hline $\mathrm{C} 18$ & -5.2864522283 & 1.1634343865 & -0.4909480226 \\
\hline 019 & -6.7905870624 & 2.8396800130 & -1.3412141365 \\
\hline $\mathrm{C} 20$ & -2.4761820504 & 0.1875465213 & 1.6454343449 \\
\hline $\mathrm{C} 21$ & -1.4855820587 & -0.5882284097 & 2.5737816932 \\
\hline 022 & -1.8533558045 & -0.4753692194 & 3.9463885638 \\
\hline N23 & -3.8282406093 & 0.0601408658 & 2.2117400703 \\
\hline $\mathrm{C} 24$ & -2.6422547581 & -2.7757507531 & 4.0677339740 \\
\hline $\mathrm{C} 25$ & -3.9872296831 & -2.6531123215 & 3.6558832082 \\
\hline $\mathrm{C} 26$ & -1.8002413352 & -1.7072222326 & 4.7041273506 \\
\hline $\mathrm{C} 27$ & -2.2498576245 & -0.2834819884 & 0.2181967139 \\
\hline $\mathrm{C} 28$ & -2.4603055161 & -1.6198125182 & -0.1593623338 \\
\hline $\mathrm{C} 29$ & -2.1758900896 & -2.0459495899 & -1.4571277477 \\
\hline $\mathrm{C} 30$ & -1.6627539497 & -1.1466016058 & -2.3975957312 \\
\hline C31 & -1.4389416037 & 0.1808958894 & -2.0304275557 \\
\hline C32 & -1.7320218186 & 0.6062631453 & -0.7312523816 \\
\hline H33 & -7.3585364869 & -6.2781495167 & 0.4461166309 \\
\hline H34 & -5.4671719406 & -6.1418042470 & 2.0767032209 \\
\hline H35 & -8.3275800448 & -4.1377017492 & -0.4672913316 \\
\hline H36 & -2.8745622080 & -5.3511554519 & 2.3946927398 \\
\hline H37 & -4.8436434229 & -0.9503145221 & -0.1057890764 \\
\hline H38 & -8.0163468535 & 0.5904918509 & -1.3765105786 \\
\hline H39 & -8.5118197238 & 0.8805001032 & 0.3016039083 \\
\hline $\mathrm{H} 4 \mathrm{O}$ & -6.6579634958 & 2.3662698502 & 0.6763946181 \\
\hline $\mathrm{H} 41$ & -4.4400308711 & 1.7614813824 & -0.1496541754 \\
\hline $\mathrm{H} 42$ & -5.1475163189 & 0.9485737499 & -1.5557313295 \\
\hline $\mathrm{H} 43$ & -7.5659927376 & 3.3829208047 & -1.1499496541 \\
\hline H4 4 & -2.2042000146 & 1.2519224043 & 1.6854645719 \\
\hline H4 5 & -0.4804550651 & -0.1714566484 & 2.4254739164 \\
\hline H4 6 & -1.4646311591 & -1.6381633043 & 2.2741400309 \\
\hline $\mathrm{H} 47$ & -3.7906024757 & 0.2034109099 & 3.2163504597 \\
\hline $\mathrm{H} 48$ & -4.6849345053 & -1.8533553258 & 3.8451077383 \\
\hline $\mathrm{H} 49$ & -0.7653971752 & -2.0606111301 & 4.7938657454 \\
\hline $\mathrm{H} 50$ & -2.1646434814 & -1.4262986578 & 5.6953272267 \\
\hline H51 & -2.8614859339 & -2.3248635614 & 0.5627941103 \\
\hline H52 & -2.3520888207 & -3.0812391601 & -1.7350035927 \\
\hline H53 & -1.4384434324 & -1.4803671469 & -3.4064288406 \\
\hline H5 4 & -1.0389894015 & 0.8872275584 & -2.751893828 \\
\hline H5 5 & -1.5555311954 & 1.6423472791 & -0.453358968 \\
\hline
\end{tabular}

\section{Computational data for DFT-optimized 50-cis}

Number of imaginary frequencies: 0

Total energy: $\quad-1465.03001451047$ hartrees

Final geometry:

$\begin{array}{lc}\text { atom } & x \\ \text { C1 } & -3.5444432253 \\ \text { C2 } & -2.4879906314 \\ \text { C3 } & -1.6403083617\end{array}$

$\begin{array}{cc}\text { angstroms } & \\ y & z \\ -7.2252927256 & -5.4399934582 \\ -7.8423829727 & -4.7690177933 \\ -7.0540129727 & -3.9713661485\end{array}$




\begin{tabular}{|c|c|c|c|}
\hline N4 & -1.8325083157 & -5.7313234694 & -3.8297259227 \\
\hline $\mathrm{C} 5$ & -2.8470731926 & -5.1501508780 & -4.4800762860 \\
\hline $\mathrm{C} 6$ & -3.7279518984 & -5.8484183230 & -5.3111296941 \\
\hline $\mathrm{C} 7$ & -2.9120438542 & -3.6458801477 & -4.3180427224 \\
\hline 08 & -2.9316892248 & -2.9162491576 & -5.3102403971 \\
\hline $\mathrm{C} 9$ & -0.5009533735 & -7.6187079467 & -3.2311592479 \\
\hline N10 & -0.5582719236 & -8.8370347324 & -2.6192252361 \\
\hline N11 & 0.5803165293 & -9.1725574945 & -1.9797562952 \\
\hline N12 & -2.8339022359 & -3.1871735678 & -3.0355920513 \\
\hline C13 & -2.6075376026 & -1.7688080867 & -2.7291589298 \\
\hline C14 & -1.1191168535 & -1.4588234117 & -2.4113228850 \\
\hline 015 & -0.8143577180 & -0.4052802609 & -1.8575410409 \\
\hline C16 & -3.2193084512 & -3.9429887299 & -1.8233318585 \\
\hline C17 & -3.3672964340 & -2.8635047843 & -0.7313997758 \\
\hline C18 & -3.4975838517 & -1.5384283310 & -1.5029648969 \\
\hline 019 & -4.4956604935 & -3.2031166706 & 0.0716772765 \\
\hline $\mathrm{C} 20$ & 1.2070398586 & -2.3383023174 & -2.5331957725 \\
\hline C21 & 1.8473985757 & -3.5924615398 & -3.1482457194 \\
\hline C22 & 1.4323278667 & -5.8921365984 & -3.5135521126 \\
\hline 023 & 1.1954574321 & -4.7615589361 & -2.6652294137 \\
\hline N24 & -0.2261269375 & -2.4016103557 & -2.8110355336 \\
\hline C25 & 1.3973774114 & -8.1461665212 & -2.2110917389 \\
\hline C26 & 0.7844903595 & -7.1316758417 & -2.9854089047 \\
\hline C27 & 1.8926390772 & -1.1079798980 & -3.1129250063 \\
\hline C28 & 2.8990882505 & -0.4651019291 & -2.3837871493 \\
\hline C29 & 3.5917109538 & 0.6190438014 & -2.9281848555 \\
\hline C30 & 3.2805124048 & 1.0744241628 & -4.2113397503 \\
\hline C31 & 2.2735741105 & 0.4396980585 & -4.9439443276 \\
\hline C32 & 1.5864815518 & -0.6466483240 & -4.3996436717 \\
\hline H33 & -4.2066924430 & -7.8116098234 & -6.0698428529 \\
\hline H3 4 & -2.3026121886 & -8.9053501609 & -4.8886201155 \\
\hline H35 & -4.5168434597 & -5.3221552370 & -5.8368299259 \\
\hline H3 6 & -1.3635164045 & -9.4383773191 & -2.5261319365 \\
\hline H37 & -2.8891527563 & -1.1565479406 & -3.5892336437 \\
\hline H38 & -2.4692127723 & -4.6938995911 & -1.5667543055 \\
\hline H39 & -4.1842459337 & -4.4395780770 & -1.9675059777 \\
\hline $\mathrm{H} 40$ & -2.4634247243 & -2.8427108039 & -0.1073564243 \\
\hline $\mathrm{H} 41$ & -3.1800424253 & -0.6647207441 & -0.9304766417 \\
\hline $\mathrm{H} 42$ & -4.5391256570 & -1.4113075168 & -1.8170551725 \\
\hline $\mathrm{H} 43$ & -4.5164219489 & -2.6263312688 & 0.8463215782 \\
\hline H 44 & 1.3683072334 & -2.3498226037 & -1.4470740318 \\
\hline H 45 & 1.7516251914 & -3.5331972706 & -4.2435149316 \\
\hline H 46 & 2.9188588148 & -3.6112228570 & -2.9025905645 \\
\hline H 47 & 1.0545405403 & -5.6663249795 & -4.5214305898 \\
\hline H 48 & 2.5166327756 & -6.0628403465 & -3.5946099609 \\
\hline H 49 & -0.5853436006 & -3.3187332332 & -3.053159608 \\
\hline $\mathrm{H} 50$ & 2.4067140196 & -8.1658128102 & -1.821466699 \\
\hline H51 & 3.1314738266 & -0.8036689874 & -1.376959582 \\
\hline H52 & 4.3654991243 & 1.1121775108 & -2.346839195 \\
\hline H53 & 3.8122709401 & 1.9217184267 & -4.634598717 \\
\hline H54 & 2.0187920353 & 0.7933663456 & -5.939021192 \\
\hline H55 & 0.7942593133 & -1.1257378644 & -4.96832142 \\
\hline
\end{tabular}

\section{Computational data for DFT-optimized 50-trans}

Number of imaginary frequencies: 0

Total energy: $\quad-1465.02974590530$ hartrees 
Final geometry:

\begin{tabular}{|c|c|c|c|}
\hline atom & $x$ & $y$ & z \\
\hline $\mathrm{C} 1$ & -0.8651751351 & -6.9772907933 & -0.5669938189 \\
\hline $\mathrm{C} 2$ & -0.4548524473 & -7.3102044792 & -1.8603761266 \\
\hline $\mathrm{C} 3$ & -1.0241207176 & -6.6288597355 & -2.9431043529 \\
\hline N4 & -1.9342568662 & -5.6495596953 & -2.7823786284 \\
\hline $\mathrm{C} 5$ & -2.3184115392 & -5.3374764295 & -1.5386174353 \\
\hline C6 & -1.8243326085 & -5.9853669437 & -0.3985159843 \\
\hline $\mathrm{C} 7$ & -3.3801343203 & -4.2783076062 & -1.3471634651 \\
\hline 08 & -4.2174624277 & -4.4333298914 & -0.4514803606 \\
\hline $\mathrm{C} 9$ & -0.6325246939 & -6.9618332820 & -4.3351732387 \\
\hline $\mathrm{N} 10$ & -1.4510626867 & -7.6405577933 & -5.1822986161 \\
\hline N11 & -0.9100113615 & -7.8499735106 & -6.4014128588 \\
\hline N12 & -3.4083938912 & -3.1955169571 & -2.1681401472 \\
\hline C13 & -2.4086156133 & -2.7500499133 & -3.1497547205 \\
\hline C14 & -0.9746996725 & -2.7427419644 & -2.5915877835 \\
\hline 015 & -0.7297957347 & -2.5619788920 & -1.4041584654 \\
\hline C16 & -4.4938098853 & -2.2206585796 & -1.9952127000 \\
\hline C17 & -4.3656045396 & -1.3140605709 & -3.2212771511 \\
\hline C18 & -2.8598480773 & -1.3025716354 & -3.4750551052 \\
\hline 019 & -4.9804725969 & -1.8694858049 & -4.3877407273 \\
\hline $\mathrm{C} 20$ & 1.4034639750 & -3.0145227595 & -3.1849777894 \\
\hline $\mathrm{C} 21$ & 2.1670143203 & -3.4902394599 & -4.4466521138 \\
\hline C22 & 1.6811113072 & -5.8574706593 & -4.6257268808 \\
\hline 023 & 1.4876406761 & -4.5254840846 & -5.1429122065 \\
\hline N24 & -0.0068919153 & -2.8729517395 & -3.5476170810 \\
\hline C25 & 0.2893919160 & -7.2779762638 & -6.3296244135 \\
\hline $\mathrm{C} 26$ & 0.5298661396 & -6.7027880151 & -5.0560479368 \\
\hline $\mathrm{C} 27$ & 2.0358996969 & -1.7326081572 & -2.6665539903 \\
\hline $\mathrm{C} 28$ & 1.8164226818 & -0.5071916407 & -3.3095665457 \\
\hline C29 & 2.4502369539 & 0.6528165350 & -2.8608706928 \\
\hline C30 & 3.3169611787 & 0.6015299831 & -1.7655366384 \\
\hline C31 & 3.5406489231 & -0.6162331788 & -1.1192374276 \\
\hline C32 & 2.9011878121 & -1.7742960075 & -1.5674979204 \\
\hline H33 & -0.4453267229 & -7.4920556916 & 0.2920963065 \\
\hline H34 & 0.2786190679 & -8.0903594934 & -2.0364629643 \\
\hline H3 5 & -2.1949523931 & -5.6973591624 & 0.5778738046 \\
\hline H36 & -2.3805225120 & -7.9826511957 & -4.9872732199 \\
\hline H37 & -2.4601908655 & -3.3783995914 & -4.0429064335 \\
\hline H38 & -5.4568025347 & -2.7355627098 & -1.9300428604 \\
\hline H39 & -4.3584967029 & -1.6524957827 & -1.0650790651 \\
\hline $\mathrm{H} 40$ & -4.7607720426 & -0.3090146808 & -3.0238326785 \\
\hline $\mathrm{H} 41$ & -2.3803277022 & -0.5996532287 & -2.7859352517 \\
\hline $\mathrm{H} 42$ & -2.6217577732 & -1.0144790983 & -4.5009246300 \\
\hline $\mathrm{H} 43$ & -5.9218973229 & -2.0068386904 & -4.2192865273 \\
\hline H 44 & 1.4855485500 & -3.7720916453 & -2.3943814376 \\
\hline H 45 & 2.2438737606 & -2.6607380041 & -5.1561544684 \\
\hline H 46 & 3.1847168113 & -3.7888184617 & -4.1611420212 \\
\hline $\mathrm{H} 47$ & 2.6325572202 & -6.2483543114 & -5.0125526521 \\
\hline $\mathrm{H} 48$ & 1.7436078651 & -5.8328316788 & -3.5307752991 \\
\hline H49 & -0.2691634218 & -3.3277317174 & -4.4158094314 \\
\hline $\mathrm{H} 50$ & 0.9415565903 & -7.2870251294 & -7.1927303129 \\
\hline H51 & 1.1339657744 & -0.4597648245 & -4.1537495614 \\
\hline H5 2 & 2.2656876664 & 1.5974718066 & -3.3646738668 \\
\hline H53 & 3.8077446694 & 1.5047169580 & -1.4148570235 \\
\hline H5 4 & 4.2032995341 & -0.6640127687 & -0.2598804234 \\
\hline
\end{tabular}


H55

$3.0597508100 \quad-2.7150327631$

$-1.0458975209$

\section{Computational data for DFT-optimized s-trans-51}

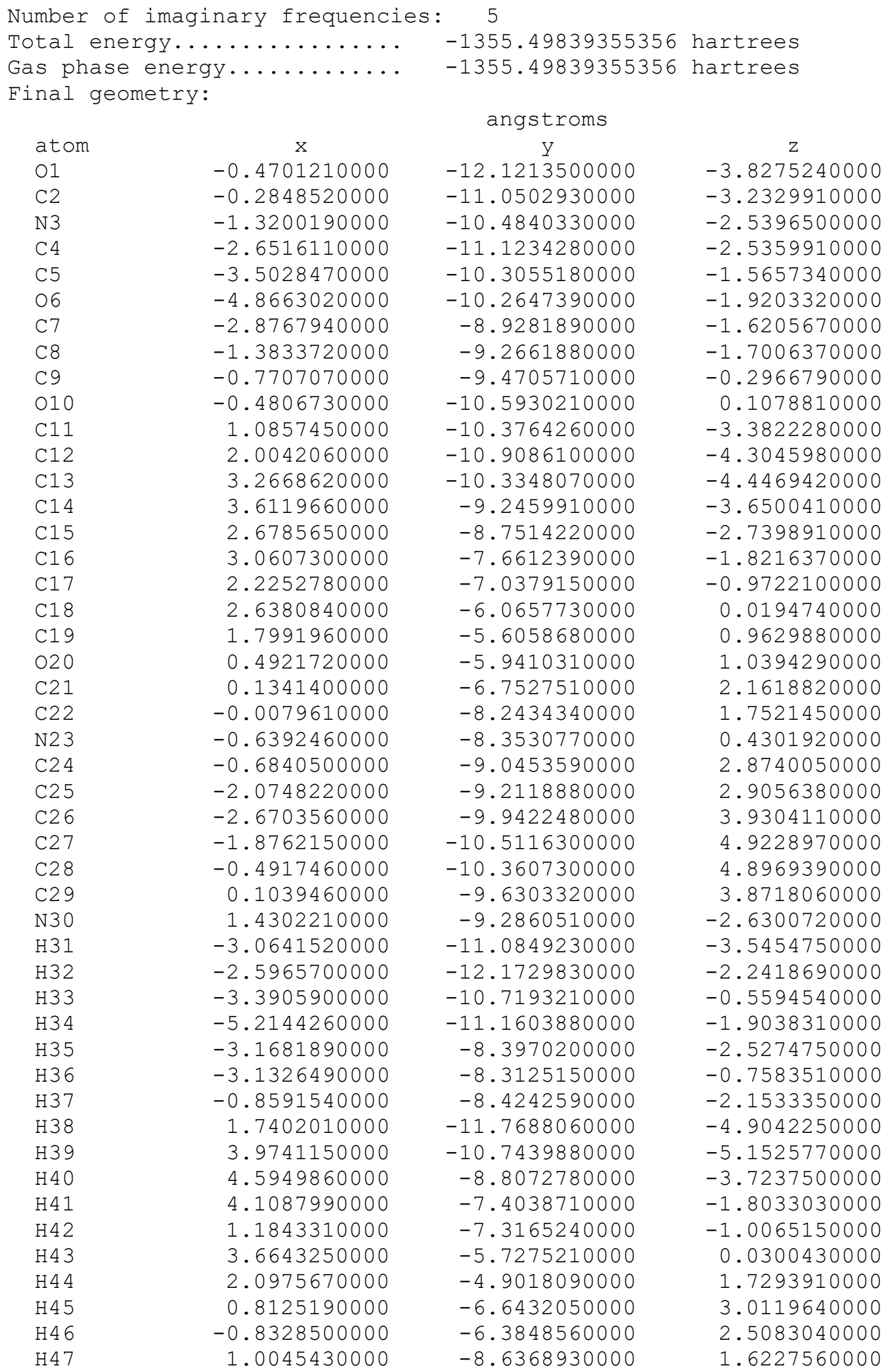




$\begin{array}{lrrr}\text { H48 } & -0.8271800000 & -7.4682280000 & -0.0166640000 \\ \text { H49 } & -2.6943400000 & -8.7817880000 & 2.1322050000 \\ \text { H50 } & -3.7426260000 & -10.0734900000 & 3.9501630000 \\ \text { H51 } & -2.3377350000 & -11.0844180000 & 5.7144920000 \\ \text { H52 } & 0.1178640000 & -10.8164330000 & 5.6639110000 \\ \text { H53 } & 1.1796100000 & -9.5291910000 & 3.8529790000\end{array}$

\section{Computational data for DFT-optimized $s$-cis-51}

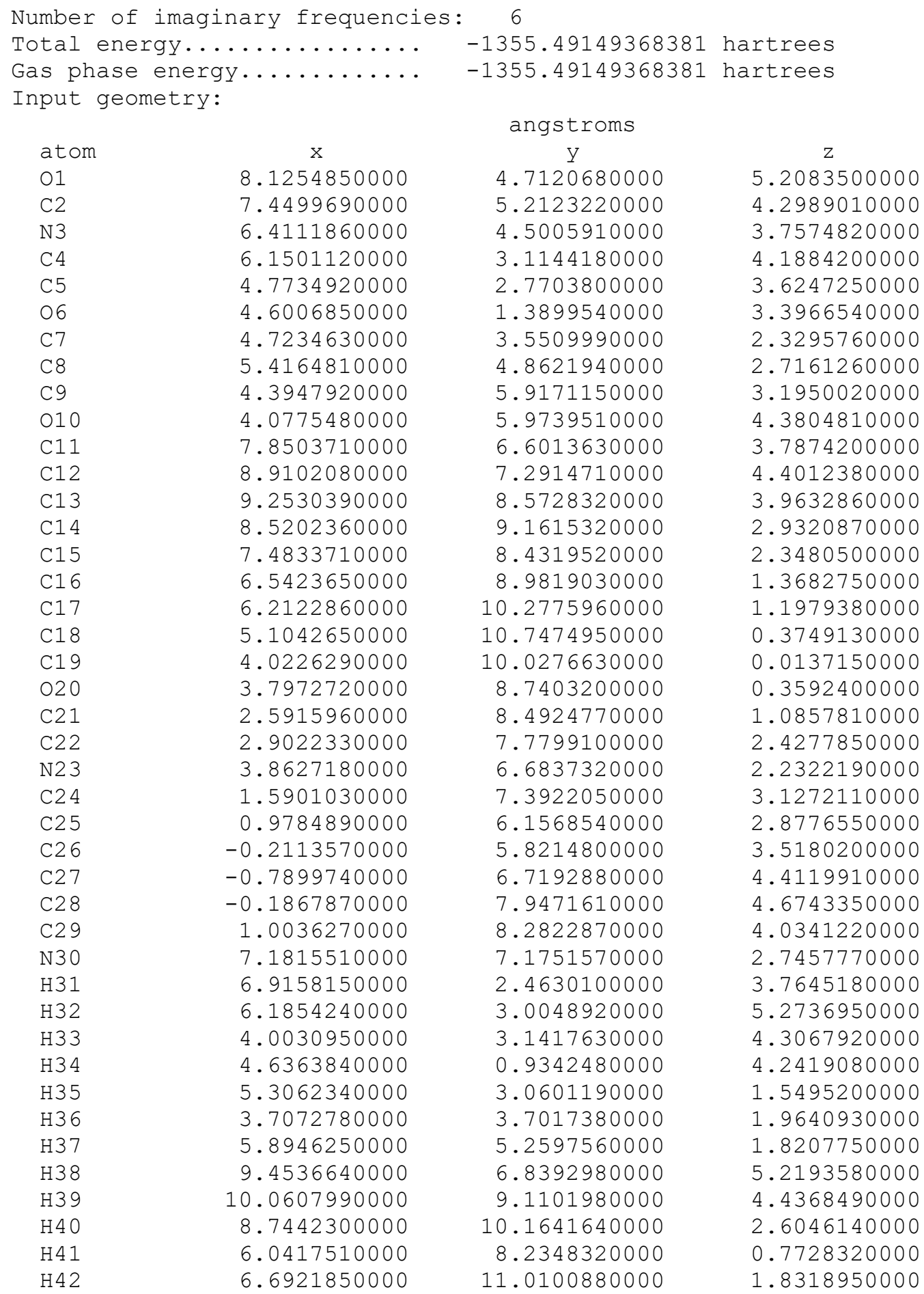




$\begin{array}{lrrr}\text { H43 } & 5.1678120000 & 11.7578640000 & -0.0026160000 \\ \text { H44 } & 3.2380060000 & 10.4327960000 & -0.6128880000 \\ \text { H45 } & 2.0049740000 & 9.3954460000 & 1.2766320000 \\ \text { H46 } & 1.9647280000 & 7.8509790000 & 0.4645170000 \\ \text { H47 } & 3.4204110000 & 8.5056180000 & 3.0610490000 \\ \text { H48 } & 4.3067780000 & 6.6687680000 & 1.3268200000 \\ \text { H49 } & 1.4303550000 & 5.4532630000 & 2.1937870000 \\ \text { H50 } & -0.6778850000 & 4.8655100000 & 3.3288280000 \\ \text { H51 } & -1.7096370000 & 6.4561140000 & 4.9145870000 \\ \text { H52 } & -0.6357250000 & 8.6324180000 & 5.3788390000 \\ \text { H53 } & 1.4712380000 & 9.2314980000 & 4.2529560000\end{array}$

\section{Computational data for DFT-optimized $\mathbf{5 2}$}

Number of imaginary frequencies: 0 Total energy: $\quad-1233.21762038054$ hartrees Final geometry:

\begin{tabular}{|c|c|c|c|}
\hline & & & \\
\hline atom & $\mathrm{x}$ & $\mathrm{y}$ & $z$ \\
\hline N1 & -2.7222305972 & 2.7341571337 & 3.6188783163 \\
\hline $\mathrm{C} 2$ & -3.4452331972 & 1.7234001331 & 4.3682643807 \\
\hline C3 & -4.2493192726 & 0.6952270577 & 3.5229606612 \\
\hline C4 & -5.5319937498 & 1.3434958287 & 2.8998711799 \\
\hline C5 & -5.3617921659 & 2.2371928670 & 1.6745160979 \\
\hline N6 & -5.9668499037 & 1.7982913907 & 0.5389158412 \\
\hline C7 & -5.9498469207 & 2.5404592638 & -0.7204966551 \\
\hline $\mathrm{C} 8$ & -5.6091608092 & 6.1878487615 & -1.5646048435 \\
\hline C9 & -4.4624614290 & 7.0478311313 & -2.0202062181 \\
\hline 010 & -4.7378622940 & 3.3078569361 & 1.6987124462 \\
\hline C11 & -4.7225559084 & -0.4009758194 & 4.5235616356 \\
\hline C12 & -5.3006711714 & -1.6670995523 & 3.8693593597 \\
\hline C13 & -4.3269206041 & -2.2619882218 & 2.8418915066 \\
\hline C14 & -3.9197445941 & -1.2115962773 & 1.7986074708 \\
\hline C15 & -3.3304627949 & 0.0469947214 & 2.4575813065 \\
\hline C16 & -0.7449174374 & 3.9850621839 & 2.8899029002 \\
\hline C17 & -1.4072101188 & 4.6605165465 & 1.8593699877 \\
\hline C18 & -1.4128173306 & 6.4051010169 & 0.0253587802 \\
\hline C19 & -1.3732877393 & 2.8907494112 & 3.7231841215 \\
\hline 020 & -0.6775003820 & 2.1922610202 & 4.4694618464 \\
\hline $\mathrm{C} 21$ & 0.5882335567 & 4.3128302942 & 3.1863176625 \\
\hline C22 & 1.2382654950 & 5.3109224181 & 2.4639742669 \\
\hline C23 & 0.5706970694 & 5.9830579853 & 1.4398752689 \\
\hline C24 & -0.7637047172 & 5.6714244655 & 1.1166289466 \\
\hline C25 & -3.2114093074 & 7.0831370719 & -1.5111060458 \\
\hline C26 & -7.1324860683 & 3.5104322241 & -0.8608125950 \\
\hline $\mathrm{C} 27$ & -7.1831323259 & 4.2336704317 & -2.2184929123 \\
\hline $\mathrm{C} 28$ & -5.9448539696 & 5.0741462263 & -2.5801620128 \\
\hline C29 & -2.6937779494 & 6.3042926960 & -0.4000669028 \\
\hline H3O & -3.2712125252 & 3.3042119468 & 2.9839453844 \\
\hline H31 & -2.6957274622 & 1.1907523659 & 4.9575132916 \\
\hline H32 & -4.1336288341 & 2.2118074963 & 5.0740521034 \\
\hline H33 & -6.0053534965 & 1.9650499809 & 3.6703221402 \\
\hline H34 & -6.2500579945 & 0.5540747125 & 2.6586291142 \\
\hline H35 & -6.5011785238 & 0.9433218606 & 0.5822031252 \\
\hline H36 & -5.9544389853 & 1.8103586723 & -1.5383158270 \\
\hline H37 & -5.0007515190 & 3.0788290173 & -0.7592067420 \\
\hline H38 & -5.3956993701 & 5.7482914562 & 001289 \\
\hline
\end{tabular}




$\begin{array}{lrrr}\text { H39 } & -6.5004044324 & 6.8197823307 & -1.4328717633 \\ \text { H40 } & -4.6728275206 & 7.6926754803 & -2.8734819170 \\ \text { H41 } & -3.8546112182 & -0.6990915641 & 5.1267563290 \\ \text { H42 } & -5.4503321027 & 0.0294430186 & 5.2250406762 \\ \text { H43 } & -5.5268433023 & -2.4019777191 & 4.6515807154 \\ \text { H44 } & -6.2592471166 & -1.4477899818 & 3.3781143649 \\ \text { H45 } & -3.4265487588 & -2.6189398933 & 3.3622084081 \\ \text { H46 } & -4.7744890781 & -3.1361132144 & 2.3530016285 \\ \text { H47 } & -3.1826291416 & -1.6323626789 & 1.1040588296 \\ \text { H48 } & -4.7951179681 & -0.9484387351 & 1.1874363899 \\ \text { H49 } & -3.0629111314 & 0.7856037417 & 1.6940983874 \\ \text { H50 } & -2.3886040787 & -0.2259830177 & 2.9520351258 \\ \text { H51 } & -2.4283663766 & 4.4007990907 & 1.6029106524 \\ \text { H52 } & -0.7660871674 & 7.1151566693 & -0.4913881620 \\ \text { H53 } & 1.0876558231 & 3.7691428546 & 3.9803948977 \\ \text { H54 } & 2.2679794828 & 5.5661678814 & 2.6972179243 \\ \text { H55 } & 1.0842418993 & 6.7600418727 & 0.8788269782 \\ \text { H56 } & -2.4974493404 & 7.7612887344 & -1.9782387324 \\ \text { H57 } & -8.0669419452 & 2.9492845899 & -0.7265271226 \\ \text { H58 } & -7.0852359564 & 4.2346259040 & -0.0397028327 \\ \text { H59 } & -7.3437628385 & 3.4911029550 & -3.0129185750 \\ \text { H60 } & -8.0693489058 & 4.8823479236 & -2.2266890521 \\ \text { H61 } & -6.1128166808 & 5.5290214284 & -3.5652432555 \\ \text { H62 } & -5.0654583443 & 4.4283952603 & -2.6981117572 \\ \text { H63 } & -3.3723018005 & 5.6144096022 & 0.0953886160\end{array}$

Computational data for DFT-optimized 53

Number of imaginary frequencies: 0

Total energy: $\quad-1382.22825148044$ hartrees

Final geometry:

$\begin{array}{lccc}\text { atom } & \text { X } & \text { angstroms } & \text { Z } \\ \text { N1 } & -4.1659990441 & -2.1091289337 & 1.7258267843 \\ \text { C2 } & -3.7165098647 & -1.7974566882 & 0.3813907125 \\ \text { C3 } & -3.7372322702 & -0.2722208266 & 0.0241518431 \\ \text { C4 } & -3.4451505891 & 0.6227240961 & 1.2640608657 \\ \text { C5 } & -2.1538251090 & 0.3910696180 & 2.0467586243 \\ \text { N6 } & -1.4248362885 & 1.5094325118 & 2.3148445371 \\ \text { C7 } & -0.8050058584 & 2.7920052420 & 5.2957903567 \\ \text { C8 } & -3.4422582769 & -0.2280139830 & 6.4893884603 \\ \text { O9 } & -1.8150472886 & -0.7199578314 & 2.4741552149 \\ \text { C10 } & -5.6851362647 & -2.5803940504 & 3.5557533280 \\ \text { C11 } & -4.9059901725 & -1.8839152097 & 4.4804932730 \\ \text { C12 } & -4.3666472120 & -1.1593160284 & 6.8209778345 \\ \text { C13 } & -5.4229059825 & -2.4965833932 & 2.0696424704 \\ \text { O14 } & -6.3073575884 & -2.7474844396 & 1.2450462538 \\ \text { C15 } & -6.7717310953 & -3.3431409921 & 4.0104866962 \\ \text { C16 } & -7.0404495722 & -3.4140985526 & 5.3774493931 \\ \text { C17 } & -6.2525629154 & -2.7141058636 & 6.2957780365 \\ \text { C18 } & -5.1757199755 & -1.9205352233 & 5.8609108128 \\ \text { C19 } & -2.6078579348 & 0.5034685534 & 7.4144016955 \\ \text { C20 } & -0.2271481791 & 1.5124289009 & 3.1629036297 \\ \text { C21 } & -0.1757395154 & 2.7496126071 & 4.0340001335 \\ \text { O22 } & -1.4366995782 & 1.6386704249 & 5.7001802118 \\ \text { C23 } & -0.8034849578 & 3.9623571874 & 6.0618196772\end{array}$




$\begin{array}{lrrr}\text { C24 } & -0.1446645325 & 5.0994360394 & 5.5872608322 \\ \text { C25 } & 0.5059673798 & 5.0742243780 & 4.3540706205 \\ \text { C26 } & 0.4802583655 & 3.9033459531 & 3.5906319721 \\ \text { C27 } & -5.1461206164 & 0.1398402749 & -0.4914063965 \\ \text { C28 } & -5.5081377985 & -0.4497405072 & -1.8647120176 \\ \text { C29 } & -4.4298268540 & -0.1569445627 & -2.9184026881 \\ \text { C30 } & -3.0448256848 & -0.6181519945 & -2.4407108651 \\ \text { C31 } & -2.6891868949 & 0.0097036654 & -1.0820678424 \\ \text { C32 } & -1.6777362434 & 1.4019146004 & 7.0332675637 \\ \text { H33 } & -3.4605016741 & -1.9573155055 & 2.4359689176 \\ \text { H34 } & -2.6933327056 & -2.1732592254 & 0.2911586131 \\ \text { H35 } & -4.3435997324 & -2.3549400396 & -0.3151900591 \\ \text { H36 } & -4.2594446297 & 0.4866440023 & 1.9870676228 \\ \text { H37 } & -3.4954810429 & 1.6688938329 & 0.9401856000 \\ \text { H38 } & -1.7415584126 & 2.3948889936 & 1.9462512967 \\ \text { H39 } & -3.2770706004 & 0.0083279855 & 5.4432320417 \\ \text { H40 } & -4.0698112453 & -1.2878514809 & 4.1361882537 \\ \text { H41 } & -4.5442002966 & -1.3766527242 & 7.8743684426 \\ \text { H42 } & -7.3851456343 & -3.8651694291 & 3.2838905364 \\ \text { H43 } & -7.8729936649 & -4.0147577001 & 5.7326010354 \\ \text { H44 } & -6.4801515583 & -2.7715790142 & 7.3574325546 \\ \text { H45 } & -2.7020566881 & 0.3157538513 & 8.4804477819 \\ \text { H46 } & -0.2615658179 & 0.5986972021 & 3.7577658690 \\ \text { H47 } & 0.6709755198 & 1.4716883468 & 2.5341253607 \\ \text { H48 } & -1.3320874901 & 3.9960440602 & 7.0076805086 \\ \text { H49 } & -0.1495896911 & 6.0054803852 & 6.1858726601 \\ \text { H50 } & 1.0252038750 & 5.9539710513 & 3.9874060289 \\ \text { H51 } & 0.9883469522 & 3.8766841380 & 2.6292736776 \\ \text { H52 } & -5.9032948801 & -0.1521226865 & 0.2452571776 \\ \text { H53 } & -5.1791669538 & 1.2373816020 & -0.5658216048 \\ \text { H54 } & -5.6571756499 & -1.5331861988 & -1.7786856050 \\ \text { H55 } & -6.4734650241 & -0.0393827434 & -2.1850914966 \\ \text { H56 } & -4.6857980621 & -0.6405570956 & -3.8691296136 \\ \text { H57 } & -4.3978953287 & 0.9253581580 & -3.1154186974 \\ \text { H58 } & -3.0278751314 & -1.7139322364 & -2.3663078857 \\ \text { H59 } & -2.2779650675 & -0.3487920463 & -3.1776326726 \\ \text { H60 } & -2.6126083822 & 1.0991757695 & -1.2182062543 \\ \text { H61 } & -1.7004599321 & -0.3348929586 & -0.7513045999 \\ \text { H62 } & -1.0325721021 & 1.9252856730 & 7.7313615157 \\ & & & \end{array}$

\section{Computational data for DFT-optimized $\mathbf{5 8}$}

\begin{tabular}{|c|c|c|c|}
\hline \multicolumn{4}{|c|}{$\begin{array}{l}\text { Total energy: } \\
\text { Final geometry: }\end{array}$} \\
\hline atom & $\mathrm{x}$ & $\mathrm{y}$ & $z$ \\
\hline $\mathrm{C} 1$ & 11.7659926544 & 0.3520752424 & 2.1948456868 \\
\hline $\mathrm{C} 2$ & 10.9195261921 & 0.1557548605 & 0.9112030022 \\
\hline 03 & 11.5649012539 & 0.7755881338 & -0.1984139232 \\
\hline N4 & 12.2104050740 & 5.2499178463 & 4.5027800888 \\
\hline C5 & 12.9384519990 & 4.5580480043 & 5.5531560072 \\
\hline c6 6 & 12.6043658796 & 3.0353425163 & 5.6999350888 \\
\hline C7 & 13.1715618746 & 2.1533808541 & 4.5231597340 \\
\hline C8 & 12.8580372427 & 2.5487757621 & 3.0888842418 \\
\hline N9 & 12.0462347407 & 1.7953132427 & 2.2808239902 \\
\hline 010 & 13.3203978907 & 3.5988107227 & 2.6132637455 \\
\hline
\end{tabular}




\begin{tabular}{|c|c|c|c|}
\hline $\mathrm{C} 11$ & 10.3730460056 & 6.3675387512 & 3.4127729875 \\
\hline C12 & 10.2447991813 & 5.3800728489 & 2.4321796551 \\
\hline C13 & 9.4126146097 & 4.4174947192 & 0.353355841 \\
\hline C14 & 11.1460435601 & 6.0771118380 & 4.681587291 \\
\hline 015 & 10.7843381198 & 6.5303883046 & 5.771273558 \\
\hline N1 6 & 8.2929159241 & 3.6971897167 & 0.026817526 \\
\hline C17 & 9.6974649835 & 7.5818860112 & 3.23985762 \\
\hline C18 & 8.9415350208 & 7.7978711652 & 2.085167019 \\
\hline C19 & 8.8190690021 & 6.8038281542 & 1.10619352 \\
\hline $\mathrm{C} 20$ & 9.4649327314 & 5.5715096896 & 1.280508515 \\
\hline N21 & 8.6059232836 & 2.5338629348 & -0.597887364 \\
\hline $\mathrm{C} 22$ & 9.9414189058 & 2.5206968487 & -0.69633962 \\
\hline C23 & 10.4916633497 & 3.6869245244 & -0.129900175 \\
\hline C24 & 10.6782463076 & 1.3121102691 & -1.201889217 \\
\hline C25 & 6.8875621044 & 3.9807533686 & 0.30993305 \\
\hline C26 & 6.0021594535 & 4.0324422402 & -0.961357998 \\
\hline 027 & 5.9011613336 & 2.7236892237 & -1.531491153 \\
\hline C28 & 4.5804577289 & 4.4216886072 & -0.547721594 \\
\hline C29 & 11.0818950077 & 2.8040356480 & $5.85586177 \varepsilon$ \\
\hline C30 & 10.4923133192 & 3.3491107658 & 7.168907334 \\
\hline C31 & 11.2210463763 & 2.7662406901 & 8.387928938 \\
\hline C32 & 12.7317535662 & 3.0274660774 & 8.304226622 \\
\hline C33 & 13.3249255242 & 2.5169045751 & 6.979575042 \\
\hline C34 & 6.5616090426 & 5.0172717865 & -2.000391051 \\
\hline C35 & 11.0104516229 & -0.2629927569 & 3.361699200 \\
\hline C36 & 11.5036507574 & -1.4180837846 & 3.981669974 \\
\hline C37 & 10.7798989787 & -2.0483744376 & 4.998393193 \\
\hline C38 & 9.5480163735 & -1.5302114569 & 5.40276648 \\
\hline C39 & 9.0473155130 & -0.3778372164 & 4.789043195 \\
\hline C40 & 9.7738382374 & 0.2512569858 & 3.777572876 \\
\hline H4 1 & 12.7104462595 & -0.1941239410 & 2.063821069 \\
\hline H 42 & 10.7977430437 & -0.9199536182 & 0.728305176 \\
\hline H4 3 & 9.9281248031 & 0.5953502181 & 1.069721628 \\
\hline H 44 & 12.5081191065 & 5.0164621293 & 3.562193000 \\
\hline H4 5 & 14.0118804518 & 4.6662600152 & 5.359223996 \\
\hline H4 6 & 12.7157010992 & 5.0726787381 & 6.488418560 \\
\hline H4 7 & 12.8648096487 & 1.1211569738 & 4.711503291 \\
\hline H4 8 & 14.2668858877 & 2.1875898500 & 4.583478127 \\
\hline H 49 & 12.0448268046 & 2.2005867985 & 1.348371445 \\
\hline H5O & 10.7249677428 & 4.4192606684 & 2.567344838 \\
\hline H51 & 9.7686527565 & 8.3416069304 & 4.011494760 \\
\hline H52 & 8.4403806801 & 8.7506820310 & 1.942069239 \\
\hline H53 & 8.2381886990 & 6.9965454170 & 0.208809500 \\
\hline H5 4 & 11.5322565654 & 3.9635461701 & -0.044074544 \\
\hline H55 & 9.9612896563 & 0.5517952722 & -1.536347104 \\
\hline H56 & 11.3376018711 & 1.5629813687 & -2.036758216 \\
\hline H57 & 6.5021638174 & 3.1957165706 & 0.968901212 \\
\hline H58 & 6.8518772611 & 4.9320278468 & 0.842540310 \\
\hline H59 & 6.7928055090 & 2.3308852808 & -1.539219154 \\
\hline $\mathrm{H} 60$ & 3.9218971547 & 4.3622216926 & -1.418808730 \\
\hline H61 & 4.1964661519 & 3.7334304567 & 0.211806728 \\
\hline H62 & 4.5445255664 & 5.4426150585 & -0.153421845 \\
\hline H63 & 10.5493653868 & 3.2332242975 & 4.999455333 \\
\hline H6 4 & 10.9010067194 & 1.7237214745 & 5.819981686 \\
\hline H65 & 10.5462119945 & 4.4435952015 & 7.184663610 \\
\hline H6 6 & 9.4260579124 & 3.0933795750 & 7.210726349 \\
\hline H67 & 10.8157369087 & 3.1905436274 & 9.314552952 \\
\hline
\end{tabular}




$\begin{array}{lrrr}\text { H68 } & 11.0445599076 & 1.6809315669 & 8.4337984936 \\ \text { H69 } & 12.9214677074 & 4.1021920584 & 8.4198117302 \\ \text { H70 } & 13.2509642857 & 2.5367685167 & 9.1370695228 \\ \text { H71 } & 13.2693571364 & 1.4190514400 & 6.9787353770 \\ \text { H72 } & 14.3929383068 & 2.7700103562 & 6.9239950676 \\ \text { H73 } & 5.9127002764 & 5.0193035371 & -2.8804593716 \\ \text { H74 } & 6.6087823426 & 6.0386257126 & -1.6042032633 \\ \text { H75 } & 7.5661145033 & 4.7255399004 & -2.3216336681 \\ \text { H76 } & 12.4597274797 & -1.8291746193 & 3.6662171516 \\ \text { H77 } & 11.1777301455 & -2.9421040634 & 5.4702698390 \\ \text { H78 } & 8.9819298410 & -2.0184890288 & 6.1906521110 \\ \text { H79 } & 8.0916534551 & 0.0331689397 & 5.1012163377 \\ \text { H80 } & 9.3850680536 & 1.1552103805 & 3.3185918318\end{array}$

\section{Computational data for DFT-optimized 59}

\begin{tabular}{|c|c|c|c|}
\hline \multicolumn{4}{|c|}{$\begin{array}{l}\text { Number of imagin } \\
\text { Total energy: } \\
\text { Final geometry: }\end{array}$} \\
\hline atom & $x$ & Y & z \\
\hline C1 & 3.6802831191 & -2.4152136048 & -6.7023341461 \\
\hline $\mathrm{C} 2$ & 3.8532965875 & -2.1746028585 & -5.1793475734 \\
\hline 03 & 4.2732068000 & -0.8596437496 & -4.8297062775 \\
\hline N4 & 4.1313324737 & -0.1325726258 & -11.2949185306 \\
\hline $\mathrm{C} 5$ & 4.9349188785 & -1.2240422976 & -11.8259718281 \\
\hline C6 & 4.6280361554 & -2.6236825723 & -11.1940497408 \\
\hline C7 & 4.2110271628 & -2.5313668665 & -9.7005022070 \\
\hline C8 & 5.1461312435 & -1.8244308285 & -8.7265121195 \\
\hline N9 & 4.9150418716 & -1.9920449762 & -7.3749393853 \\
\hline 010 & 6.0843327718 & -1.1112649770 & -9.0914524787 \\
\hline C11 & 2.1845332631 & 1.2429257960 & -10.9034222886 \\
\hline C12 & 2.2810511466 & 1.1002874362 & -9.5183633556 \\
\hline C13 & 1.6882924087 & 1.5775217475 & -7.1937857512 \\
\hline C14 & 2.9374528274 & 0.2924020897 & -11.8015922621 \\
\hline 015 & 2.4744951644 & -0.1003679727 & -12.8750803658 \\
\hline N16 & 0.7098371546 & 1.7231349064 & -6.2936823602 \\
\hline C17 & 1.3058938874 & 2.2029638011 & -11.4215340762 \\
\hline C18 & 0.5678450995 & 3.0046167905 & -10.5458630098 \\
\hline C19 & 0.6698259769 & 2.8499757333 & -9.1583675670 \\
\hline C20 & 1.5330863022 & 1.8817331807 & -8.6274313682 \\
\hline N21 & 1.2003323087 & 1.1872835473 & -5.1412755282 \\
\hline $\mathrm{C} 22$ & 2.4742334741 & 0.7078858244 & -5.2979578864 \\
\hline C23 & 2.8225785291 & 0.9631681991 & -6.6072058798 \\
\hline C24 & 3.3007983716 & 0.0119368244 & -4.2487783851 \\
\hline C25 & 0.3514313862 & 1.1920054612 & -3.9610124577 \\
\hline C26 & 0.6232068722 & 2.3313900835 & -2.9568458449 \\
\hline C27 & -0.4224429037 & 2.2558076857 & -1.8347814355 \\
\hline 028 & 1.9305341984 & 2.0355643099 & -2.4216886313 \\
\hline C29 & 5.8798258495 & -3.5298480546 & -11.3335074014 \\
\hline C30 & 6.2059299793 & -3.9080395845 & -12.7886394023 \\
\hline C31 & 5.0084445391 & -4.5863043532 & -13.4707372222 \\
\hline C32 & 3.7495641814 & -3.7104301607 & -13.3833679340 \\
\hline C33 & 3.4360916955 & -3.2977247691 & -11.9356027223 \\
\hline C34 & 0.6275598135 & 3.7090919521 & -3.6261387930 \\
\hline C35 & 3.3166429403 & -3.8811281636 & -6.8950387522 \\
\hline C36 & 1.9740689546 & -4.2416453414 & -7.0696388958 \\
\hline
\end{tabular}




\begin{tabular}{|c|c|c|c|}
\hline C37 & 1.6054964268 & -5.5838826162 & -7.1895579401 \\
\hline C38 & 2.5797966663 & -6.5833395626 & -7.1357410765 \\
\hline C39 & 3.9216359430 & -6.2322903508 & -6.9600084141 \\
\hline C40 & 4.2878840628 & -4.8901612572 & -6.838086242 \\
\hline H4 1 & 2.8393296711 & -1.8020210834 & -7.049629483 \\
\hline H 42 & 2.9096655645 & -2.4307588789 & -4.682413786 \\
\hline H4 3 & 4.6285534073 & -2.8567509956 & -4.81012495 \\
\hline H 44 & 4.4963343606 & 0.3187501636 & $-10.46903467 \varepsilon$ \\
\hline H4 5 & 4.7720426367 & -1.2549867222 & -12.90378075 \\
\hline H4 6 & 5.9793195683 & -0.9683748880 & -11.641162923 \\
\hline H4 7 & 4.0340257254 & -3.5470474118 & -9.33508887 \\
\hline H4 8 & 3.2351669979 & -2.0295851899 & -9.629444833 \\
\hline H49 & 5.5073308427 & -1.3798768262 & -6.82143825 \\
\hline H5O & 2.9047112285 & 0.3166849781 & -9.108332203 \\
\hline H51 & 1.2017664130 & 2.3039215333 & -12.496885623 \\
\hline H52 & -0.1031234228 & 3.7578555363 & -10.948821636 \\
\hline H53 & 0.0796218669 & 3.4692598027 & -8.490205433 \\
\hline H5 4 & 3.7714055785 & 0.7328481053 & -7.063853103 \\
\hline H55 & 2.6656365693 & -0.5394270578 & -3.542442795 \\
\hline H5 6 & 3.8681015028 & 0.7387593509 & -3.66269763 \\
\hline H5 7 & -0.6741060789 & 1.2622223835 & -4.327904262 \\
\hline H5 8 & 0.4688442016 & 0.2387338753 & -3.435533469 \\
\hline H59 & -0.2087737489 & 3.0072417460 & -1.066508913 \\
\hline H6O & -1.4314868887 & 2.4466476372 & -2.215695635 \\
\hline H61 & -0.4072550696 & 1.2711953519 & -1.356722248 \\
\hline H62 & 2.2119740332 & 2.7681421406 & -1.85730848 \\
\hline H63 & 6.7393733233 & -3.0408439385 & -10.862182176 \\
\hline H64 & 5.6955996351 & -4.4579863653 & -10.772034484 \\
\hline H65 & 6.4939808019 & -3.0128736657 & -13.356753716 \\
\hline H6 6 & 7.0790127578 & -4.5724757126 & -12.802996145 \\
\hline H67 & 5.2426286887 & -4.8127237026 & -14.518450320 \\
\hline H68 & 4.8151052489 & -5.5501132951 & -12.976129926 \\
\hline H69 & 3.8752228252 & -2.8174209546 & -14.008179854 \\
\hline H7O & 2.8852839925 & -4.2446559757 & -13.796646502 \\
\hline H71 & 3.1480478041 & -4.1980597924 & -11.372773169 \\
\hline H72 & 2.5703452764 & -2.6259667043 & -11.92641323 \\
\hline H73 & 0.8262481090 & 4.4884882584 & -2.880186583 \\
\hline H7 4 & 1.3959913622 & 3.7630447609 & -4.400677240 \\
\hline H75 & -0.3392159553 & 3.9270836514 & -4.089950795 \\
\hline H7 6 & 1.2132993835 & -3.4664068926 & $-7.12780211 \varepsilon$ \\
\hline H77 & 0.5614765485 & -5.8460841633 & $-7.33474212 \varepsilon$ \\
\hline H78 & 2.2968021045 & -7.6269749477 & -7.236580044 \\
\hline H79 & 4.6847576469 & -7.0043267437 & -6.922294636 \\
\hline H8 & 5.3333855023 & -4.6193305638 & -6.721142794 \\
\hline
\end{tabular}

\section{Computational data for DFT-optimized $\mathbf{6 1}$}

Number of imaginary frequencies: 0 Total energy: $\quad-1566.93385216292$ hartrees Final geometry:

$\begin{array}{lc}\text { atom } & x \\ \text { N1 } & -2.9314110327 \\ \text { C2 } & -2.8345549623 \\ \text { C3 } & -1.8110046127 \\ \text { O4 } & -1.7074262659 \\ \text { C5 } & -0.5159366575\end{array}$

angstroms Y

1.5012762290

2.5964675821

3.6775364140

4.0550449077

0.9849940525
$-7.1895579401$

$-7.1357410765$

$-6.9600084141$

$-6.8380862425$

$-7.0496294838$

$-4.6824137868$

$-12.9037807587$

11.6411629235

$-9.6294448334$

$-6.8214382570$

$-9.1083322033$

$-12.4968856233$

$-8.4902054337$

$-7.0638531038$

$-3.5424427958$

$-3.6626976315$

$-4.3279042628$

$-3.4355334692$

$-1.0665089131$

$-2.2156956357$

$-1.3567222488$

$-1.8573084810$

$-10.7720344843$

$-13.3567537166$

$-12.8029961451$

$-14.5184503206$

.9761299268

$-13.7966465026$

$-11.3727731694$

$-4.4006772407$

$-4.0899507959$

$-7.1278021180$

$-7.3347421289$

$-6.9222$

$-6.7211427949$ 


\begin{tabular}{|c|c|c|c|}
\hline C6 & -0.1976300123 & 0.9635352855 & -3.0446367172 \\
\hline C7 & -1.9252244422 & 0.8496823171 & -1.1813628341 \\
\hline ०8 & -2.1778001040 & 0.1474306375 & -0.1997114640 \\
\hline C9 & 0.5144413435 & 1.0919735598 & -0.7395143739 \\
\hline C10 & 1.8364355754 & 1.2173415357 & -1.1613737133 \\
\hline C11 & 2.1431389784 & 1.2402761786 & -2.5259865347 \\
\hline C12 & 1.1259908050 & 1.1086578386 & -3.4802385593 \\
\hline C13 & 1.5374717541 & 2.3750637485 & -5.7220719532 \\
\hline C14 & 1.8452277329 & 1.8971148330 & -6.9860854632 \\
\hline C15 & 1.4230519297 & 1.1900786407 & -4.9334667955 \\
\hline J1 6 & 1.6250114445 & 0.0908411853 & -5.6633861471 \\
\hline N17 & 1.8791542402 & 0.5352328459 & -6.9193241138 \\
\hline C18 & -4.3126020372 & 1.2819238475 & -1.3895534467 \\
\hline C19 & -5.1584363244 & 2.0249359795 & -2.4159362176 \\
\hline C20 & -4.2591061734 & 3.2066003226 & -2.8013777293 \\
\hline 021 & -5.3846038992 & 1.1368130233 & -3.5170697281 \\
\hline C22 & 1.4283702307 & 3.8108780584 & -5.2758142615 \\
\hline C23 & 0.1204965173 & 4.5953553502 & -5.6262336283 \\
\hline C24 & -1.1158093651 & 4.0293494454 & -4.8801035763 \\
\hline C25 & -0.1897676619 & 4.5060533204 & -7.1333184960 \\
\hline C26 & 0.3462923621 & 6.0798558988 & -5.2663199178 \\
\hline N27 & -1.0741306008 & 4.2503168929 & -3.4349655055 \\
\hline C28 & 2.1519624217 & -0.3913353176 & -7.9645049837 \\
\hline C29 & 1.9632866004 & -0.0363844916 & -9.3004109760 \\
\hline C30 & 2.2527485520 & -0.9443611219 & -10.3250686318 \\
\hline C31 & 2.7162074992 & -2.2261107712 & -10.0073940778 \\
\hline C32 & 2.8865367640 & -2.5856562168 & -8.6607875364 \\
\hline C33 & 2.6126110273 & -1.6781972938 & -7.6472264094 \\
\hline 034 & 3.0224751472 & -3.1928326431 & -10.9234906237 \\
\hline C35 & 2.8762771418 & -2.8934336323 & -12.3057544148 \\
\hline H3 6 & -2.6347579527 & 2.1968246513 & -3.8210540651 \\
\hline H37 & -0.9765879028 & 0.8167792140 & -3.7853853455 \\
\hline H38 & 0.2618302727 & 1.0804009486 & 0.3151177417 \\
\hline H39 & 2.6322115964 & 1.3056182299 & -0.4274716922 \\
\hline $\mathrm{H} 4 \mathrm{O}$ & 3.1729938934 & 1.3516971831 & -2.8534177080 \\
\hline H4 1 & 2.0738747060 & 2.4228022630 & -7.9002053833 \\
\hline H4 2 & -4.5419482672 & 0.2148820399 & -1.3616947810 \\
\hline H4 3 & -4.4549721721 & 1.6913770429 & -0.3817936060 \\
\hline H 44 & -6.1149590167 & 2.3537468553 & -1.9900146506 \\
\hline H4 5 & -4.3138041694 & 3.9735790099 & -2.0211795208 \\
\hline H4 6 & -4.5330282644 & 3.6605089854 & -3.7593430011 \\
\hline H4 7 & -5.9491145519 & 1.5774759792 & -4.1659315793 \\
\hline H4 8 & 1.5689594538 & 3.8456191467 & -4.1899826961 \\
\hline H4 9 & 2.2627025028 & 4.3782071498 & -5.7082365560 \\
\hline $\mathrm{H} 5 \mathrm{O}$ & -2.0142171919 & 4.4973931050 & -5.3097935693 \\
\hline H51 & -1.1754950471 & 2.9565047162 & -5.0836295758 \\
\hline H52 & -1.0306018361 & 5.1573694801 & -7.3972304853 \\
\hline H53 & 0.6747545711 & 4.8276765330 & -7.7251102765 \\
\hline H5 4 & -0.4442626726 & 3.4856975967 & -7.4359573218 \\
\hline H55 & -0.5752272901 & 6.6624390892 & -5.3794971237 \\
\hline H5 6 & 0.7122992575 & 6.2169740981 & -4.2420296580 \\
\hline H57 & 1.1010186516 & 6.5184817269 & -5.9278522627 \\
\hline H58 & -0.5197824039 & 5.0261269681 & -3.0950818556 \\
\hline H59 & 1.5726872227 & 0.9414064129 & -9.5615366567 \\
\hline $\mathrm{H} 60$ & 2.0969213827 & -0.6422155813 & -11.3536128993 \\
\hline H61 & 3.2423223572 & -3.5843423744 & -8.429612035 \\
\hline H62 & 2.7434024734 & -1.9519713418 & -6.607407673 \\
\hline
\end{tabular}




$\begin{array}{llll}\text { H63 } & 1.8342855524 & -2.6567872201 & -12.5561377765 \\ \text { H64 } & 3.5239569760 & -2.0594004167 & -12.6048889053 \\ \text { H65 } & 3.1795374663 & -3.7954901713 & -12.8387822125\end{array}$

\section{Computational data for DFT-optimized 63}






$\begin{array}{lrrr}\text { H47 } & -4.6178587231 & 3.3786300935 & 2.0138940083 \\ \text { H48 } & -6.9081118062 & 5.2249253417 & 1.2155348080 \\ \text { H49 } & -5.6611985851 & 5.5519461881 & 2.4494916638 \\ \text { H50 } & -6.4003983378 & 5.5424587948 & -0.9314472115 \\ \text { H51 } & -5.1773327629 & 2.0270500617 & 0.3423655804 \\ \text { H52 } & -4.8306592718 & 5.3409072024 & -2.7609729489 \\ \text { H53 } & -3.2675394806 & 7.9392518199 & -1.2203223247 \\ \text { H54 } & -3.3498517920 & 7.3223378331 & 0.4295631026 \\ \text { H55 } & -1.8433846194 & 8.0725077422 & -0.1515503421 \\ \text { H56 } & 0.7761551066 & 2.6313509171 & -1.6057685790 \\ \text { H57 } & -0.7218265905 & 1.6722709864 & -1.7125778836 \\ \text { H58 } & -0.0868499539 & 2.4783766913 & -3.1478417283 \\ \text { H59 } & -4.6652593145 & 2.4402004961 & 4.0810662760 \\ \text { H60 } & -5.8571495373 & 1.6790829225 & 6.1095101958 \\ \text { H61 } & -8.3380804660 & 1.8414167790 & 6.2336093081 \\ \text { H62 } & -9.6072628908 & 2.7748486291 & 4.3083640305 \\ \text { H63 } & -8.4149426910 & 3.5232720928 & 2.2722458408\end{array}$




\section{Select NMR Spectra}

Crude compound 5 was used without further purification. The crude ${ }^{1} \mathrm{H}-\mathrm{NMR}$ (shown below) is consistent with the chemical shifts reported in: Jacobi, P.A.; Liu, H. Synthesis of 1,2,3,7,8,9Hexahydrodipyrrins and Secocorrins: Important Precursors for the Construction of Corrins. Org. Lett. 1999, 1, 341-344.

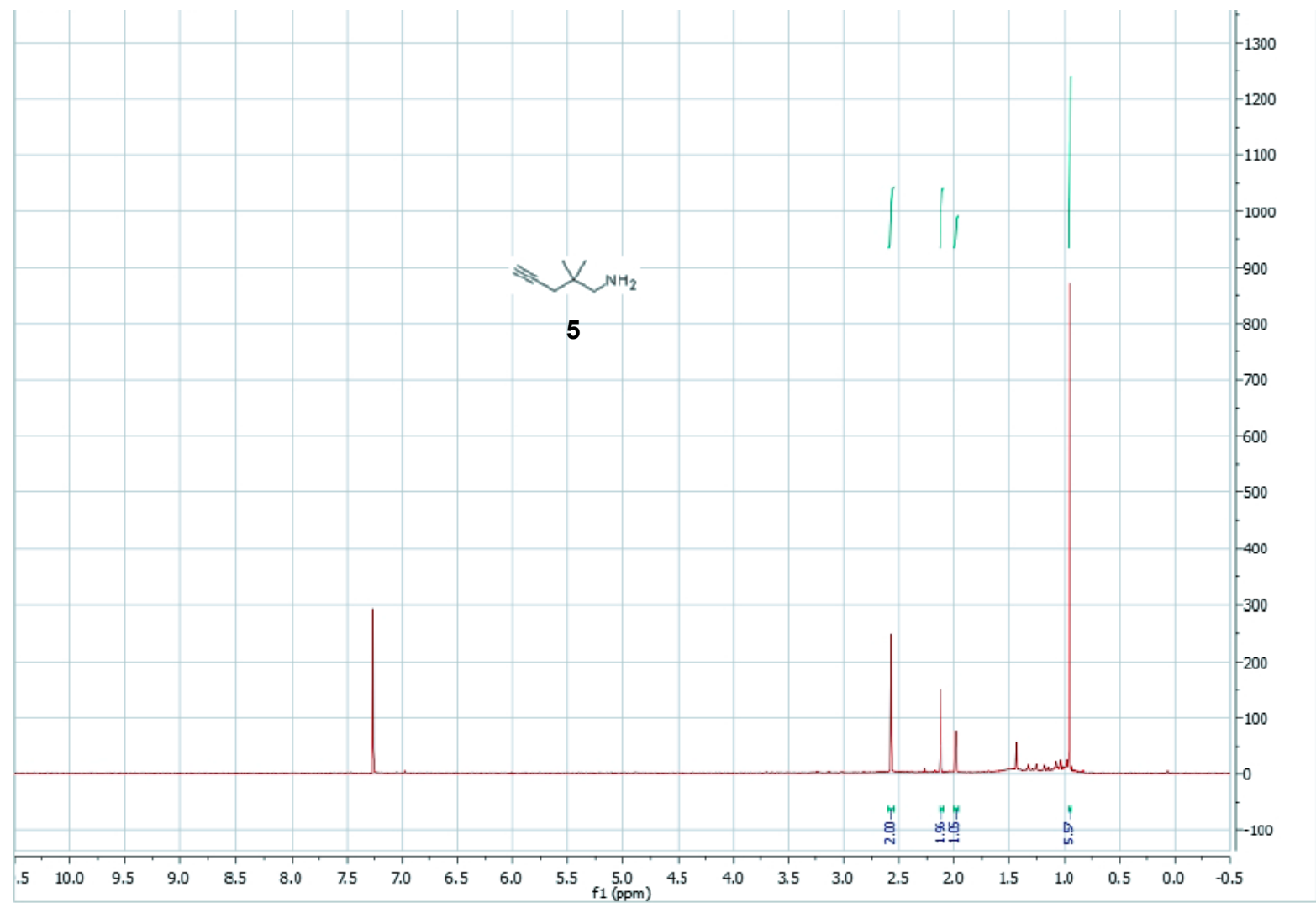




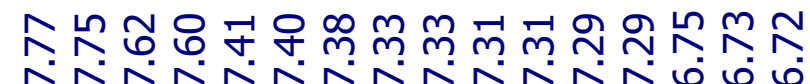

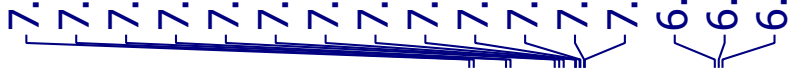

\begin{tabular}{|ll|}
\hline \multicolumn{1}{|c|}{ Parameter } & \multicolumn{1}{c|}{ Value } \\
1 Solvent & cdcl3 \\
2 Experiment & $1 \mathrm{D}$ \\
3 Number of Scans & 8 \\
4 Relaxation Delay & 1.0000 \\
5 Acquisition Time & 2.5592 \\
6 Spectrometer Frequency & 400.10 \\
7 Nucleus & $1 \mathrm{H}$ \\
\hline
\end{tabular}

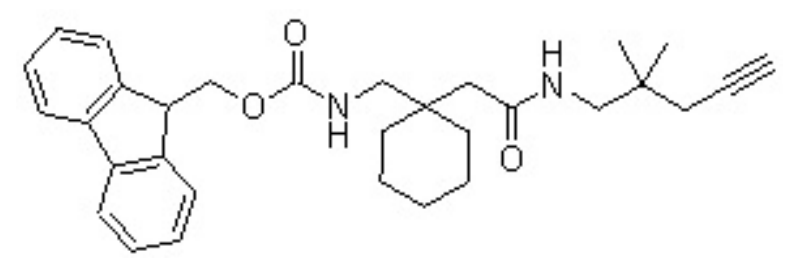

$6 a$
ชิ ถิำ

เก เก เ

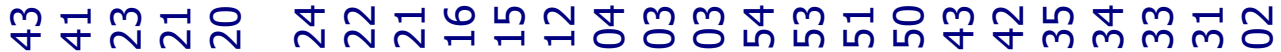



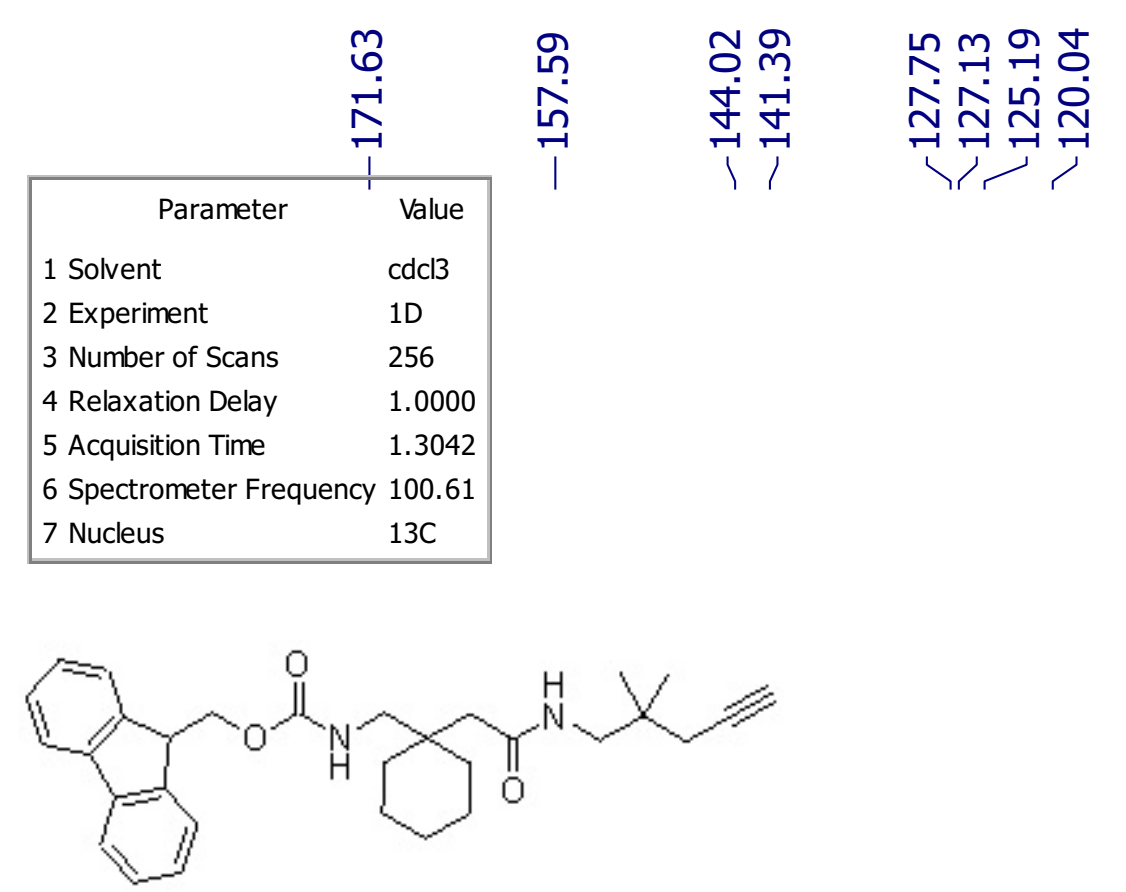

$6 a$

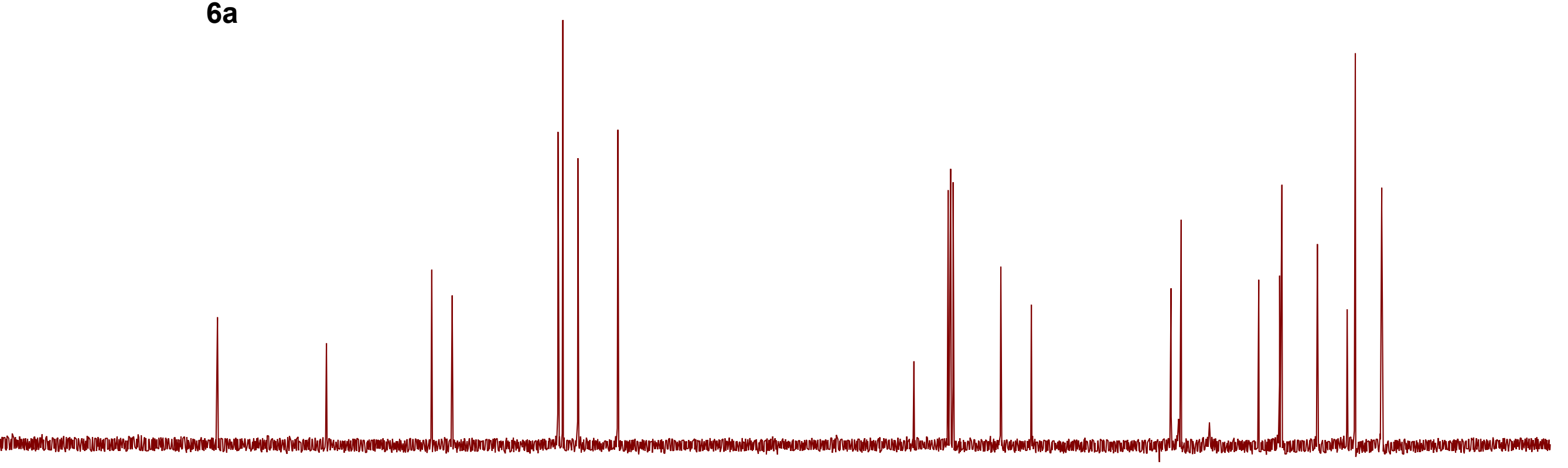




\begin{tabular}{|ll|}
\hline \multicolumn{1}{|c|}{ Parameter } & \multicolumn{1}{c|}{ Value } \\
1 Solvent & cdcl3 \\
2 Experiment & $1 \mathrm{D}$ \\
3 Number of Scans & 8 \\
4 Relaxation Delay & 1.0000 \\
5 Acquisition Time & 2.5592 \\
6 Spectrometer Frequency & 400.10 \\
7 Nucleus & $1 \mathrm{H}$ \\
\hline
\end{tabular}

으름ㄱ

NN

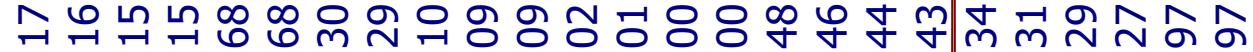

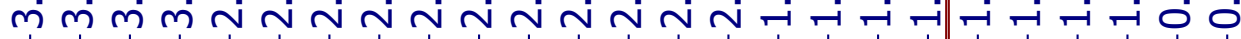

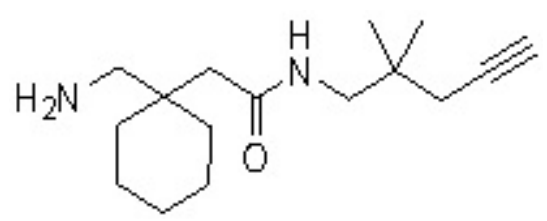

6

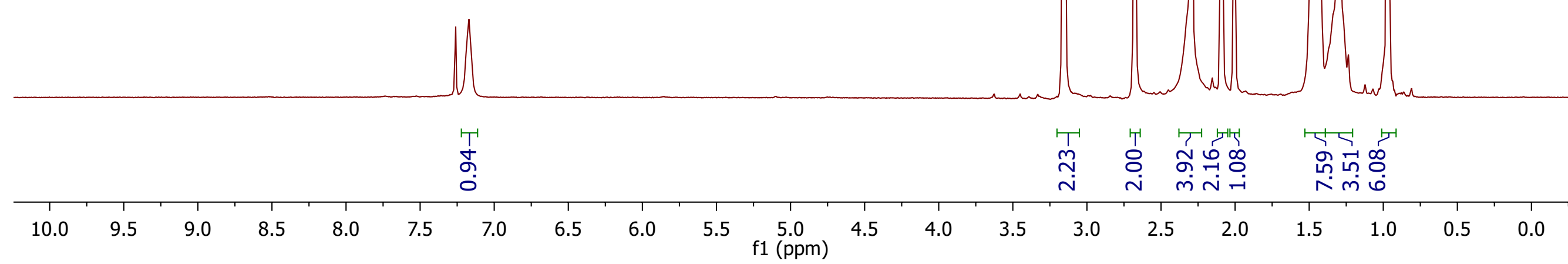




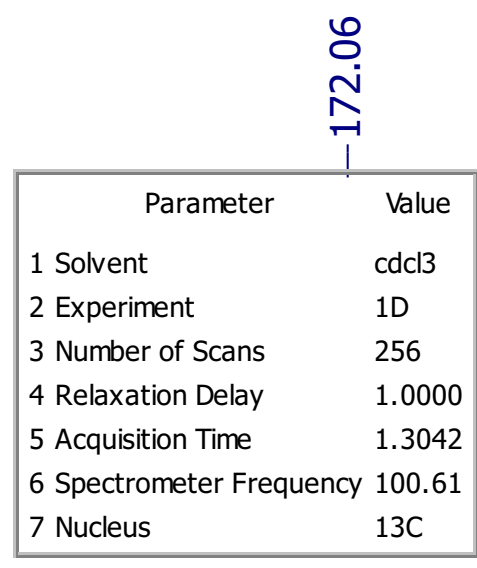<smiles>C#CCC(C)(C)CNC(=O)CC1(CN)CCCCC1</smiles>

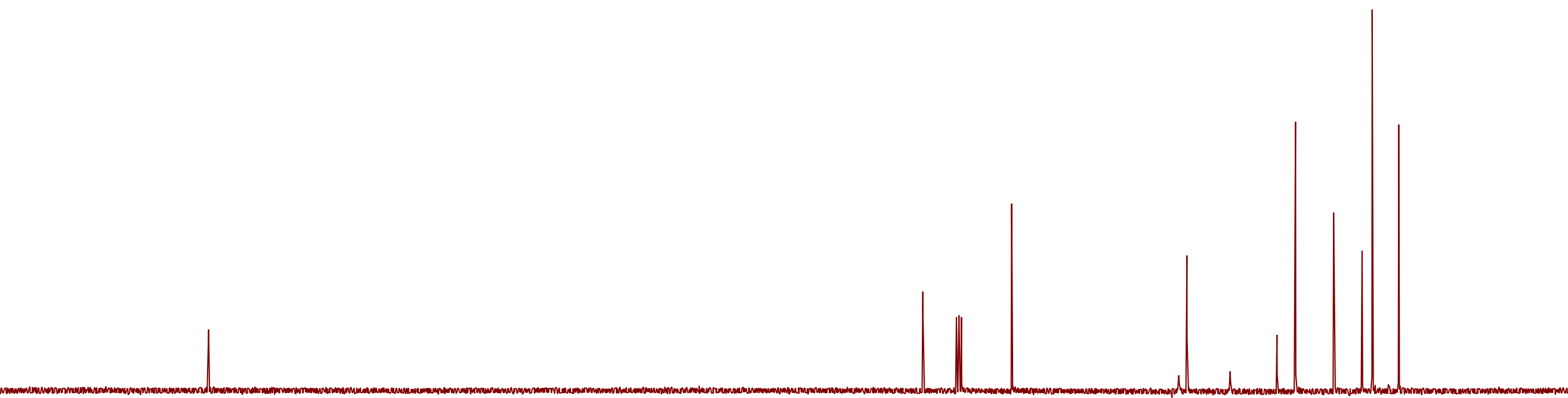

$190 \quad 180 \quad 170$

160

$60 \quad 150$

50

140

130

120

110

$90 \quad 80$
f1 (ppm)

70

60

$50 \quad 40$



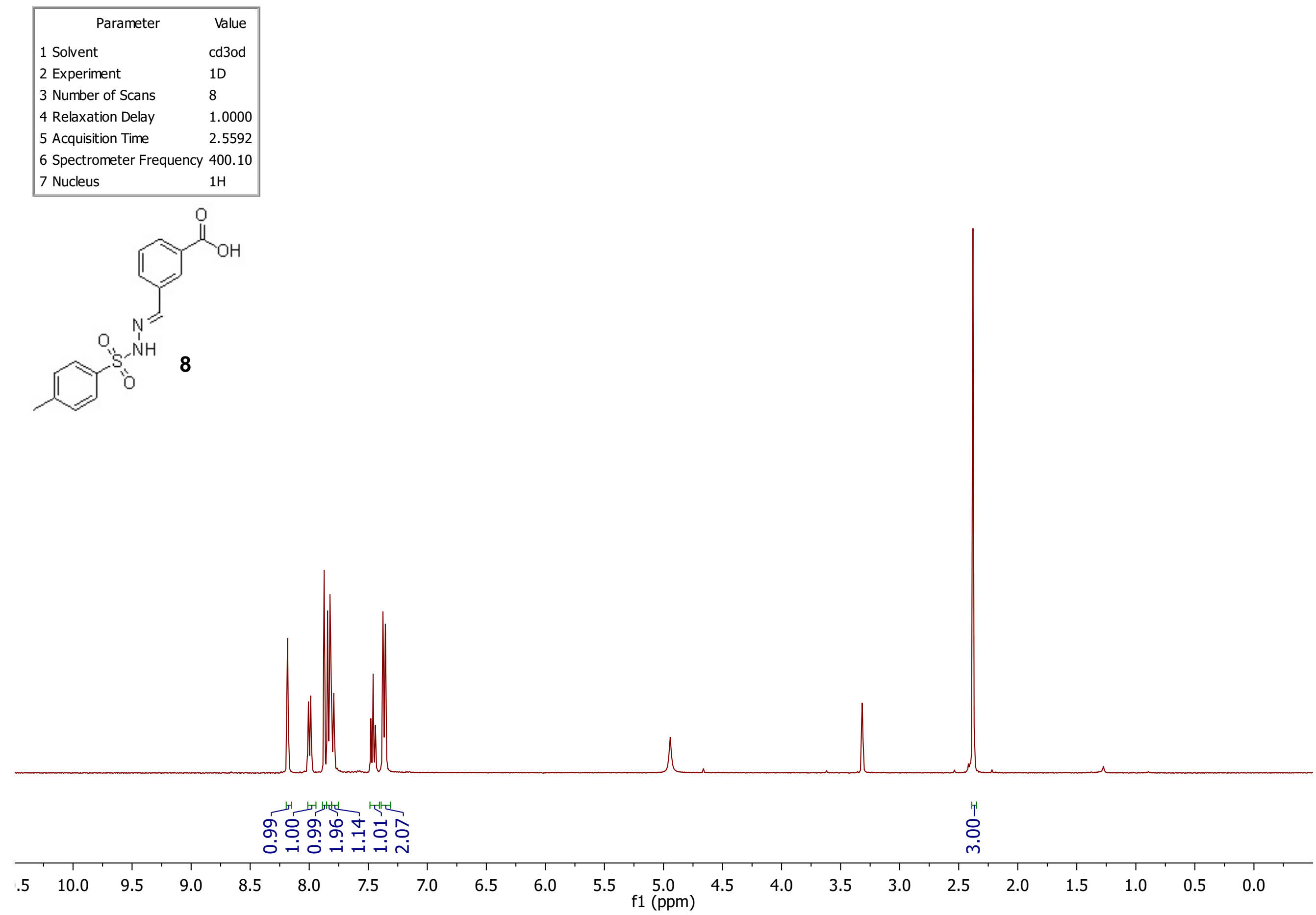


\begin{tabular}{|ll|}
\hline 1 Solvent & \multicolumn{1}{|c|}{ Value } \\
2 Experiment & $1 \mathrm{~cd} 3 \mathrm{od}$ \\
3 Number of Scans & 256 \\
4 Relaxation Delay & 1.0000 \\
5 Acquisition Time & 1.3042 \\
6 Spectrometer Frequency 100.62 \\
7 Nucleus
\end{tabular}

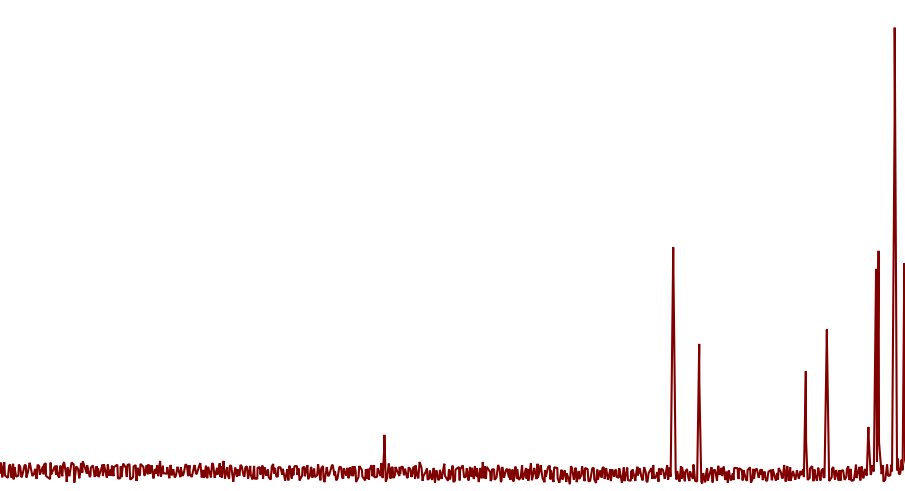

$190 \quad 180 \quad 170$

70

60


$\infty$ \&

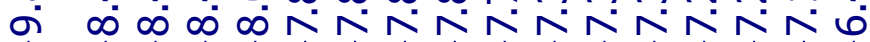

I $\underbrace{\infty}_{m} \underbrace{\infty}_{1}$

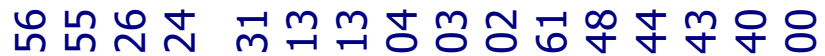

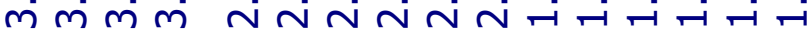

\begin{tabular}{|ll|}
\hline \multicolumn{1}{|c|}{ Parameter } & \multicolumn{1}{c|}{ Value } \\
1 Solvent & cdcl3 \\
2 Experiment & 1D \\
3 Number of Scans & 8 \\
4 Relaxation Delay & 1.0000 \\
5 Acquisition Time & 2.5592 \\
6 Spectrometer Frequency & 400.10 \\
7 Nucleus & $1 \mathrm{H}$ \\
\hline
\end{tabular}
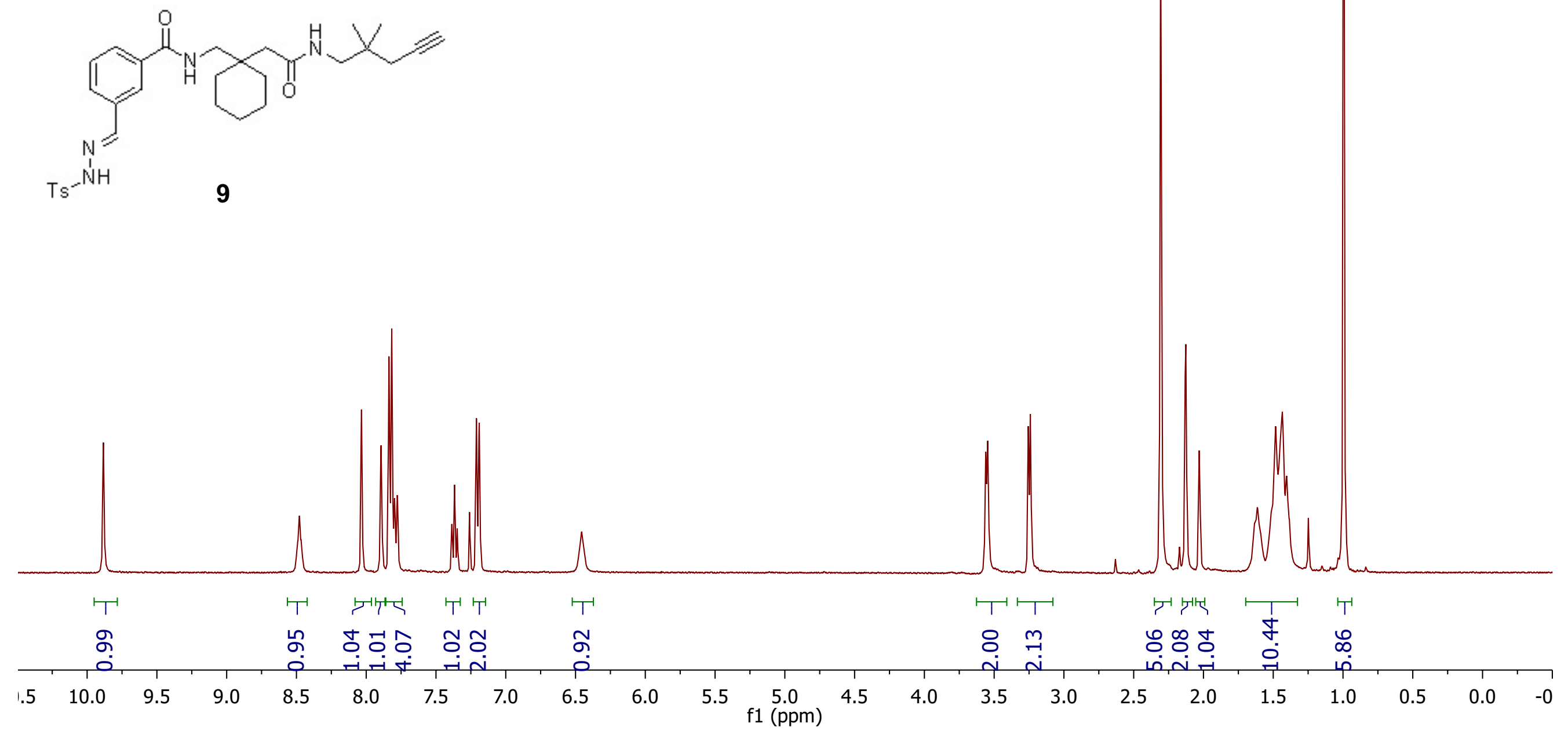


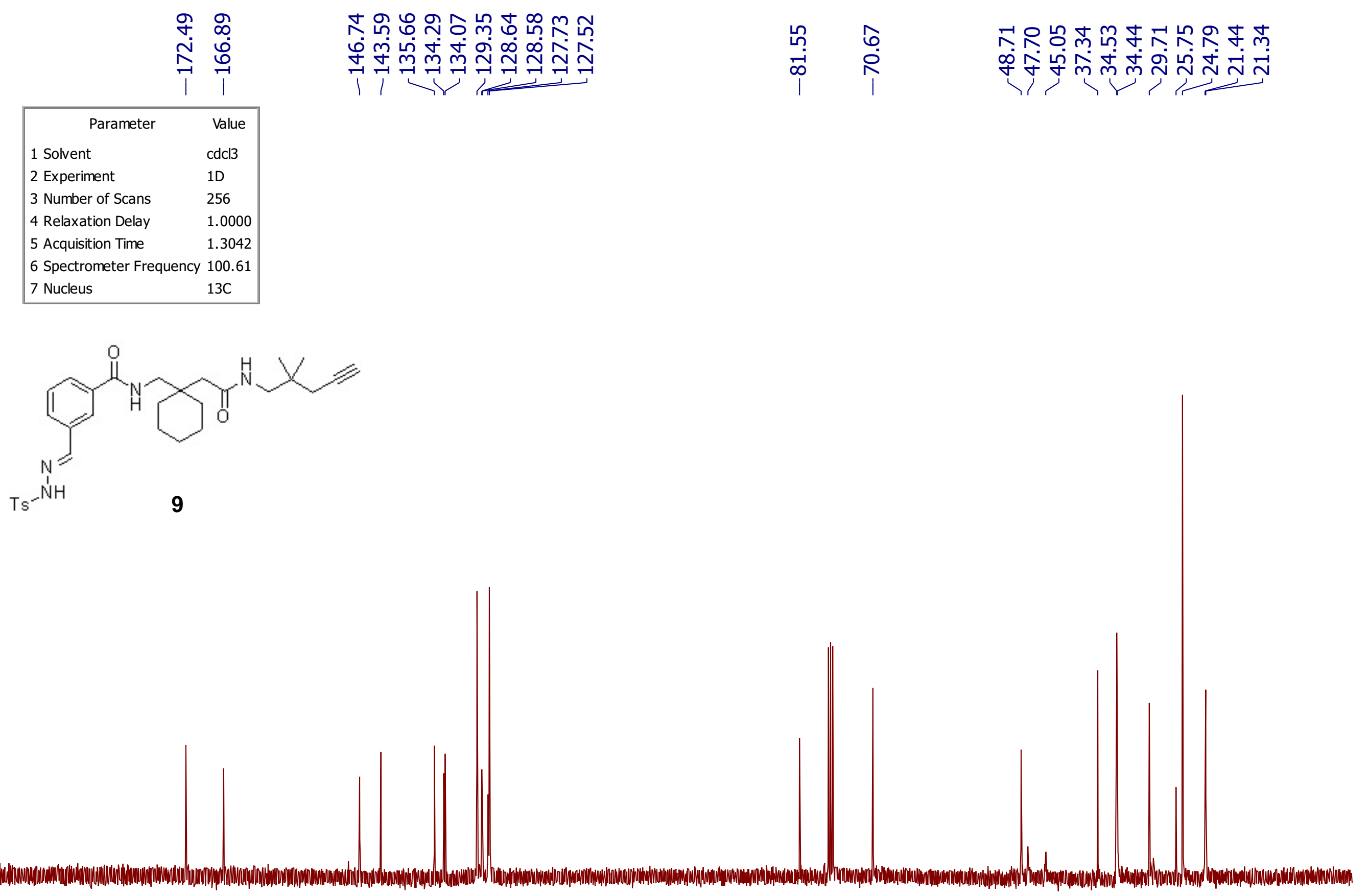

\begin{tabular}{|c|c|c|c|c|c|c|c|c|c|c|c|c|c|c|c|c|c|c|c|}
\hline )0 & 190 & 180 & 170 & 160 & 150 & 140 & 130 & 120 & 110 & $\begin{array}{c}100 \\
\mathrm{f} 1(\mathrm{ppm})\end{array}$ & 90 & 80 & 70 & 60 & 50 & 40 & 30 & 20 & 10 \\
\hline
\end{tabular}




\section{걱ન 겊}

\begin{tabular}{|ll|}
\hline \multicolumn{1}{|c|}{ Parameter } & \multicolumn{1}{c|}{ Value } \\
1 Solvent & cdcl3 \\
2 Experiment & $1 \mathrm{D}$ \\
3 Number of Scans & 8 \\
4 Relaxation Delay & 1.0000 \\
5 Acquisition Time & 2.5592 \\
6 Spectrometer Frequency & 400.09 \\
7 Nucleus & $1 \mathrm{H}$ \\
\hline
\end{tabular}

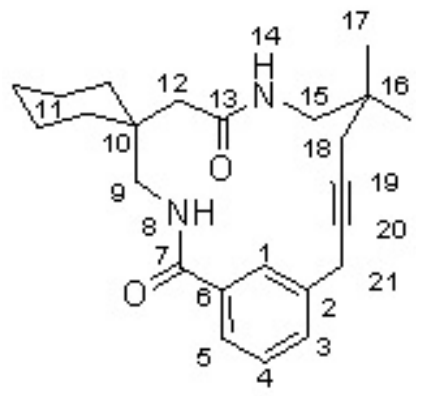

11

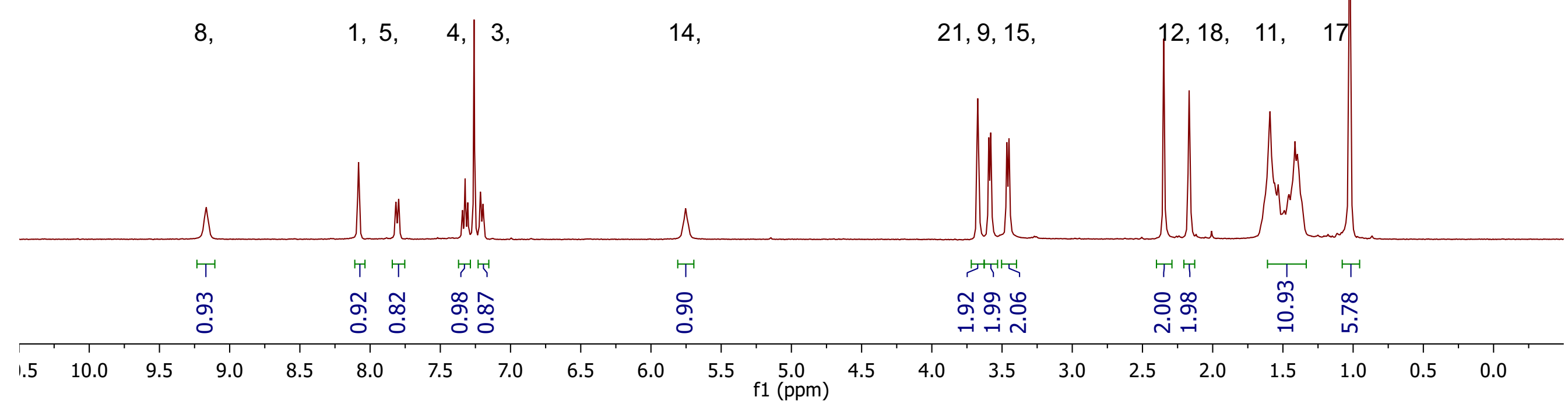




\begin{tabular}{|ll|}
\hline \multicolumn{1}{|c|}{ Parameter } & \multicolumn{1}{c|}{ Value } \\
1 Solvent & cdcl3 \\
2 Experiment & $1 \mathrm{D}$ \\
3 Number of Scans & 3000 \\
4 Relaxation Delay & 1.0000 \\
5 Acquisition Time & 1.3107 \\
6 Spectrometer Frequency & 100.59 \\
7 Nucleus & $13 \mathrm{C}$ \\
\hline
\end{tabular}

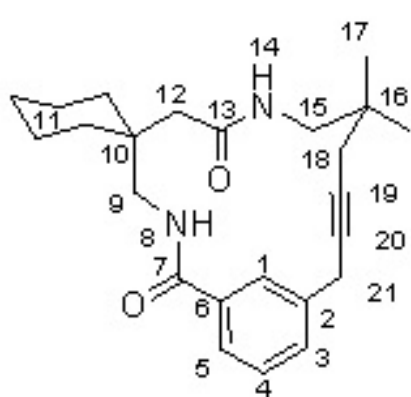

11
11,17

13,7

$2,6, \quad 3,4,5,1$,

19,20,

$12,9,15, \quad 16,11$

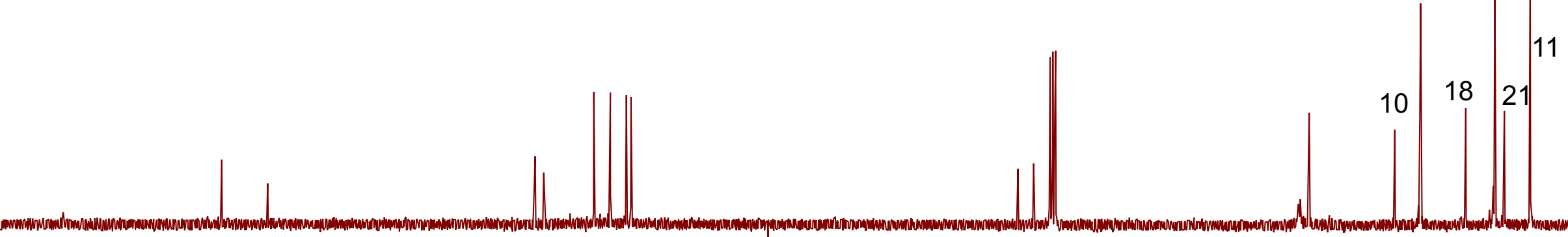




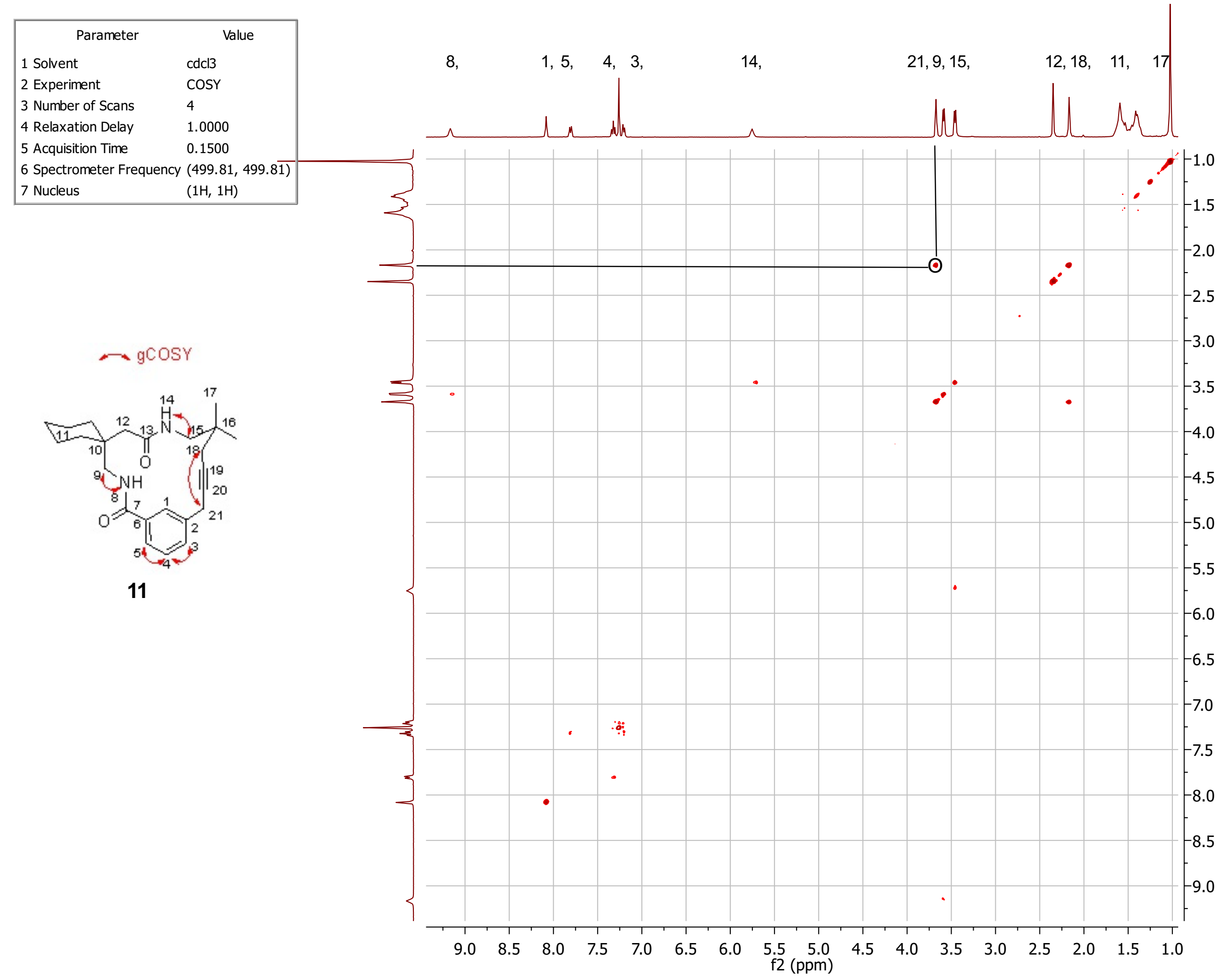

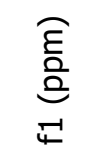




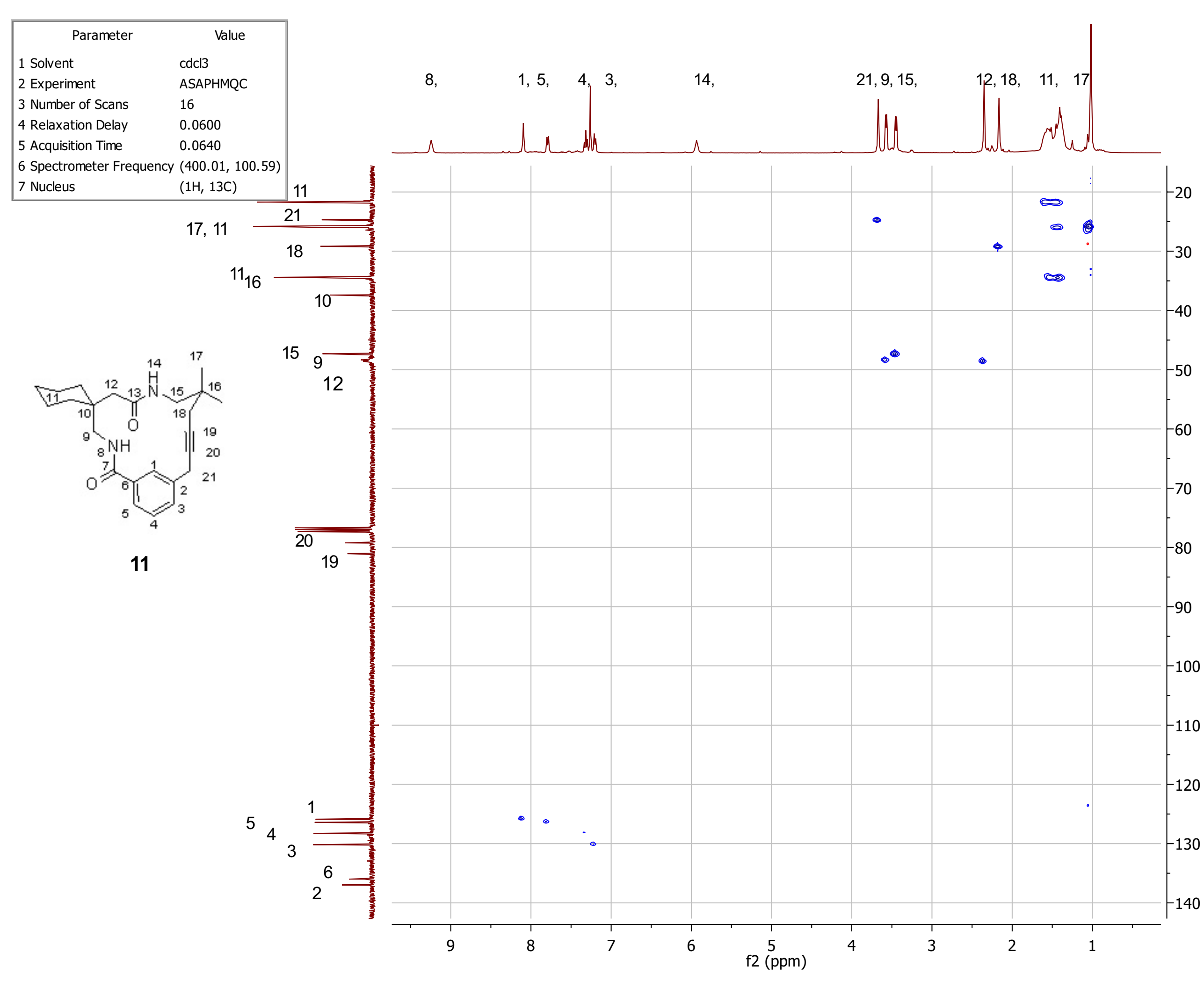




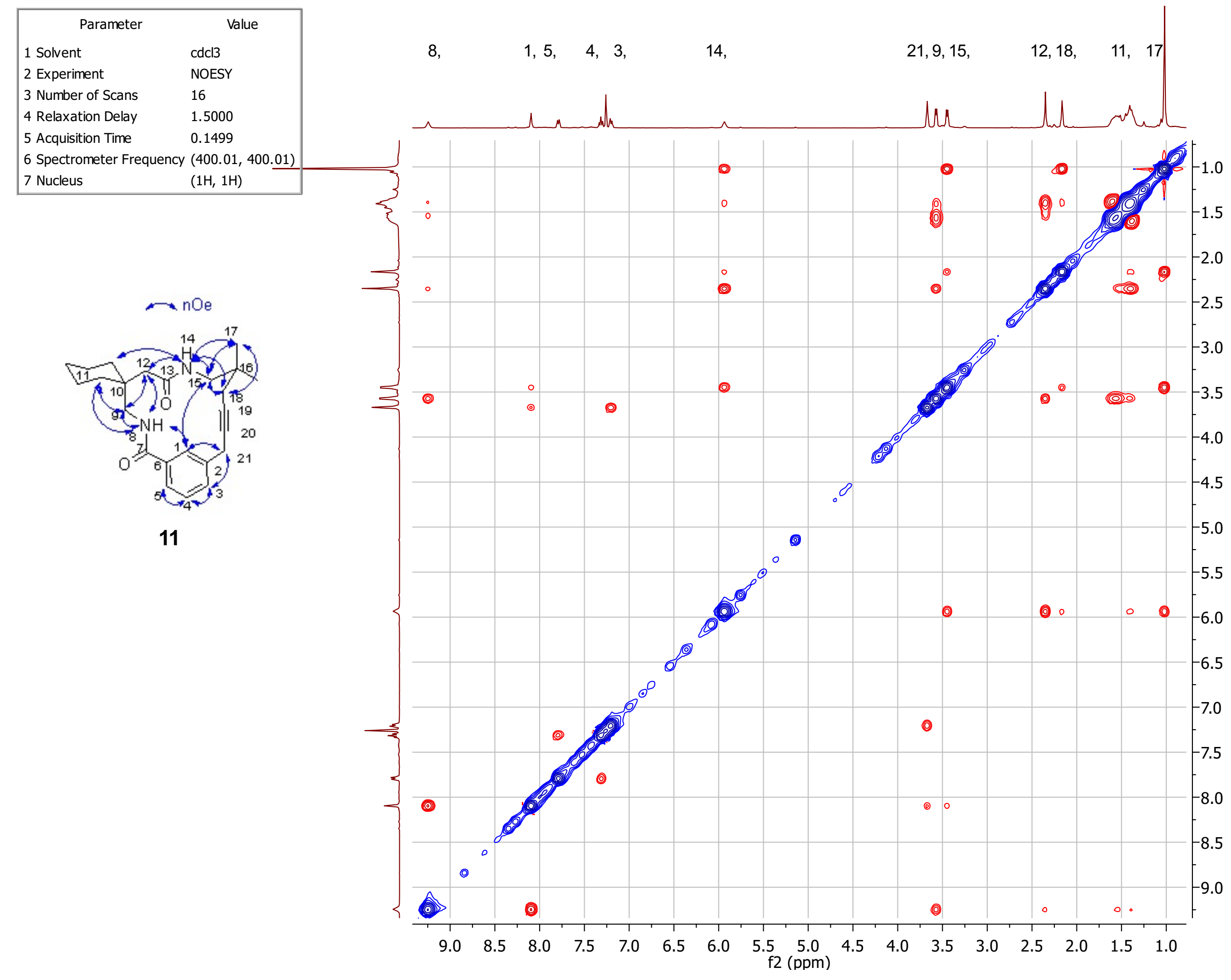

$\widehat{\varepsilon}$
는 


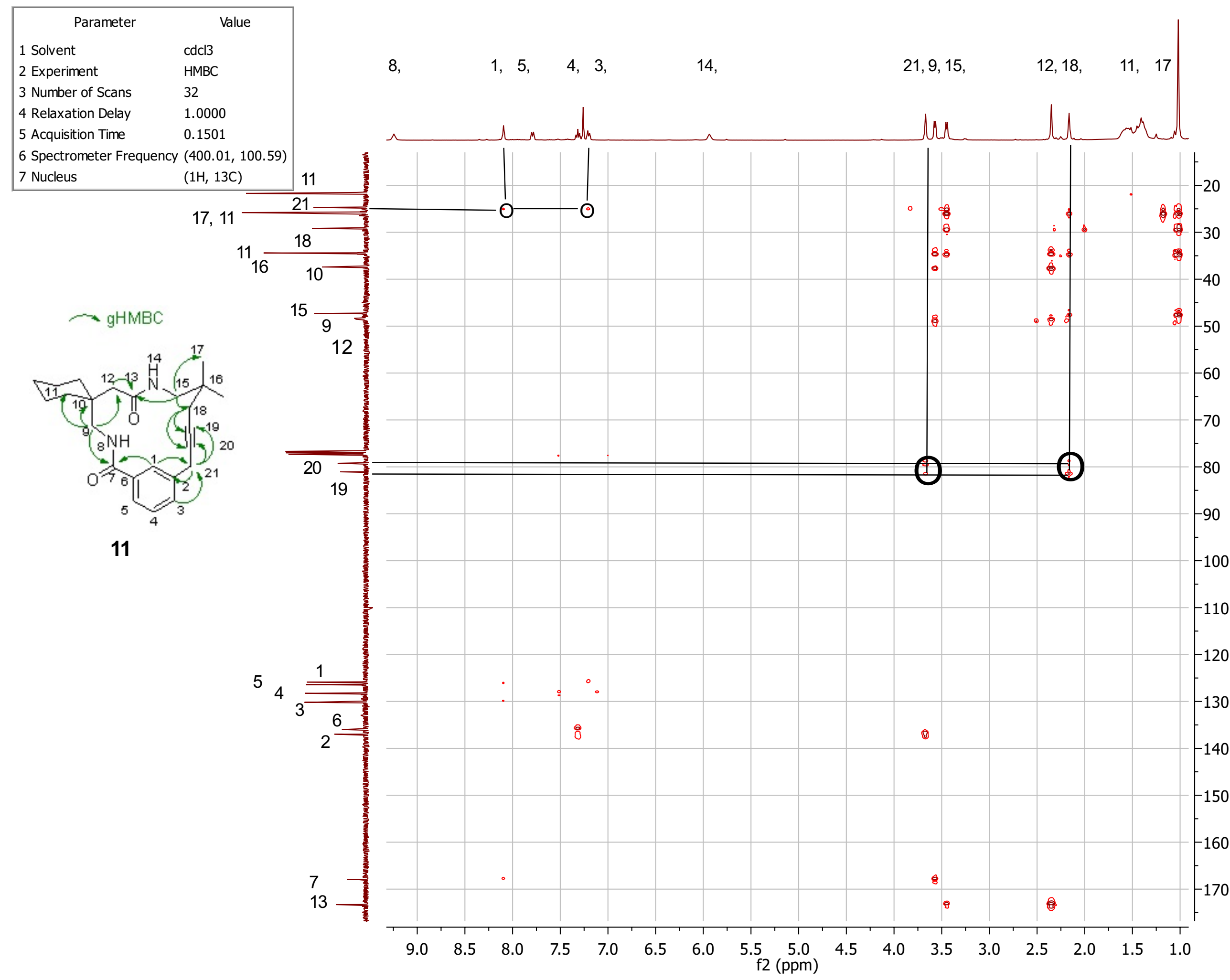

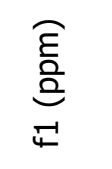




\begin{tabular}{|ll|}
\hline \multicolumn{1}{|c|}{ Parameter } & \multicolumn{1}{c|}{ Value } \\
1 Solvent & cdcl3 \\
2 Experiment & $1 \mathrm{D}$ \\
3 Number of Scans & 8 \\
4 Relaxation Delay & 1.0000 \\
5 Acquisition Time & 2.0447 \\
6 Spectrometer Frequency & 499.81 \\
7 Nucleus & $1 \mathrm{H}$ \\
\hline
\end{tabular}

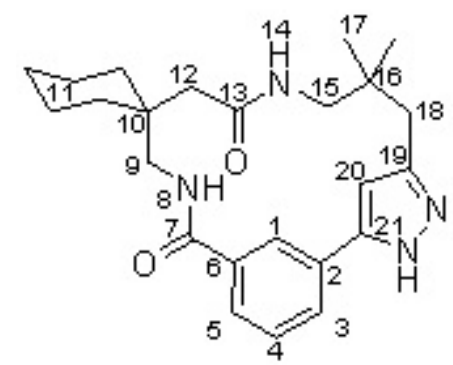

17

8 ,

$1,3,5,4, \quad 20$,

14 ,

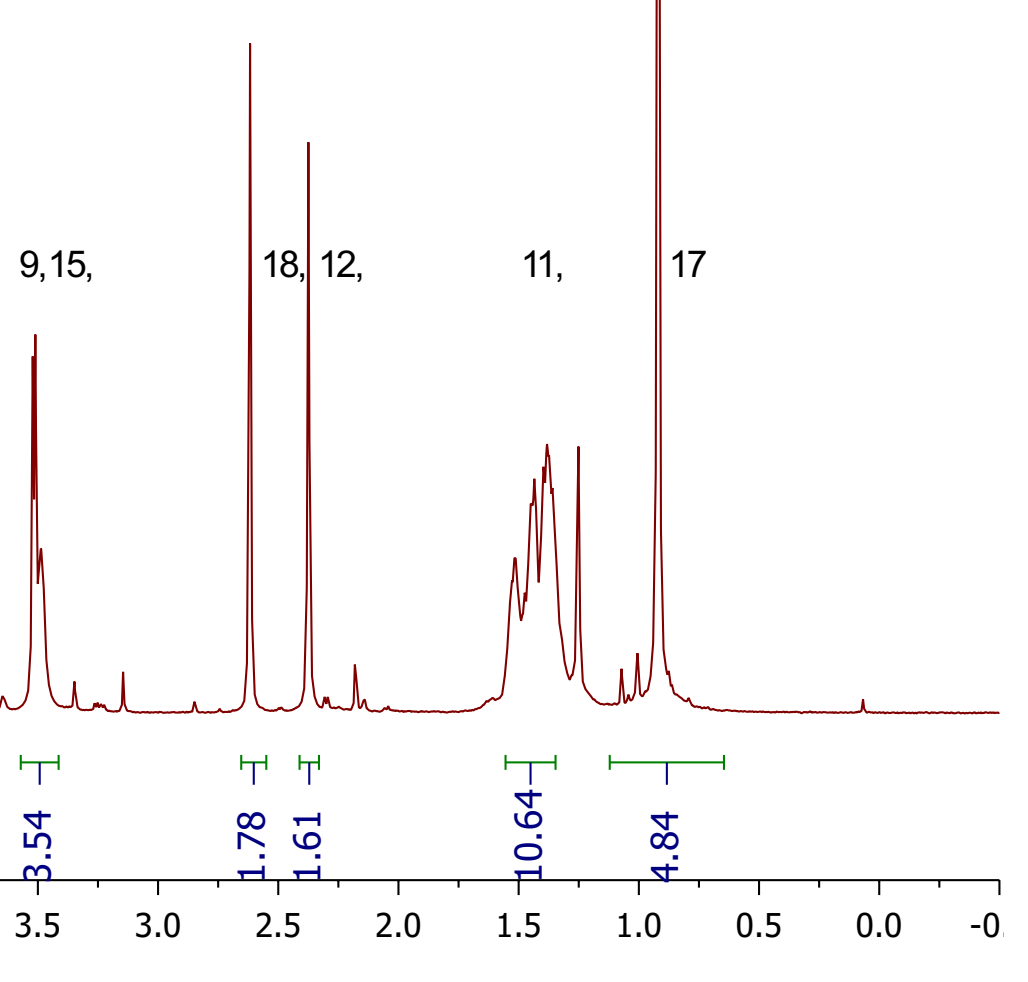




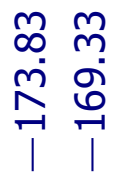

Paramete

1 Solvent

2 Experiment

3 Relaxation Delay

5 Nucleus

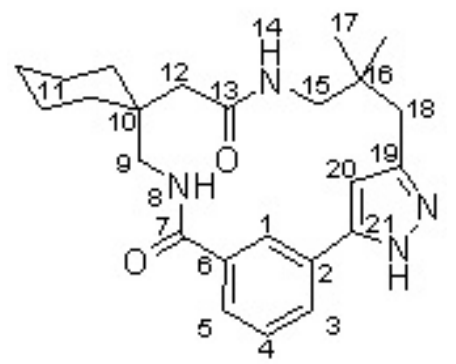

17

13,7 ,

21, $19, \quad 6,1,4,3,5$,

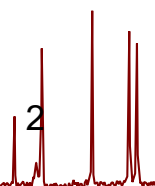




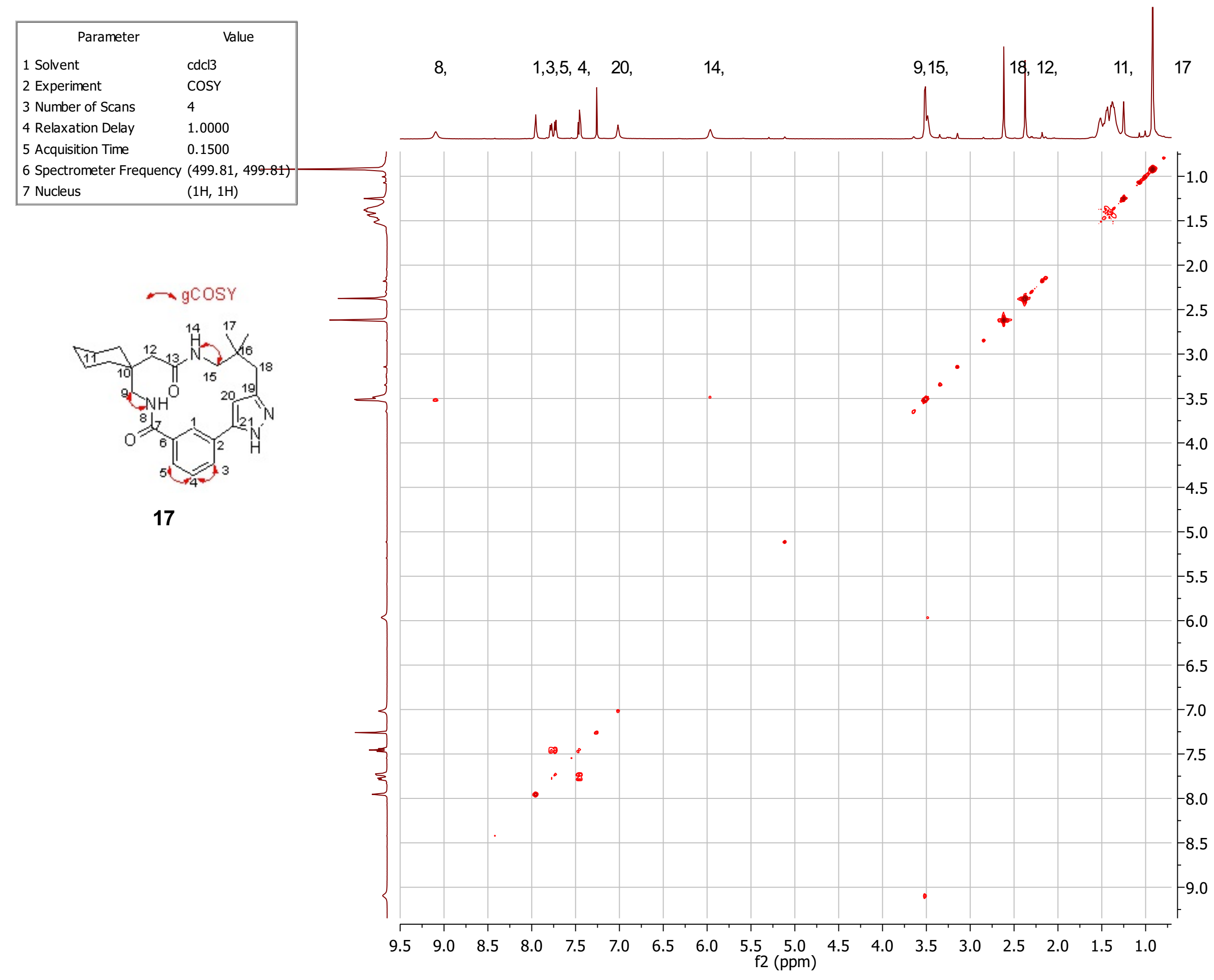

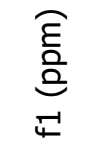




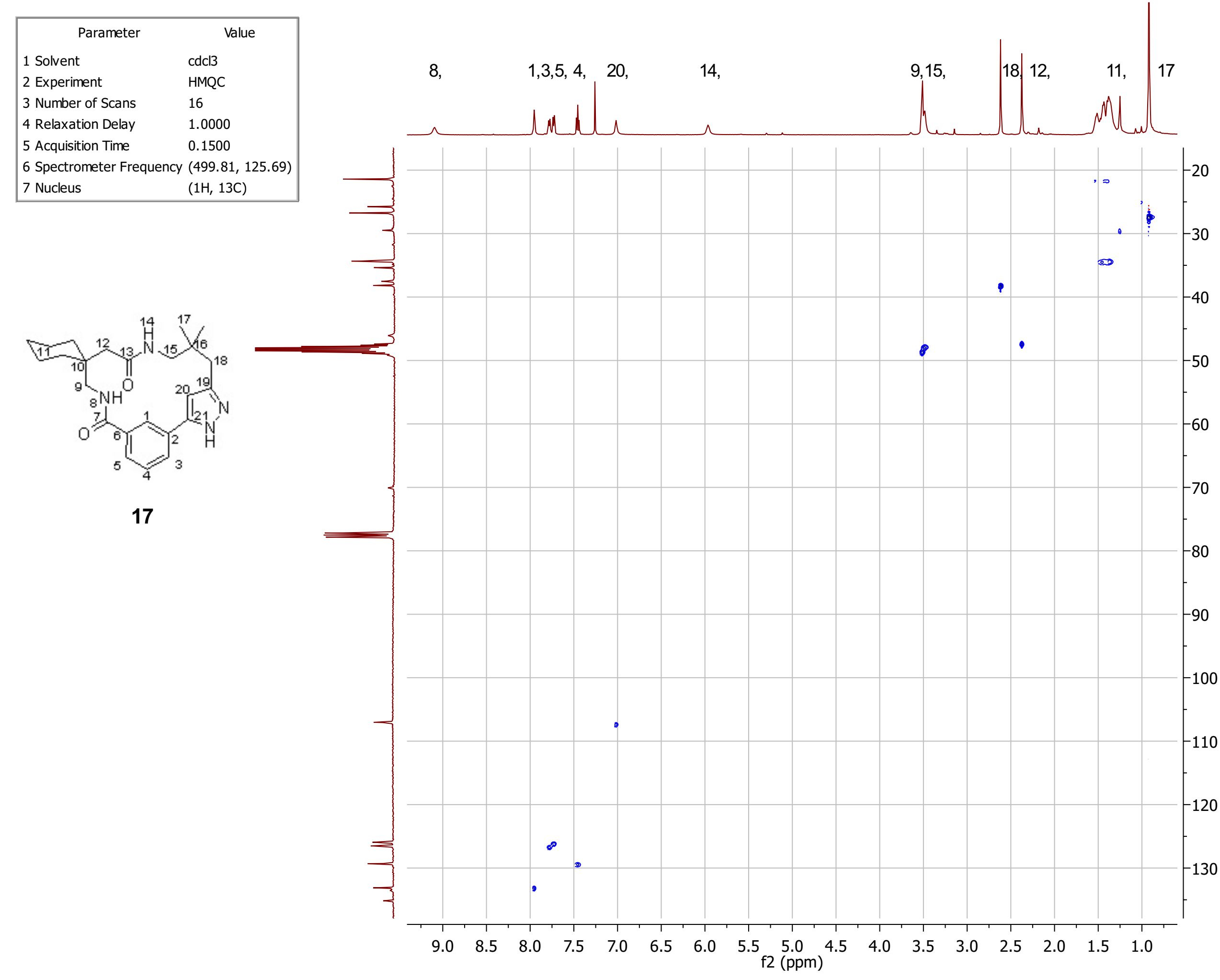




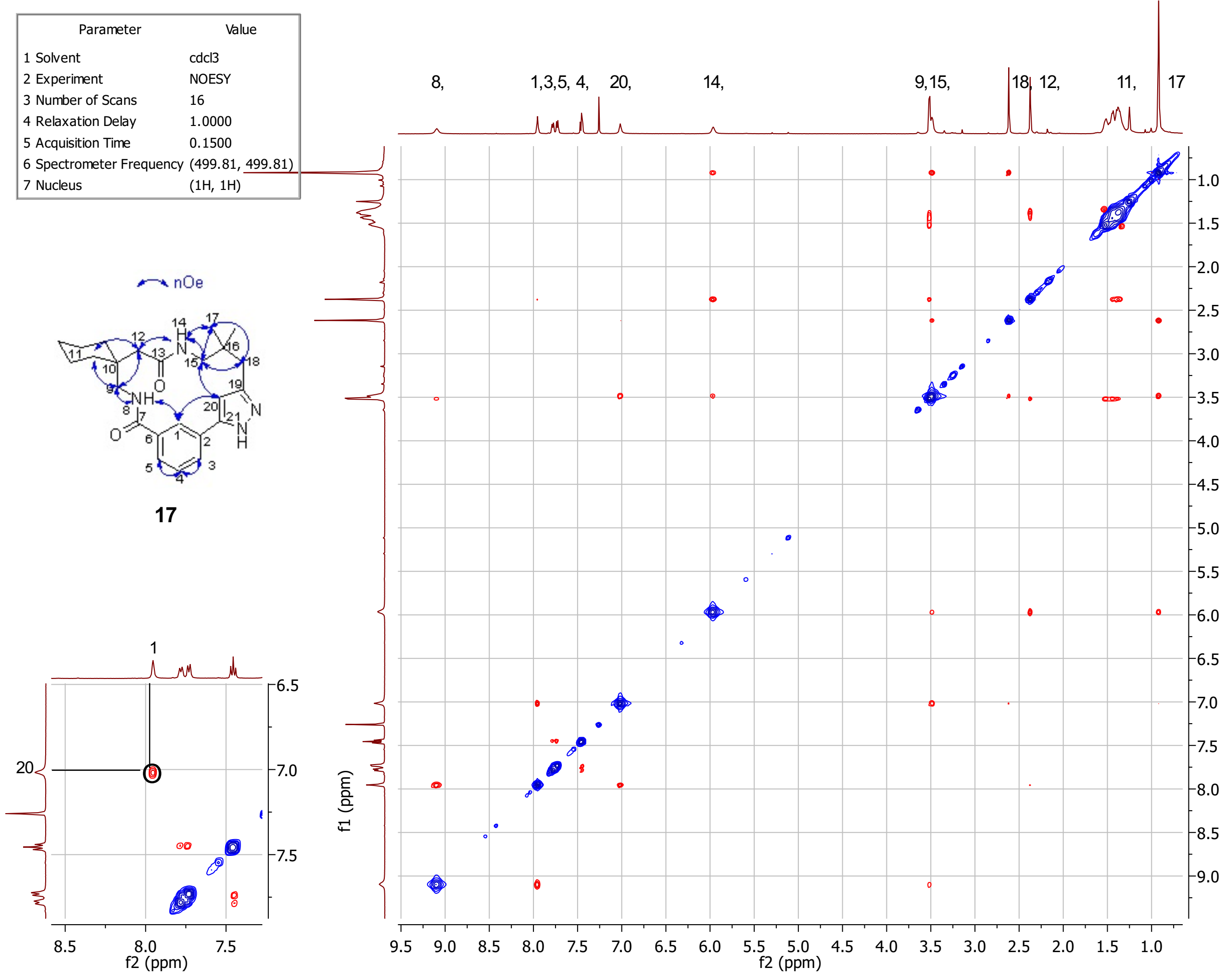

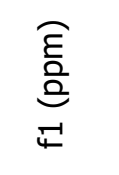




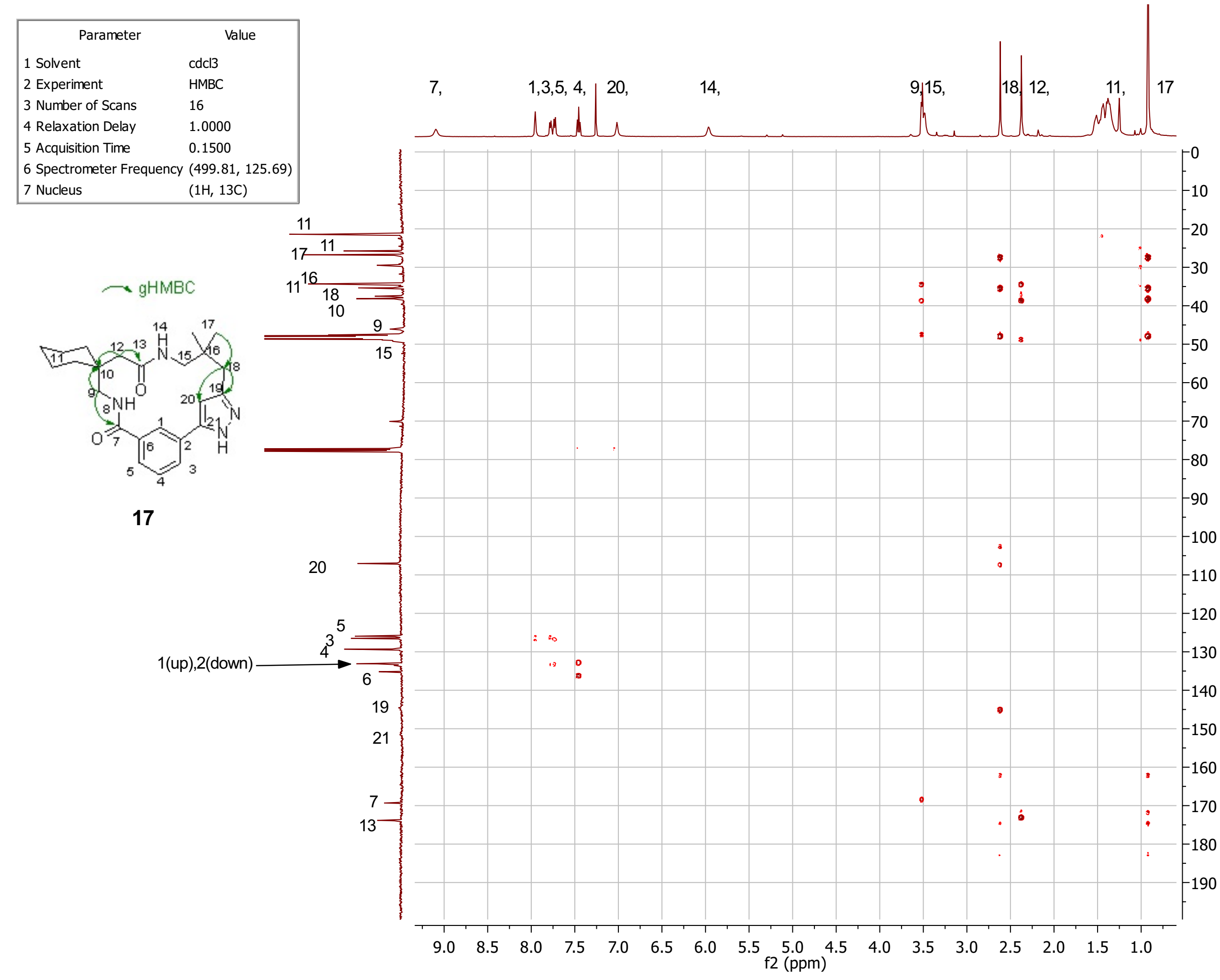




\begin{tabular}{|ll|}
\hline \multicolumn{1}{|c|}{ Parameter } & \multicolumn{1}{c|}{ Value } \\
1 Solvent & cdcl3 \\
2 Experiment & $1 \mathrm{D}$ \\
3 Number of Scans & 8 \\
4 Relaxation Delay & 1.0000 \\
5 Acquisition Time & 2.5592 \\
6 Spectrometer Frequency & 400.09 \\
7 Nucleus & $1 \mathrm{H}$ \\
\hline
\end{tabular}

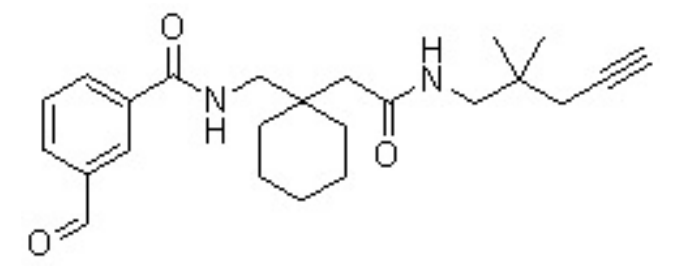

12

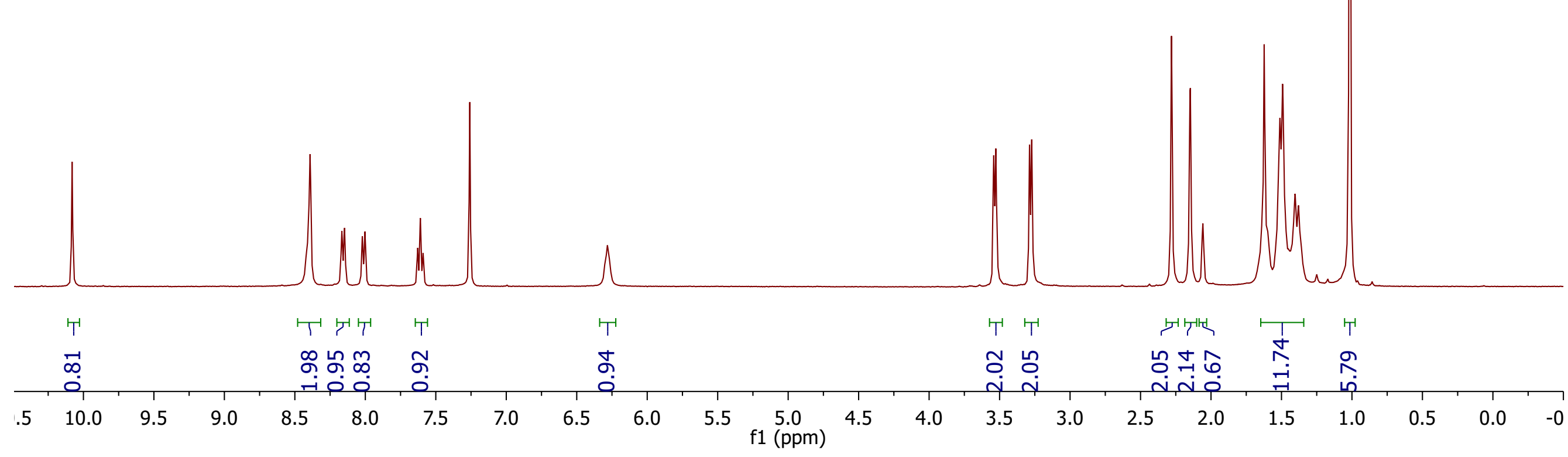




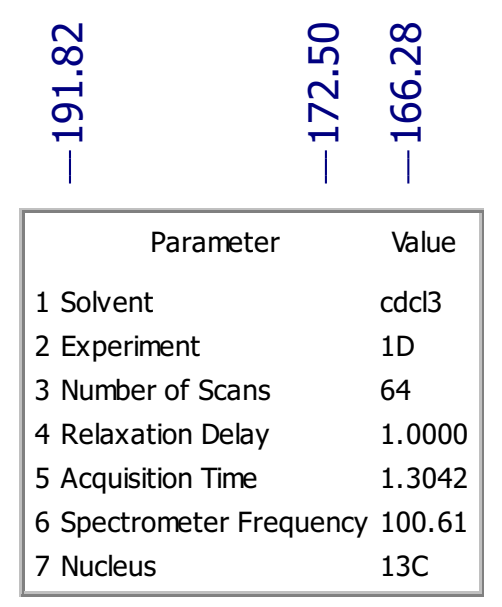

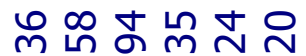

ஸึ่

实示芯

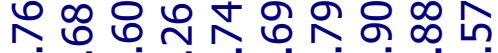

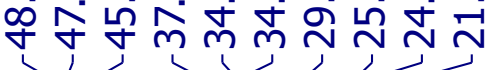

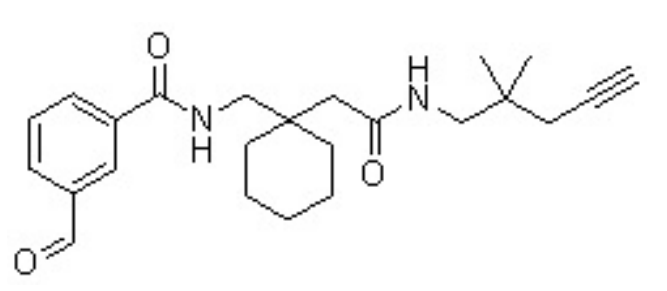

12

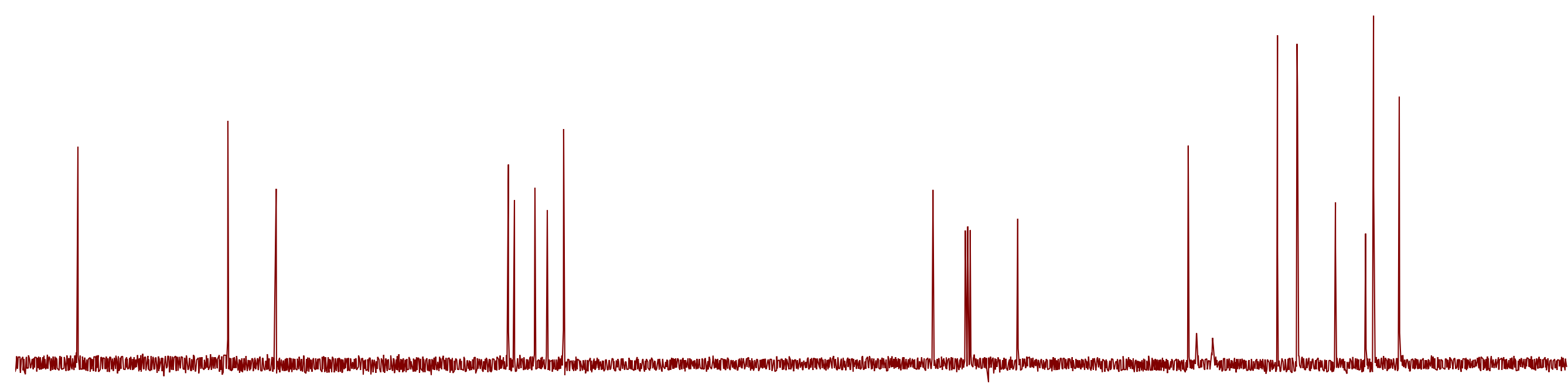




\begin{tabular}{|ll|}
\hline \multicolumn{1}{|c|}{ Parameter } & \multicolumn{1}{c|}{ Value } \\
1 Solvent & cdcl3 \\
2 Experiment & $1 \mathrm{D}$ \\
3 Number of Scans & 8 \\
4 Relaxation Delay & 1.0000 \\
5 Acquisition Time & 2.5592 \\
6 Spectrometer Frequency & 400.09 \\
7 Nucleus & $1 \mathrm{H}$ \\
\hline
\end{tabular}

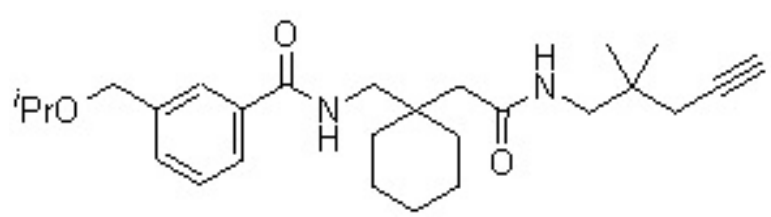

13
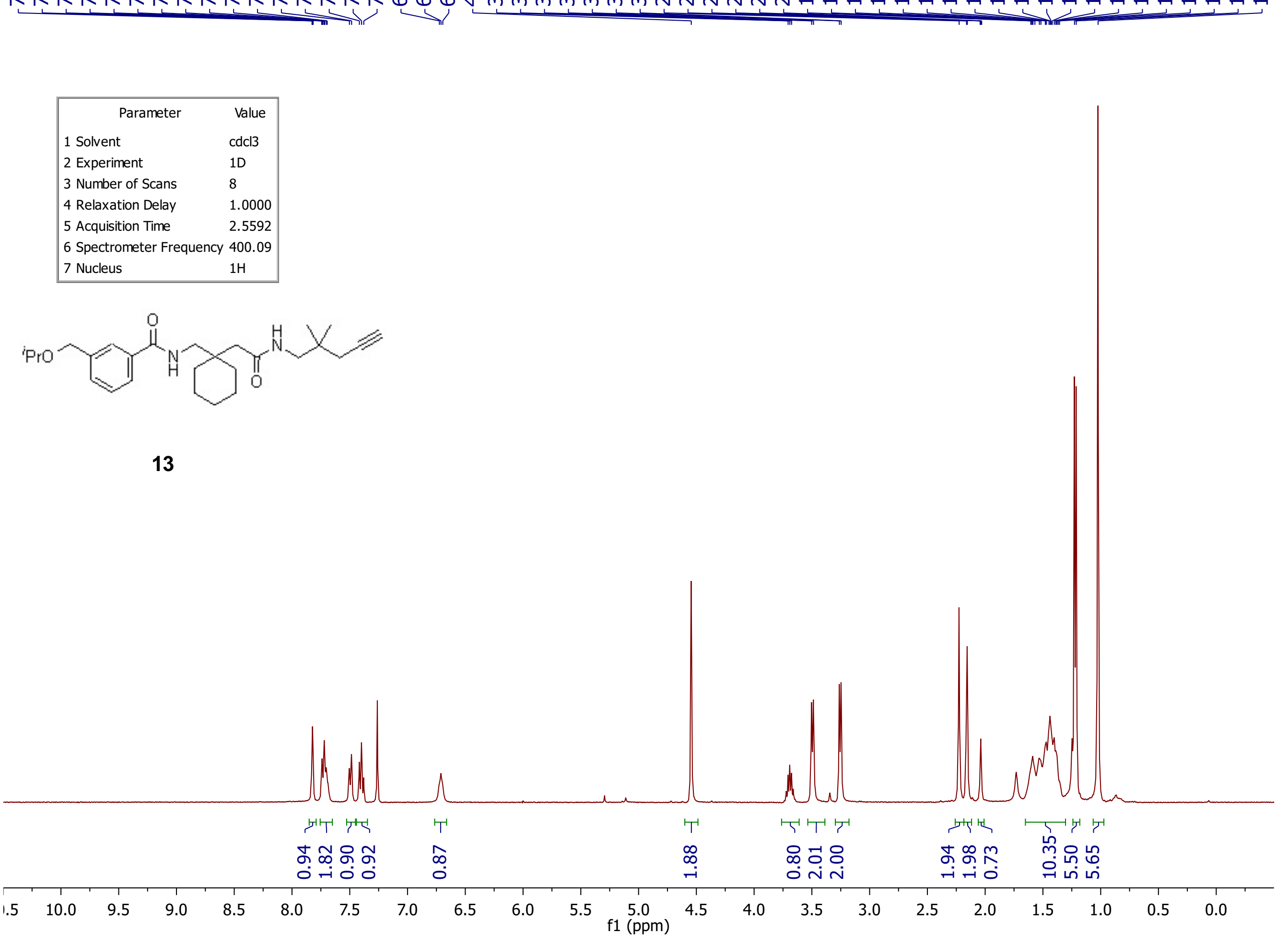


\begin{tabular}{|ll|}
\hline \multicolumn{1}{|c}{ Parameter } & \multicolumn{1}{c|}{ Value } \\
1 Solvent & cdcl3 \\
2 Experiment & $1 \mathrm{D}$ \\
3 Number of Scans & 10000 \\
4 Relaxation Delay & 1.0000 \\
5 Acquisition Time & 1.3042 \\
6 Spectrometer Frequency & 100.61 \\
7 Nucleus & $13 \mathrm{C}$ \\
\hline
\end{tabular}

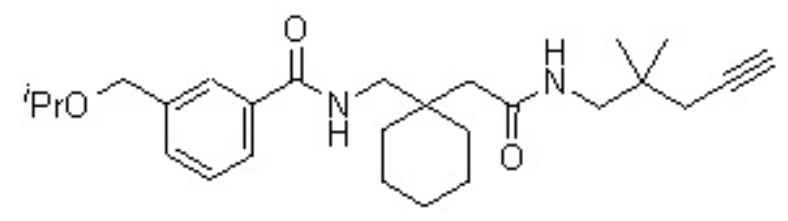

13

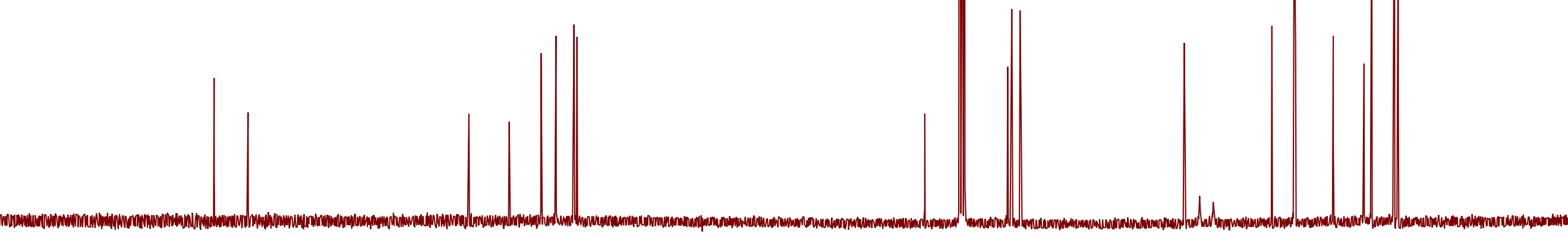

\begin{tabular}{|c|c|c|c|c|c|c|c|c|c|c|c|c|c|c|c|c|c|c|}
\hline 190 & 180 & 170 & 160 & 150 & 140 & 130 & 120 & 110 & 100 & 90 & 80 & 70 & 60 & 50 & 40 & 30 & 20 & 10 \\
\hline
\end{tabular}




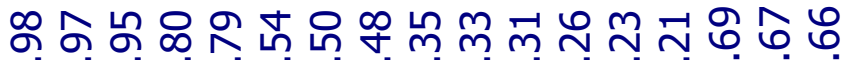

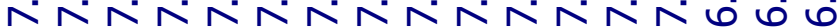

\begin{tabular}{|ll|}
\hline \multicolumn{1}{|c|}{ Parameter } & \multicolumn{1}{c|}{ Value } \\
1 Solvent & cdcl3 \\
2 Experiment & $1 \mathrm{D}$ \\
3 Number of Scans & 8 \\
4 Relaxation Delay & 1.0000 \\
5 Acquisition Time & 2.5592 \\
6 Spectrometer Frequency & 400.09 \\
7 Nucleus & $1 \mathrm{H}$ \\
\hline
\end{tabular}

14



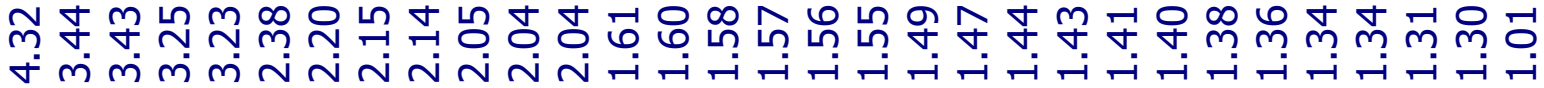




\begin{tabular}{|ll|}
\hline \multicolumn{1}{|c|}{ Parameter } & \multicolumn{1}{c|}{ Value } \\
1 Solvent & cdcl3 \\
2 Experiment & 1D \\
3 Number of Scans & 256 \\
4 Relaxation Delay & 1.0000 \\
5 Acquisition Time & 1.3042 \\
6 Spectrometer Frequency & 100.61 \\
7 Nucleus & $13 \mathrm{C}$ \\
\hline
\end{tabular}

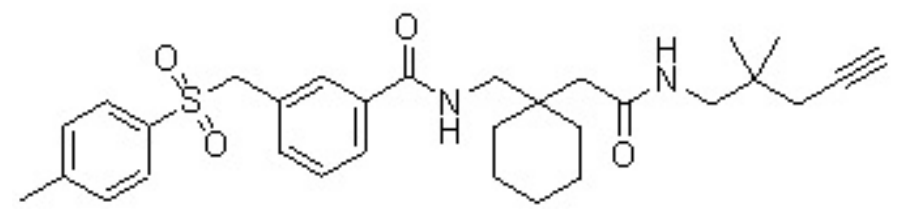

14

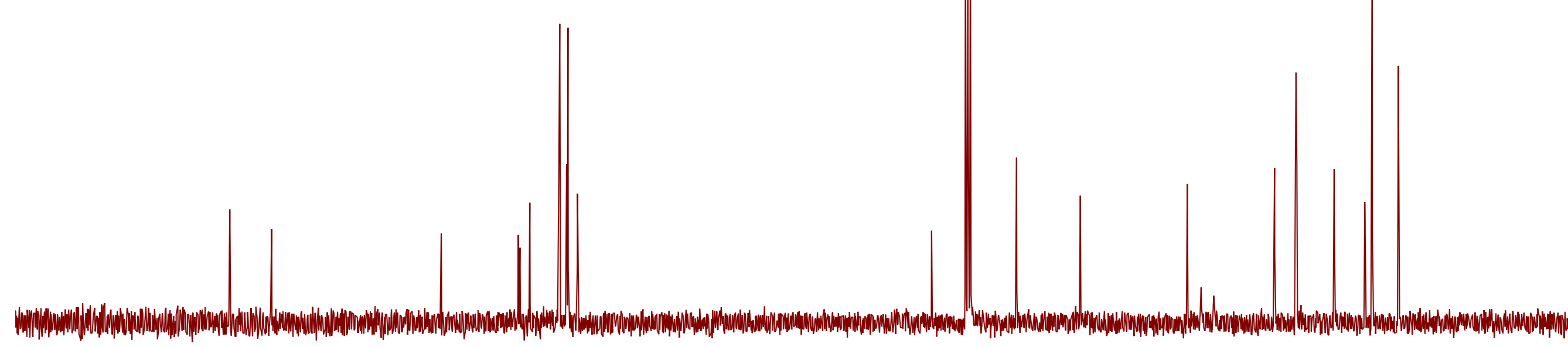

\begin{tabular}{|c|c|c|c|c|c|c|c|c|c|c|c|c|c|c|c|c|c|c|c|}
\hline 10 & 190 & 180 & 170 & 160 & 150 & 14 & 130 & 120 & 110 & $\begin{array}{c}100 \\
\mathrm{f} 1(\mathrm{ppm})\end{array}$ & 90 & 80 & 70 & 60 & 50 & 40 & 30 & 20 & 10 \\
\hline
\end{tabular}




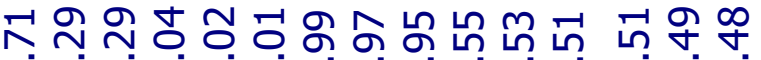
$\infty \infty \infty \infty \infty \infty)$

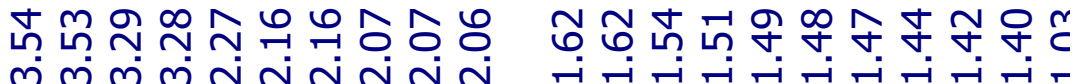
m

\begin{tabular}{|ll|}
\hline \multicolumn{1}{|c|}{ Parameter } & \multicolumn{1}{c|}{ Value } \\
1 Solvent & cdcl3 \\
2 Experiment & $1 \mathrm{D}$ \\
3 Number of Scans & 8 \\
4 Relaxation Delay & 1.0000 \\
5 Acquisition Time & 2.5592 \\
6 Spectrometer Frequency & 400.09 \\
7 Nucleus & $1 \mathrm{H}$ \\
\hline
\end{tabular}

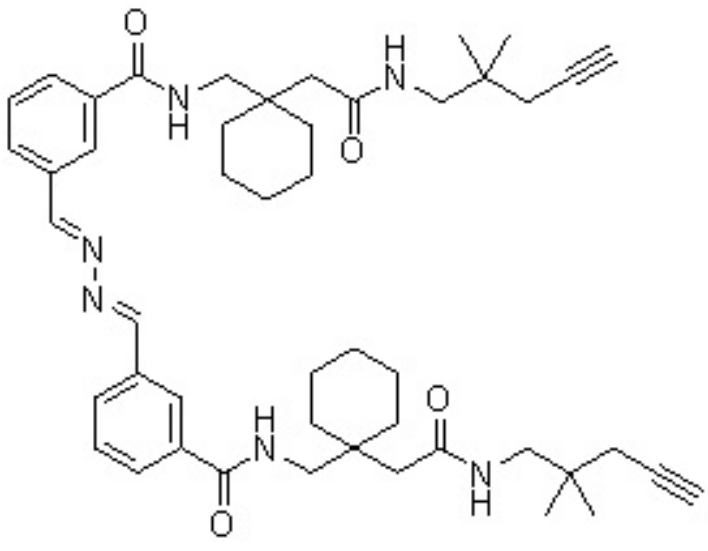

15

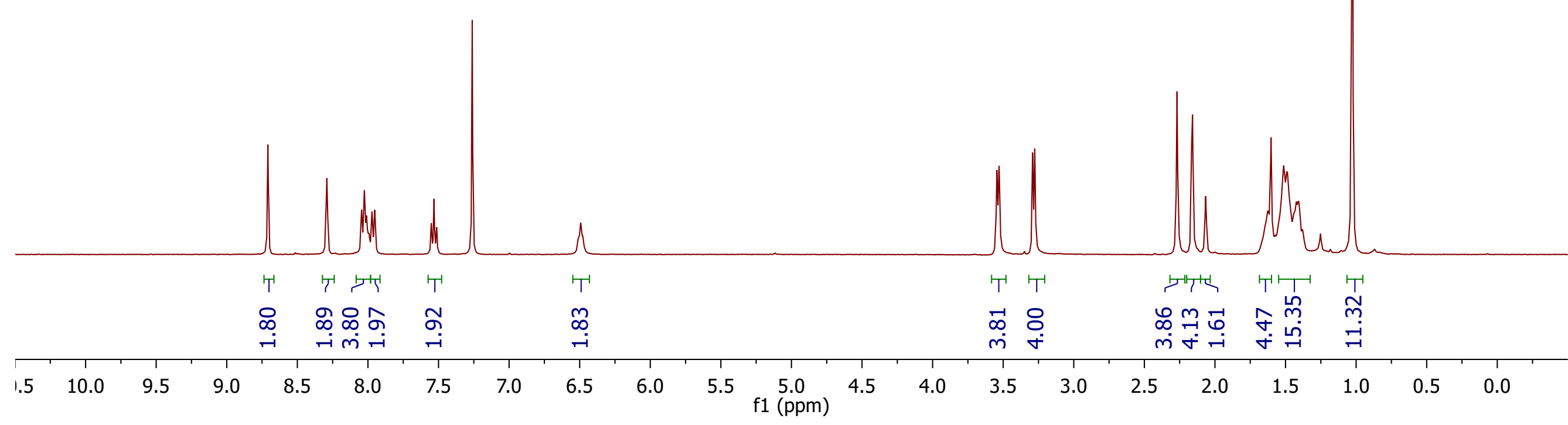




\begin{tabular}{|ll|}
\hline \multicolumn{1}{|c|}{ Parameter } & \multicolumn{1}{c|}{ Value } \\
1 Solvent & cdcl3 \\
2 Experiment & 1D \\
3 Number of Scans & 512 \\
4 Relaxation Delay & 1.0000 \\
5 Acquisition Time & 1.3042 \\
6 Spectrometer Frequency & 100.61 \\
7 Nucleus & $13 \mathrm{C}$ \\
\hline
\end{tabular}

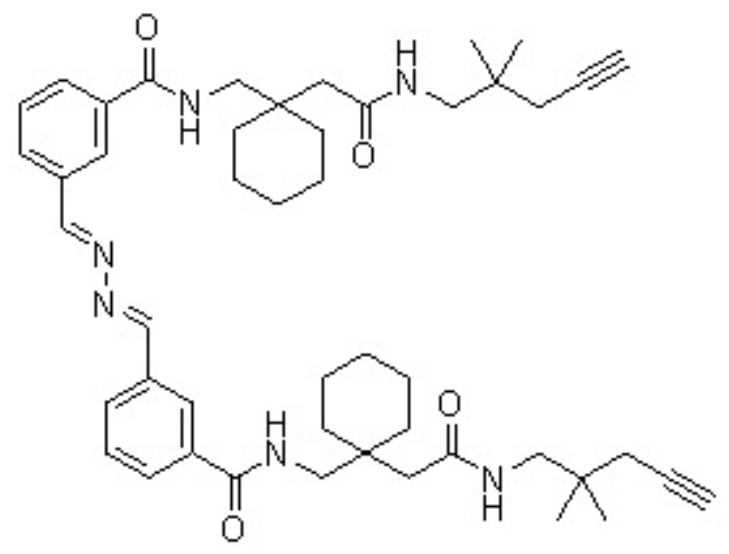

$180 \quad 170$

160

150

140

130

$80 \quad 70$

70

60




\begin{tabular}{|ll|}
\hline \multicolumn{1}{|c|}{ Parameter } & \multicolumn{1}{c|}{ Value } \\
1 Solvent & cdcl3 \\
2 Experiment & $1 \mathrm{D}$ \\
3 Number of Scans & 8 \\
4 Relaxation Delay & 1.0000 \\
5 Acquisition Time & 2.5592 \\
6 Spectrometer Frequency & 400.09 \\
7 Nucleus & $1 \mathrm{H}$ \\
\hline
\end{tabular}

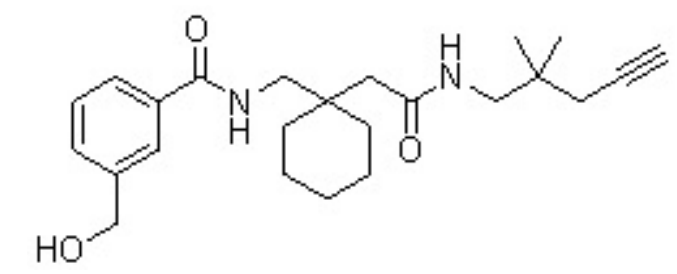

18

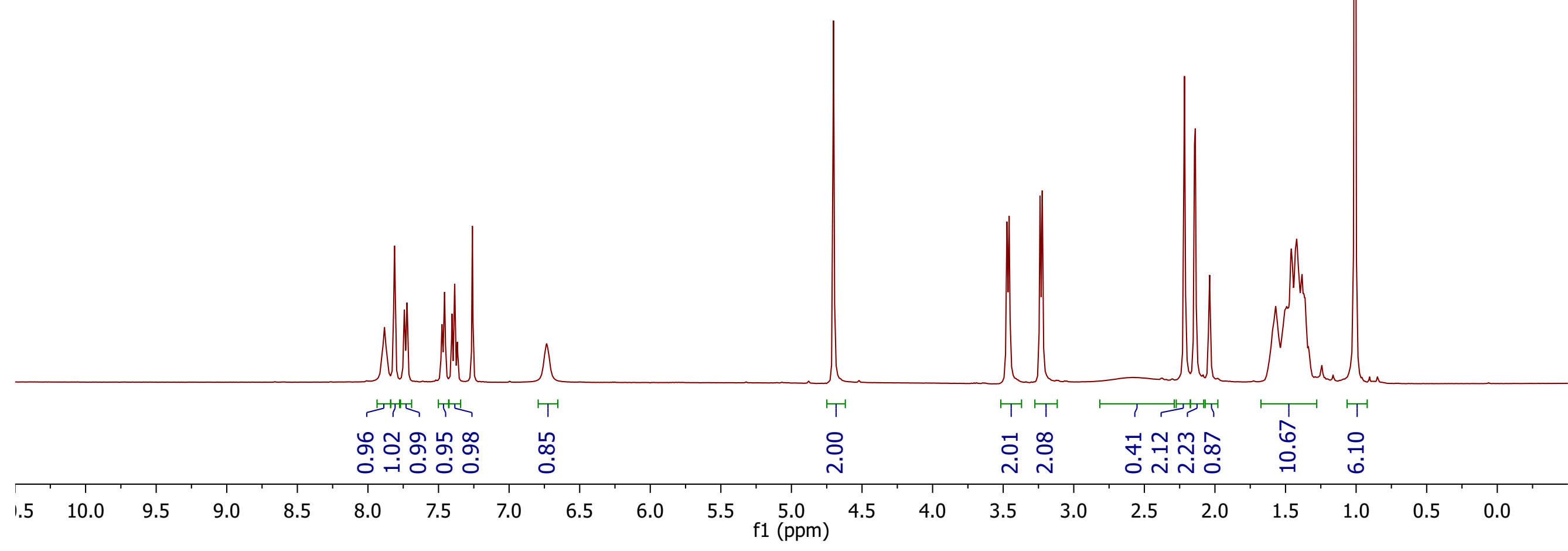


Parameter

Value

1 Solvent $\mathrm{cdcl} 3$

2 Experiment

3 Number of Scans

1D

4 Relaxation Delay

128

5 Acquisition Time

1.0000

7 Nucleus

$13 \mathrm{C}$

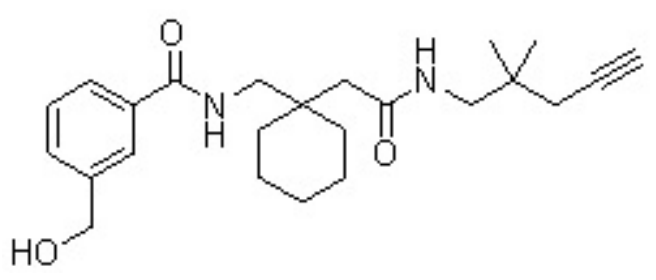

18

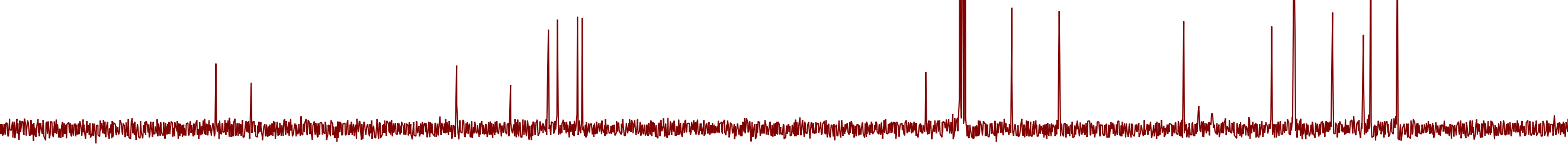

\begin{tabular}{|c|c|c|c|c|c|c|c|c|c|c|c|c|c|c|c|c|c|c|c|}
\hline & $T$ & $T$ & $T$ & $T$ & $T$ & $T$ & $T$ & $T$ & $T$ & $T$ & $T$ & $T$ & $T$ & $T$ & $T$ & $T$ & $T$ & $T$ & 1 \\
\hline$) 0$ & 190 & 180 & 170 & 160 & 150 & 140 & 130 & 120 & 110 & $\begin{array}{l}100 \\
\mathrm{f} 1(\mathrm{ppm})\end{array}$ & 90 & 80 & 70 & 60 & 50 & 40 & 30 & 20 & 10 \\
\hline
\end{tabular}




\begin{tabular}{ll|}
1 Parameter & \multicolumn{1}{|c|}{ Value } \\
2 Experiment & dmso \\
3 Number of Scans & $1 \mathrm{D}$ \\
4 Relaxation Delay & 1.0000 \\
5 Acquisition Time & 2.5592 \\
6 Spectrometer Frequency 400.09 \\
7 Nucleus
\end{tabular}

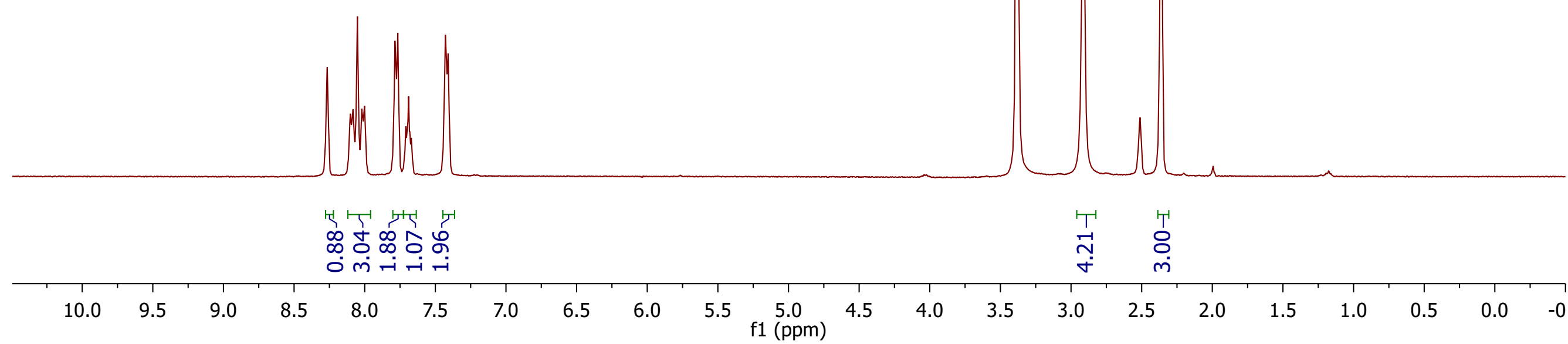




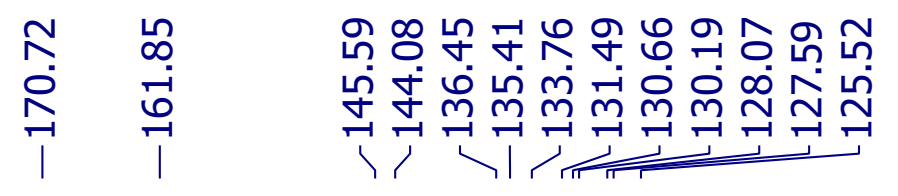

Parameter

Value

1 Solvent

dmso

2 Experiment

3 Number of Scans

4 Relaxation Delay

1D

5 Acquisition Time

Spectrometer Frequency 100.61

7 Nucleus $13 \mathrm{C}$
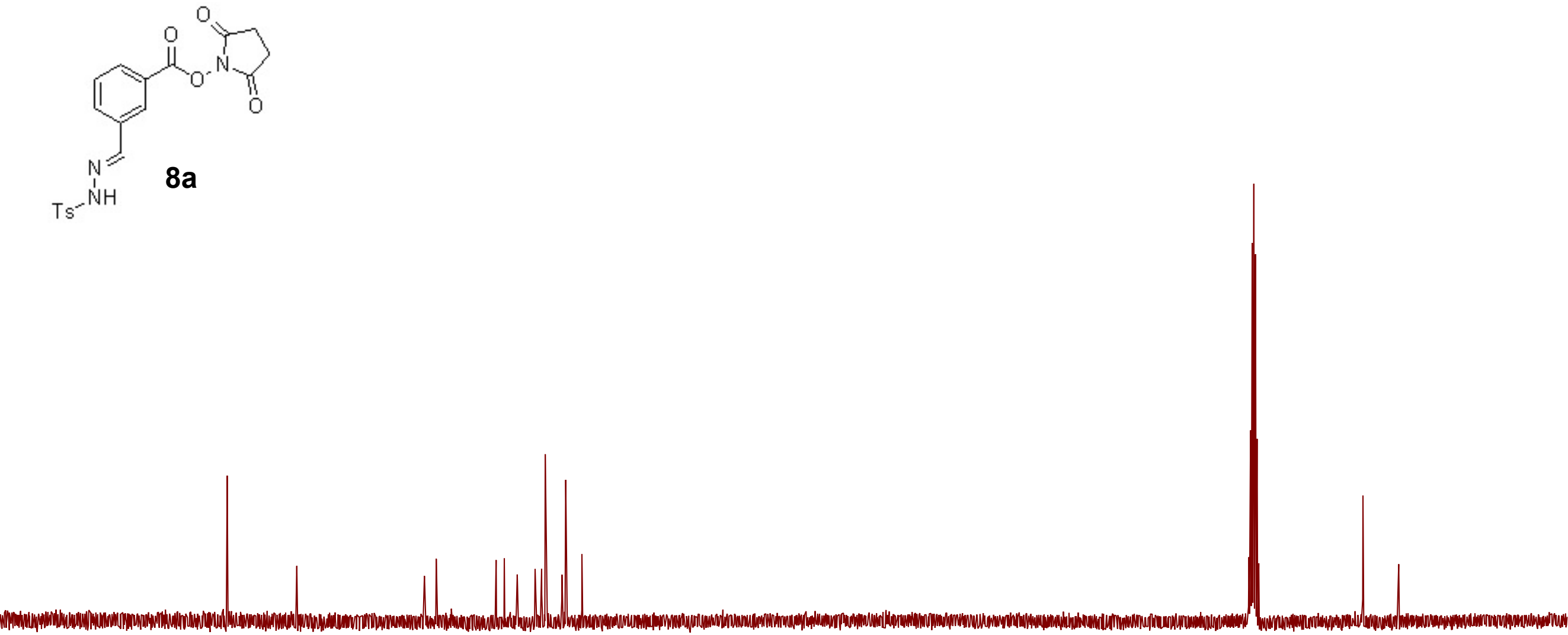


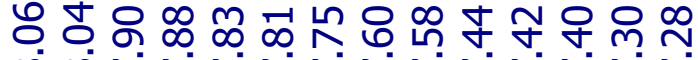 \\ $\infty \infty N N$ N}

\begin{tabular}{|ll|}
\hline \multicolumn{1}{|c}{ Parameter } & \multicolumn{1}{c}{ Value } \\
1 Solvent & cdcl3 \\
2 Experiment & $1 \mathrm{D}$ \\
3 Number of Scans & 8 \\
4 Relaxation Delay & 1.0000 \\
5 Acquisition Time & 2.5592 \\
6 Spectrometer Frequency & 400.09 \\
7 Nucleus & $1 \mathrm{H}$ \\
\hline
\end{tabular}

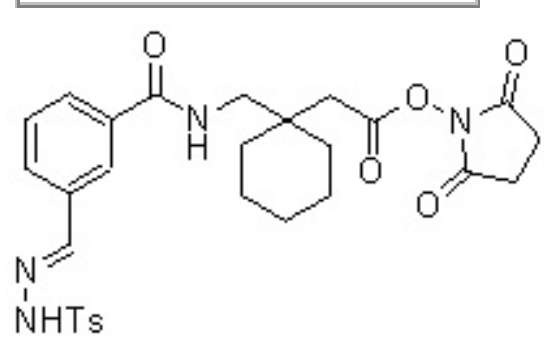

$8 b$

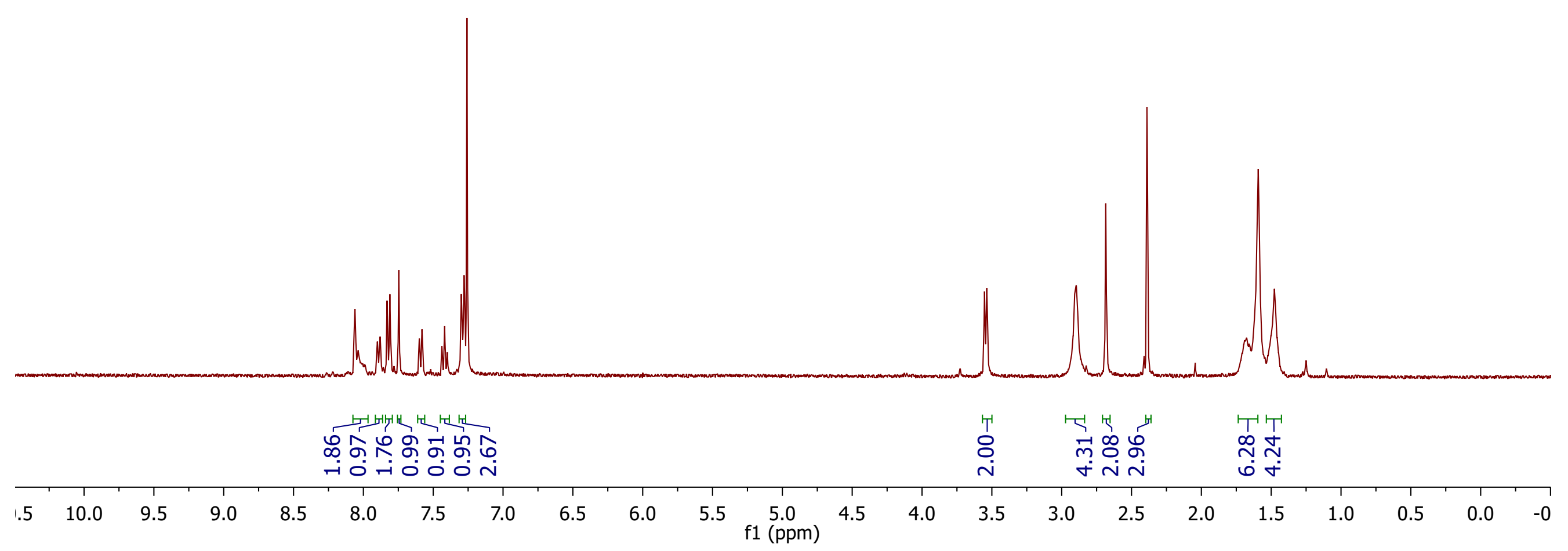


$8 \%$

مَ

ᄀ广

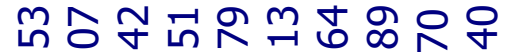

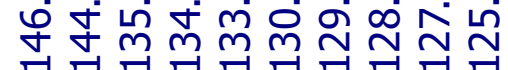

국

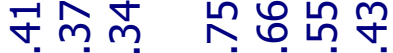

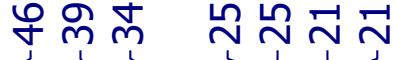

\begin{tabular}{|ll|}
\hline \multicolumn{1}{|c|}{ Parameter } & \multicolumn{1}{c|}{ Value } \\
1 Solvent & cdcl3 \\
2 Experiment & $1 \mathrm{D}$ \\
3 Number of Scans & 64 \\
4 Relaxation Delay & 1.0000 \\
5 Acquisition Time & 1.3042 \\
6 Spectrometer Frequency & 100.61 \\
7 Nucleus & $13 \mathrm{C}$ \\
\hline
\end{tabular}<smiles>O=C(CC1(CNC(=O)c2cccc(/C=N/NC(F)(F)F)c2)CCCCC1)ON1C(=O)CCC1=O</smiles>

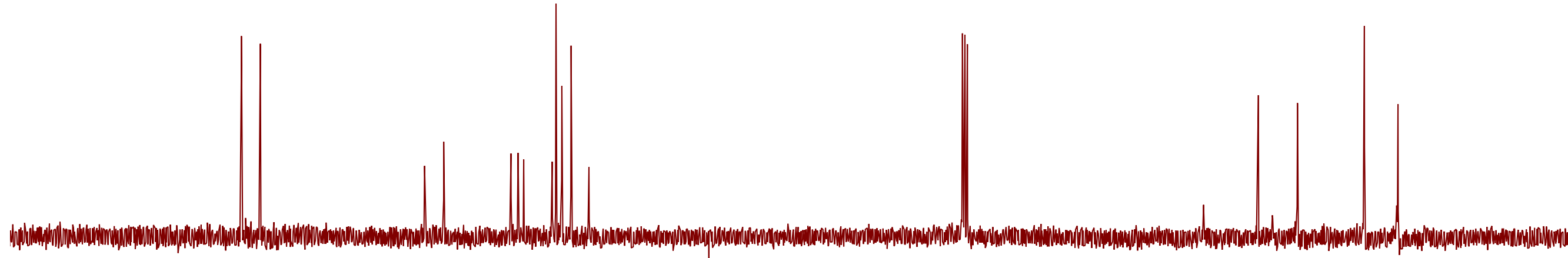


ஜ

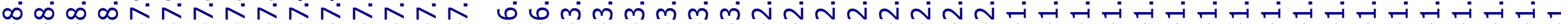

\begin{tabular}{|ll|}
\hline \multicolumn{1}{|c|}{ Parameter } & \multicolumn{1}{c|}{ Value } \\
1 Solvent & cdcl3 \\
2 Experiment & $1 \mathrm{D}$ \\
3 Number of Scans & 8 \\
4 Relaxation Delay & 1.0000 \\
5 Acquisition Time & 2.5592 \\
6 Spectrometer Frequency & 400.09 \\
7 Nucleus & $1 \mathrm{H}$ \\
\hline
\end{tabular}

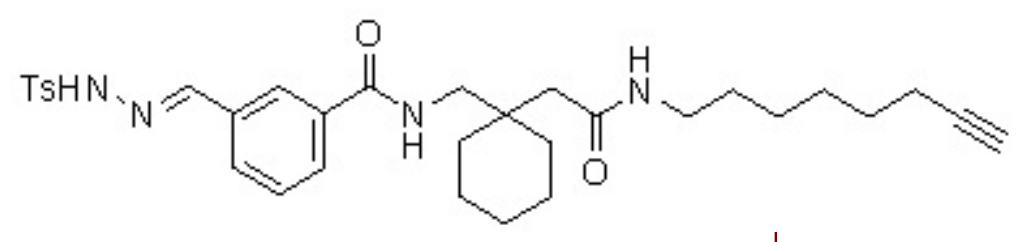

$19 a$

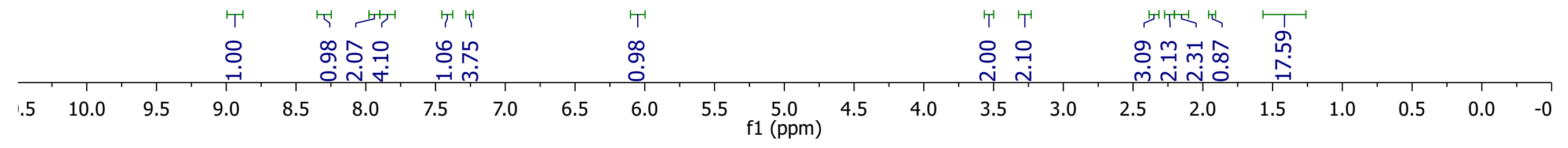




\begin{tabular}{|l|l|}
\hline \multicolumn{1}{|c|}{ Parameter } & Value \\
1 Solvent & cdcl3 \\
2 Experiment & $1 \mathrm{D}$ \\
3 Number of Scans & 64 \\
4 Relaxation Delay & 1.0000 \\
5 Acquisition Time & 1.3042 \\
6 Spectrometer Frequency & 100.61 \\
7 Nucleus & $13 \mathrm{C}$ \\
\hline
\end{tabular}

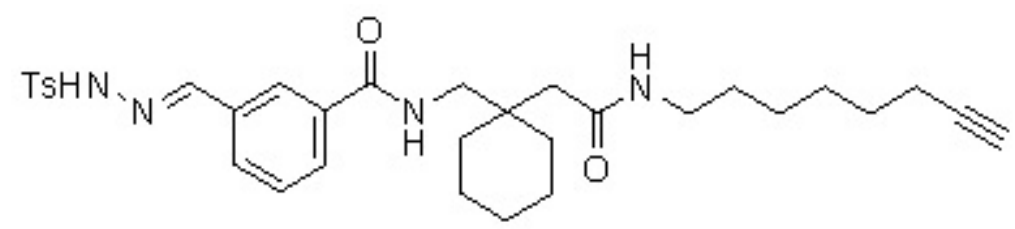

$19 a$

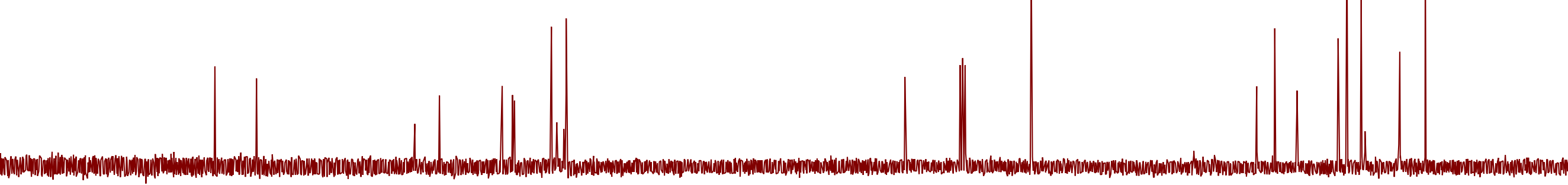




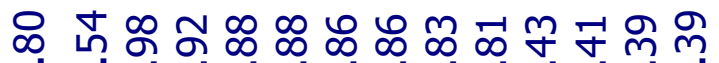

$\infty \infty N N$ N

112

Parameter

1 Solvent

2 Experiment

3 Number of Scans

4 Relaxation Delay

5 Acquisition Time

7 Nucleus

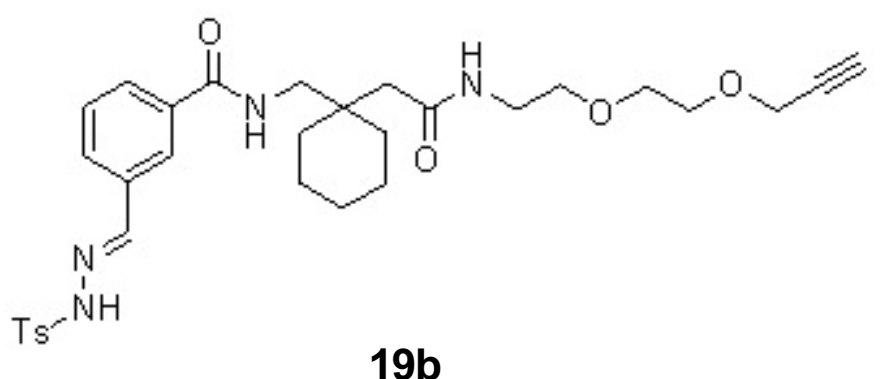

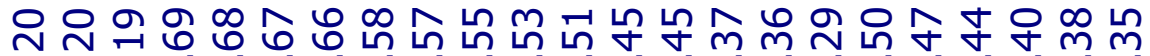
ナ

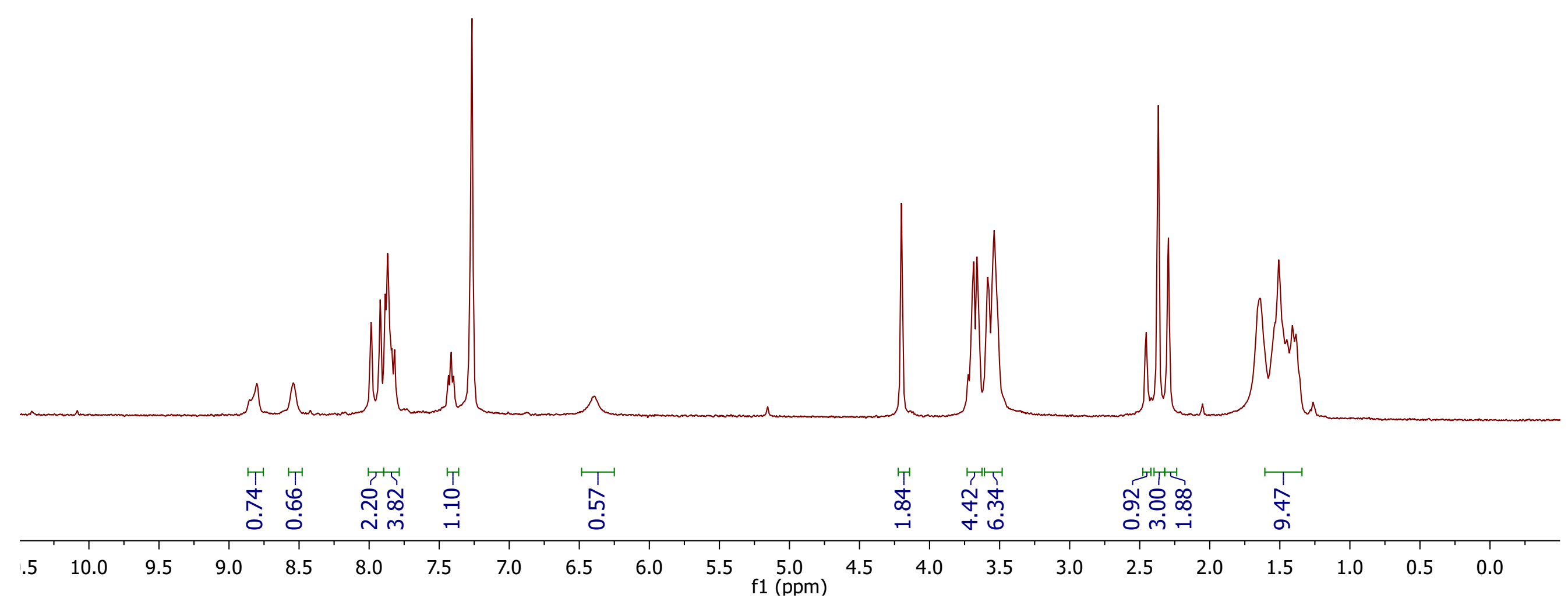




\begin{tabular}{|ll|}
\hline \multicolumn{1}{|c|}{ Parameter } & Value \\
1 Solvent & cdcl3 \\
2 Experiment & $1 \mathrm{D}$ \\
3 Number of Scans & 64 \\
4 Relaxation Delay & 1.0000 \\
5 Acquisition Time & 1.3042 \\
6 Spectrometer Frequency & 100.61 \\
7 Nucleus & $13 \mathrm{C}$ \\
\hline
\end{tabular}

m

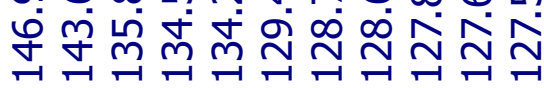
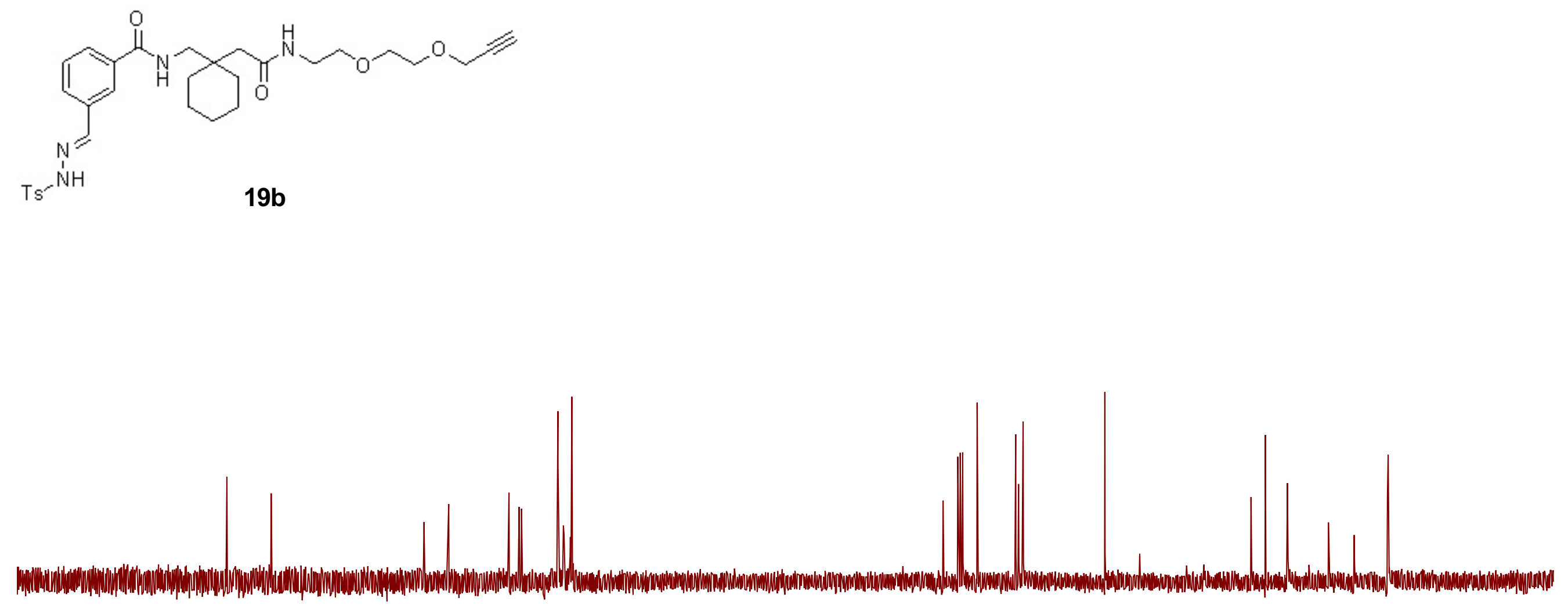

$90 \quad 80$

$80-70$

60

50


1 Solvent

2 Experiment

3 Number of Scans

4 Relaxation Delay

5 Acquisition Time

6 Spectrometer Frequency 400.10

7 Nucleus $1 \mathrm{H}$<smiles>C#CCOc1ccccc1CNC(=O)O</smiles>

\section{$19 \mathrm{cb}$}

\begin{tabular}{|c|c|c|c|c|c|c|c|c|c|c|c|c|c|c|c|c|c|c|c|c|c|}
\hline & & & & & & & 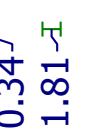 & & & & $\begin{array}{ll}T & T \\
m & 0 \\
0 & 0 \\
0 & 0\end{array}$ & $\begin{array}{l}T \\
\text { J' } \\
\text { N }\end{array}$ & & & & $\begin{array}{l}\mathfrak{T}^{\prime} \\
\stackrel{m}{0} \\
0\end{array}$ & & 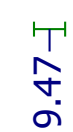 & & & \\
\hline 1.5 & 10.0 & 9.5 & 9.0 & 8.5 & 8.0 & 7.5 & 7.0 & 6.5 & 6.0 & 5.5 & $\begin{array}{l}5.0 \\
\mathrm{f} 1(\mathrm{ppm})\end{array}$ & 4.5 & 4.0 & 3.5 & 3.0 & 2.5 & 2.0 & 1.5 & 1.0 & 0.5 & 0.0 \\
\hline
\end{tabular}


3 Number of Scans

5 Acquisition Time

6 Spectrometer Frequency 100.61

7 Nucleus $13 \mathrm{C}$

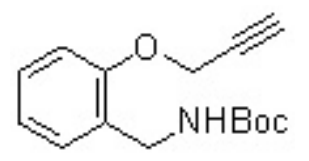

守守市

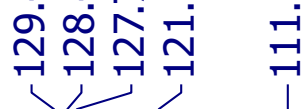

雍约

央年

$19 \mathrm{cb}$

$190 \quad 180 \quad 170$



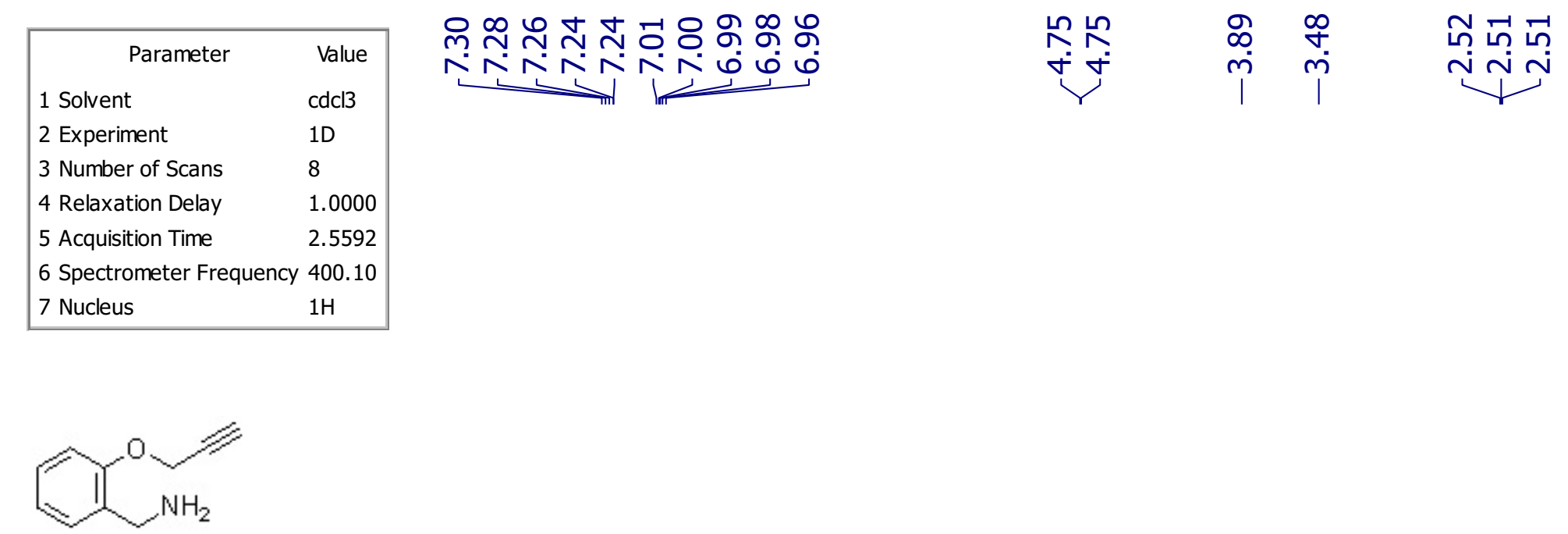

$19 c c$

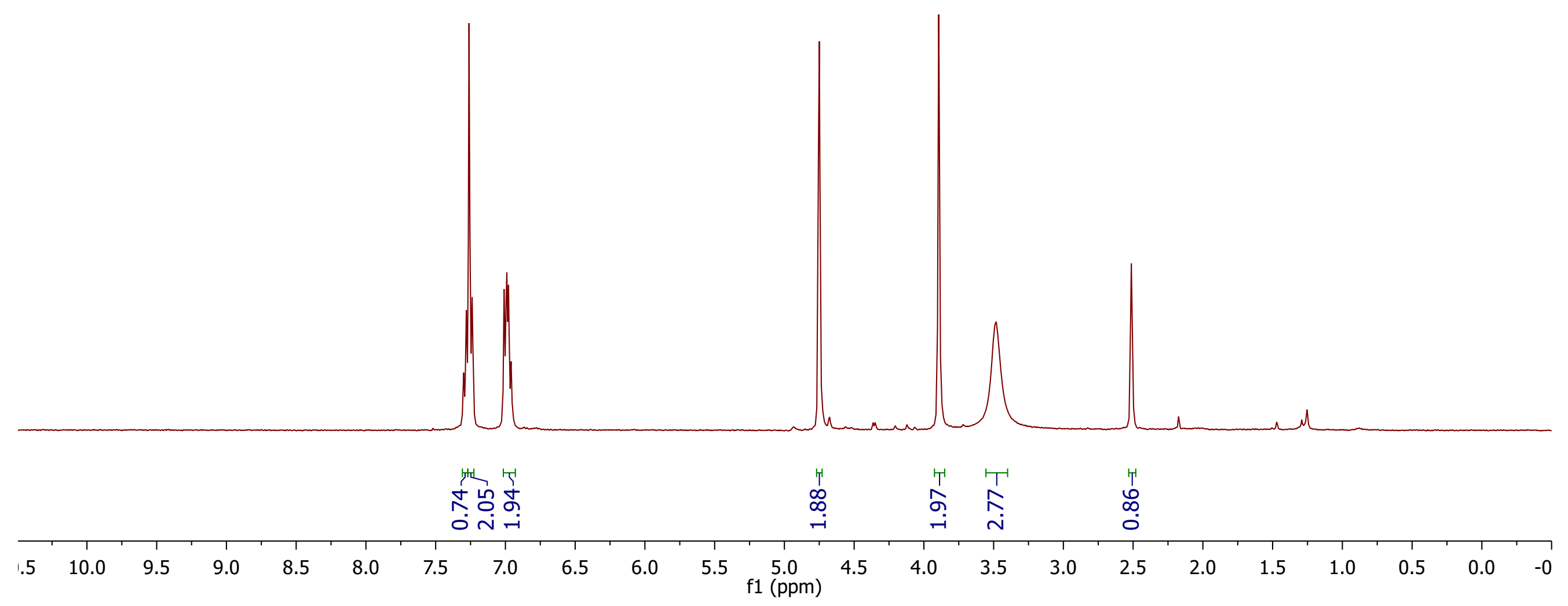




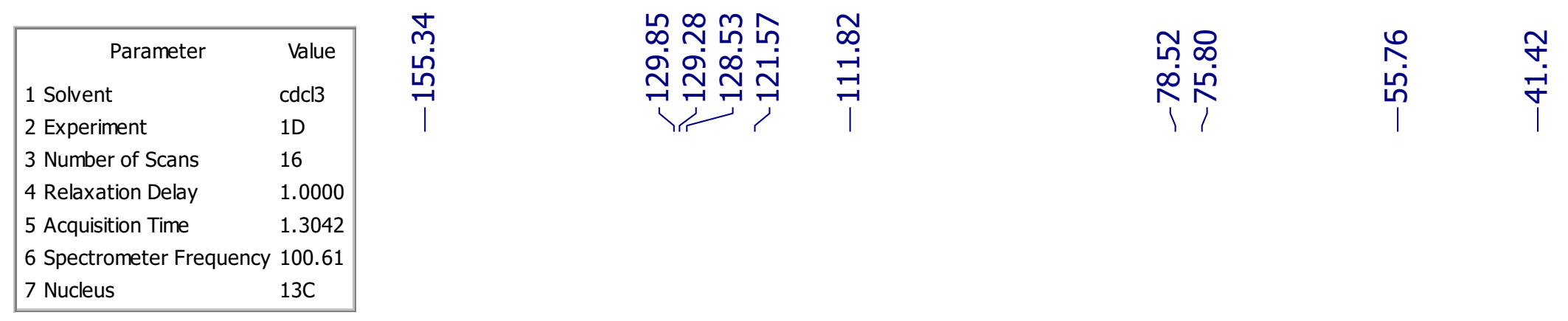

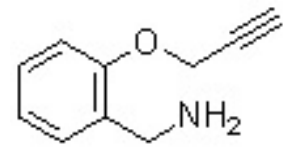

19cc

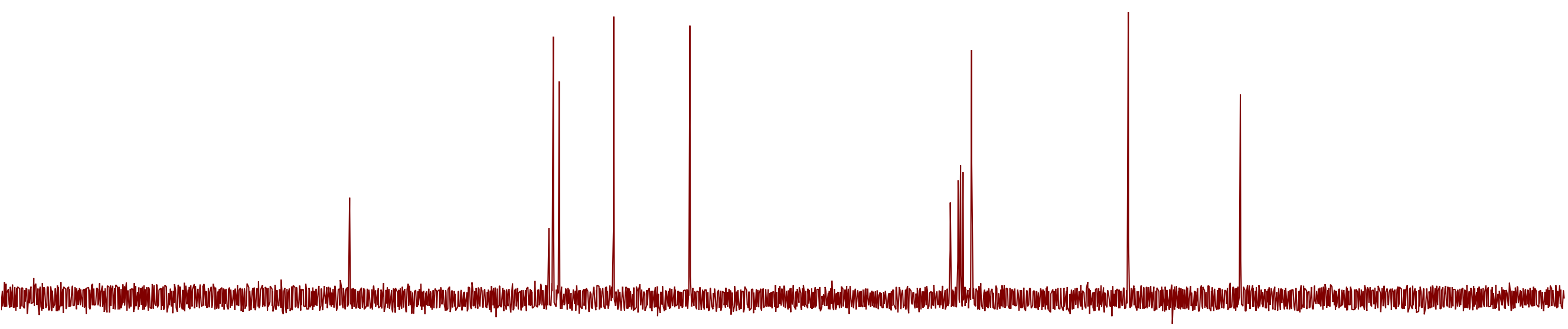

$180 \quad 170$

160

150

140

130

120

110

100

90

80

70

60

50

40

30




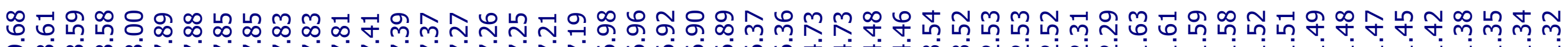

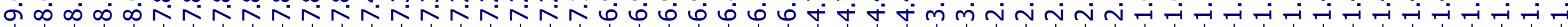

\begin{tabular}{|ll|}
\hline \multicolumn{1}{|c|}{ Parameter } & \multicolumn{1}{c|}{ Value } \\
1 Solvent & cdcl3 \\
2 Experiment & $1 \mathrm{D}$ \\
3 Number of Scans & 8 \\
4 Relaxation Delay & 1.0000 \\
5 Acquisition Time & 2.5592 \\
6 Spectrometer Frequency & 400.10 \\
7 Nucleus & $1 \mathrm{H}$ \\
\hline
\end{tabular}
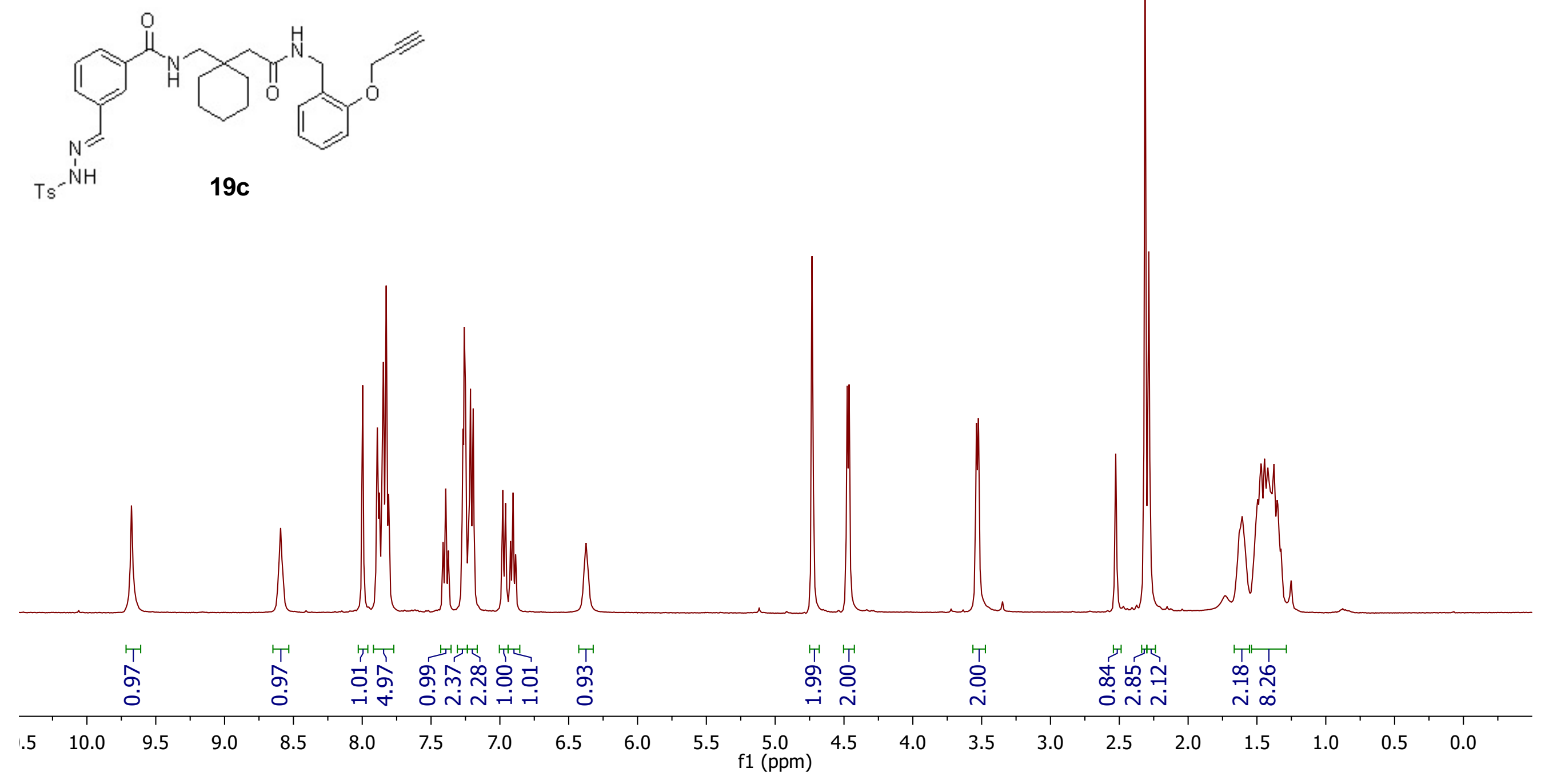

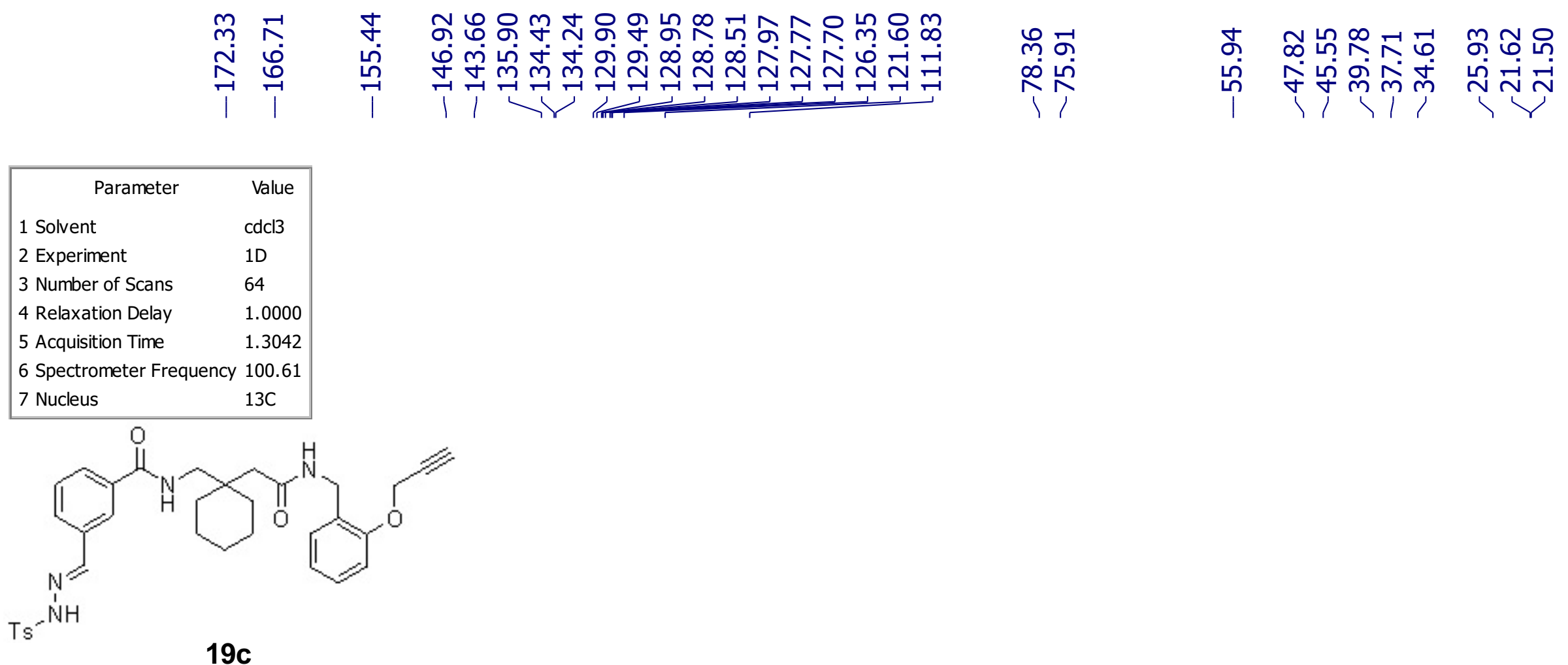

$19 c$

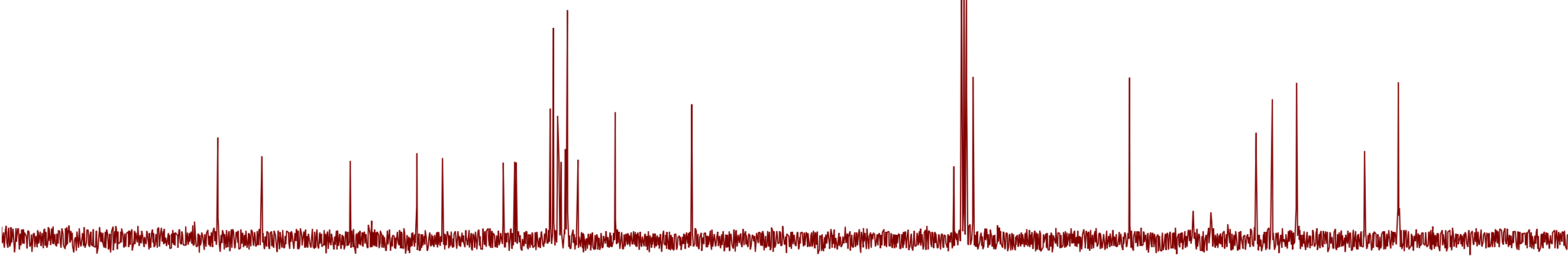

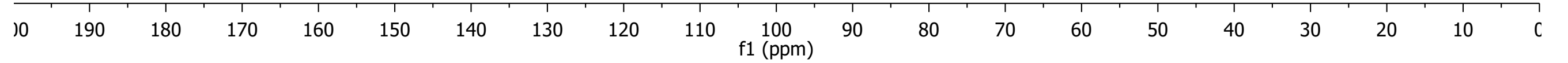




\begin{tabular}{|ll|}
\hline \multicolumn{1}{|c|}{ Parameter } & \multicolumn{1}{c|}{ Value } \\
1 Solvent & cdcl3 \\
2 Experiment & $1 \mathrm{D}$ \\
3 Number of Scans & 8 \\
4 Relaxation Delay & 1.0000 \\
5 Acquisition Time & 2.0447 \\
6 Spectrometer Frequency & 499.81 \\
7 Nucleus & $1 \mathrm{H}$ \\
\hline
\end{tabular}
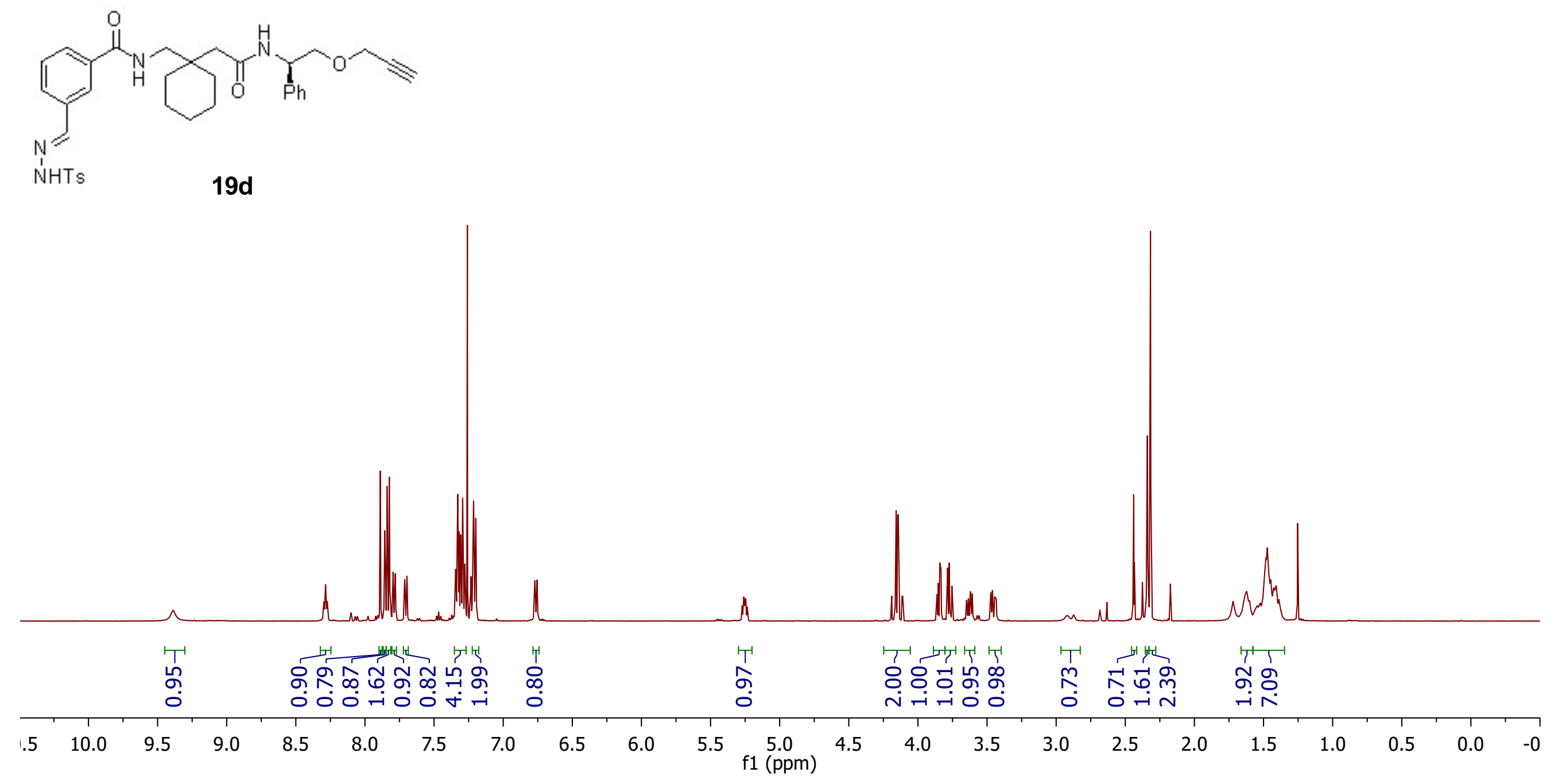


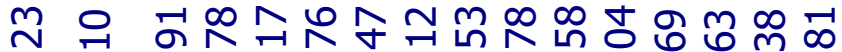

송

I

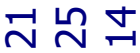

ำกㄴำ

1)

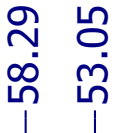

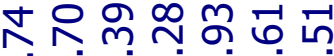

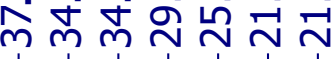

\begin{tabular}{|ll|}
\hline \multicolumn{1}{|c|}{ Parameter } & \multicolumn{1}{c|}{ Value } \\
1 Solvent & cdcl3 \\
2 Experiment & 1D \\
3 Number of Scans & 64 \\
4 Relaxation Delay & 1.0000 \\
5 Acquisition Time & 1.3042 \\
6 Spectrometer Frequency & 100.61 \\
7 Nucleus & $13 \mathrm{C}$ \\
\hline
\end{tabular}<smiles>C#CCOCC(NC(=O)CC1(CNC(=O)c2cccc(CN=[NH2+])c2)CCCCC1)c1ccccc1</smiles>

19d

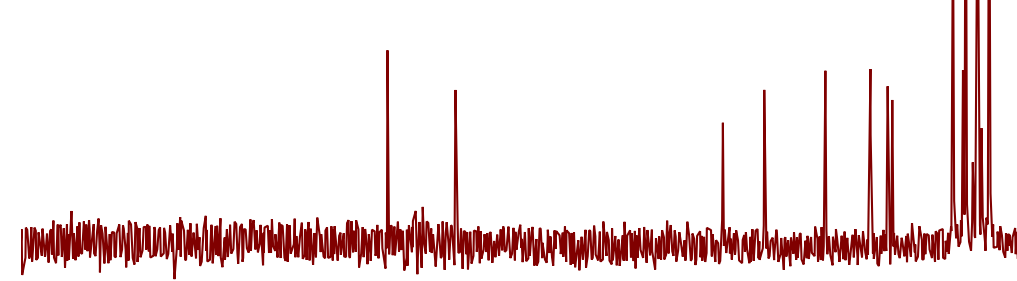

$190 \quad 180$

170

160

150

140

130

120

110

100
f1 (ppm)

90

80

70

60

50

40

30




\begin{tabular}{|ll|}
\hline \multicolumn{1}{|c|}{ Parameter } & \multicolumn{1}{c|}{ Value } \\
1 Solvent & cdcl3 \\
2 Experiment & $1 \mathrm{D}$ \\
3 Number of Scans & 8 \\
4 Relaxation Delay & 1.0000 \\
5 Acquisition Time & 2.5592 \\
6 Spectrometer Frequency & 400.09 \\
7 Nucleus & $1 \mathrm{H}$ \\
\hline
\end{tabular}

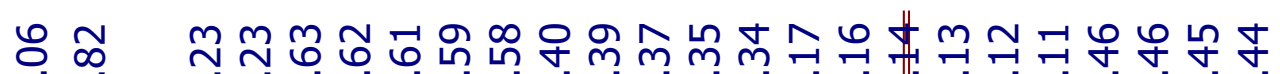

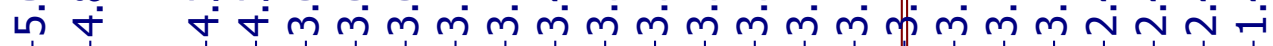<smiles>C#CCOC(CNC(=O)OCc1ccccc1)CNC(=O)OCc1ccccc1</smiles>

$19 \mathrm{eb}$

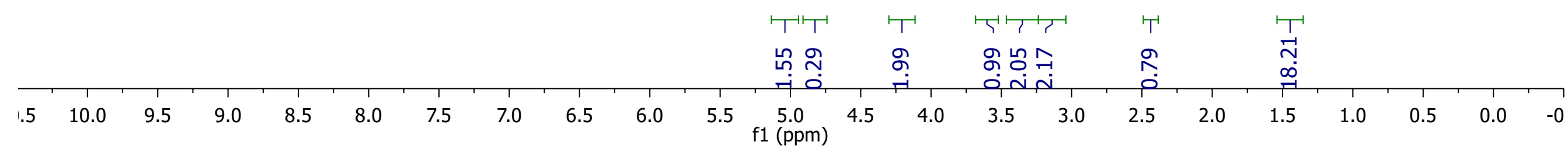




\begin{tabular}{|ll|}
\hline \multicolumn{1}{|c}{ Parameter } & Value \\
1 Solvent & cdcl3 \\
2 Experiment & $1 \mathrm{D}$ \\
3 Number of Scans & 32 \\
4 Relaxation Delay & 1.0000 \\
5 Acquisition Time & 1.3042 \\
6 Spectrometer Frequency & 100.61 \\
7 Nucleus & $13 \mathrm{C}$ \\
\hline
\end{tabular}

ำㄷำ

ס่

ก

$1 / 1$

$\stackrel{\infty}{\circ}$

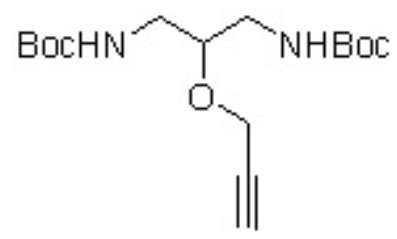

19 eb

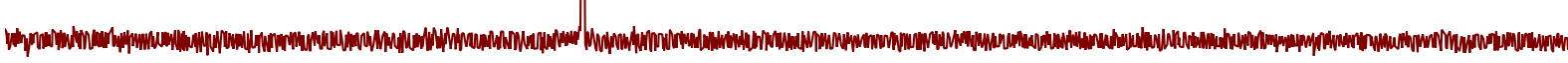

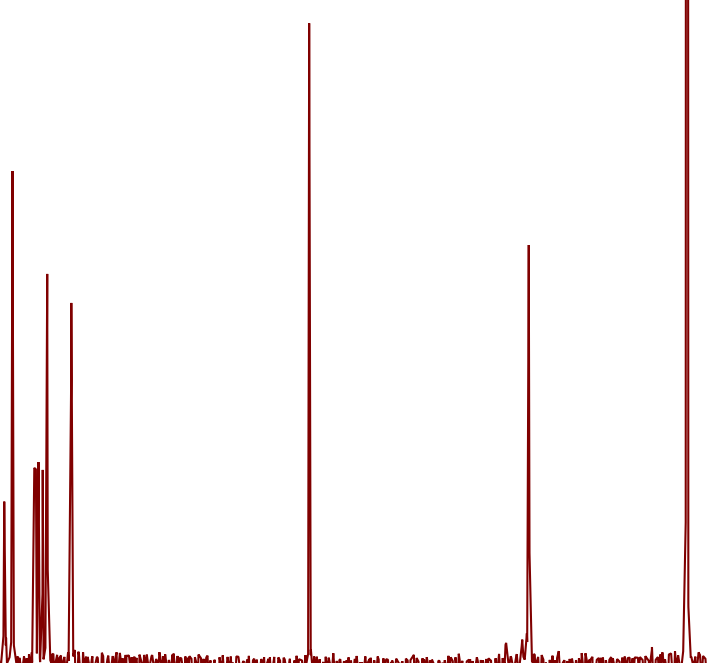

80

160

150

140

130

120

$110 \quad 100$

90

80

70

60

50

40

30

20 


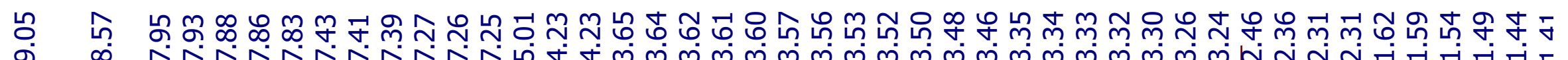

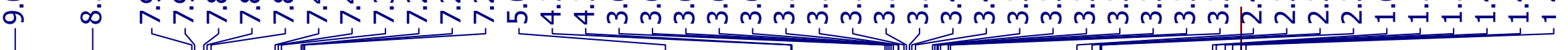

\begin{tabular}{|ll|}
\hline \multicolumn{1}{|c|}{ Parameter } & \multicolumn{1}{c|}{ Value } \\
1 Solvent & cdcl3 \\
2 Experiment & $1 \mathrm{D}$ \\
3 Number of Scans & 8 \\
4 Relaxation Delay & 1.0000 \\
5 Acquisition Time & 2.5592 \\
6 Spectrometer Frequency & 400.09 \\
7 Nucleus & $1 \mathrm{H}$ \\
\hline
\end{tabular}

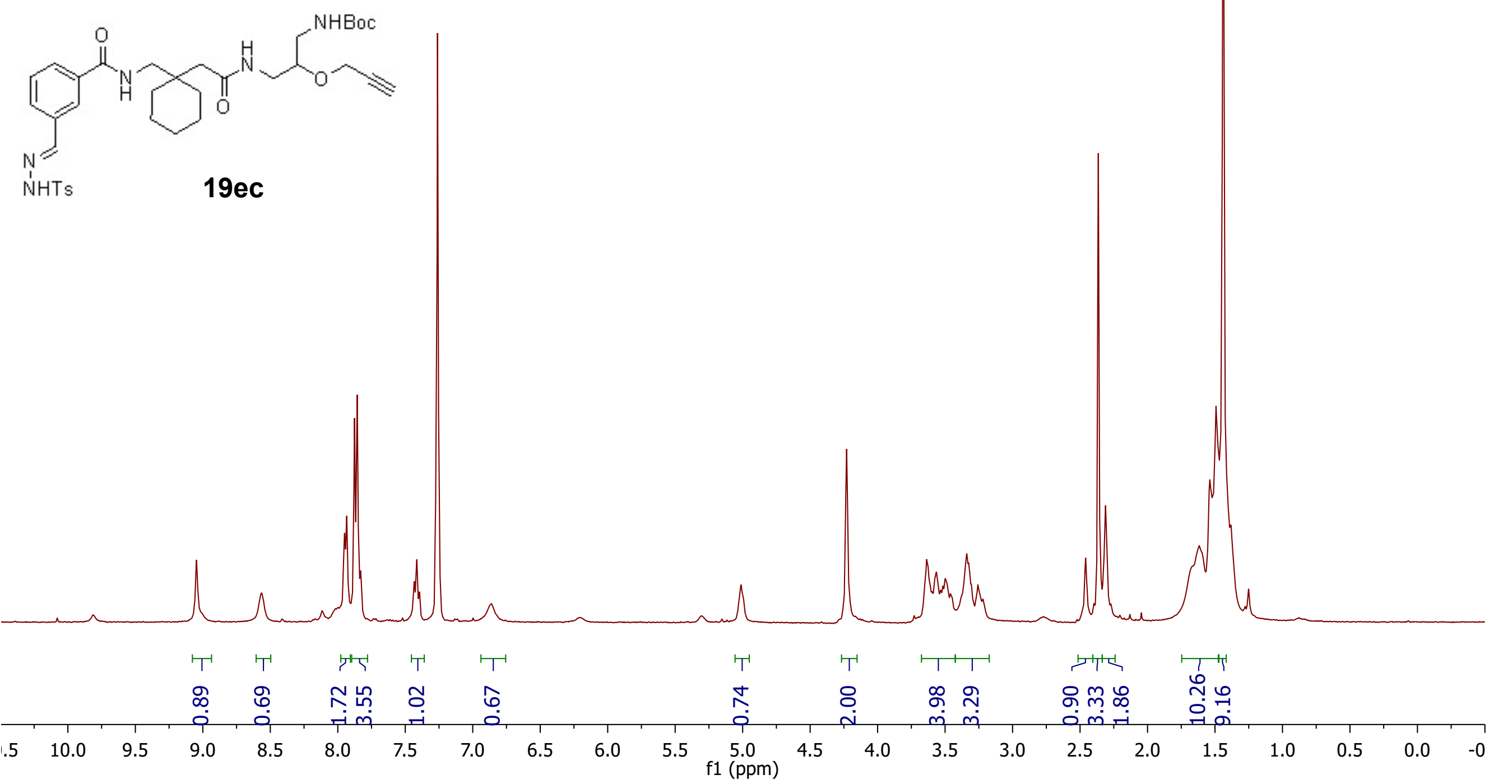




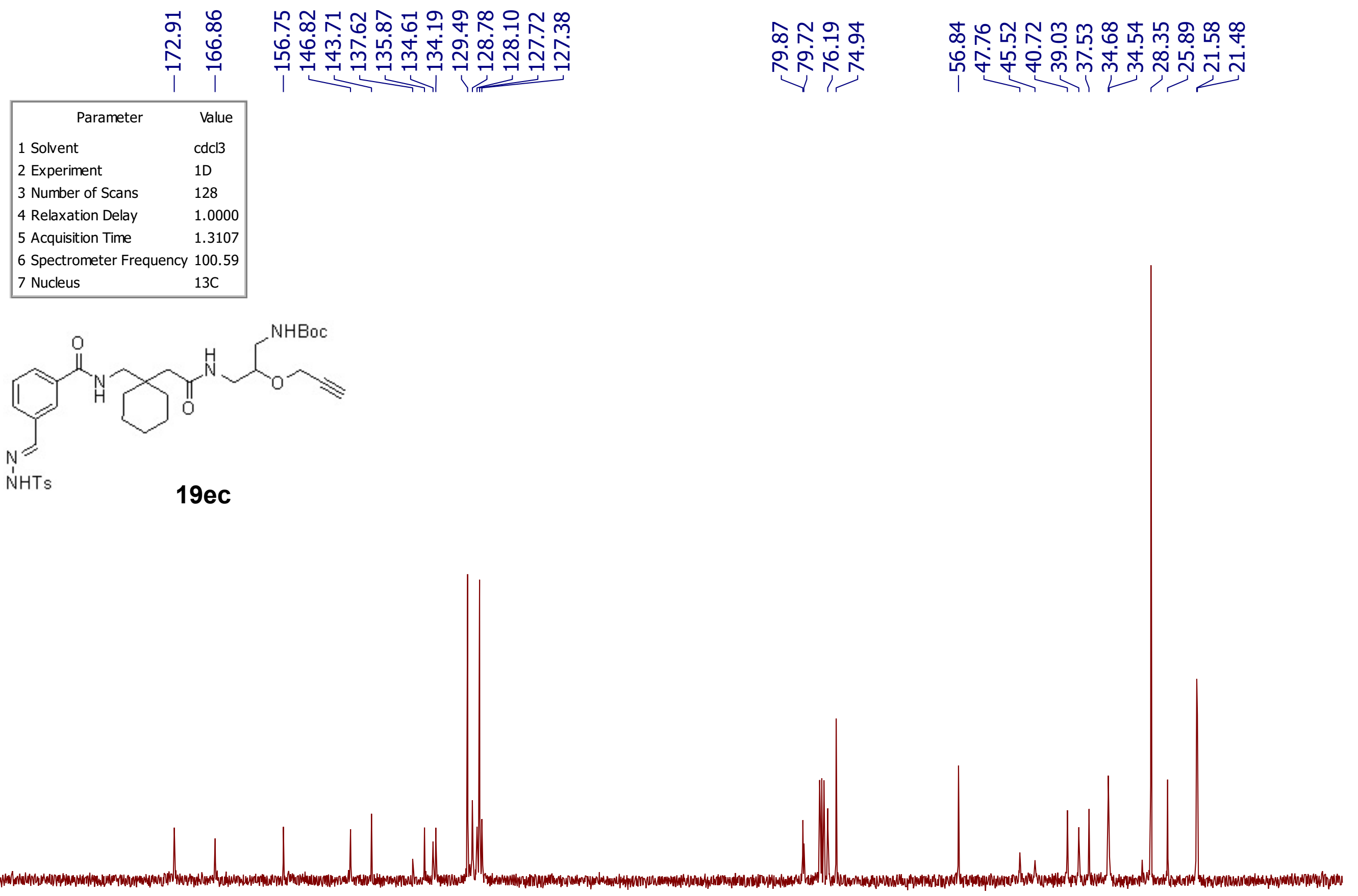

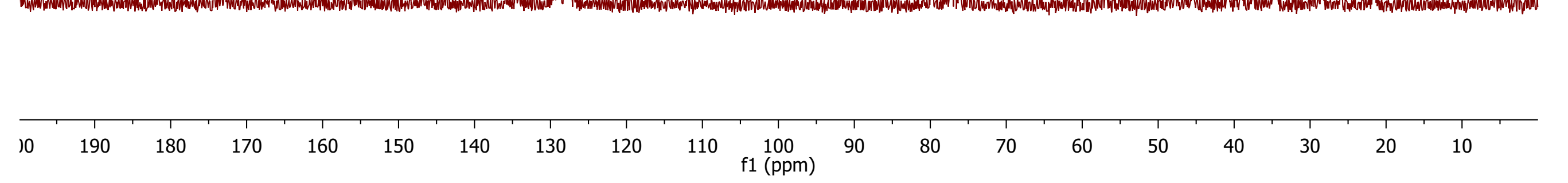




\begin{tabular}{|ll|}
\hline \multicolumn{1}{|c|}{ Parameter } & \multicolumn{1}{c|}{ Value } \\
1 Solvent & cd3od \\
2 Experiment & $1 \mathrm{D}$ \\
3 Number of Scans & 8 \\
4 Relaxation Delay & 1.0000 \\
5 Acquisition Time & 2.5592 \\
6 Spectrometer Frequency & 400.09 \\
7 Nucleus & $1 \mathrm{H}$ \\
\hline
\end{tabular}

NHTs
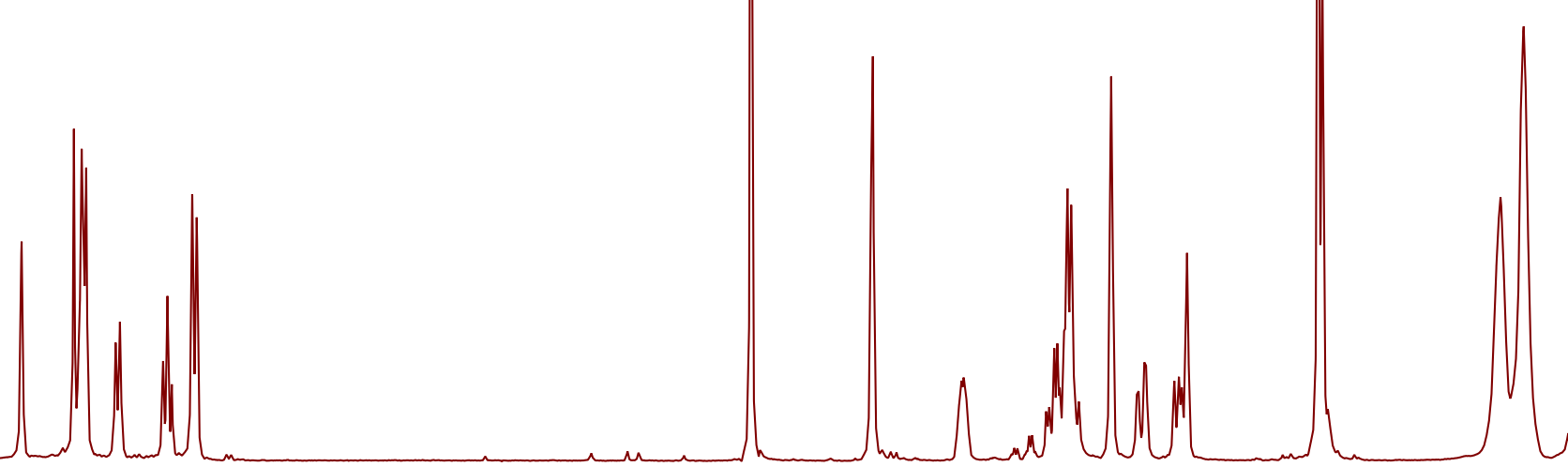

T田师办

T फ ए म

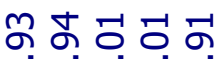
0 लिं

으 नำ 융

$\begin{array}{lll}9.5 & 9.0 & 8.5\end{array}$

8.0

7.5

$\begin{array}{lll}4.5 & 4.0 & 3.5\end{array}$

3.0, 2.5

\begin{tabular}{|c|c|c|c|c|}
\hline & $\begin{array}{l}1 \\
\infty \\
\sigma \\
0\end{array}$ & T & T & \\
\hline 2.0 & 1.5 & 1.0 & 0.5 & 0.0 \\
\hline
\end{tabular}




\section{ก กิ \\ 亲 \\ I ।}

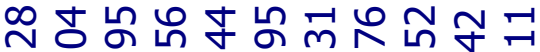

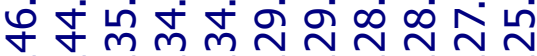

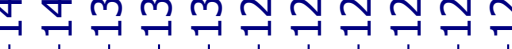

மீ $\stackrel{\infty}{\wedge}$ ำ

र广

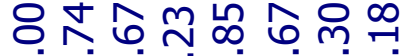
mㅇํㅇ

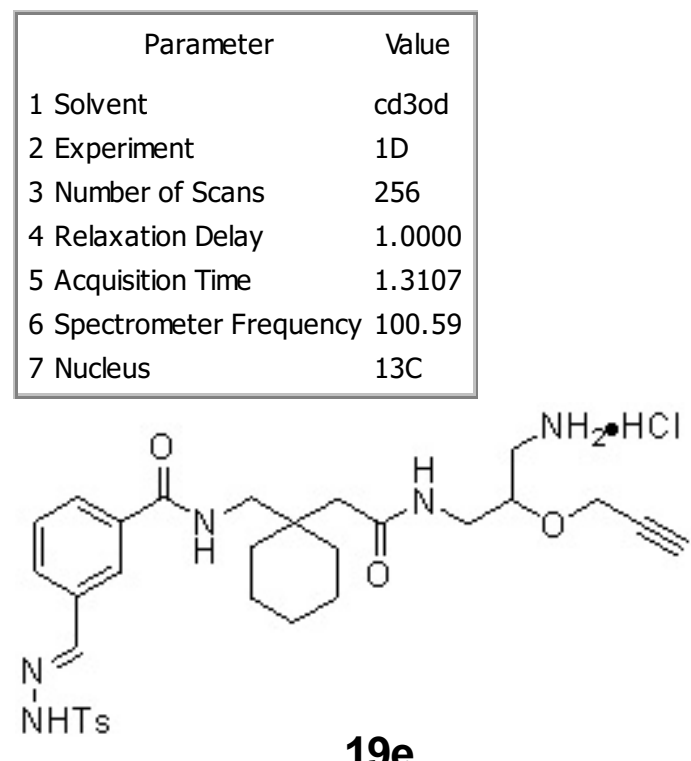

$19 e$

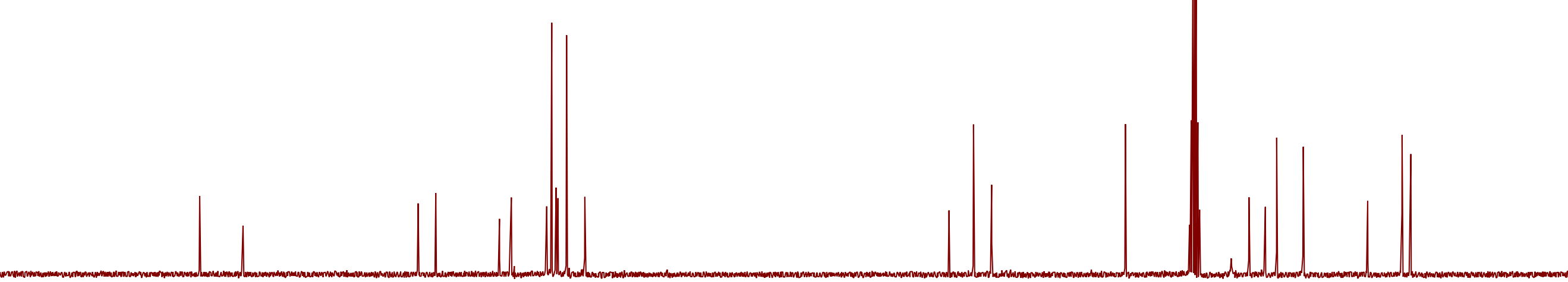

$190 \quad 180$


하 유 m

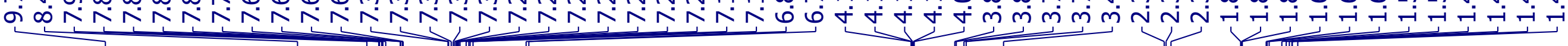

\begin{tabular}{|ll|}
\hline \multicolumn{1}{|c|}{ Parameter } & \multicolumn{1}{c|}{ Value } \\
1 Solvent & cdcl3 \\
2 Experiment & $1 \mathrm{D}$ \\
3 Number of Scans & 8 \\
4 Relaxation Delay & 1.0000 \\
5 Acquisition Time & 2.5592 \\
6 Spectrometer Frequency & 400.09 \\
7 Nucleus & $1 \mathrm{H}$ \\
\hline
\end{tabular}

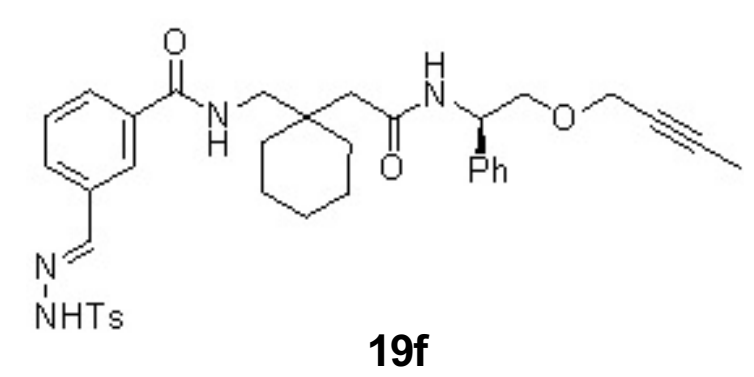

NHTs $19 f$

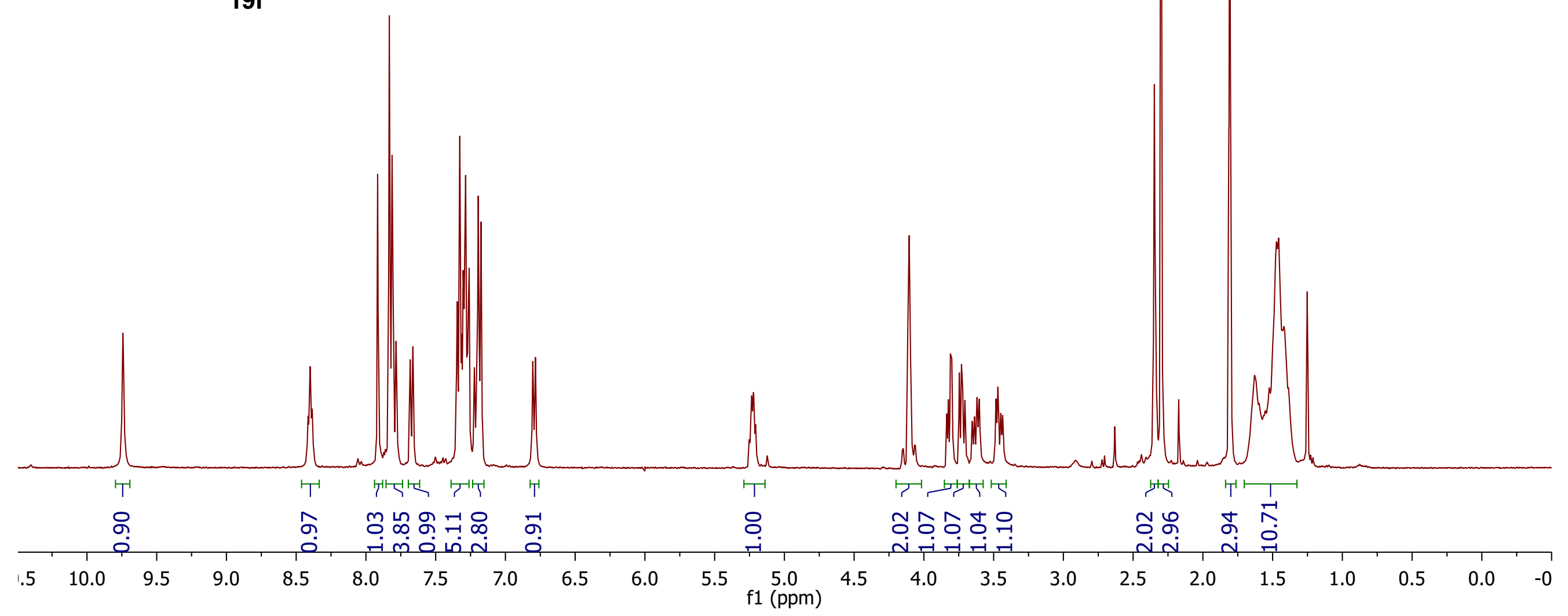




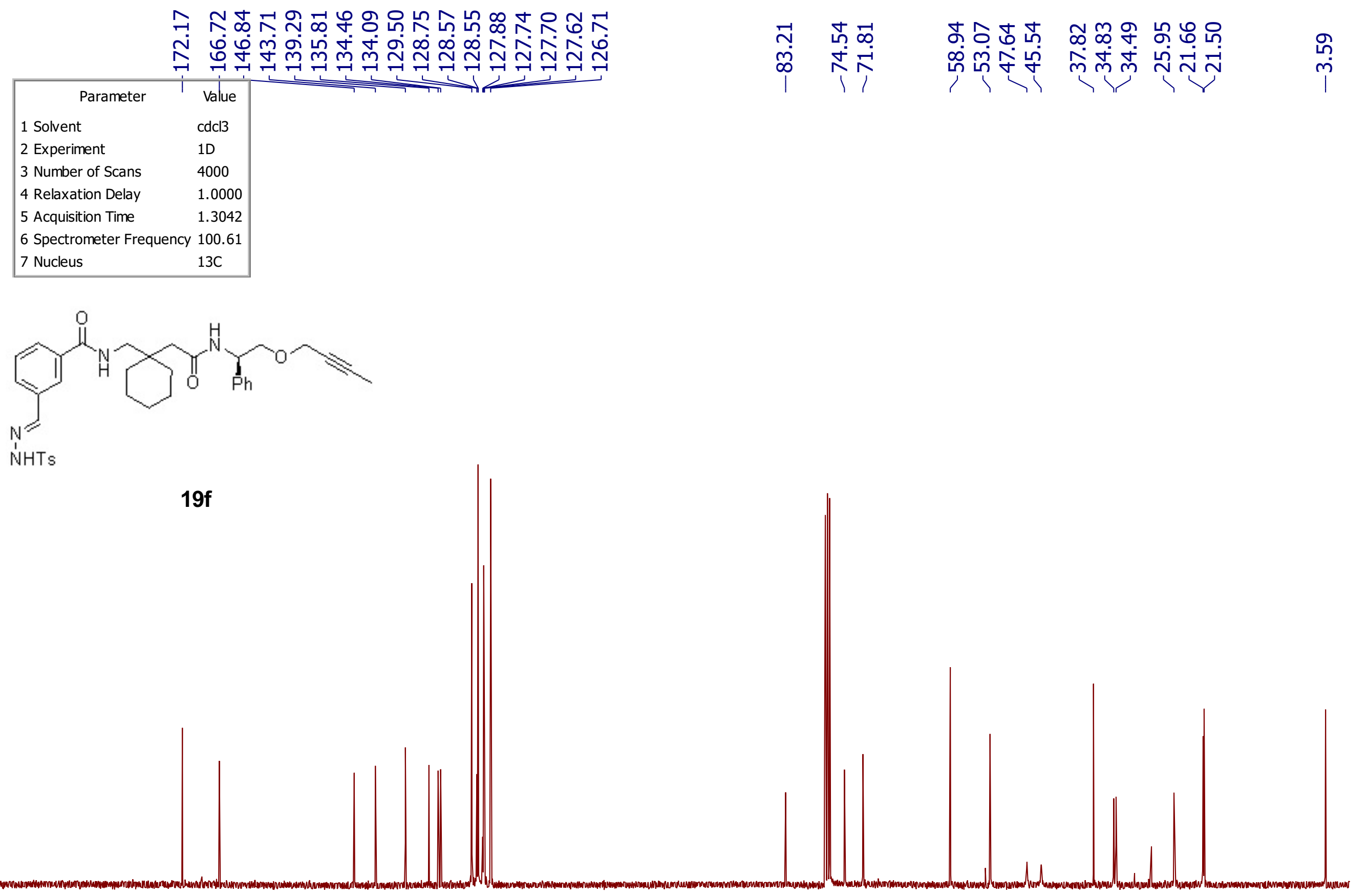

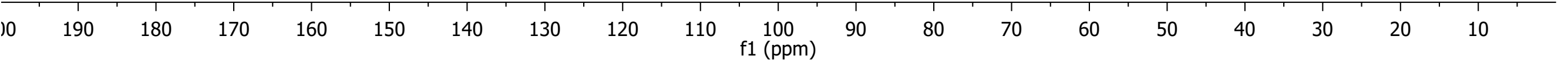




\begin{tabular}{|ll|}
\hline \multicolumn{1}{|c|}{ Parameter } & \multicolumn{1}{c|}{ Value } \\
1 Solvent & cdcl3 \\
2 Experiment & $1 \mathrm{D}$ \\
3 Number of Scans & 8 \\
4 Relaxation Delay & 1.0000 \\
5 Acquisition Time & 2.5592 \\
6 Spectrometer Frequency & 400.09 \\
7 Nucleus & $1 \mathrm{H}$ \\
\hline
\end{tabular}

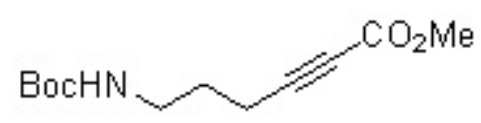

$19 \mathrm{gb}$

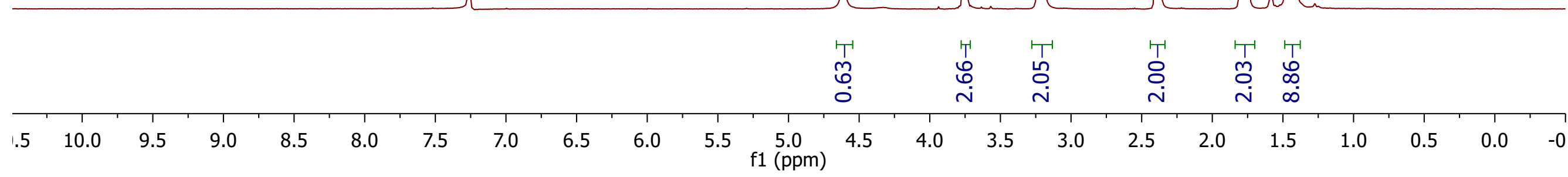




\begin{tabular}{|ll|}
\hline \multicolumn{1}{|c}{ Parameter } & Value \\
1 Solvent & $\mathrm{cdcl3}$ \\
2 Experiment & $1 \mathrm{D}$ \\
3 Number of Scans & 64 \\
4 Relaxation Delay & 1.0000 \\
5 Acquisition Time & 1.3042 \\
6 Spectrometer Frequency & 100.61 \\
7 Nucleus & $13 \mathrm{C}$ \\
\hline
\end{tabular}

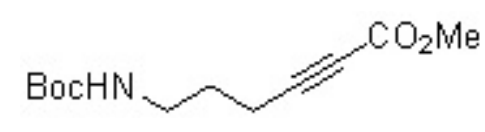

$19 g b$

$180 \quad 170$

160

150

140

130

120




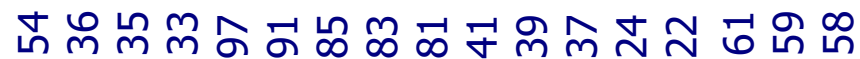

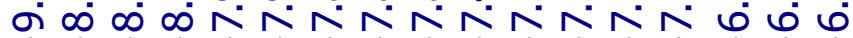

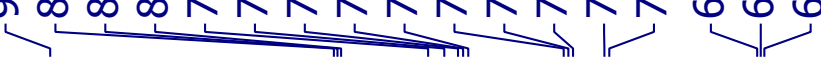

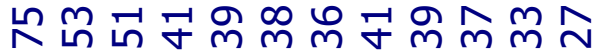
minm $m$ m $\sim \sim \sim N$

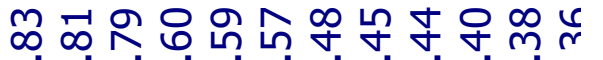

\begin{tabular}{|ll|}
\hline \multicolumn{1}{|c|}{ Parameter } & \multicolumn{1}{c|}{ Value } \\
1 Solvent & cdcl3 \\
2 Experiment & $1 \mathrm{D}$ \\
3 Number of Scans & 8 \\
4 Relaxation Delay & 1.0000 \\
5 Acquisition Time & 2.5592 \\
6 Spectrometer Frequency & 400.09 \\
7 Nucleus & $1 \mathrm{H}$ \\
\hline
\end{tabular}
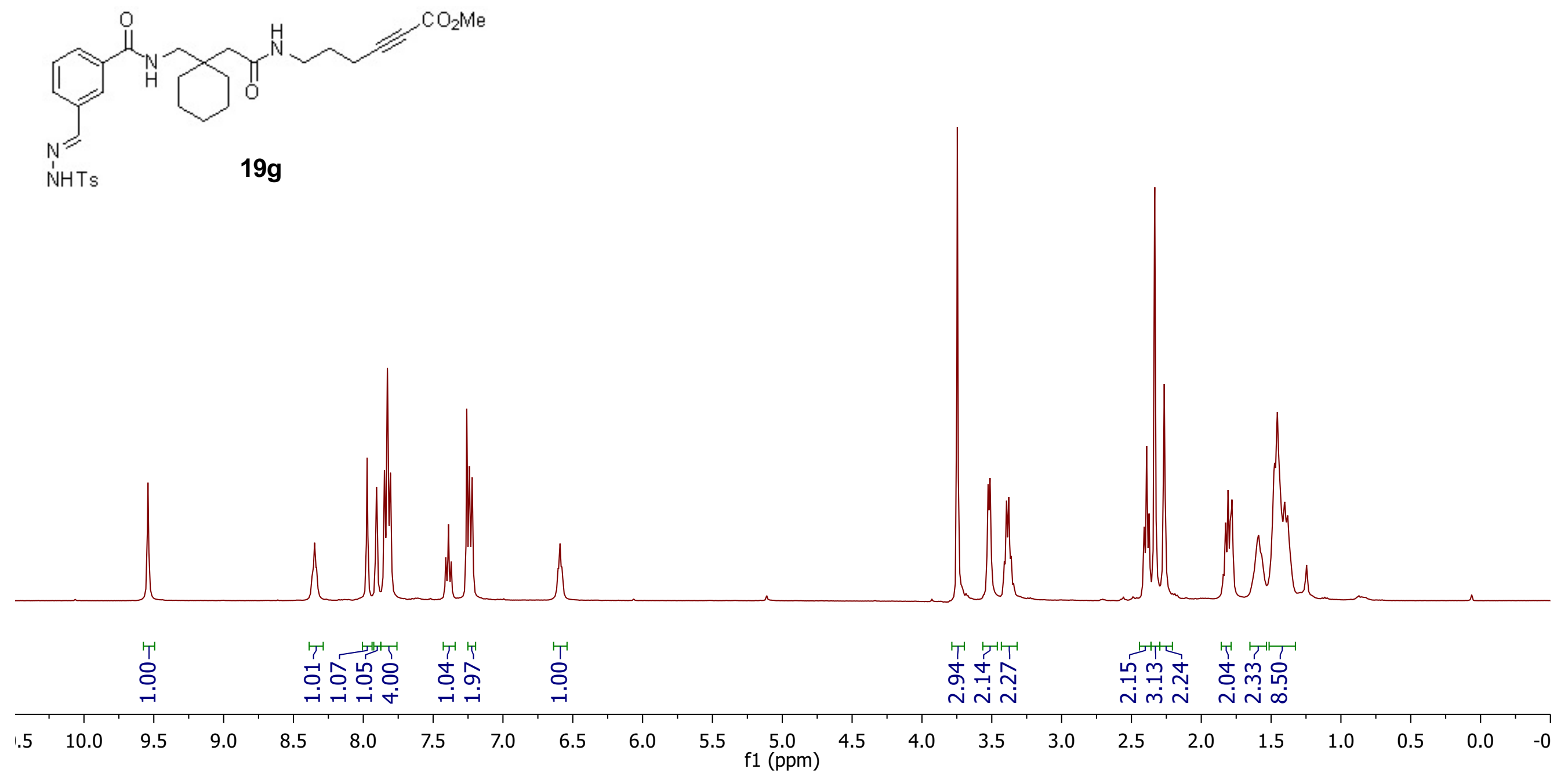


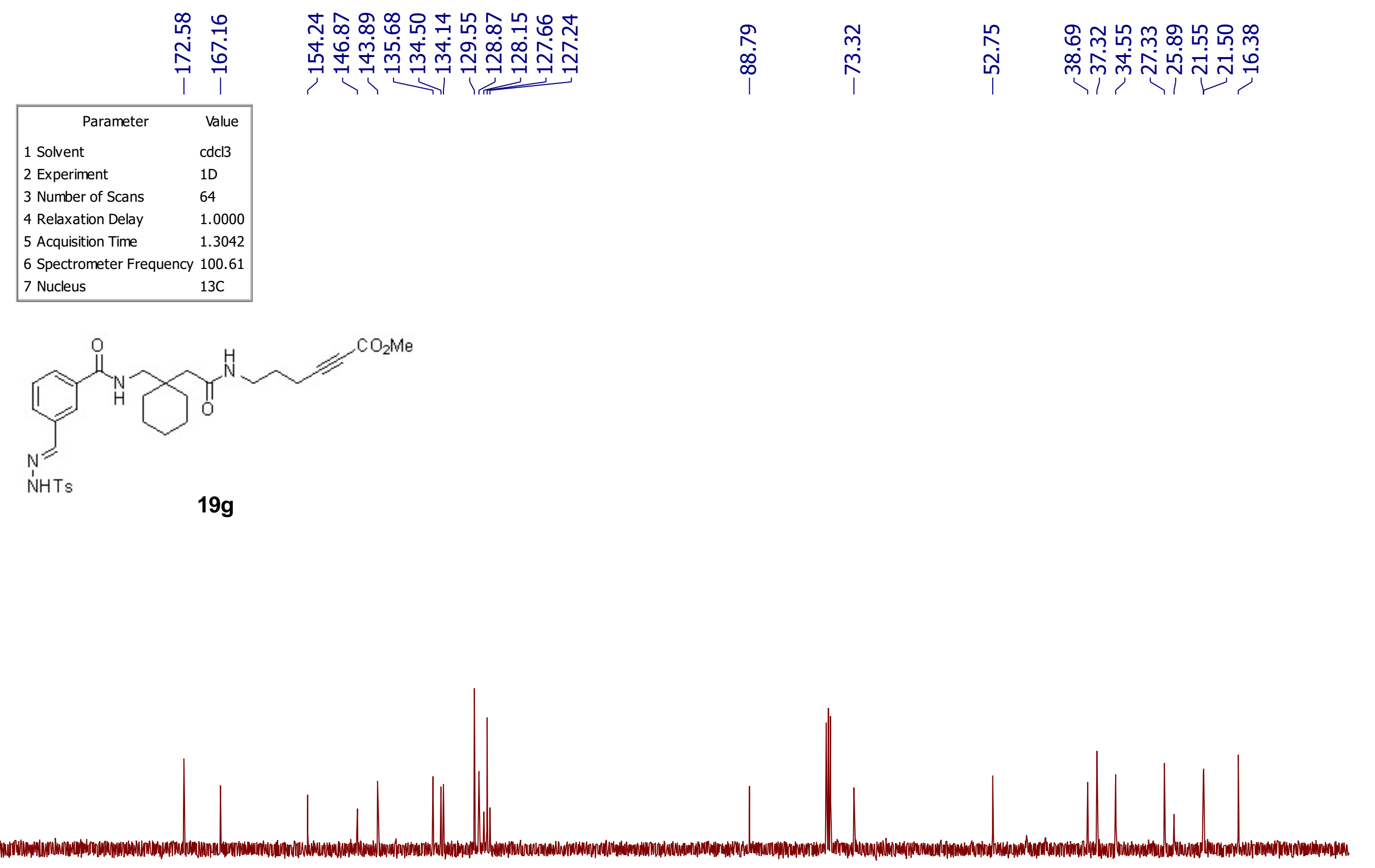

윰

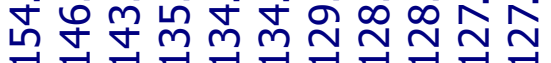

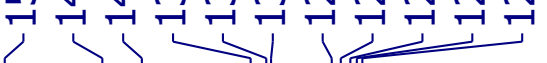

2 Experiment

Spectrometer Frequency 100.6

7 Nucleus

$190 \quad 180$

170

160

150

140

130

120

110

100

90

80

70

60

50

40

30 


\begin{tabular}{|ll|}
\hline \multicolumn{1}{|c|}{ Parameter } & \multicolumn{1}{c|}{ Value } \\
1 Solvent & cdcl3 \\
2 Experiment & $1 \mathrm{D}$ \\
3 Number of Scans & 8 \\
4 Relaxation Delay & 1.0000 \\
5 Acquisition Time & 2.0447 \\
6 Spectrometer Frequency & 499.81 \\
7 Nucleus & $1 \mathrm{H}$ \\
\hline
\end{tabular}

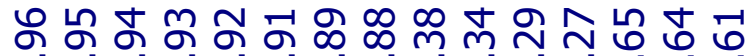
ก ம்

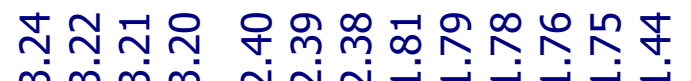

mmm NNN

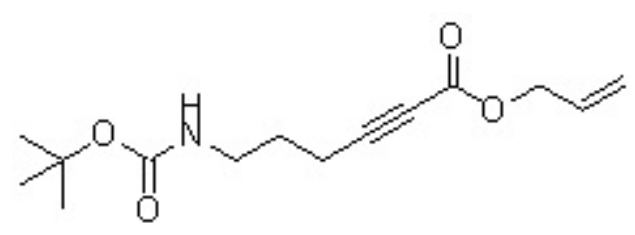

19ha

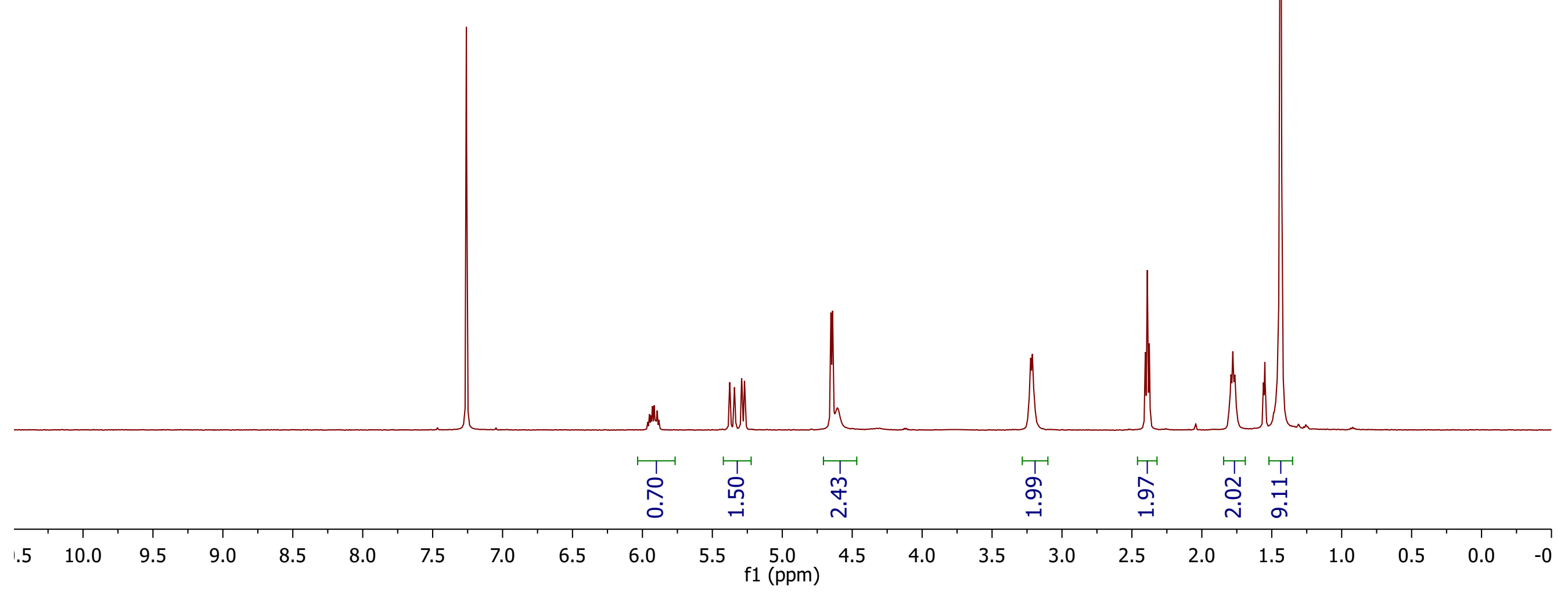




\begin{tabular}{|ll|}
\hline \multicolumn{1}{|c|}{ Parameter } & \multicolumn{1}{c|}{ Value } \\
1 Solvent & cdcl3 \\
2 Experiment & $1 \mathrm{D}$ \\
3 Number of Scans & 8 \\
4 Relaxation Delay & 1.0000 \\
5 Acquisition Time & 2.5592 \\
6 Spectrometer Frequency & 400.09 \\
7 Nucleus & $1 \mathrm{H}$ \\
\hline
\end{tabular}
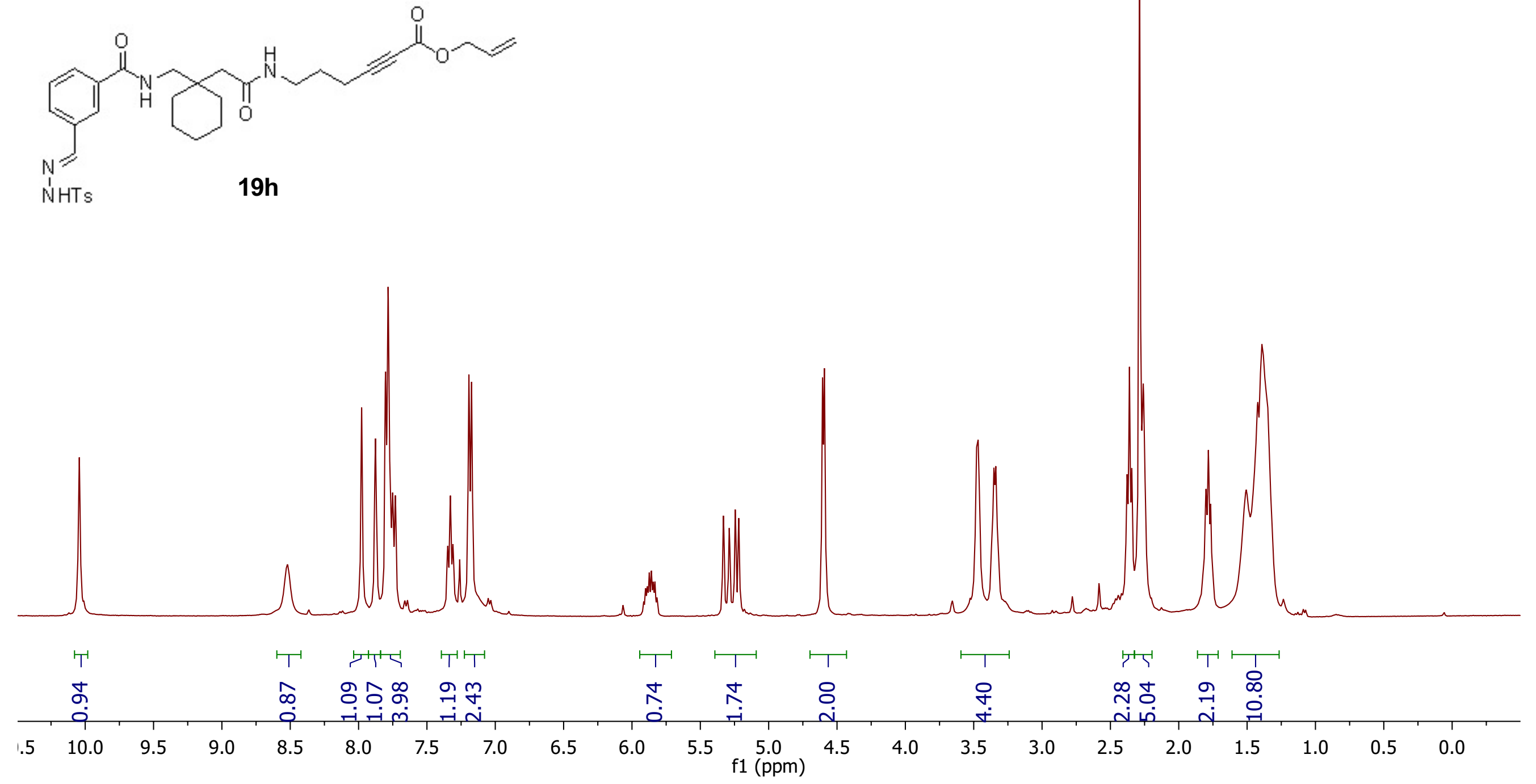

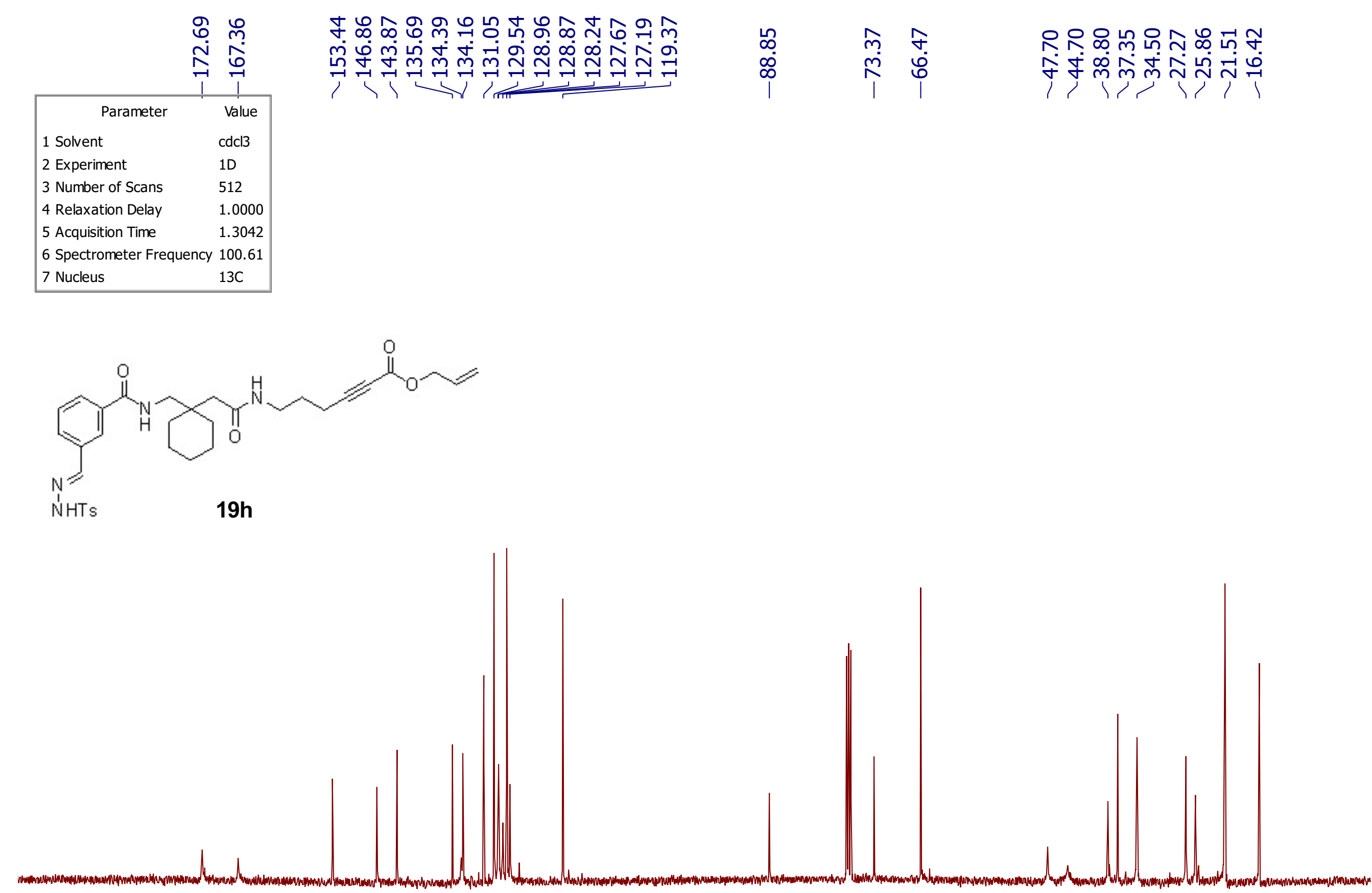

$190 \quad 180$

150

$140 \quad 130$

120

110

100

90

80

70

60

50

40

30

20

10 
mิ

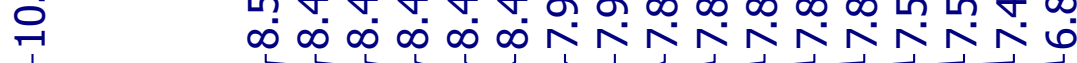

1

Parameter

Value

1 Solvent

2 Experiment

3 Number of Scans

4 Relaxation Delay

5 Acquisition Time

7 Nucleus cd3od

1D

8

1.0000

2.0447

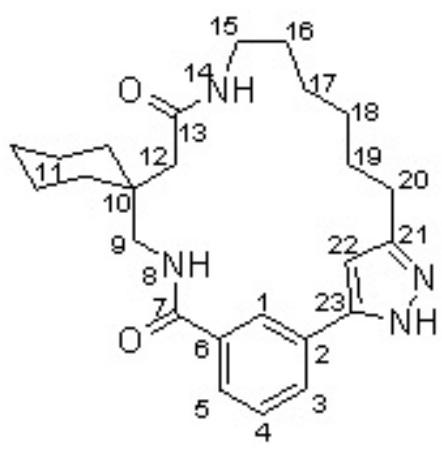

20a

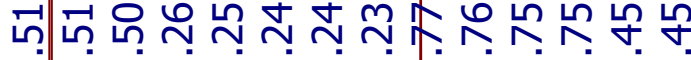

m

象象

ન

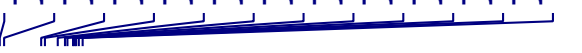

$17,18,11$
12, 19, 16,

$9, \quad 15, \quad 20$

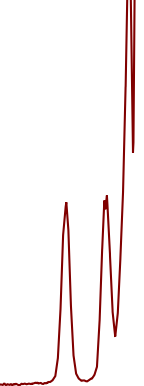

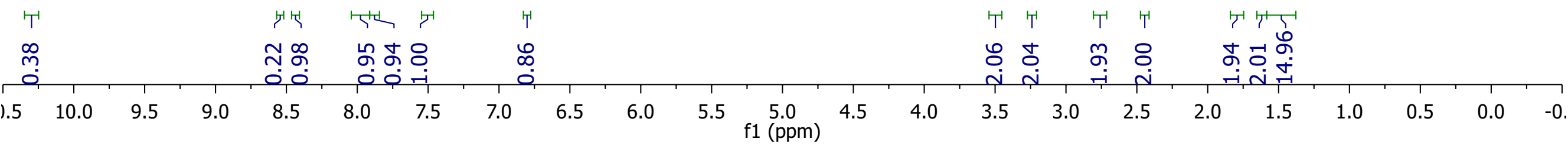




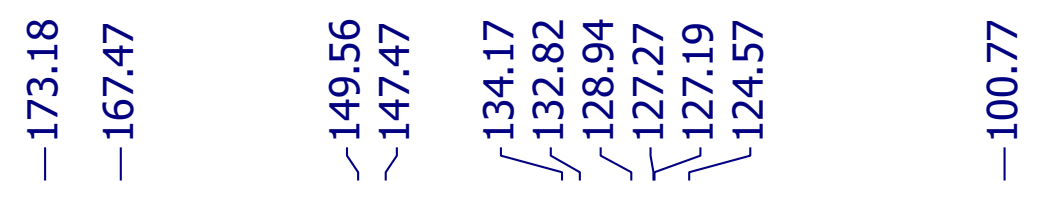

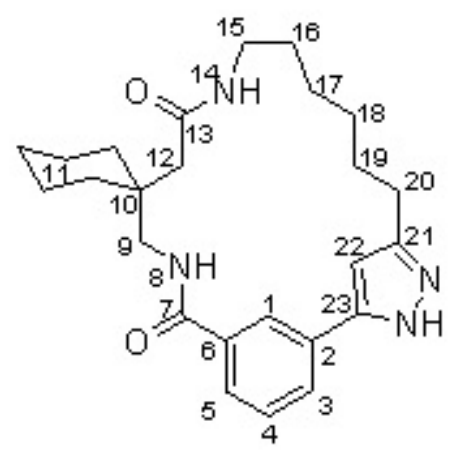

20a

13, 7,

23,21

$6,2, \quad 4,5,3,1$,

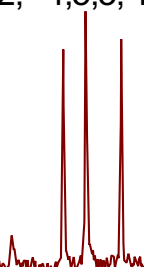

22

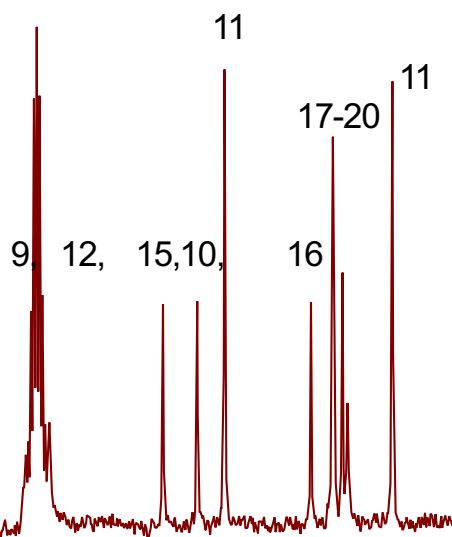




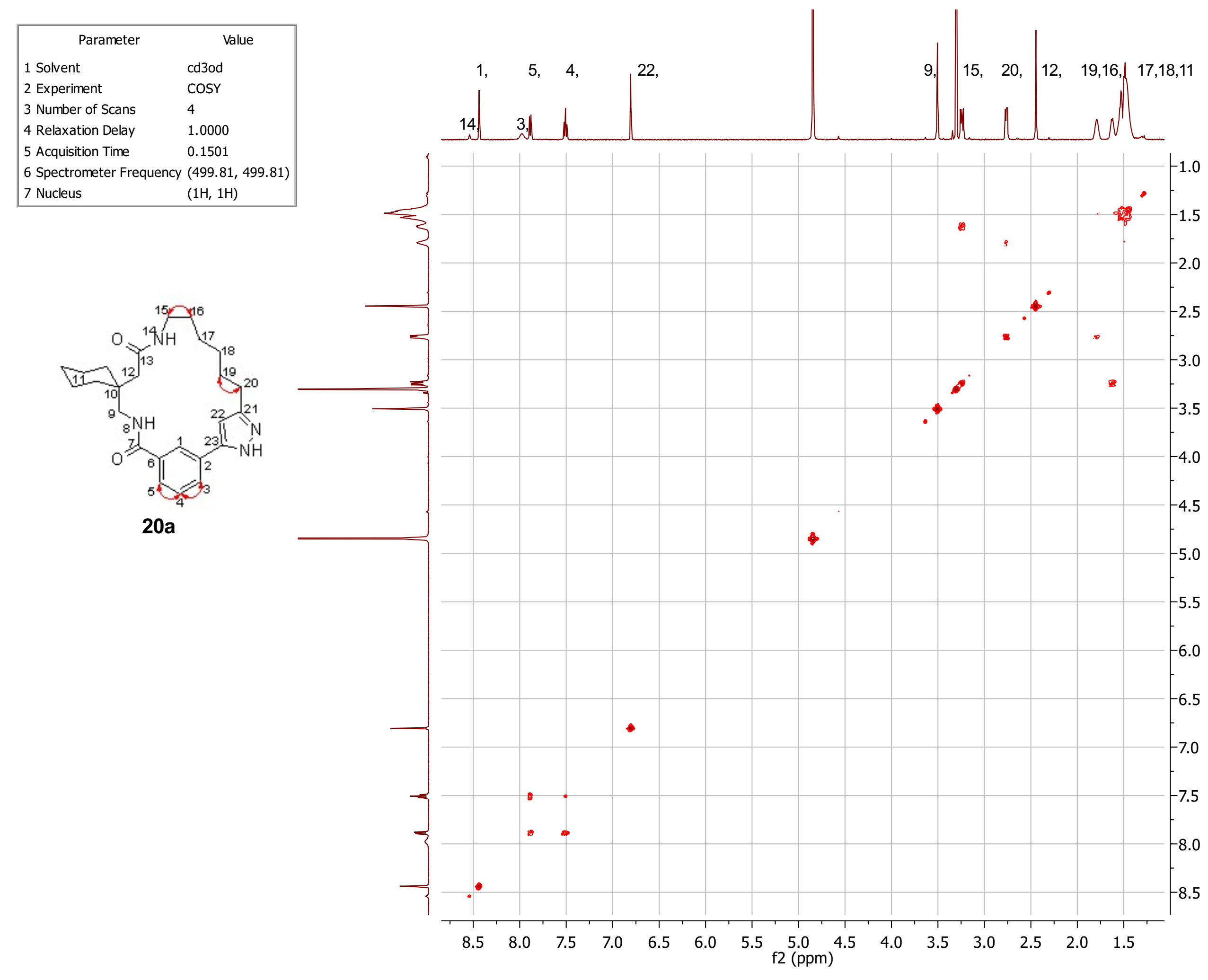




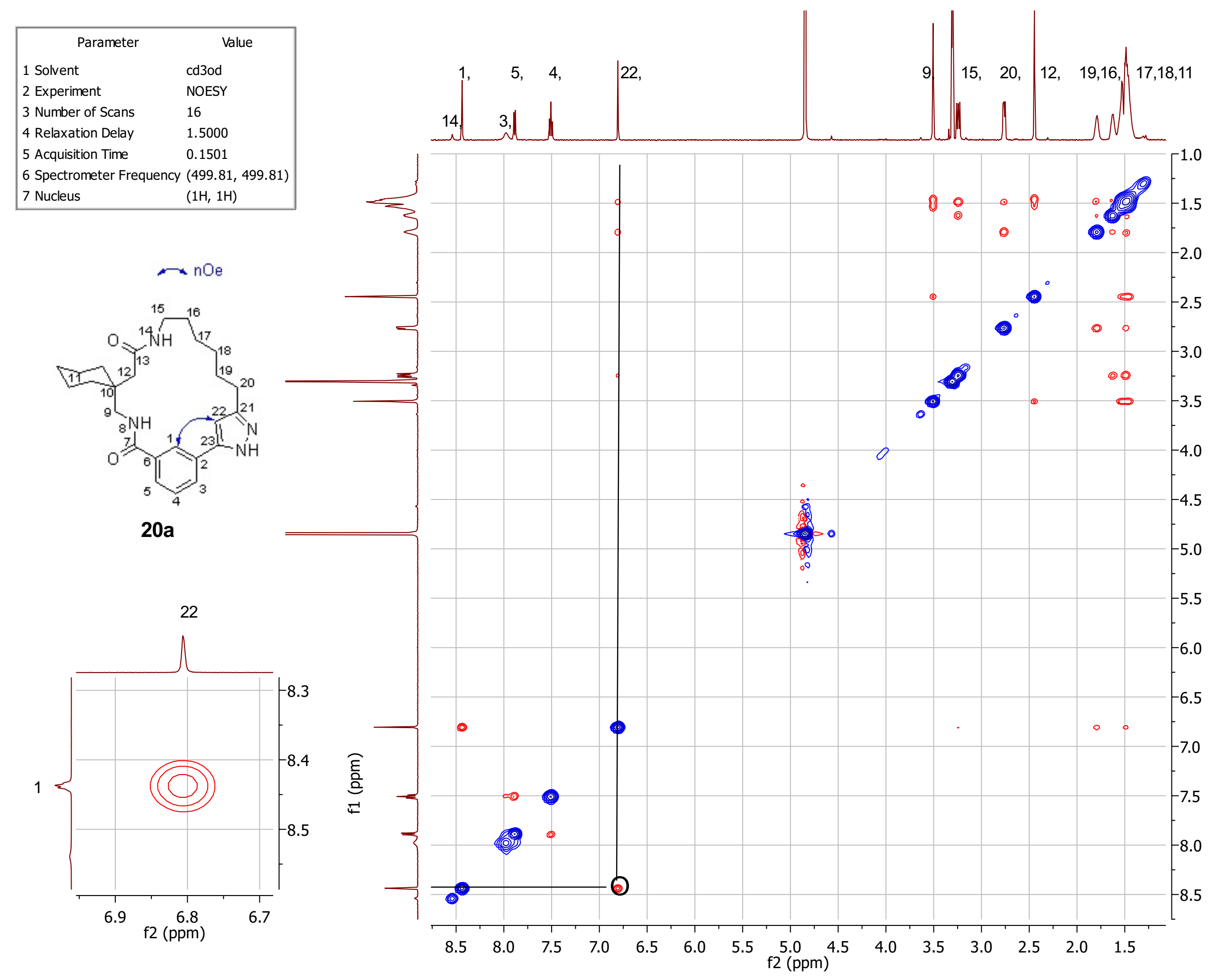


นกำ $\infty$ ベN゙ベN゙N゙N゙N

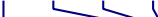

Paramete

1 Solvent

2 Experiment

3 Number of Scans

4 Relaxation Delay

5 Acquisition Time

6 Spectrometer Frequency 499.81

7 Nucleus

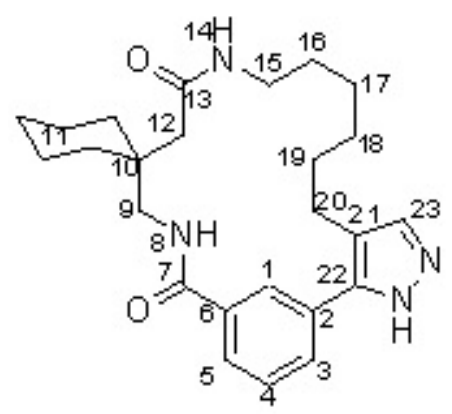

21a

$1,5,4,23$ 3

Tा माना श्राप

స

○, m m m m m m m 


\begin{tabular}{|l|l|}
\hline \multicolumn{1}{|c|}{ Parameter } & Value \\
1 Solvent & cd3od \\
2 Experiment & $1 \mathrm{D}$ \\
3 Number of Scans & 19000 \\
4 Relaxation Delay & 1.0000 \\
5 Acquisition Time & 1.3107 \\
6 Spectrometer Frequency & 100.59 \\
7 Nucleus & $13 \mathrm{C}$ \\
\hline
\end{tabular}

চ

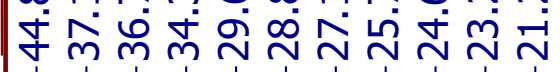

党

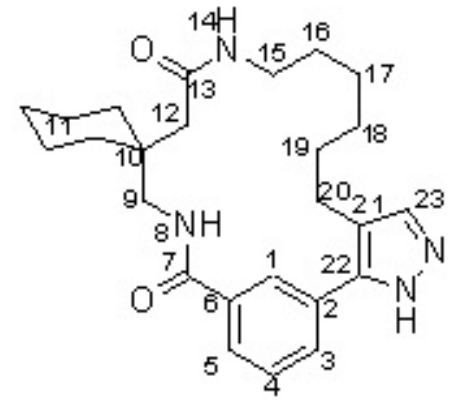

21a

13, 7,

22, $\quad 23$

$6,3,4$,

21,

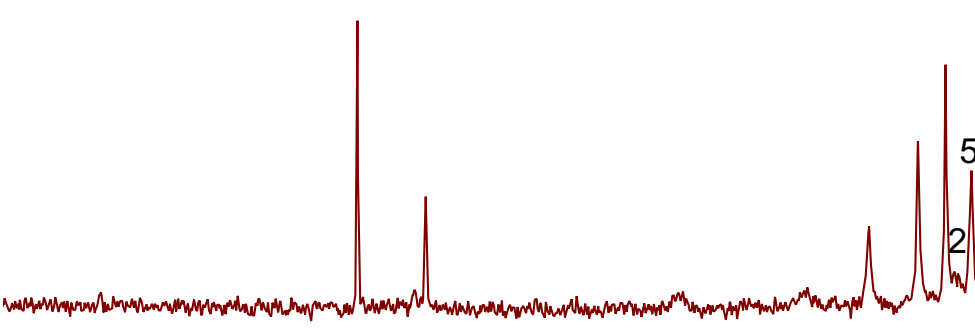

(1) 


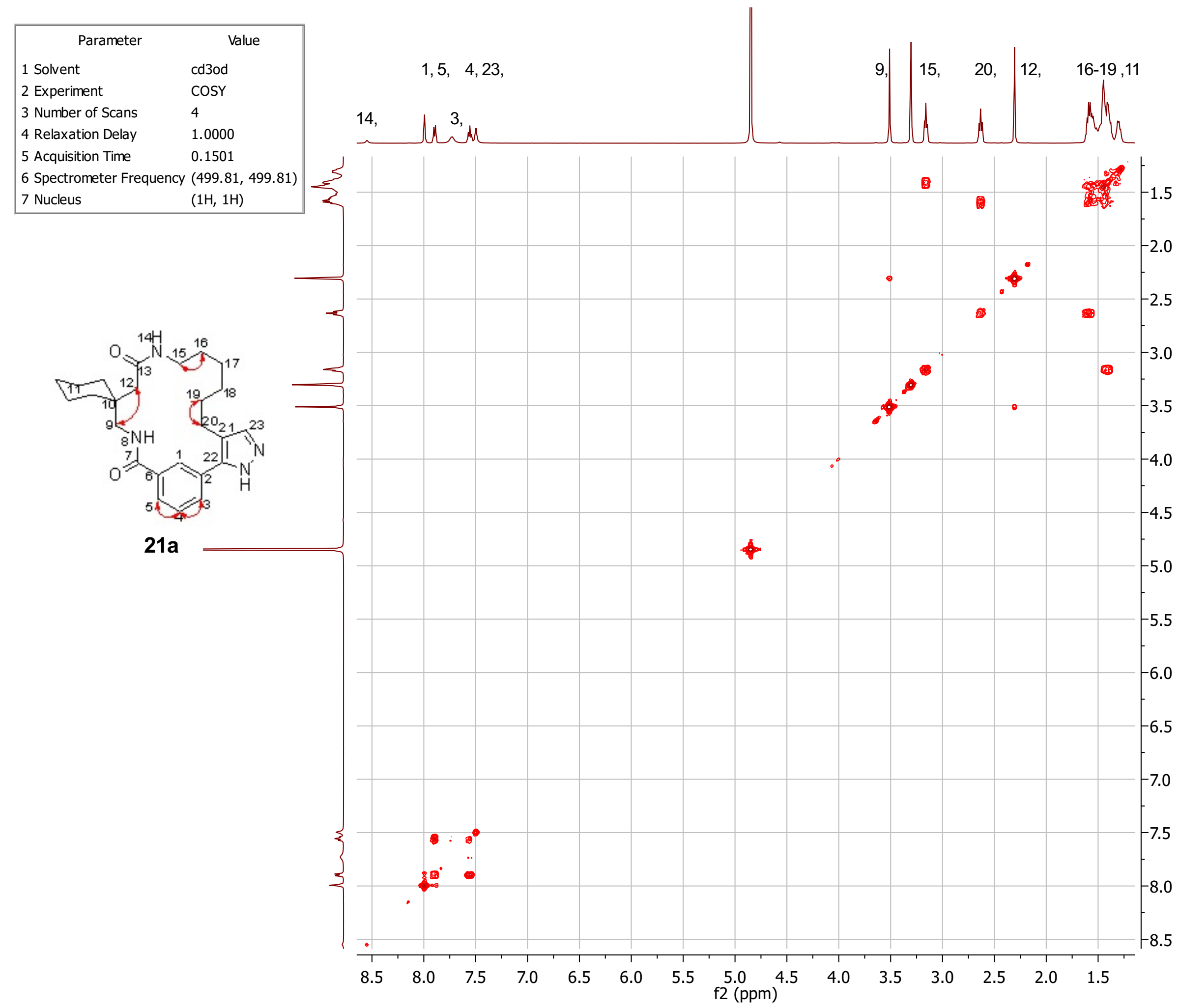




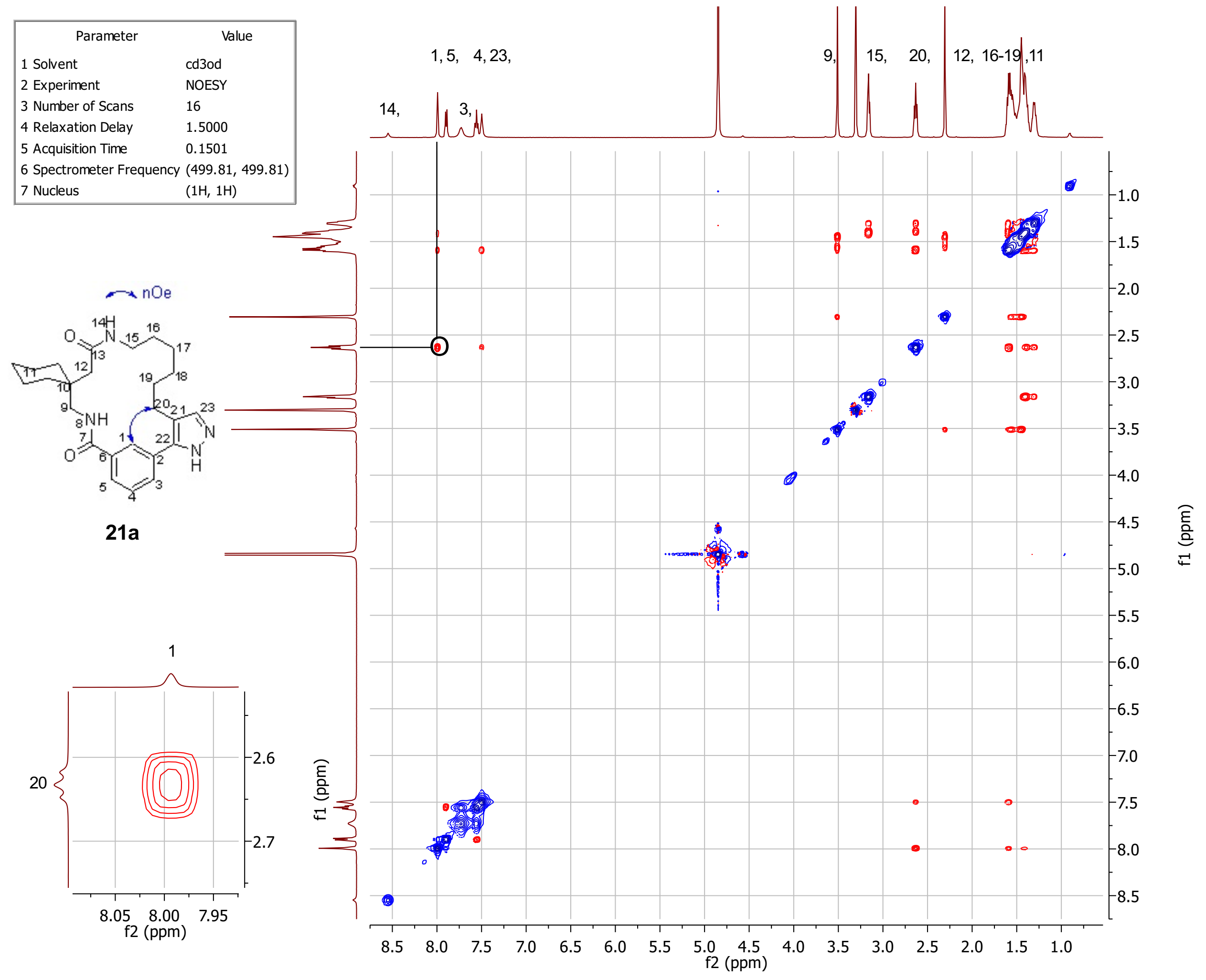




\begin{tabular}{|ll|}
\hline \multicolumn{1}{|c|}{ Parameter } & \multicolumn{1}{c|}{ Value } \\
1 Solvent & cd3od \\
2 Experiment & $1 \mathrm{D}$ \\
3 Number of Scans & 8 \\
4 Relaxation Delay & 1.0000 \\
5 Acquisition Time & 2.0447 \\
6 Spectrometer Frequency & 499.81 \\
7 Nucleus & $1 \mathrm{H}$ \\
\hline
\end{tabular}
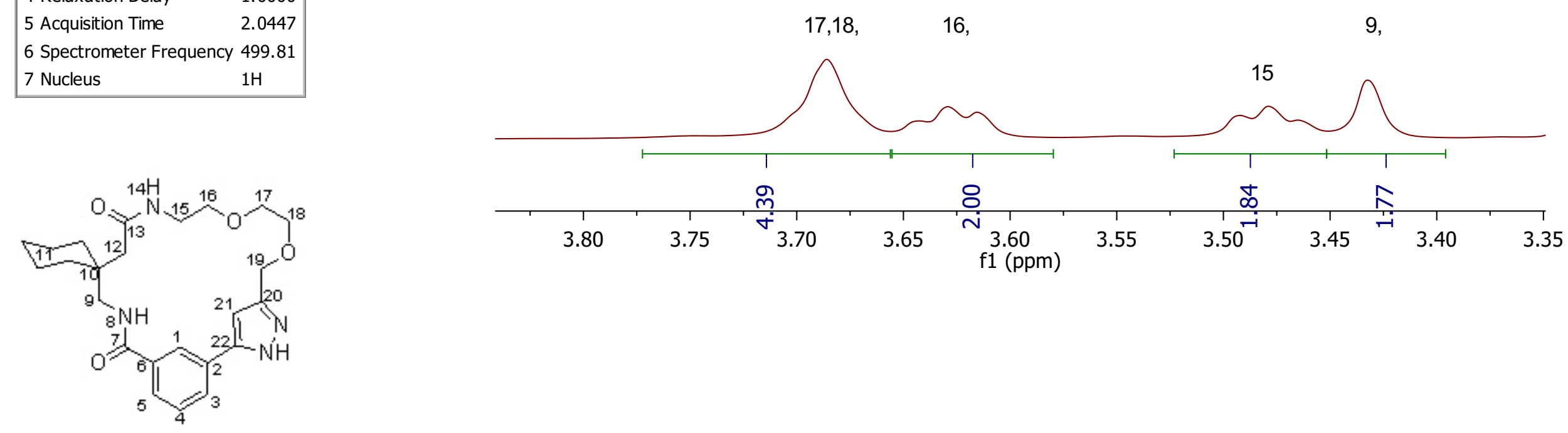

$20 b$

$1, \quad 5, \quad 4, \quad 21$,
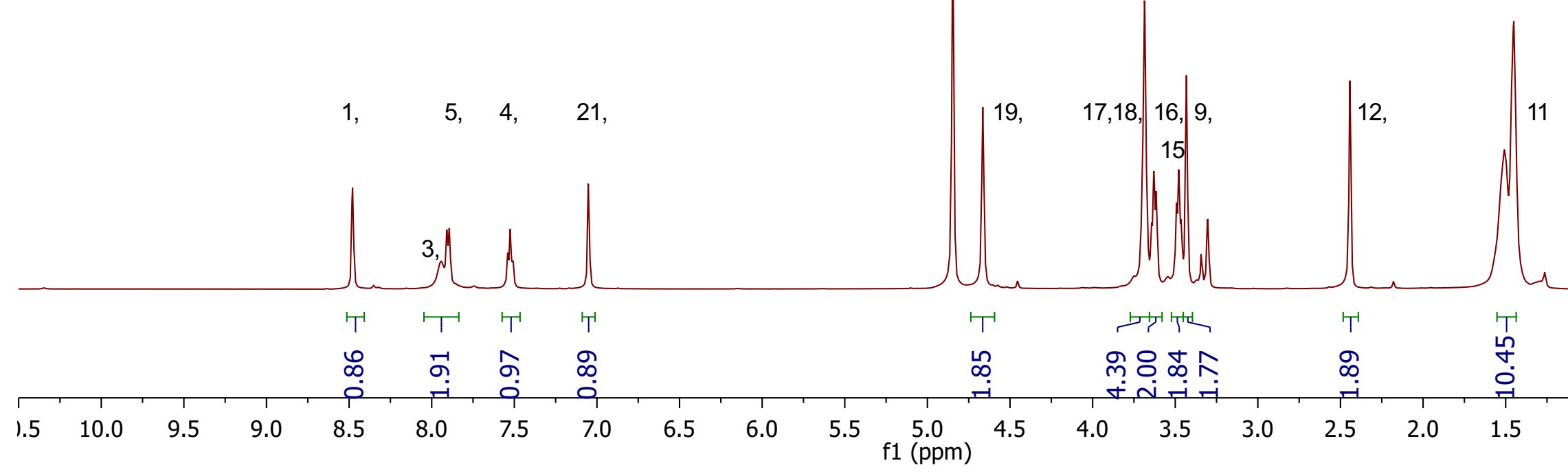


\section{ติ ก \\ ก}

\begin{tabular}{|c|c|}
\hline Parameter & Value \\
\hline 1 Solvent & cd3od \\
\hline 2 Experiment & 1D \\
\hline 3 Number of Scans & 10000 \\
\hline 4 Relaxation Delay & 1.0000 \\
\hline 5 Acquisition Time & 1.3042 \\
\hline 6 Spectrometer Frequency & 100.61 \\
\hline 7 Nucleus & $13 C$ \\
\hline
\end{tabular}

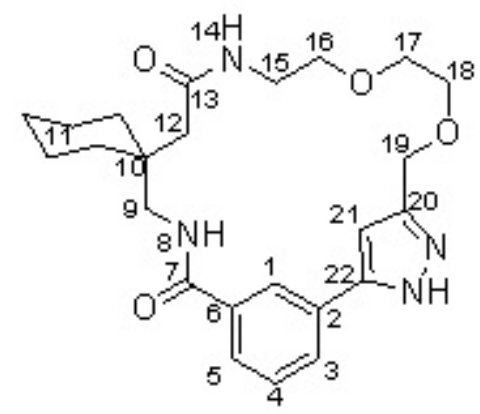

20b

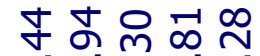

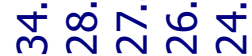

각각

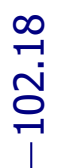

당우능

ㅊํㅇ

र

13

21

16

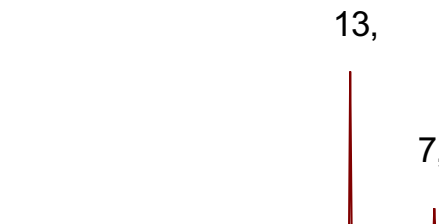

4
5
$j^{3}$

17,18 


\begin{tabular}{|ll|}
\hline \multicolumn{1}{|c|}{ Parameter } & \multicolumn{1}{c|}{ Value } \\
1 Solvent & cd3od \\
2 Experiment & COSY \\
3 Number of Scans & 4 \\
4 Relaxation Delay & 1.0000 \\
5 Acquisition Time & 0.1501 \\
6 Spectrometer Frequency & $(499.81,499.81)$ \\
7 Nucleus & $(1 \mathrm{H}, 1 \mathrm{H})$ \\
\hline
\end{tabular}<smiles></smiles>

20b

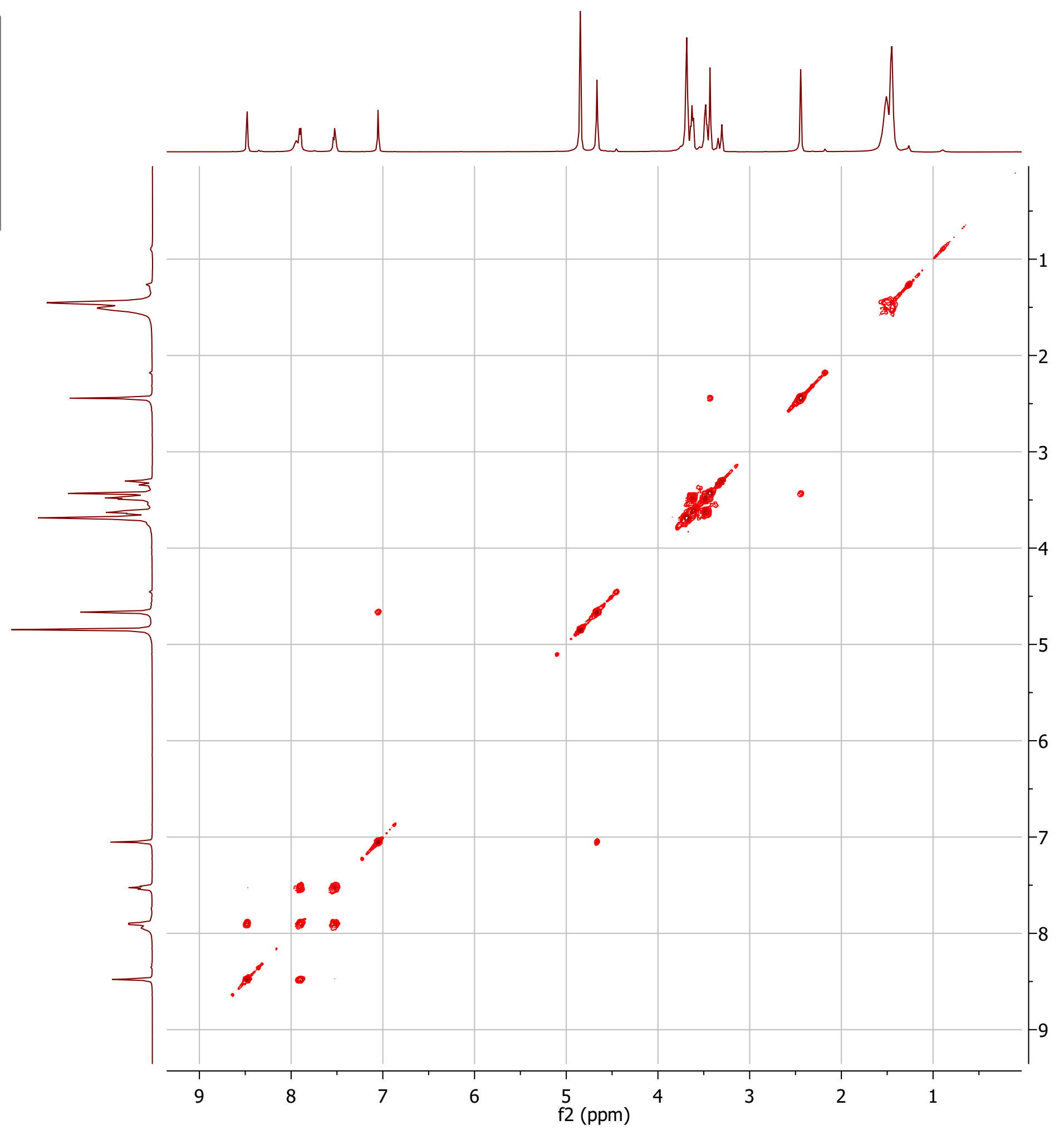

छิ
는 


\begin{tabular}{|ll|}
\hline \multicolumn{1}{|c|}{ Parameter } & \multicolumn{1}{c|}{ Value } \\
1 Solvent & cd3od \\
2 Experiment & NOESY \\
3 Number of Scans & 16 \\
4 Relaxation Delay & 1.5000 \\
5 Acquisition Time & 0.1501 \\
6 Spectrometer Frequency & $(499.81,499.81)$ \\
7 Nucleus & $(1 \mathrm{H}, 1 \mathrm{H})$ \\
\hline
\end{tabular}

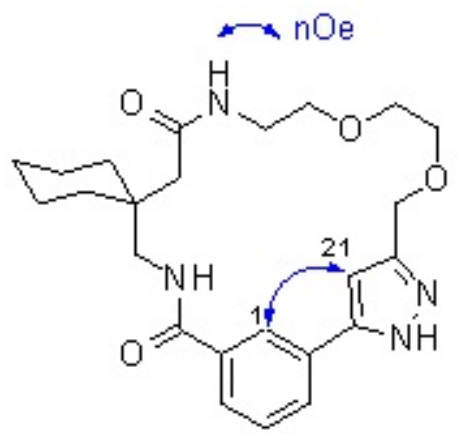

20b
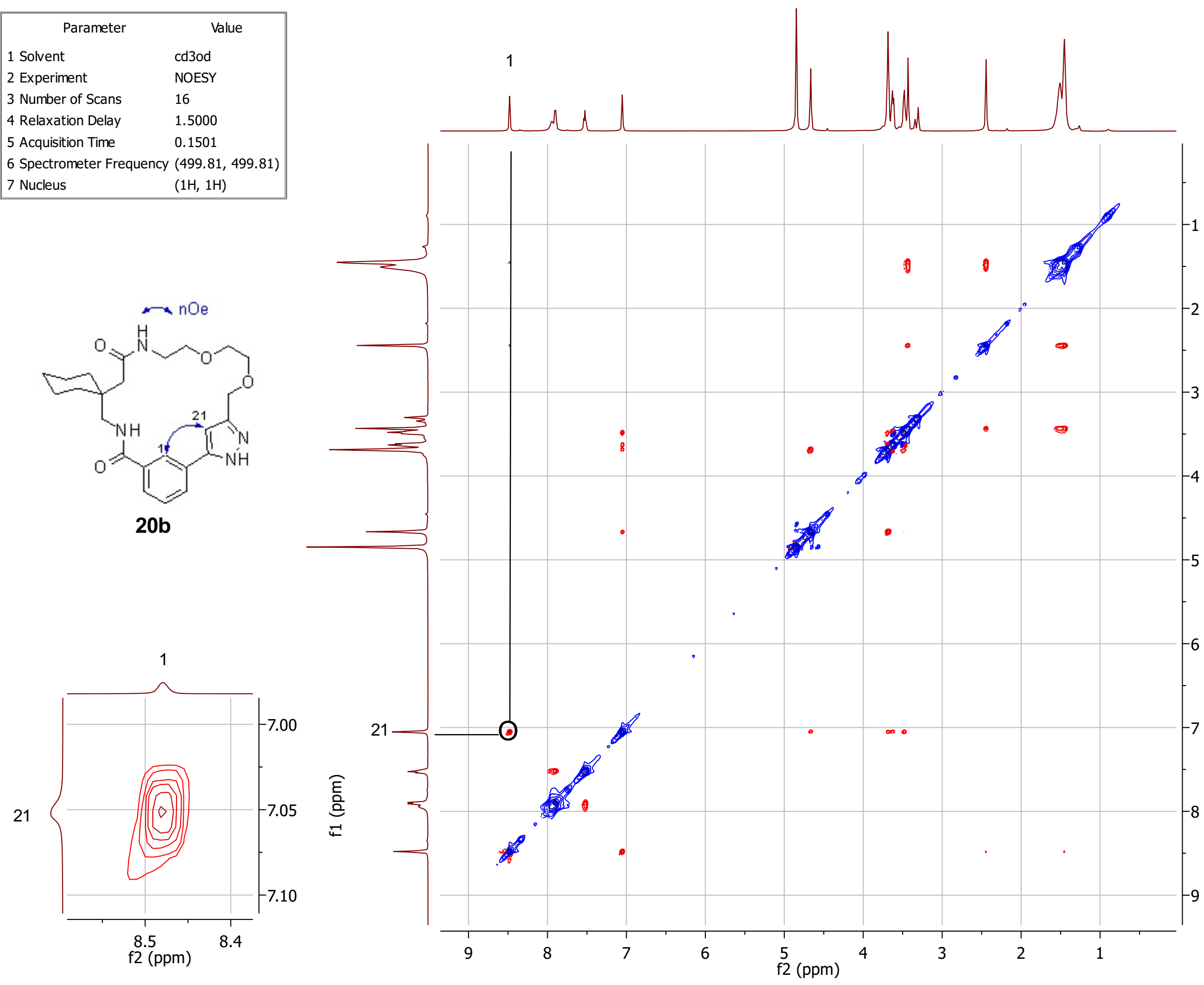

흘
건 


\begin{tabular}{|ll|}
\hline \multicolumn{1}{|c|}{ Parameter } & \multicolumn{1}{c|}{$\begin{array}{r}\text { Value } \\
\text { 1 Solvent }\end{array}$} \\
2 Experiment & 1D \\
3 Number of Scans & 8 \\
4 Relaxation Delay & 1.0000 \\
5 Acquisition Time & 2.0447 \\
6 Spectrometer Frequency & 499.81 \\
7 Nucleus & $1 \mathrm{H}$ \\
\hline
\end{tabular}
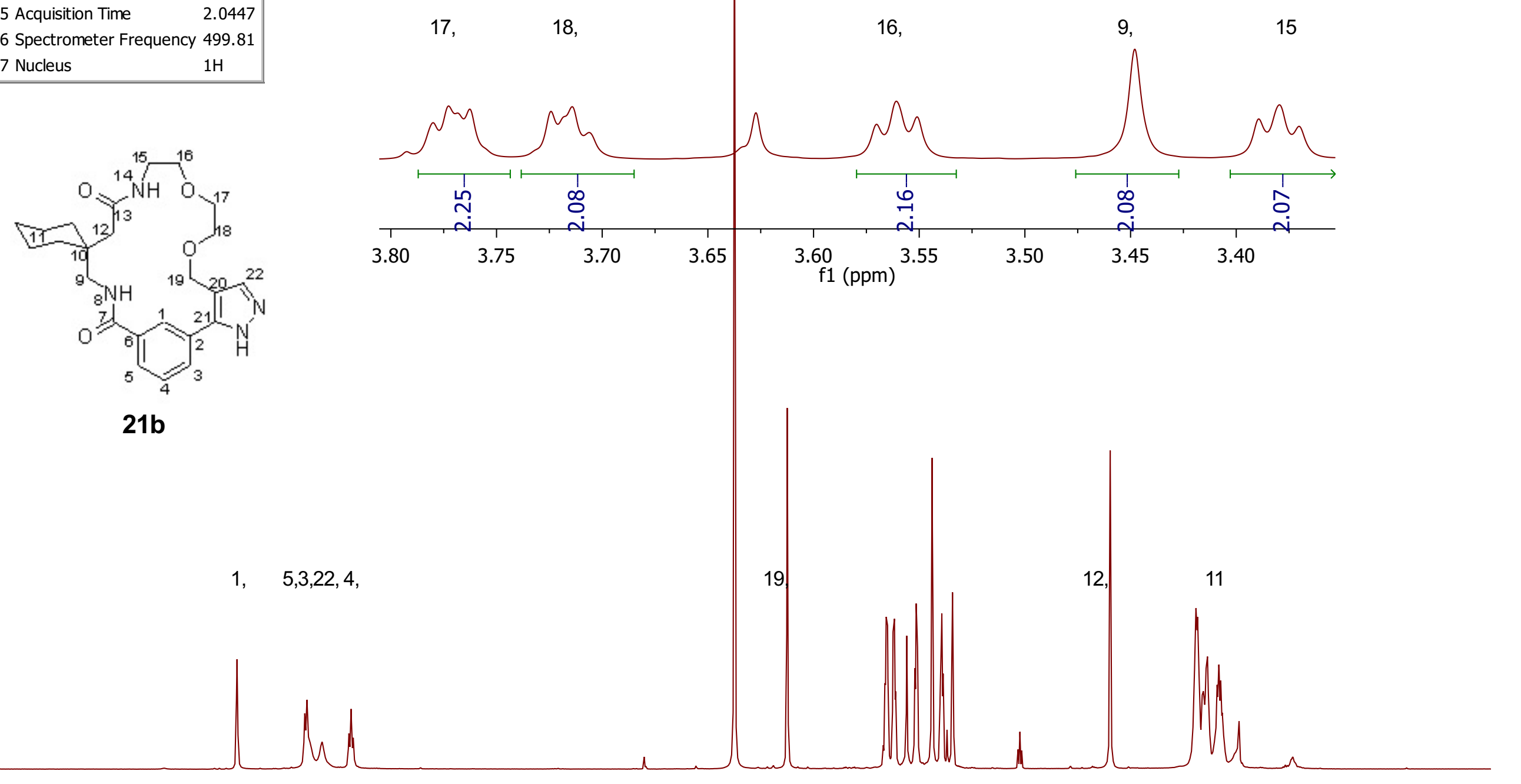

$1, \quad 5,3,22,4$,

:




\section{$\forall$ \\ ㅊํㄱ}

ㄲํㅇㅎํํํำ 웅

苛

긍ํํㅇ

रंशें

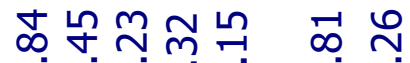

परा

\begin{tabular}{|ll|}
\hline \multicolumn{1}{|c|}{ Parameter } & \multicolumn{1}{c|}{ Value } \\
1 Solvent & cd3od \\
2 Experiment & 1D \\
3 Number of Scans & 2176 \\
4 Relaxation Delay & 1.0000 \\
5 Acquisition Time & 1.3042 \\
6 Spectrometer Frequency & 100.61 \\
7 Nucleus & $13 \mathrm{C}$ \\
\hline \multicolumn{2}{r}{} \\
\end{tabular}

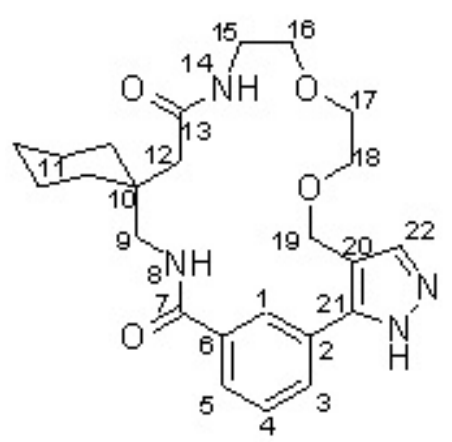

21b
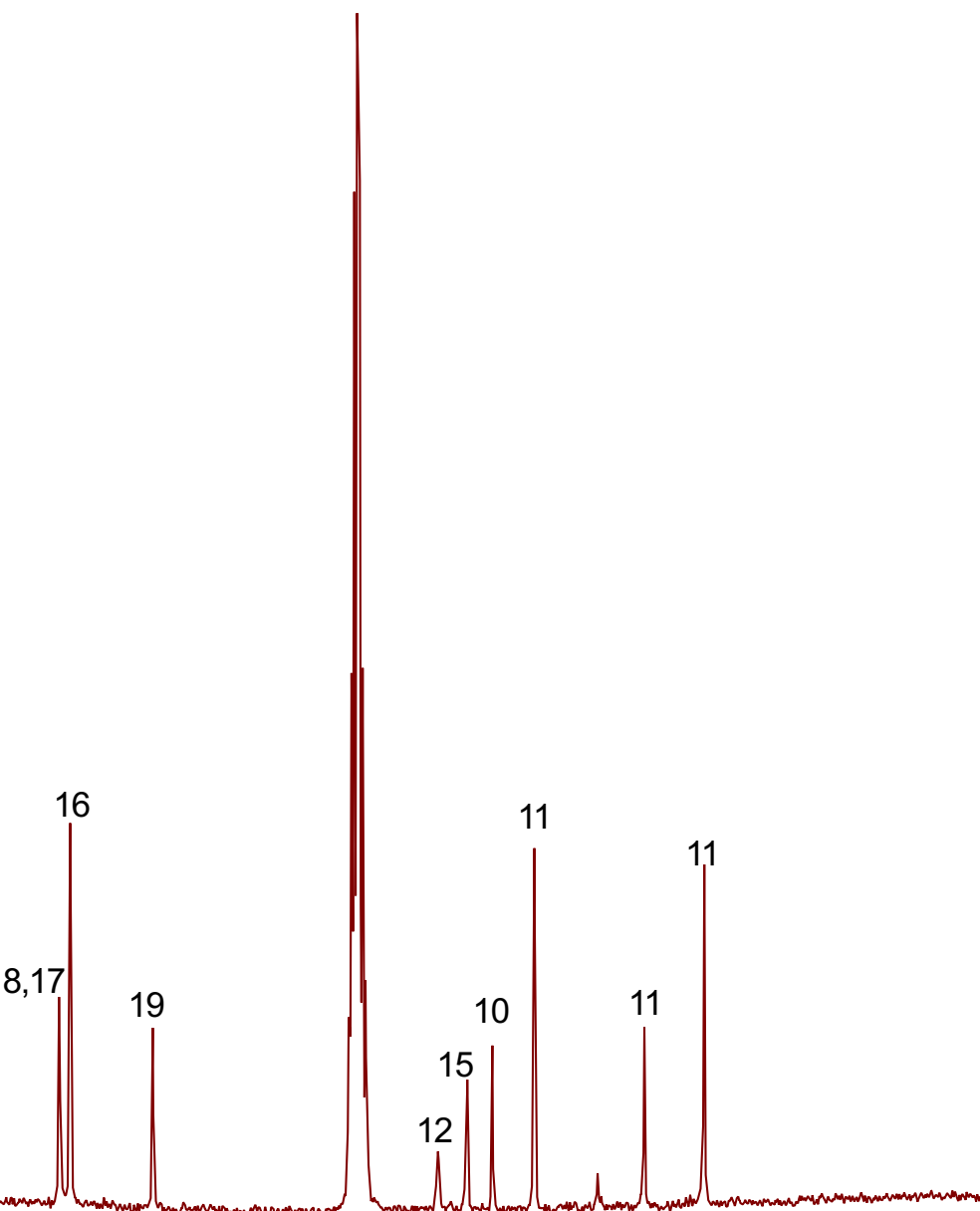


\begin{tabular}{|ll|}
\hline \multicolumn{1}{|c|}{ Parameter } & \multicolumn{1}{c|}{ Value } \\
1 Solvent & cd3od \\
2 Experiment & COSY \\
3 Number of Scans & 4 \\
4 Relaxation Delay & 1.0000 \\
5 Acquisition Time & 0.1499 \\
6 Spectrometer Frequency & $(499.81,499.81)$ \\
7 Nucleus & $(1 \mathrm{H}, 1 \mathrm{H})$ \\
\hline
\end{tabular}

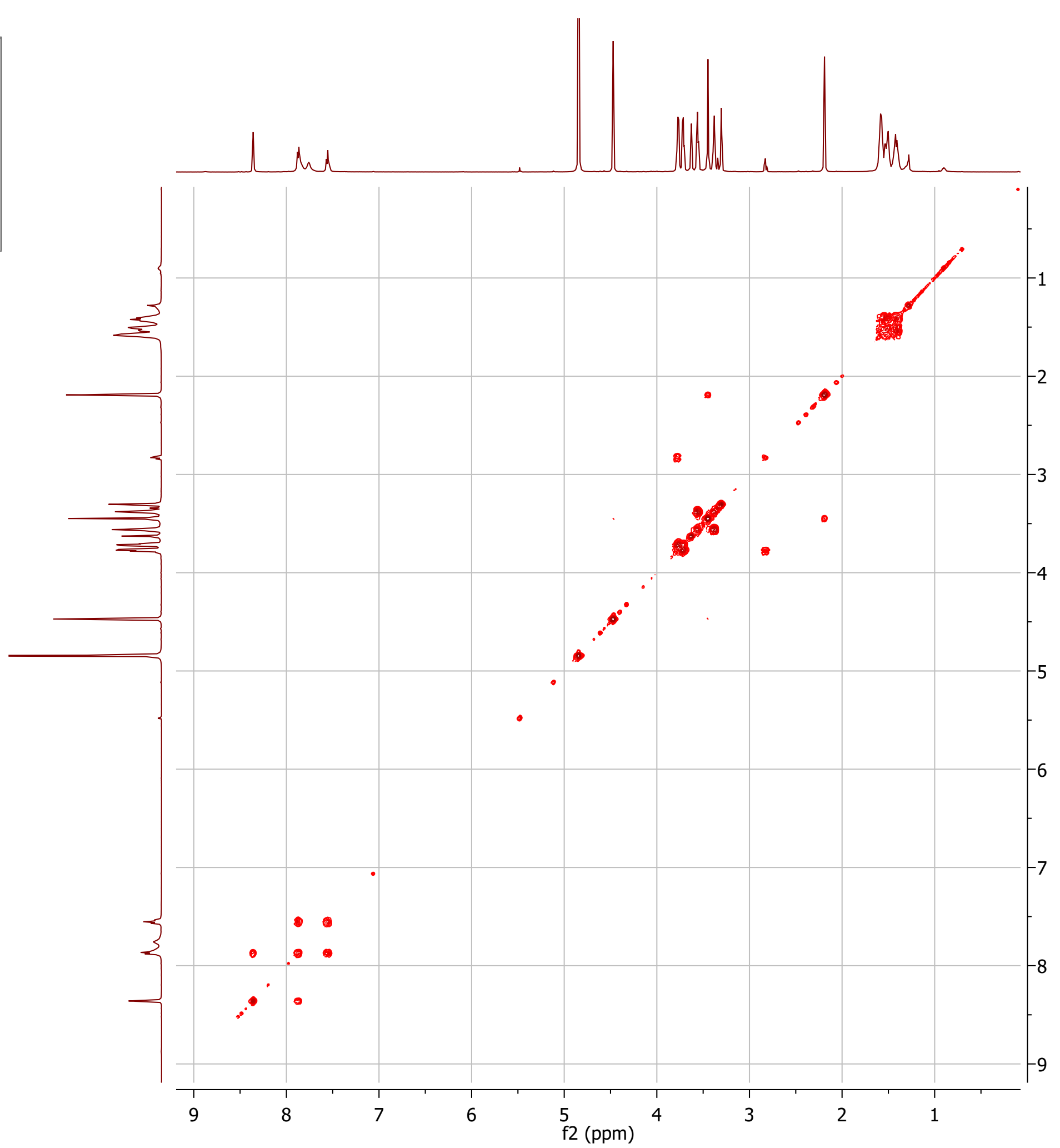

$\hat{E}$
흔 


\begin{tabular}{|ll|}
\hline \multicolumn{1}{|c|}{ Parameter } & \multicolumn{1}{c|}{ Value } \\
1 Solvent & cd3od \\
2 Experiment & NOESY \\
3 Number of Scans & 16 \\
4 Relaxation Delay & 1.5000 \\
5 Acquisition Time & 0.1499 \\
6 Spectrometer Frequency & $(499.81,499.81)$ \\
7 Nucleus & $(1 \mathrm{H}, 1 \mathrm{H})$ \\
\hline
\end{tabular}

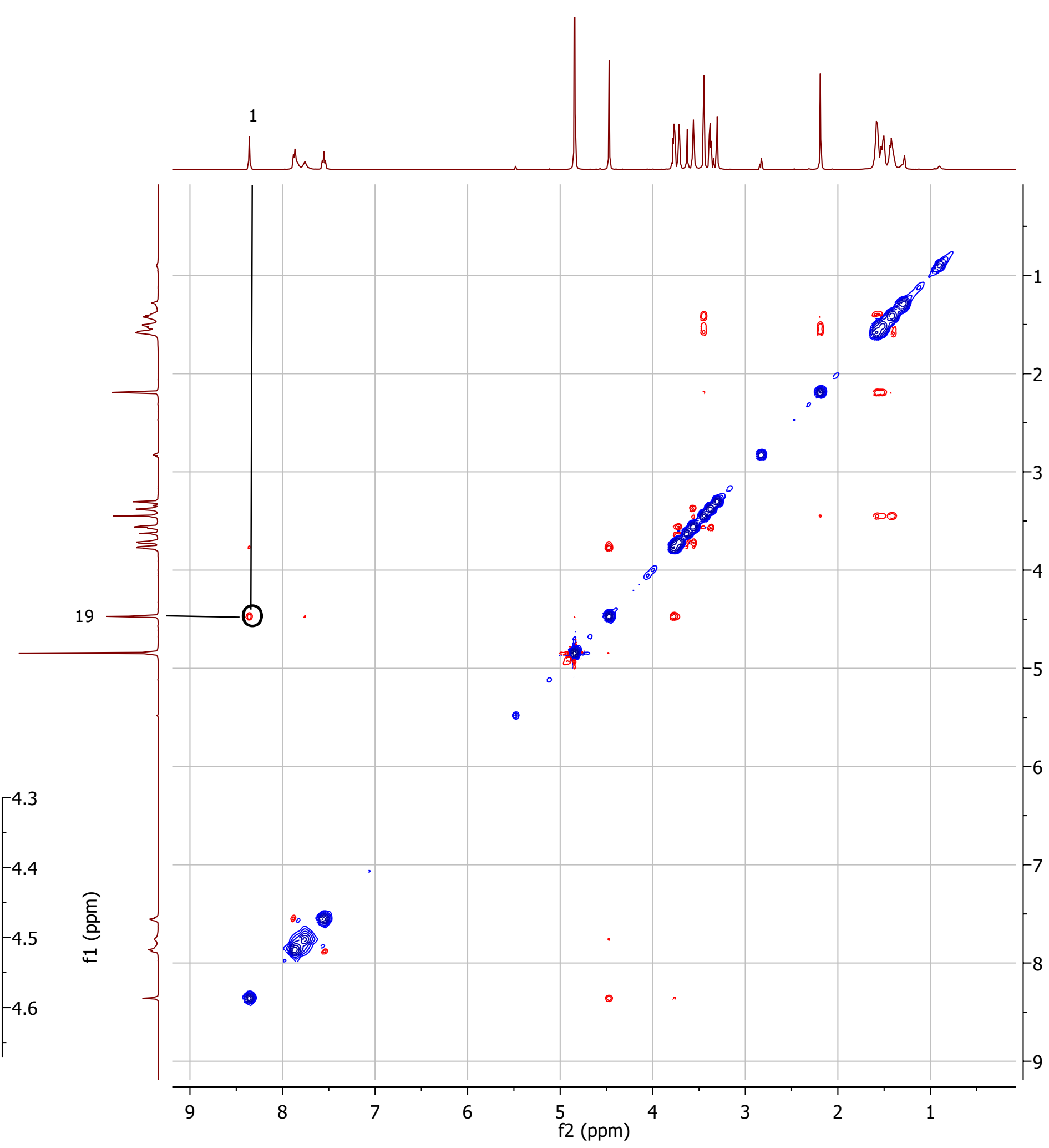




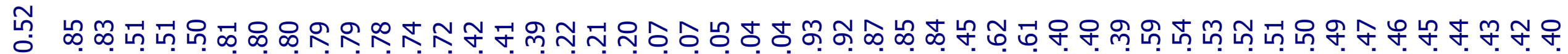

自

\begin{tabular}{|ll|}
\hline \multicolumn{1}{|c|}{ Parameter } & \multicolumn{1}{c|}{$\begin{array}{c}\text { Value } \\
\text { cd3od }\end{array}$} \\
2 Solvent & $1 \mathrm{D}$ \\
3 Numberiment & 8 \\
4 Relaxation Delay & 1.0000 \\
5 Acquisition Time & 2.0447 \\
6 Spectrometer Frequency & 499.81 \\
7 Nucleus & $1 \mathrm{H}$ \\
\hline
\end{tabular}
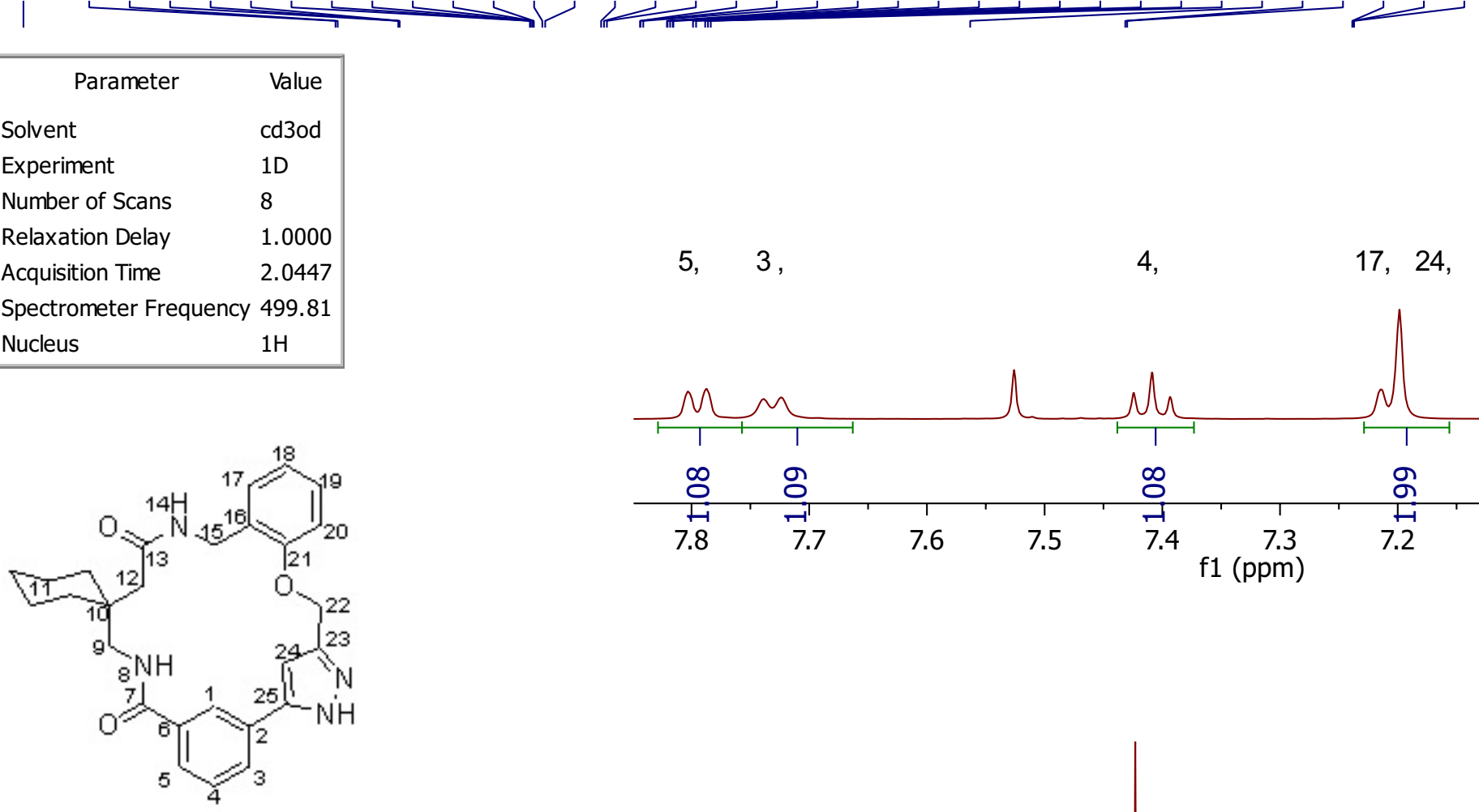
$5, \quad 3$,
4
17, 24,
19
20, 18,
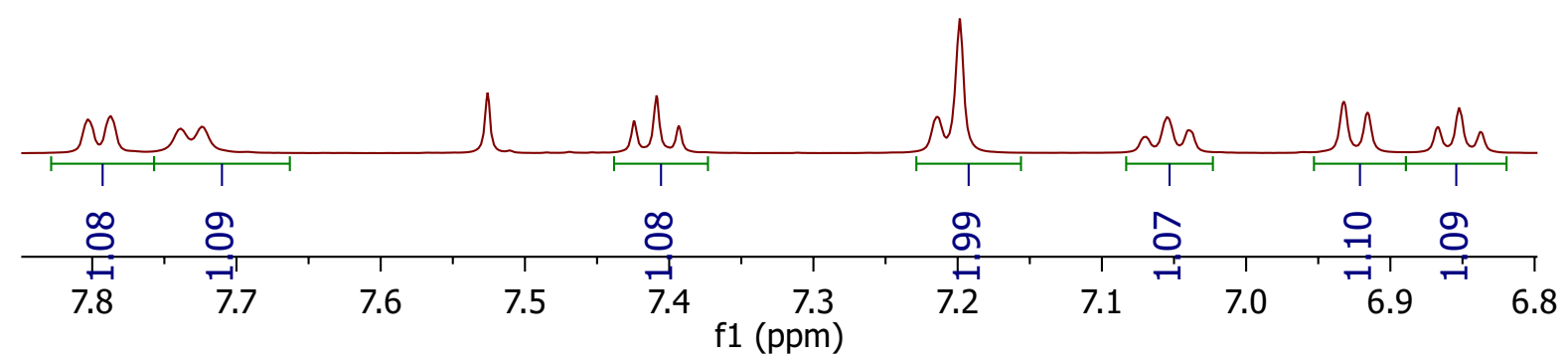

20c

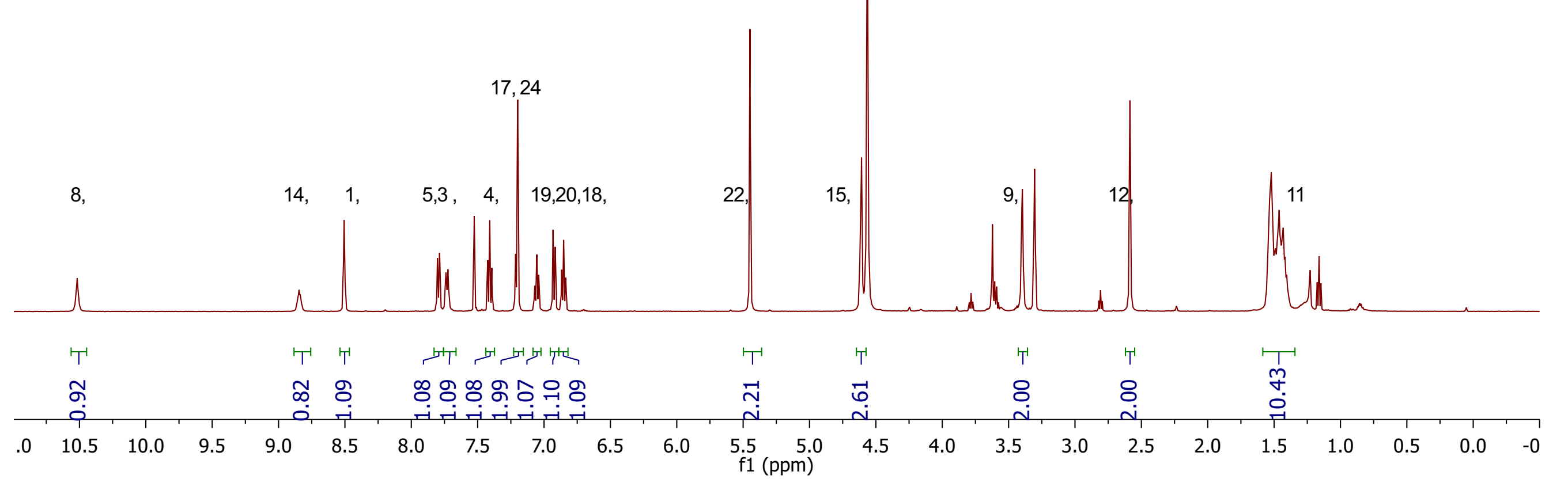




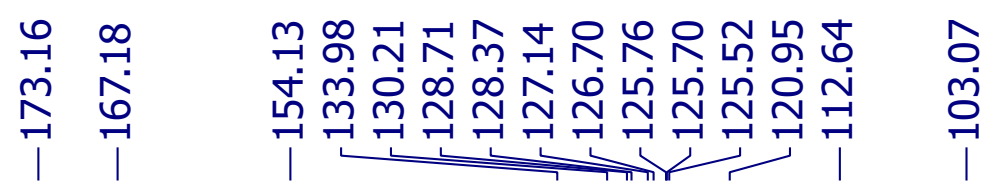

\begin{tabular}{|ll|}
\hline \multicolumn{1}{|c|}{ Parameter } & \multicolumn{1}{c|}{ Value } \\
1 Solvent & cd3od/cdcl3=1:1 \\
2 Experiment & 1D \\
3 Number of Scans & 14000 \\
4 Relaxation Delay & 1.0000 \\
5 Acquisition Time & 1.3042 \\
6 Spectrometer Frequency & 100.61 \\
7 Nucleus & $13 \mathrm{C}$ \\
\hline
\end{tabular}
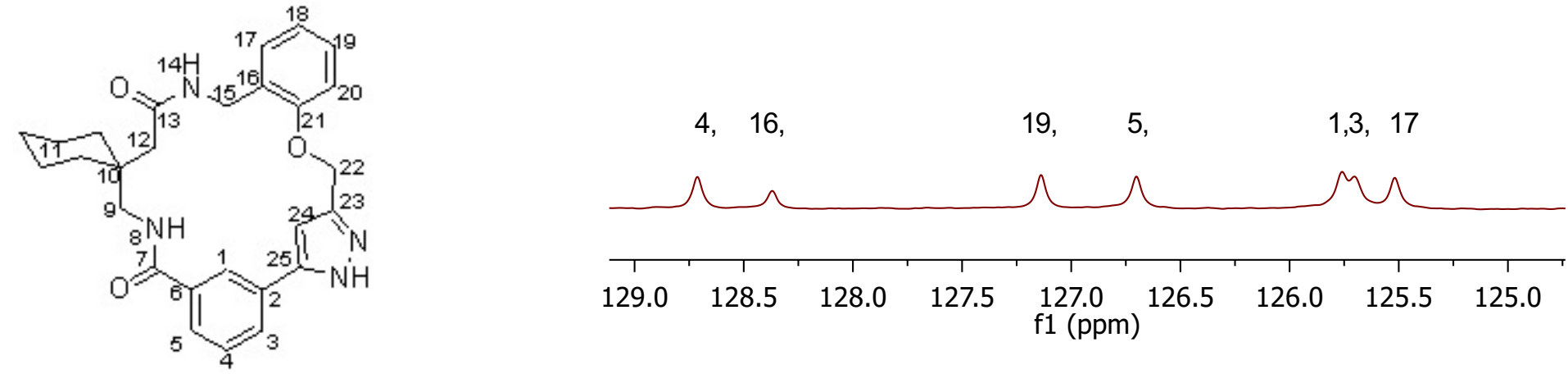

20c

190

180

170

160

150

140

130

120

$110 \quad 100$ f1 (ppm)

$90 \quad 80$

18, 20,

24

13,7 ,

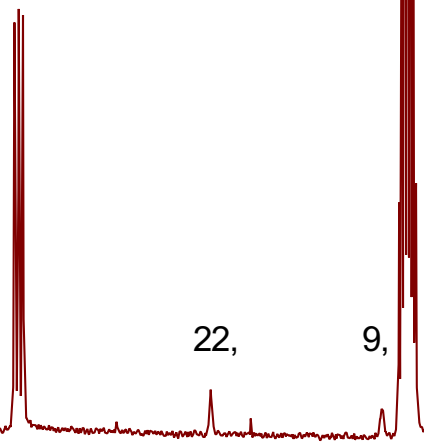

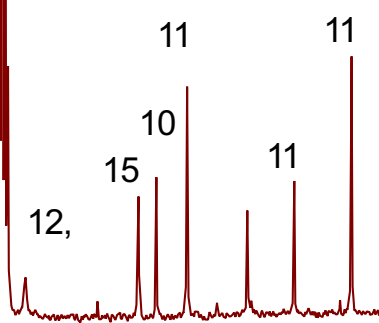

8070

60

50

40

30

20

10 C 


\begin{tabular}{|ll|}
\hline \multicolumn{1}{|c|}{ Parameter } & \multicolumn{1}{c|}{ Value } \\
1 Solvent & cd3od \\
2 Experiment & COSY \\
3 Number of Scans & 4 \\
4 Relaxation Delay & 1.0000 \\
5 Acquisition Time & 0.1501 \\
6 Spectrometer Frequency & $(499.81,499.81)$ \\
7 Nucleus & $(1 \mathrm{H}, 1 \mathrm{H})$ \\
\hline
\end{tabular}

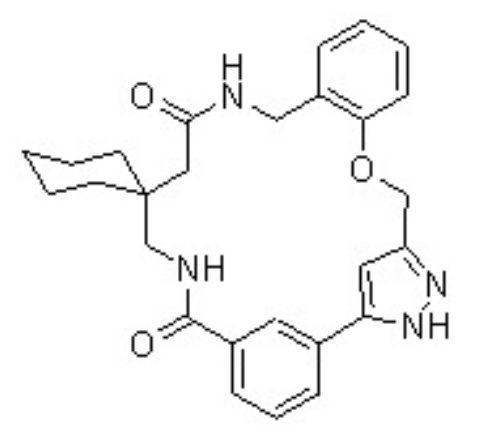

20c

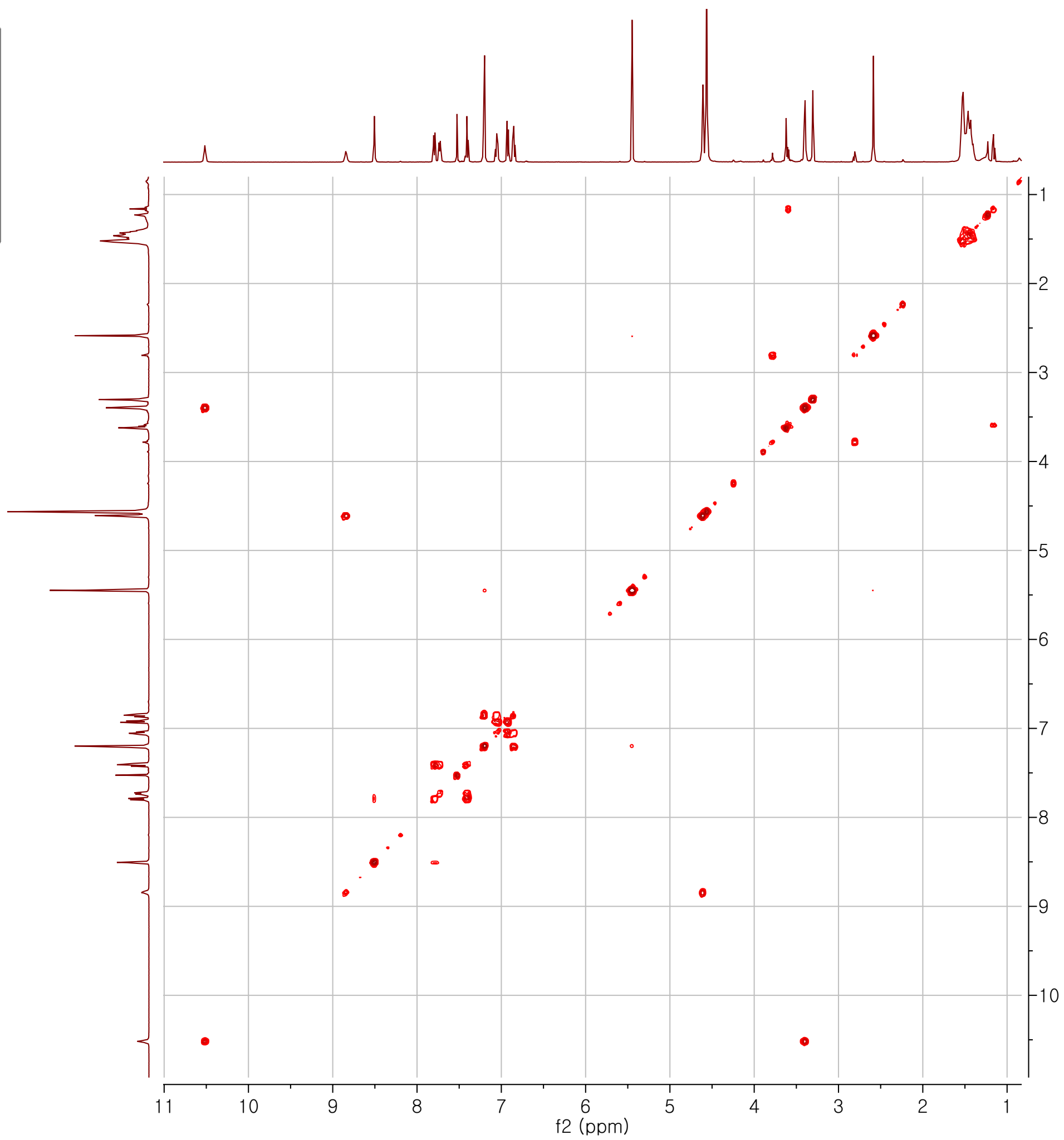




\begin{tabular}{|ll|}
\hline \multicolumn{1}{|c|}{ Parameter } & \multicolumn{1}{c|}{ Value } \\
1 Solvent & cd3od \\
2 Experiment & ASAPHMQC \\
3 Number of Scans & 32 \\
4 Relaxation Delay & 0.0600 \\
5 Acquisition Time & 0.0640 \\
6 Spectrometer Frequency & $(499.81,125.69)$ \\
7 Nucleus & $(1 \mathrm{H}, 13 \mathrm{C})$ \\
\hline
\end{tabular}

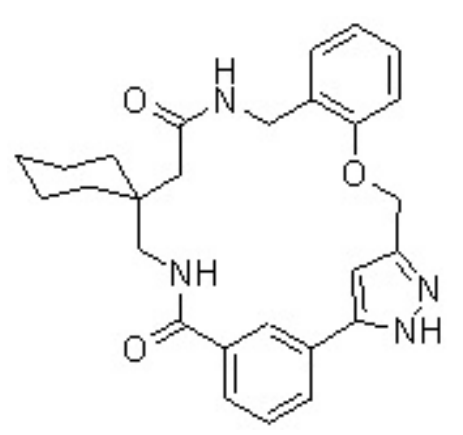

20c

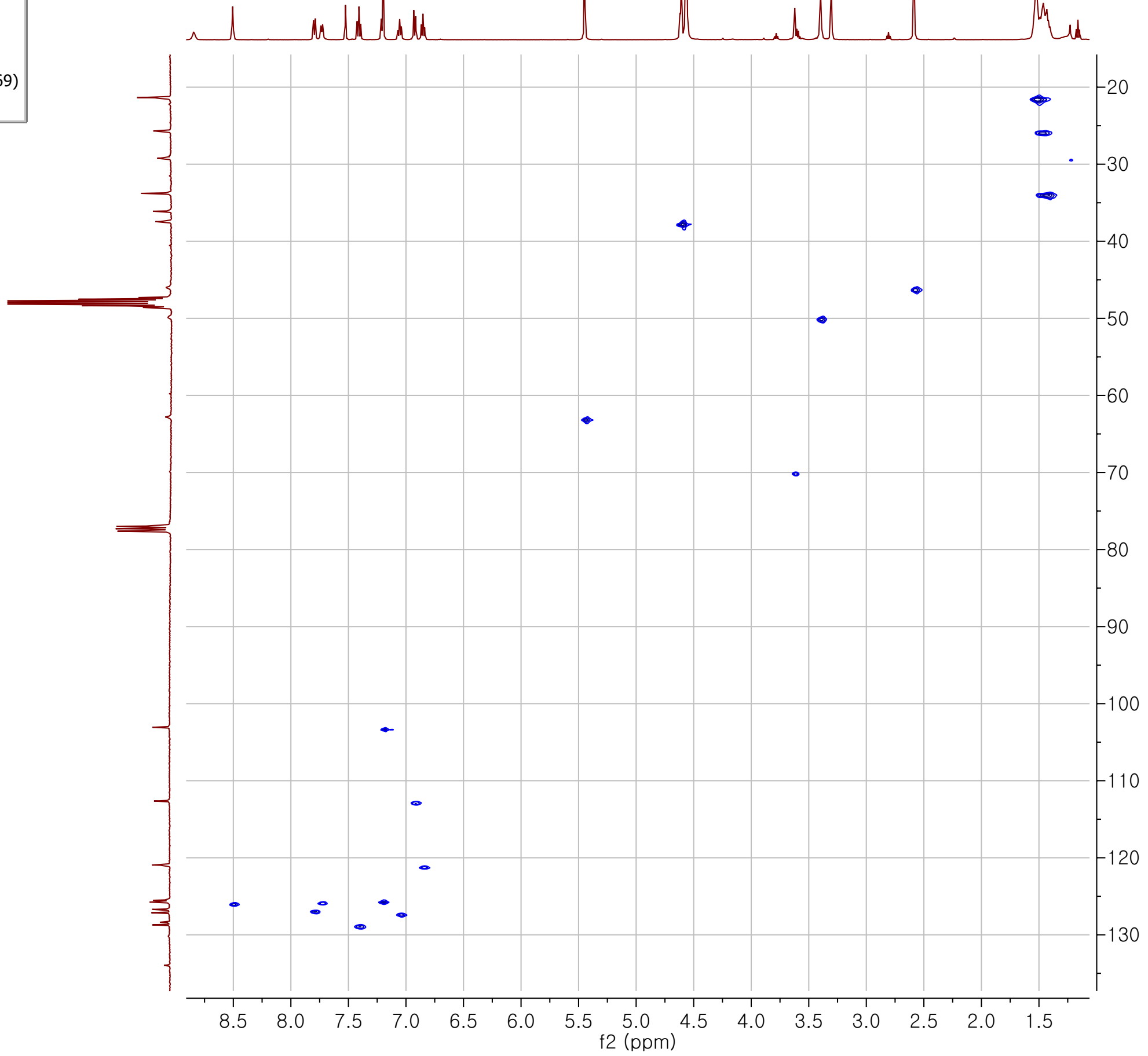




\begin{tabular}{|ll|}
\hline \multicolumn{1}{|c|}{ Parameter } & \multicolumn{1}{c|}{ Value } \\
1 Solvent & cd3od \\
2 Experiment & NOESY \\
3 Number of Scans & 16 \\
4 Relaxation Delay & 1.5000 \\
5 Acquisition Time & 0.1501 \\
6 Spectrometer Frequency & $(499.81,499.81)$ \\
7 Nucleus & $(1 \mathrm{H}, 1 \mathrm{H})$ \\
\hline
\end{tabular}

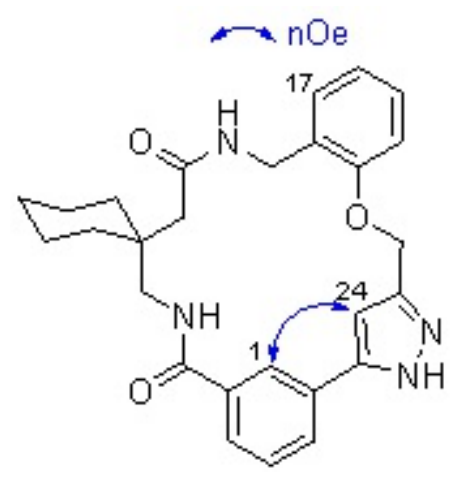

20c

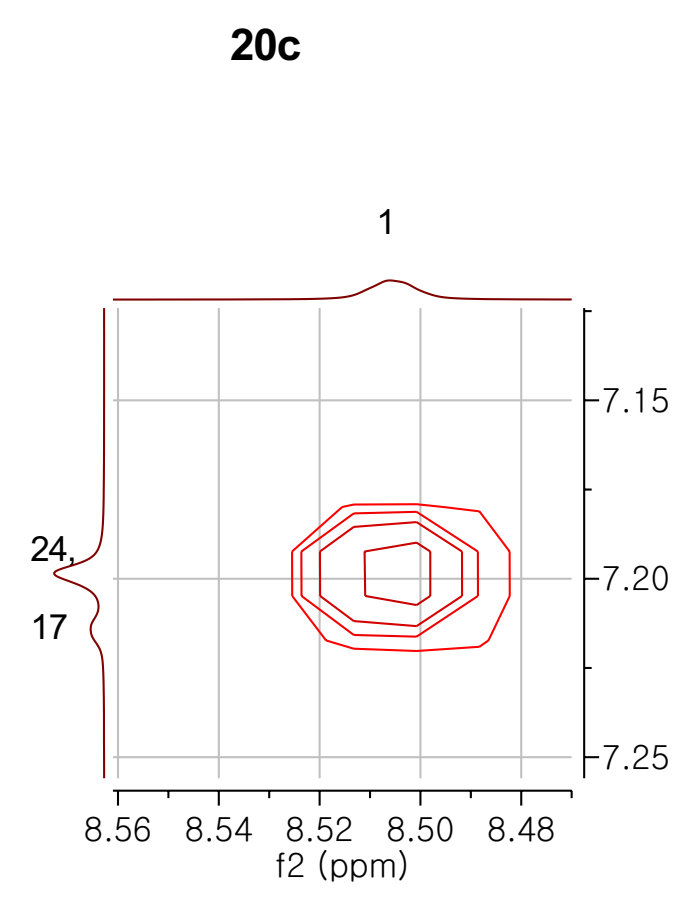




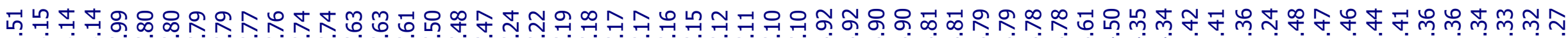

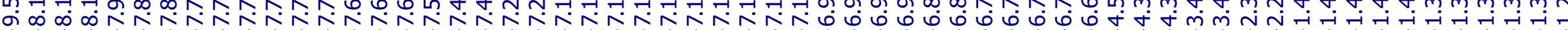

\begin{tabular}{|ll|}
\hline \multicolumn{1}{|c|}{ Parameter } & \multicolumn{1}{c|}{ Value } \\
1 Solvent & cdcl3 \\
2 Experiment & $1 \mathrm{D}$ \\
3 Number of Scans & 8 \\
4 Relaxation Delay & 1.0000 \\
5 Acquisition Time & 2.0447 \\
6 Spectrometer Frequency & 499.81 \\
7 Nucleus & $1 \mathrm{H}$ \\
\hline
\end{tabular}

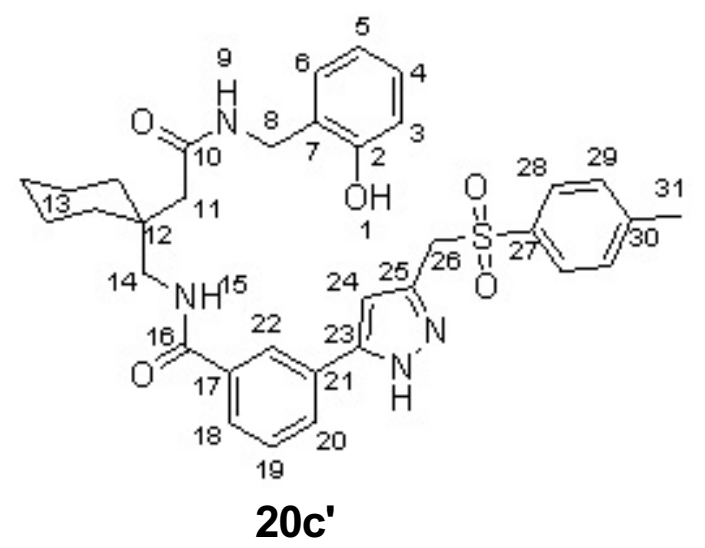
22
$9, \quad 18,15,20, \quad 28, \quad 19$,
$29,4,6$,
$3, \quad 5$,
24

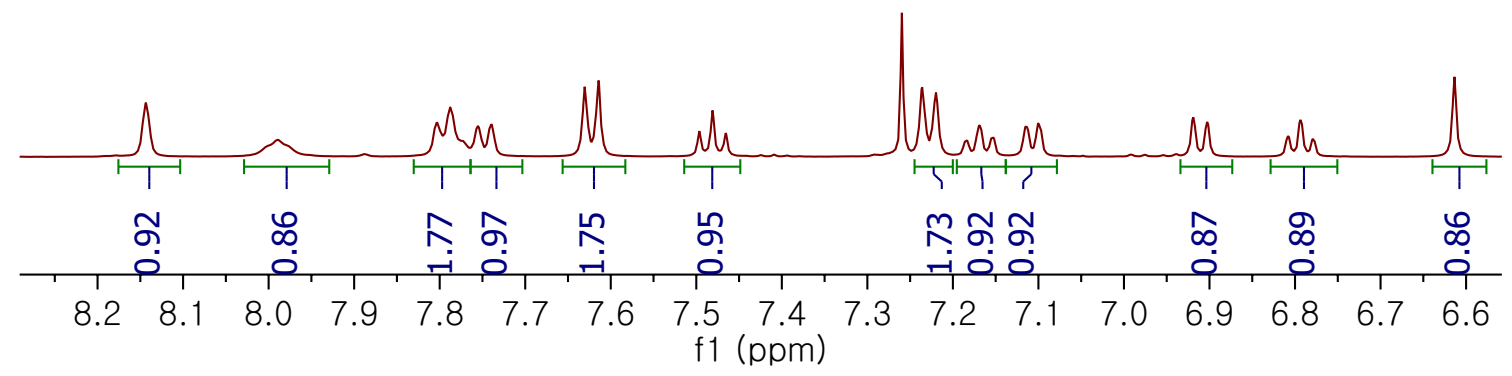

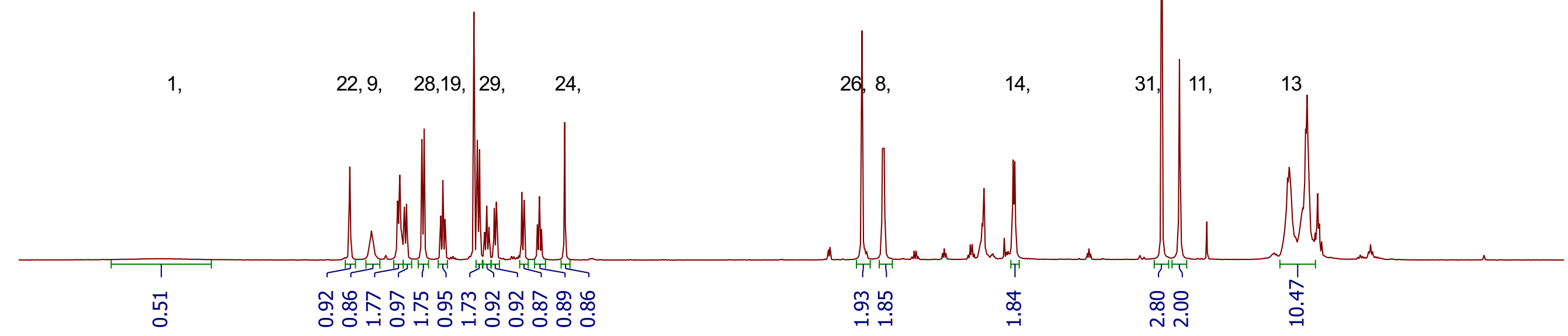

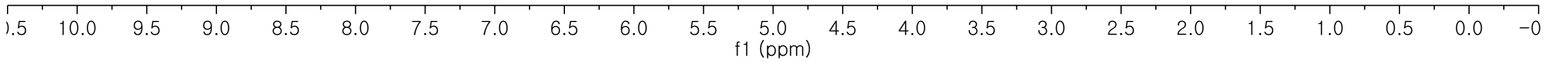




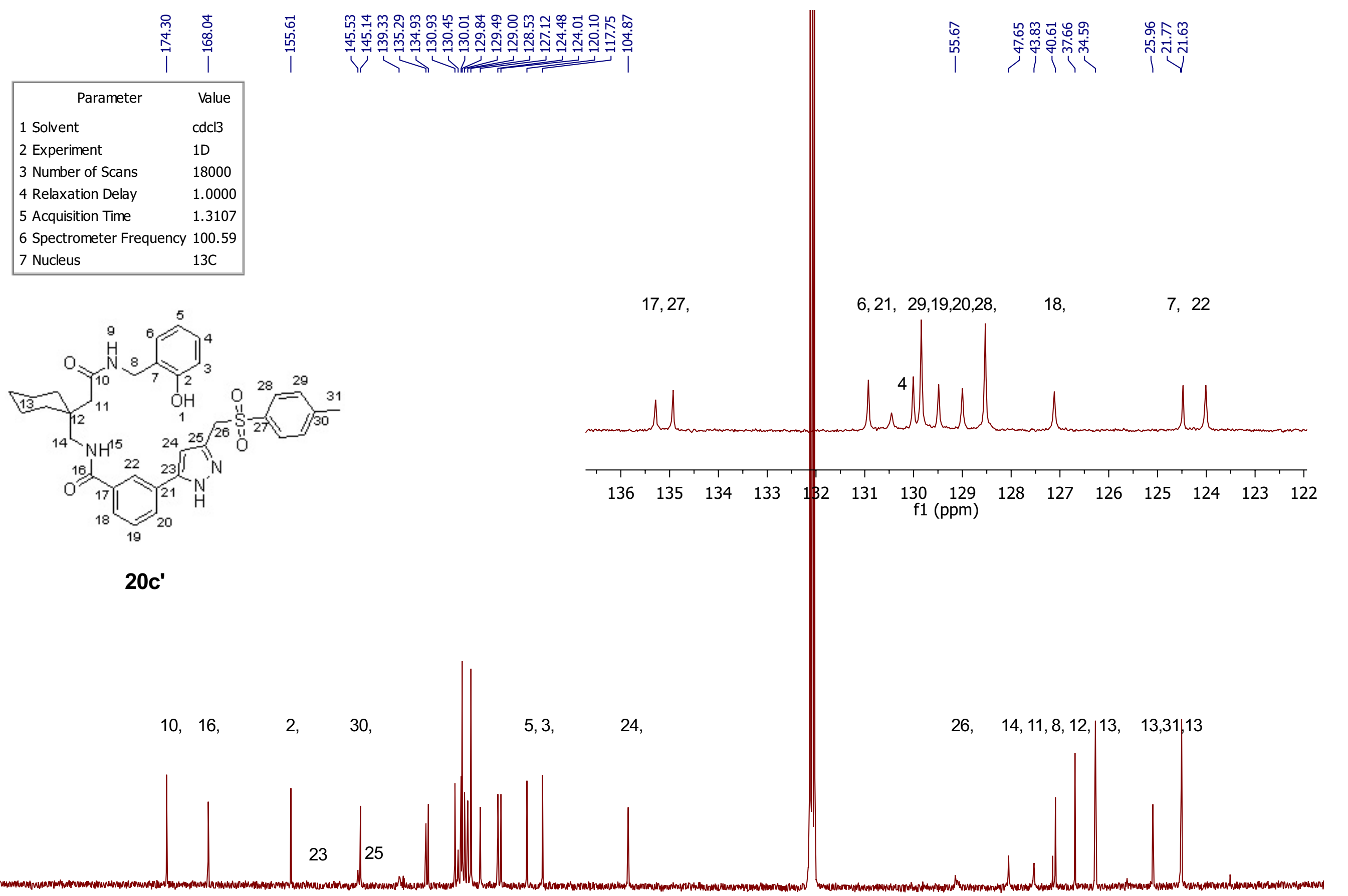

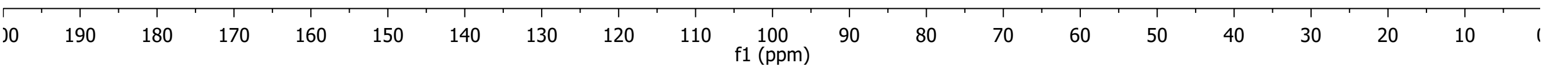




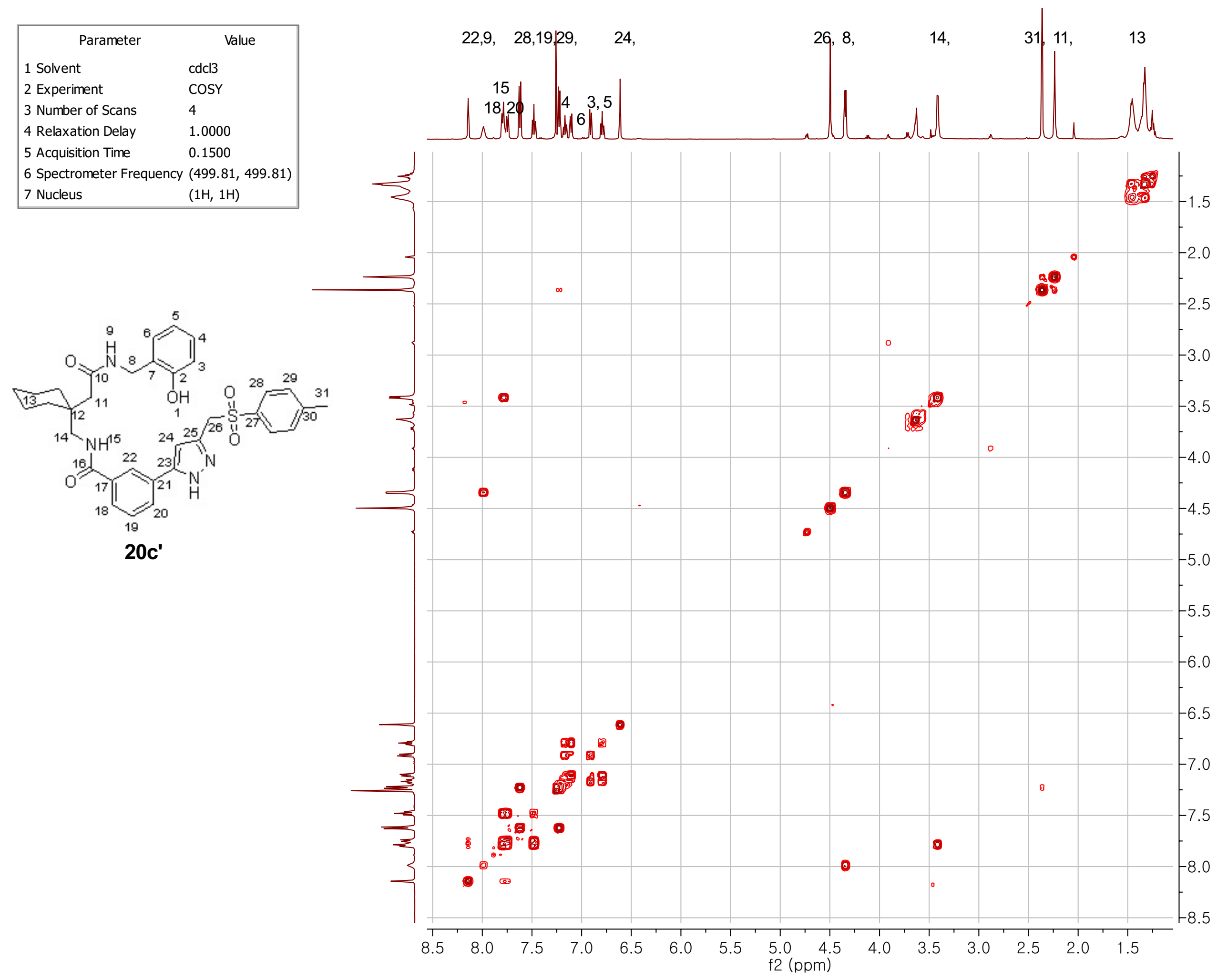




\begin{tabular}{|ll|}
\hline \multicolumn{1}{|c|}{ Parameter } & \multicolumn{1}{c|}{ Value } \\
1 Solvent & cdcl3 \\
2 Experiment & ASAPHMQC \\
3 Number of Scans & 32 \\
4 Relaxation Delay & 0.0600 \\
5 Acquisition Time & 0.0640 \\
6 Spectrometer Frequency & $(499.81,125.69)$ \\
7 Nucleus & $(1 \mathrm{H}, 13 \mathrm{C})$ \\
\hline
\end{tabular}
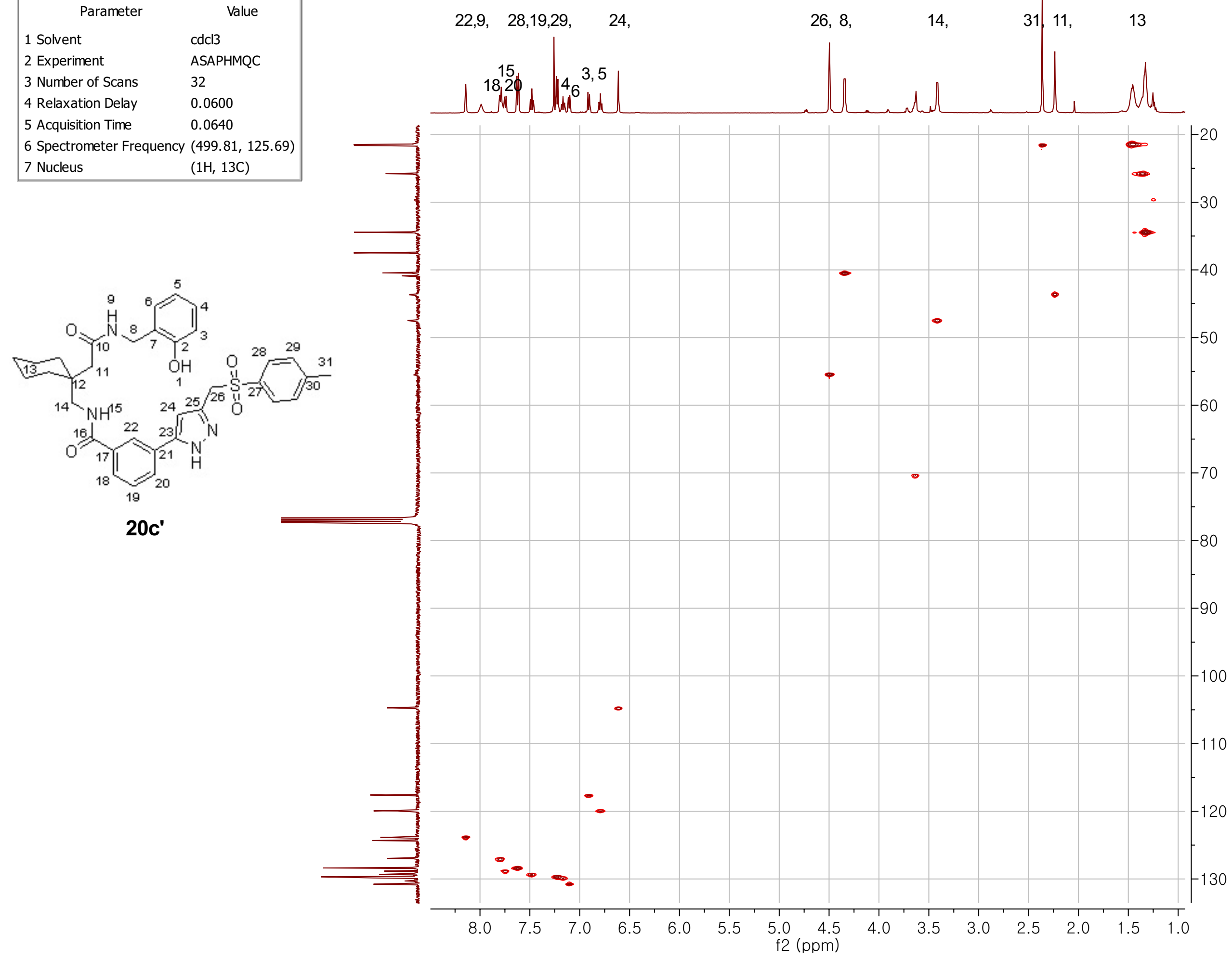


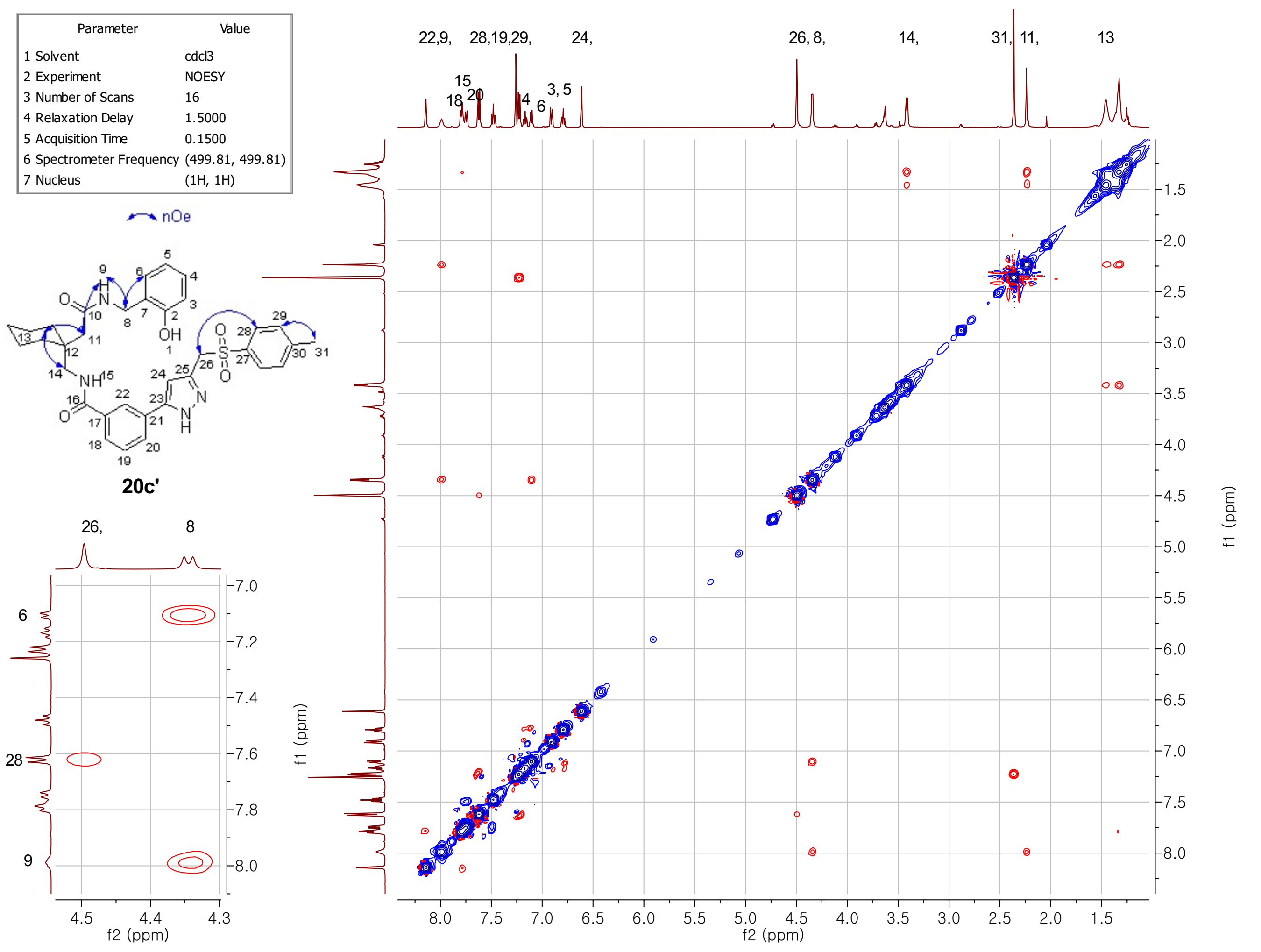




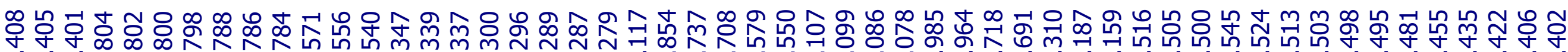

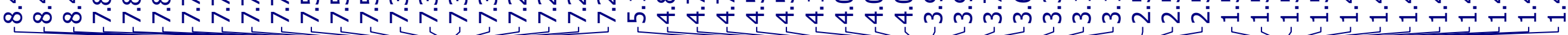

\begin{tabular}{|ll|}
\hline \multicolumn{1}{|c|}{ Parameter } & \multicolumn{1}{c|}{ Value } \\
1 Solvent & cd3od \\
2 Experiment & $1 \mathrm{D}$ \\
3 Number of Scans & 8 \\
4 Relaxation Delay & 1.0000 \\
5 Acquisition Time & 2.0447 \\
6 Spectrometer Frequency & 499.81 \\
7 Nucleus & $1 \mathrm{H}$ \\
\hline
\end{tabular}

16 ,

20 ,
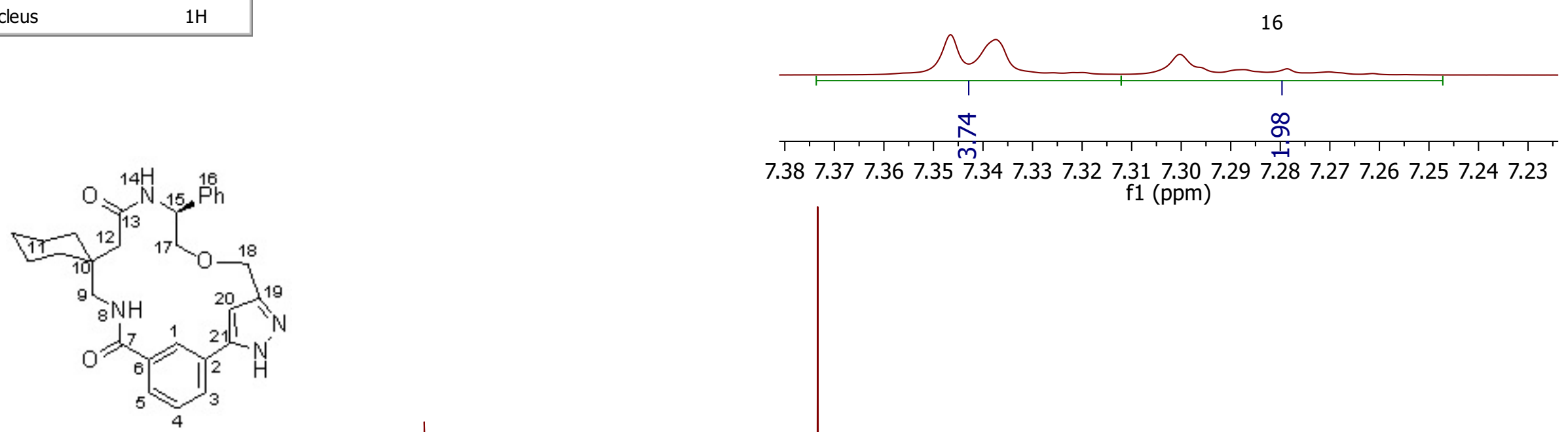

7.387 .37 7.36 7.35 7.34 7.33 7.32 7.31 7.30 7.29 7.28 7.27 7.26 7.25 7.24 7.23

f1 (ppm)

20d

8

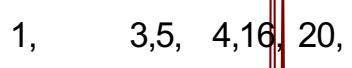

15. $\quad 18,18, \quad 17,17,9$
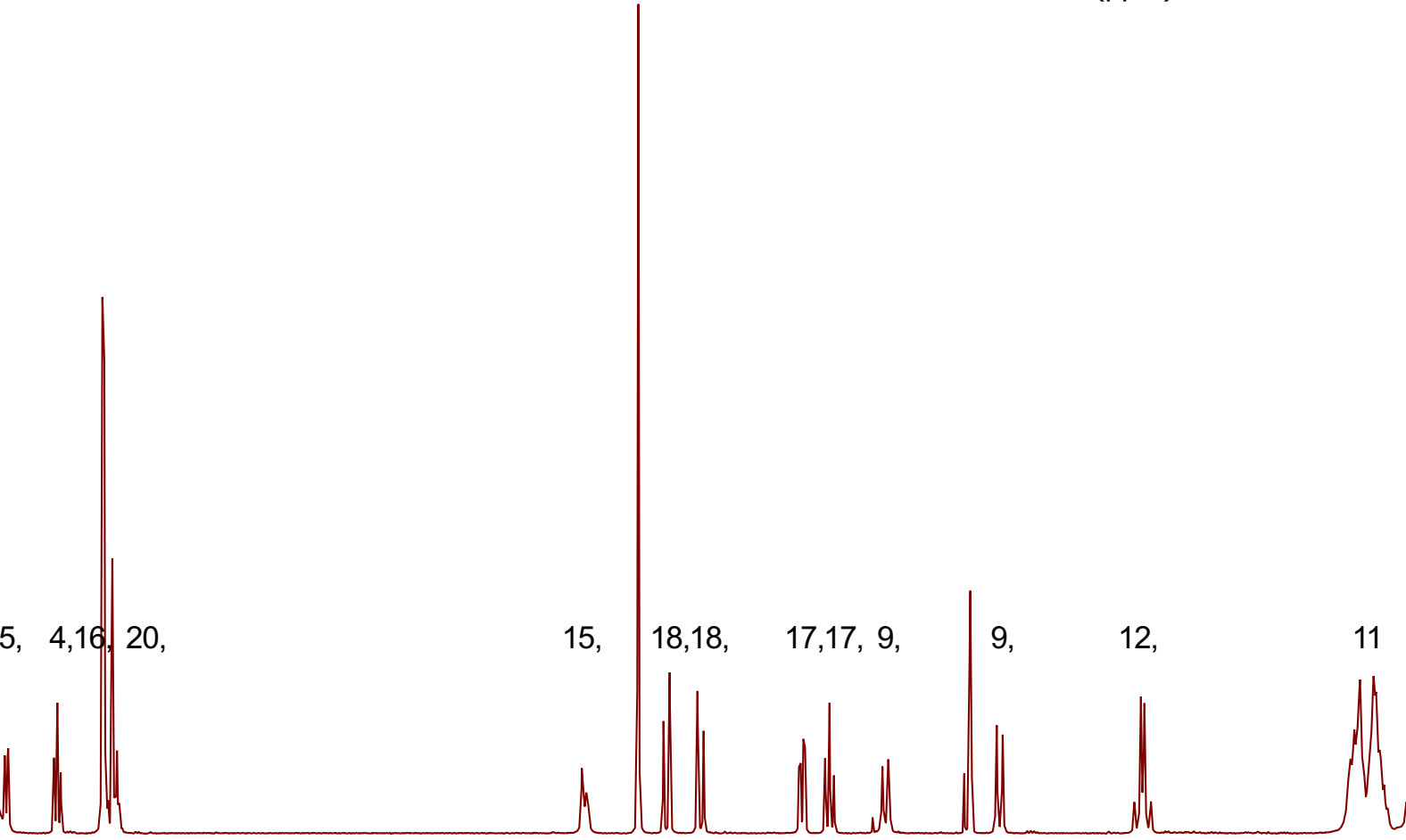

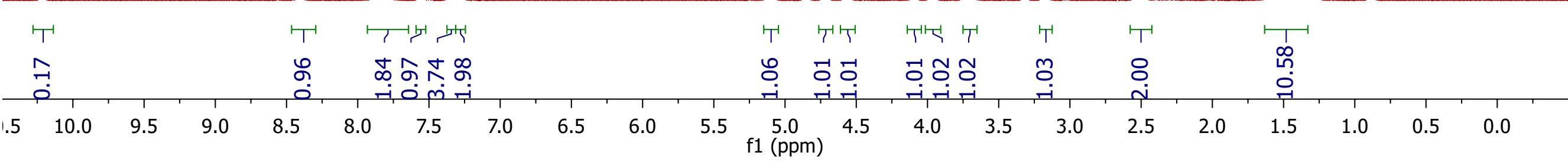


ขึ

m

ता

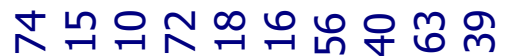

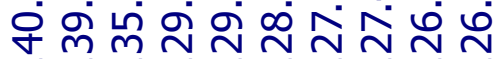

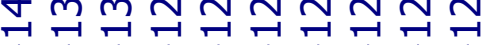

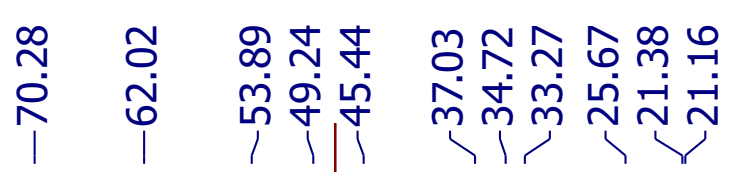

\begin{tabular}{|ll|}
\hline \multicolumn{1}{|c}{ Parameter } & \multicolumn{1}{c|}{ Value } \\
1 Solvent & cd3od \\
2 Experiment & $1 \mathrm{D}$ \\
3 Number of Scans & 17000 \\
4 Relaxation Delay & 1.0000 \\
5 Acquisition Time & 1.3042 \\
6 Spectrometer Frequency & 100.61 \\
7 Nucleus & $13 \mathrm{C}$ \\
\hline
\end{tabular}
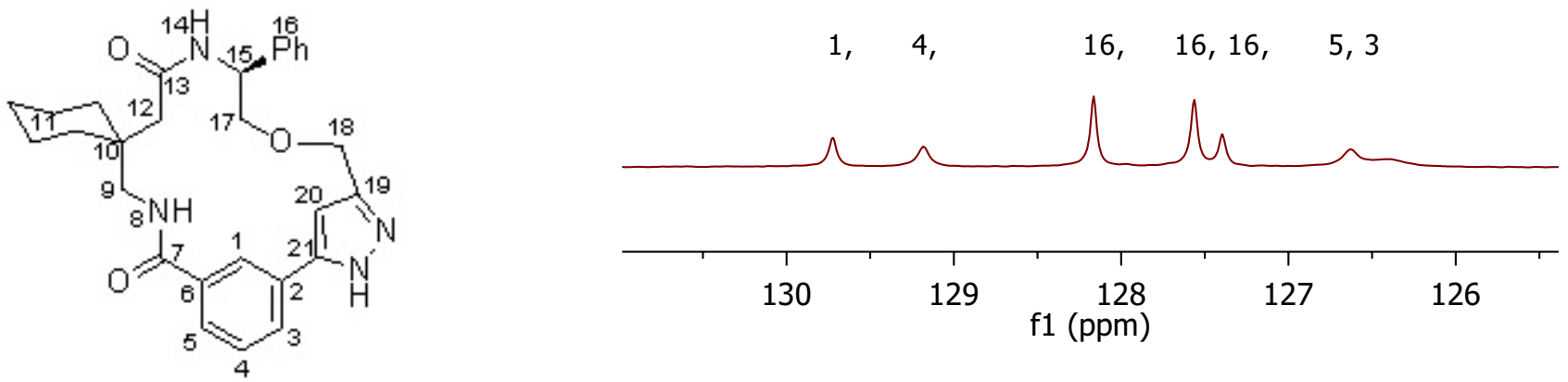

20d

13,7
190

180
10,1

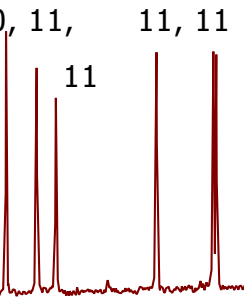




\begin{tabular}{|ll|}
\hline \multicolumn{1}{|c|}{ Parameter } & \multicolumn{1}{c|}{ Value } \\
1 Solvent & cd3od \\
2 Experiment & COSY \\
3 Number of Scans & 4 \\
4 Relaxation Delay & 1.0000 \\
5 Acquisition Time & 0.1500 \\
6 Spectrometer Frequency & $(499.81,499.81)$ \\
7 Nucleus & $(1 \mathrm{H}, 1 \mathrm{H})$ \\
\hline
\end{tabular}

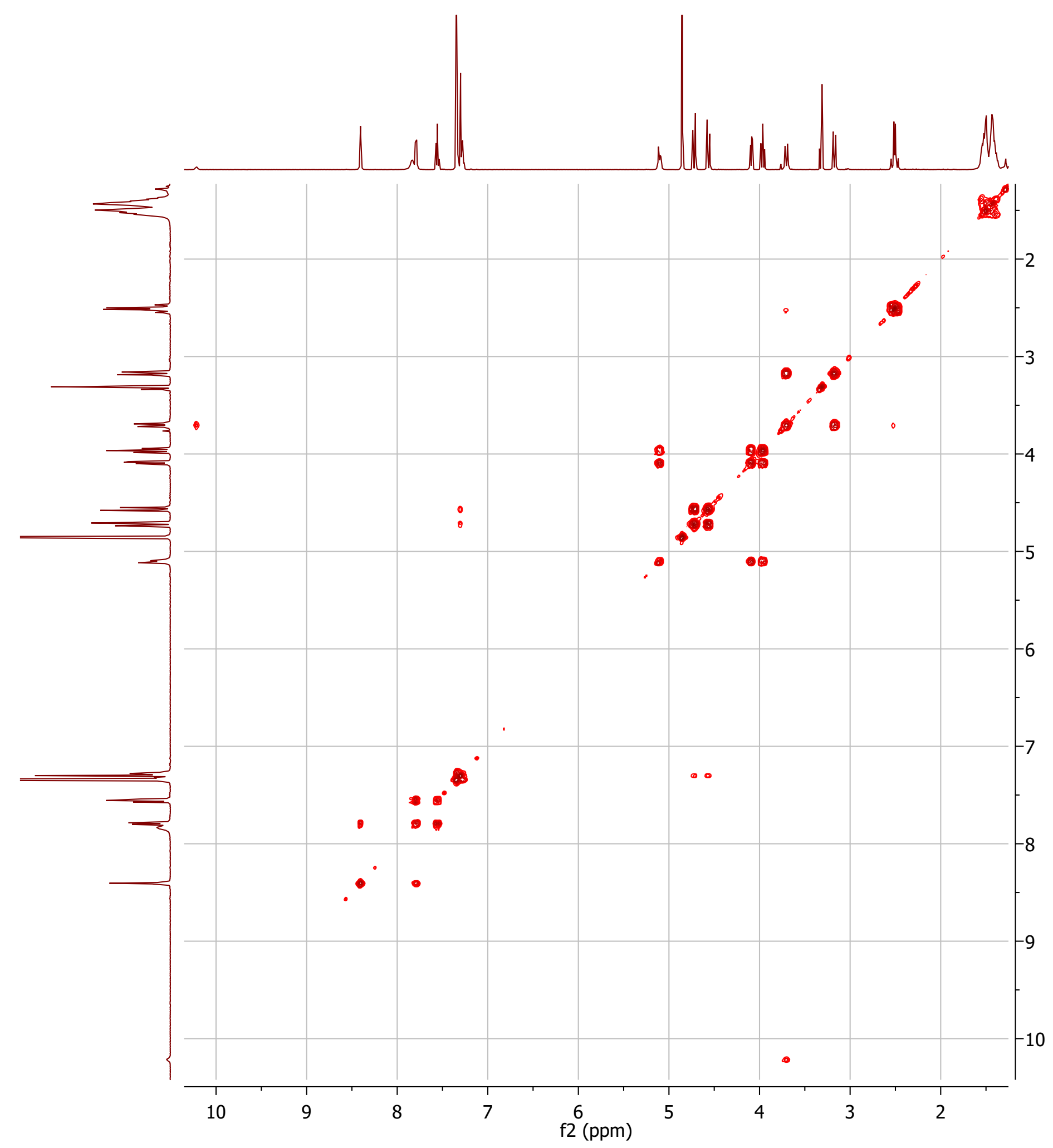

$\widehat{\varepsilon}$
을
난

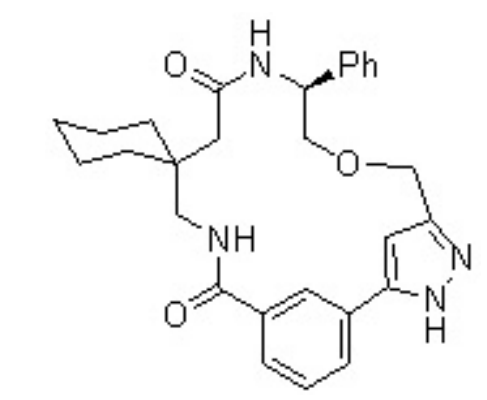

20d 


\begin{tabular}{|ll|}
\hline \multicolumn{1}{|c|}{ Parameter } & \multicolumn{1}{c|}{ Value } \\
1 Solvent & cd3od \\
2 Experiment & NOESY \\
3 Number of Scans & 16 \\
4 Relaxation Delay & 1.5000 \\
5 Acquisition Time & 0.1500 \\
6 Spectrometer Frequency & $(499.81,499.81)$ \\
7 Nucleus & $(1 \mathrm{H}, 1 \mathrm{H})$ \\
\hline
\end{tabular}

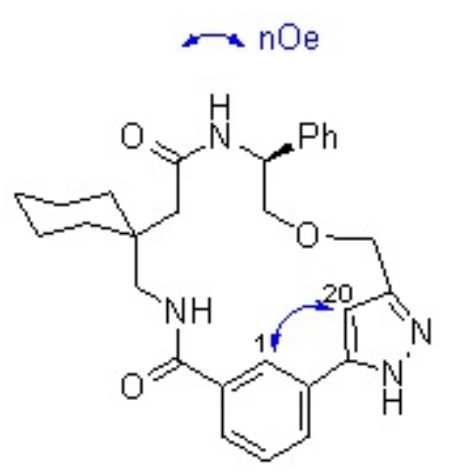

20d

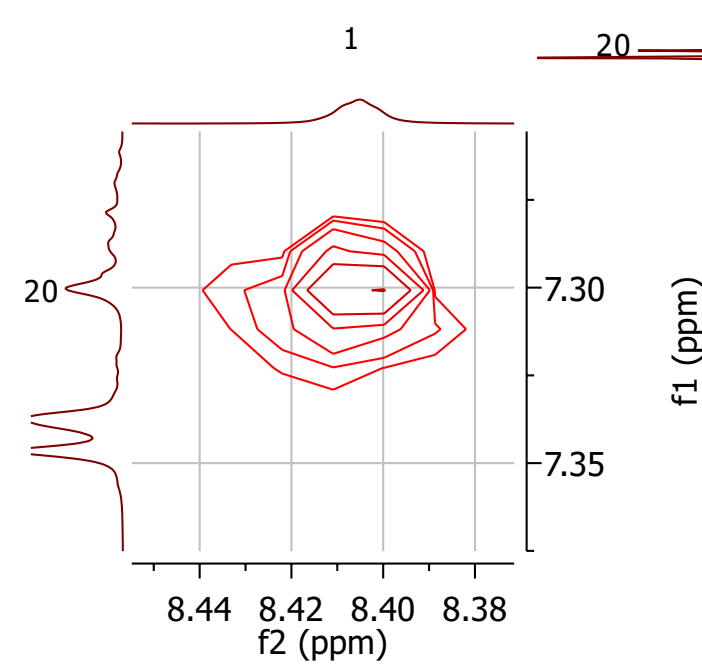

f2 (ppm)

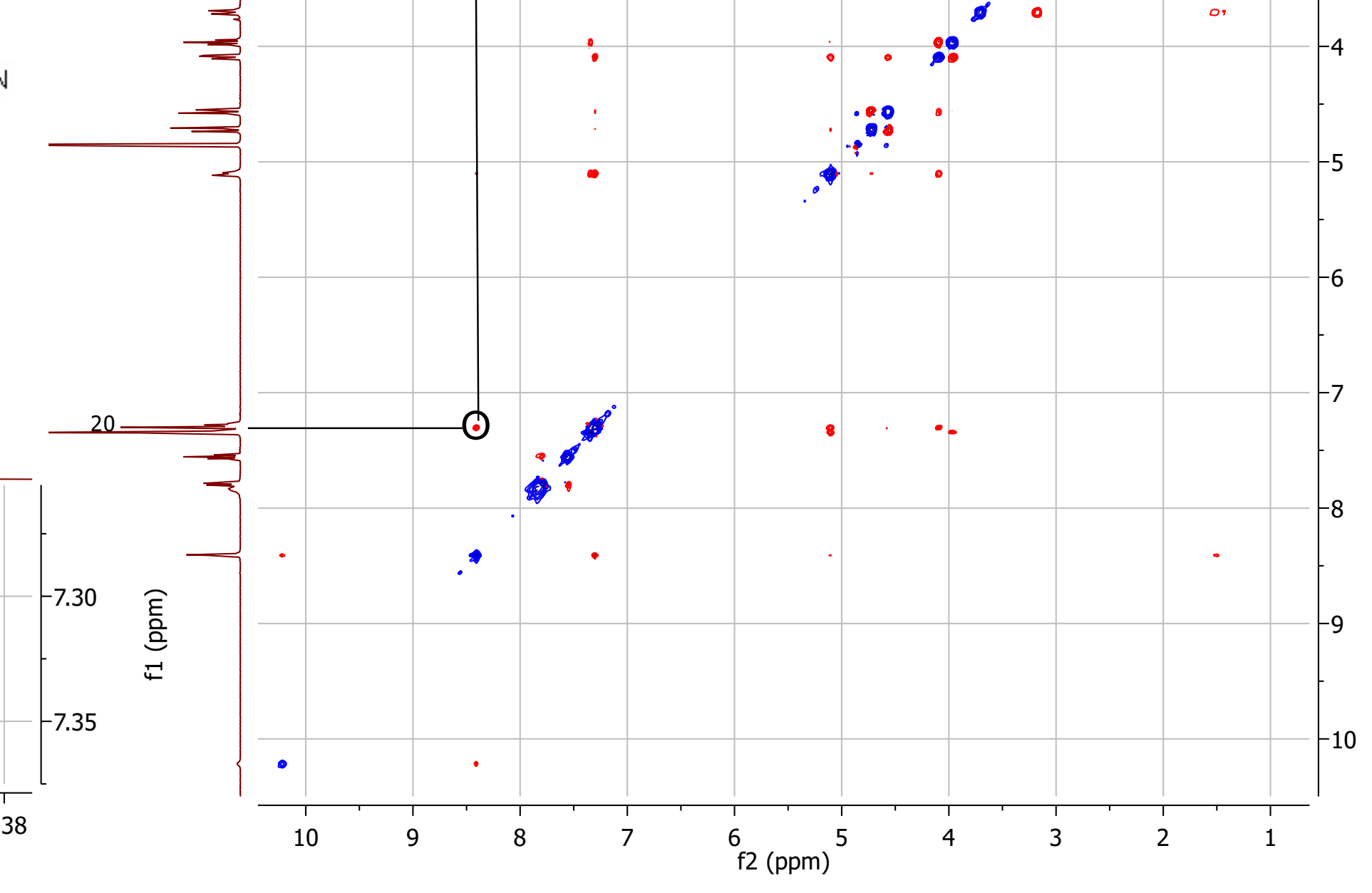




\begin{tabular}{|ll|}
\hline \multicolumn{1}{|c|}{ Parameter } & \multicolumn{1}{c|}{ Value } \\
1 Solvent & cd3od \\
2 Experiment & $1 \mathrm{D}$ \\
3 Number of Scans & 8 \\
4 Relaxation Delay & 1.0000 \\
5 Acquisition Time & 2.0447 \\
6 Spectrometer Frequency & 499.81 \\
7 Nucleus & $1 \mathrm{H}$ \\
\hline
\end{tabular}

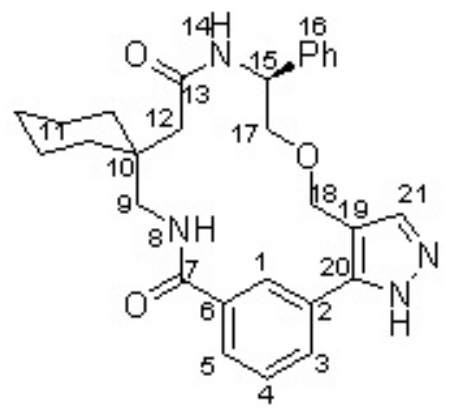

21d

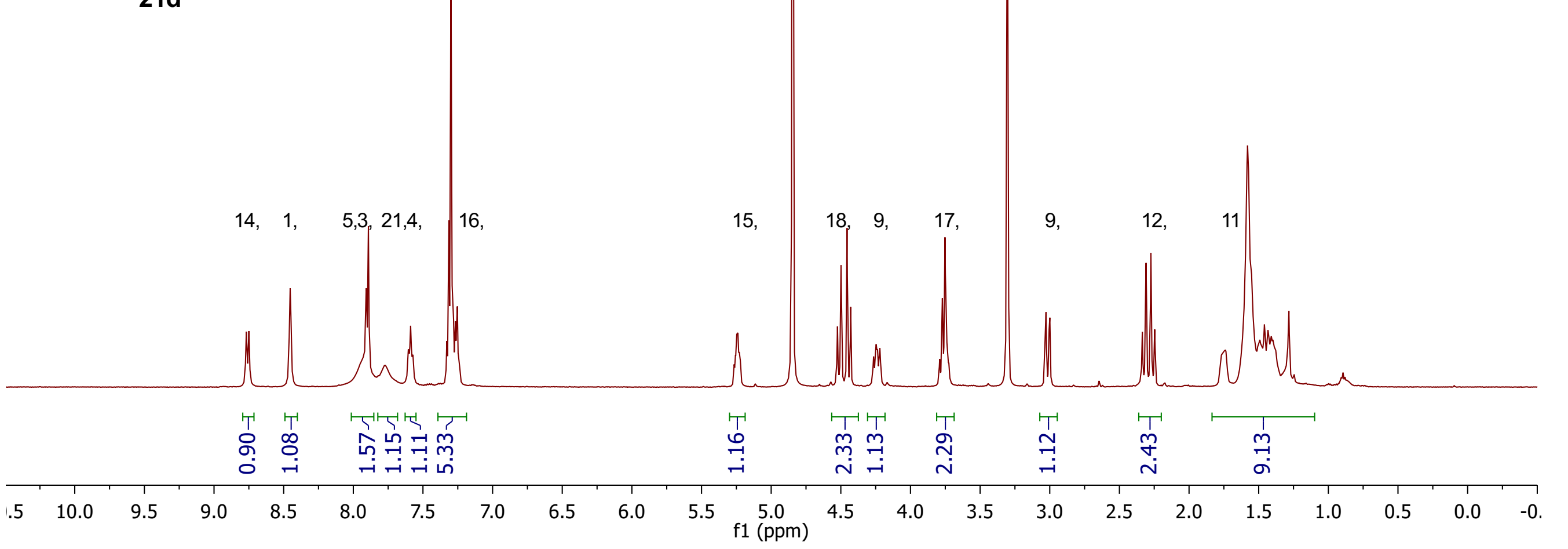




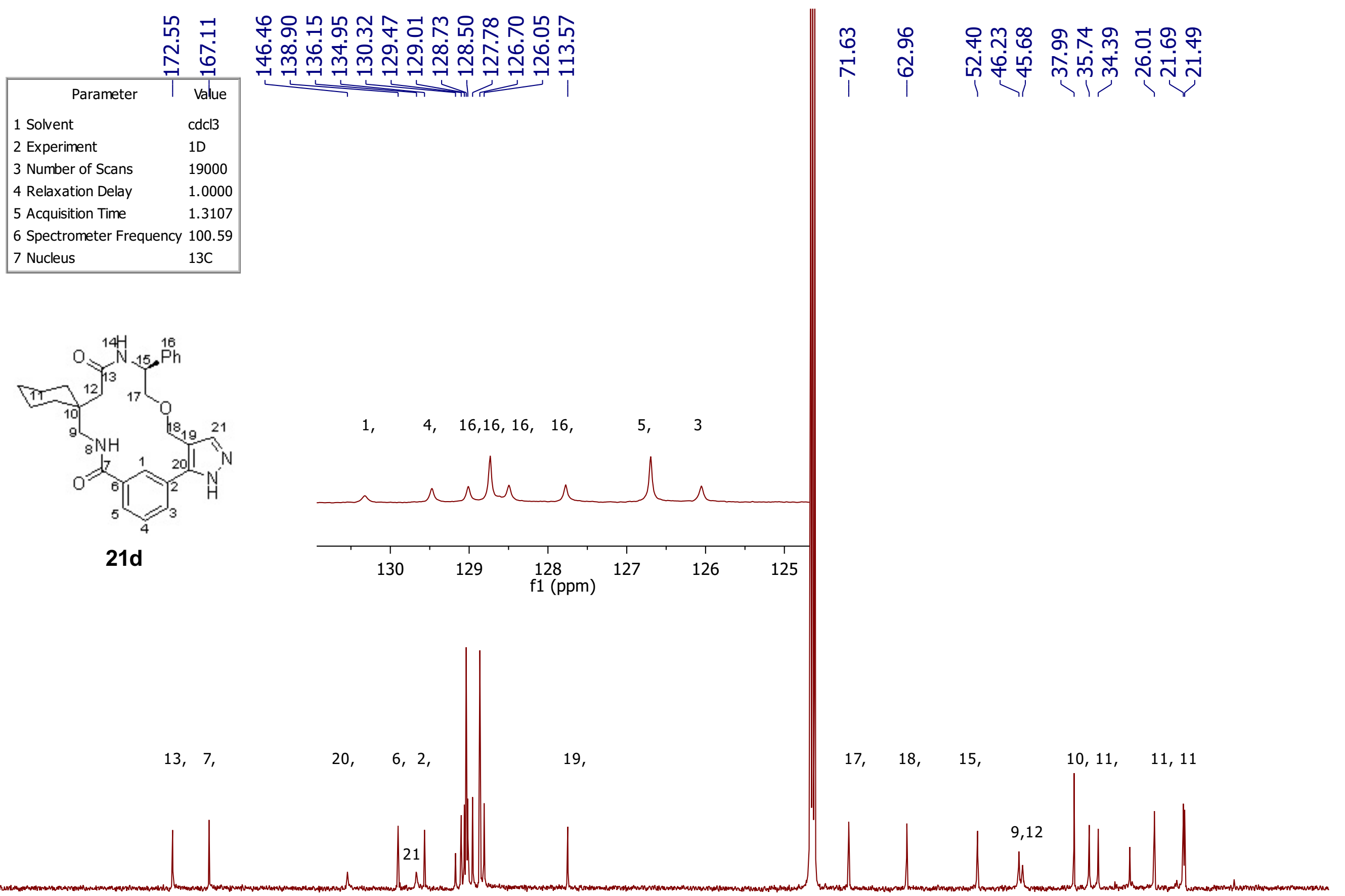

$190 \quad 180 \quad 170$

$\begin{array}{rrrrr}1 & & & \\ 160 & 150 & 140 & 130\end{array}$

$\begin{array}{llll}130 & 120 & 110 & 100 \\ \mathrm{f} 1(\mathrm{ppm})\end{array}$




\begin{tabular}{|ll|}
\hline \multicolumn{1}{|c|}{ Parameter } & \multicolumn{1}{c|}{ Value } \\
1 Solvent & cd3od \\
2 Experiment & COSY \\
3 Number of Scans & 4 \\
4 Relaxation Delay & 1.0000 \\
5 Acquisition Time & 0.1500 \\
6 Spectrometer Frequency & $(499.81,499.81)$ \\
7 Nucleus & $(1 \mathrm{H}, 1 \mathrm{H})$ \\
\hline
\end{tabular}

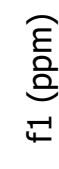




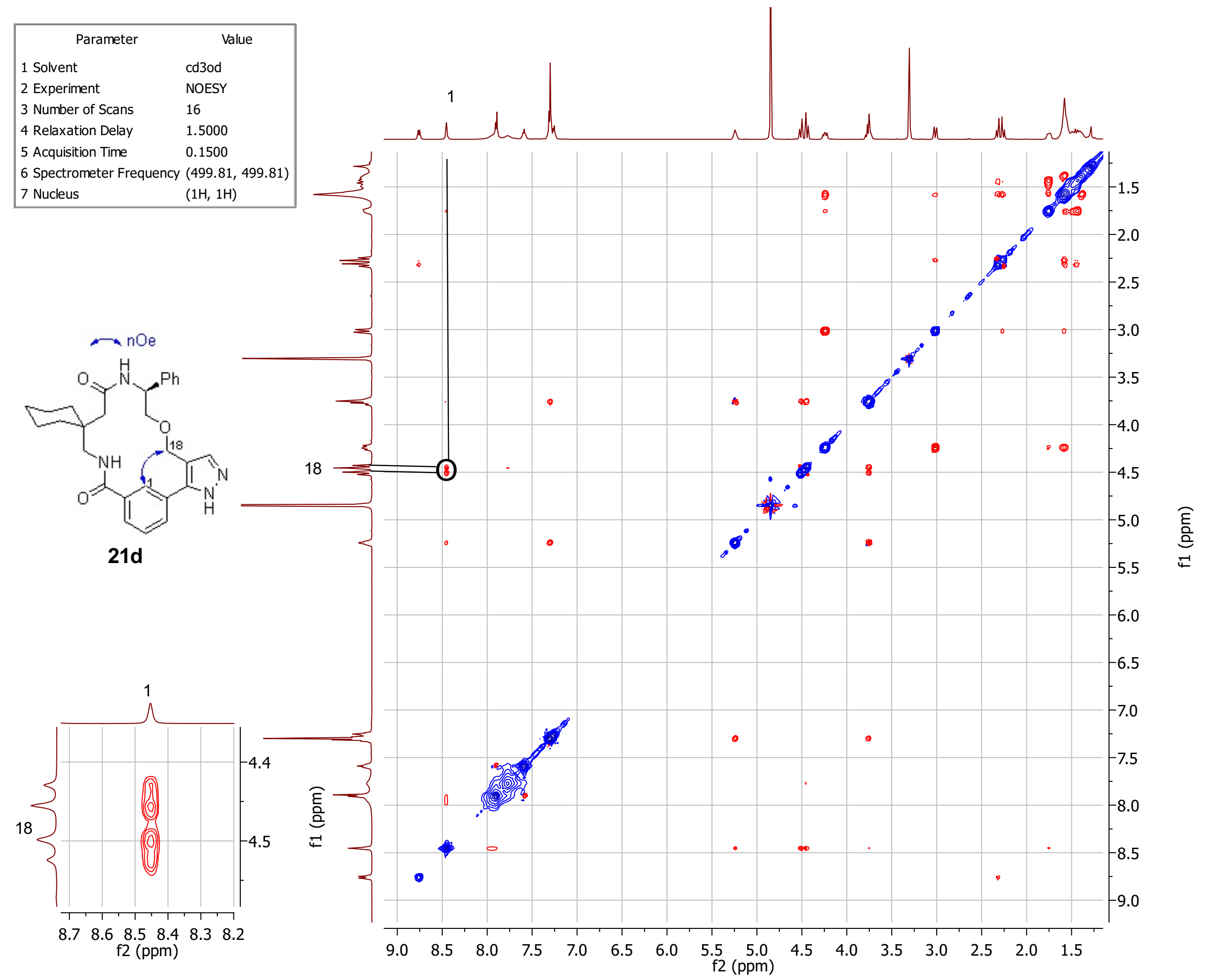




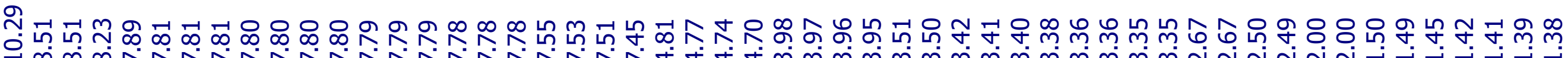

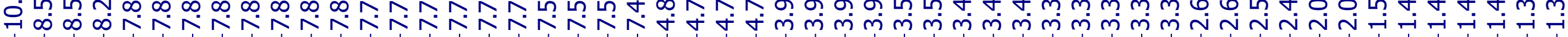

$\left.\begin{array}{ll}\text { Parameter } & \multicolumn{1}{c}{\text { Value }} \\ \text { 1 Solvent } & \text { cd3od } \\ 2 \text { Experiment } & 1 \mathrm{D} \\ \text { 3 Number of Scans } & 8 \\ \text { 4 Relaxation Delay } & 1.0000 \\ 5 \text { Acquisition Time } & 2.5559 \\ 6 \text { Spectrometer Frequency } & 400.02 \\ 7 \text { Nucleus } & 1 \mathrm{H}\end{array}\right]$
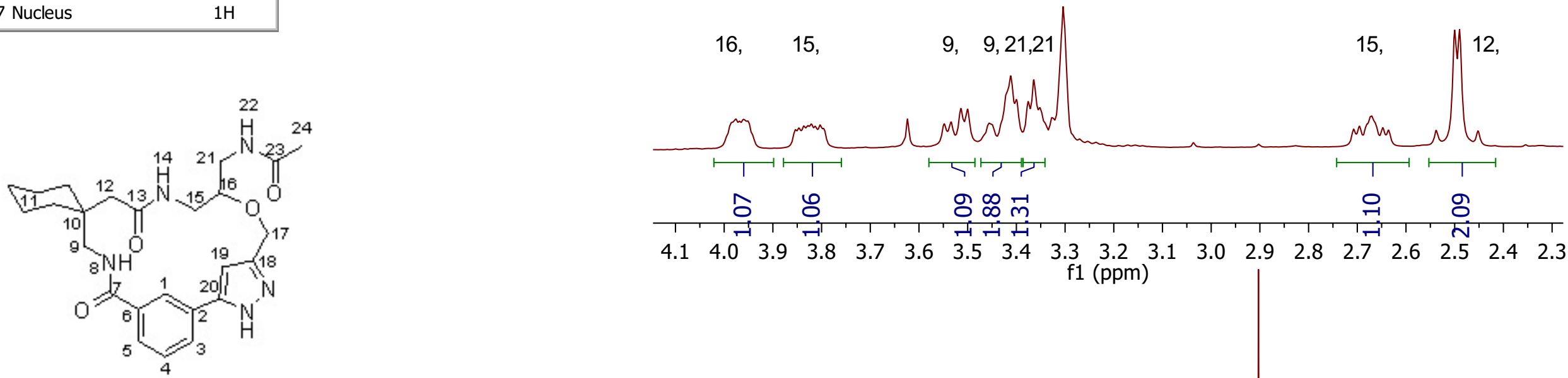

$20 \mathrm{e}$

8

$1,14,22, \quad 5,3,4,19$

$17, \quad 16,15$

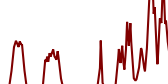

15,12
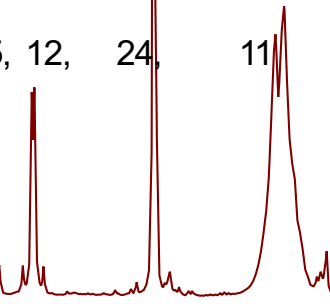

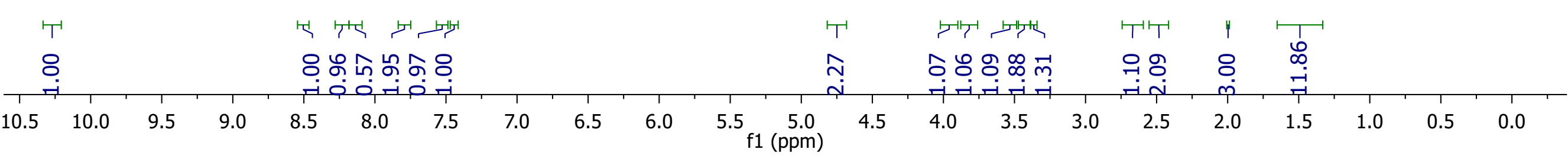




\begin{tabular}{|lc|}
\hline \multicolumn{1}{|c|}{ Parameter } & Value \\
1 Solvent & cd3od \\
2 Experiment & $1 \mathrm{D}$ \\
3 Number of Scans & 512 \\
4 Relaxation Delay & 1.0000 \\
5 Acquisition Time & 1.3042 \\
6 Spectrometer Frequency & 100.61 \\
7 Nucleus & $13 \mathrm{C}$ \\
\hline
\end{tabular}

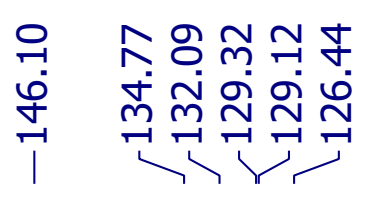

กิ
กิ่
|

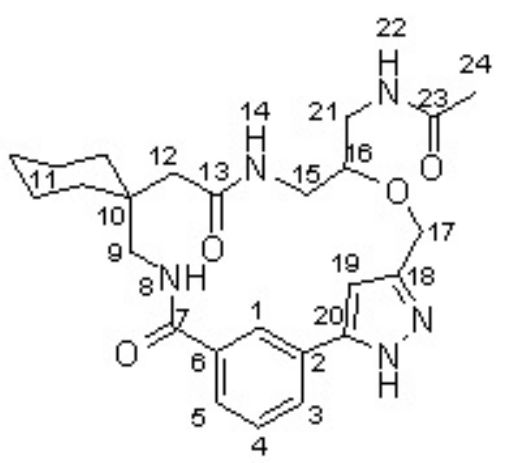

20 e

$23,13,7$,

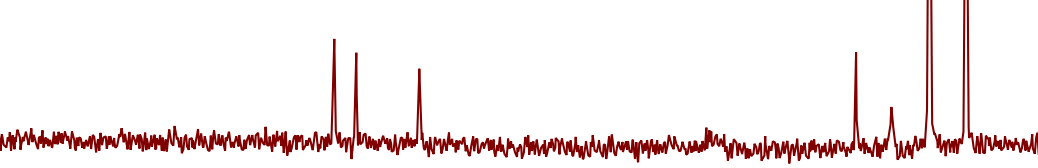

5,3 ,
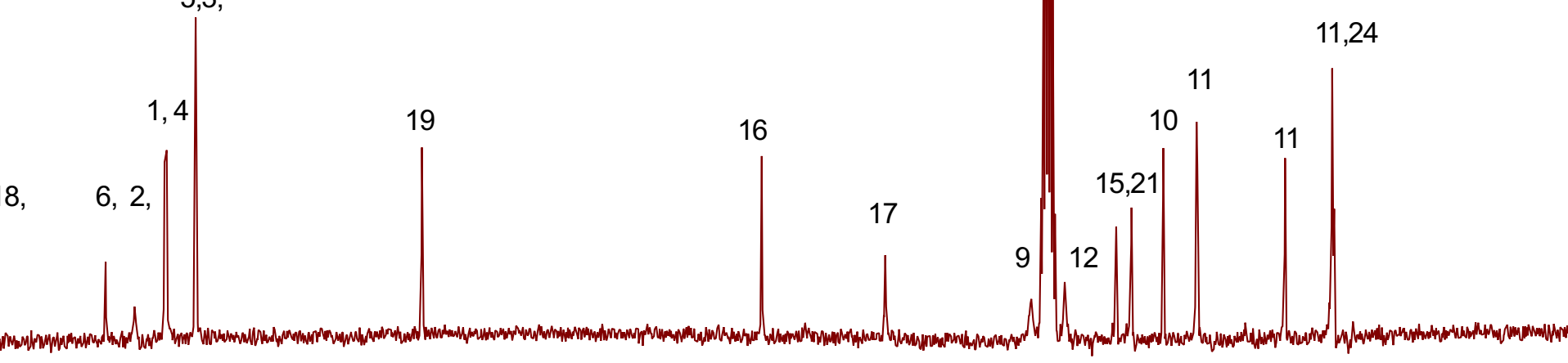


\begin{tabular}{|ll|}
\hline \multicolumn{1}{|c|}{ Parameter } & \multicolumn{1}{c|}{ Value } \\
1 Solvent & cd3od \\
2 Experiment & COSY \\
3 Number of Scans & 4 \\
4 Relaxation Delay & 1.0000 \\
5 Acquisition Time & 0.1500 \\
6 Spectrometer Frequency & $(400.02,400.02)$ \\
7 Nucleus & $(1 \mathrm{H}, 1 \mathrm{H})$ \\
\hline
\end{tabular}

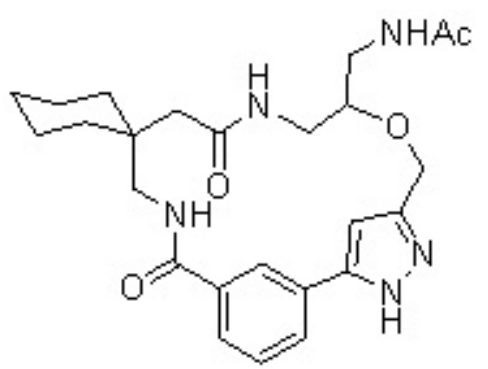

$20 \mathrm{e}$

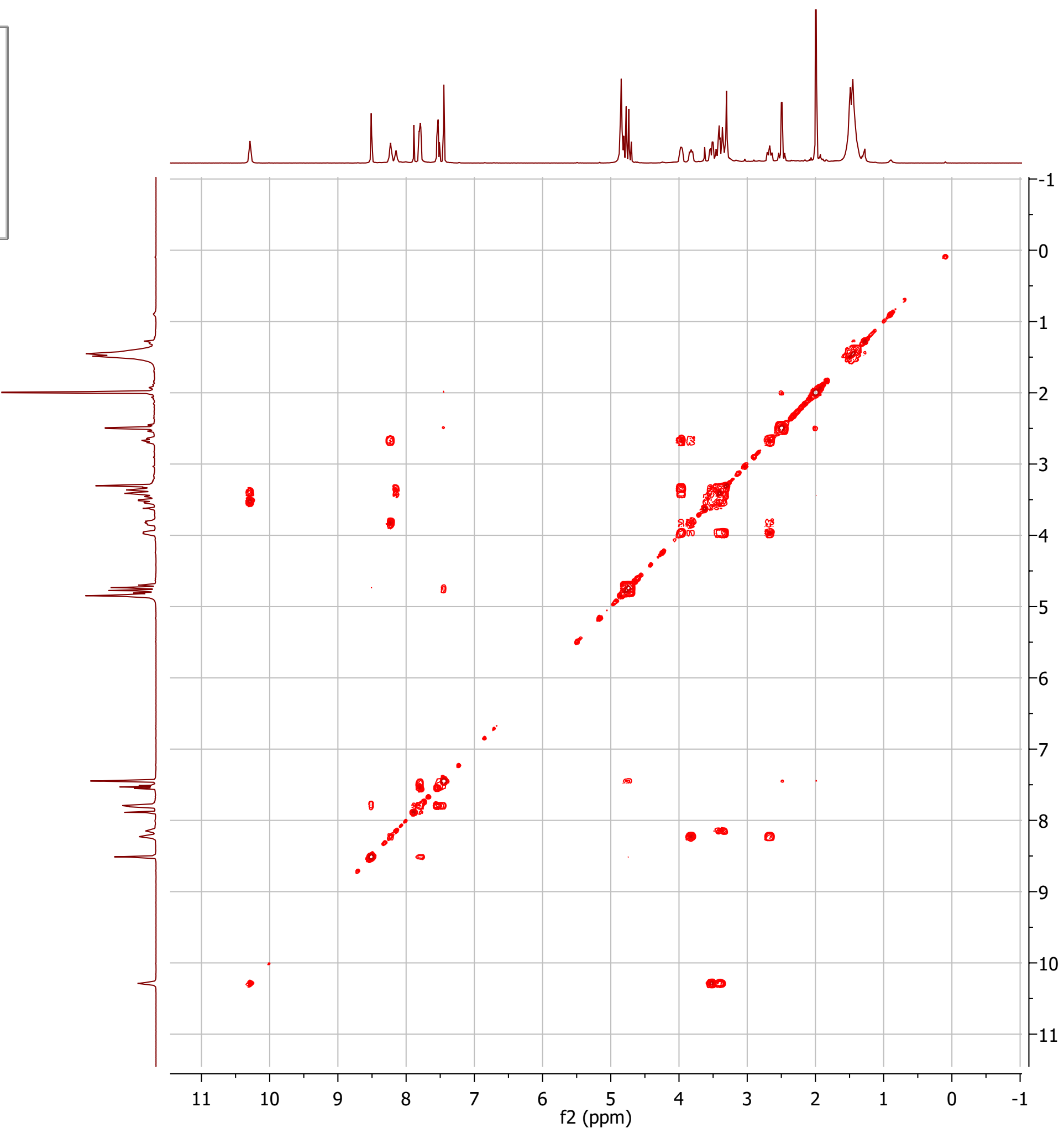




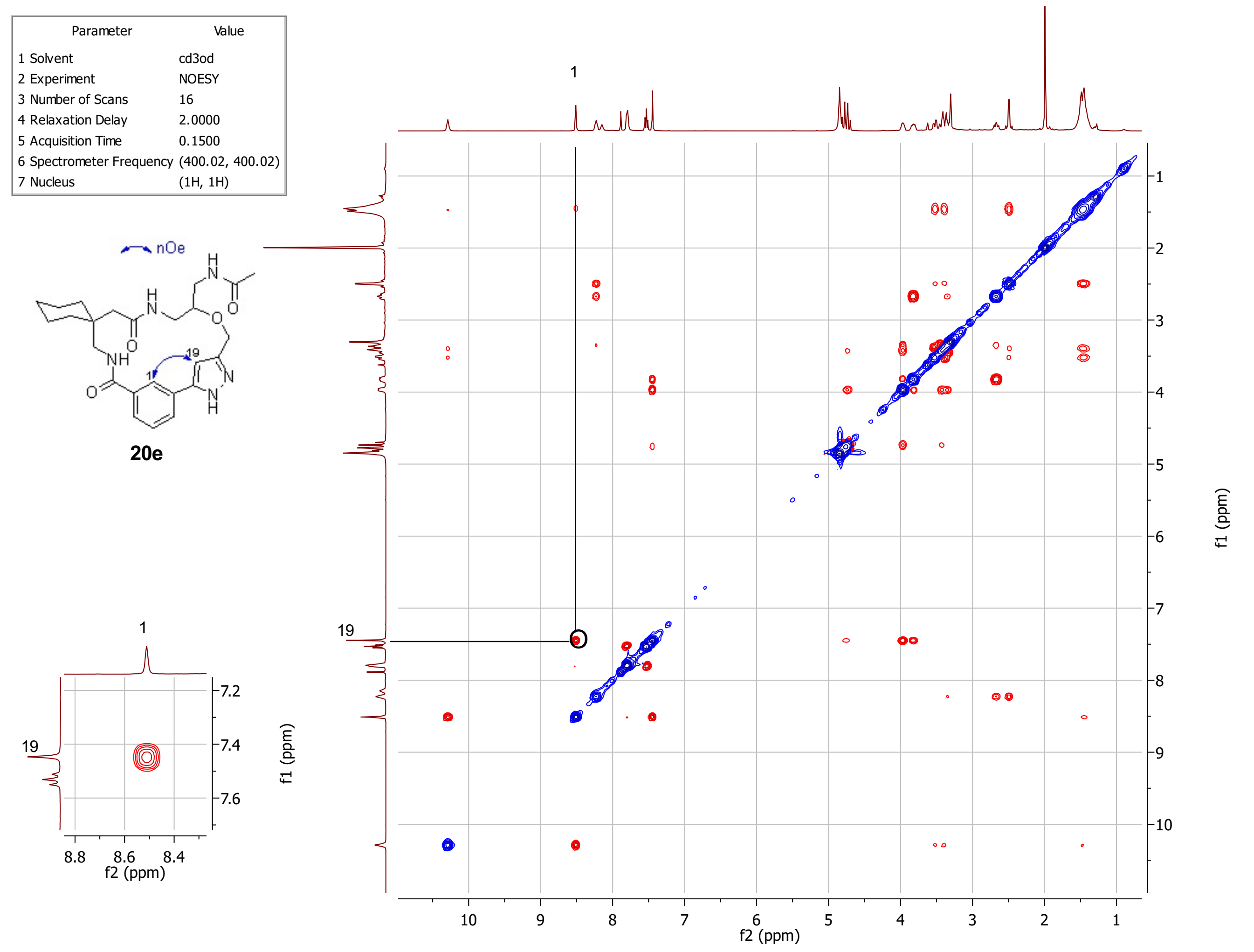


늠 ๓ क人र

\begin{tabular}{|ll|}
\hline \multicolumn{1}{|c|}{ Parameter } & \multicolumn{1}{c|}{ Value } \\
1 Solvent & cd3od \\
2 Experiment & $1 \mathrm{D}$ \\
3 Number of Scans & 8 \\
4 Relaxation Delay & 1.0000 \\
5 Acquisition Time & 2.5592 \\
6 Spectrometer Frequency & 400.09 \\
7 Nucleus & $1 \mathrm{H}$ \\
\hline
\end{tabular}

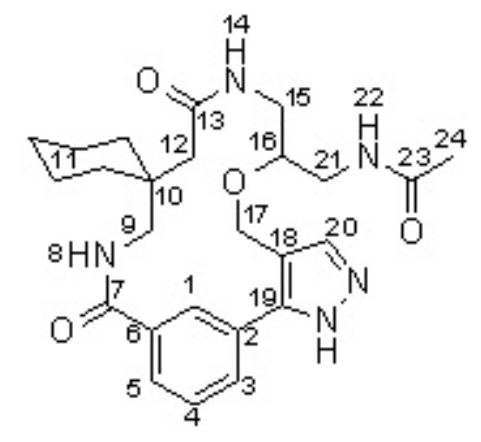

$21 e$

$1, \quad 5,3,4$,

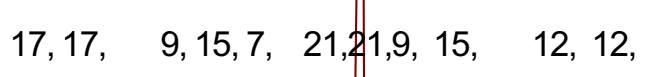

$17,17, \quad 9,15,7, \quad 21,21,9,15, \quad 12,12$
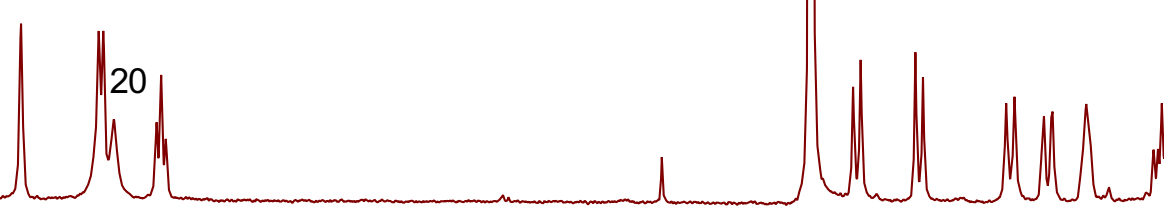

Tा माT

ஸ⿻

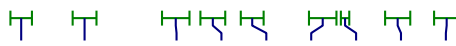

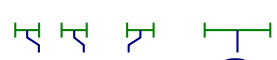

远通

नَ

๗

8.0

$8.0 \quad 7.5$

$\begin{array}{lcc}6.0 & 5.5 & 5.0 \\ & & f 1(\mathrm{ppm})\end{array}$

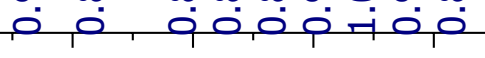
$0 \mathrm{~m}$ 


\begin{tabular}{|l|l|}
\hline \multicolumn{1}{|c|}{ Parameter } & dmso \\
1 Solvent & Value \\
2 Experiment & $1 \mathrm{D}$ \\
3 Number of Scans & 20000 \\
4 Relaxation Delay & 1.0000 \\
5 Acquisition Time & 1.0486 \\
6 Spectrometer Frequency & 125.69 \\
7 Nucleus & $13 \mathrm{C}$ \\
\hline \multicolumn{2}{|c|}{} \\
\hline
\end{tabular}

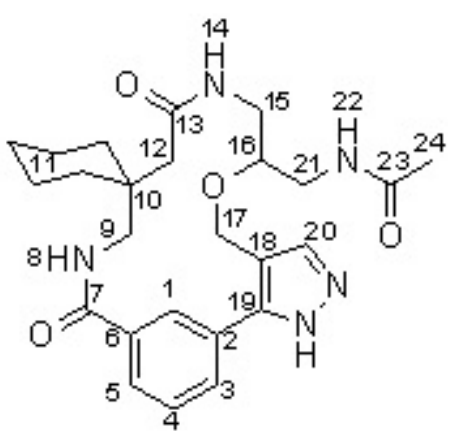

21e

23,13

18
17

16
19

2,4
1

$6, \quad 3,5$ 


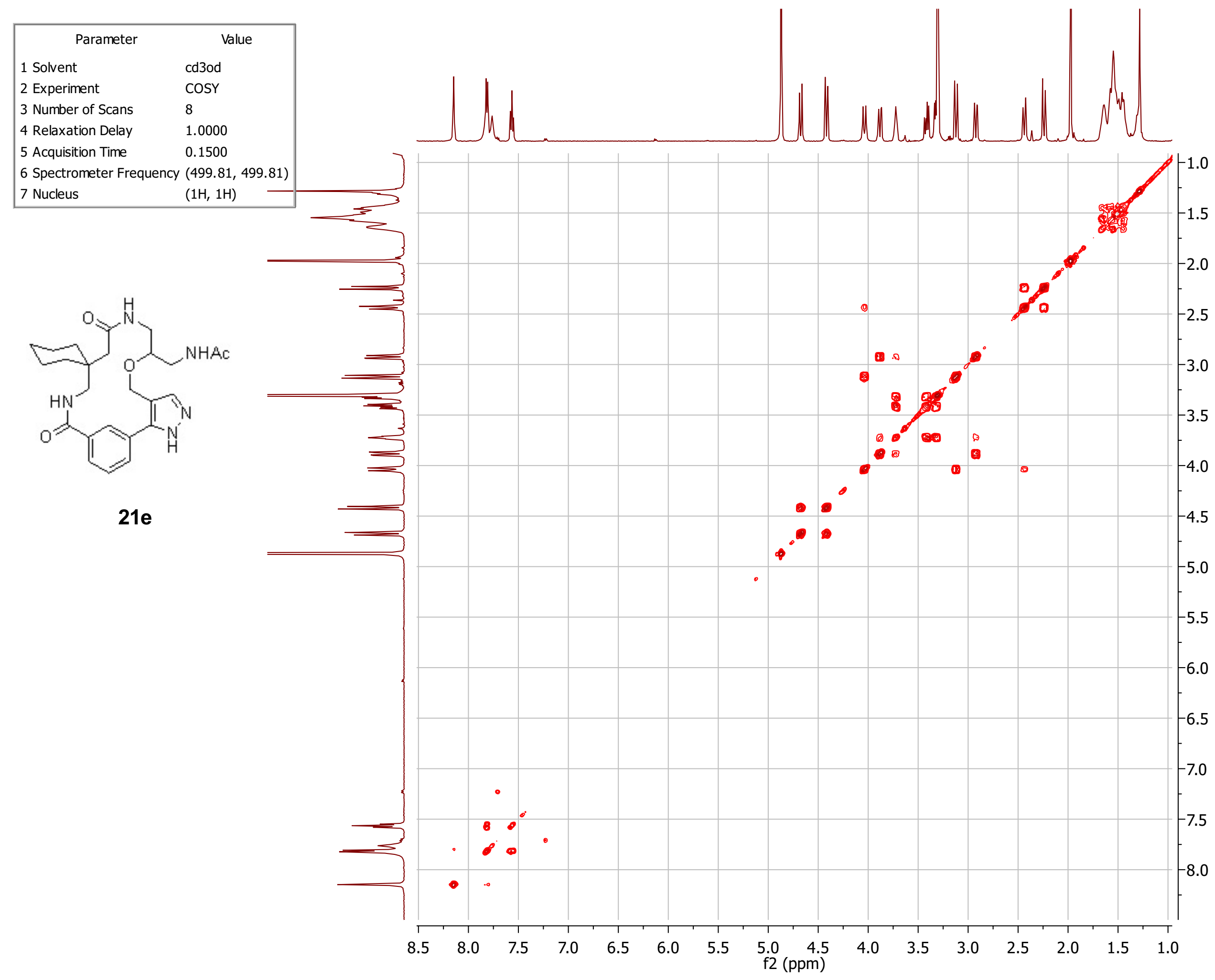

$\underset{\square}{\stackrel{\Xi}{0}}$ 


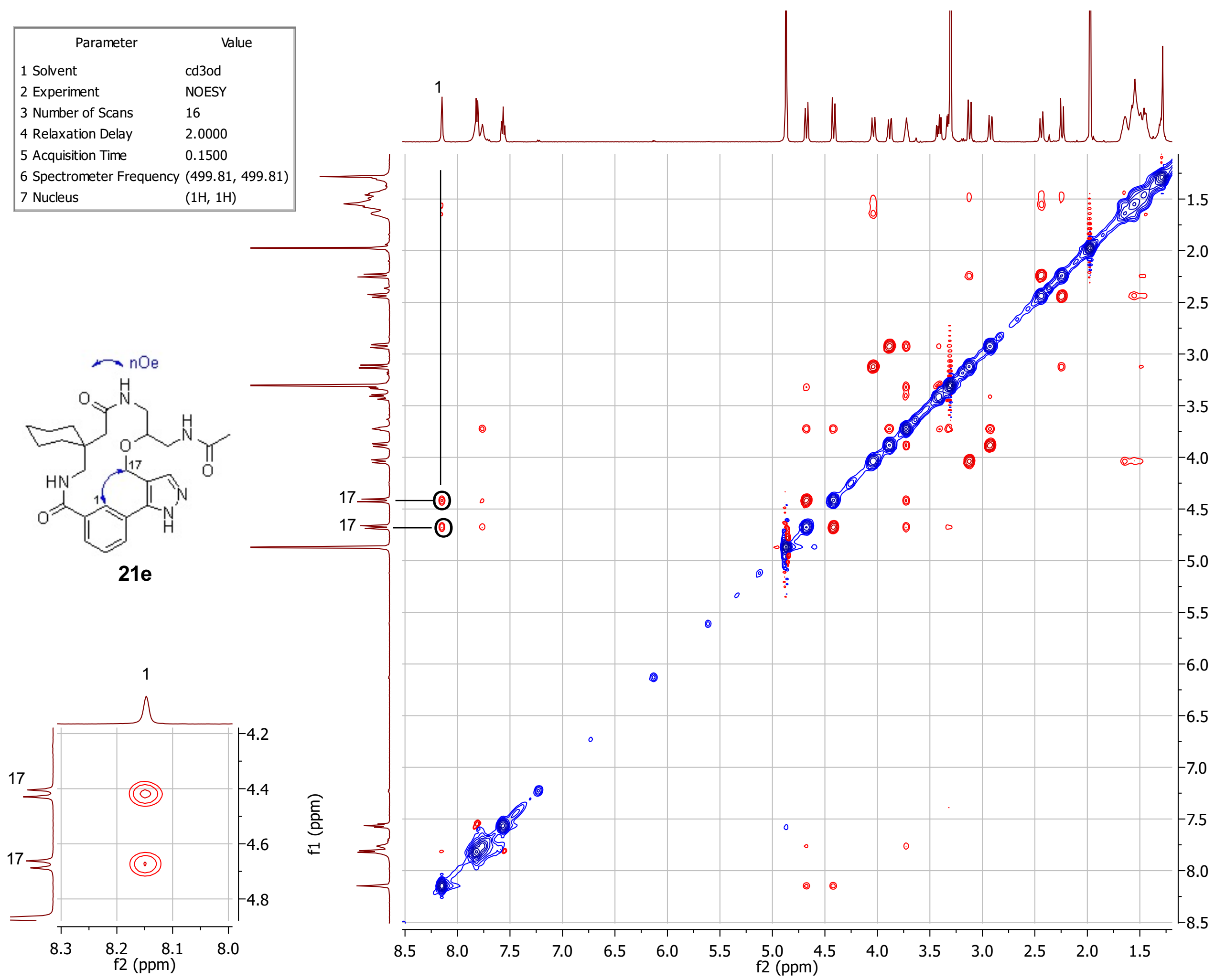




\begin{tabular}{|ll|}
\hline \multicolumn{1}{|c|}{ Parameter } & \multicolumn{1}{c|}{ Value } \\
1 Solvent & cdc13 \\
2 Experiment & 1D \\
3 Number of Scans & 8 \\
4 Relaxation Delay & 1.0000 \\
5 Acquisition Time & 2.5559 \\
6 Spectrometer Frequency & 400.01 \\
7 Nucleus & $1 \mathrm{H}$ \\
\hline \multicolumn{2}{r}{} \\
\hline
\end{tabular}
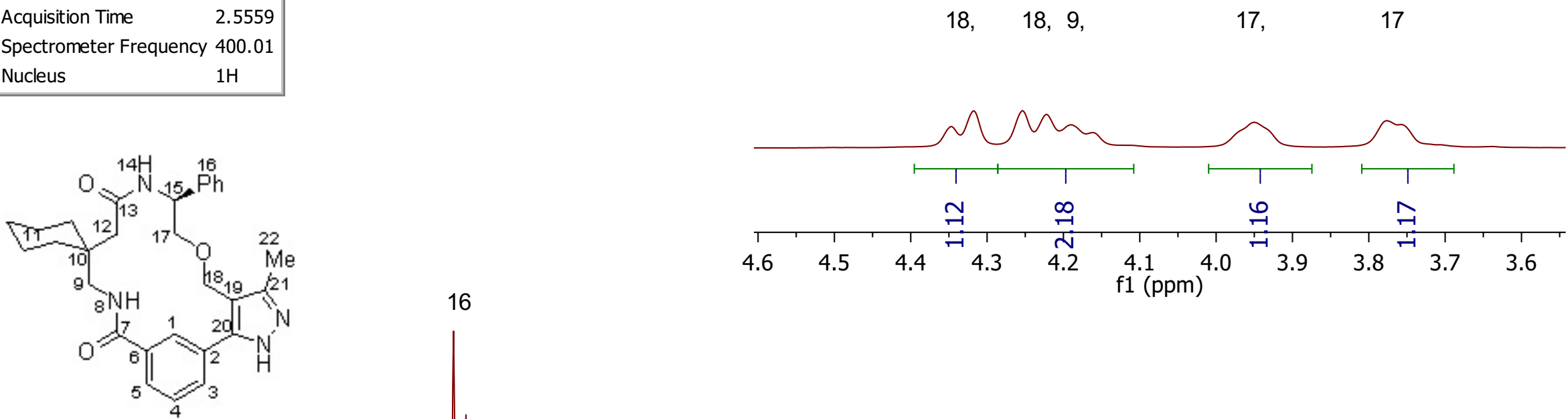

\section{$21 f$}

15

18, 17, 17,

9

12,12

22, 11
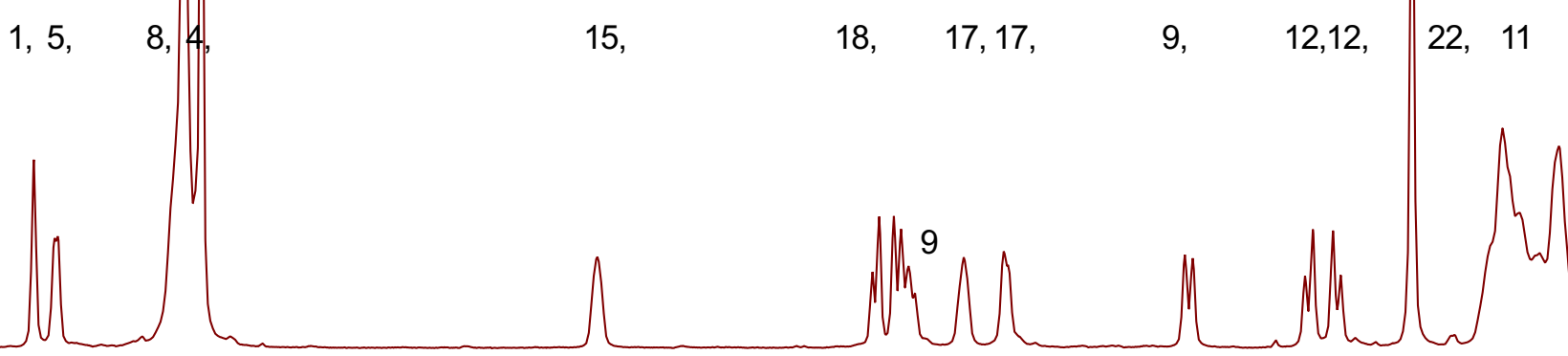

邢 可

응 nิ

9.0

8.5

8.0

7.5

6.5

6.0

†

L

$\stackrel{10}{\longrightarrow}$

$\begin{array}{ll}5.5 & 5.0 \\ \mathrm{f} 1 & (\mathrm{ppm})\end{array}$

गम पा

구국국

$4.0 \quad 3.5$

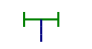

एक ?

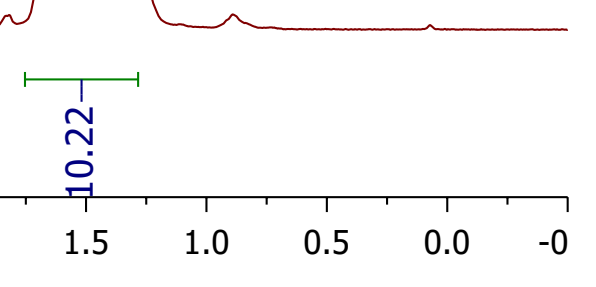




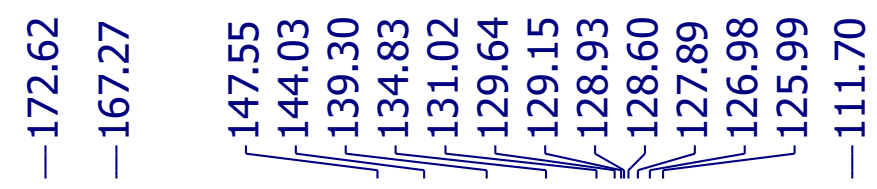

\begin{tabular}{|ll|}
\hline \multicolumn{1}{|c}{ Parameter } & \multicolumn{1}{c|}{ Value } \\
1 Solvent & cdcl3 \\
2 Experiment & $1 \mathrm{D}$ \\
3 Number of Scans & 3200 \\
4 Relaxation Delay & 1.0000 \\
5 Acquisition Time & 1.3107 \\
6 Spectrometer Frequency & 100.59 \\
7 Nucleus & $13 \mathrm{C}$ \\
\hline
\end{tabular}
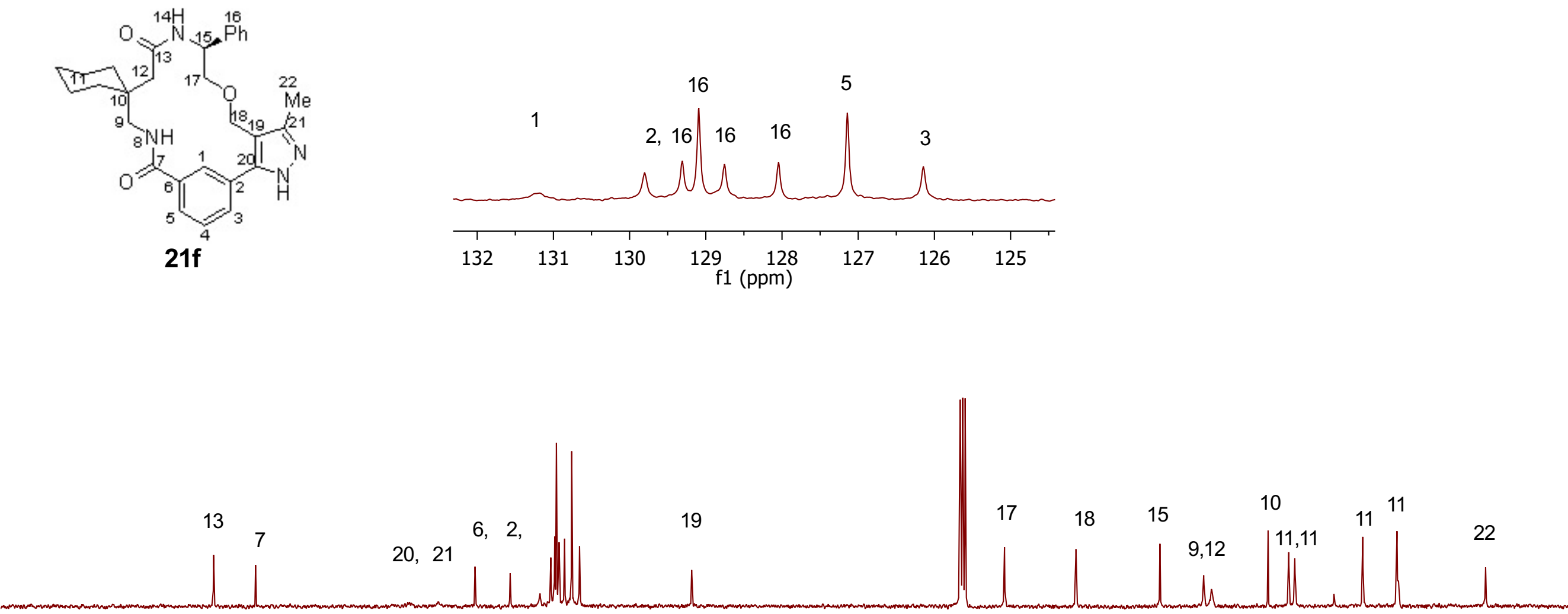

$90 \quad 80$

80

70

60

50

40

30




\begin{tabular}{|ll|}
\hline \multicolumn{1}{|c|}{ Parameter } & \multicolumn{1}{c|}{ Value } \\
1 Solvent & cdcl3 \\
2 Experiment & COSY \\
3 Number of Scans & 4 \\
4 Relaxation Delay & 1.0000 \\
5 Acquisition Time & 0.1500 \\
6 Spectrometer Frequency & $(400.01,400.01)$ \\
7 Nucleus & $(1 \mathrm{H}, 1 \mathrm{H})$ \\
\hline
\end{tabular}
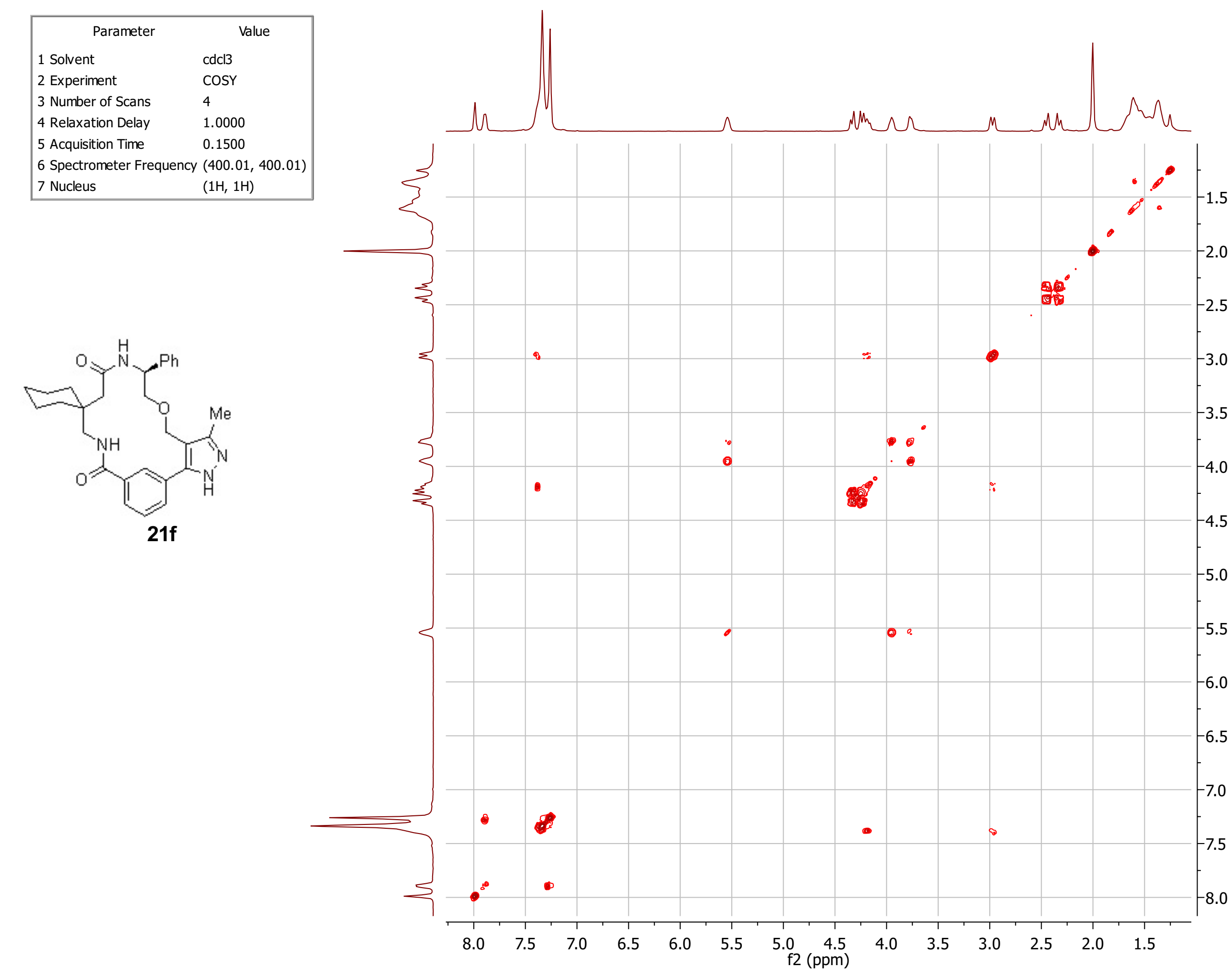


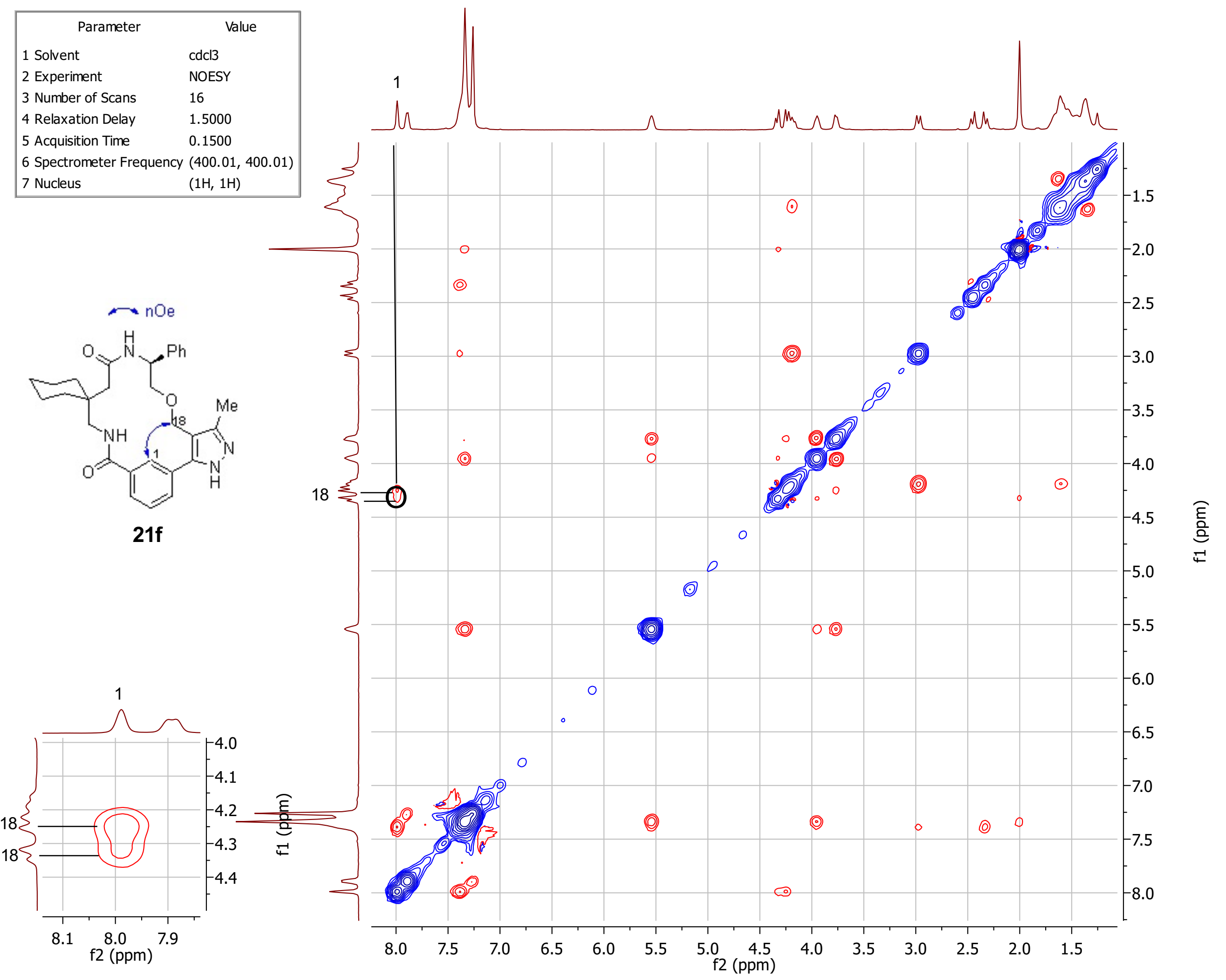




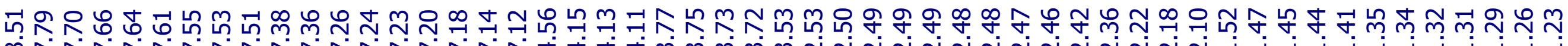

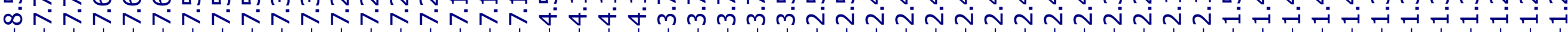

\begin{tabular}{|ll|}
\hline \multicolumn{1}{|c|}{ Parameter } & \multicolumn{1}{c|}{ Value } \\
1 Solvent & dmso at $100 \mathrm{oC}$ \\
2 Experiment & $1 \mathrm{D}$ \\
3 Number of Scans & 8 \\
4 Relaxation Delay & 1.0000 \\
5 Acquisition Time & 2.5559 \\
6 Spectrometer Frequency & 400.02 \\
7 Nucleus & $1 \mathrm{H}$ \\
\hline
\end{tabular}

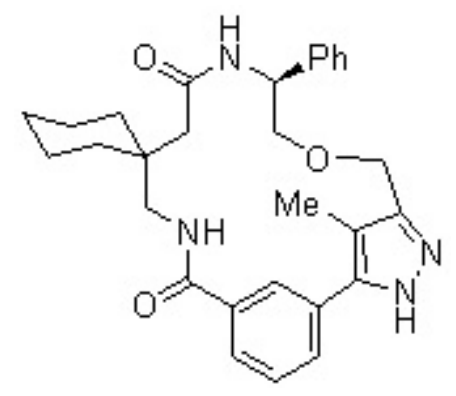

$20 f$

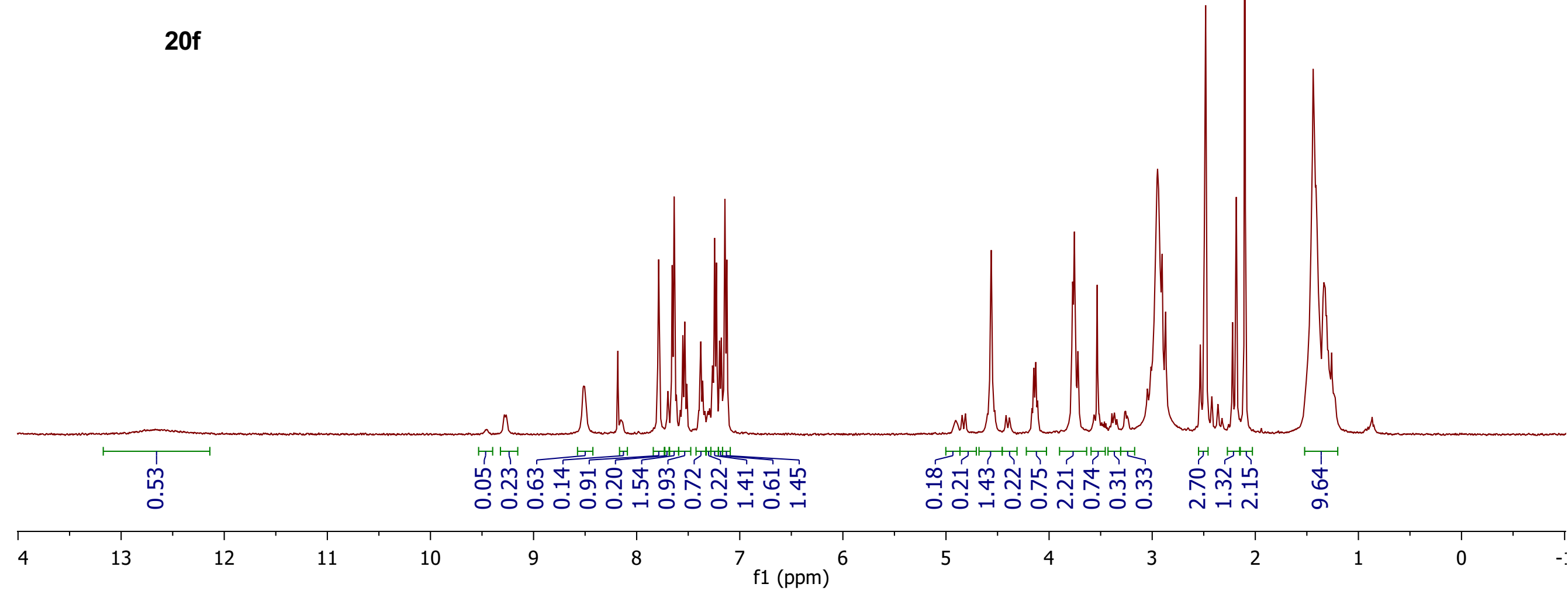




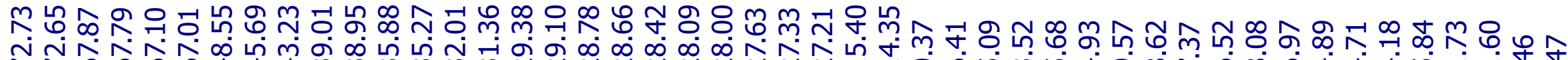

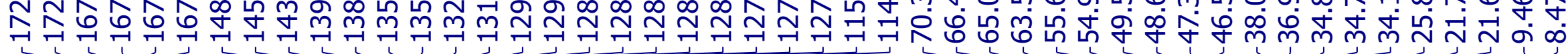

\begin{tabular}{|ll|}
\hline \multicolumn{1}{|c|}{ Parameter } & \multicolumn{1}{c|}{ Value } \\
1 Solvent & cdcl3 \\
2 Experiment & $1 \mathrm{D}$ \\
3 Number of Scans & 10000 \\
4 Relaxation Delay & 1.0000 \\
5 Acquisition Time & 1.3107 \\
6 Spectrometer Frequency & 100.59 \\
7 Nucleus & $13 \mathrm{C}$ \\
\hline
\end{tabular}

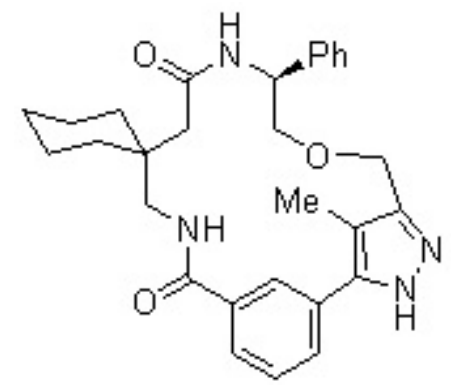

$20 f$

8070

70

60

50

40

30

20 
(CD3)2SO at $100 \mathrm{oC}$
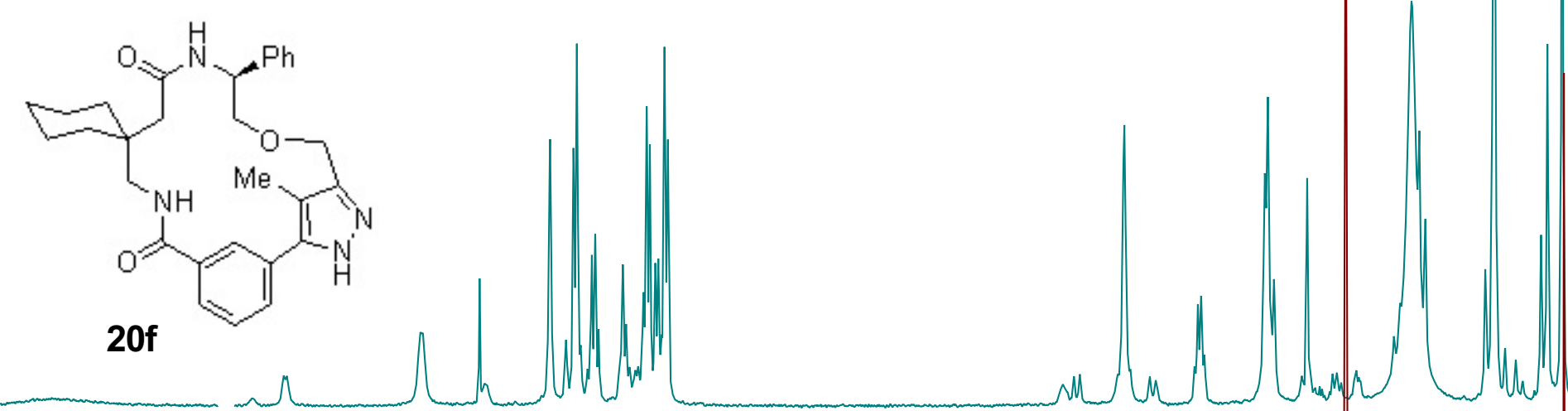

(CD3)2SO at $25 \mathrm{oC}$

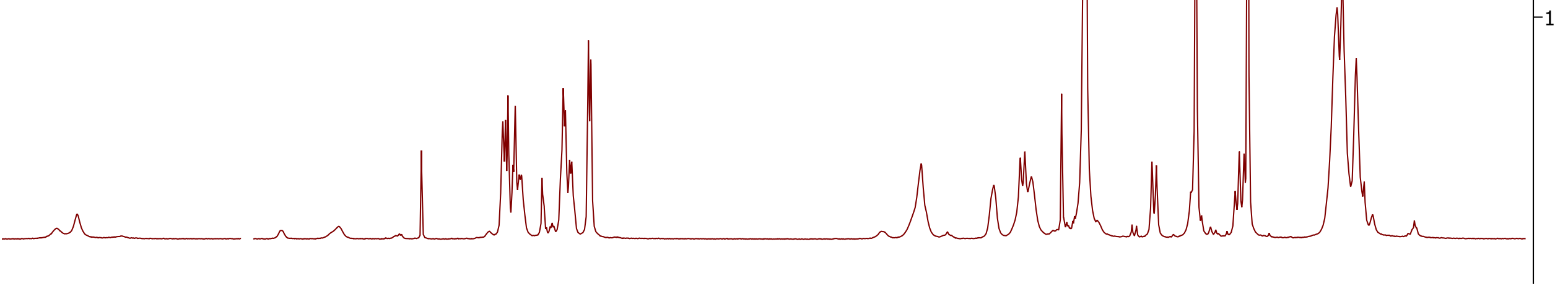

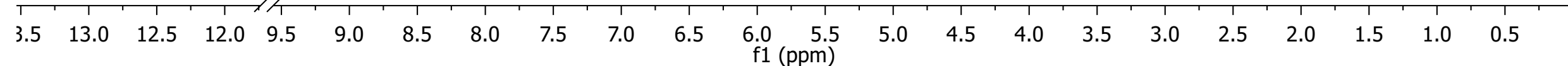




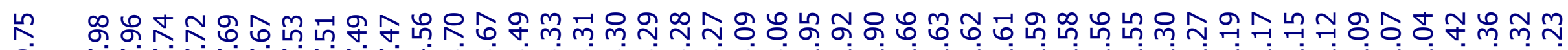

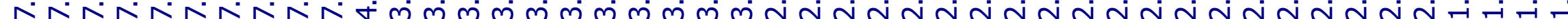

\begin{tabular}{|ll|}
\hline \multicolumn{1}{|c|}{ Parameter } & \multicolumn{1}{c|}{ Value } \\
1 Solvent & cd3od \\
2 Experiment & $1 \mathrm{D}$ \\
3 Number of Scans & 8 \\
4 Relaxation Delay & 1.0000 \\
5 Acquisition Time & 2.5559 \\
6 Spectrometer Frequency & 400.02 \\
7 Nucleus & $1 \mathrm{H}$ \\
\hline
\end{tabular}
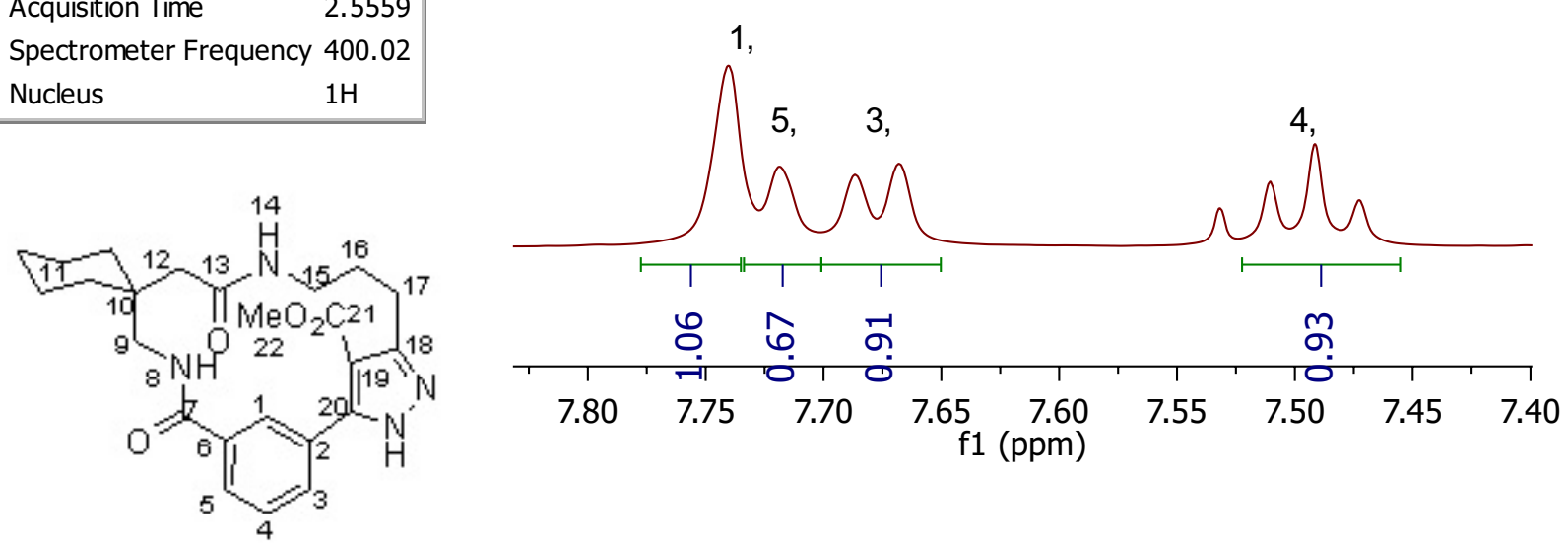

$20 \mathrm{~g}$

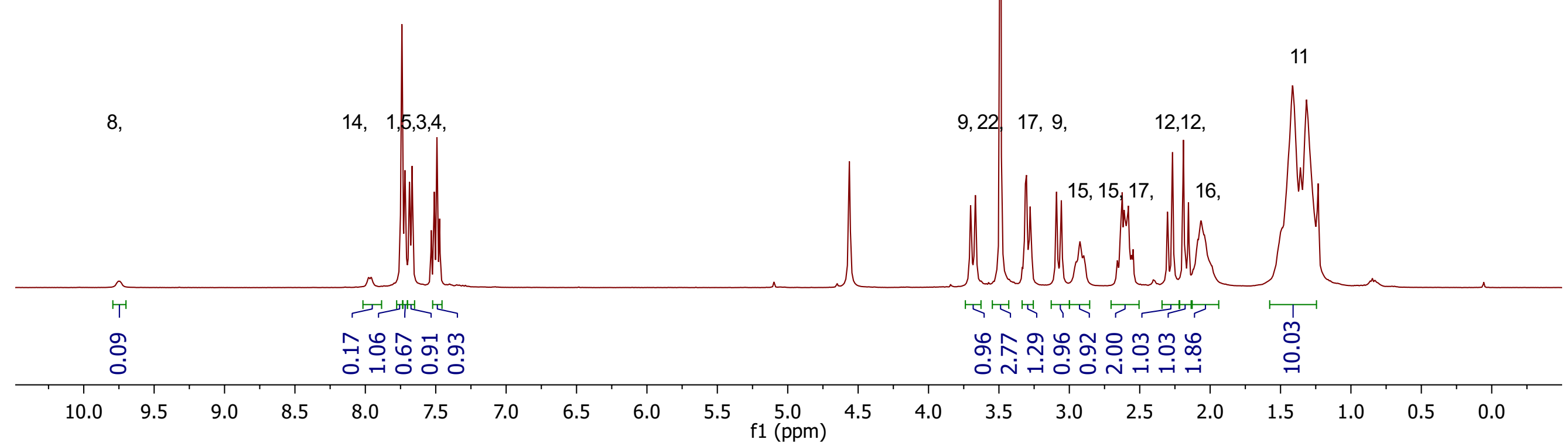


ต้ํํㅇํำ

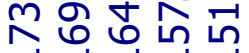

다 다

$\sim 1$

Parameter

Value

1 Solvent

$\operatorname{cd} 3$ od/cdcL3 $=1: 1$

2 Experiment

3 Number of Scans

4 Relaxation Delay

5 Acquisition Time

1D

15000

1.0000

1.3107

6 Spectrometer Frequency 100.59

7 Nucleus $13 \mathrm{C}$

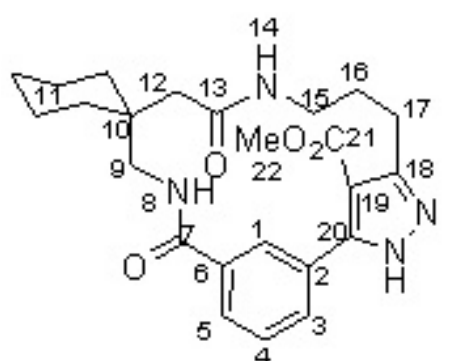

$20 \mathrm{~g}$
ষㅇํ m

ต

규 त-

O

-1

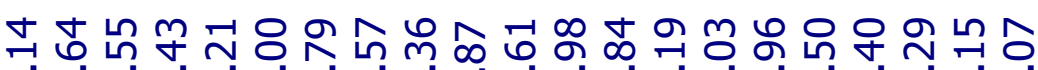
ப்

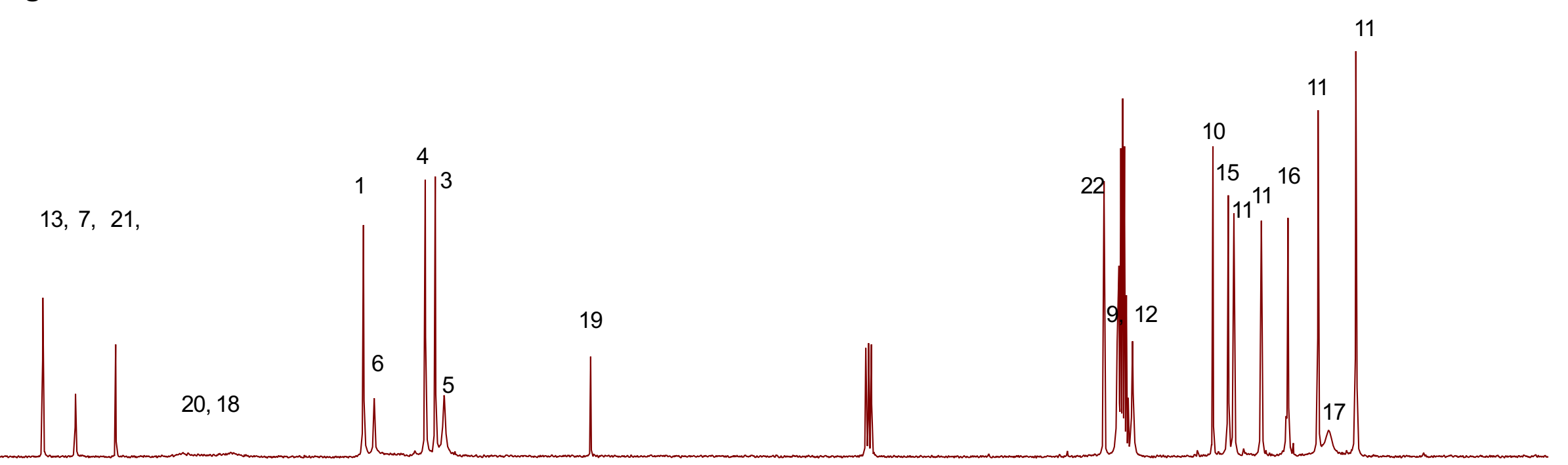




\begin{tabular}{|ll|}
\hline \multicolumn{1}{|c|}{ Parameter } & \multicolumn{1}{c|}{ Value } \\
1 Solvent & cd3od \\
2 Experiment & coSY \\
3 Number of Scans & 8 \\
4 Relaxation Delay & 1.0000 \\
5 Acquisition Time & 0.1500 \\
6 Spectrometer Frequency & $(400.02,400.02)$ \\
7 Nucleus & $(1 \mathrm{H}, 1 \mathrm{H})$ \\
\hline
\end{tabular}

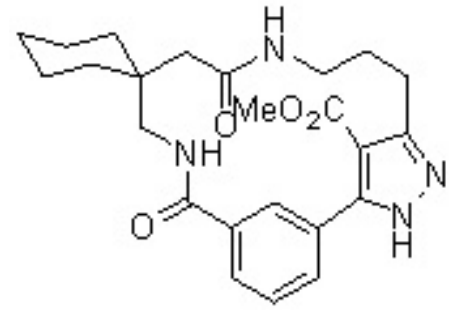

$20 \mathrm{~g}$

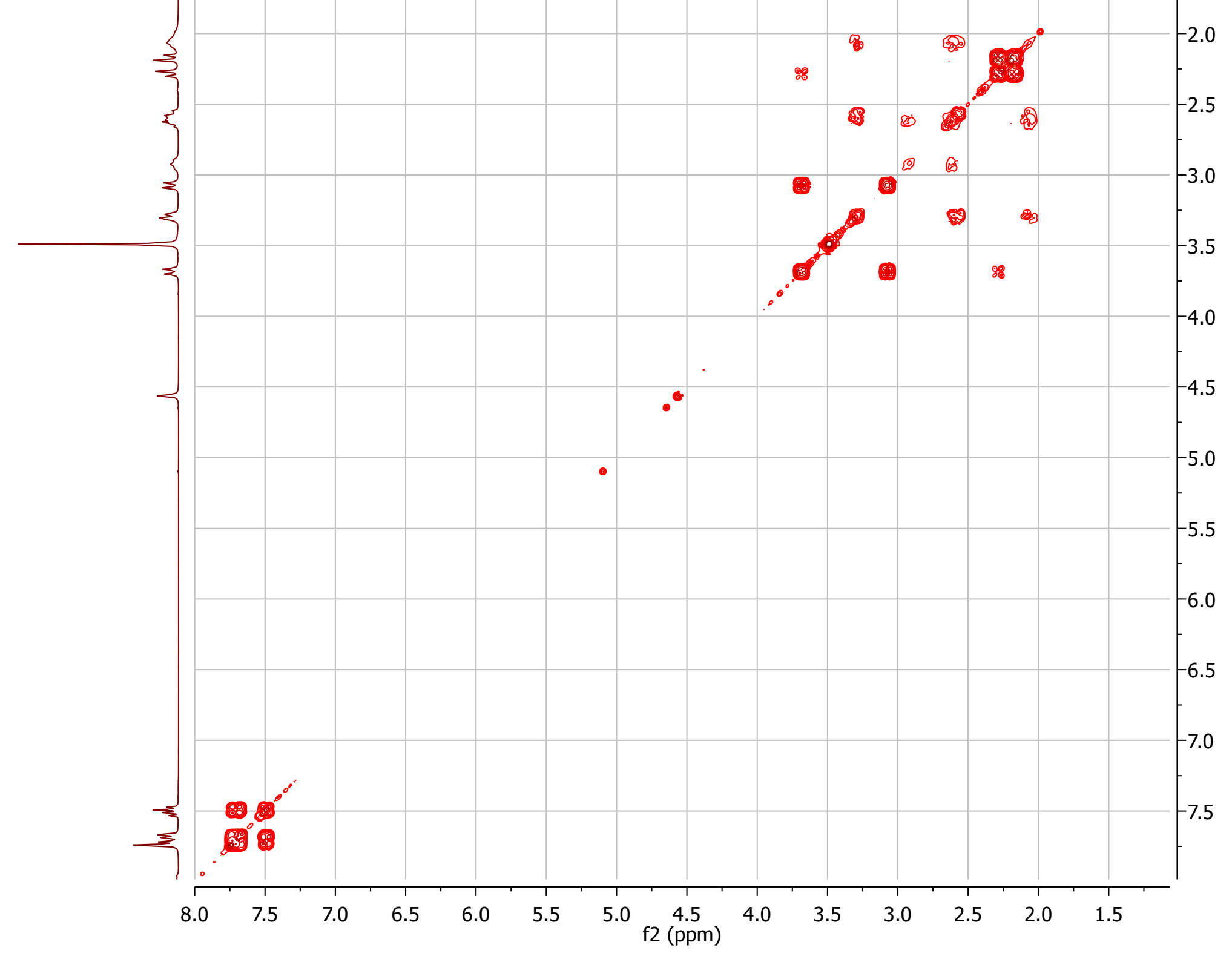




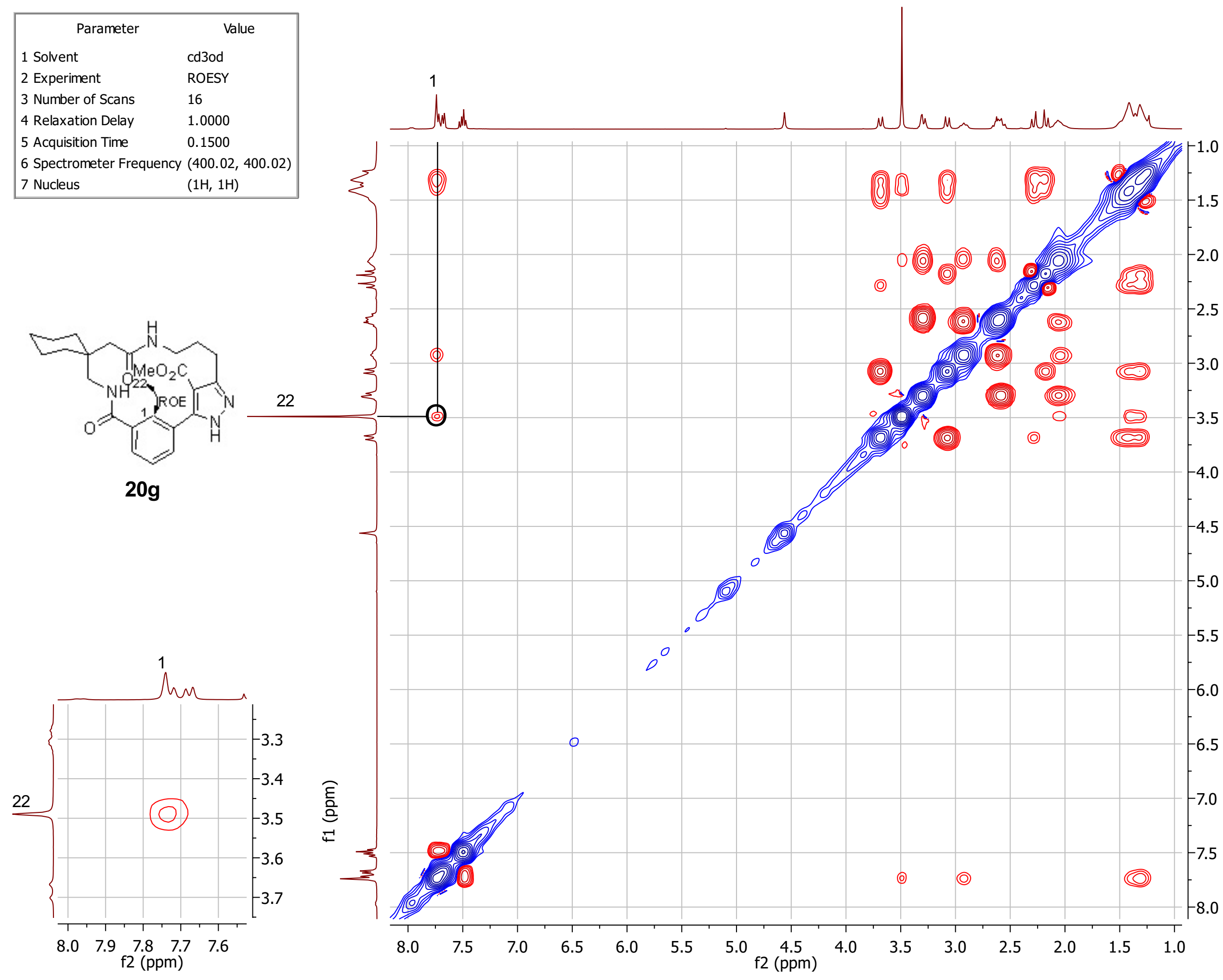




\begin{tabular}{|ll|}
\hline \multicolumn{1}{|c|}{ Parameter } & \multicolumn{1}{c|}{ Value } \\
1 Solvent & cdcl3 \\
2 Experiment & $1 \mathrm{D}$ \\
3 Number of Scans & 8 \\
4 Relaxation Delay & 1.0000 \\
5 Acquisition Time & 2.0447 \\
6 Spectrometer Frequency & 499.81 \\
7 Nucleus & $1 \mathrm{H}$ \\
\hline
\end{tabular}

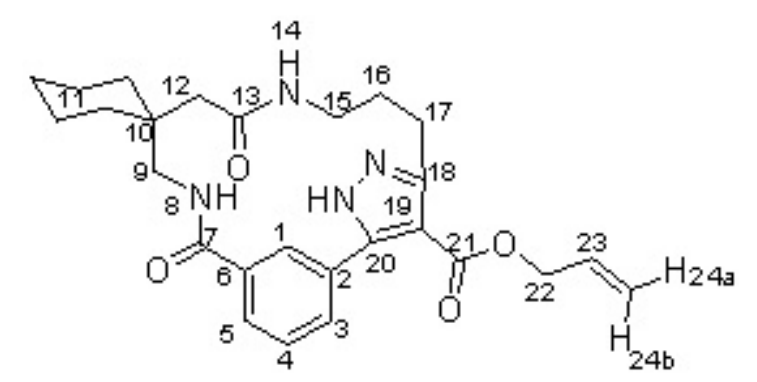

20h

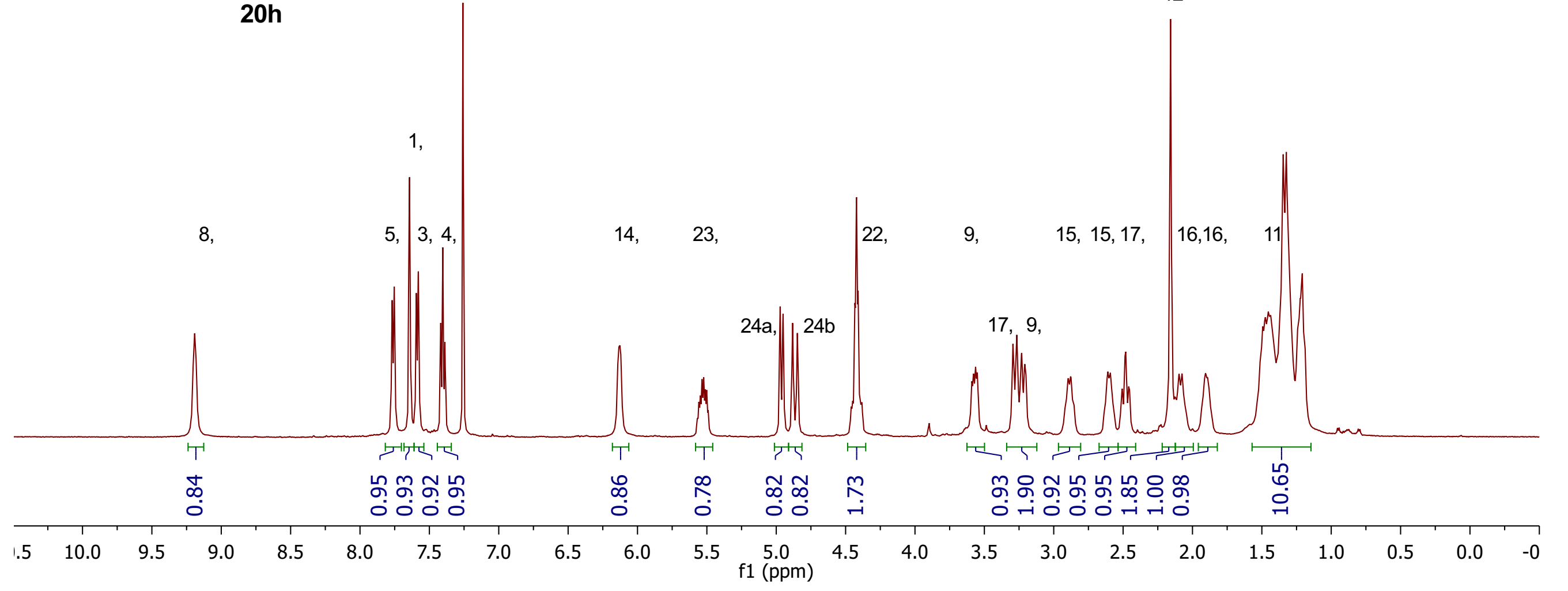




\section{กิก \\ $8 \stackrel{\circ}{\circ}$

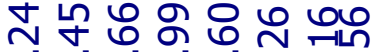 \\ 눈 \\ 든?

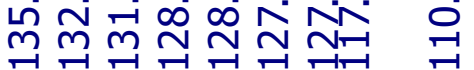

Parameter

\section{Value}

1 Solvent

cdcl3

2 Experiment

1D

3 Number of Scans

1600

4 Relaxation Delay

1.0000

5 Acquisition Time

0.2500

6 Spectrometer Frequency 125.69

7 Nucleus

$13 \mathrm{C}$

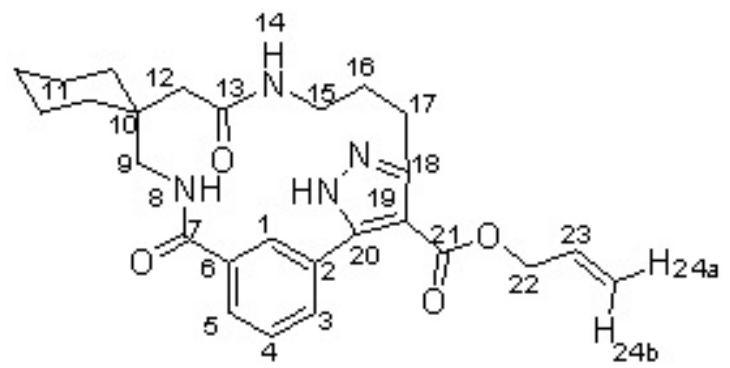

$20 \mathrm{~h}$

$13,7,21$

20,18

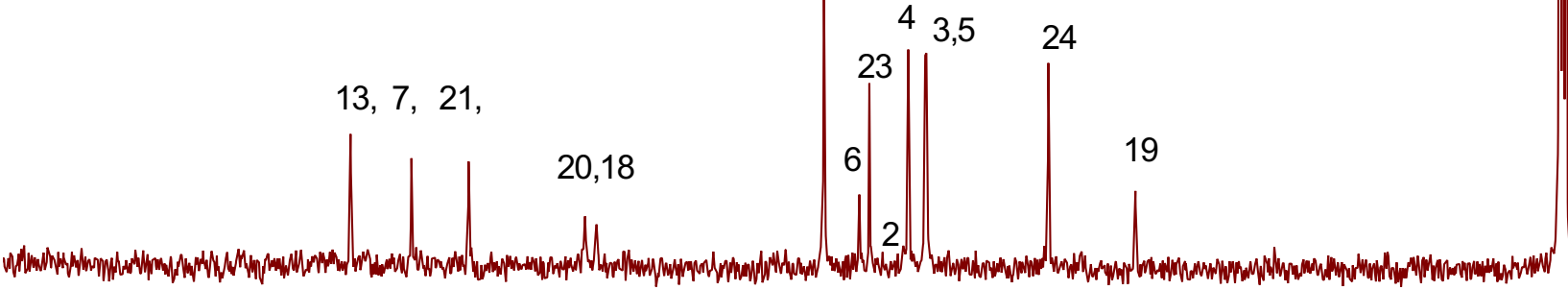

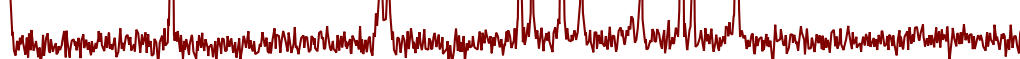

22

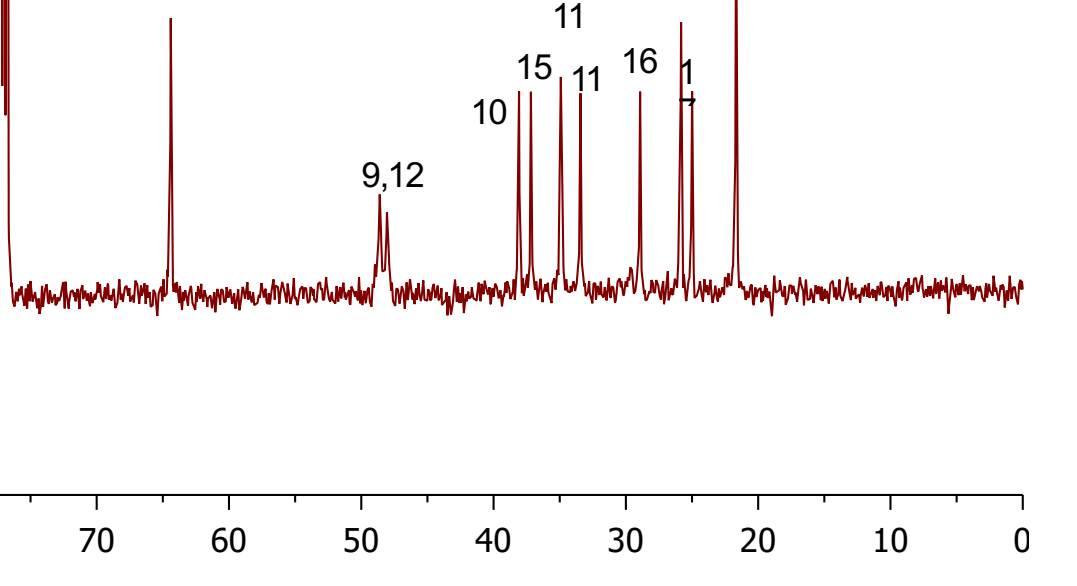




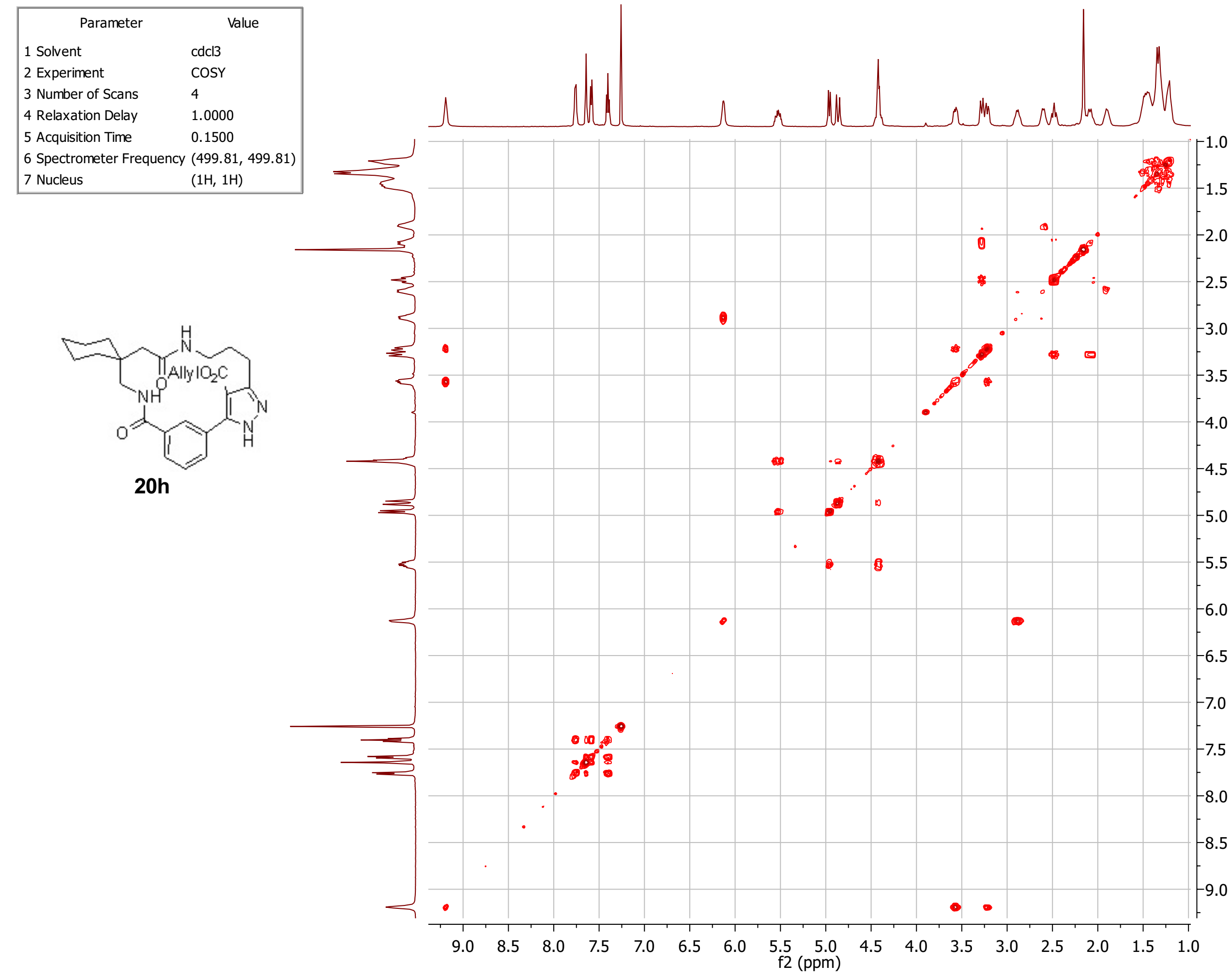

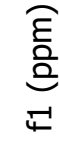




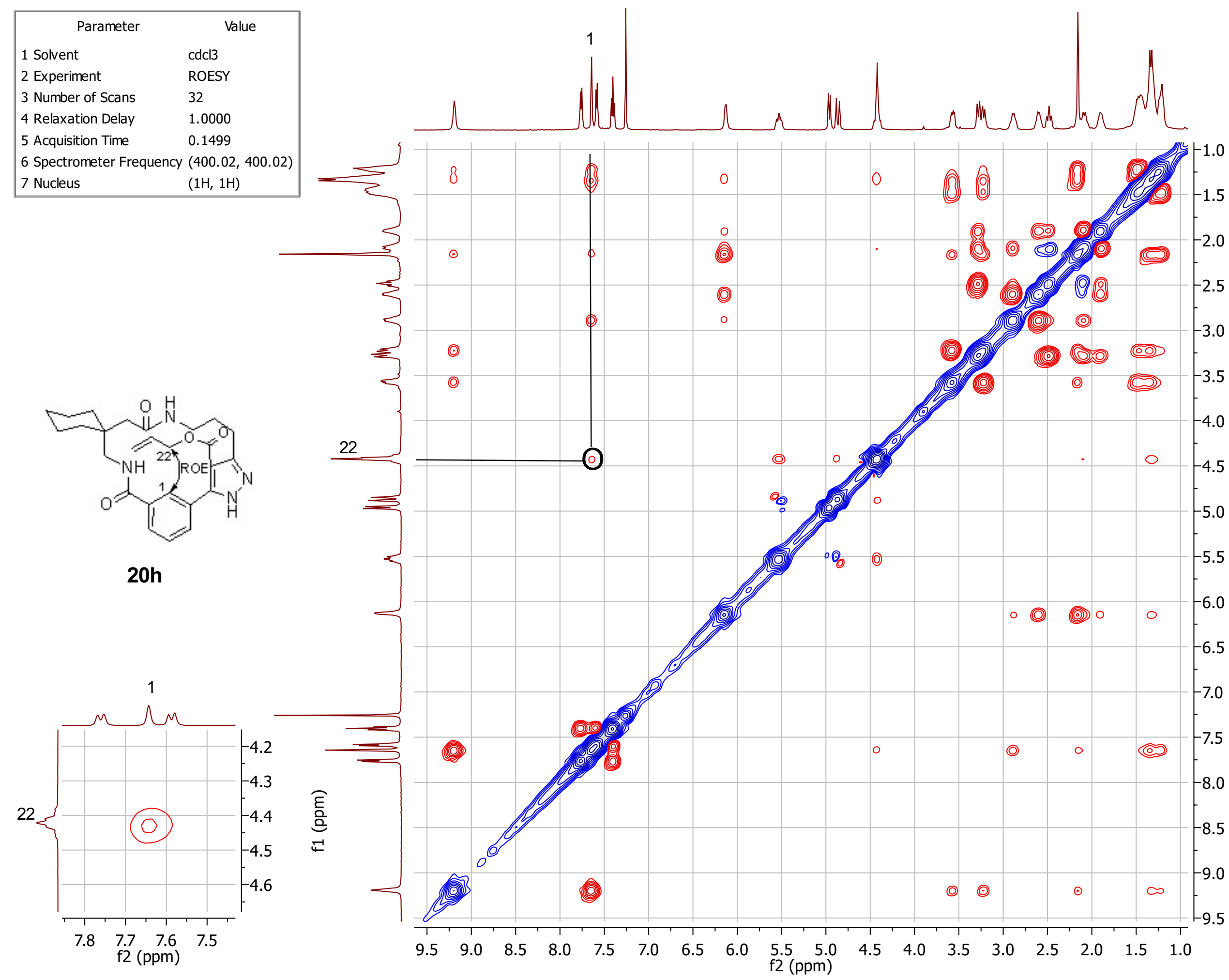

है
는 
ঢ̆

の NंN NNN

\begin{tabular}{|ll|}
\hline \multicolumn{1}{|c}{ Parameter } & \multicolumn{1}{c|}{ Value } \\
1 Solvent & cdcl3 \\
2 Experiment & $1 \mathrm{D}$ \\
3 Number of Scans & 8 \\
4 Relaxation Delay & 1.0000 \\
5 Acquisition Time & 2.0447 \\
6 Spectrometer Frequency & 499.81 \\
7 Nucleus & $1 \mathrm{H}$ \\
\hline
\end{tabular}

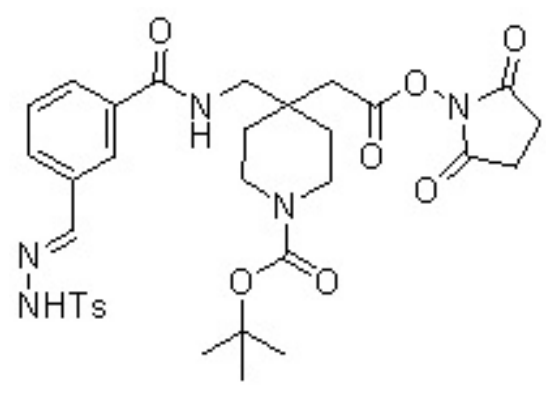

\section{$22 a b$}

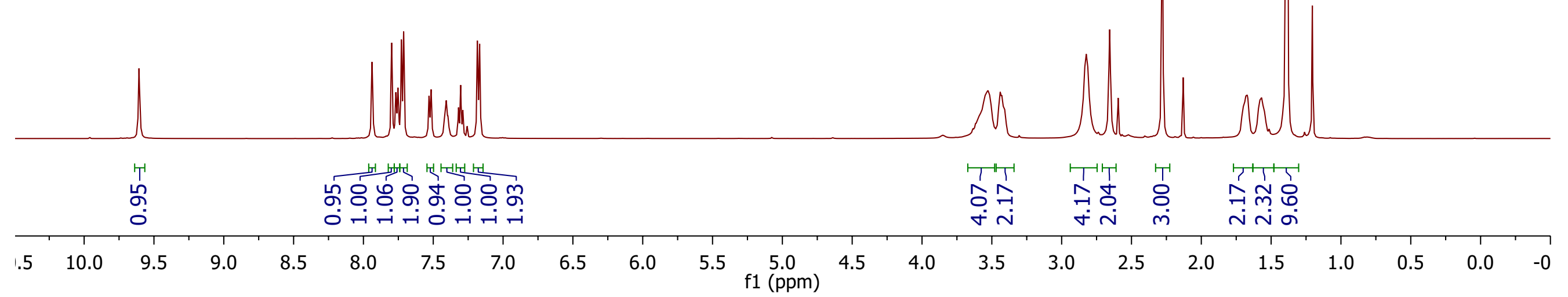



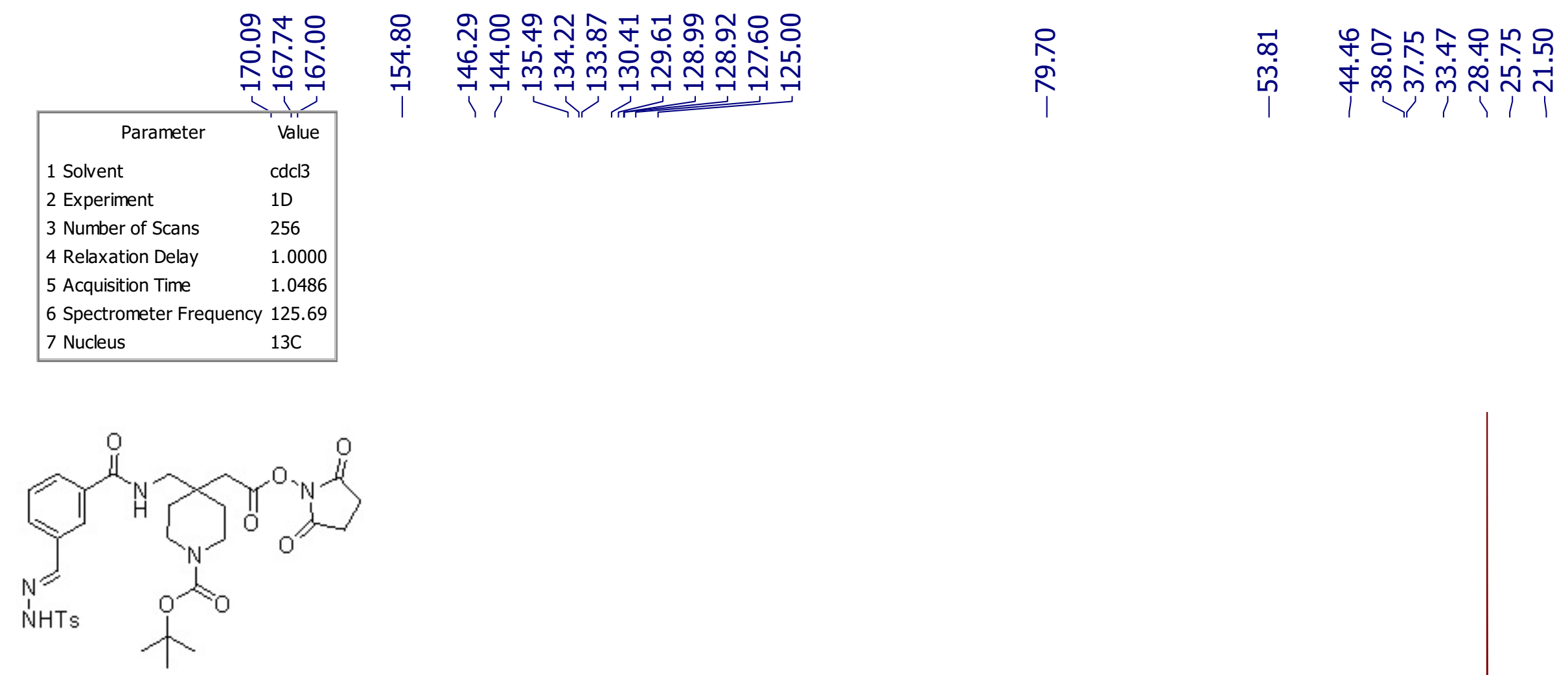

22ab 
$m$ m $\infty \mathrm{N}^{-} \mathrm{N} N \mathrm{~N} N$

\begin{tabular}{|ll|}
\hline \multicolumn{1}{|c}{ Parameter } & \multicolumn{1}{c|}{ Value } \\
1 Solvent & cdcl3 \\
2 Experiment & $1 \mathrm{D}$ \\
3 Number of Scans & 8 \\
4 Relaxation Delay & 1.0000 \\
5 Acquisition Time & 2.5592 \\
6 Spectrometer Frequency & 400.09 \\
7 Nucleus & $1 \mathrm{H}$ \\
\hline
\end{tabular}

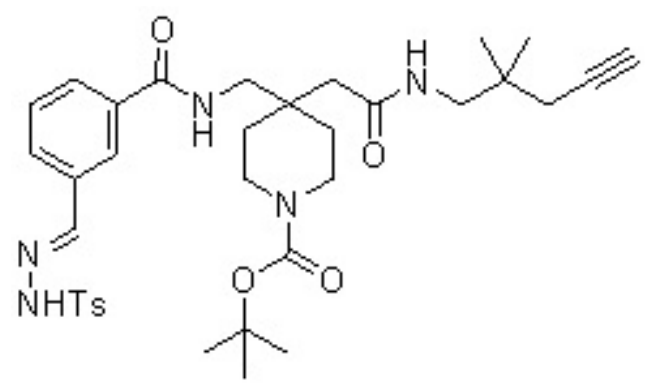

22ac

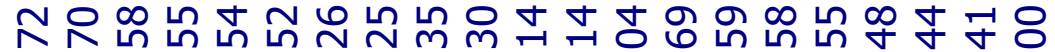



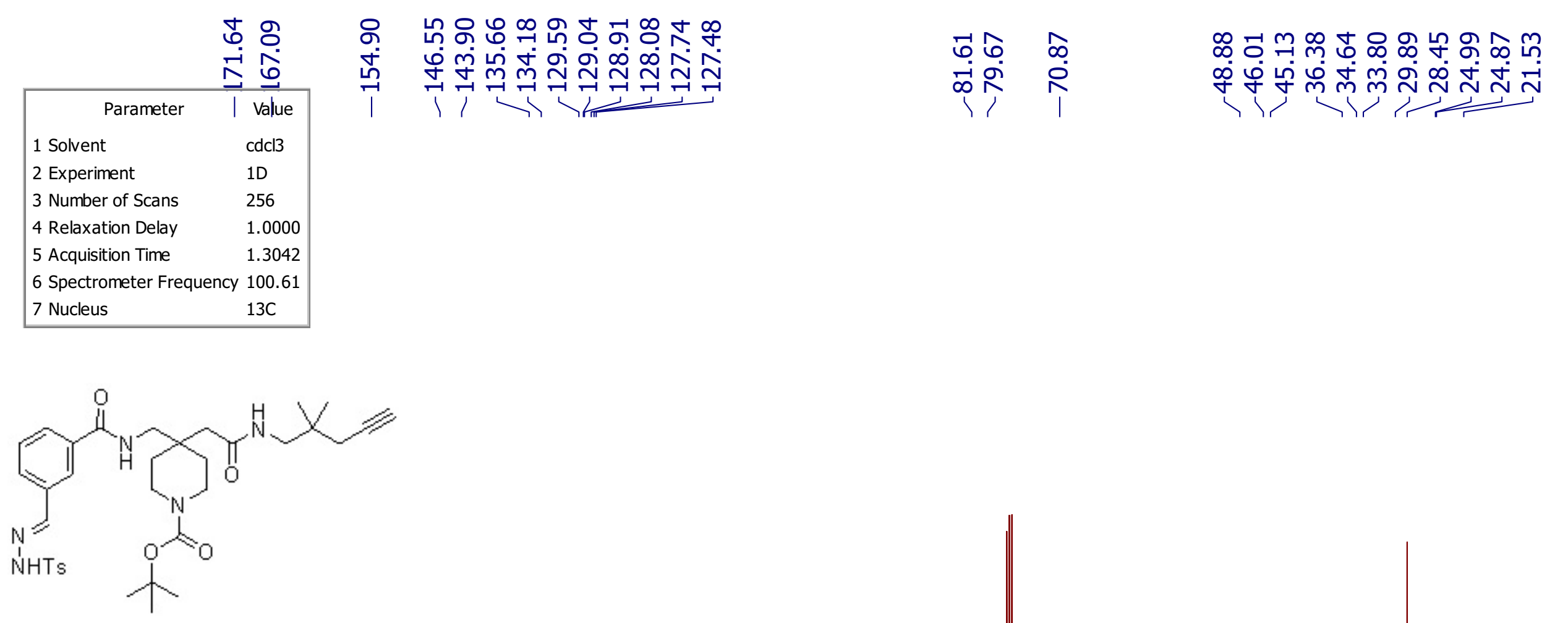

\section{2ac}

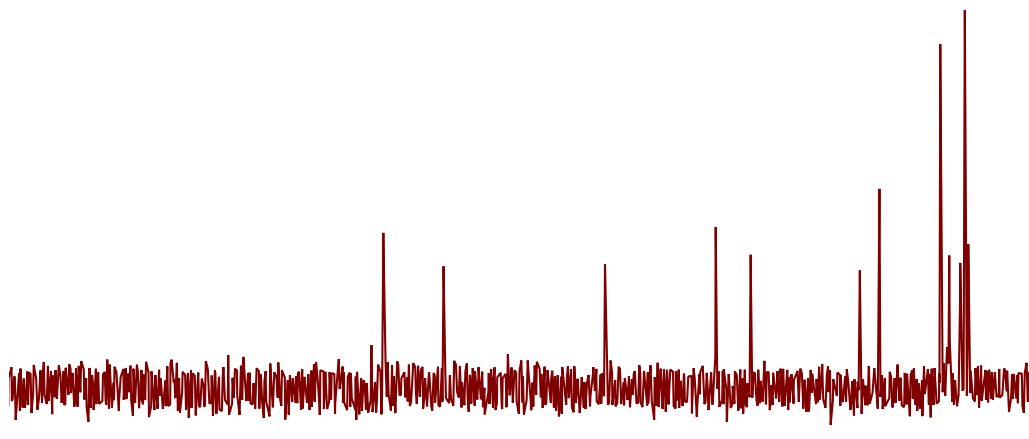

70

60




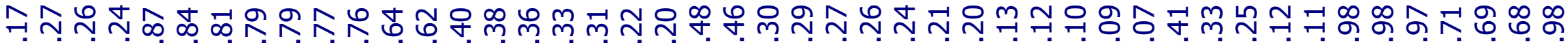

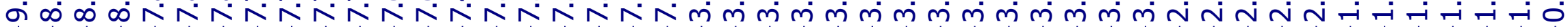

\begin{tabular}{ll|}
1 Parameter & Value \\
2 Solvent & $c d c 13$ \\
3 Experiment & $1 \mathrm{D}$ \\
4 Relaxation Delay & 1.0000 \\
5 Acquisition Time & 2.5559 \\
6 Spectrometer Frequency 400.01 \\
7 Nucleus
\end{tabular}

22a
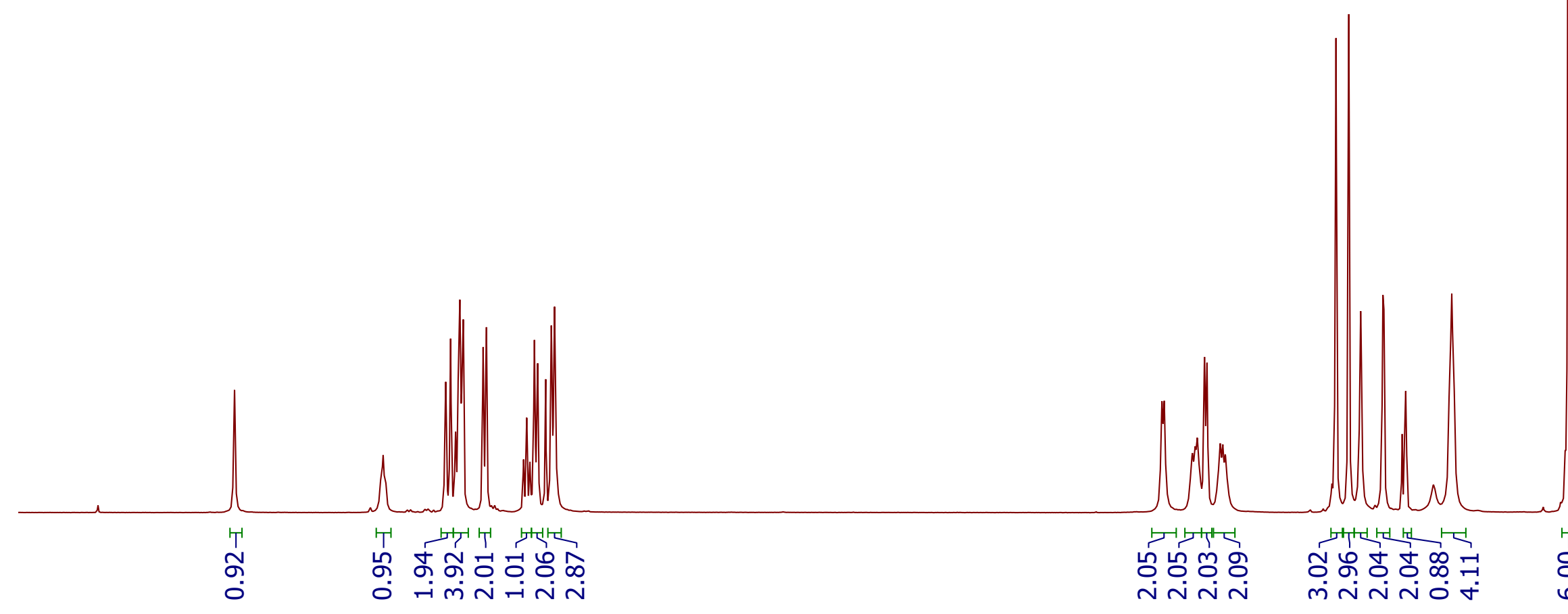

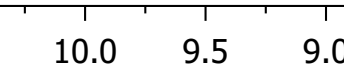

8.5

8.0

$7.5 \quad 7.0$

$7.0 \quad 6.5$




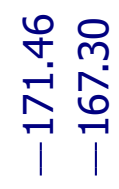

\begin{tabular}{|ll|}
\hline \multicolumn{1}{|c|}{ Parameter } & \multicolumn{1}{c|}{ Value } \\
1 Solvent & \multicolumn{1}{c}{ cdcl3 } \\
2 Experiment & $1 \mathrm{D}$ \\
3 Number of Scans & 480 \\
4 Relaxation Delay & 1.0000 \\
5 Acquisition Time & 1.3042 \\
6 Spectrometer Frequency & 100.61 \\
7 Nucleus & $13 \mathrm{C}$ \\
\hline
\end{tabular}

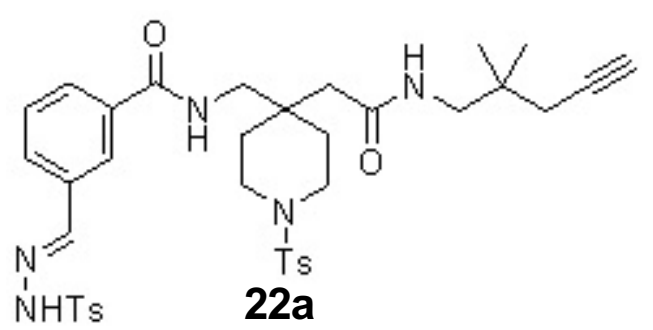

22a

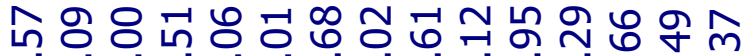

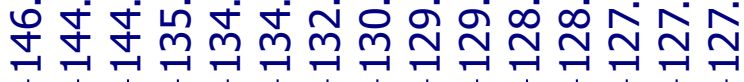

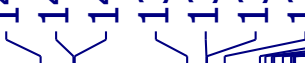

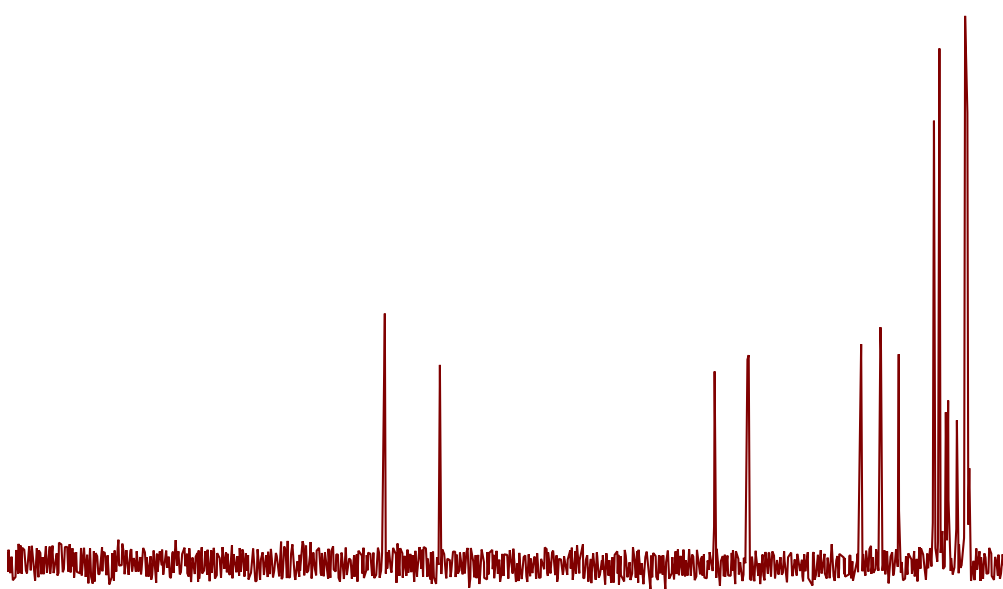

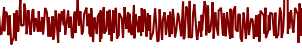

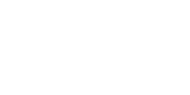


○

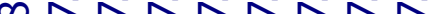

\begin{tabular}{|ll|}
\hline \multicolumn{1}{|c|}{ Parameter } & \multicolumn{1}{c|}{ Value } \\
1 Solvent & cd3od \\
2 Experiment & $1 \mathrm{D}$ \\
3 Number of Scans & 8 \\
4 Relaxation Delay & 1.0000 \\
5 Acquisition Time & 2.5592 \\
6 Spectrometer Frequency & 400.09 \\
7 Nucleus & $1 \mathrm{H}$ \\
\hline
\end{tabular}

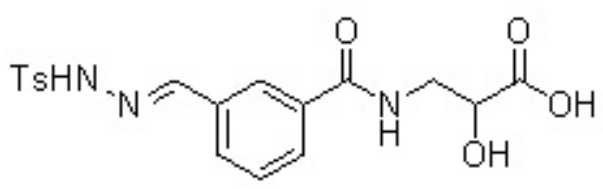

22ba

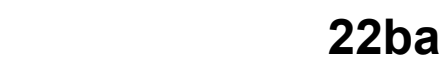

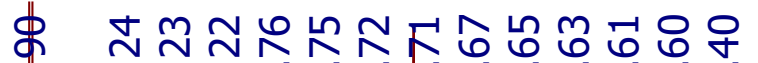

F

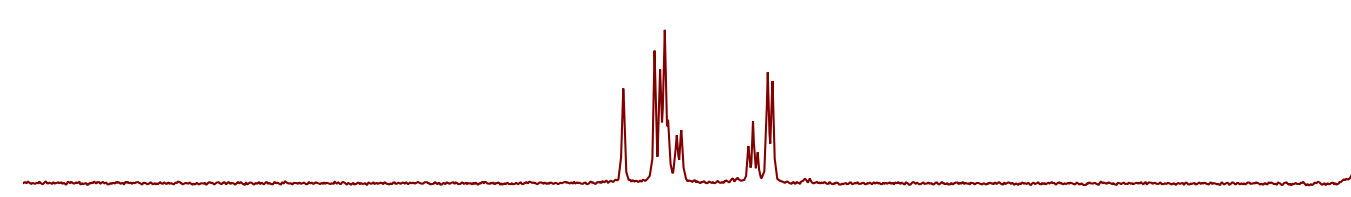

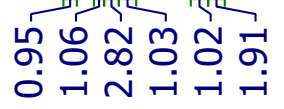

Tा ?क्त

욤

@ำ 


\begin{tabular}{|ll|}
\hline \multicolumn{1}{|c|}{ Parameter } & \multicolumn{1}{c|}{ Value } \\
1 Solvent & cd3od \\
2 Experiment & 1D \\
3 Number of Scans & 64 \\
4 Relaxation Delay & 1.0000 \\
5 Acquisition Time & 1.3042 \\
6 Spectrometer Frequency & 100.61 \\
7 Nucleus & $13 \mathrm{C}$ \\
\hline
\end{tabular}

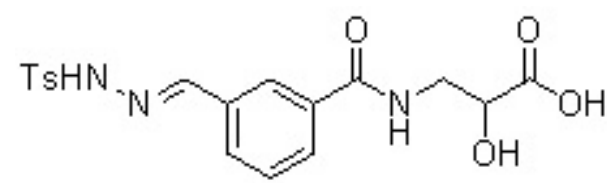

22ba

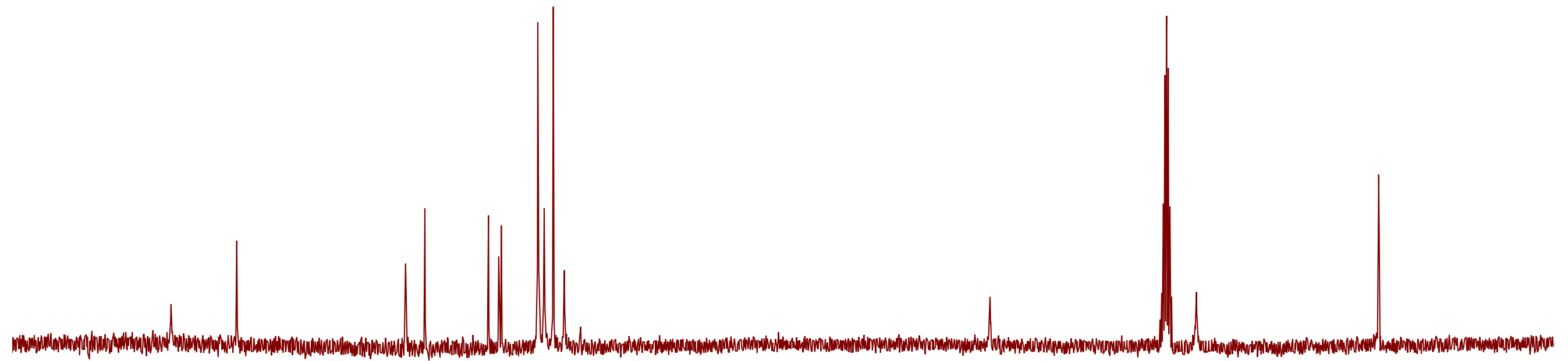

$190 \quad 180$

170




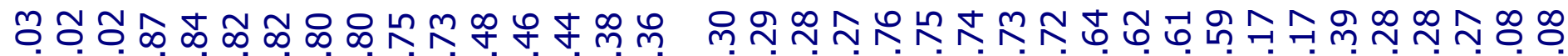

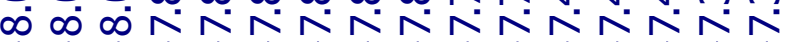

1 Solvent $\quad$ cd3od

2 Experiment

3 Number of Scans

4 Relaxation Delay

5 Acquisition Time

8

1.0000

2.5592

6 Spectrometer Frequency 400.09

7 Nucleus $1 \mathrm{H}$

TsHN

22b

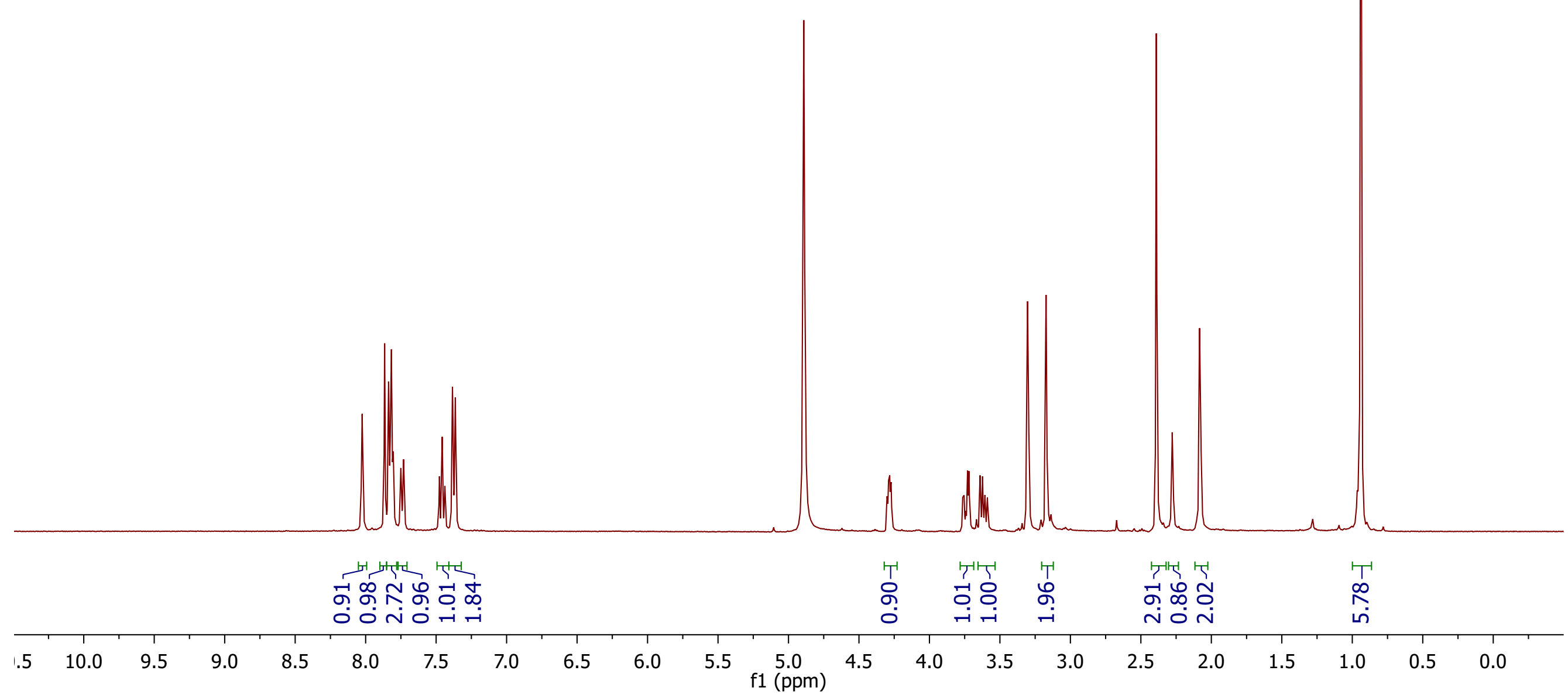



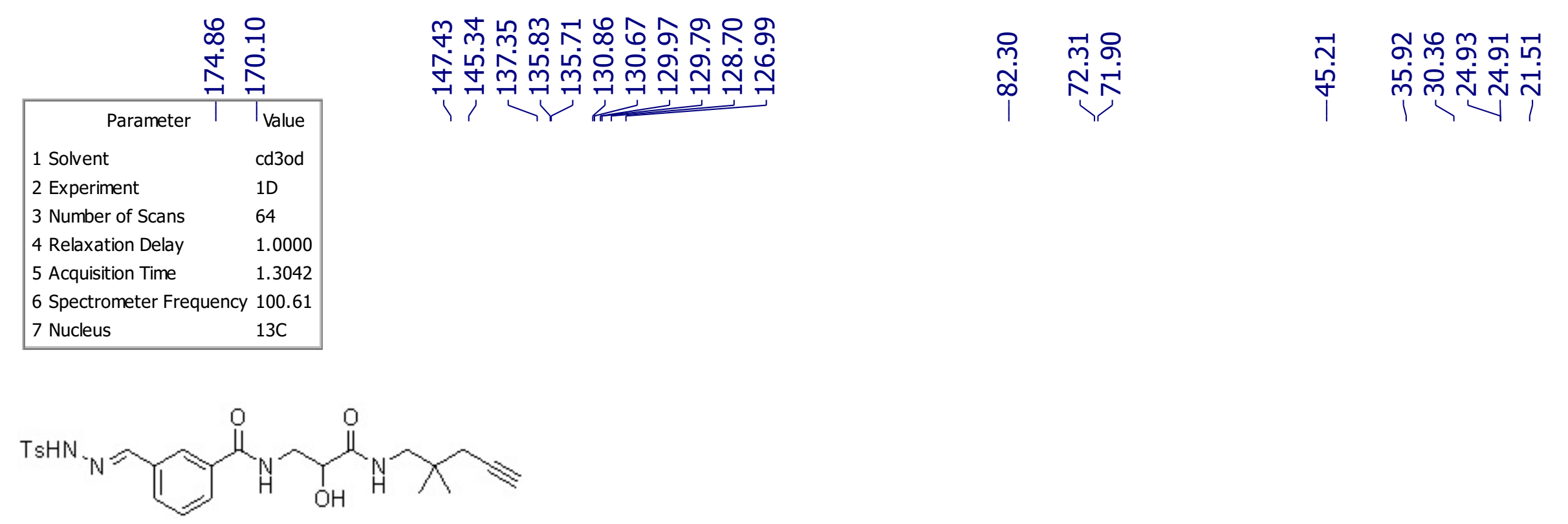

22b

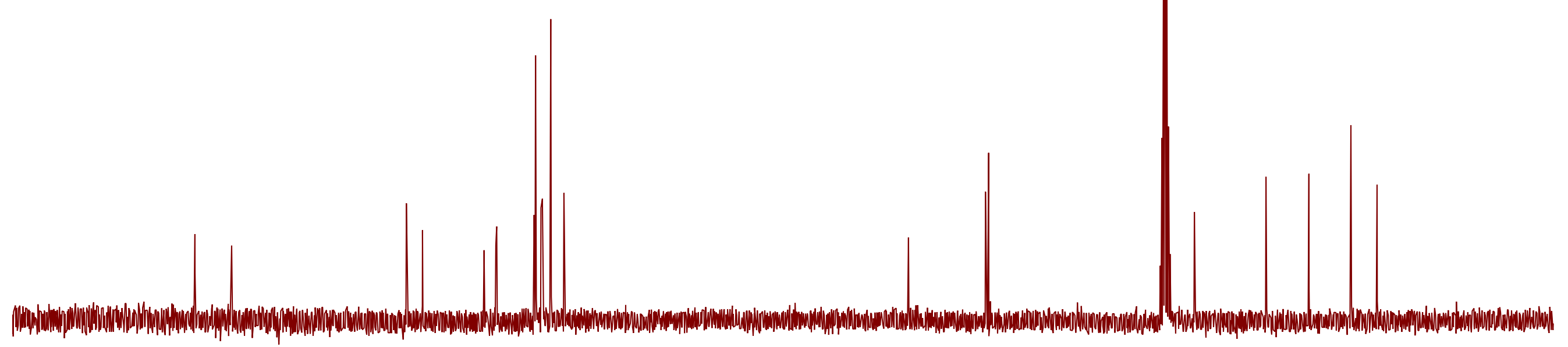

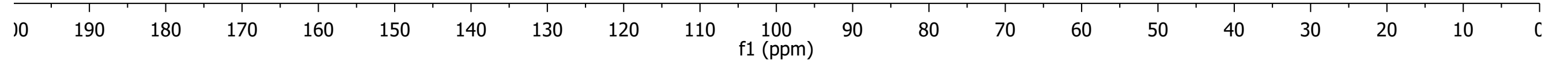




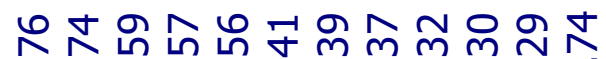

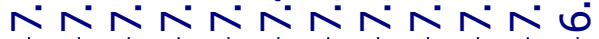

\begin{tabular}{|ll|}
\hline \multicolumn{1}{|c|}{ Parameter } & \multicolumn{1}{c|}{ Value } \\
1 Solvent & cdcl3 \\
2 Experiment & $1 \mathrm{D}$ \\
3 Number of Scans & 8 \\
4 Relaxation Delay & 1.0000 \\
5 Acquisition Time & 2.5559 \\
6 Spectrometer Frequency & 400.01 \\
7 Nucleus & $1 \mathrm{H}$ \\
\hline
\end{tabular}

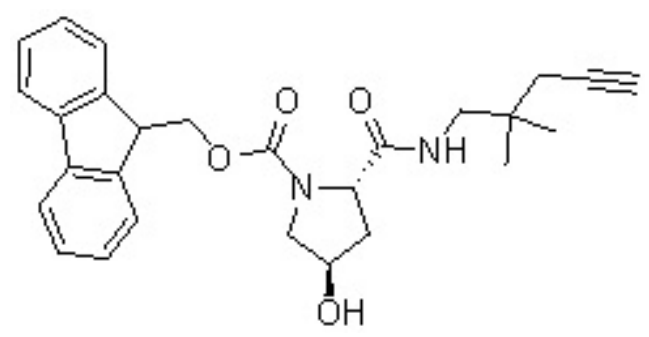

22ca

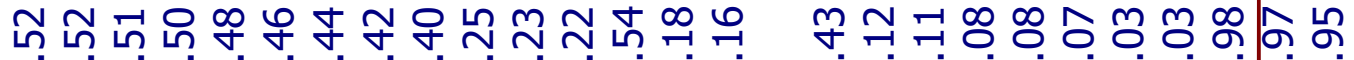

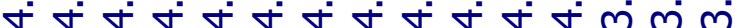

i i i ri

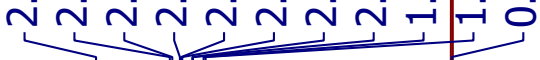




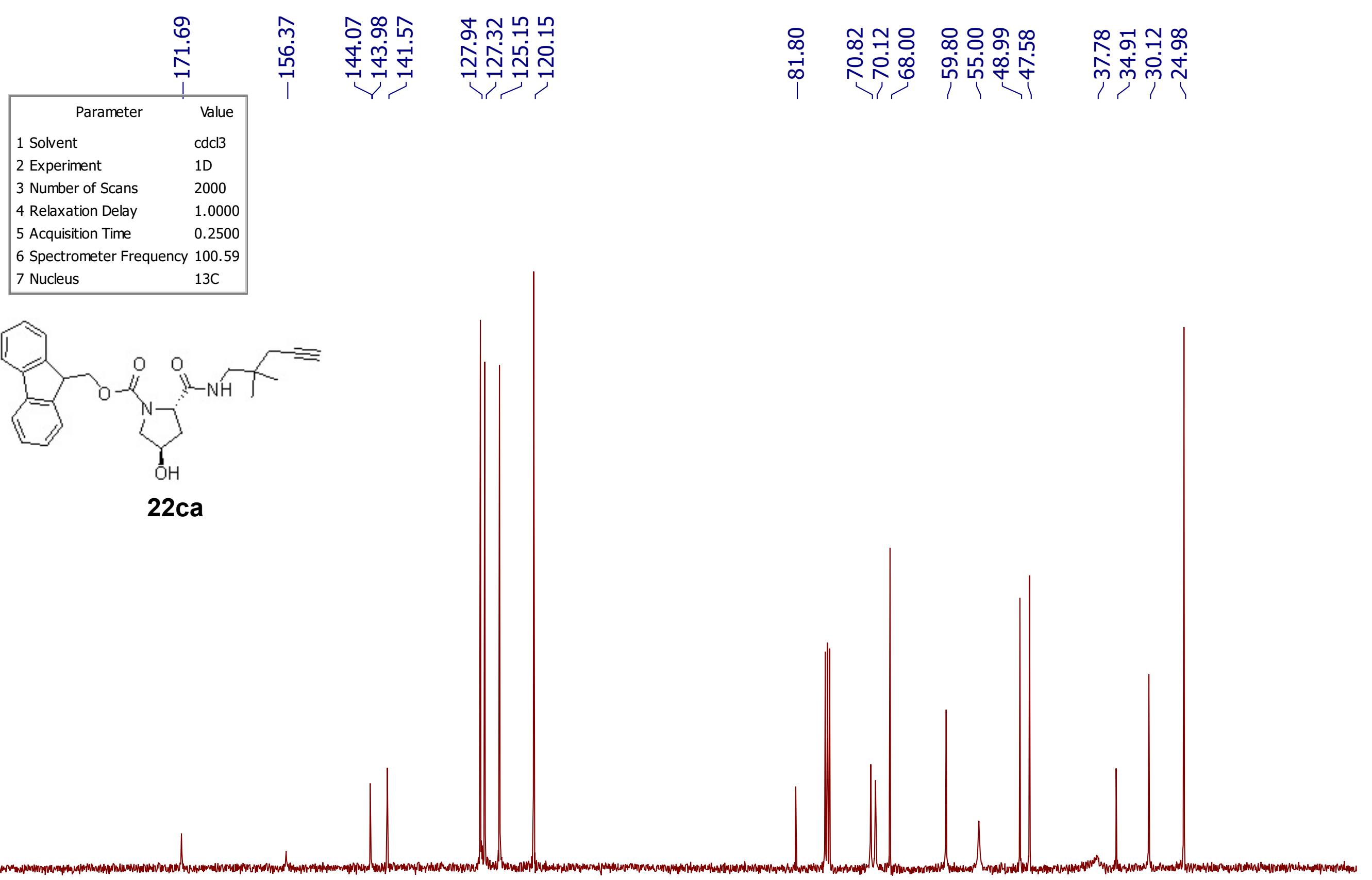

\begin{tabular}{|c|c|c|c|c|c|c|c|c|c|c|c|c|c|c|c|c|c|c|c|}
\hline 10 & 190 & 180 & 170 & 160 & 150 & 140 & 130 & 120 & 110 & $\begin{array}{c}100 \\
\mathrm{f} 1(\mathrm{ppm})\end{array}$ & 90 & 80 & 70 & 60 & 50 & 40 & 30 & 20 & ${ }_{10}^{1}$ \\
\hline
\end{tabular}




\begin{tabular}{|ll|}
\hline \multicolumn{1}{|c|}{ Parameter } & \multicolumn{1}{c|}{$\begin{array}{r}\text { Value } \\
\text { 1 Solvent }\end{array}$} \\
2 Experiment & 1D \\
3 Number of Scans & 8 \\
4 Relaxation Delay & 1.0000 \\
5 Acquisition Time & 2.5592 \\
6 Spectrometer Frequency & 400.09 \\
7 Nucleus & $1 \mathrm{H}$ \\
\hline \multicolumn{2}{|c|}{} \\
\hline
\end{tabular}<smiles>C#CCC(C)(C)CNC(=O)[C@@H]1C[C@@H](O)CN1</smiles>

$22 c b$

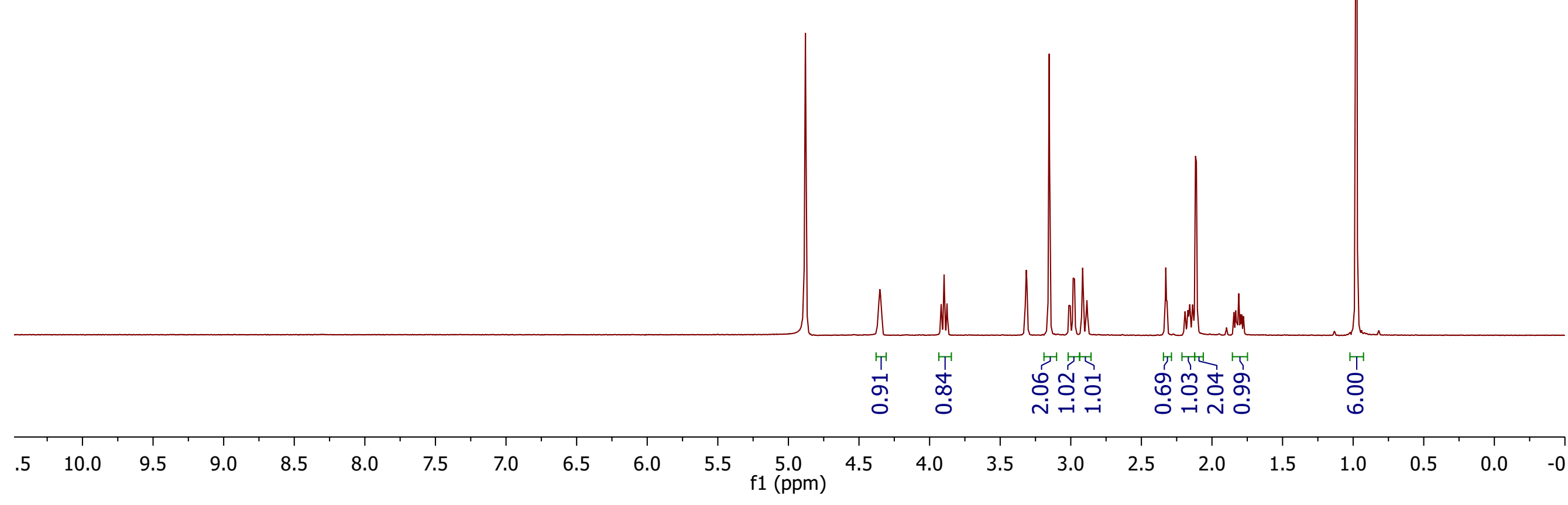




\begin{tabular}{|ll|}
\hline \multicolumn{1}{|c}{ Parameter } & \multicolumn{1}{c|}{ Value } \\
1 Solvent & cd3od \\
2 Experiment & $1 \mathrm{D}$ \\
3 Number of Scans & 256 \\
4 Relaxation Delay & 1.0000 \\
5 Acquisition Time & 1.3042 \\
6 Spectrometer Frequency & 100.61 \\
7 Nucleus & $13 \mathrm{C}$ \\
\hline
\end{tabular}

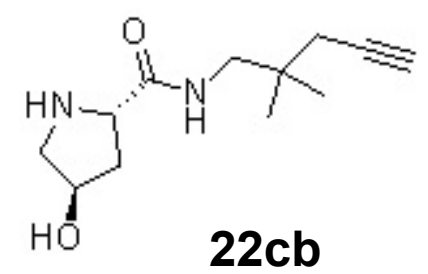

\section{2b}

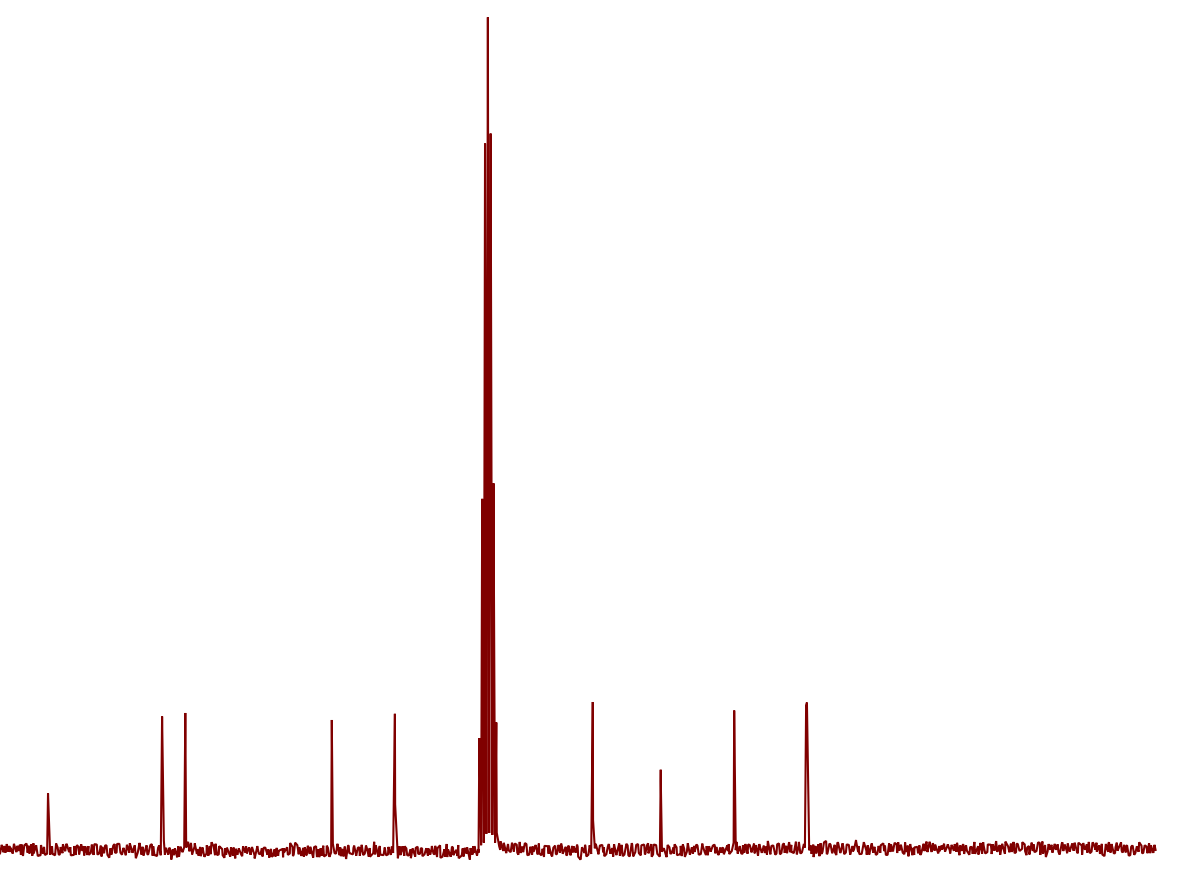




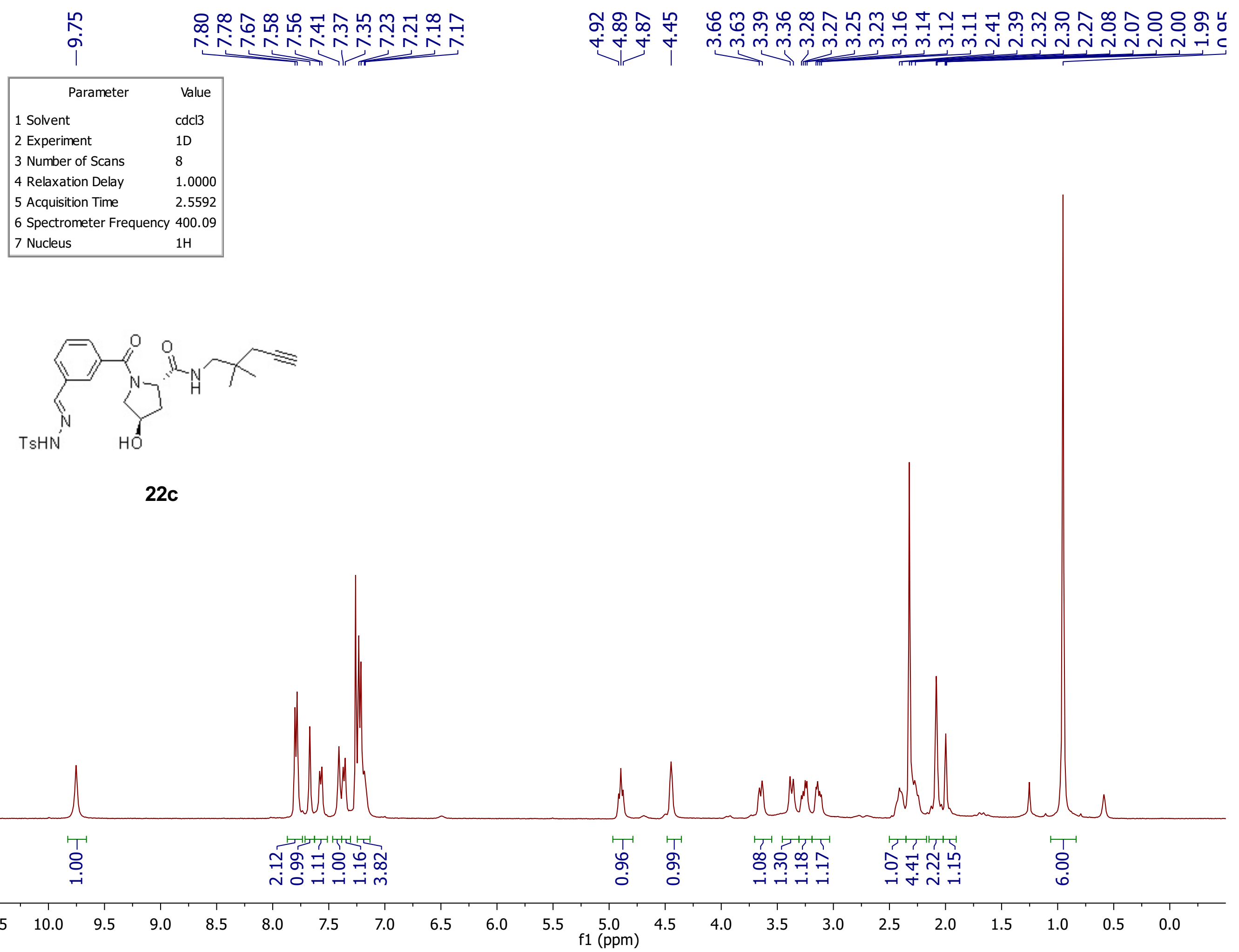




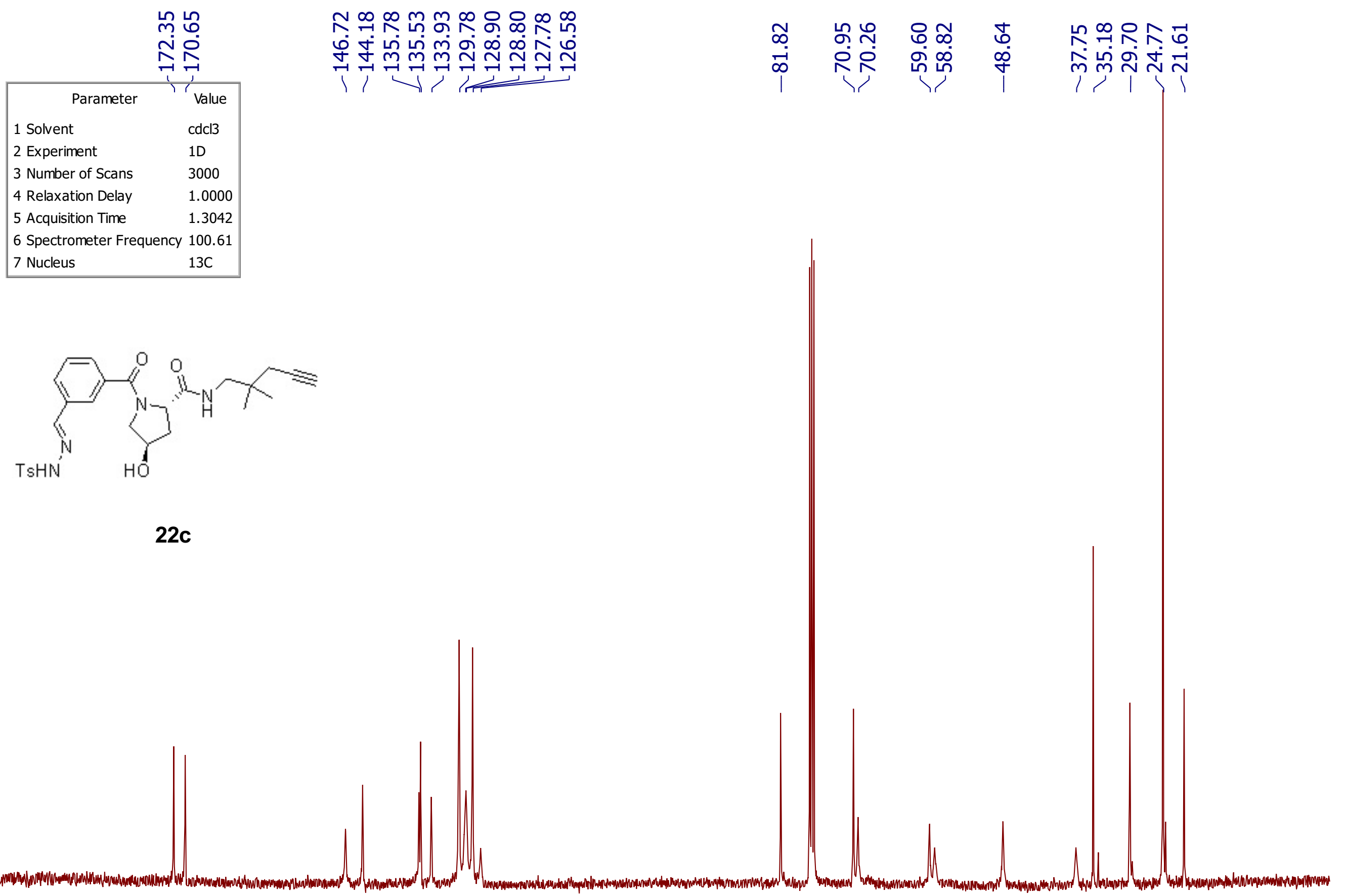

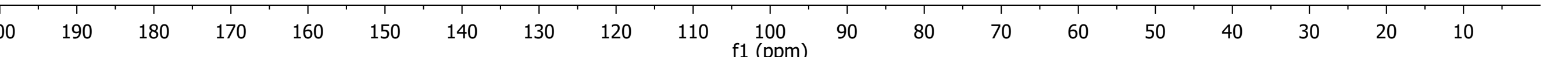




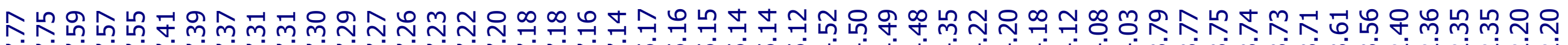

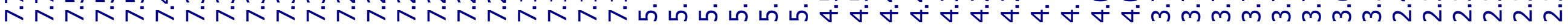

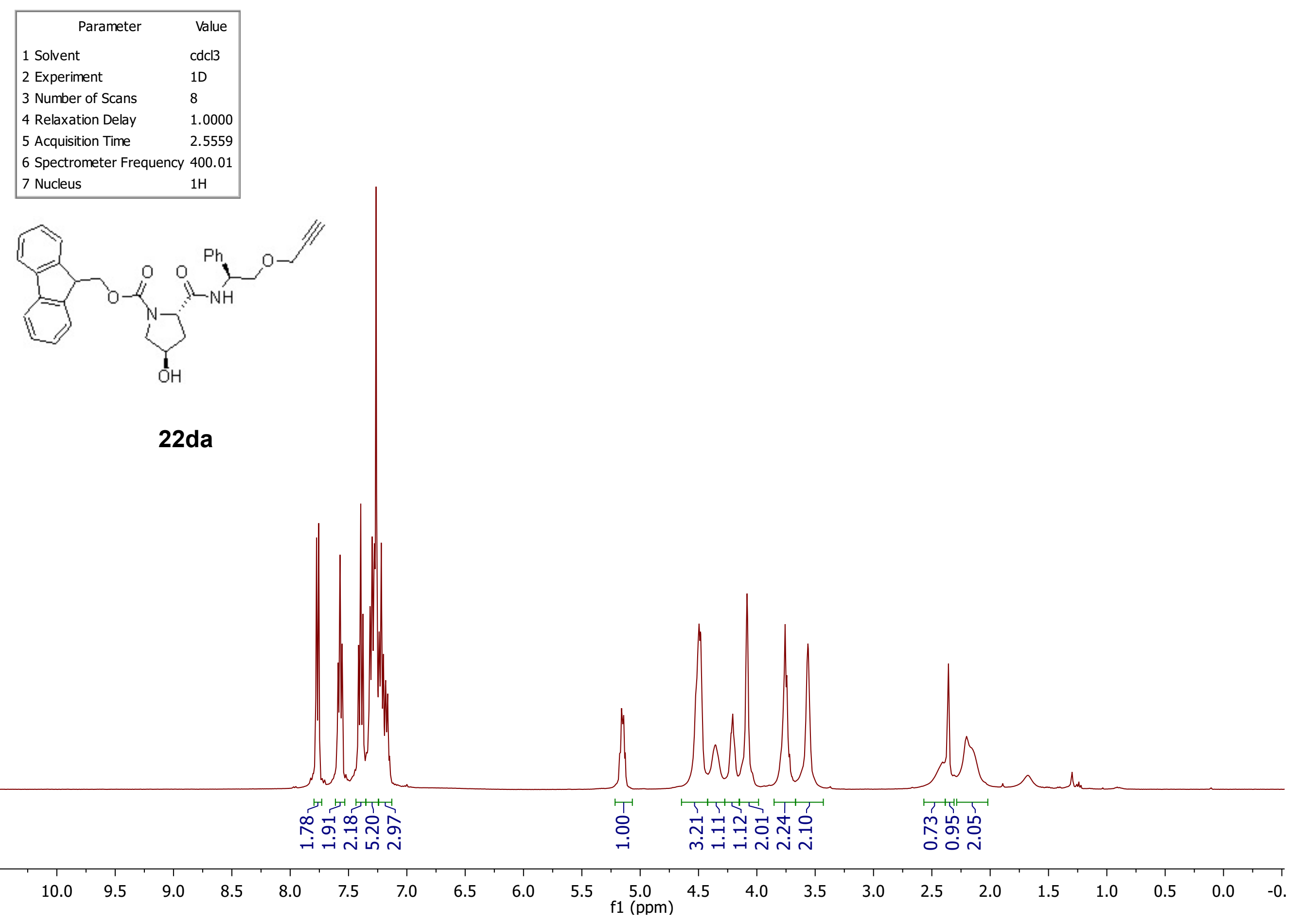




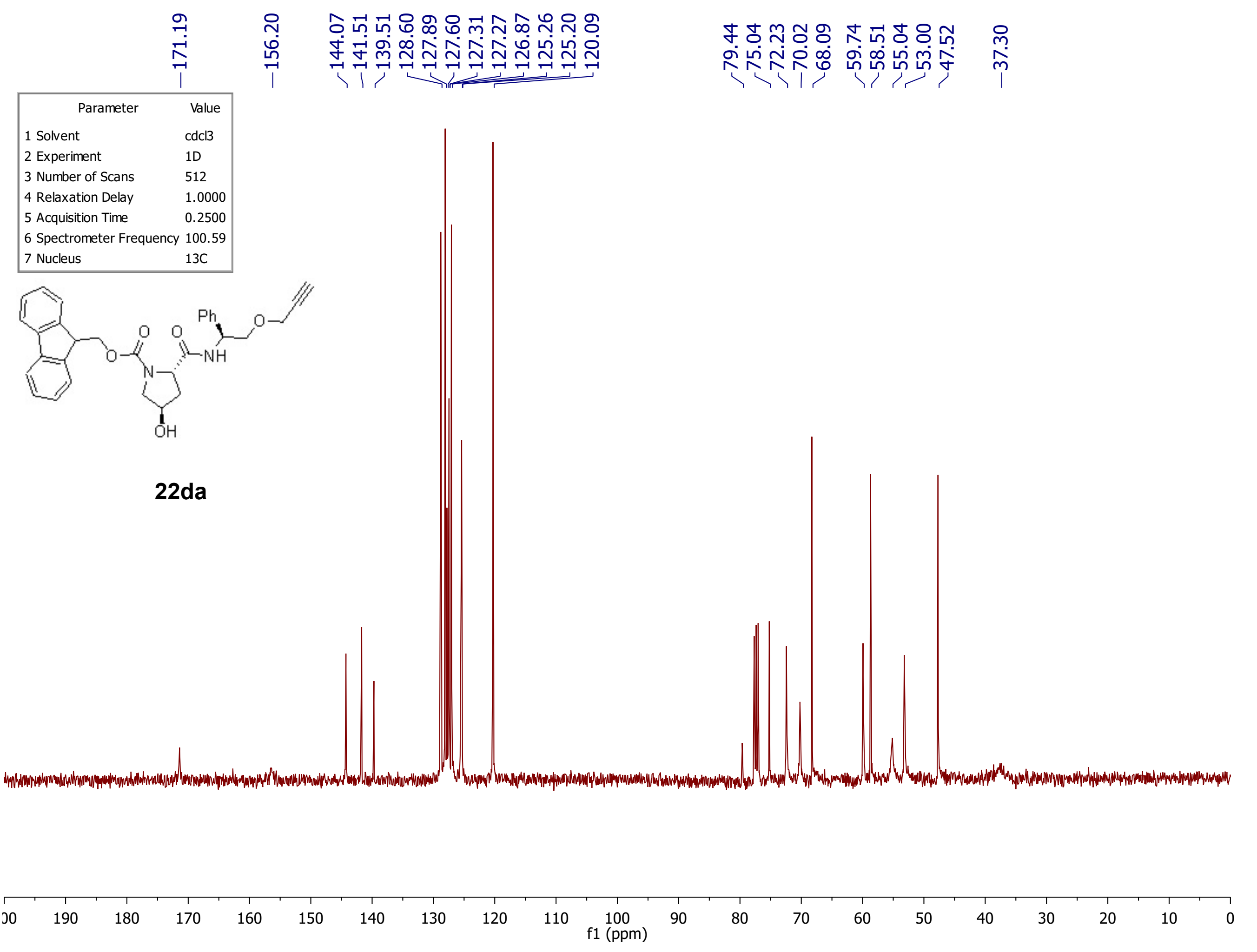

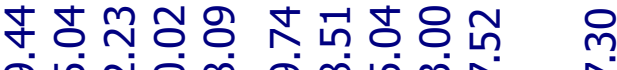

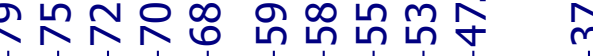




\begin{tabular}{|ll|}
\hline \multicolumn{1}{|c|}{ Parameter } & \multicolumn{1}{c|}{ Value } \\
cd Solvent & $1 \mathrm{Dod}$ \\
2 Experiment & $1 \mathrm{D}$ \\
3 Number of Scans & 8 \\
4 Relaxation Delay & 1.0000 \\
5 Acquisition Time & 2.0447 \\
6 Spectrometer Frequency & 499.81 \\
7 Nucleus & $1 \mathrm{H}$ \\
\hline
\end{tabular}

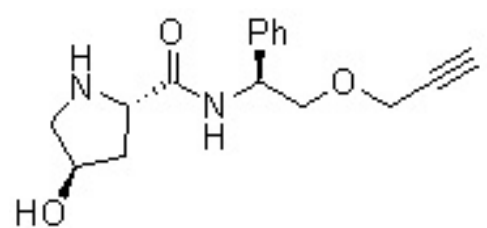

$22 \mathrm{db}$ '7

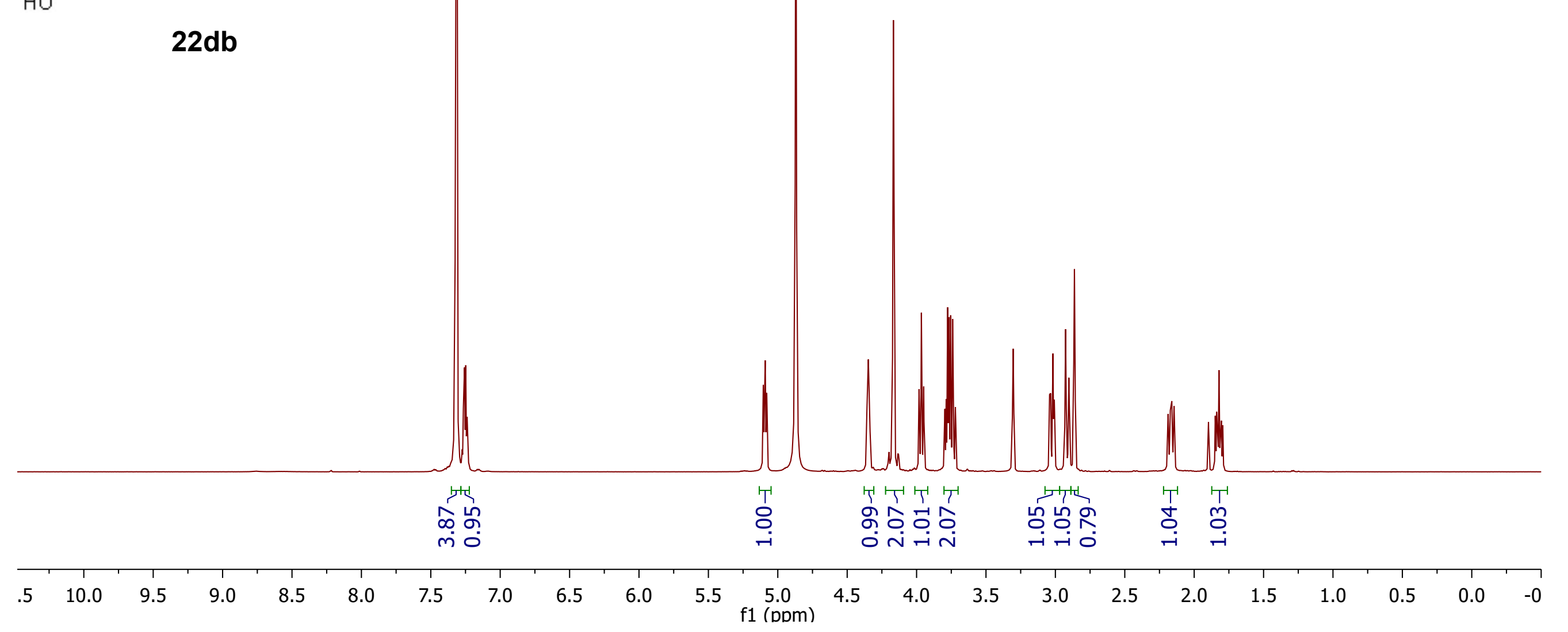




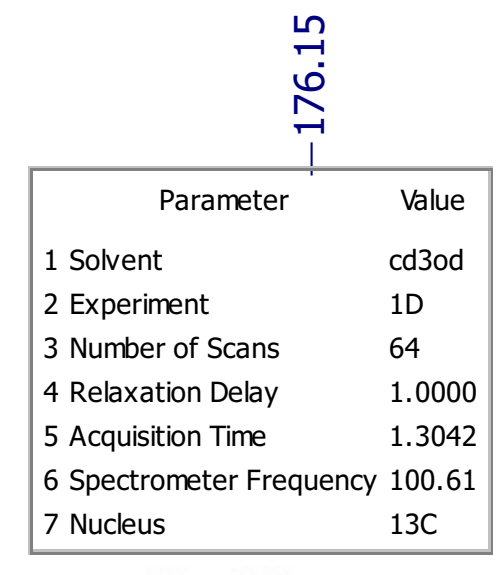

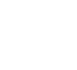

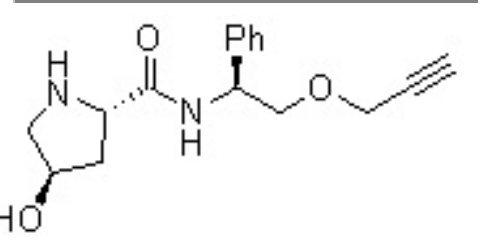

$22 d b$

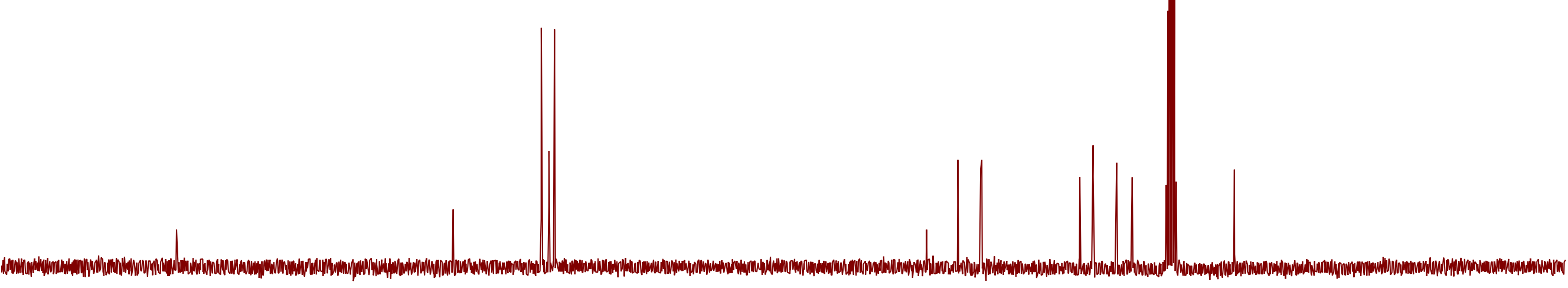

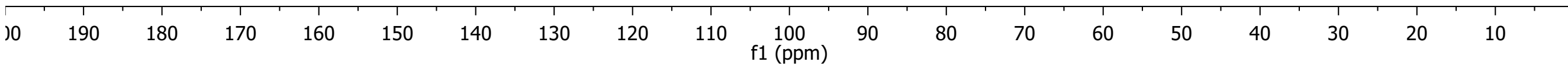




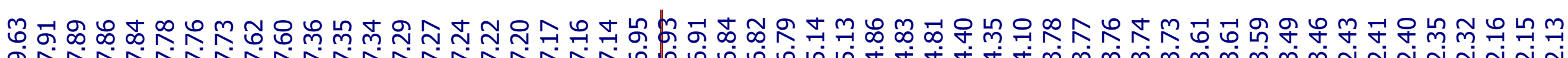

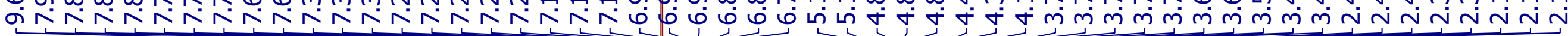

\begin{tabular}{|ll|}
\hline \multicolumn{1}{|c|}{ Parameter } & \multicolumn{1}{c|}{ Value } \\
1 Solvent & cdcl3 \\
2 Experiment & $1 \mathrm{D}$ \\
3 Number of Scans & 8 \\
4 Relaxation Delay & 1.0000 \\
5 Acquisition Time & 2.5592 \\
6 Spectrometer Frequency & 400.09 \\
7 Nucleus & $1 \mathrm{H}$ \\
\hline
\end{tabular}
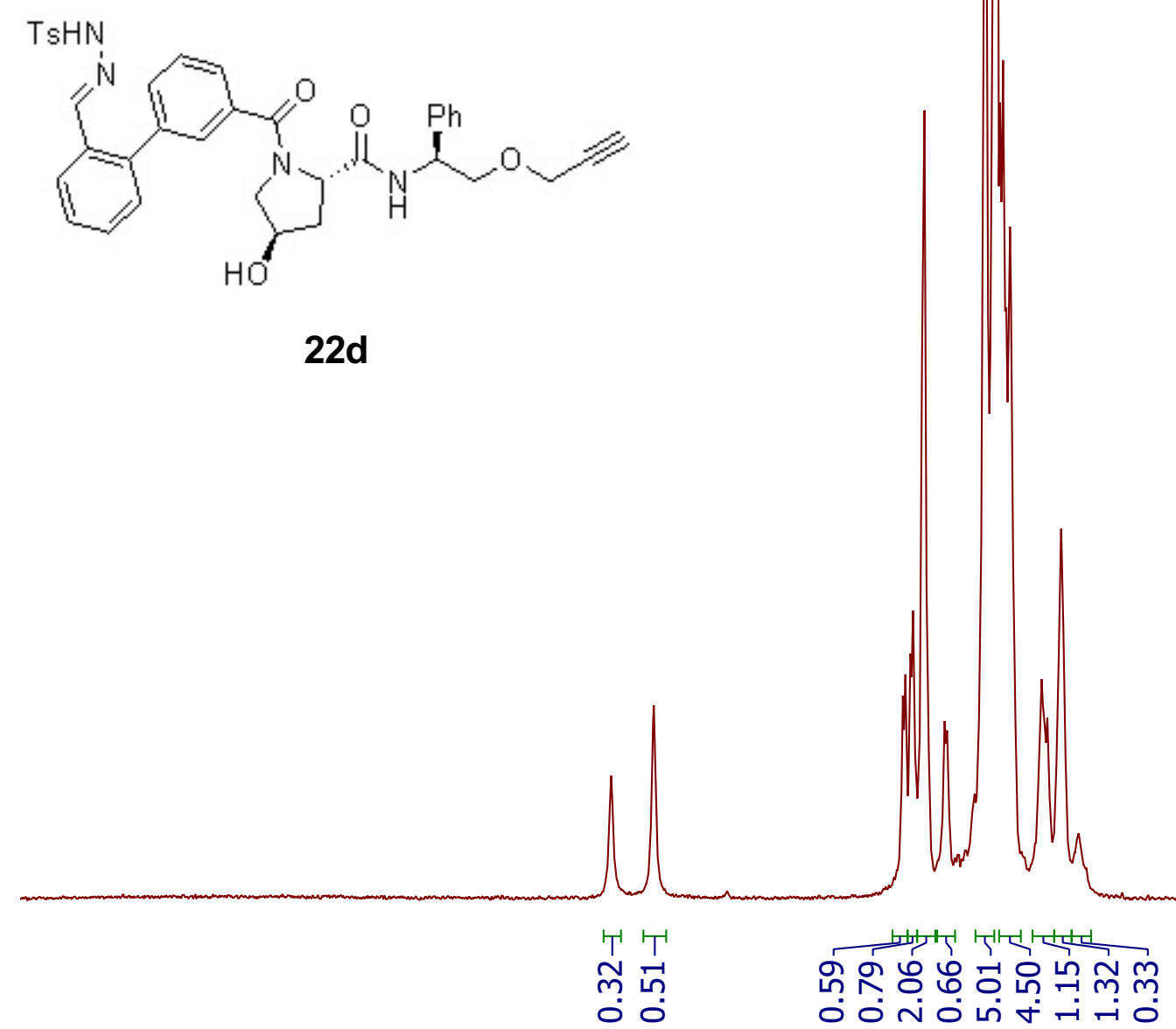

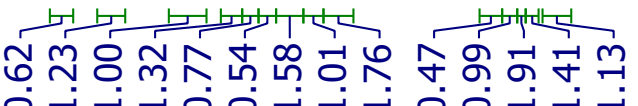

0

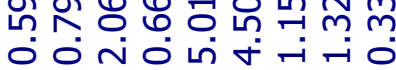

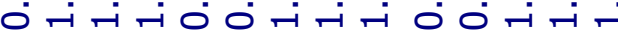




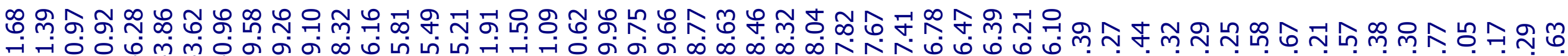

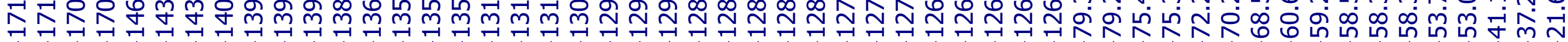

\begin{tabular}{|ll|}
\hline \multicolumn{1}{|c|}{ Parameter } & \multicolumn{1}{c|}{ Value } \\
1 Solvent & cdcl3 \\
2 Experiment & $1 \mathrm{D}$ \\
3 Number of Scans & 10000 \\
4 Relaxation Delay & 1.0000 \\
5 Acquisition Time & 1.3107 \\
6 Spectrometer Frequency & 100.59 \\
7 Nucleus & $13 \mathrm{C}$ \\
\hline
\end{tabular}

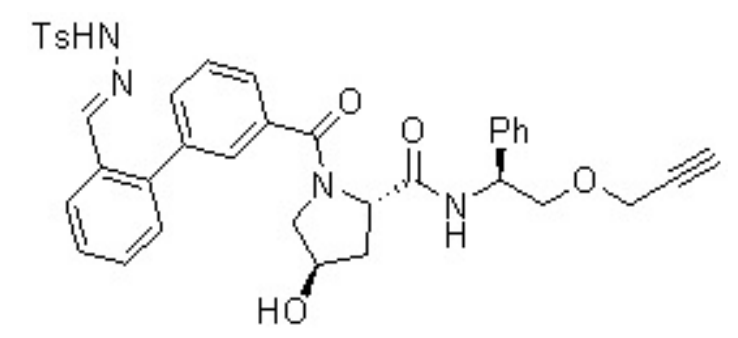

22d

(1)<smiles>C1CCCC1</smiles>

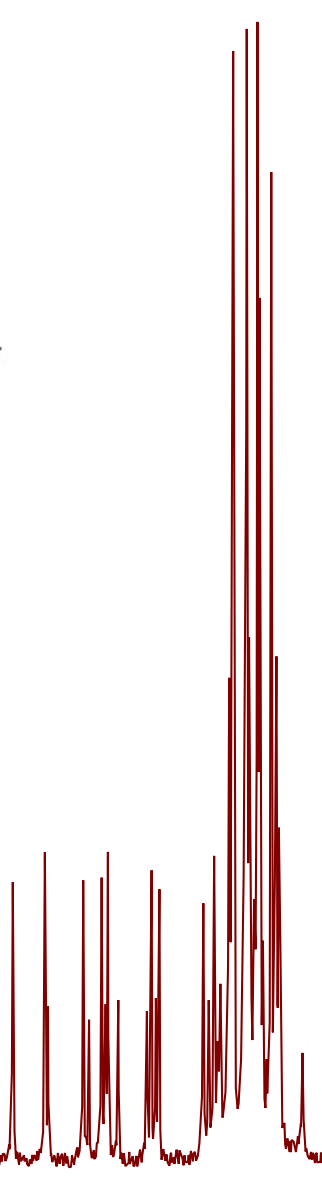

$90 \quad 180$

170

140

130

120

110

100
$\mathrm{f} 1(\mathrm{ppm})$

$90 \quad 80$

70

60

50

1
40

$40 \quad 30$

$30 \quad 20$




\begin{tabular}{|ll|}
\hline \multicolumn{1}{|c|}{ Parameter } & \multicolumn{1}{c|}{ Value } \\
1 Solvent & cdcl3 \\
2 Experiment & $1 \mathrm{D}$ \\
3 Number of Scans & 8 \\
4 Relaxation Delay & 1.0000 \\
5 Acquisition Time & 2.5559 \\
6 Spectrometer Frequency & 400.01 \\
7 Nucleus & $1 \mathrm{H}$ \\
\hline
\end{tabular}

TsHN

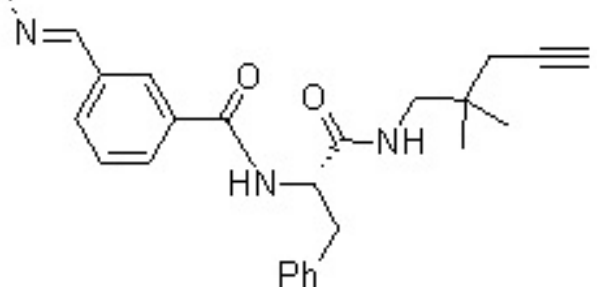

$25 \mathrm{a}$

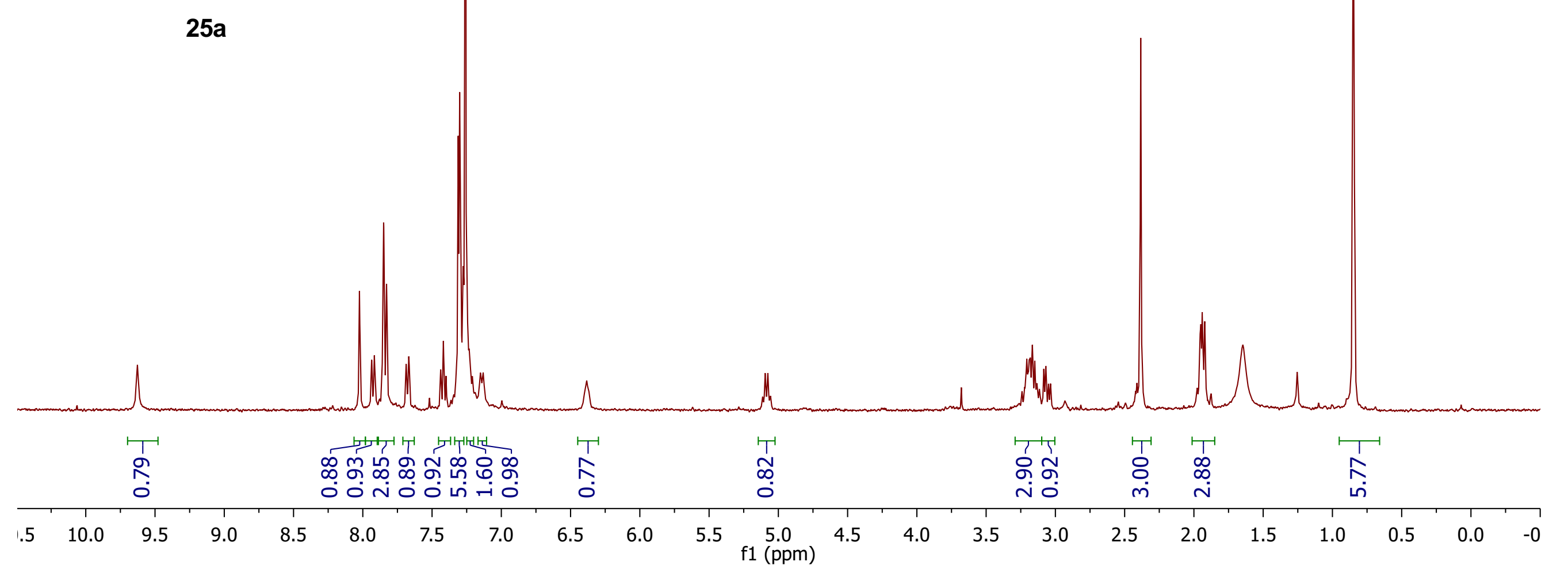




\begin{tabular}{|ll|}
\hline \multicolumn{1}{|c|}{ Parameter } & \multicolumn{1}{c|}{ Value } \\
1 Solvent & cdcl3 \\
2 Experiment & $1 \mathrm{D}$ \\
3 Number of Scans & 32 \\
4 Relaxation Delay & 1.0000 \\
5 Acquisition Time & 1.3042 \\
6 Spectrometer Frequency & 100.61 \\
7 Nucleus & $13 \mathrm{C}$ \\
\hline
\end{tabular}
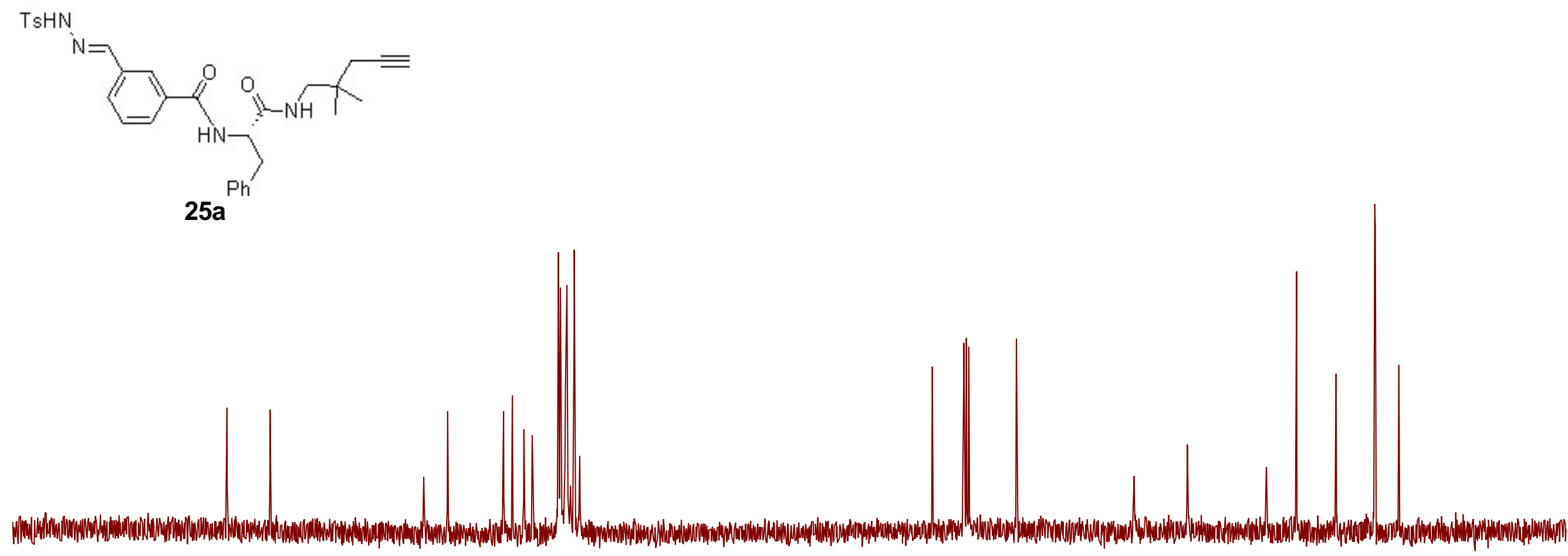

160

150

140

130

120

110

100

90

80

70

60

50

40

30

20 
ద

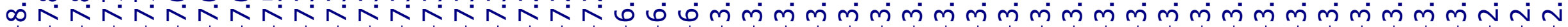

\begin{tabular}{|ll|}
\hline \multicolumn{1}{|c|}{ Parameter } & \multicolumn{1}{c|}{ Value } \\
1 Solvent & cdcl3 \\
2 Experiment & $1 \mathrm{D}$ \\
3 Number of Scans & 8 \\
4 Relaxation Delay & 1.0000 \\
5 Acquisition Time & 2.5592 \\
6 Spectrometer Frequency & 400.09 \\
7 Nucleus & $1 \mathrm{H}$ \\
\hline
\end{tabular}

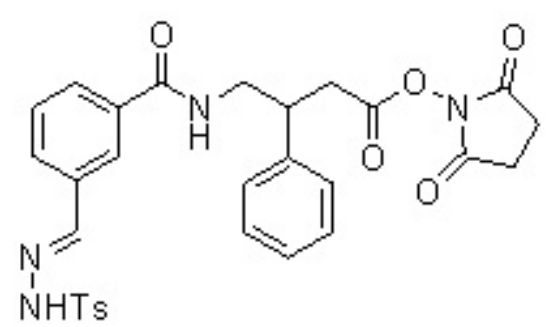

\section{$25 \mathrm{ba}$}
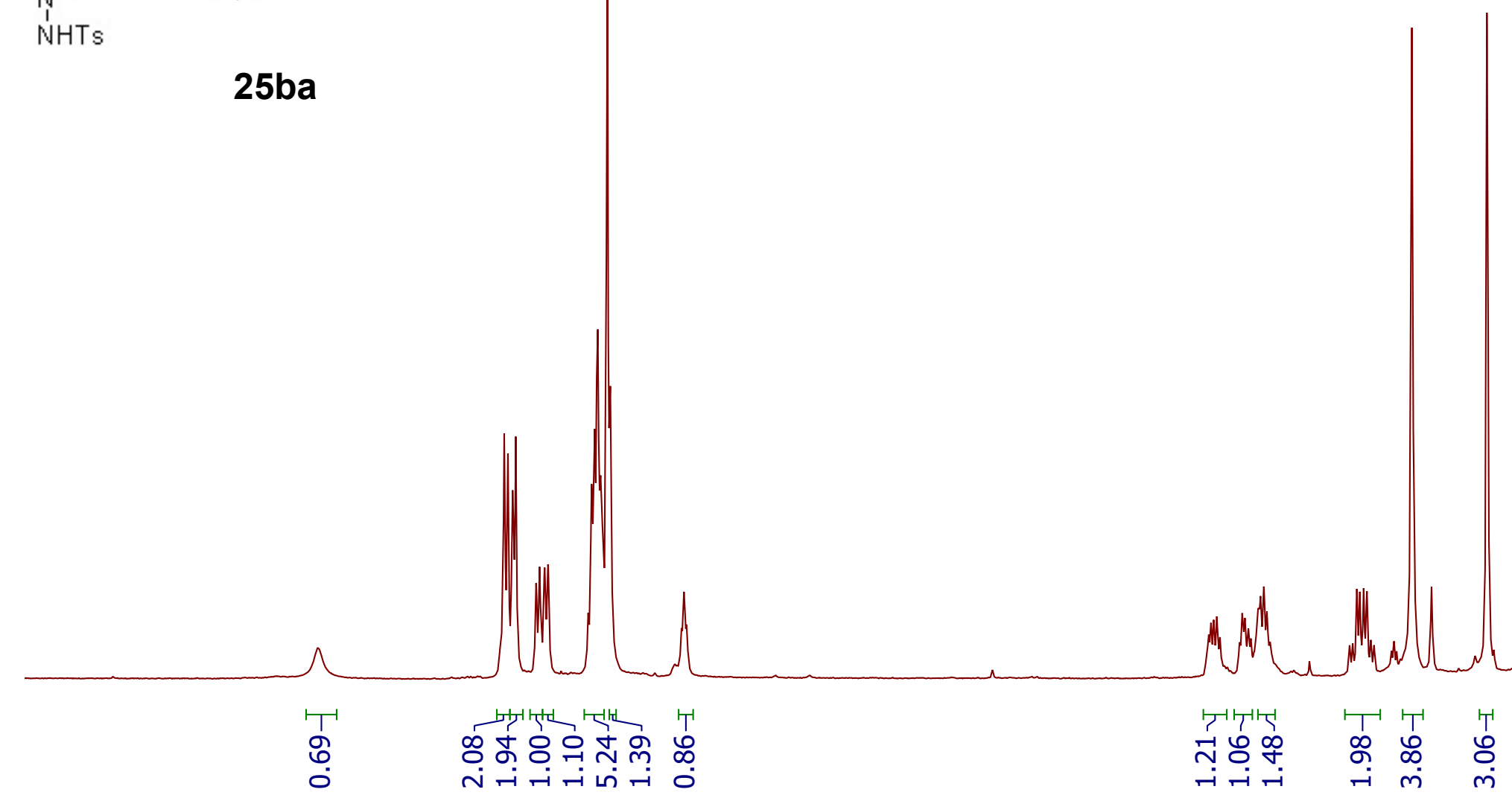

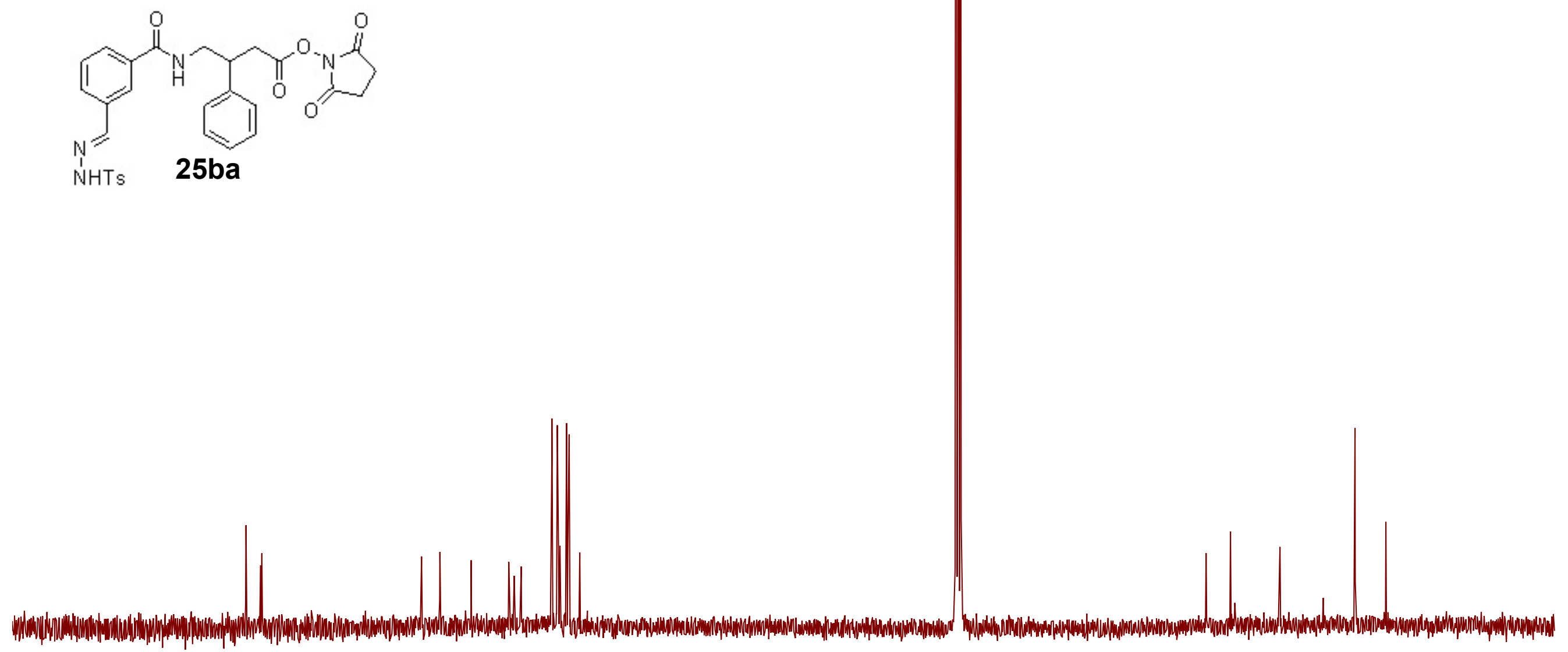

\begin{tabular}{|c|c|c|c|c|c|c|c|c|c|c|c|c|c|c|c|c|c|c|}
\hline 190 & 180 & 170 & 160 & 150 & 140 & 130 & 120 & 110 & $\begin{array}{c}100 \\
\mathrm{f} 1(\mathrm{ppm})\end{array}$ & 90 & 80 & 70 & 60 & 50 & 40 & 30 & 20 & 10 \\
\hline
\end{tabular}




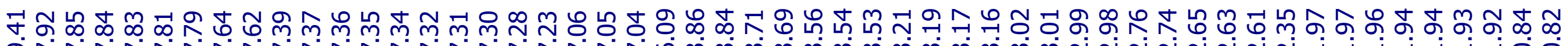

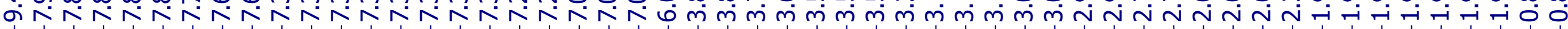

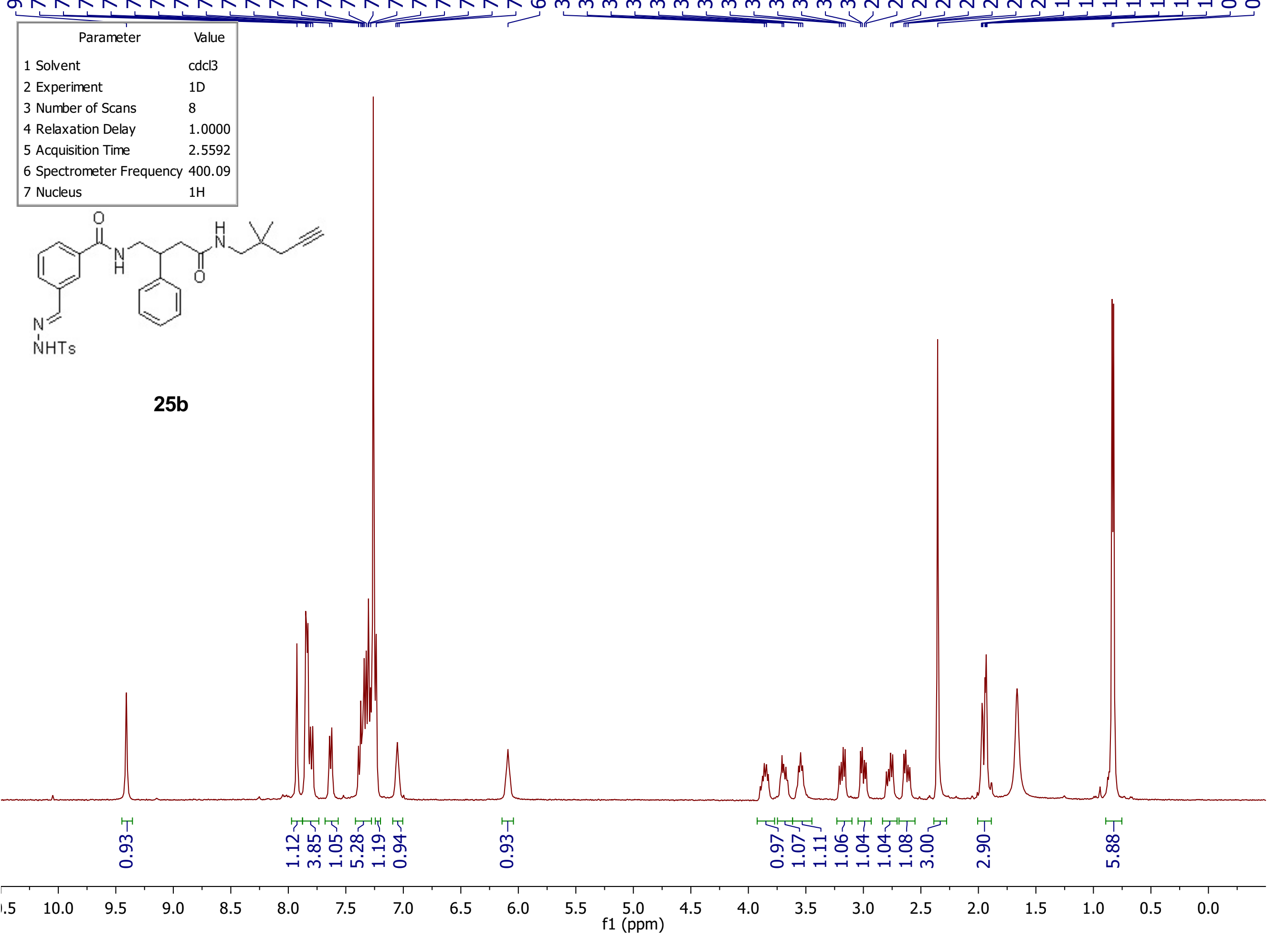


\&

손

Parameter

value

1 Solvent

cdcl3

2 Experiment

3 Number of Scans

4 Relaxation Delay

5 Acquisition Time

6 Spectrometer Frequency 100.61

7 Nucleus $13 \mathrm{C}$

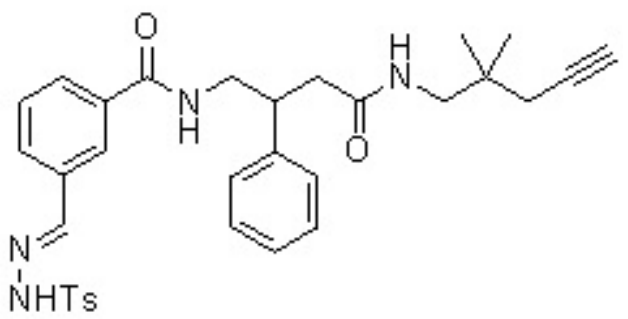

$25 b$
ホ๐ ナ

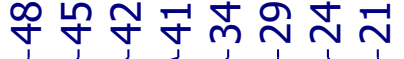

$1 / 1)$

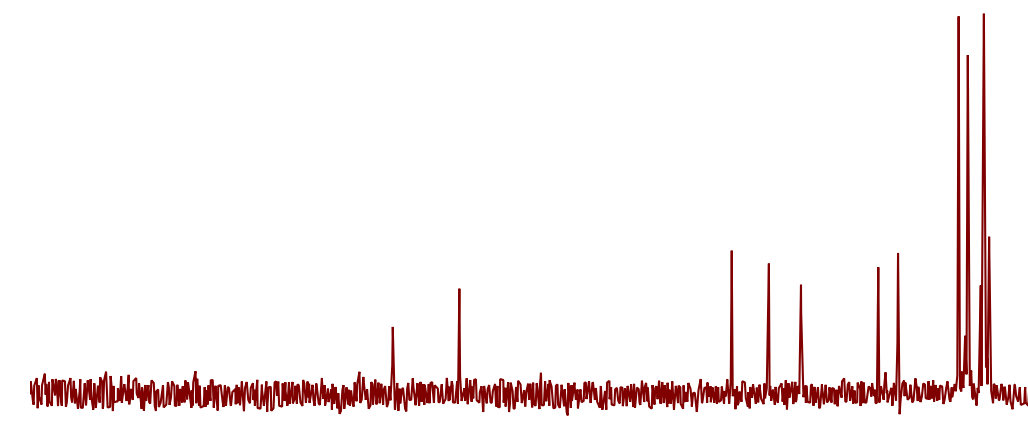

130




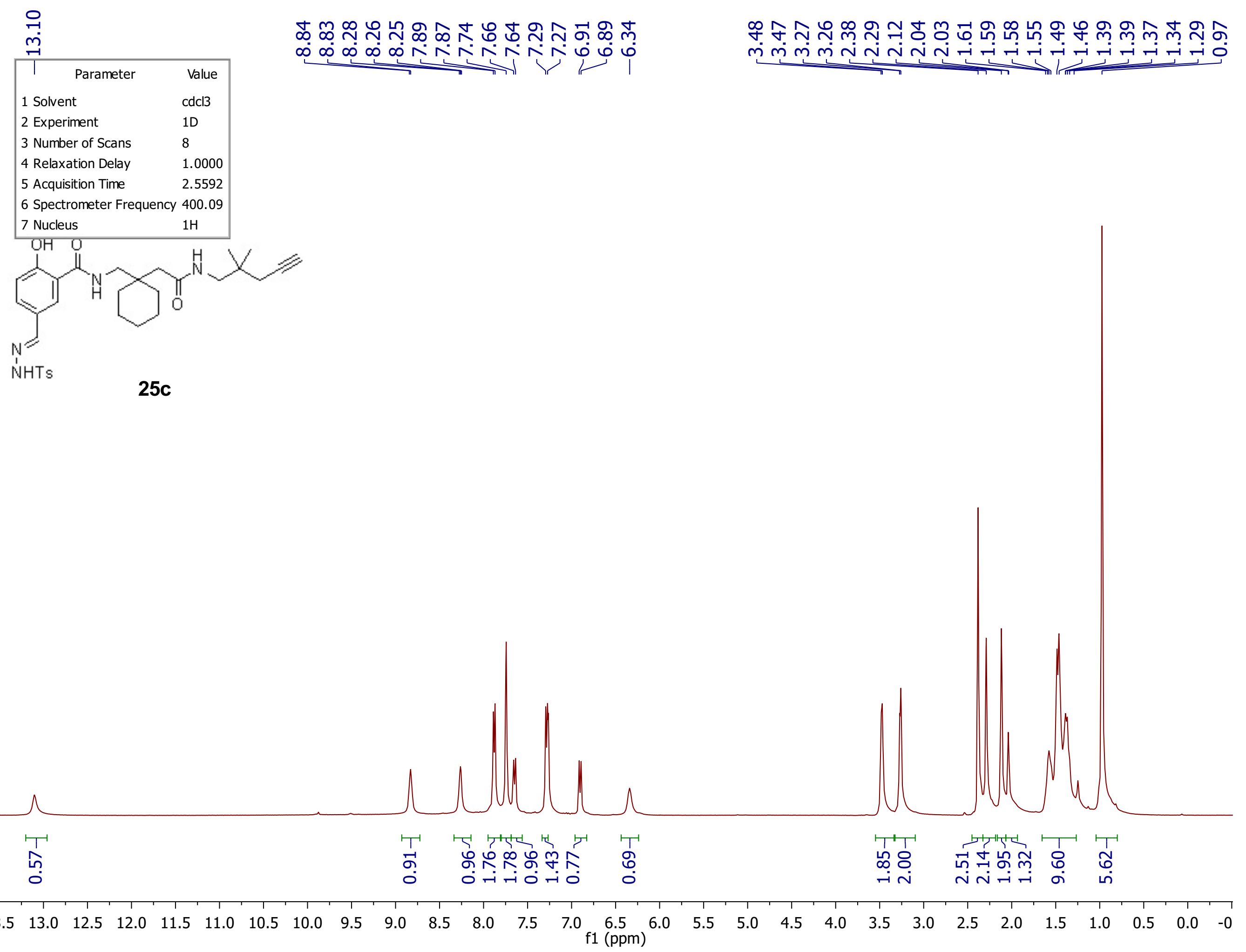




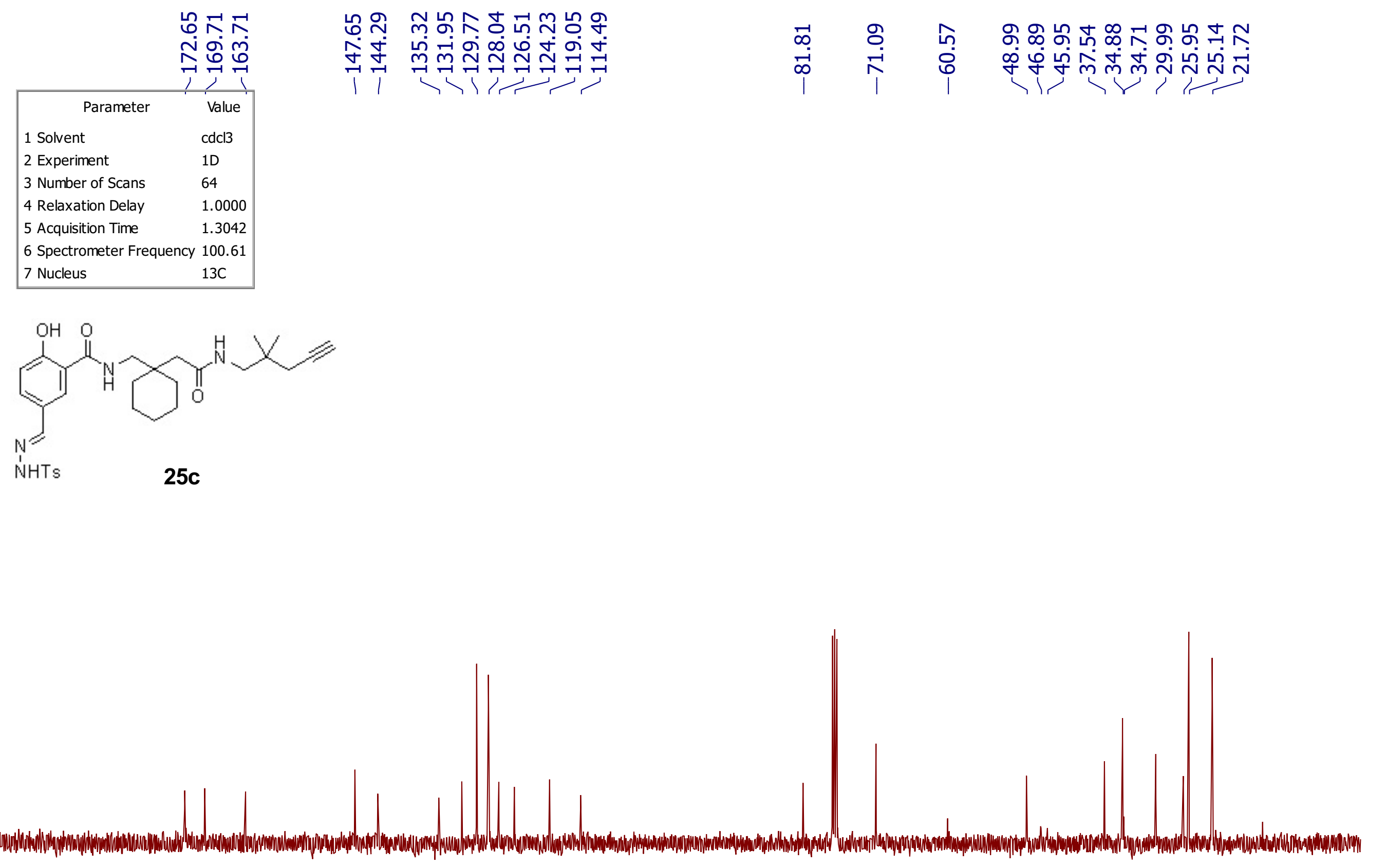

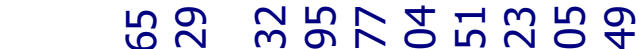
乎 离 cdcl3

1.0000

6 Spectrometer Frequency 100.61

7 Nucleus $\quad 13 \mathrm{C}$

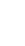

$190 \quad 180$

170

160

150

140

130

110

f1 100

$90 \quad 80$

70

60

50

40

20 


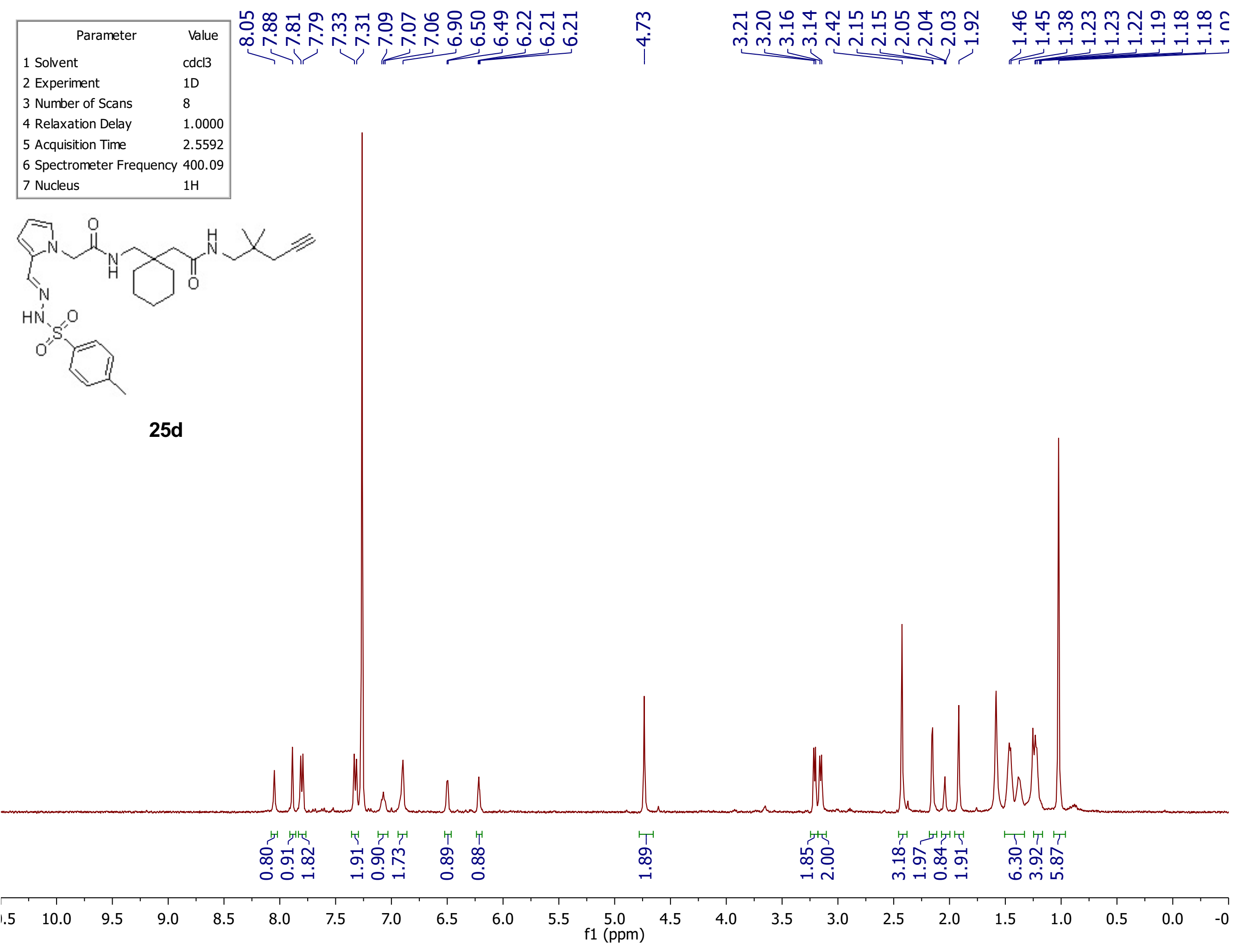


ஜ

तं

가

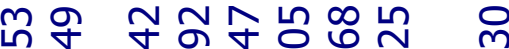

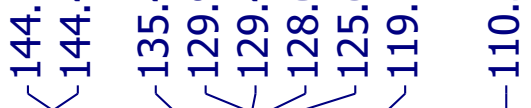

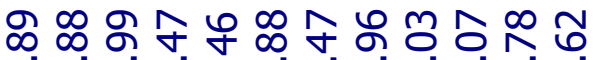

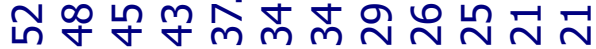

\begin{tabular}{|ll|}
\hline \multicolumn{1}{|c}{ Parameter } & \multicolumn{1}{c|}{ Value } \\
1 Solvent & cdcl3 \\
2 Experiment & $1 \mathrm{D}$ \\
3 Number of Scans & 16000 \\
4 Relaxation Delay & 1.0000 \\
5 Acquisition Time & 1.3042 \\
6 Spectrometer Frequency & 100.61 \\
7 Nucleus & $13 \mathrm{C}$ \\
\hline
\end{tabular}<smiles>C#CCC(C)(C)CNC(=O)CC1(CNC(=O)Cn2cccc2/C=N\C)CCCCC1</smiles>

$\mathrm{HN}, \mathrm{O}$

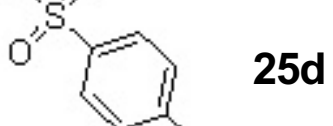

25d 


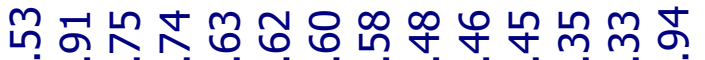

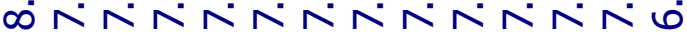

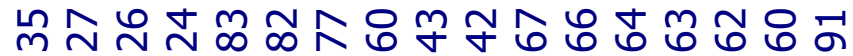
mmmmnNNNNintititio

\begin{tabular}{|ll|}
\hline \multicolumn{1}{|c|}{ Parameter } & \multicolumn{1}{c|}{ Value } \\
1 Solvent & CDCl3/CD3OD $=1: 1$ \\
2 Experiment & $1 \mathrm{D}$ \\
3 Number of Scans & 8 \\
4 Relaxation Delay & 1.0000 \\
5 Acquisition Time & 2.0447 \\
6 Spectrometer Frequency & 499.81 \\
7 Nucleus & $1 \mathrm{H}$ \\
\hline
\end{tabular}
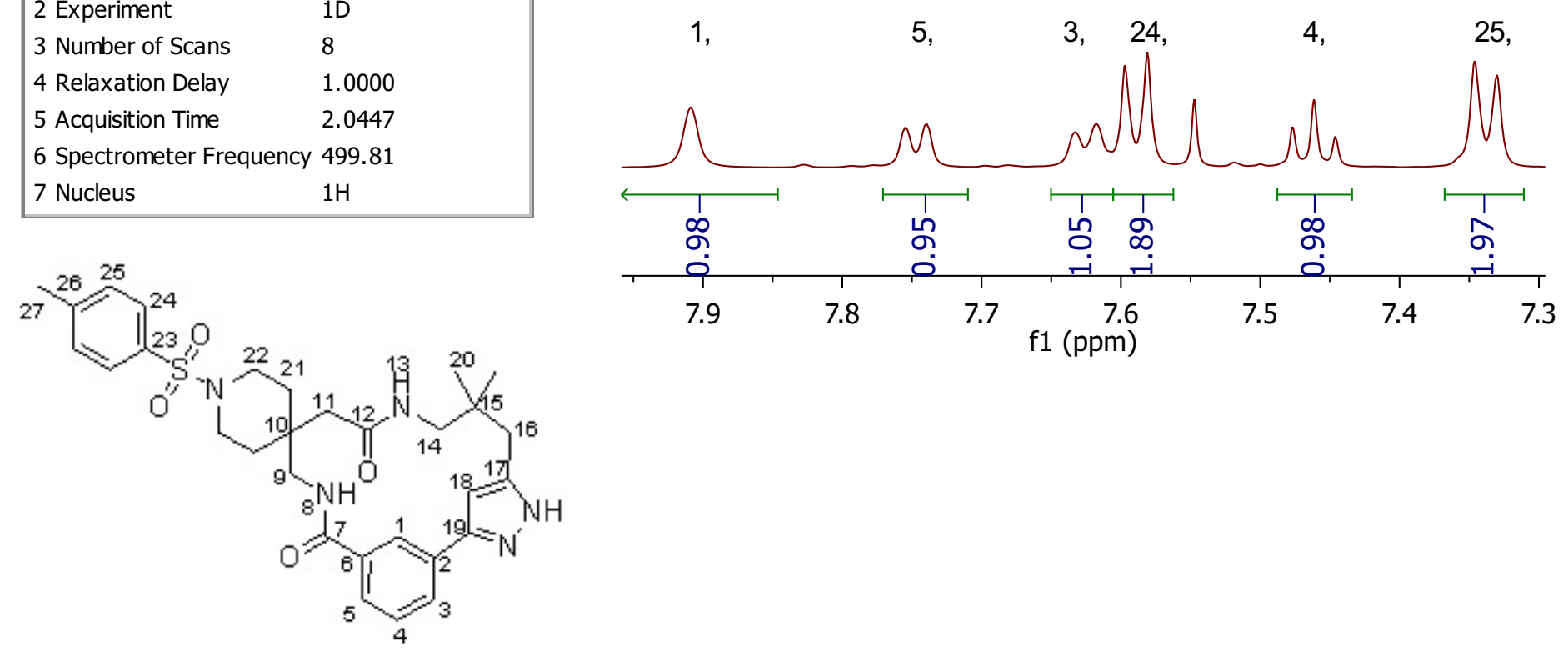

\section{$23 a$}

$5, \quad 1, \quad 25, \quad 18$,

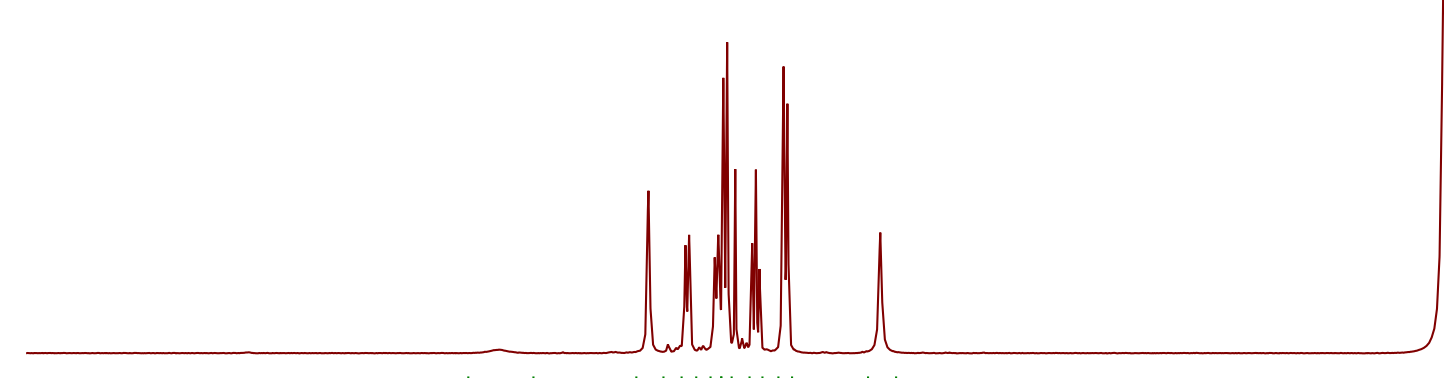

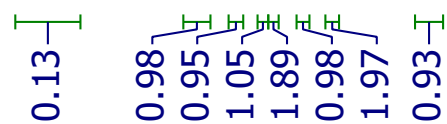

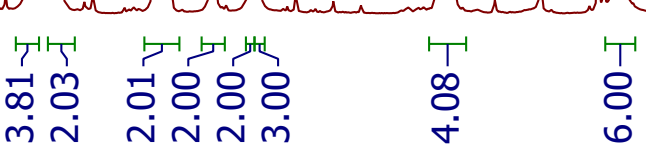

$8.5 \quad 8.0$

$\begin{array}{lll}7.5 & 7.0 & 6.5\end{array}$

$6.0 \quad 5.5$

5.0

4.5

1




\begin{tabular}{|ll|}
\hline \multicolumn{1}{|c|}{ Parameter } & \multicolumn{1}{c|}{ Value } \\
1 Solvent & CDCl3/CD3OD=1:1 \\
2 Experiment & $1 \mathrm{D}$ \\
3 Number of Scans & 2000 \\
4 Relaxation Delay & 1.0000 \\
5 Acquisition Time & 1.0486 \\
6 Spectrometer Frequency 125.69 \\
7 Nucleus & $13 \mathrm{C}$ \\
\hline
\end{tabular}

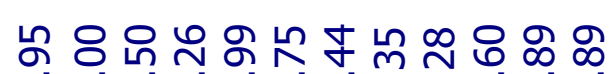

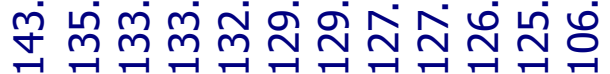

雨

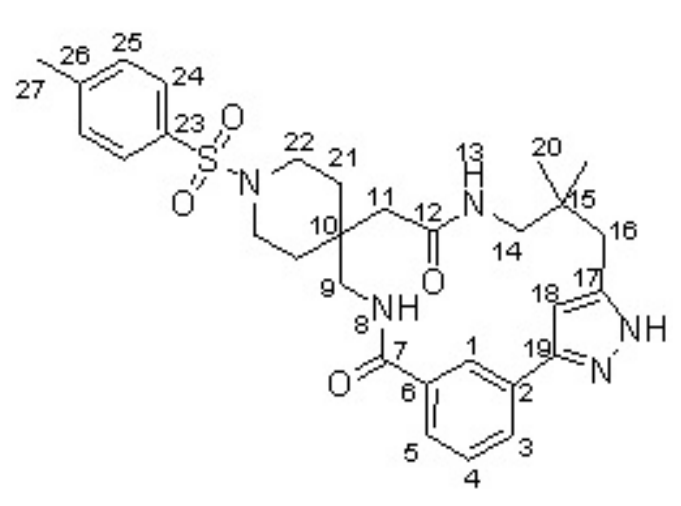

23a

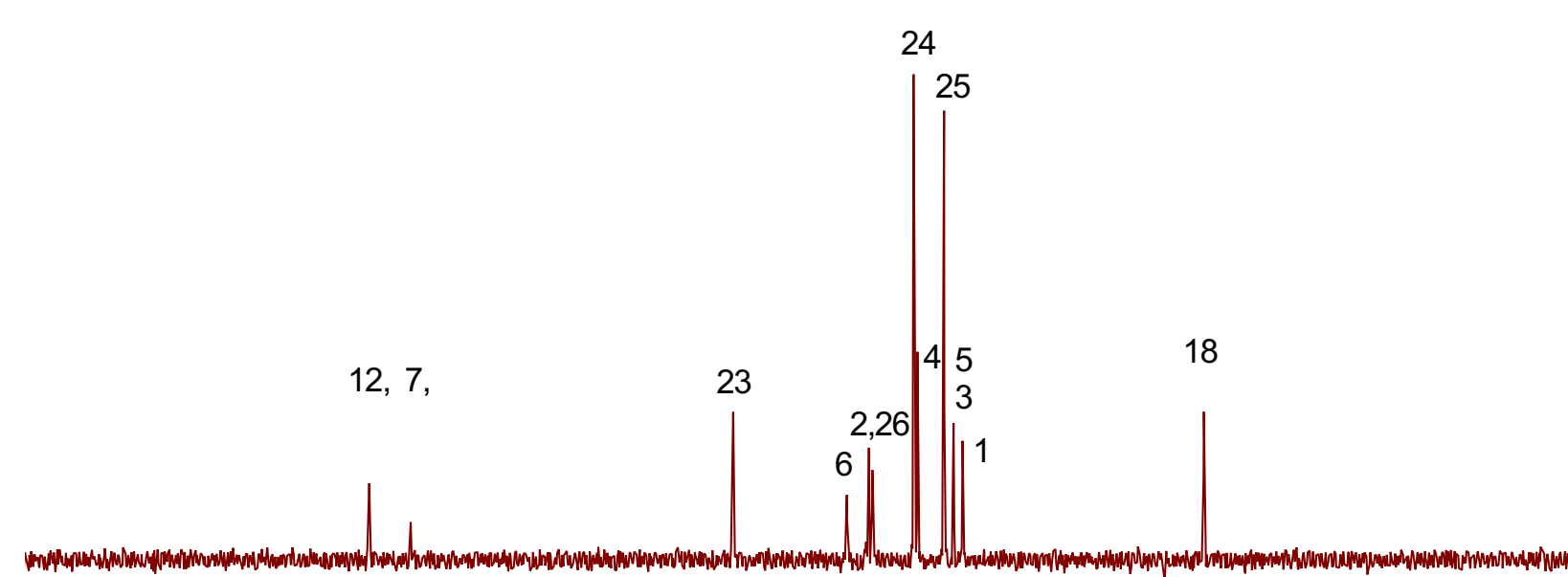

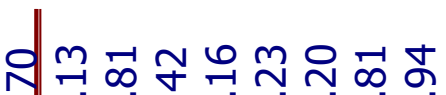

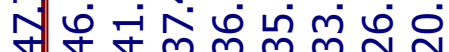
र गिm

22

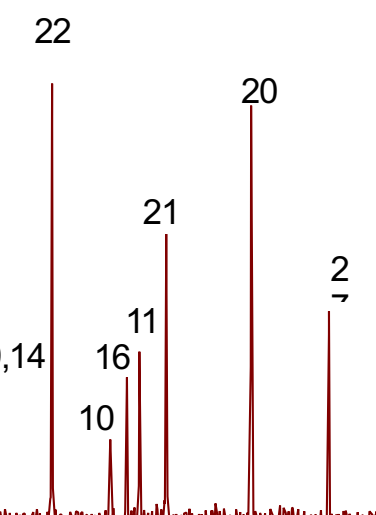

80




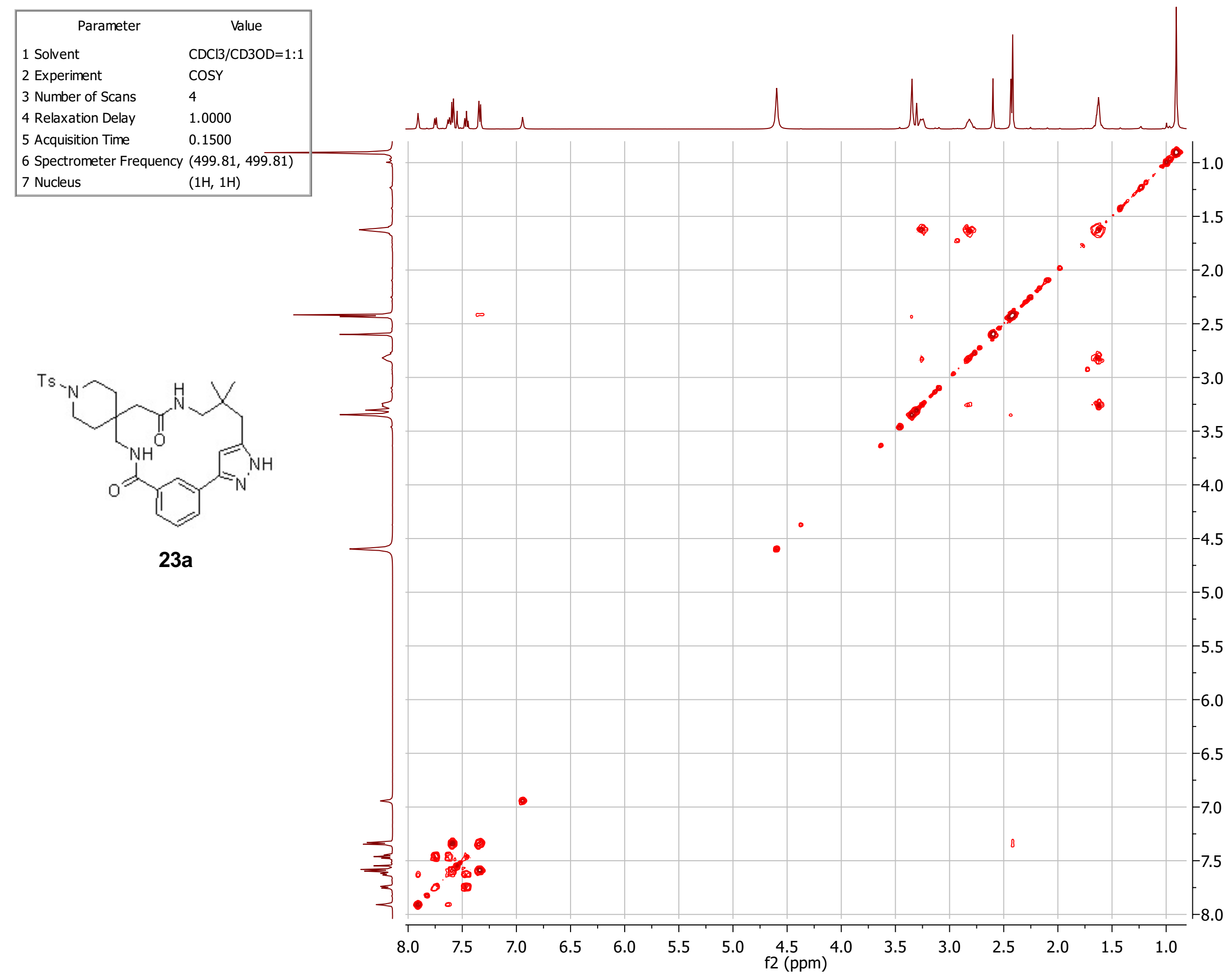




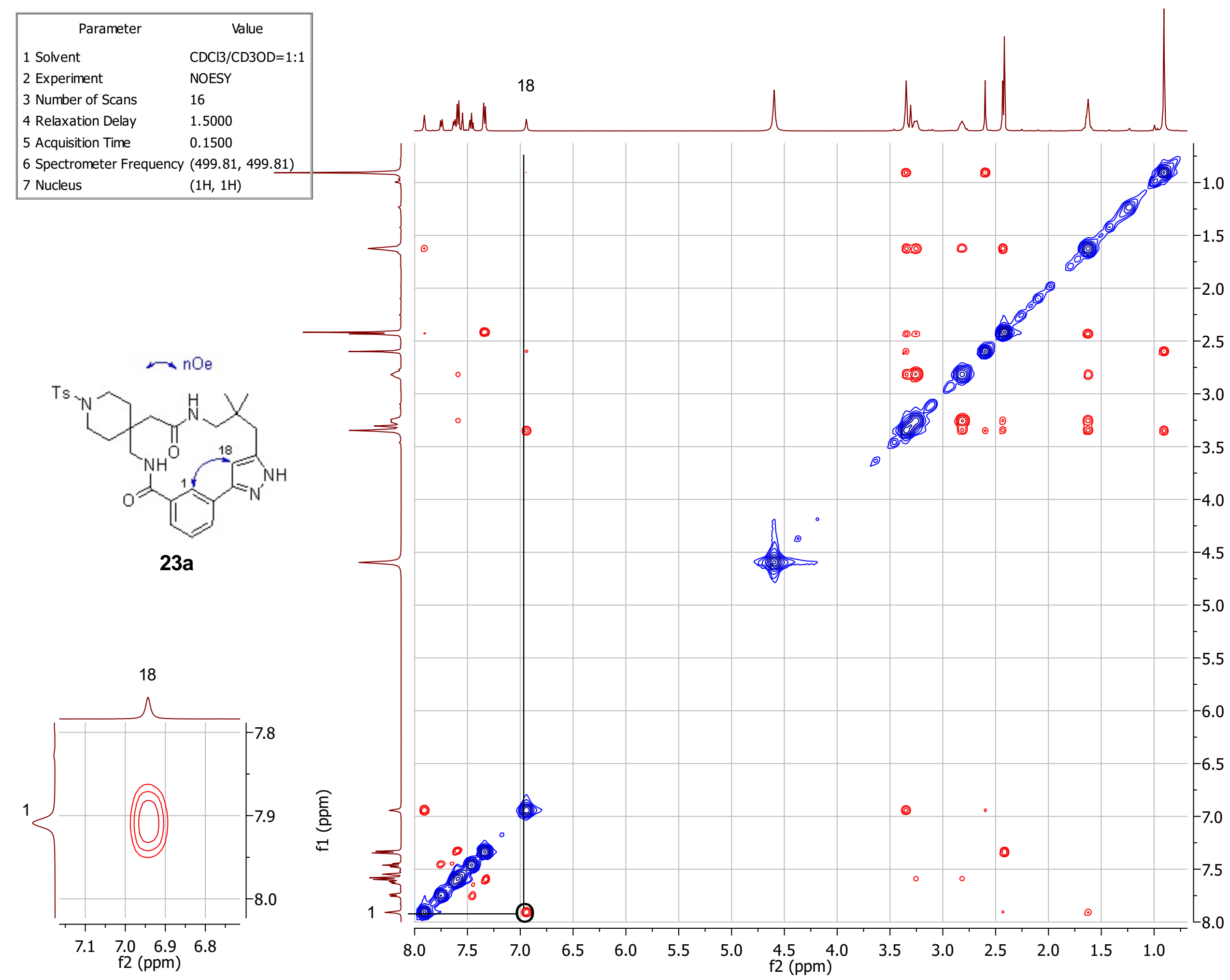

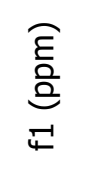




\begin{tabular}{|ll|}
\hline \multicolumn{1}{|c|}{ Parameter } & \multicolumn{1}{c|}{ Value } \\
1 Solvent & cd3od \\
2 Experiment & $1 \mathrm{D}$ \\
3 Number of Scans & 8 \\
4 Relaxation Delay & 1.0000 \\
5 Acquisition Time & 2.5559 \\
6 Spectrometer Frequency & 400.02 \\
7 Nucleus & $1 \mathrm{H}$ \\
\hline
\end{tabular}
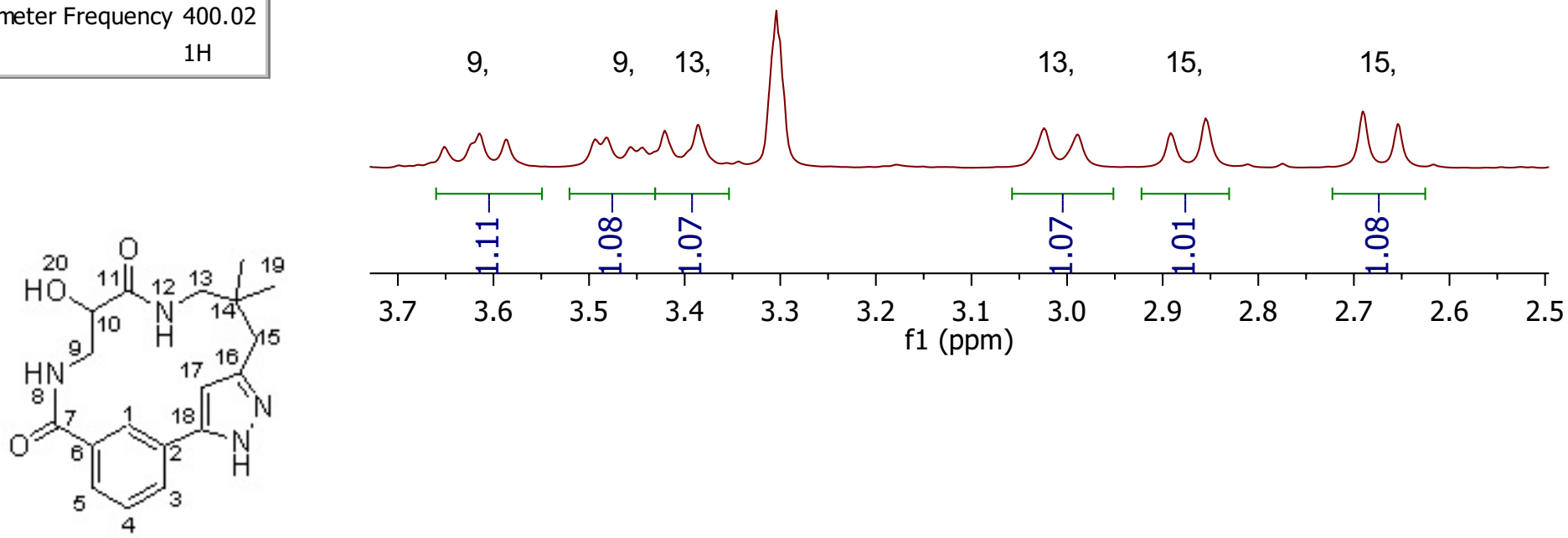

$23 b$

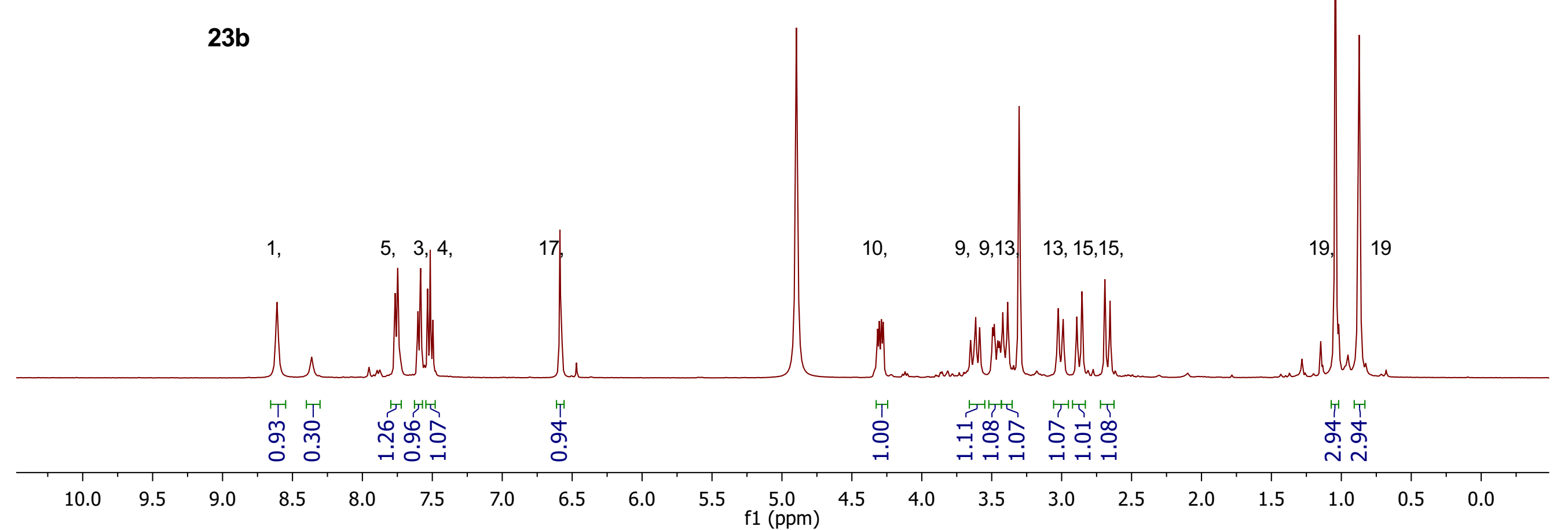




\begin{tabular}{|ll|}
\hline \multicolumn{1}{|c|}{ Parameter } & \multicolumn{1}{c|}{ Value } \\
1 Solvent & cd3od \\
2 Experiment & 1D \\
3 Number of Scans & 18500 \\
4 Relaxation Delay & 1.0000 \\
5 Acquisition Time & 1.3042 \\
6 Spectrometer Frequency & 100.61 \\
7 Nucleus & $13 \mathrm{C}$ \\
\hline
\end{tabular}

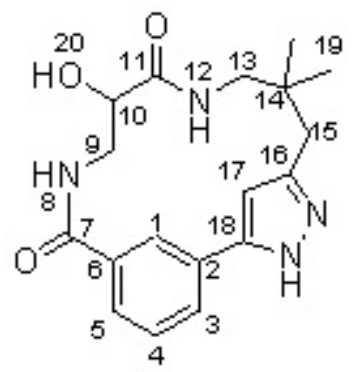

23b

11,7

18, 16,

$1,4,5$,

17 ,

10

13 ,

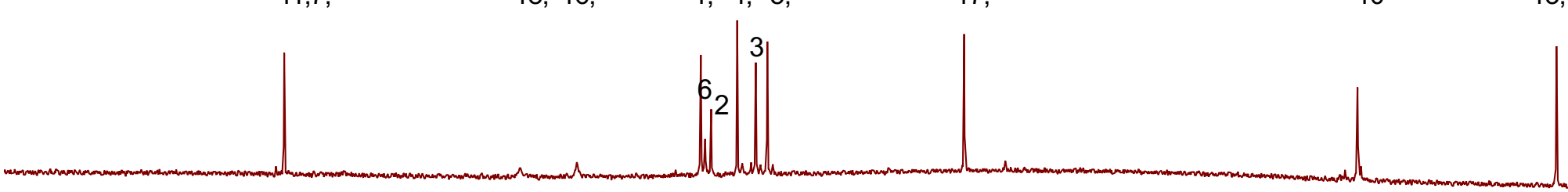

$9, \quad 15,14, \quad 19,19$

$90 \quad 80$

$80 \quad 70$

$\begin{array}{lll}1 & 1 \\ 70 & 60 & 50\end{array}$

$50 \quad 40$




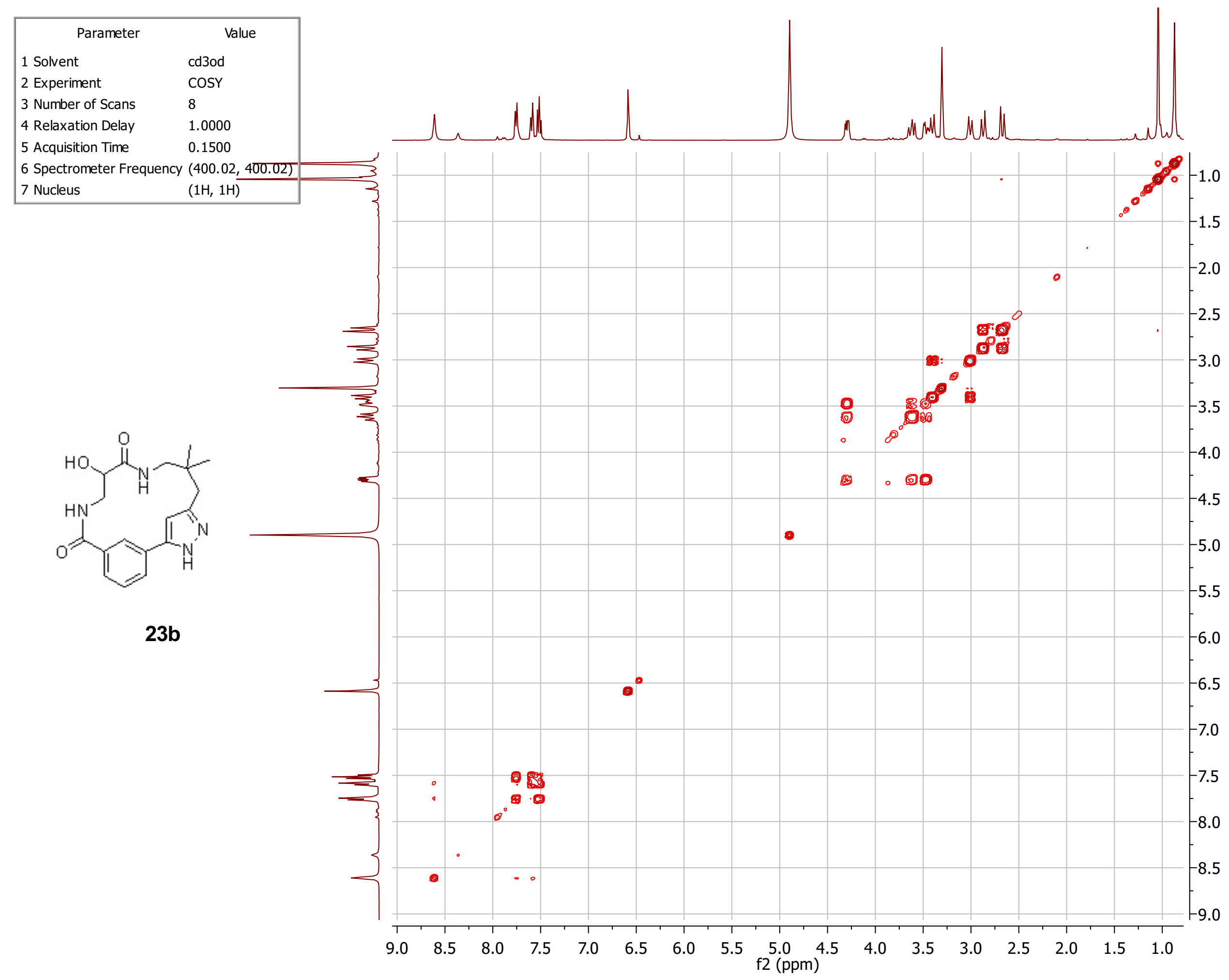

है
는 


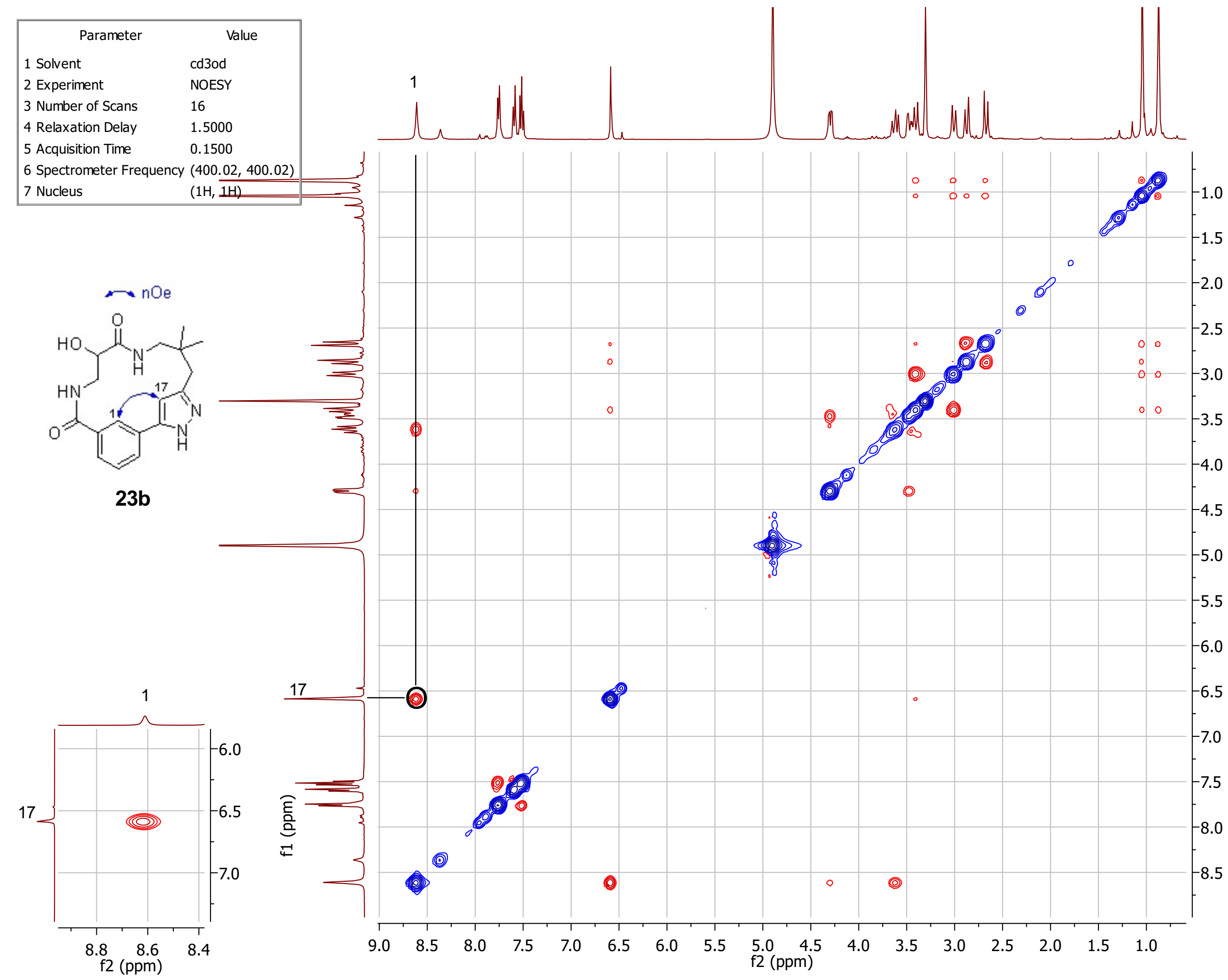

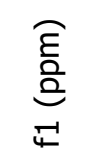




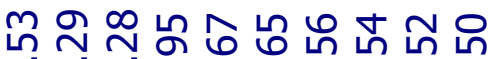

$\infty \infty \infty N N$ N

\begin{tabular}{|ll|}
\hline \multicolumn{1}{|c}{ Parameter } & \multicolumn{1}{c}{ Value } \\
1 Solvent & cd3od \\
2 Experiment & $1 \mathrm{D}$ \\
3 Number of Scans & 8 \\
4 Relaxation Delay & 1.0000 \\
5 Acquisition Time & 2.5559 \\
6 Spectrometer Frequency & 400.02 \\
7 Nucleus & $1 \mathrm{H}$ \\
\hline
\end{tabular}

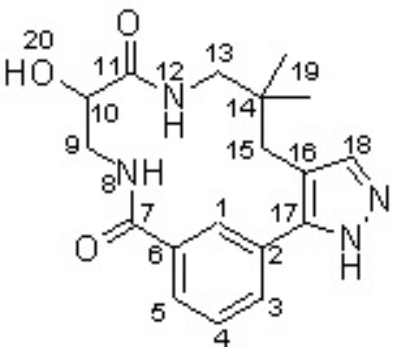

$24 b$

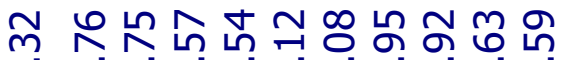

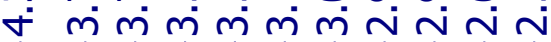

훙ำ

$\longrightarrow<$

19

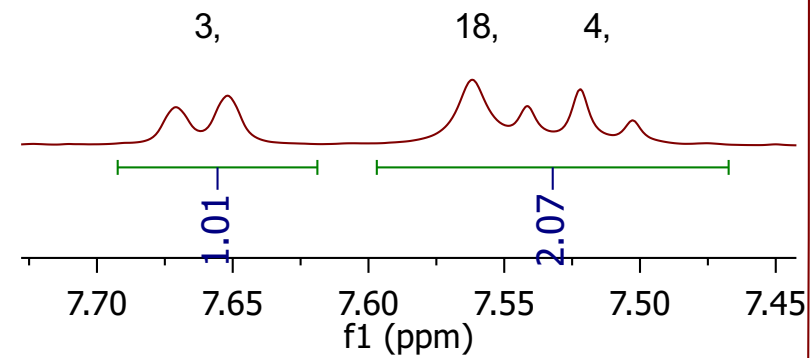

$1, \quad 5, \quad 3,18,4$

\section{एस मा 年些}

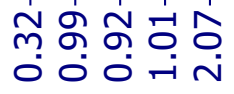

10. $9, \quad 13, \quad 15,13,15$

$15,13,15$,

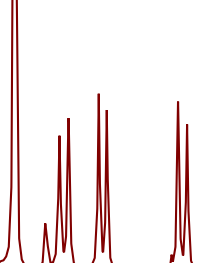

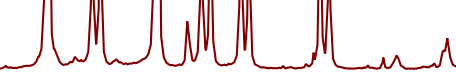

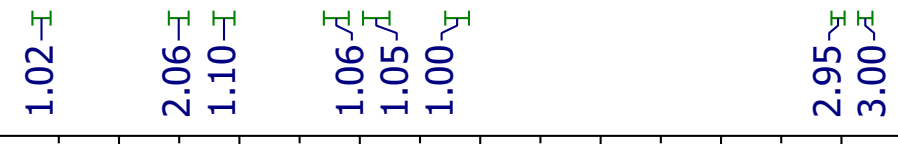

$9.5 \quad 9.0$

8.5

8.0

$\begin{array}{llllll}7.5 & 7.0 & 6.5 & 6.0 & 5.5 & \begin{array}{l}5.0 \\ \mathrm{f} 1(\mathrm{ppm})\end{array}\end{array}$




\begin{tabular}{|c|c|}
\hline Parameter & Value \\
\hline 1 Solvent & \\
\hline 2 Experiment & $1 \mathrm{D}$ \\
\hline 3 Number of Scans & 17600 \\
\hline 4 Relaxation Delay & 1.0000 \\
\hline 5 Acquisition Time & 1.3042 \\
\hline 6 Spectrometer Frec & cy 100.61 \\
\hline 7 Nucleus & $13 \mathrm{C}$ \\
\hline
\end{tabular}

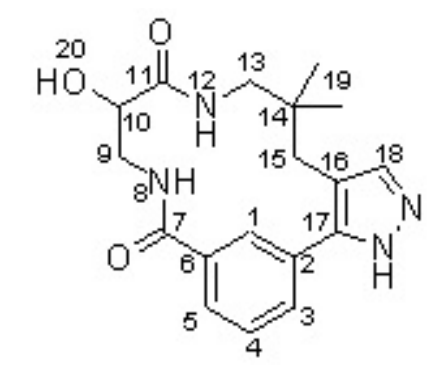

24b

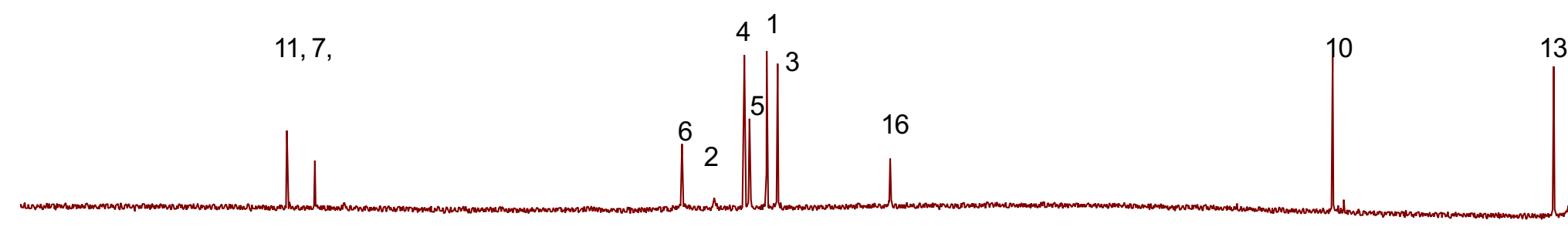

$9, \quad 15,14, \quad 19,19$

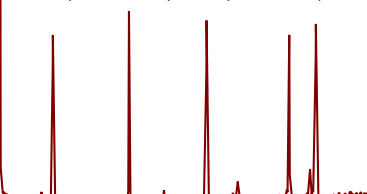

$190 \quad 180$

170

160

150

140

130

120

$90 \quad 80$

807




\begin{tabular}{|ll|}
\hline \multicolumn{1}{|c|}{ Parameter } & \multicolumn{1}{c|}{ Value } \\
1 Solvent & cd3od \\
2 Experiment & cosY \\
3 Number of Scans & 8 \\
4 Relaxation Delay & 1.0000 \\
5 Acquisition Time & 0.1500 \\
6 Spectrometer Frequency & $(400.02,400.02)$ \\
7 Nucleus & $(1 \mathrm{H}, 1 \mathrm{H})$ \\
\hline
\end{tabular}

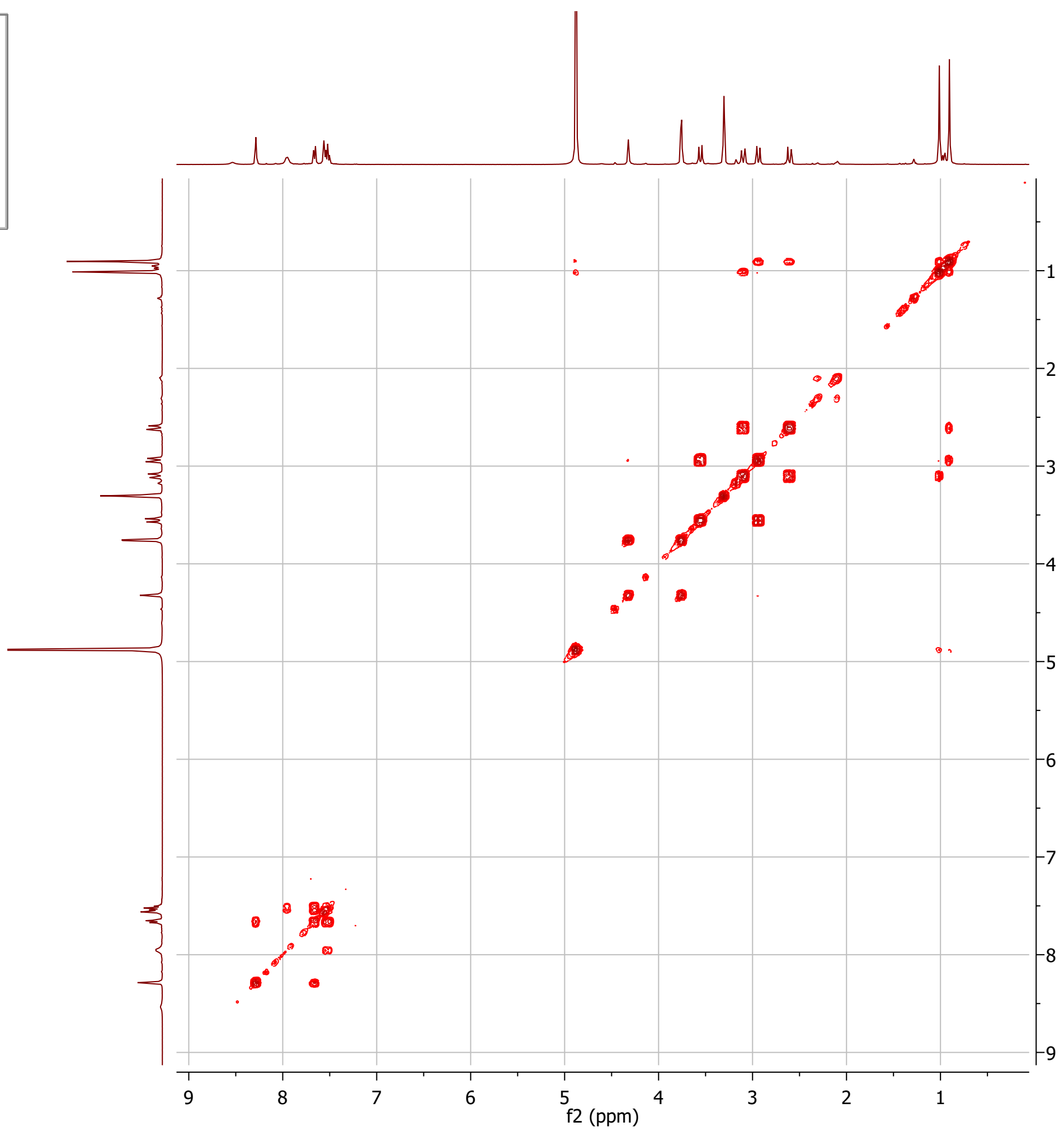

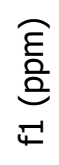

24b 


\begin{tabular}{|ll|}
\hline \multicolumn{1}{|c|}{ Parameter } & \multicolumn{1}{c|}{ Value } \\
1 Solvent & cd3od \\
2 Experiment & NOESY \\
3 Number of Scans & 16 \\
4 Relaxation Delay & 1.5000 \\
5 Acquisition Time & 0.1500 \\
6 Spectrometer Frequency & $(400.02,400.02)$ \\
7 Nucleus & $(1 \mathrm{H}, 1 \mathrm{H})$ \\
\hline
\end{tabular}
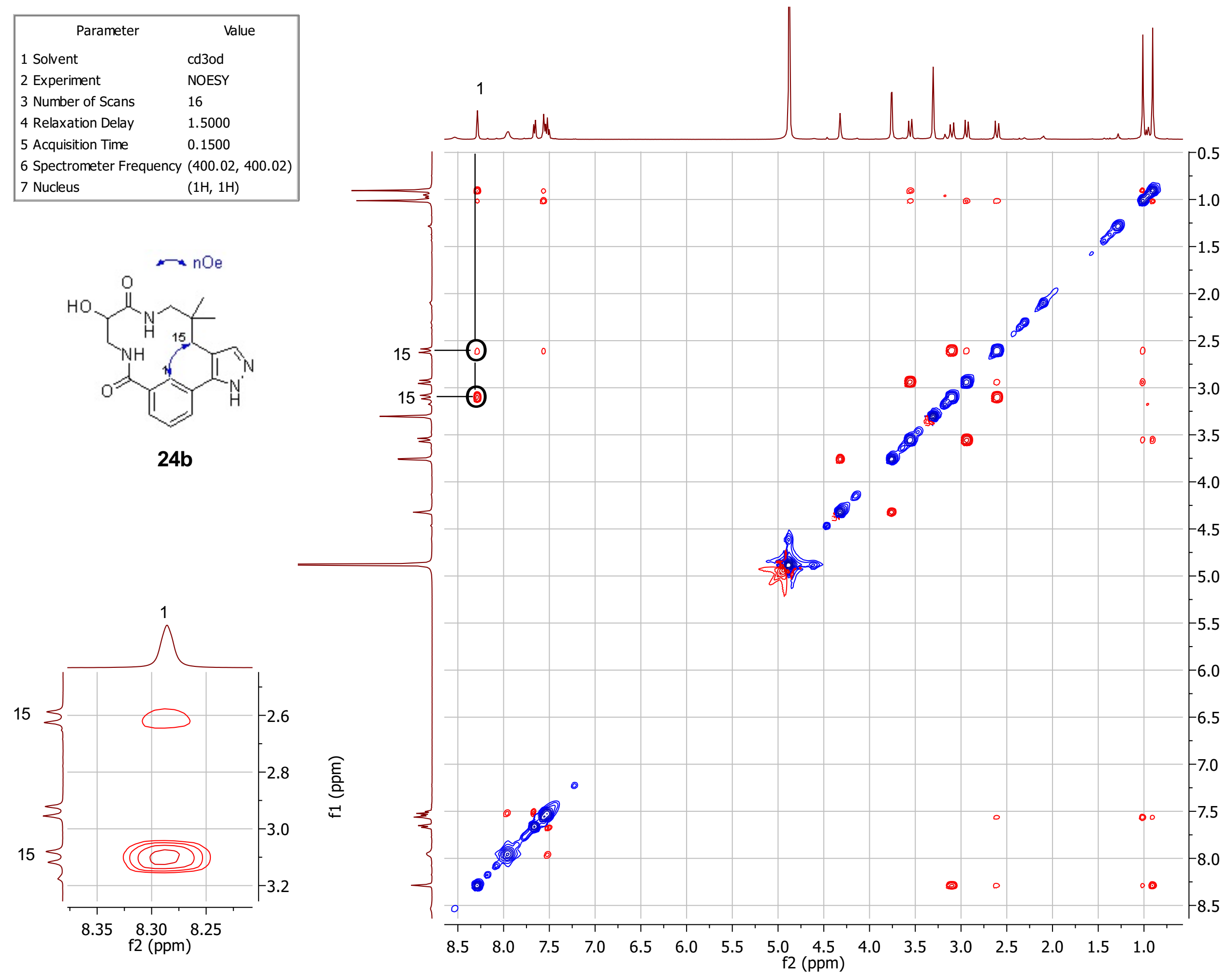

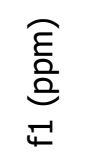




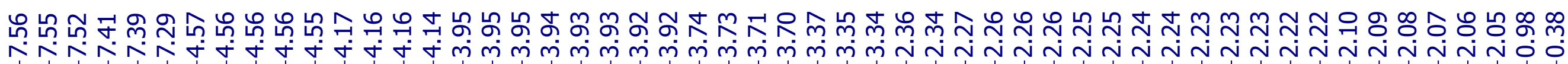

\begin{tabular}{|ll|}
\hline \multicolumn{1}{|c|}{ Parameter } & \multicolumn{1}{c|}{ Value } \\
1 Solvent & cd3od \\
2 Experiment & $1 \mathrm{D}$ \\
3 Number of Scans & 8 \\
4 Relaxation Delay & 1.0000 \\
5 Acquisition Time & 2.0447 \\
6 Spectrometer Frequency & 499.81 \\
7 Nucleus & $1 \mathrm{H}$ \\
\hline
\end{tabular}

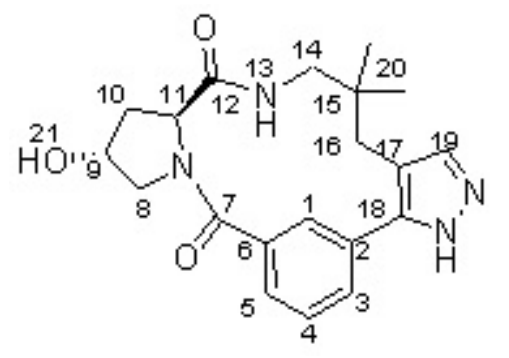

24c

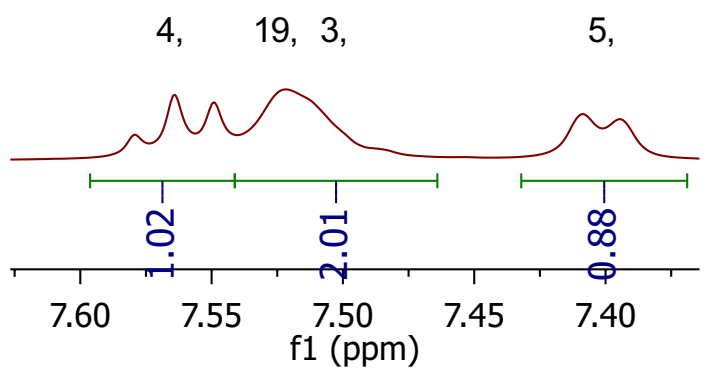

1 ,

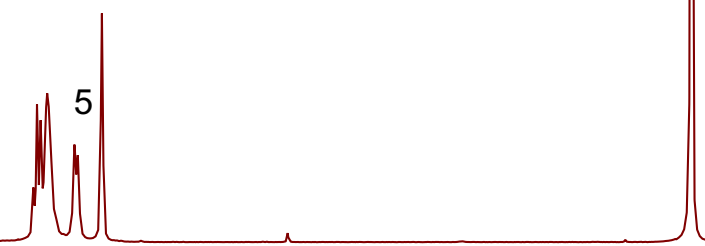

तो

กิ

- No

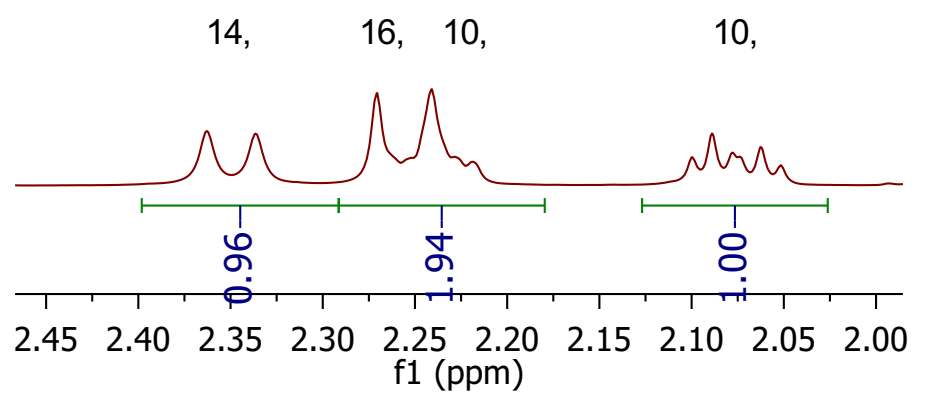

$9 . \quad 11,8,8,14, \quad 16$

14, 10,

20

20

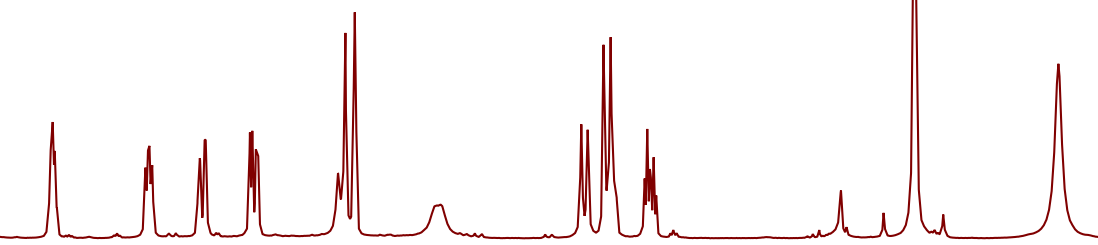

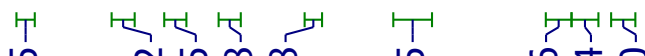

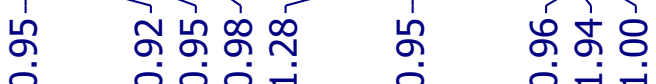

$000 \mathrm{H}$ 


\begin{tabular}{|c|c|}
\hline Parameter & Value \\
\hline 1 Solvent & cd3od \\
\hline 2 Experiment & $1 \mathrm{D}$ \\
\hline 3 Number of Scans & 18000 \\
\hline 4 Relaxation Delay & 1.0000 \\
\hline 5 Acquisition Time & 1.3107 \\
\hline 6 Spectrometer Frec & 100.59 \\
\hline 7 Nucleus & $13 \mathrm{C}$ \\
\hline
\end{tabular}

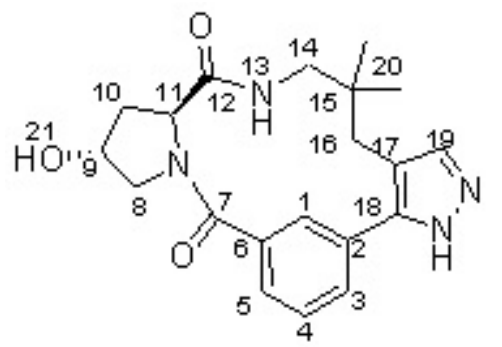

$24 c$

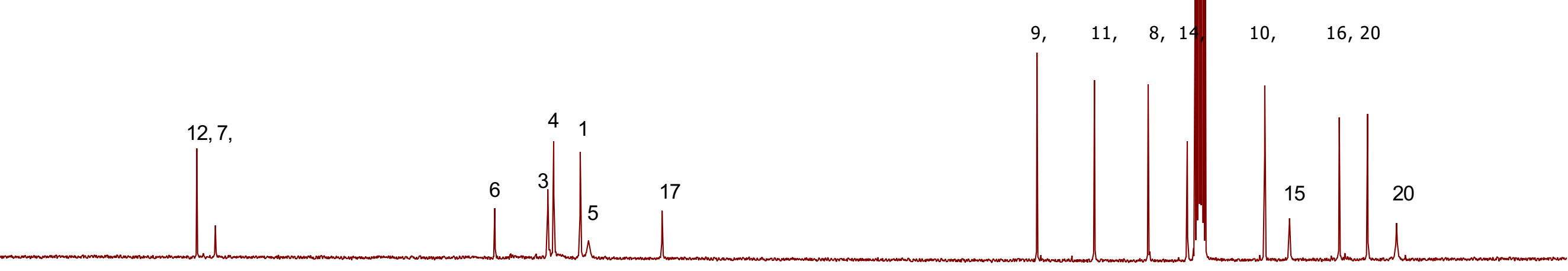




\begin{tabular}{|ll|}
\hline \multicolumn{1}{|c|}{ Parameter } & \multicolumn{1}{c|}{ Value } \\
1 Solvent & cd3od \\
2 Experiment & COSY \\
3 Number of Scans & 8 \\
4 Relaxation Delay & 1.0000 \\
5 Acquisition Time & 0.1500 \\
6 Spectrometer Frequency & $(499.81,499.81)$ \\
7 Nucleus & $(1 \mathrm{H}, 1 \mathrm{H})$ \\
\hline
\end{tabular}

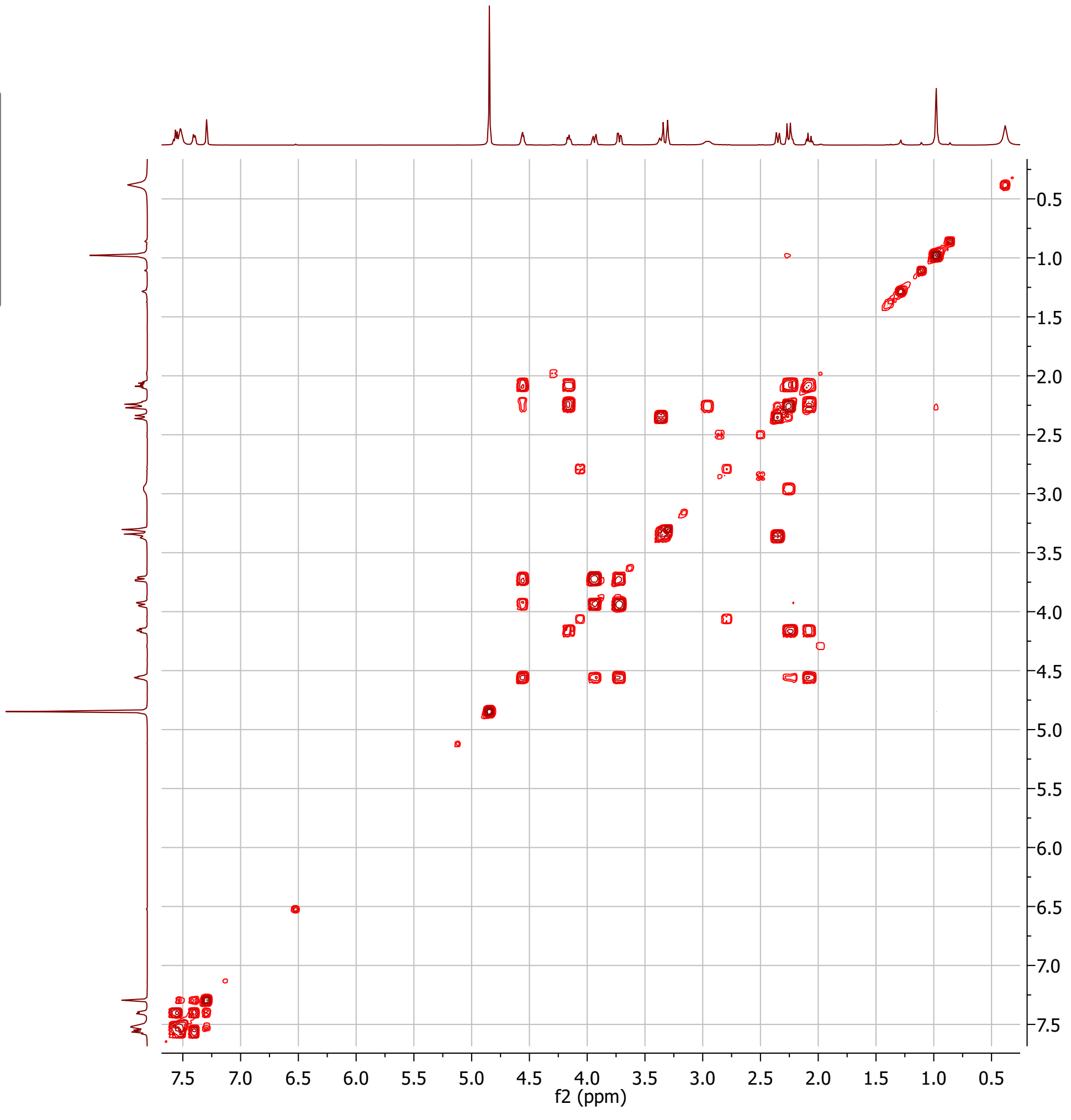

$\bar{E}$
E
$\bar{E}$

24c

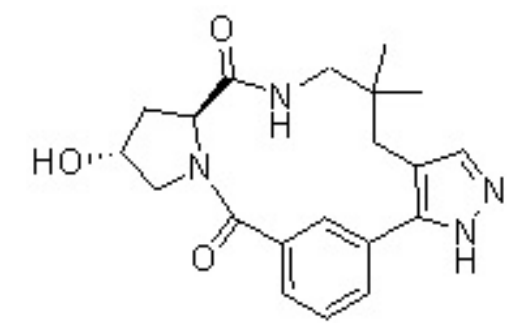




\begin{tabular}{|ll|}
\hline \multicolumn{1}{|c|}{ Parameter } & \multicolumn{1}{c|}{ Value } \\
1 Solvent & cd3od \\
2 Experiment & ASAPHMQC \\
3 Number of Scans & 32 \\
4 Relaxation Delay & 0.0600 \\
5 Acquisition Time & 0.0640 \\
6 Spectrometer Frequency & $(499.81,125.69)$ \\
7 Nucleus & $(1 \mathrm{H}, 13 \mathrm{C})$ \\
\hline
\end{tabular}

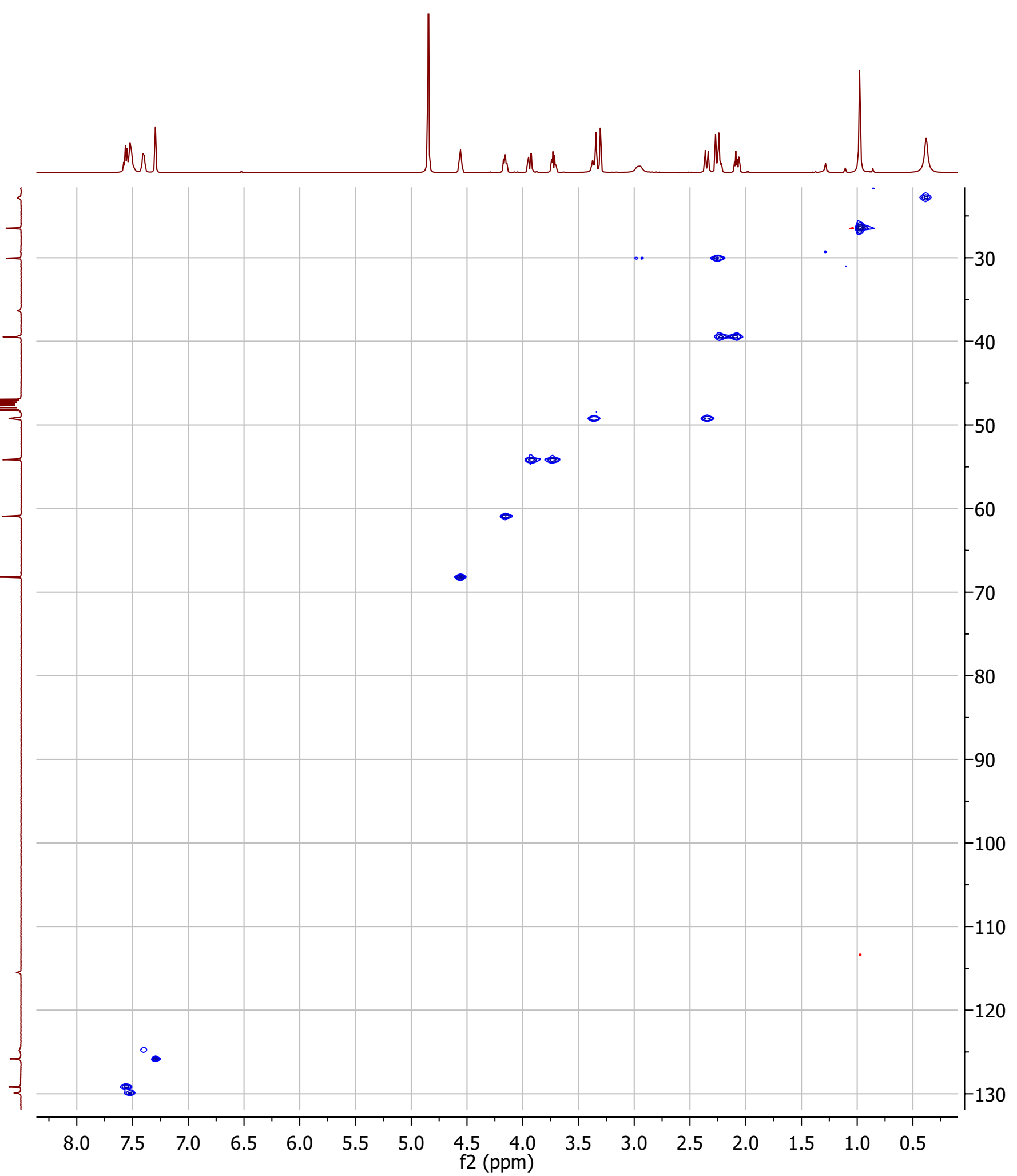

$\widehat{E}$
은
$\bar{L}$

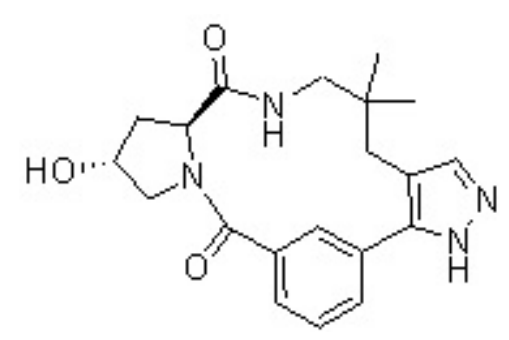

24c
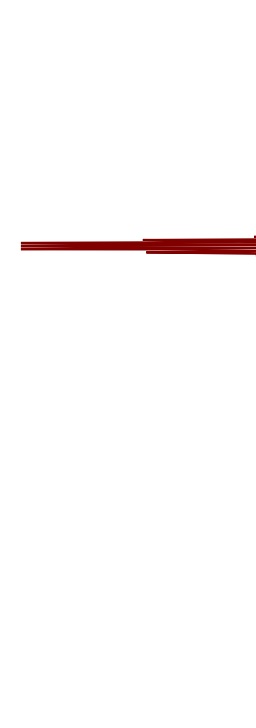


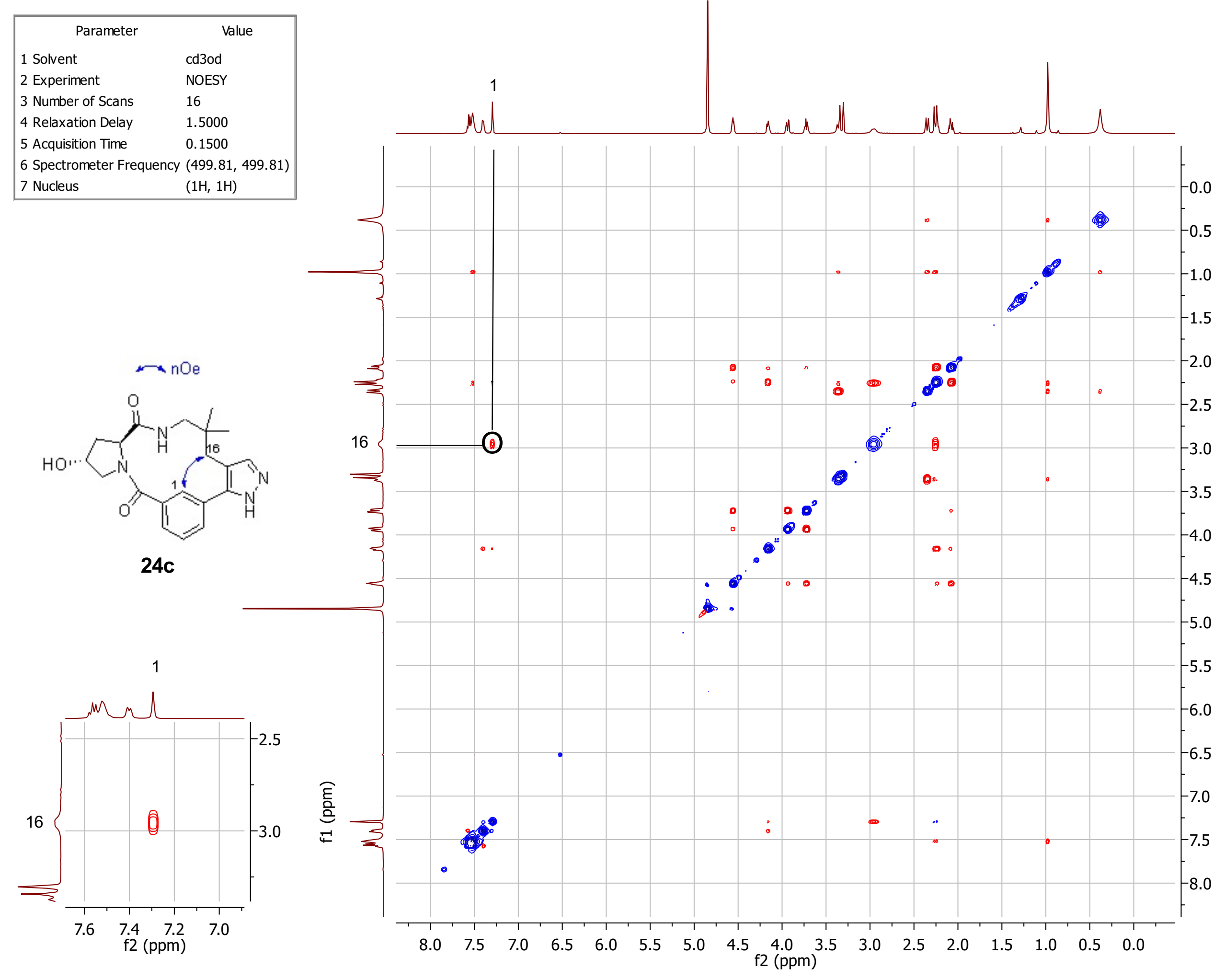




\begin{tabular}{|ll|}
\hline \multicolumn{1}{|c|}{ Parameter } & \multicolumn{1}{c|}{ Value } \\
1 Solvent & cd3od \\
2 Experiment & HMBC \\
3 Number of Scans & 16 \\
4 Relaxation Delay & 1.0000 \\
5 Acquisition Time & 0.1500 \\
6 Spectrometer Frequency & $(499.81,125.69)$ \\
7 Nucleus & $(1 \mathrm{H}, 13 \mathrm{C})$ \\
\hline
\end{tabular}

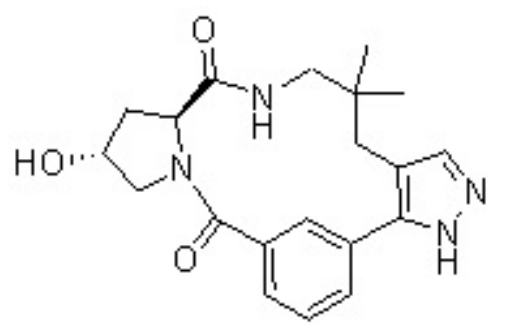

24c

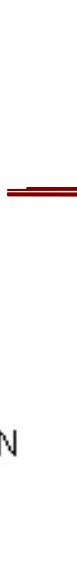




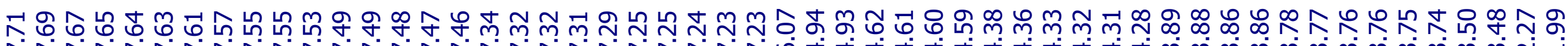

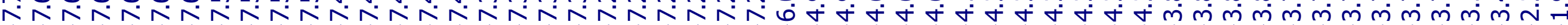

\begin{tabular}{|ll|}
\hline \multicolumn{1}{|c|}{ Parameter } & \multicolumn{1}{c|}{ Value } \\
cd3od
\end{tabular}

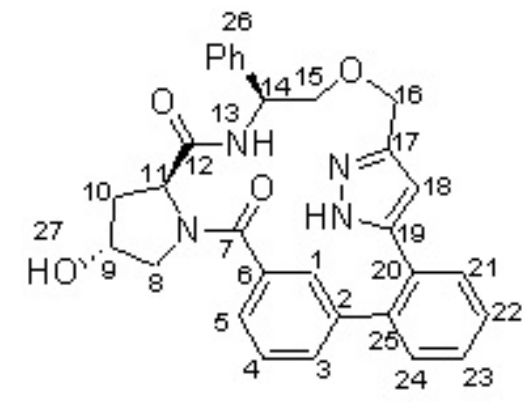

11 ,

$16, \quad 9, \quad 16$

23d
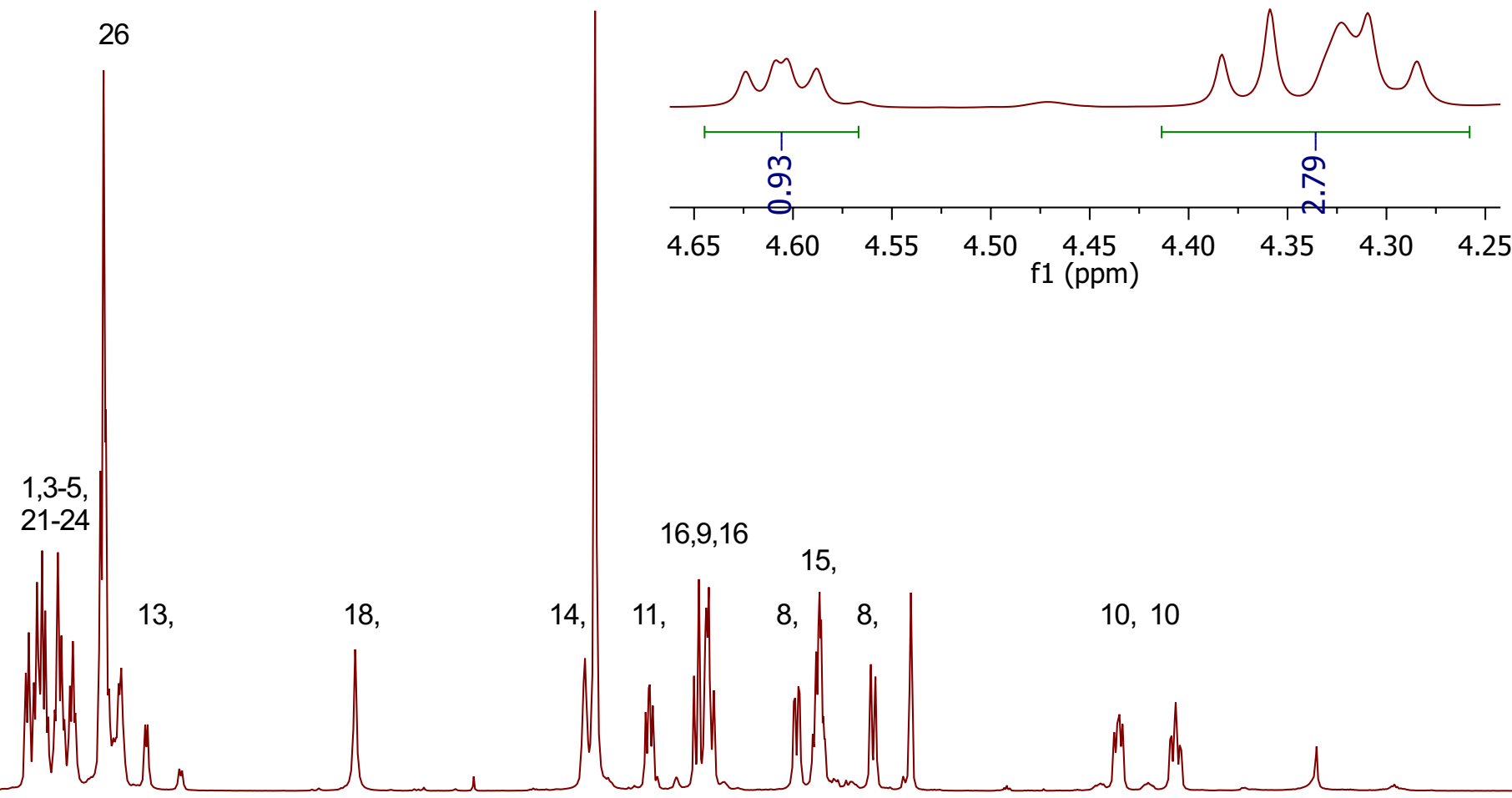

$16,9,16$

15

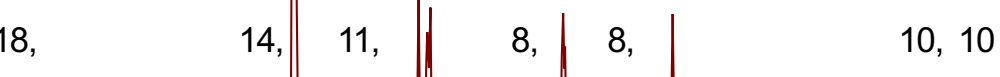

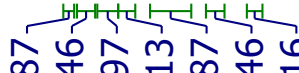

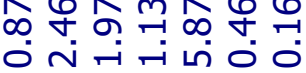

8.0

$7.5 \quad 7.0$

6.5

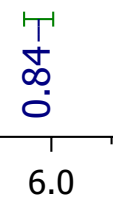

${ }_{5.0} 4.5$
f1 (ppm) 


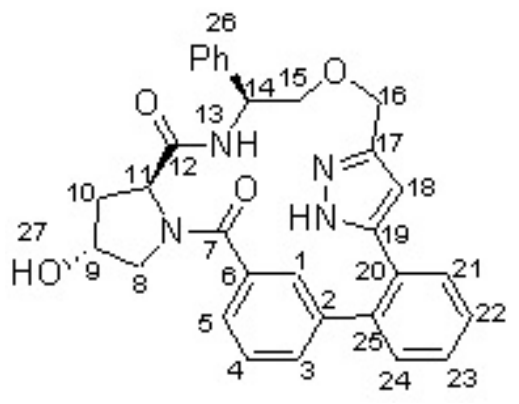

23d

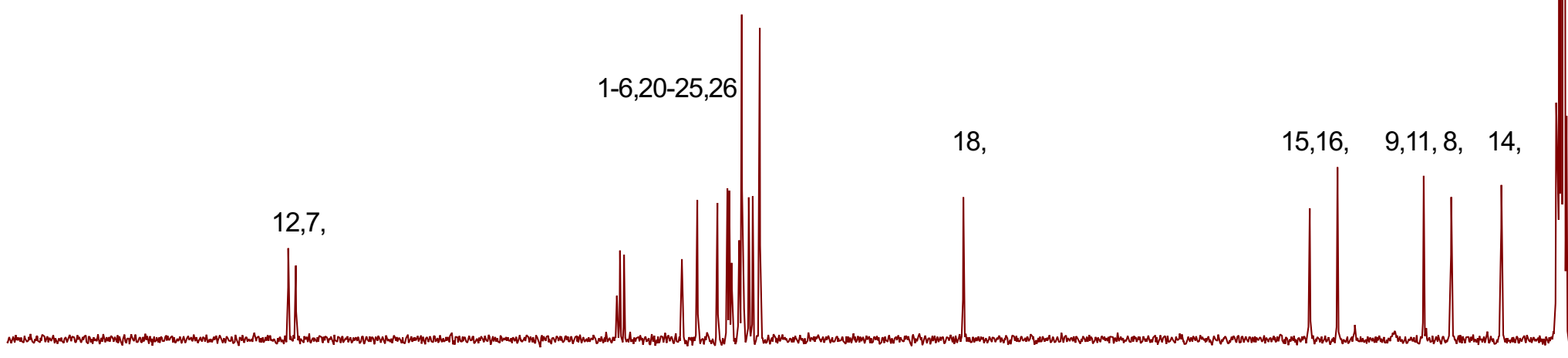




\begin{tabular}{|ll|}
\hline \multicolumn{1}{|c|}{ Parameter } & \multicolumn{1}{c|}{ Value } \\
1 Solvent & cd3od \\
2 Experiment & cosY \\
3 Number of Scans & 4 \\
4 Relaxation Delay & 1.0000 \\
5 Acquisition Time & 0.1500 \\
6 Spectrometer Frequency & $(499.81,499.81)$ \\
7 Nucleus & $(1 \mathrm{H}, 1 \mathrm{H})$ \\
\hline
\end{tabular}

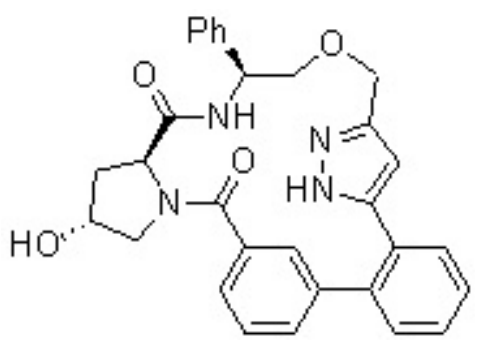

23d

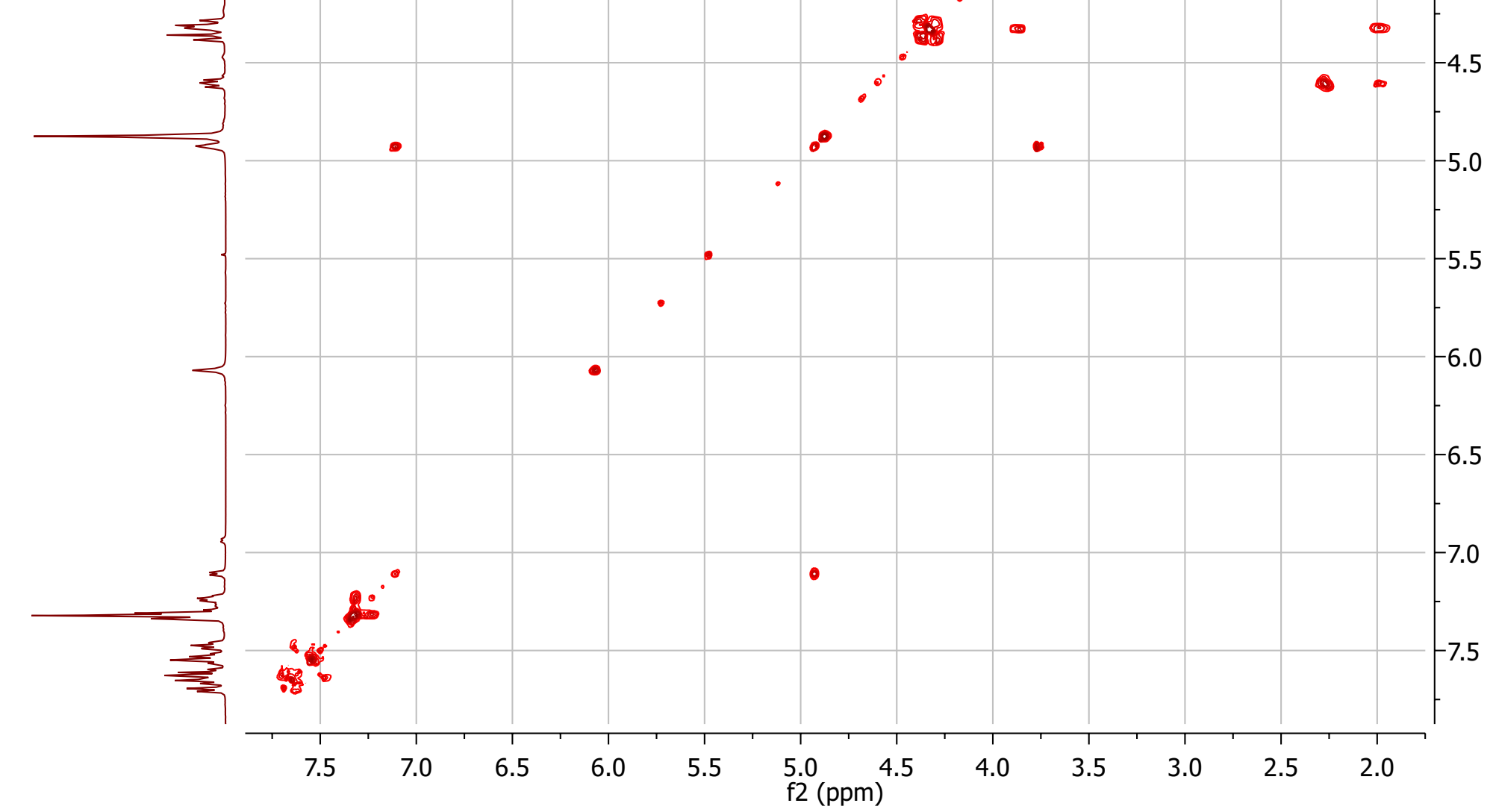




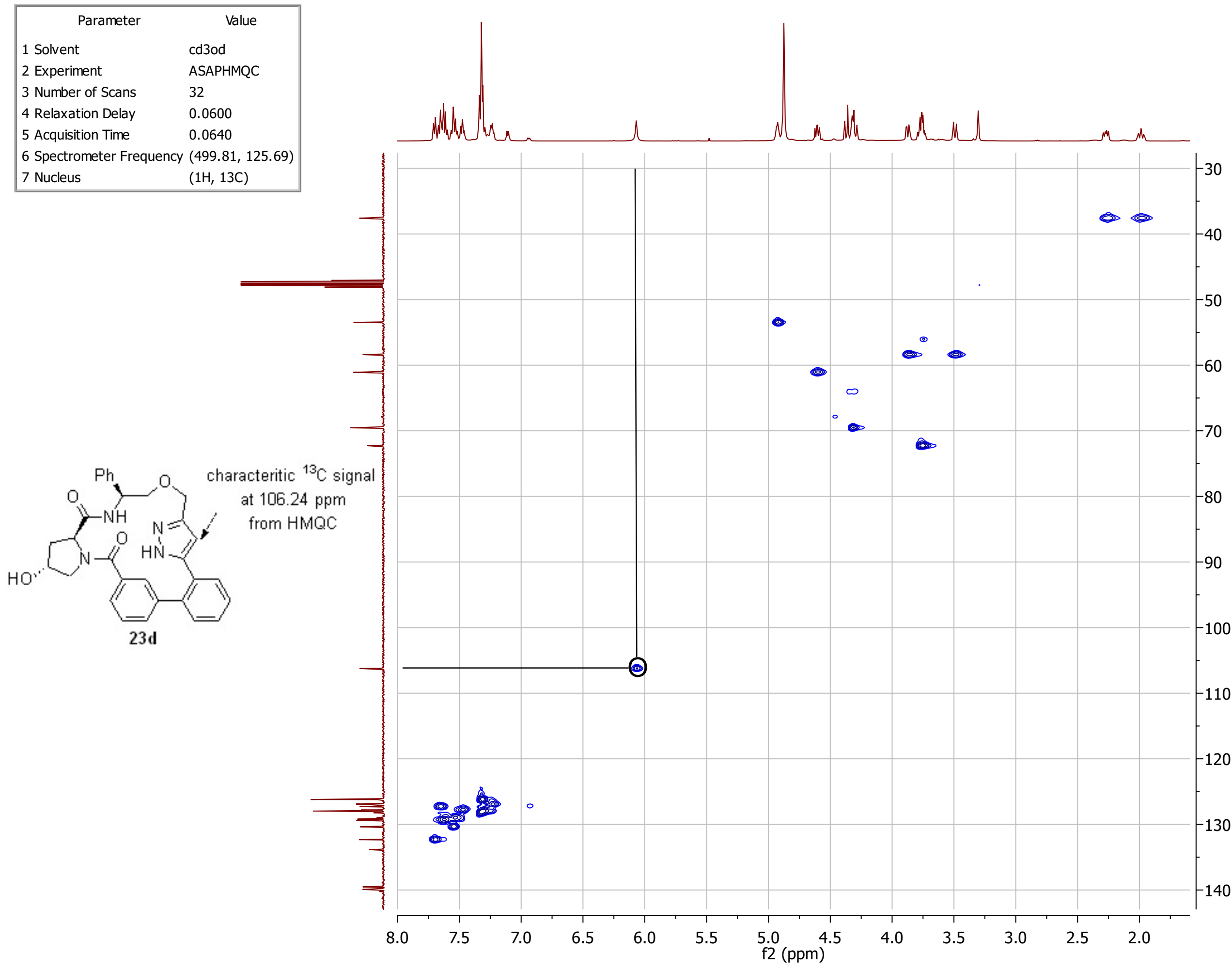




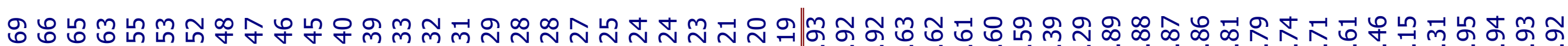

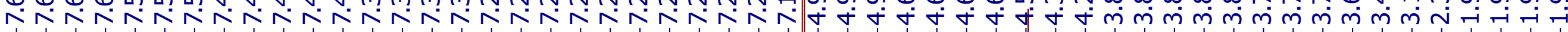

\begin{tabular}{|ll|}
\hline \multicolumn{1}{|c|}{ Parameter } & \multicolumn{1}{c|}{ Value } \\
1 Solvent & cd3od \\
2 Experiment & $1 \mathrm{D}$ \\
3 Number of Scans & 8 \\
4 Relaxation Delay & 1.0000 \\
5 Acquisition Time & 2.0447 \\
6 Spectrometer Frequency & 499.81 \\
7 Nucleus & $1 \mathrm{H}$ \\
\hline
\end{tabular}

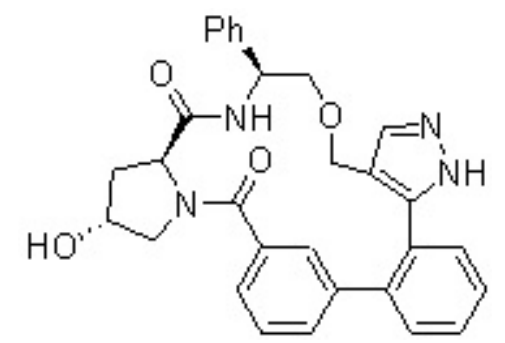

24d

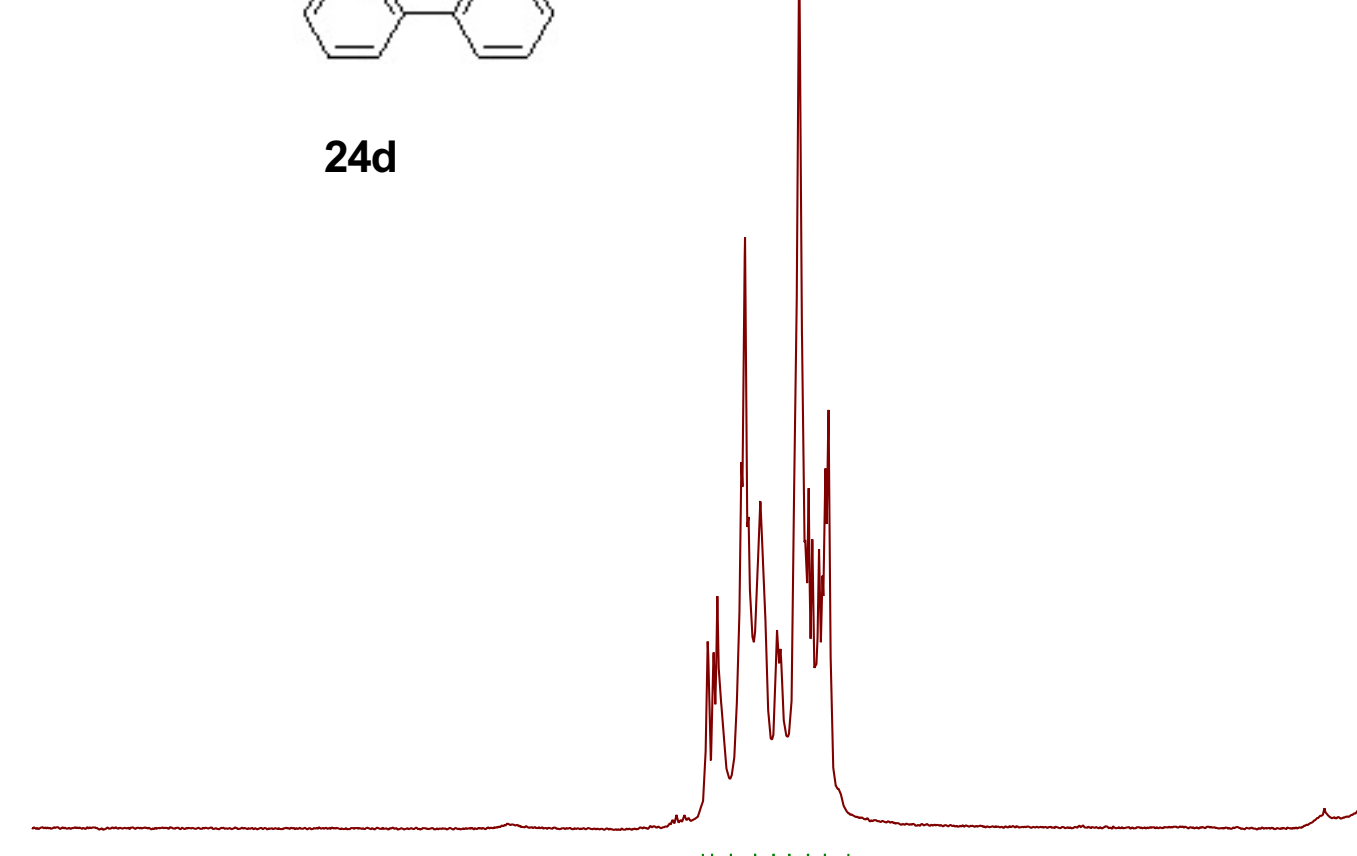

m m
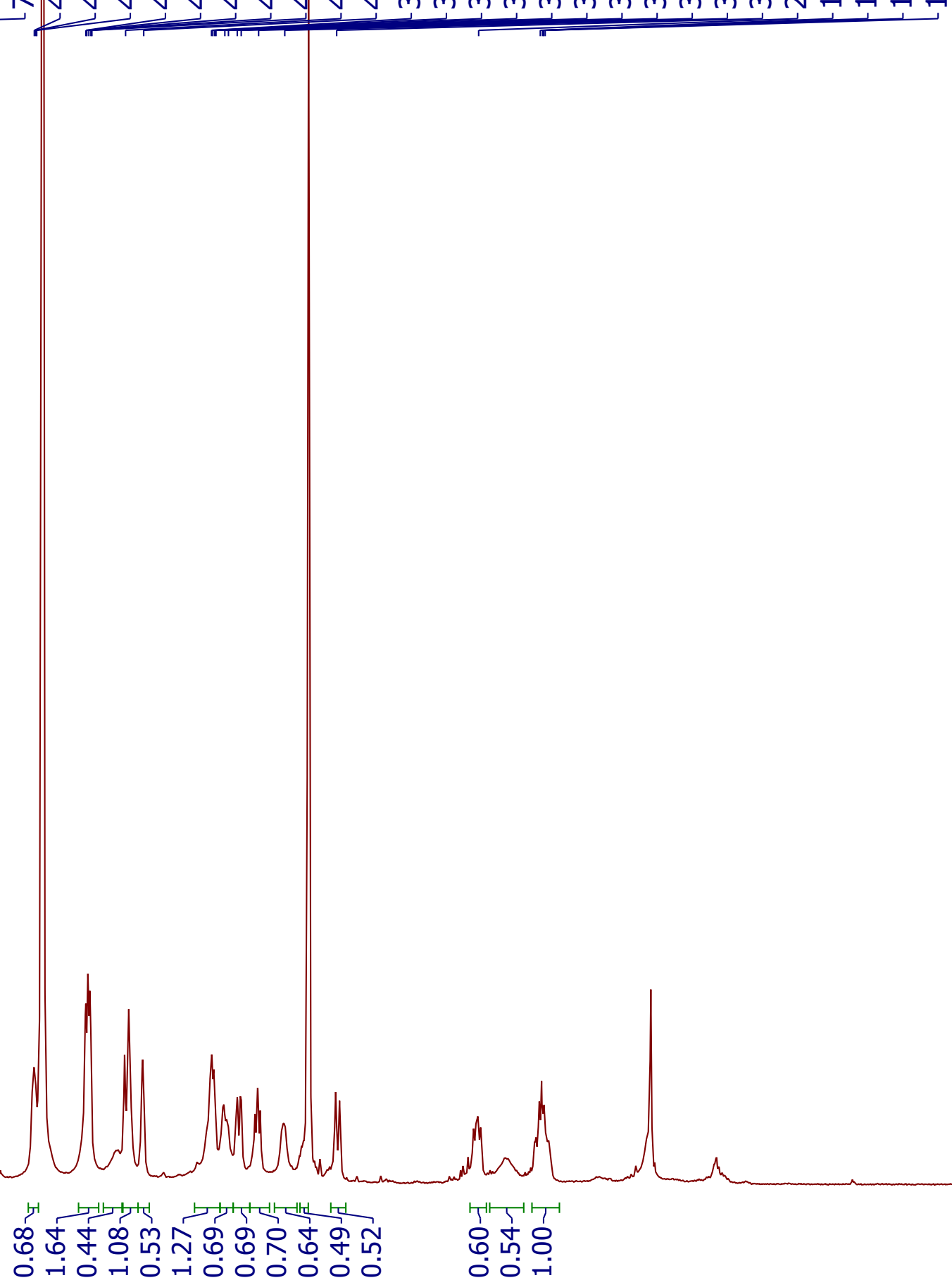

め ซே

舟

$\begin{array}{llll}10.0 & 9.5 & 9.0 & 8.5\end{array}$

$7.0 \quad 6.5$

6.5

f1 $\begin{aligned} & 5.0 \\ & (p p m)\end{aligned}$

$\begin{array}{llll}4.5 & 4.0 & 3.5 & 3.0\end{array}$

$2.5 \quad 2.0$




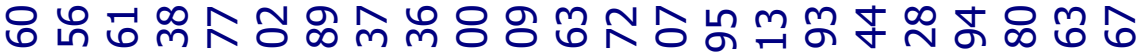

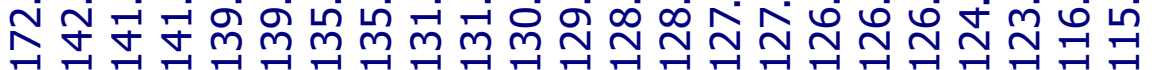

Parameter

Value

1 Solvent

2 Experiment

3 Number of Scans

4 Relaxation Delay

5 Acquisition Time

7 Nucleus

cd3od

$1 \mathrm{D}$

18000

1.0000

1.3107

100.59

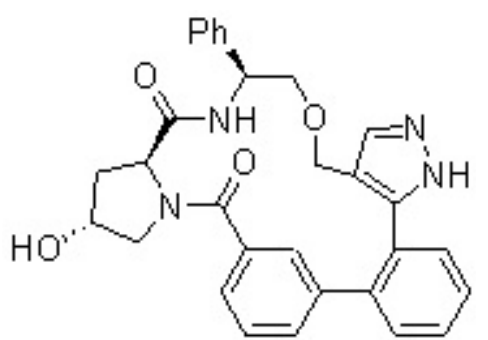

24d

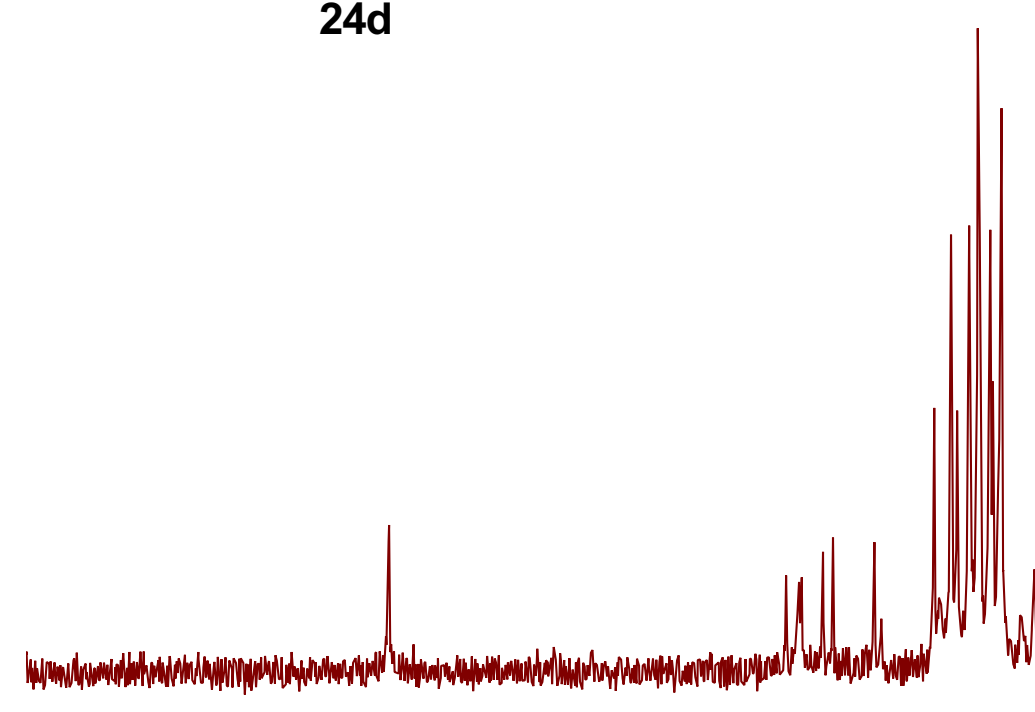


$\left(\mathrm{CD}_{3}\right)_{2} \mathrm{SO}, 85^{\circ} \mathrm{C}, 400 \mathrm{MHz}$

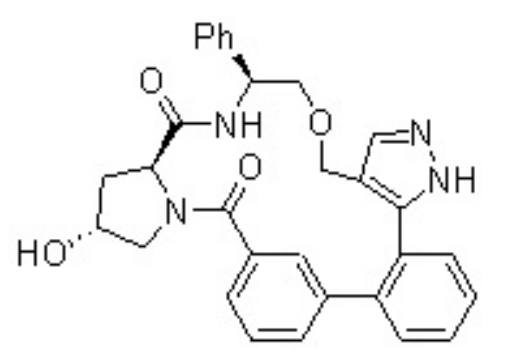

24d

$\left(\mathrm{CD}_{3}\right)_{2} \mathrm{SO}, 25^{\circ} \mathrm{C}, 400 \mathrm{MHz}$
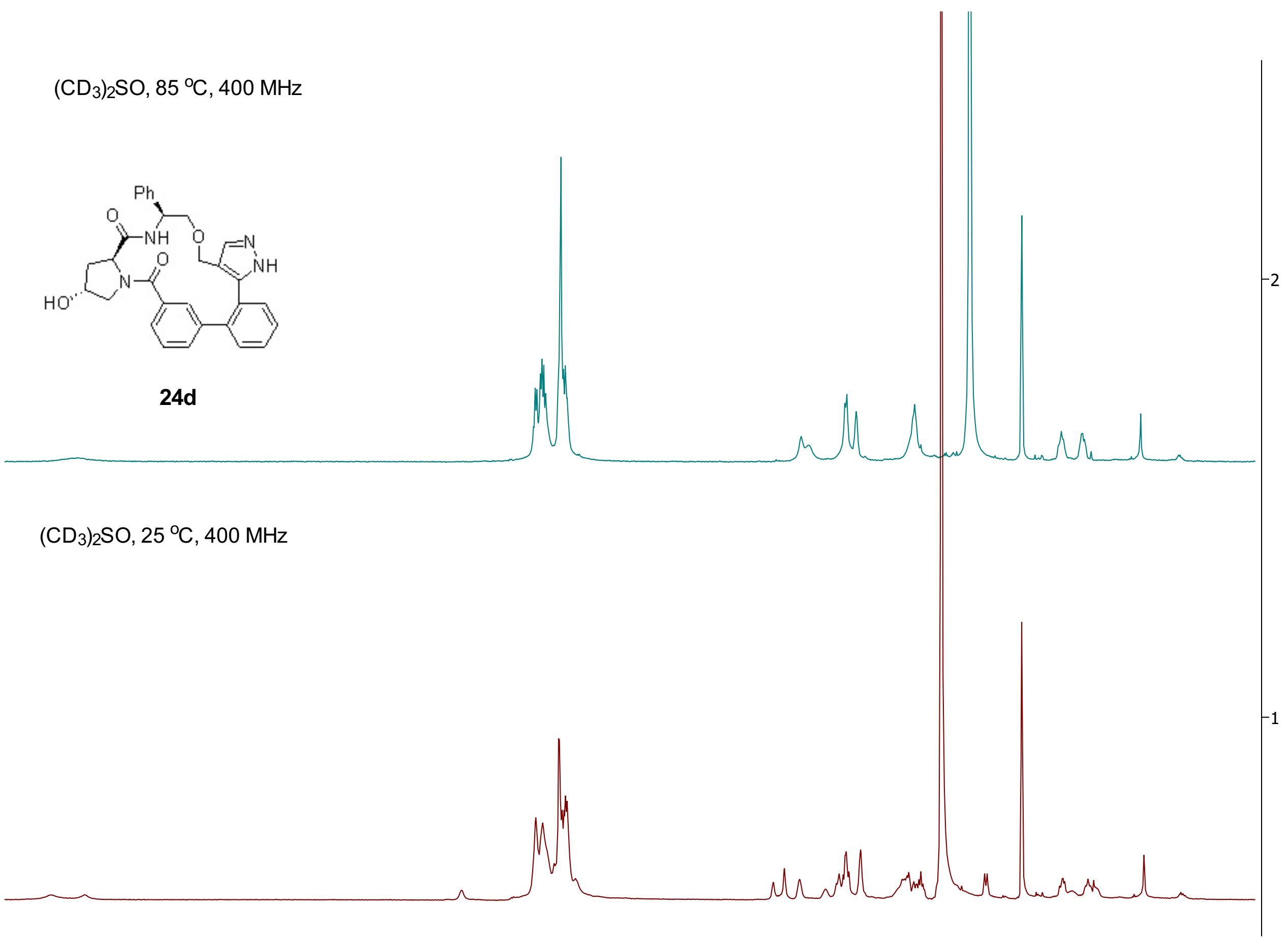

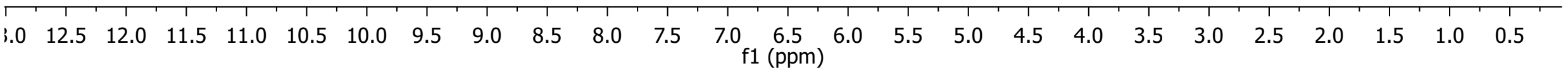




\begin{tabular}{|c|c|}
\hline Parameter & Value \\
\hline 1 Solvent & $\operatorname{cdcl} 3$ \\
\hline 2 Experiment & 1D \\
\hline 3 Number of Scans & 8 \\
\hline 4 Relaxation Delay & 1.0000 \\
\hline 5 Acquisition Time & 2.5592 \\
\hline 6 Spectrometer Frequency & 400.09 \\
\hline 7 Nucleus & $1 \mathrm{H}$ \\
\hline
\end{tabular}
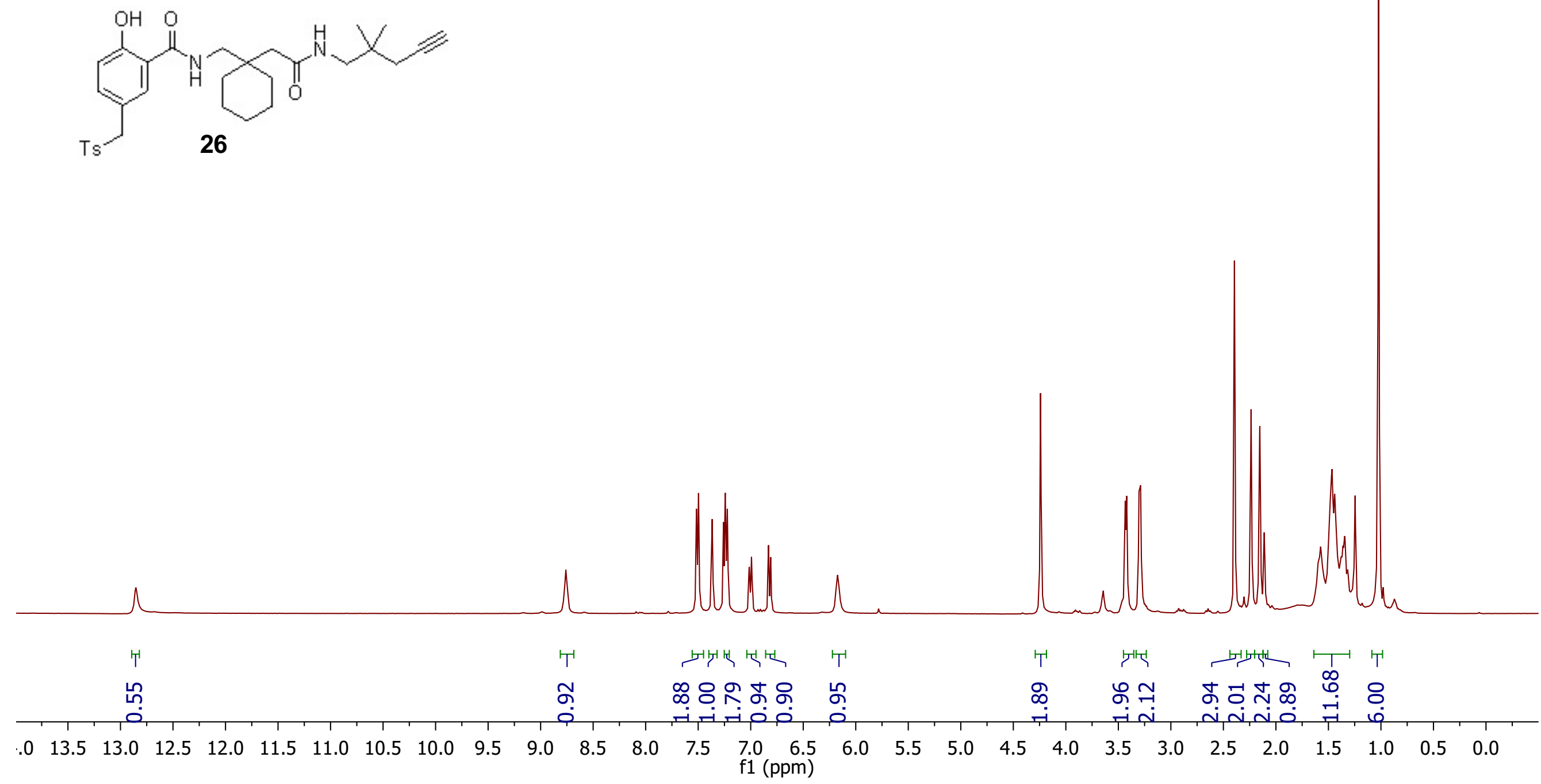
곤ํํ

ำชั

? Value

Parameter

1 Solvent

2 Experiment

3 Number of Scans

4 Relaxation Delay

5 Acquisition Time

6 Spectrometer Frequency 100.61

7 Nucleus

$13 \mathrm{C}$

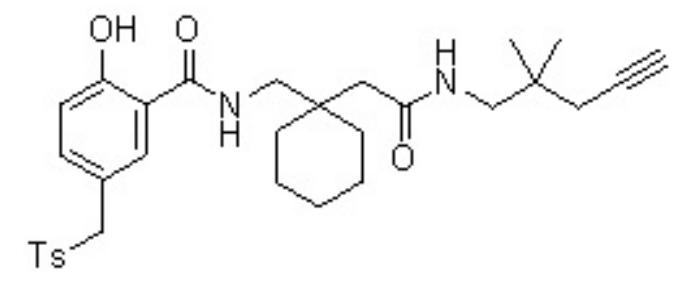

26

cdcl3

$1 \mathrm{D}$

17000

1.0000

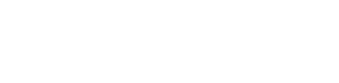

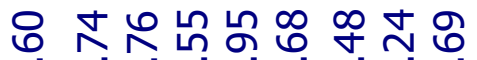

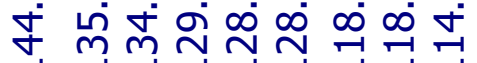

光

๓

安

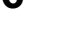


ชิ

कं

レก ๆ

$\infty \infty$ N N N

Parameter

1 Solvent

2 Experiment

3 Number of Scans

4 Relaxation Delay

5 Acquisition Time

6 Spectrometer Frequency 400.09

7 Nucleus

$1 \mathrm{H}$

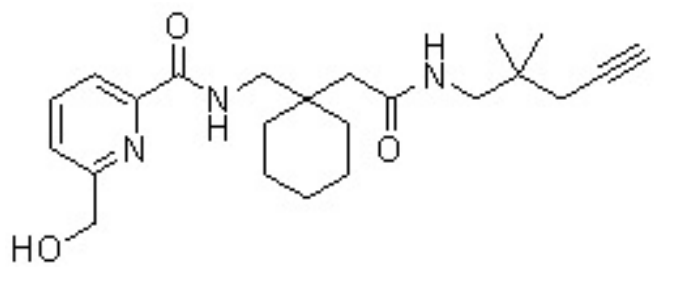

$27 b$

1D

1.0000
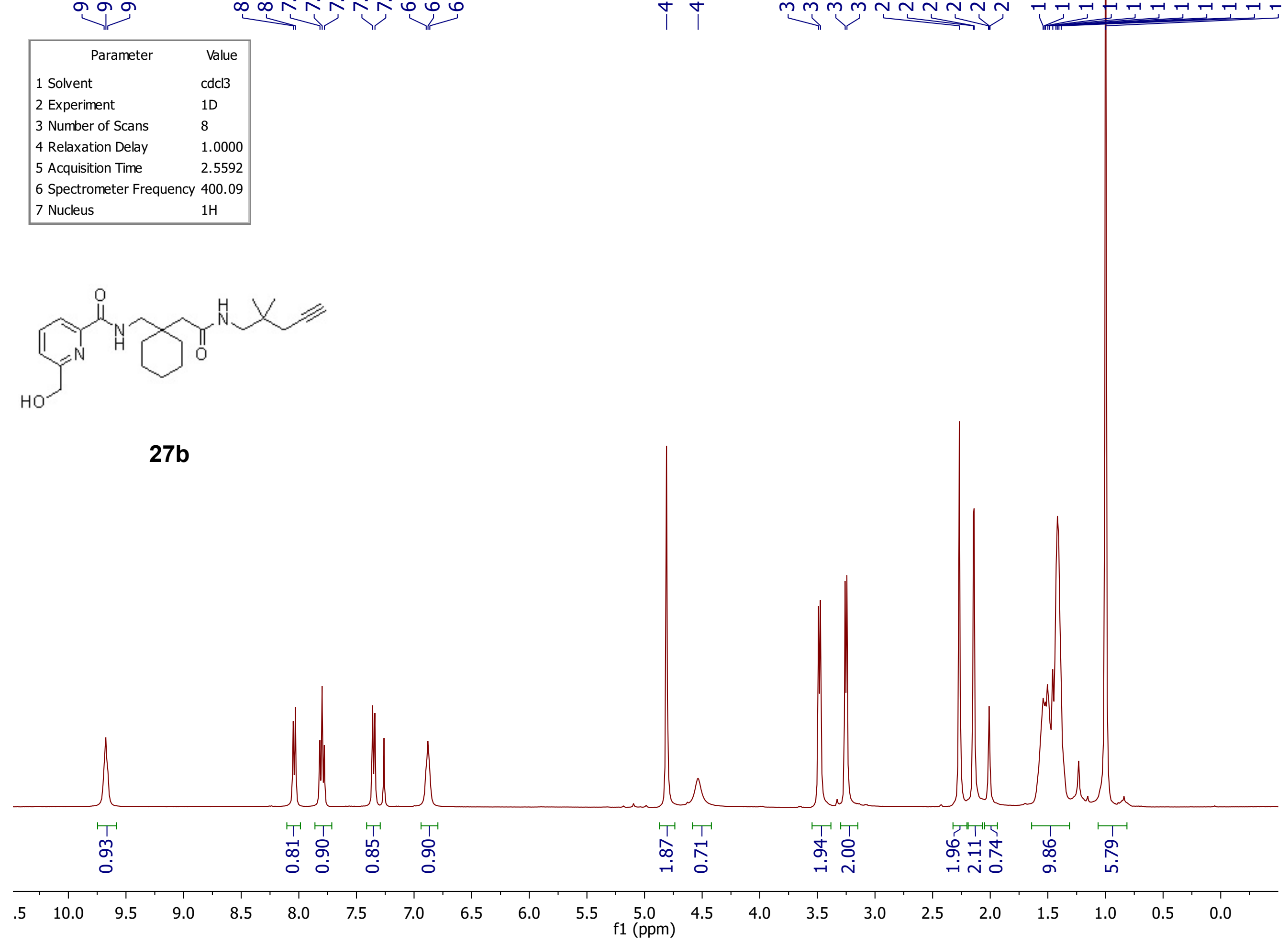

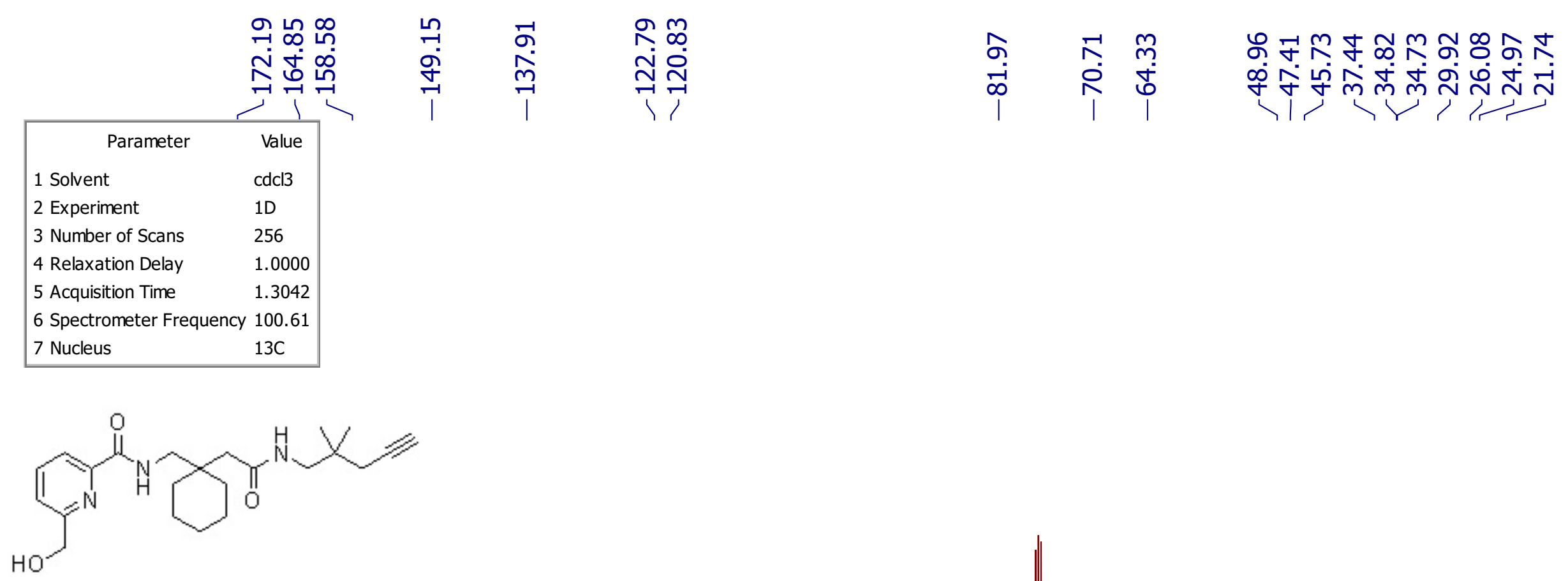

27b

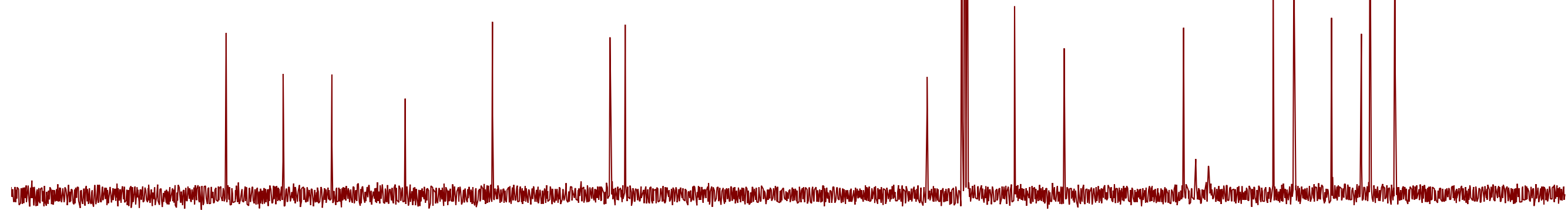

\begin{tabular}{|c|c|c|c|c|c|c|c|c|c|c|c|c|c|c|c|c|c|c|c|}
\hline 30 & 190 & 180 & 170 & 160 & 150 & 140 & 130 & 120 & 110 & $\begin{array}{c}100 \\
\mathrm{f} 1(\mathrm{ppm})\end{array}$ & 90 & 80 & 70 & 60 & 50 & 40 & 30 & 20 & 10 \\
\hline
\end{tabular}




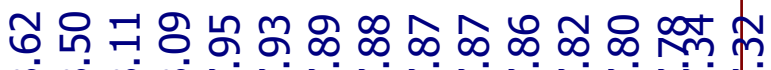

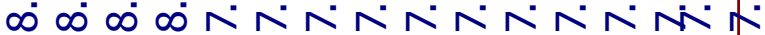

\begin{tabular}{|ll|}
\hline \multicolumn{1}{|c|}{ Parameter } & \multicolumn{1}{c|}{ Value } \\
1 Solvent & cdcl3 \\
2 Experiment & $1 \mathrm{D}$ \\
3 Number of Scans & 8 \\
4 Relaxation Delay & 1.0000 \\
5 Acquisition Time & 2.5592 \\
6 Spectrometer Frequency & 400.09 \\
7 Nucleus & $1 \mathrm{H}$ \\
\hline
\end{tabular}

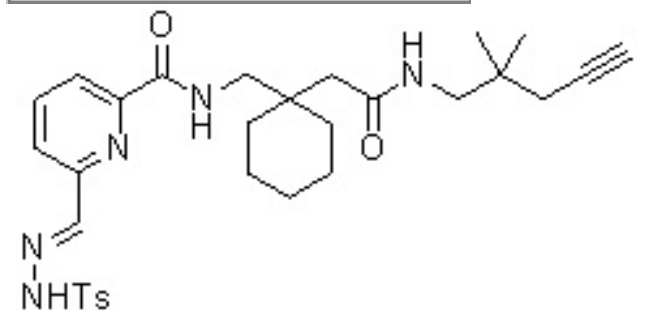

27

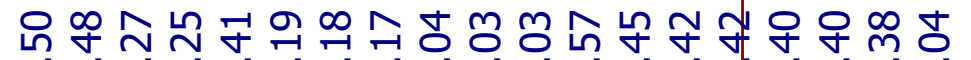

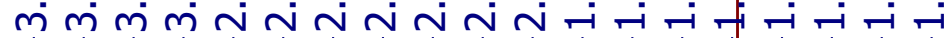

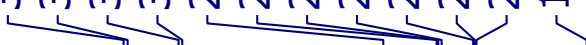




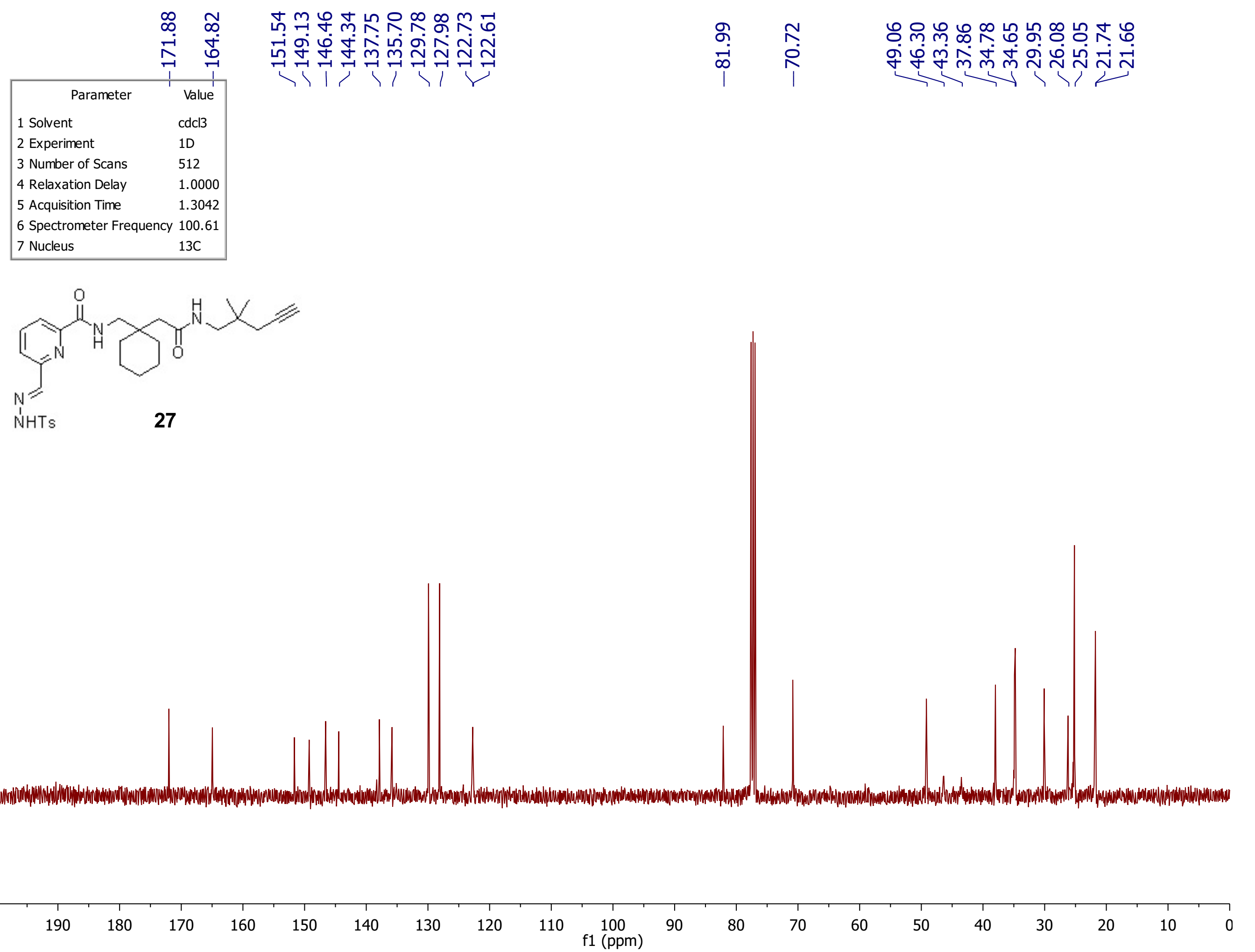

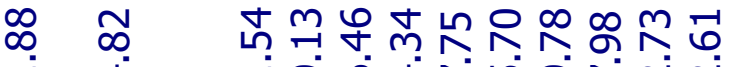

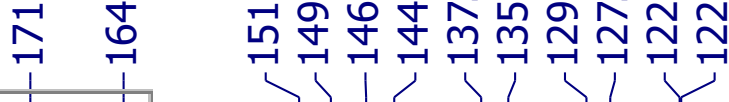

๙

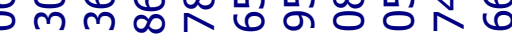

守 พั่

1 Solvent

cdcl3

1.0000

6 Spectrometer Frequency 100.61

$90 \quad 80$


ำ요 요

웅우

느늠

$\infty \infty \infty N$

Parameter

Value

1 Solvent

2 Experiment

3 Number of Scans

4 Relaxation Delay

5 Acquisition Time

6 Spectrometer Frequency 4

7 Nucleus

$1 \mathrm{H}$

cdcl3

1.0000

5592

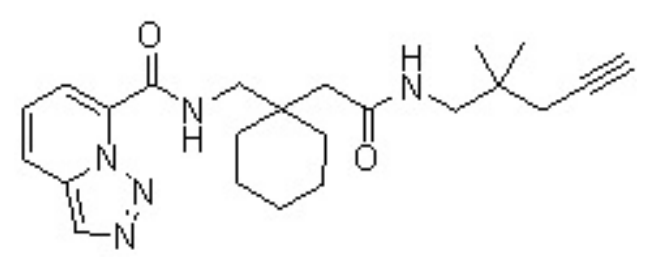

28

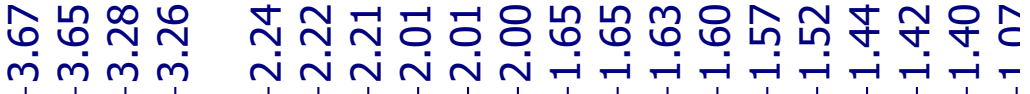




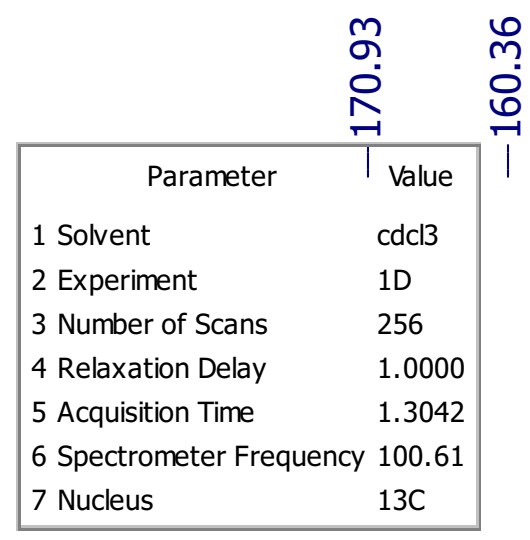

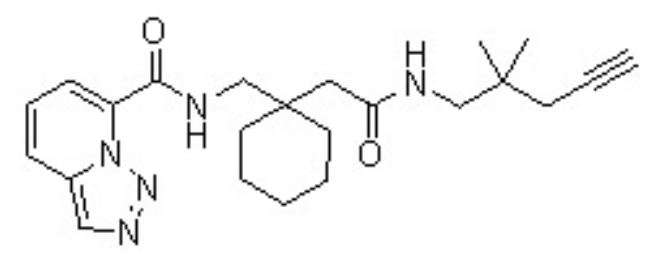

28 
mై

\begin{tabular}{|ll|}
\hline \multicolumn{1}{|c|}{ Parameter } & \multicolumn{1}{c|}{ Value } \\
1 Solvent & cdcl3 \\
2 Experiment & $1 \mathrm{D}$ \\
3 Number of Scans & 8 \\
4 Relaxation Delay & 1.0000 \\
5 Acquisition Time & 2.0447 \\
6 Spectrometer Frequency & 499.81 \\
7 Nucleus & $1 \mathrm{H}$ \\
\hline
\end{tabular}

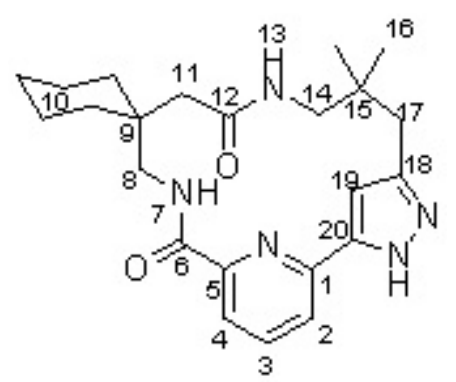

29

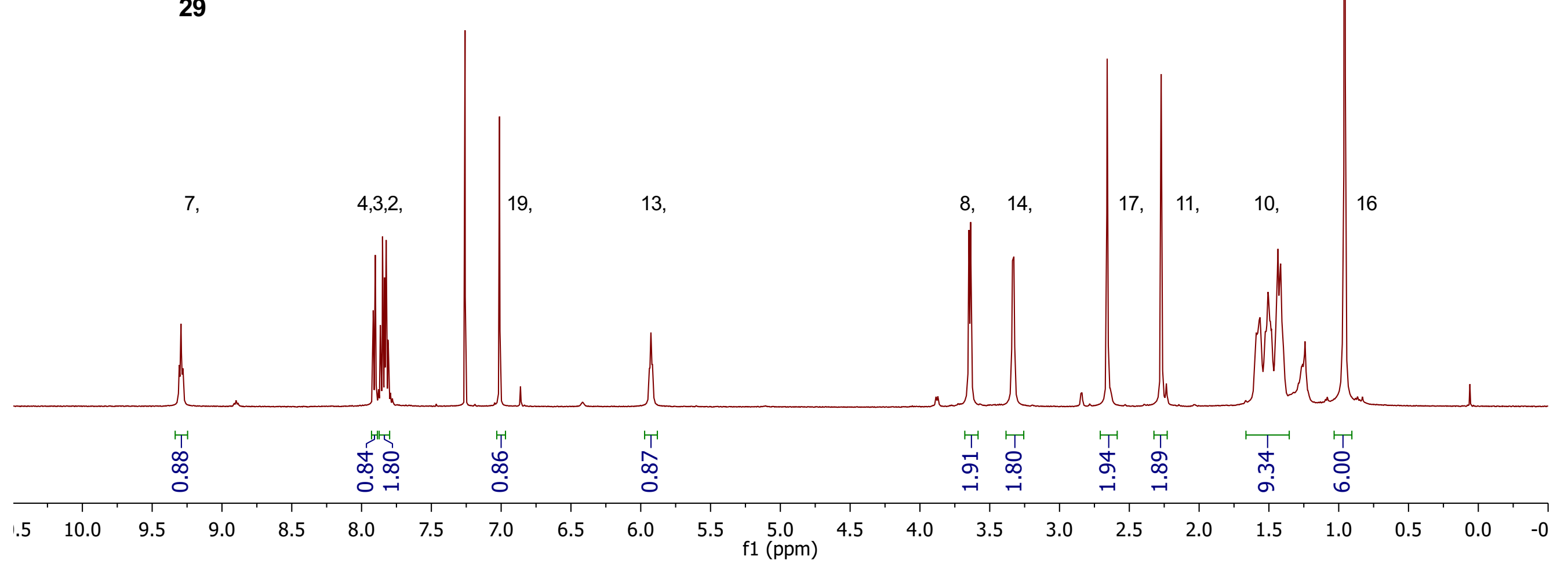

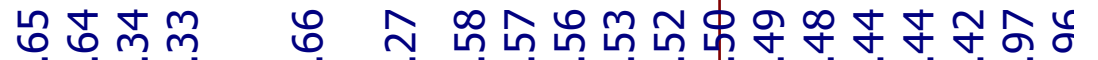

$m m m m$
N
ธัตู สิ

ம

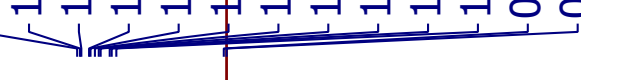




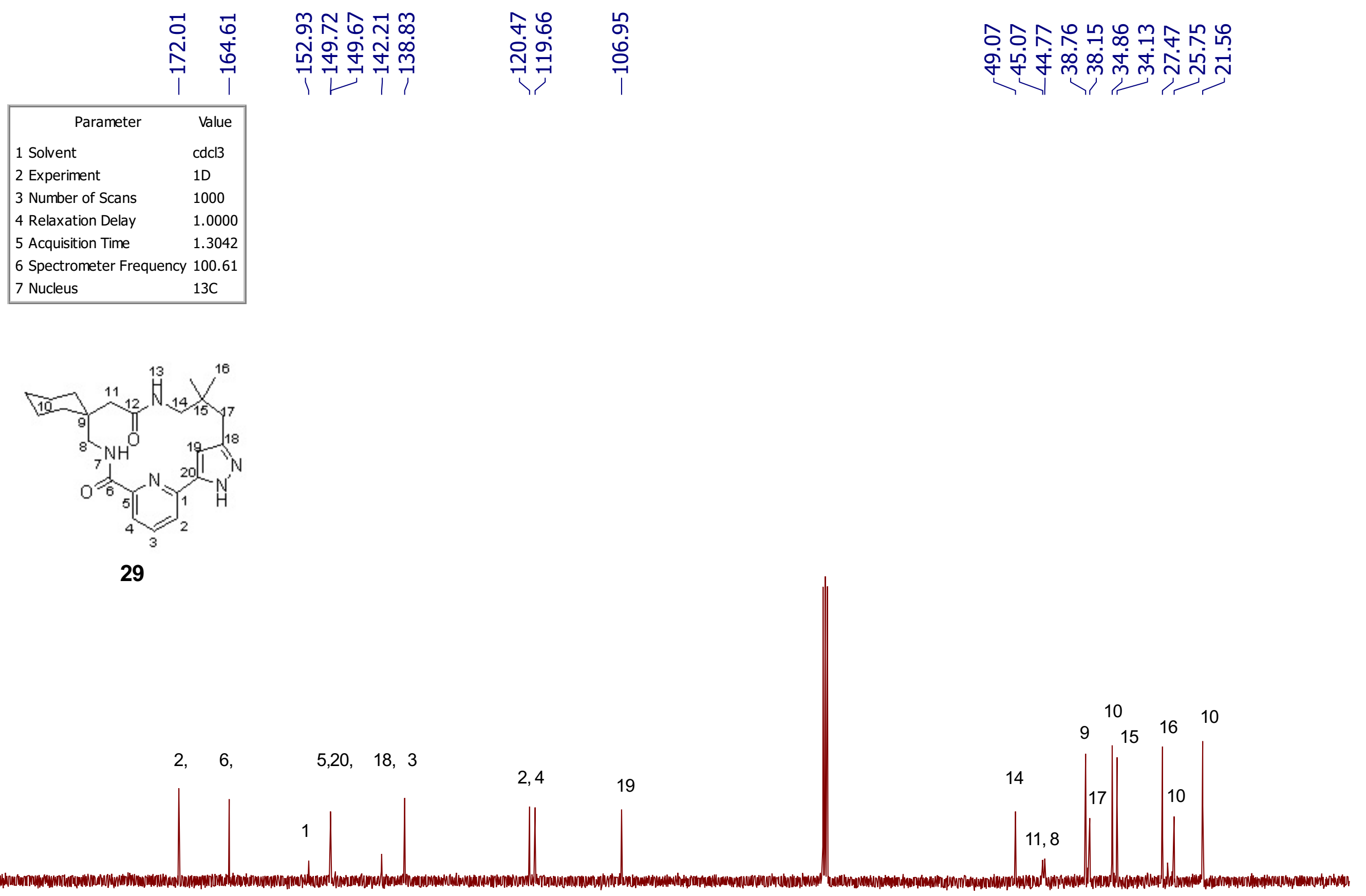

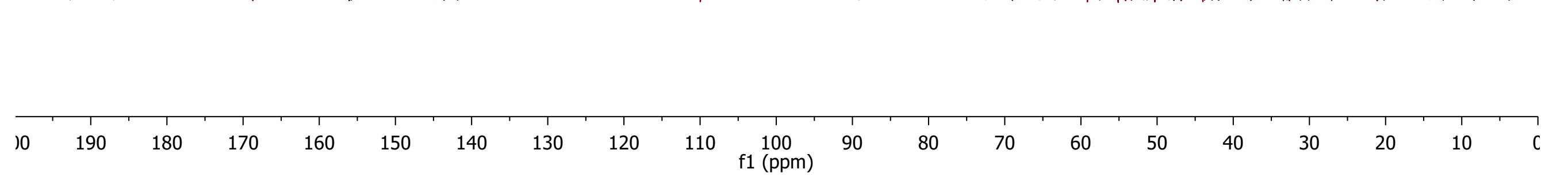




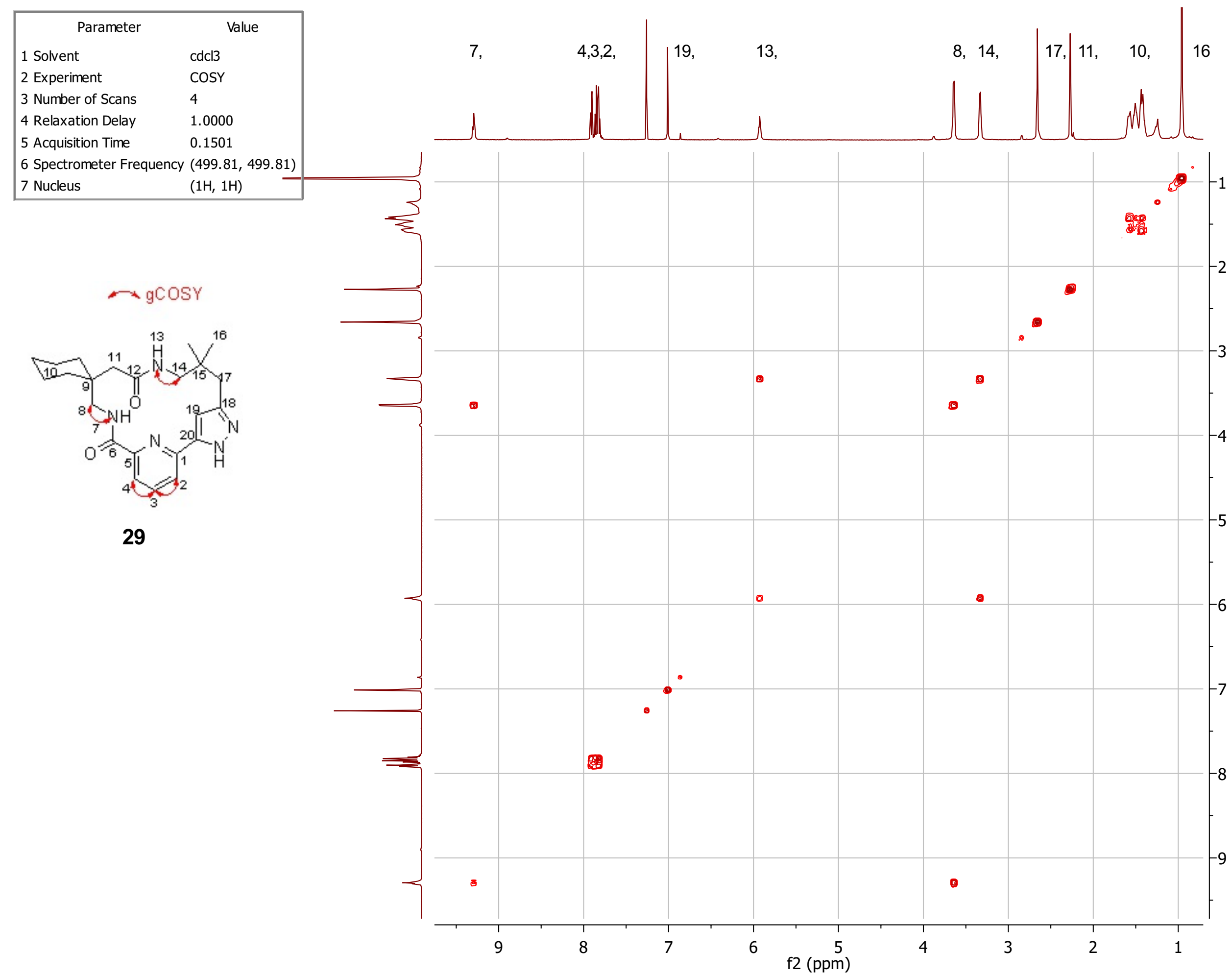




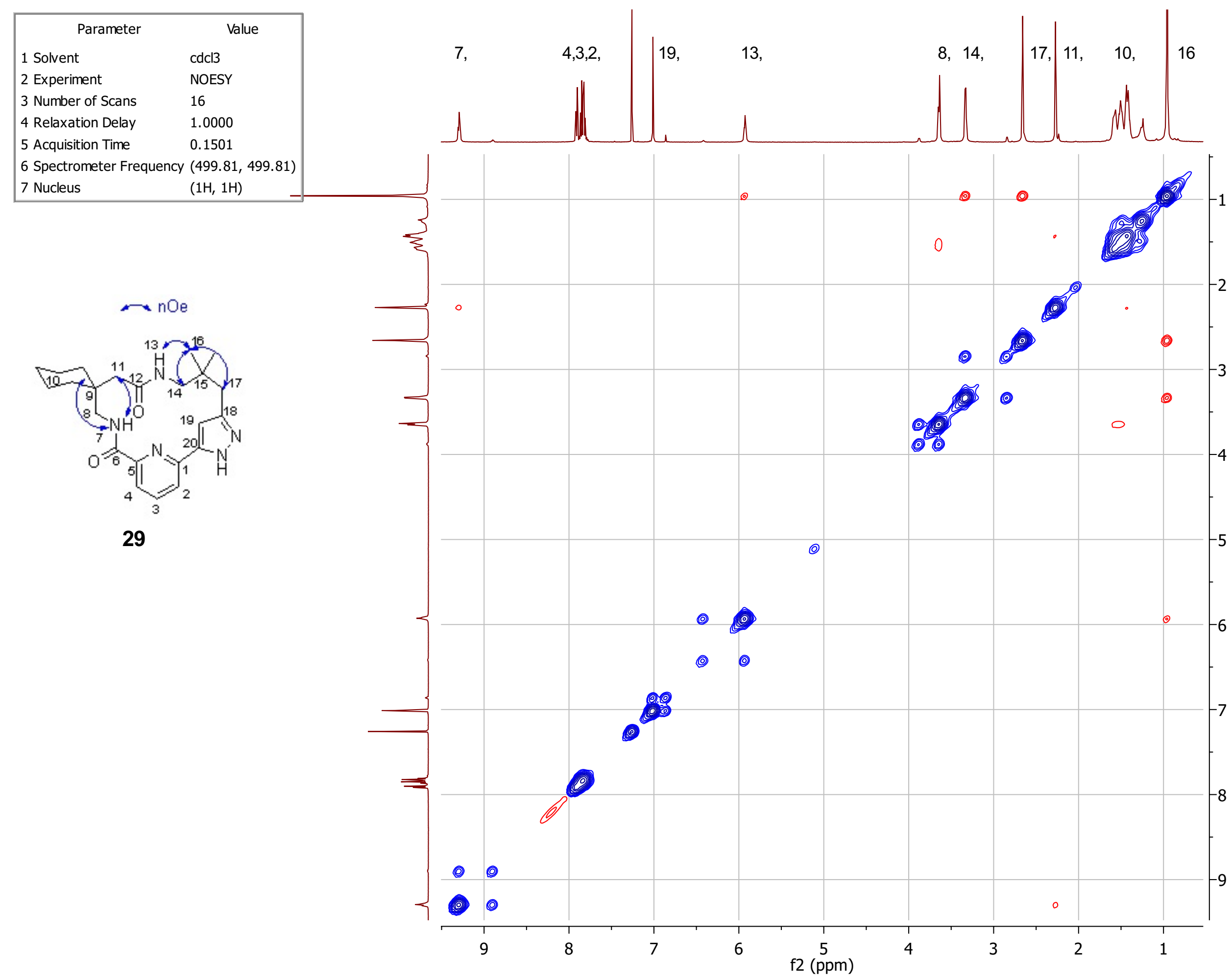

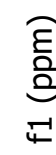




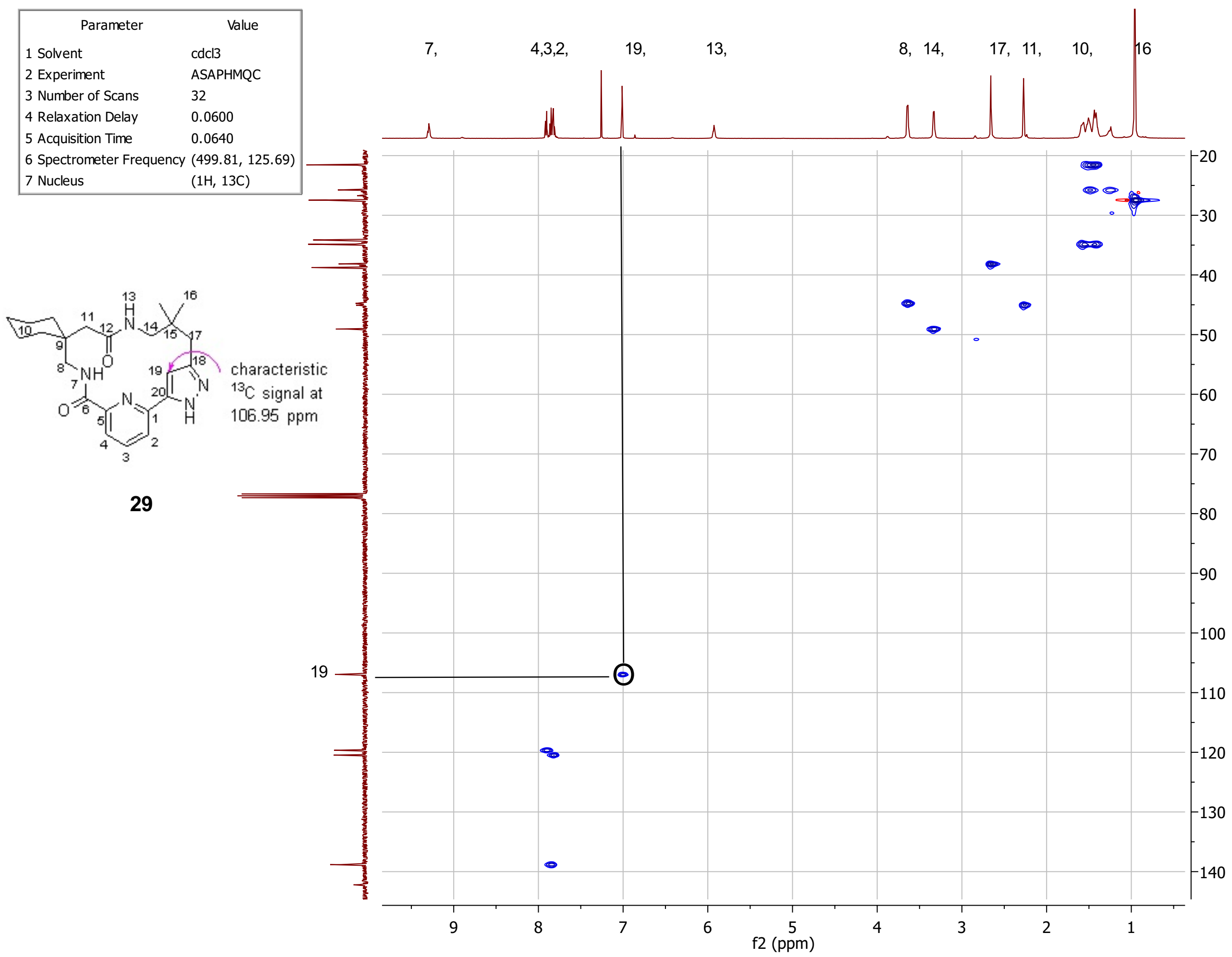

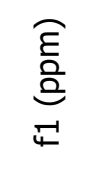




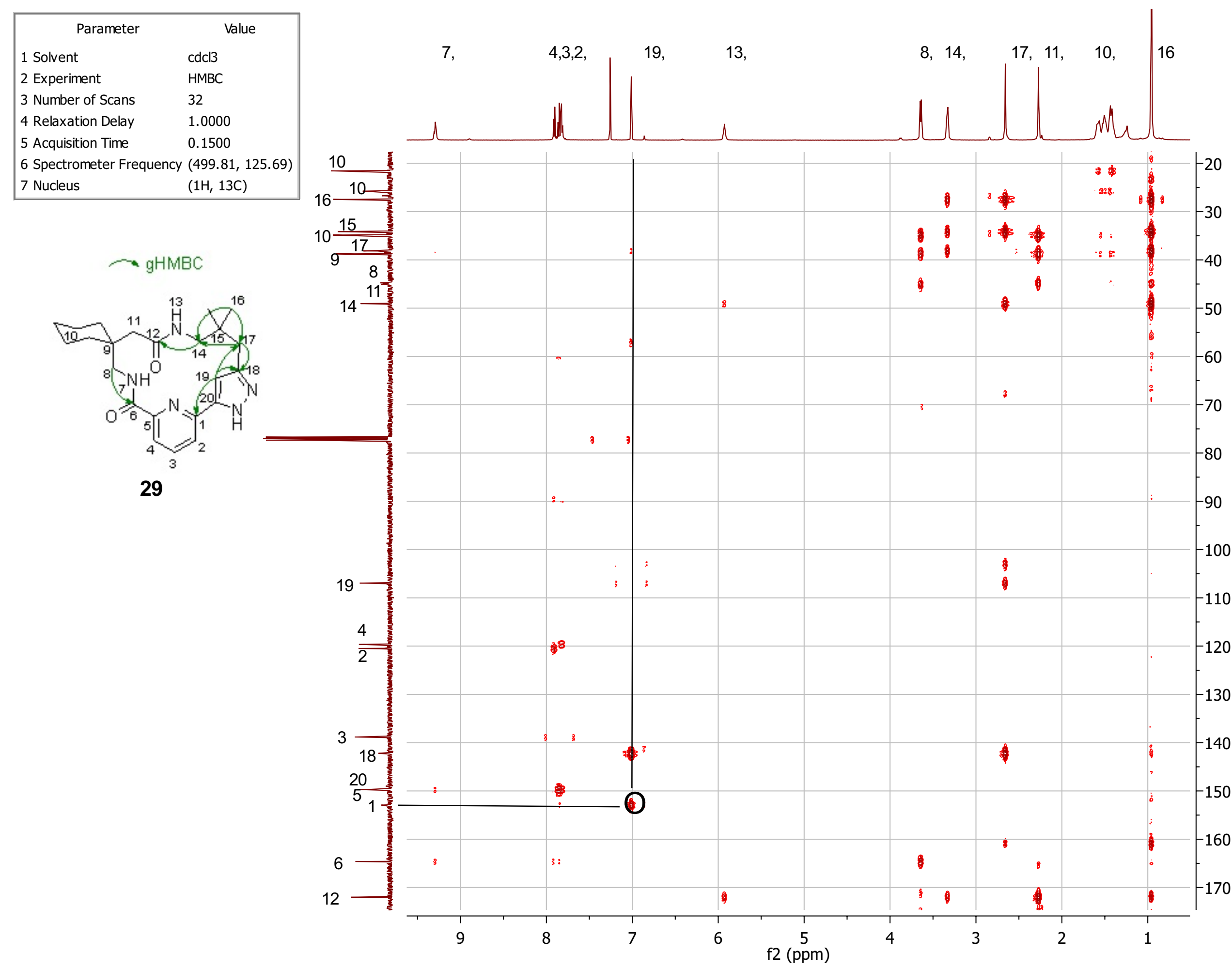




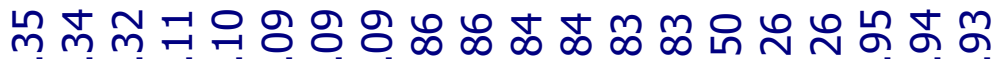

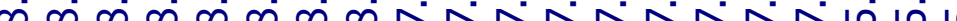

\begin{tabular}{|ll|}
\hline \multicolumn{1}{|c|}{ Parameter } & \multicolumn{1}{c|}{ Value } \\
1 Solvent & cdcl3 \\
2 Experiment & $1 \mathrm{D}$ \\
3 Number of Scans & 8 \\
4 Relaxation Delay & 1.0000 \\
5 Acquisition Time & 2.0447 \\
6 Spectrometer Frequency & 499.81 \\
7 Nucleus & $1 \mathrm{H}$ \\
\hline
\end{tabular}

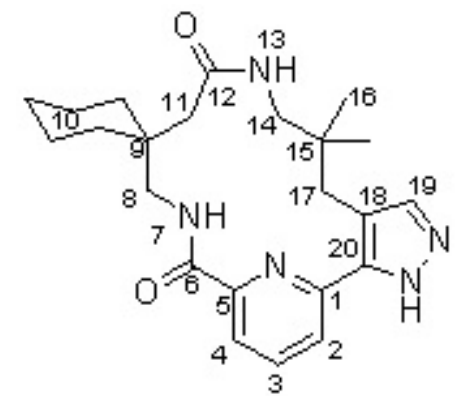

30

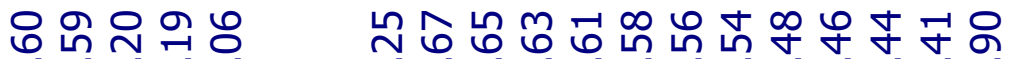

mmmm Niri-i-i-i-i-i

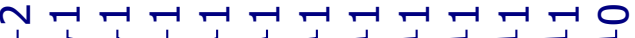




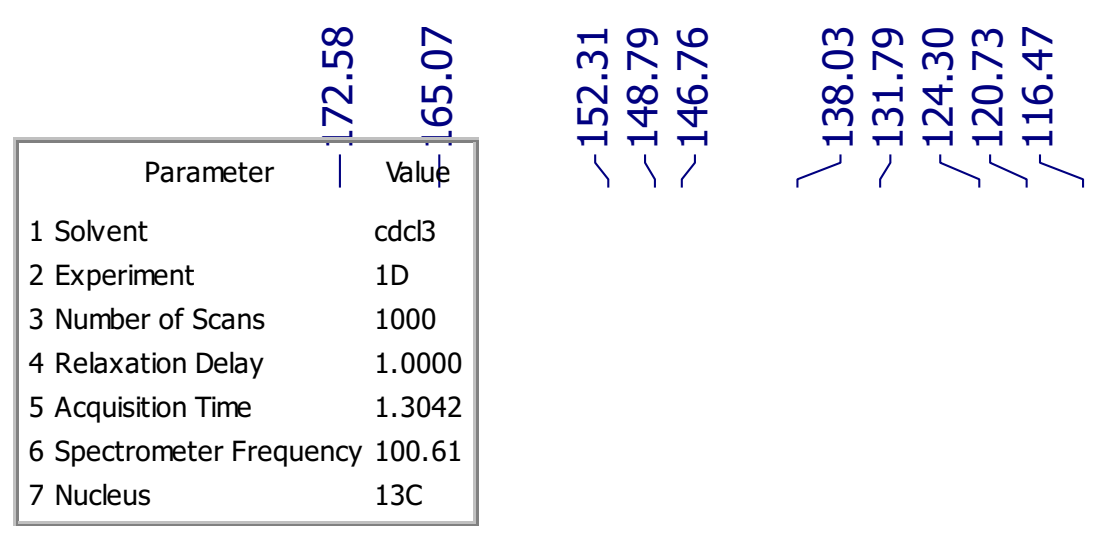

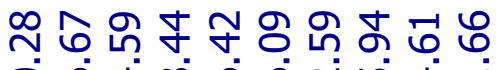
割守市

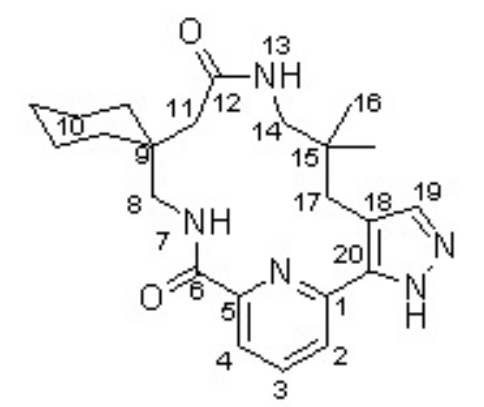

30

12, $6, \quad 5,1,20, \quad 3, \quad 19, \quad 2,4,18$

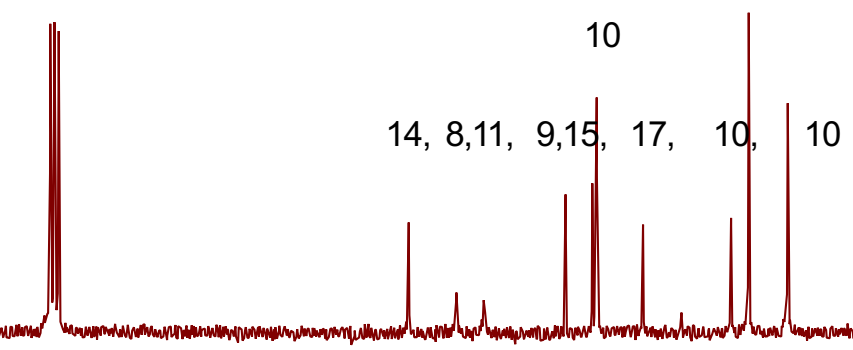

$80 \quad 170$

160

150

$140 \quad 130$

120

$110 \quad 100$ f1 (ppm)

\begin{tabular}{|c|c|c|c|c|c|c|c|c|c|}
\hline 90 & 80 & 70 & 60 & 50 & 40 & 30 & 20 & 10 & C \\
\hline
\end{tabular}




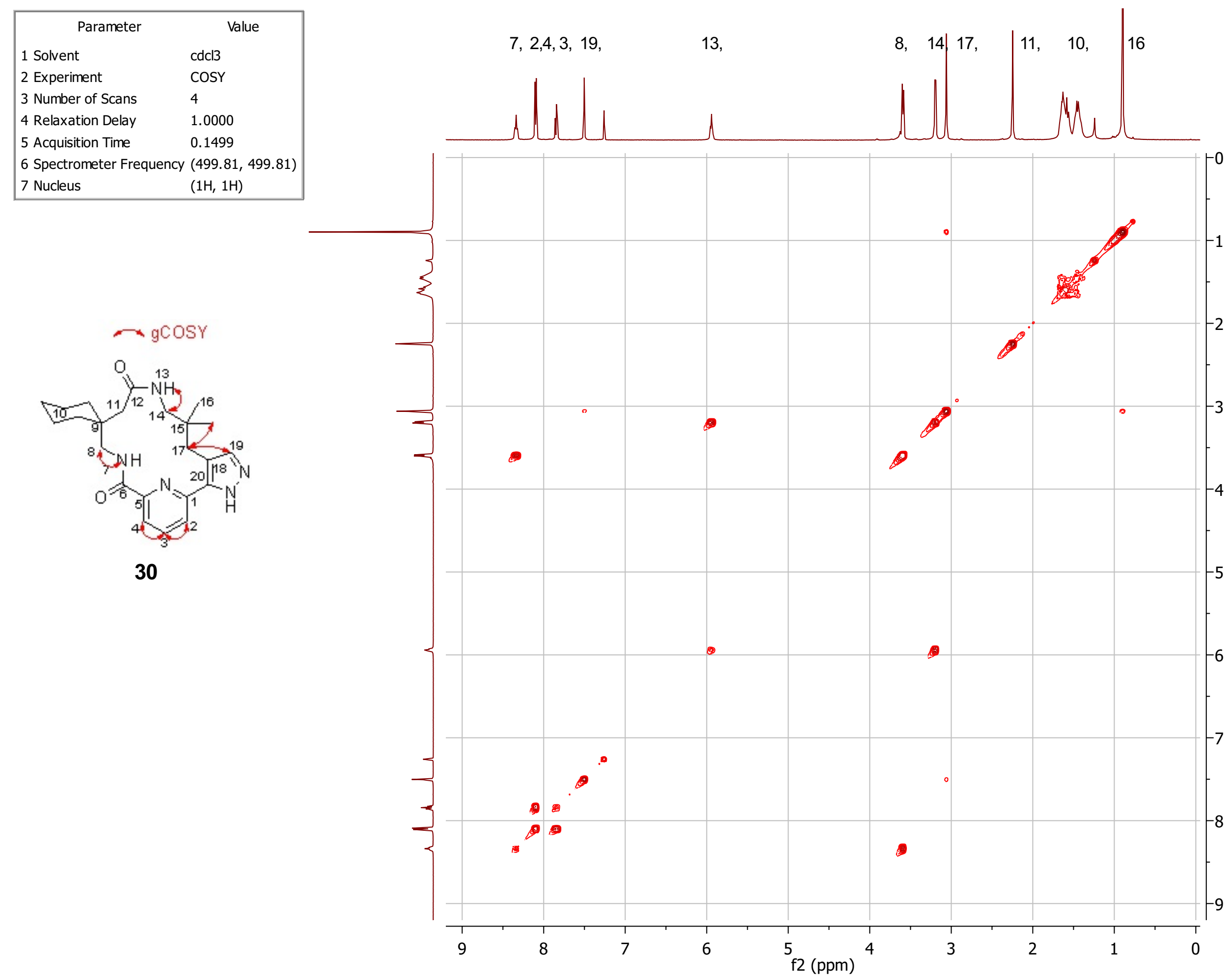

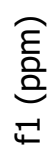




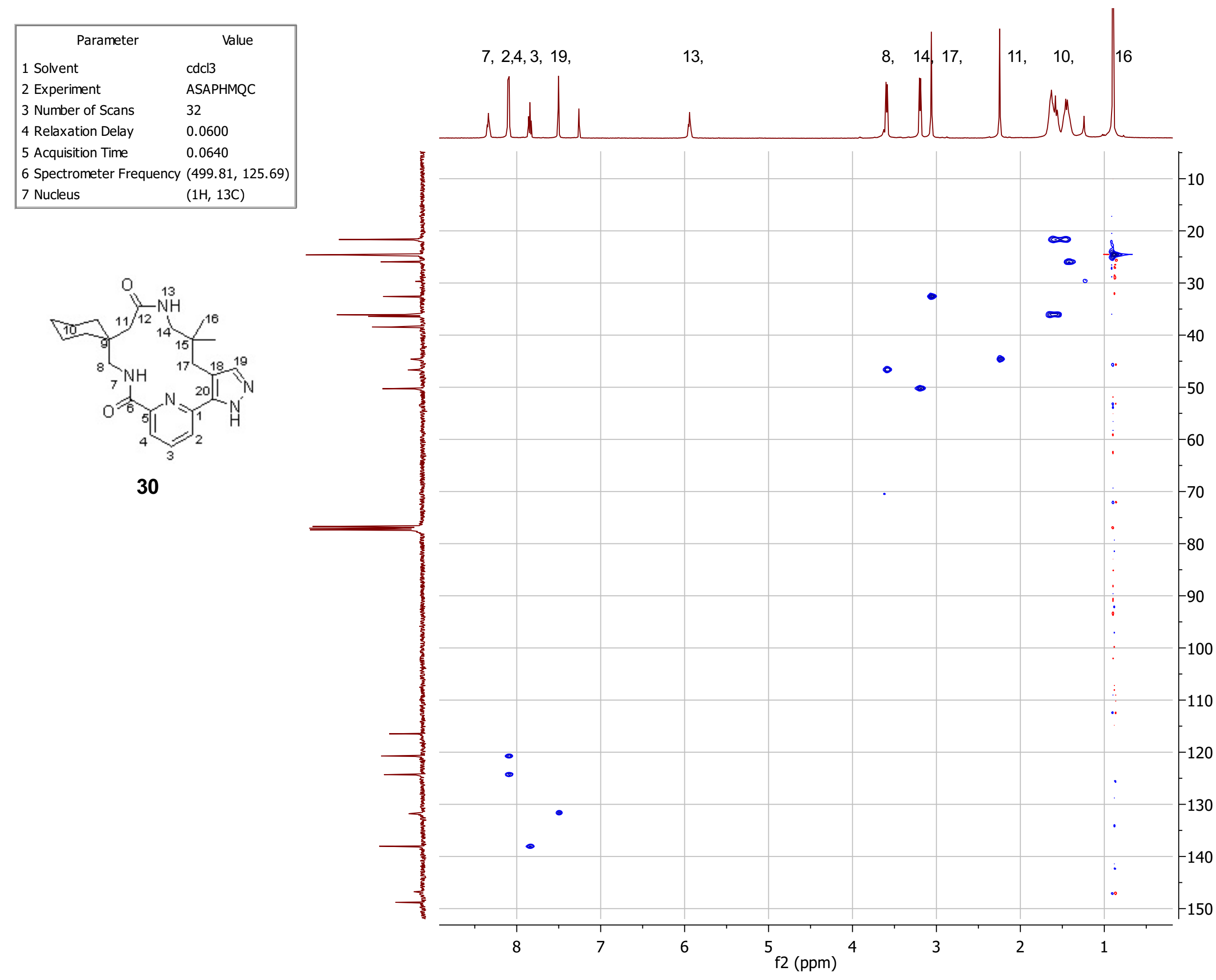




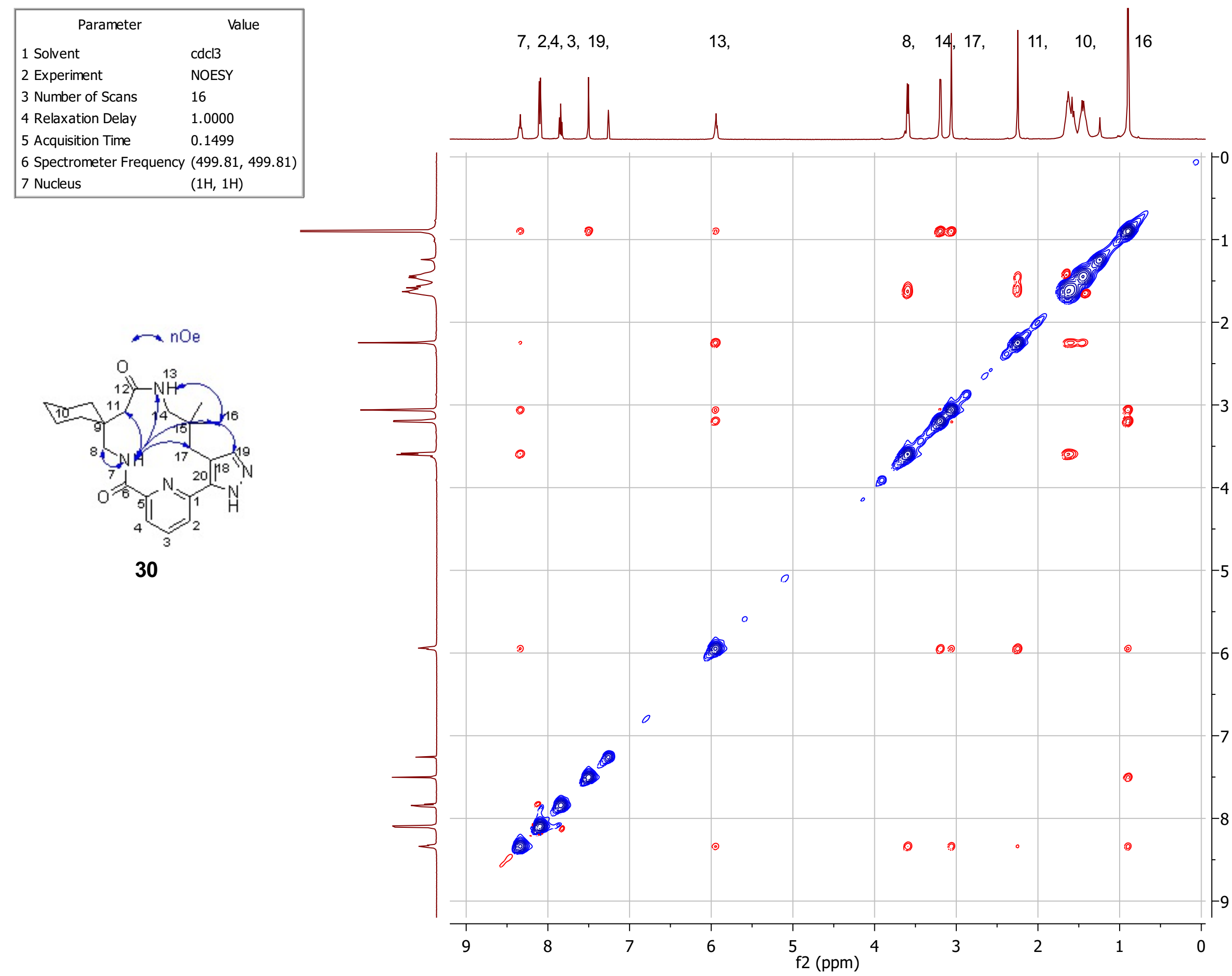

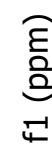




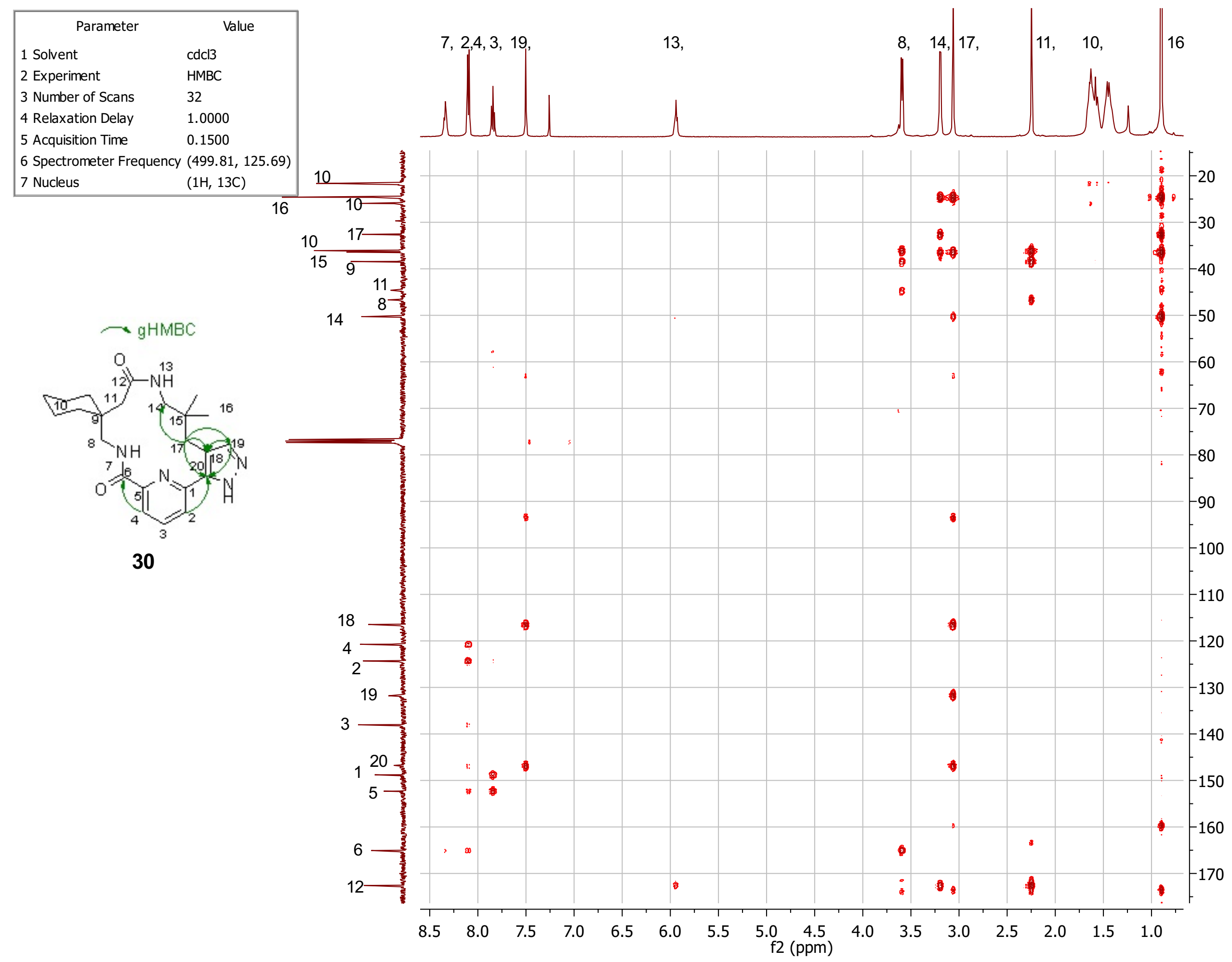




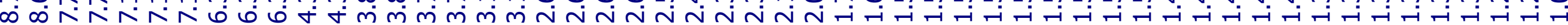

\begin{tabular}{|ll|}
\hline \multicolumn{1}{|c|}{ Parameter } & \multicolumn{1}{c|}{ Value } \\
1 Solvent & CD3OD/CDCl3 $=1: 1$ \\
2 Experiment & $1 \mathrm{D}$ \\
3 Number of Scans & 8 \\
4 Relaxation Delay & 1.0000 \\
5 Acquisition Time & 2.0447 \\
6 Spectrometer Frequency & 499.81 \\
7 Nucleus & $1 \mathrm{H}$ \\
\hline
\end{tabular}

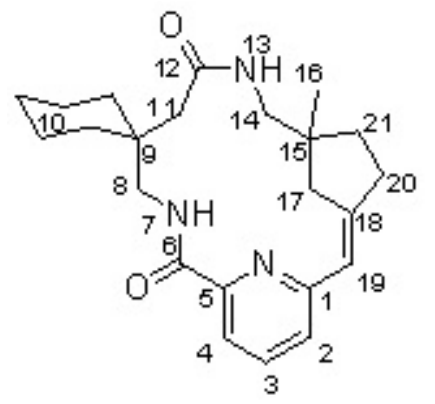

31

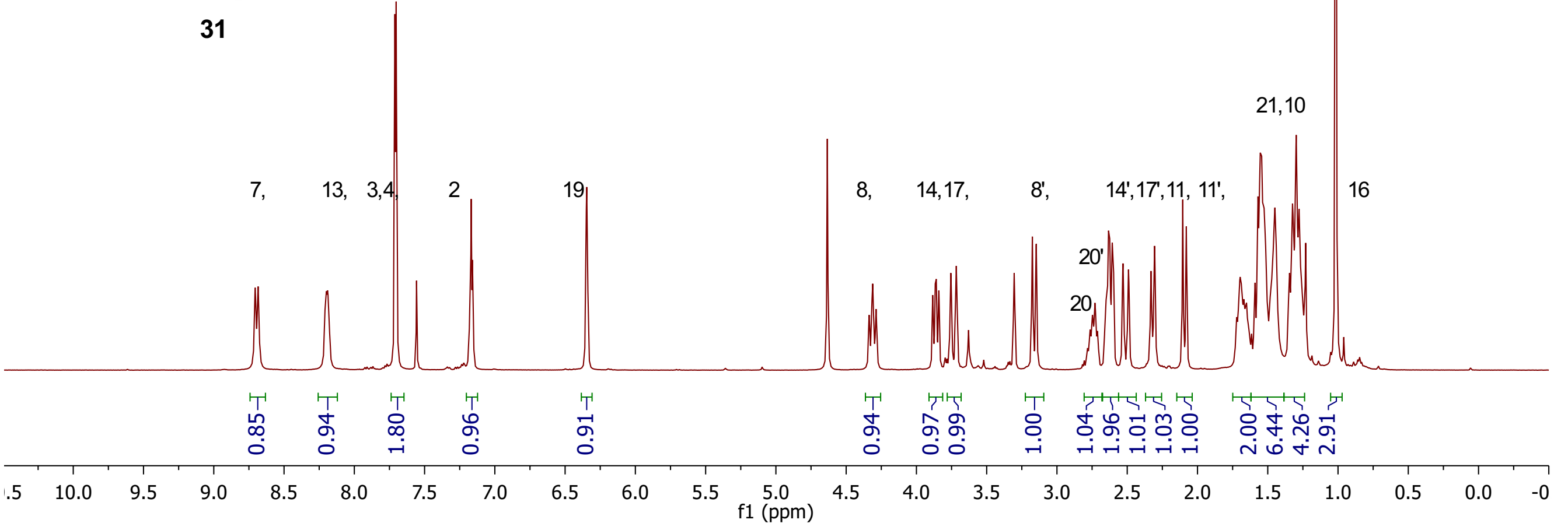




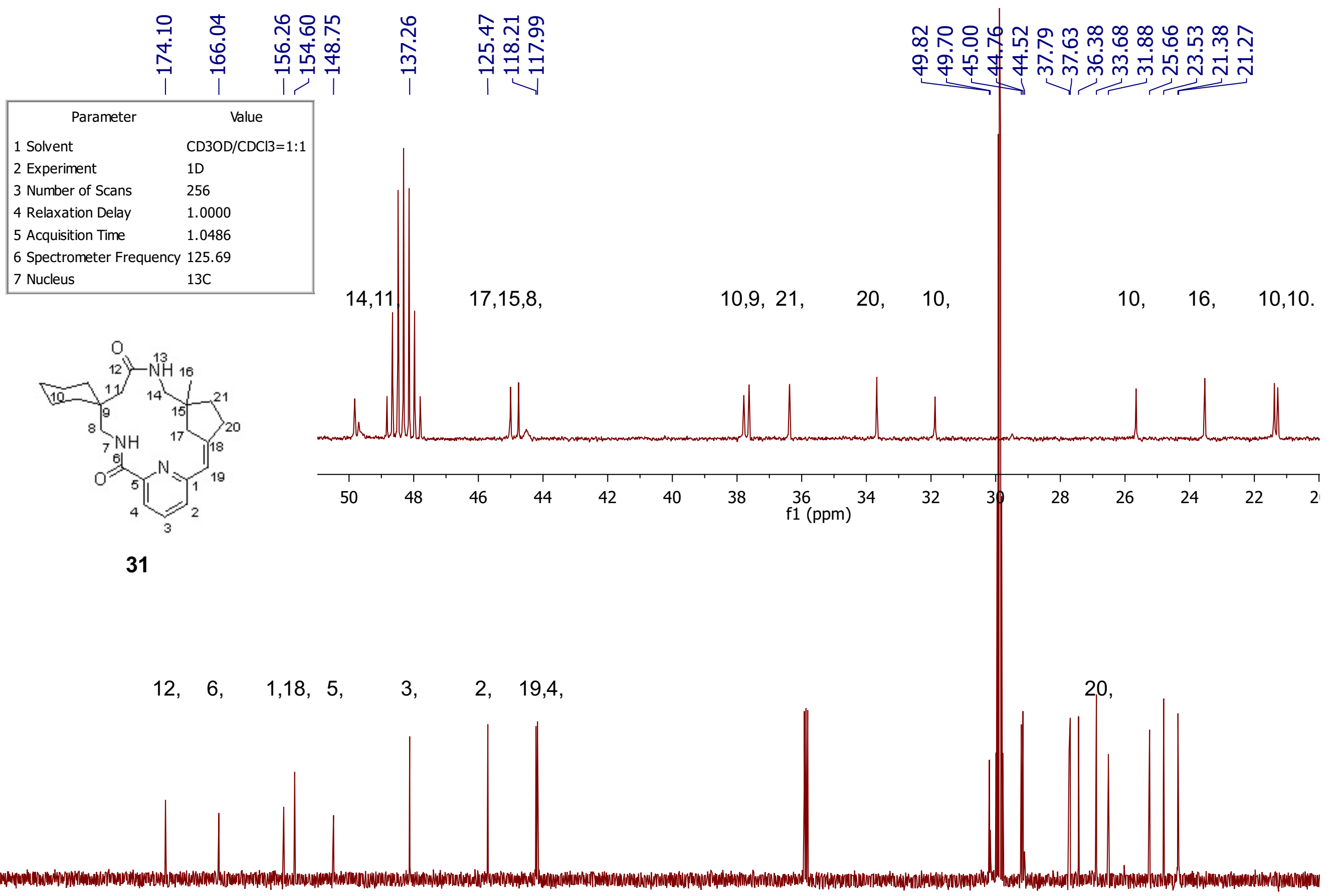

\begin{tabular}{|c|c|c|c|c|c|c|c|c|c|c|c|c|c|c|c|c|c|c|c|c|}
\hline 0 & 190 & 180 & 170 & 160 & 150 & 140 & 130 & 120 & 110 & $\begin{array}{c}100 \\
\mathrm{f} 1(\mathrm{ppm})\end{array}$ & 90 & 80 & 70 & 60 & 50 & 40 & 30 & 20 & 10 & c \\
\hline
\end{tabular}




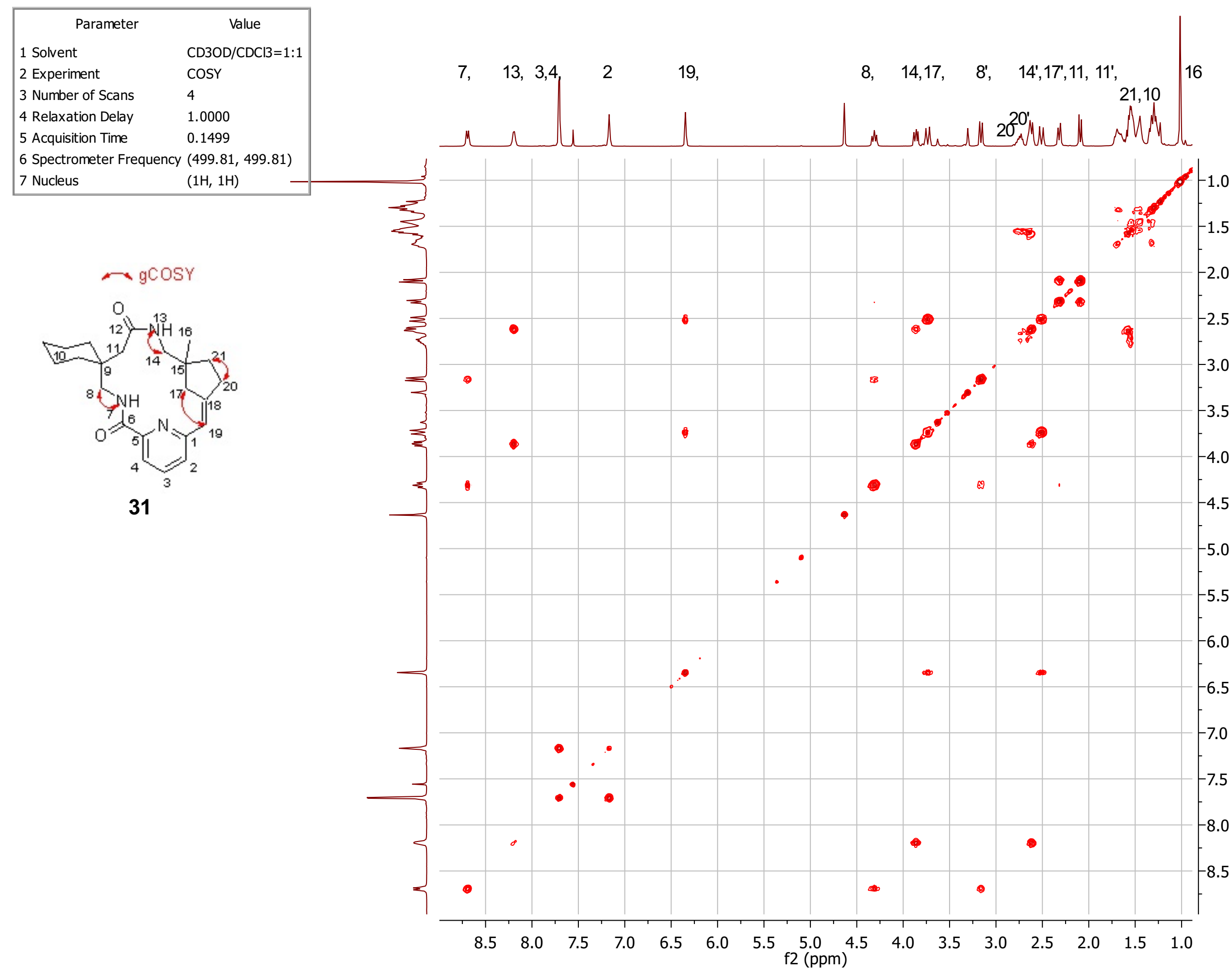

$\underbrace{\overparen{\vdots}}_{\bar{L}}$ 


\begin{tabular}{|ll|}
\hline \multicolumn{1}{|c|}{ Parameter } & \multicolumn{1}{c|}{ Value } \\
1 Solvent & CD3OD/CDCl3 $=1: 1$ \\
2 Experiment & HSQC \\
3 Number of Scans & 16 \\
4 Relaxation Delay & 1.0000 \\
5 Acquisition Time & 0.1500 \\
6 Spectrometer Frequency & $(499.81,125.69)$ \\
7 Nucleus & $(1 \mathrm{H}, 13 \mathrm{C})$ \\
\hline
\end{tabular}
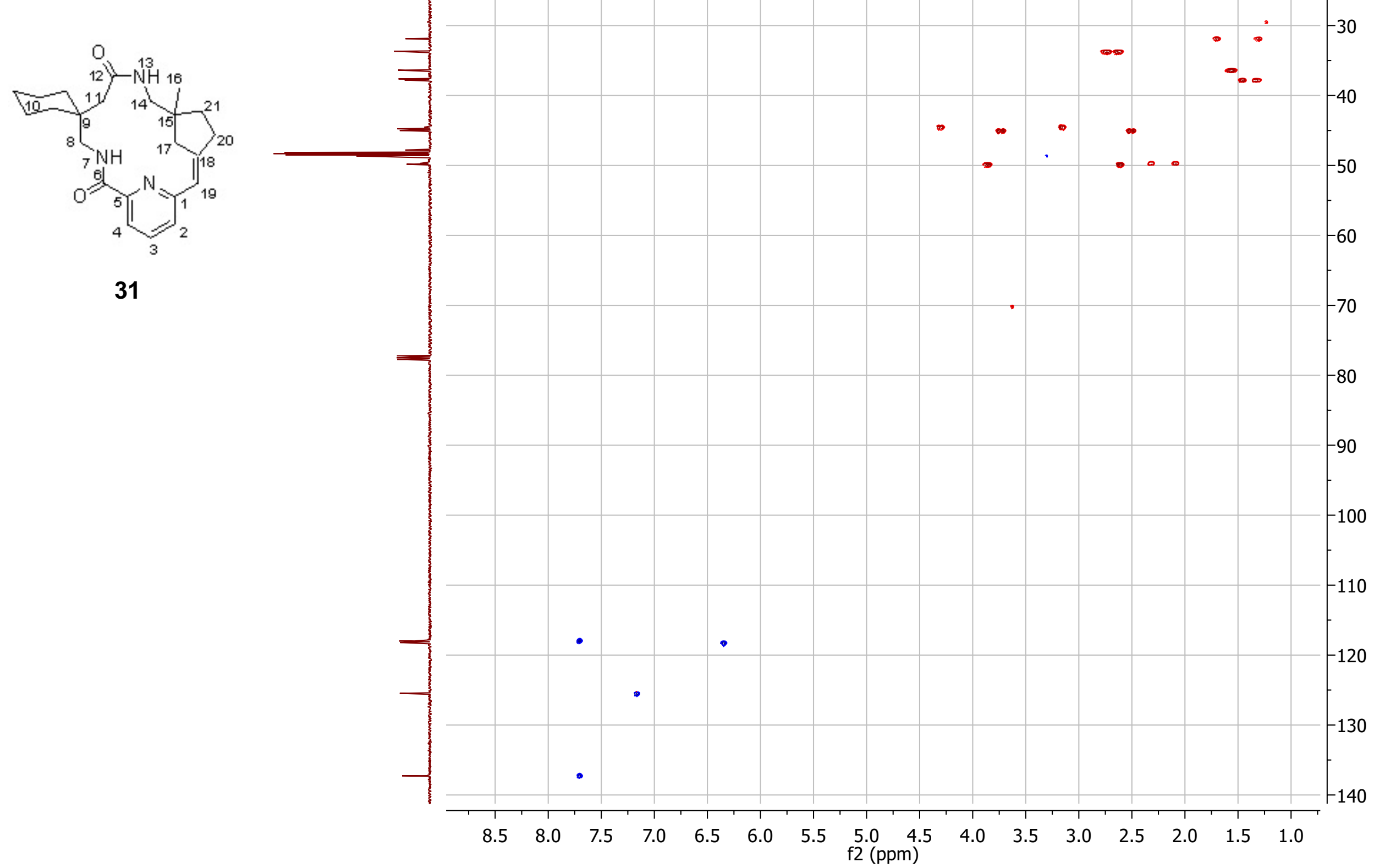


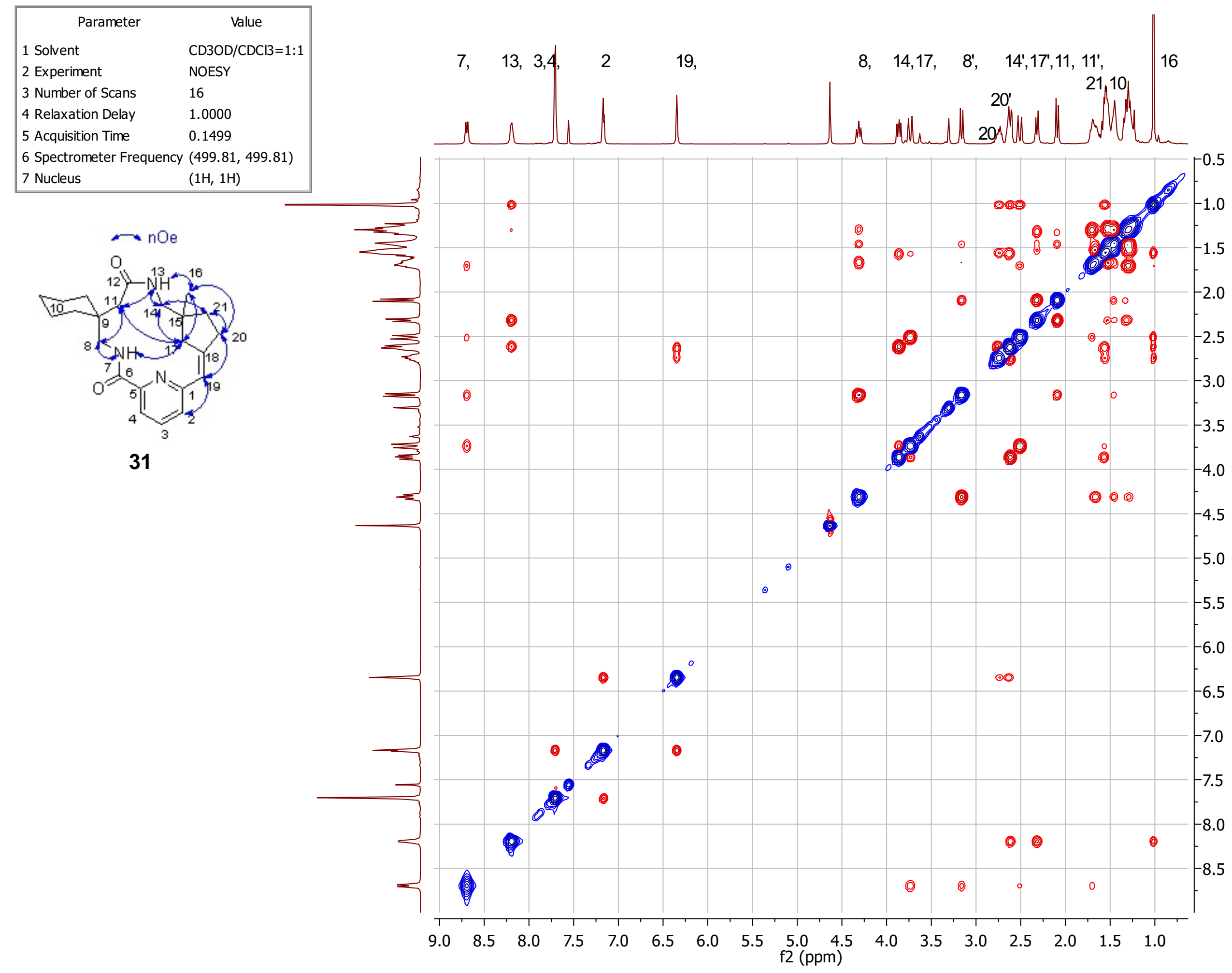

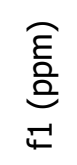




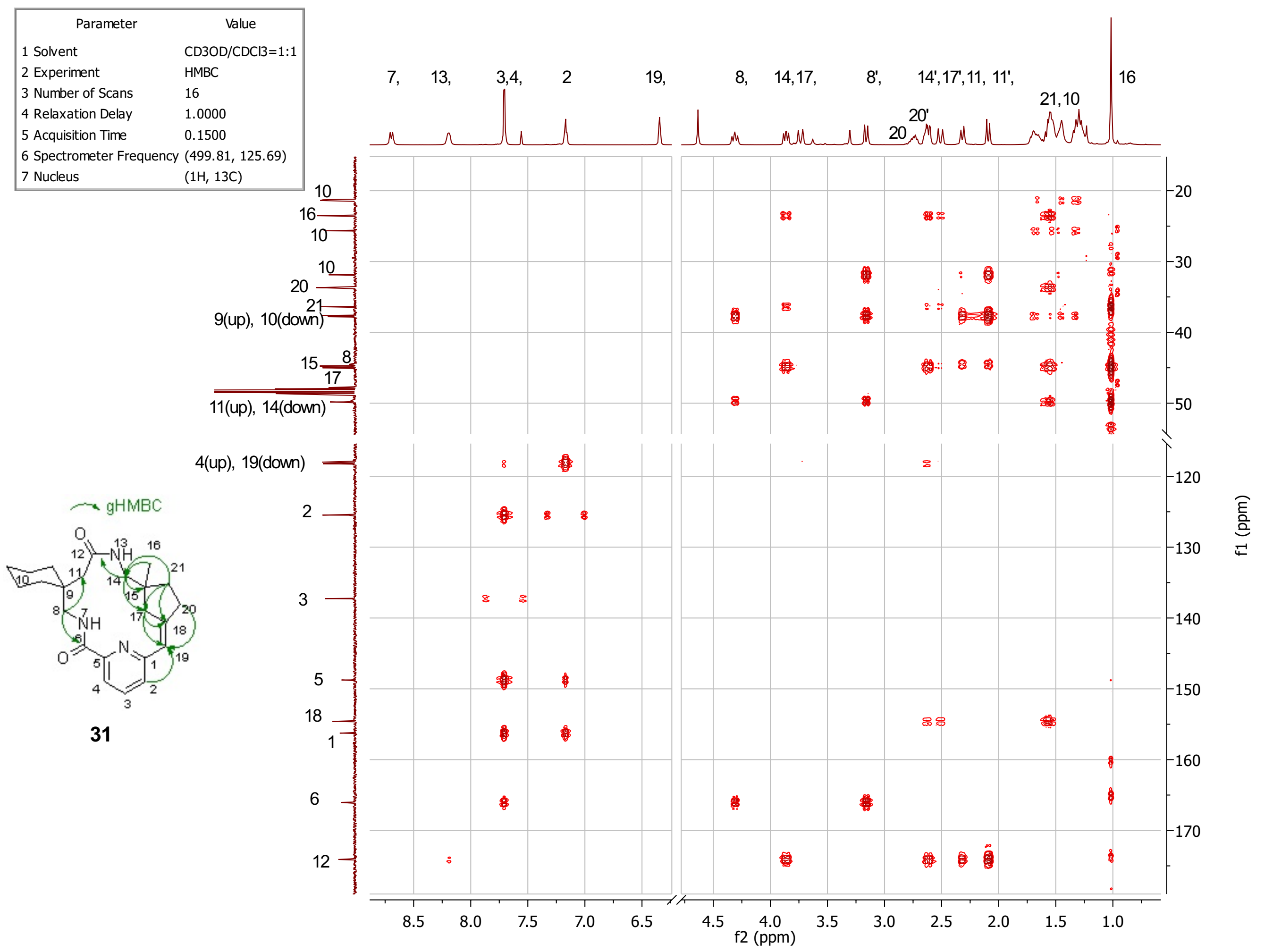




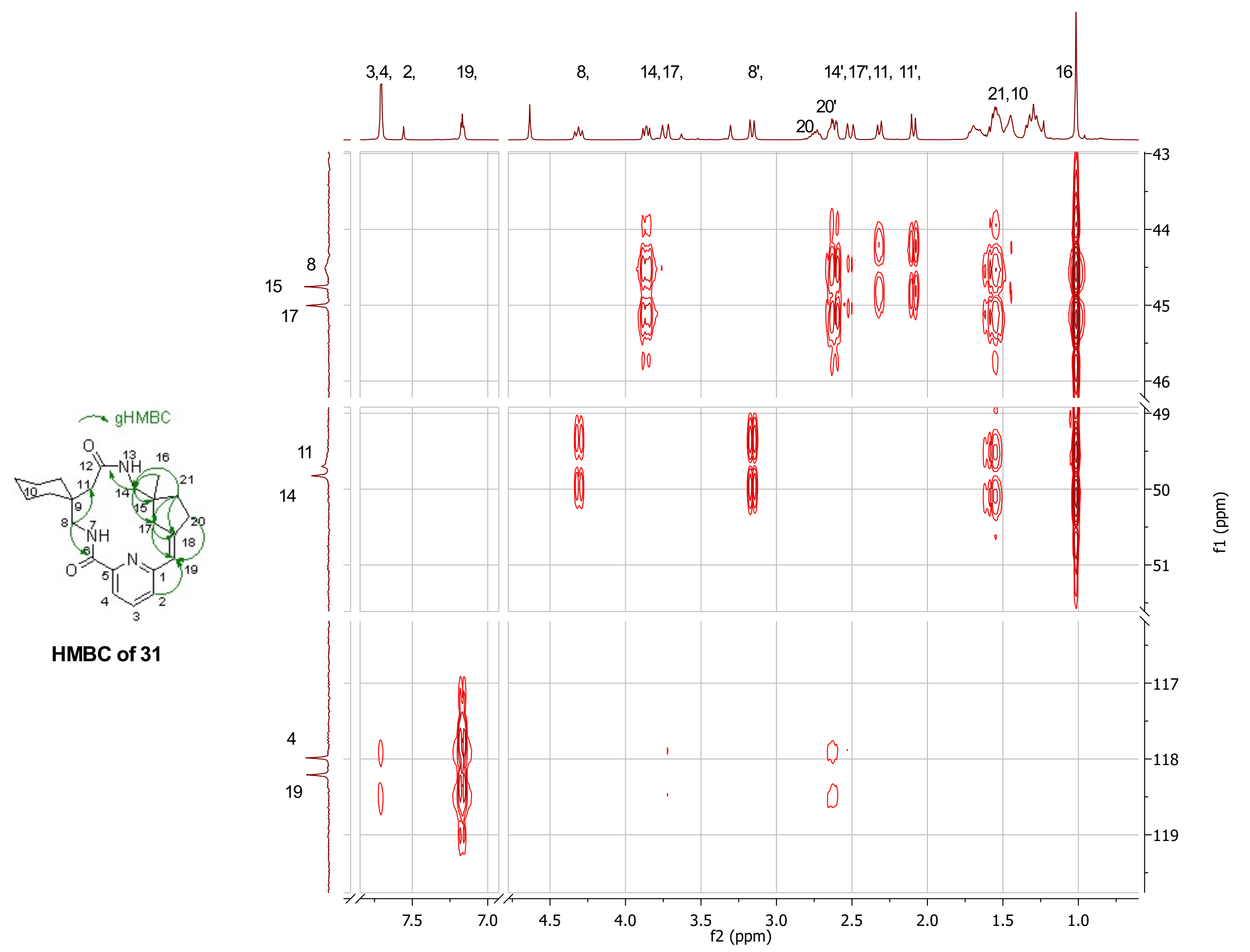




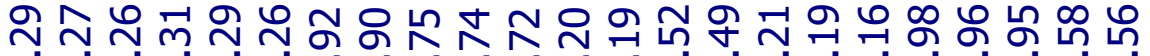

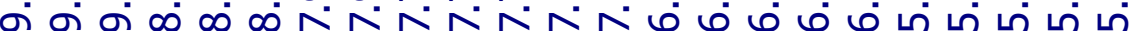

\begin{tabular}{|c|c|}
\hline Parameter & Value \\
\hline 1 Solvent & cdcl3 \\
\hline 2 Experiment & $1 \mathrm{D}$ \\
\hline 3 Number of Scans & 8 \\
\hline 4 Relaxation Delay & 1.0000 \\
\hline 5 Acquisition Time & 2.0447 \\
\hline 6 Spectrometer Frequenc & 499.81 \\
\hline 7 Nucleus & $1 \mathrm{H}$ \\
\hline
\end{tabular}

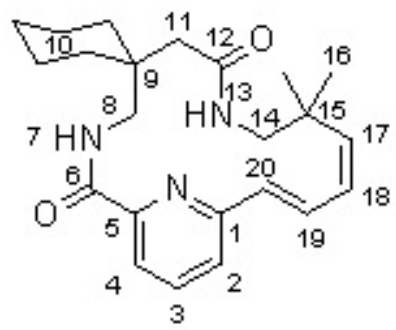

32

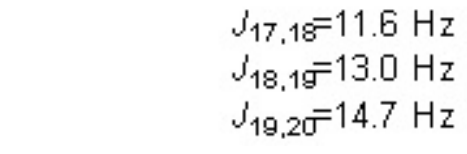

$J_{17,18}=11.6 \mathrm{~Hz}$

$J_{18,19}=13.0 \mathrm{~Hz}$

$J_{19,20}=14.7 \mathrm{~Hz}$

$\widehat{6} ซ \widetilde{6}$ $m m m$

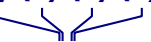

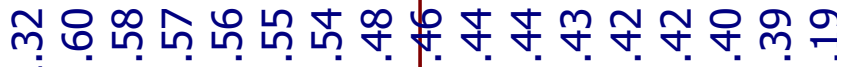
N

7,

$19,4,3$

2 ,

$20,18,13,17$,

8, 14,

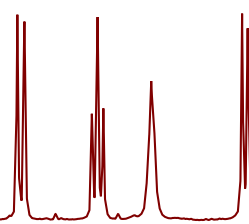

T

다 바맘

'T

祭品占

'T

สิ สู สู

ஸి 응

००० के

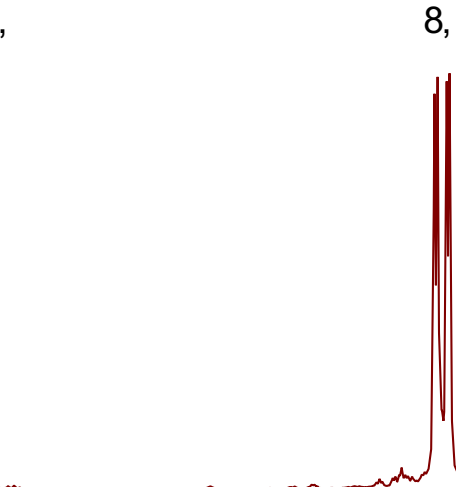

10

@

8.07 .5

7.0

6.5

$6.0 \quad 5.5$

(ppm)

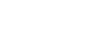




\begin{tabular}{|ll|}
\hline \multicolumn{1}{|c|}{ Parameter } & Value \\
1 Solvent & cdcl3 \\
2 Experiment & $1 \mathrm{D}$ \\
3 Number of Scans & 7200 \\
4 Relaxation Delay & 1.0000 \\
5 Acquisition Time & 1.0486 \\
6 Spectrometer Frequency & 125.69 \\
7 Nucleus & $13 \mathrm{C}$ \\
\hline
\end{tabular}

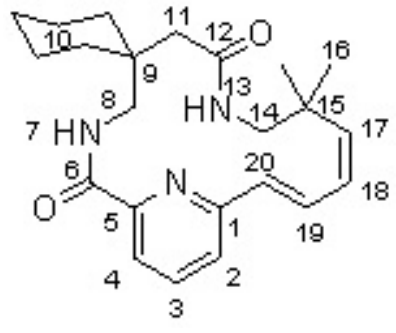

32

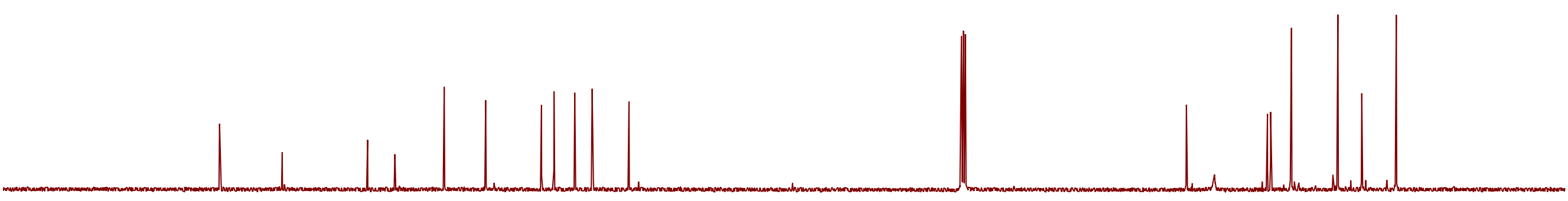

170

160

150

140

130

120

$110 \quad 100$ 1 (

$90 \quad 80$

$80-70$

$70 \quad 60$

60

50

30

$\begin{array}{lll}20 & 10 & 0\end{array}$




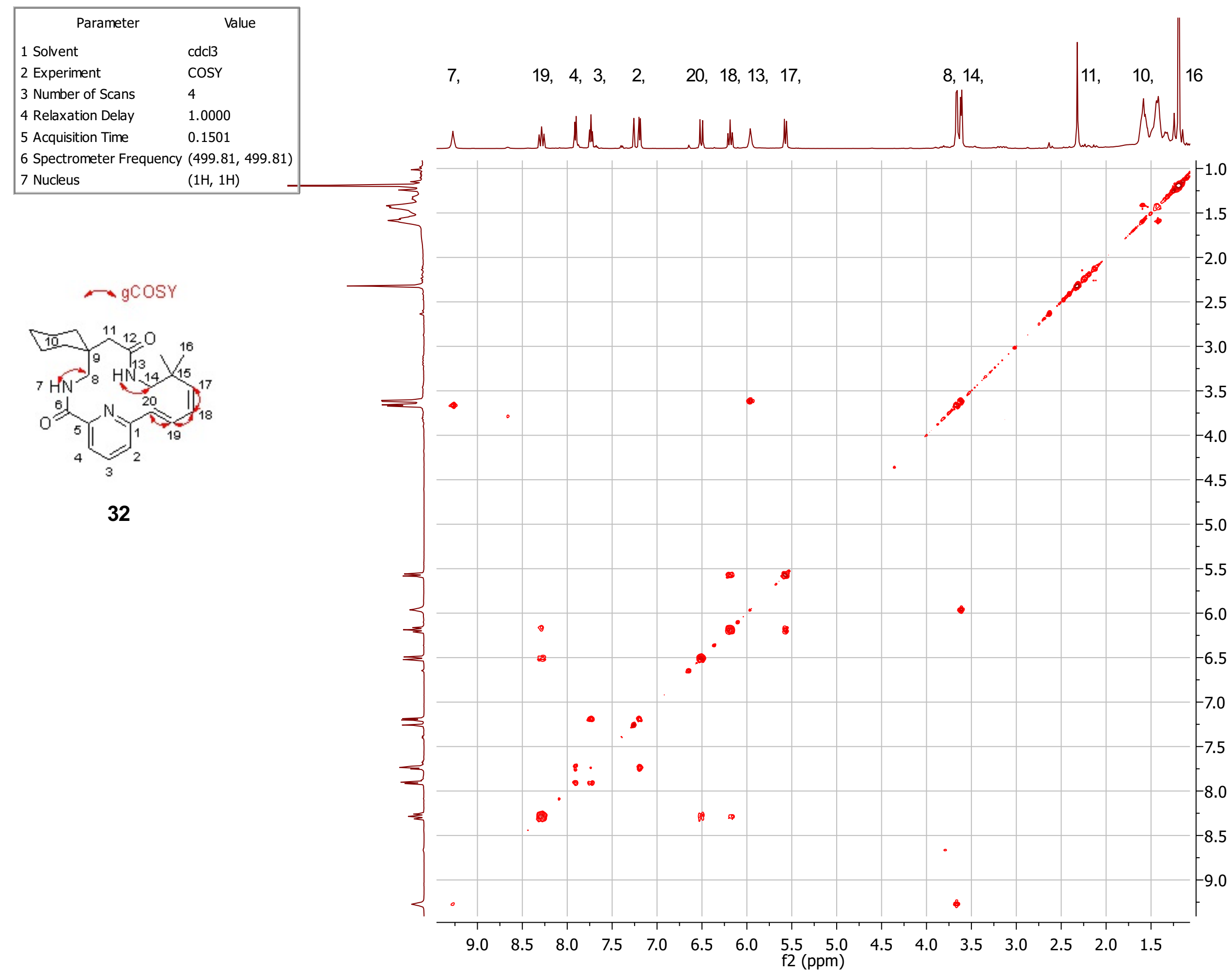

$\underbrace{\widehat{E}}_{\bar{l}}$ 


\begin{tabular}{|ll|}
\hline \multicolumn{1}{|c|}{ Parameter } & \multicolumn{1}{c|}{ Value } \\
1 Solvent & cdcl3 \\
2 Experiment & ASAPHMQC \\
3 Number of Scans & 32 \\
4 Relaxation Delay & 0.0600 \\
5 Acquisition Time & 0.0640 \\
6 Spectrometer Frequency & $(499.81,125.69)$ \\
7 Nucleus & $(1 \mathrm{H}, 13 \mathrm{C})$ \\
\hline
\end{tabular}

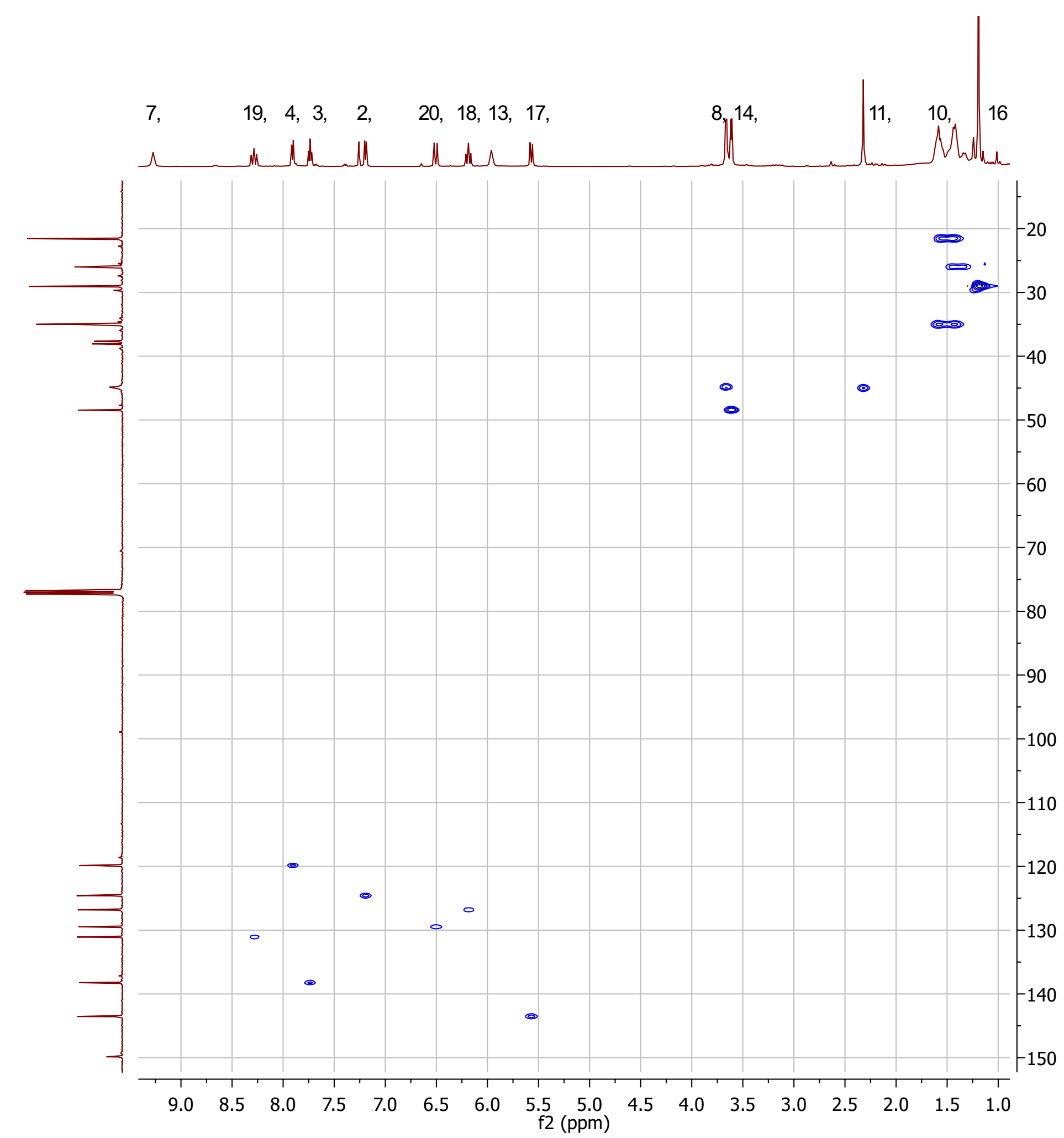




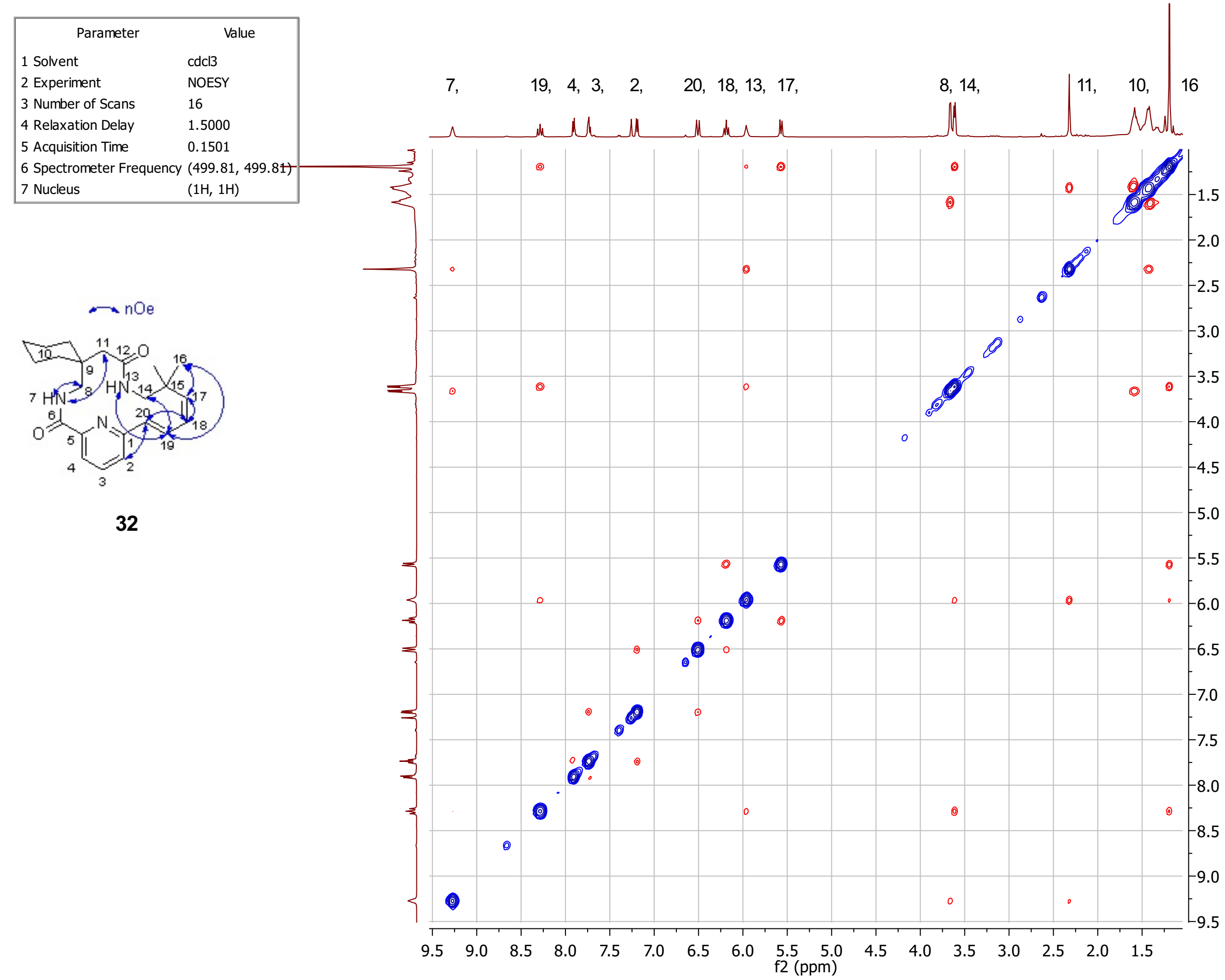




\begin{tabular}{|ll|}
\hline \multicolumn{1}{|c|}{ Parameter } & \multicolumn{1}{c|}{ Value } \\
1 Solvent & cdcl3 \\
2 Experiment & HMBC \\
3 Number of Scans & 32 \\
4 Relaxation Delay & 1.0000 \\
5 Acquisition Time & 0.1500 \\
6 Spectrometer Frequency & $(499.81,125.69)$ \\
7 Nucleus & $(1 \mathrm{H}, 13 \mathrm{C})$ \\
\hline
\end{tabular}

gHMBC

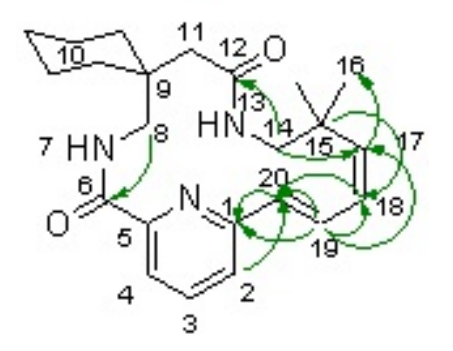

32

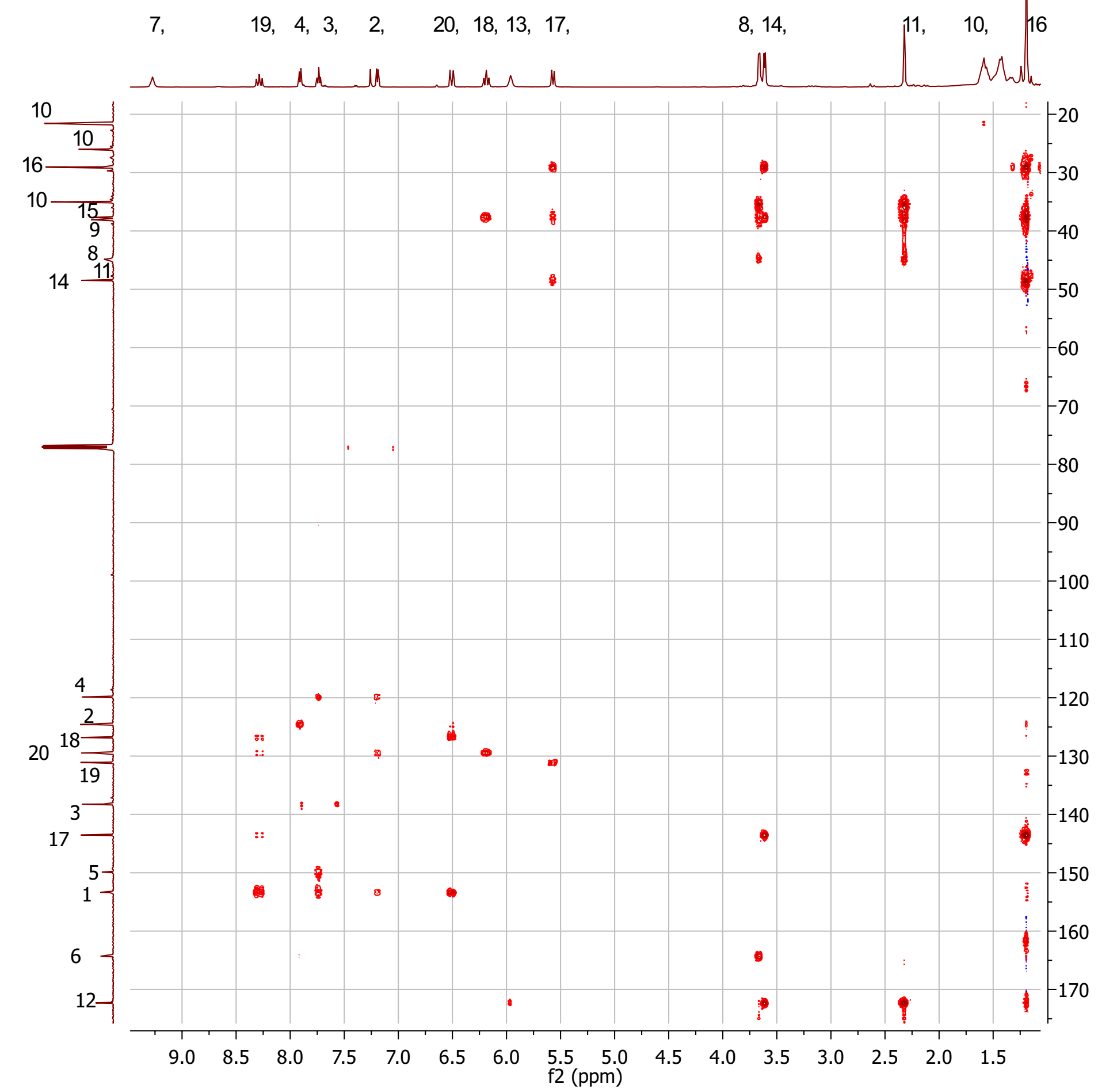

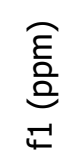




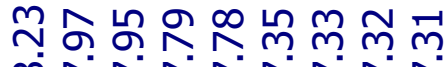

$\infty N N N$ NNN

1 Solvent
2 Experiment
3 Number of Scans
4 Relaxation Delay
5 Acquisition Time
6 Spectrometer Frequen
7 Nucleus

Value

cdcl3

1D

8

1.0000

2.5592

400.09

$38 b$

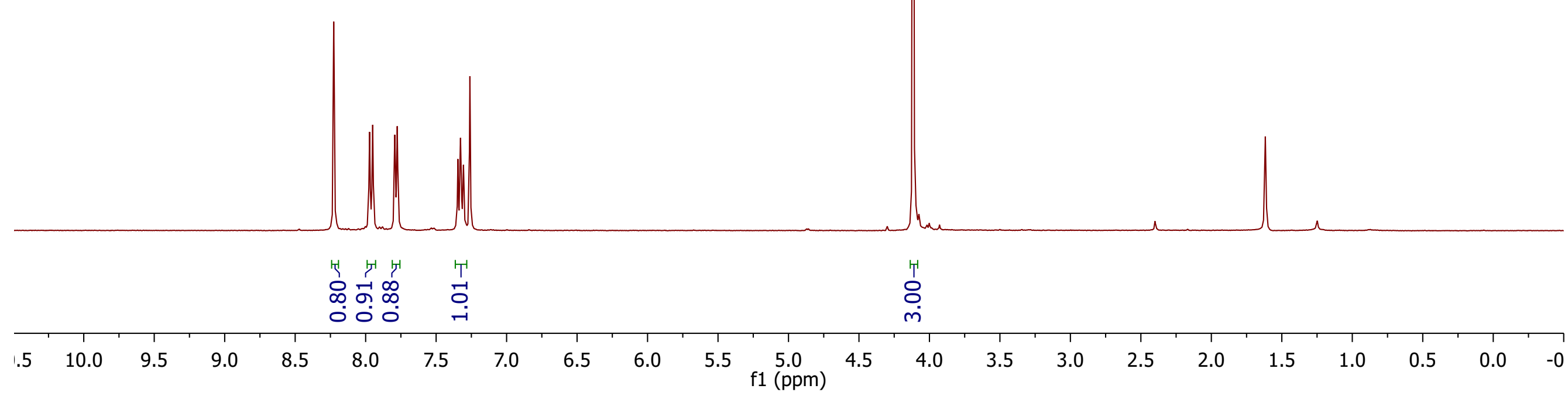




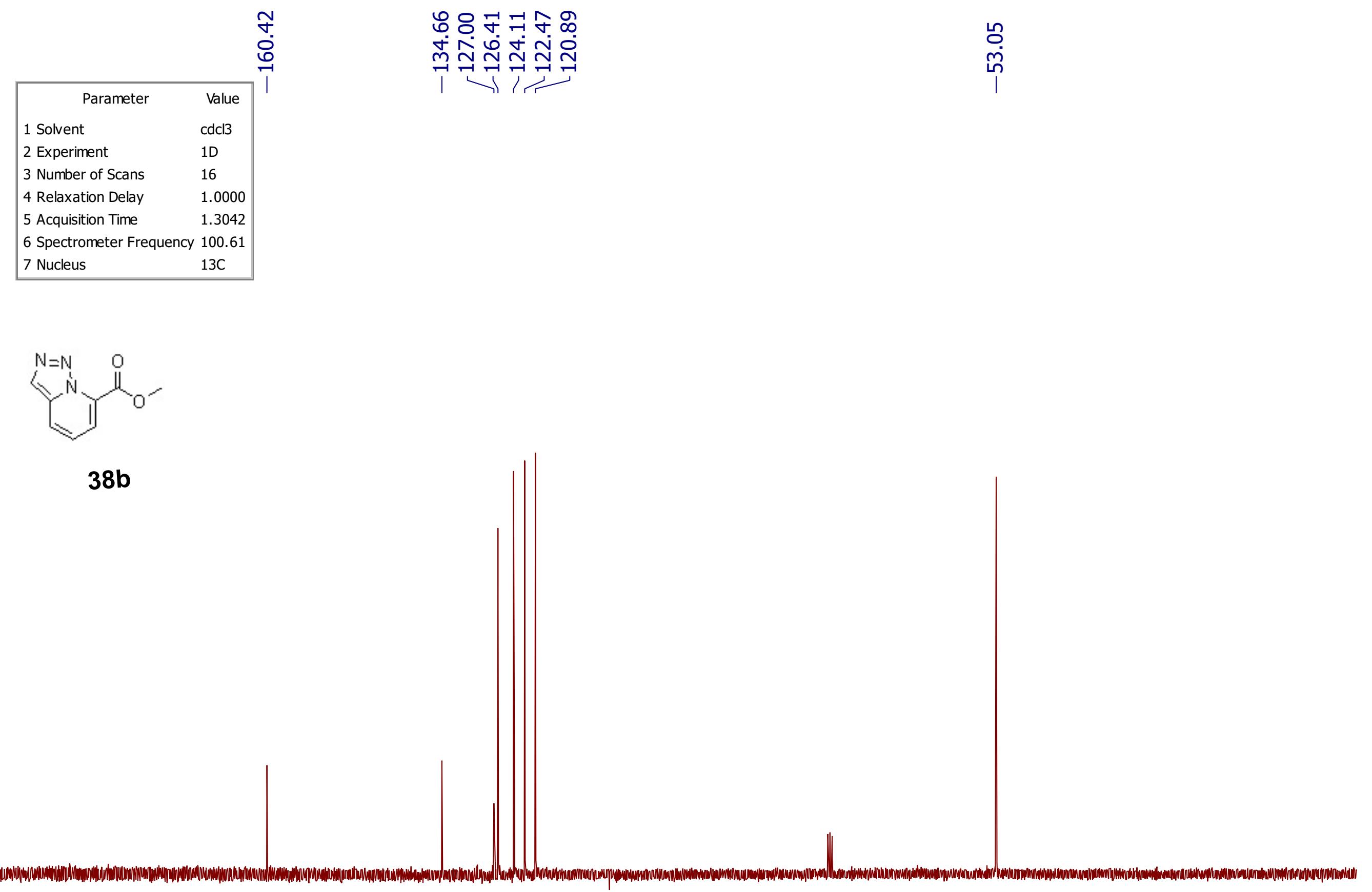

3 Number of Scans

5 Acquisition Time

6 Spectrometer Frequency

$38 b$

$190 \quad 180$




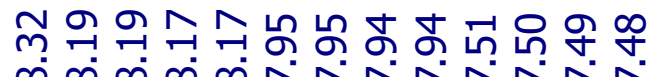

$\infty \infty_{\infty}^{\infty} \infty \infty^{\infty} \mathbf{N} \hat{N} \hat{N}$

\begin{tabular}{|c|c|}
\hline Parameter & Value \\
\hline 1 Solvent & cd3od \\
\hline 2 Experiment & 1D \\
\hline 3 Number of Scans & 8 \\
\hline 4 Relaxation Delay & 1.0000 \\
\hline 5 Acquisition Time & 2.0447 \\
\hline \multicolumn{2}{|c|}{6 Spectrometer Frequency 499.81} \\
\hline 7 Nucleus & $1 \mathrm{H}$ \\
\hline
\end{tabular}

$\underbrace{N}_{1}=\mathrm{N}$

38c

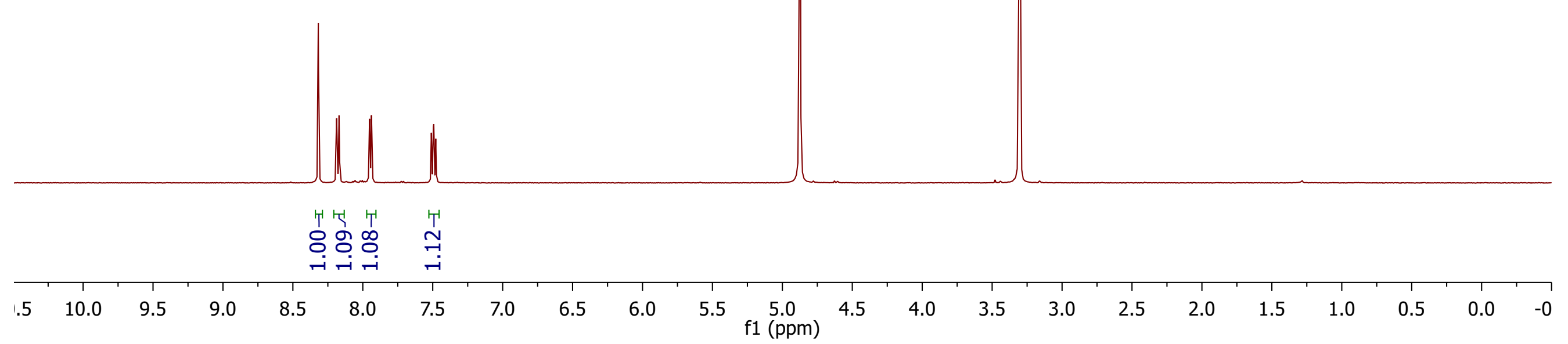




\begin{tabular}{|ll|}
\hline \multicolumn{1}{|c|}{ Parameter } & \multicolumn{1}{c|}{ Value } \\
1 Solvent & dmso \\
2 Experiment & 1D \\
3 Number of Scans & 128 \\
4 Relaxation Delay & 1.0000 \\
5 Acquisition Time & 1.3042 \\
6 Spectrometer Frequency & 100.61 \\
7 Nucleus & $13 \mathrm{C}$ \\
\hline
\end{tabular}

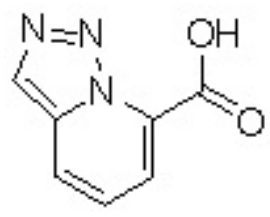

38c

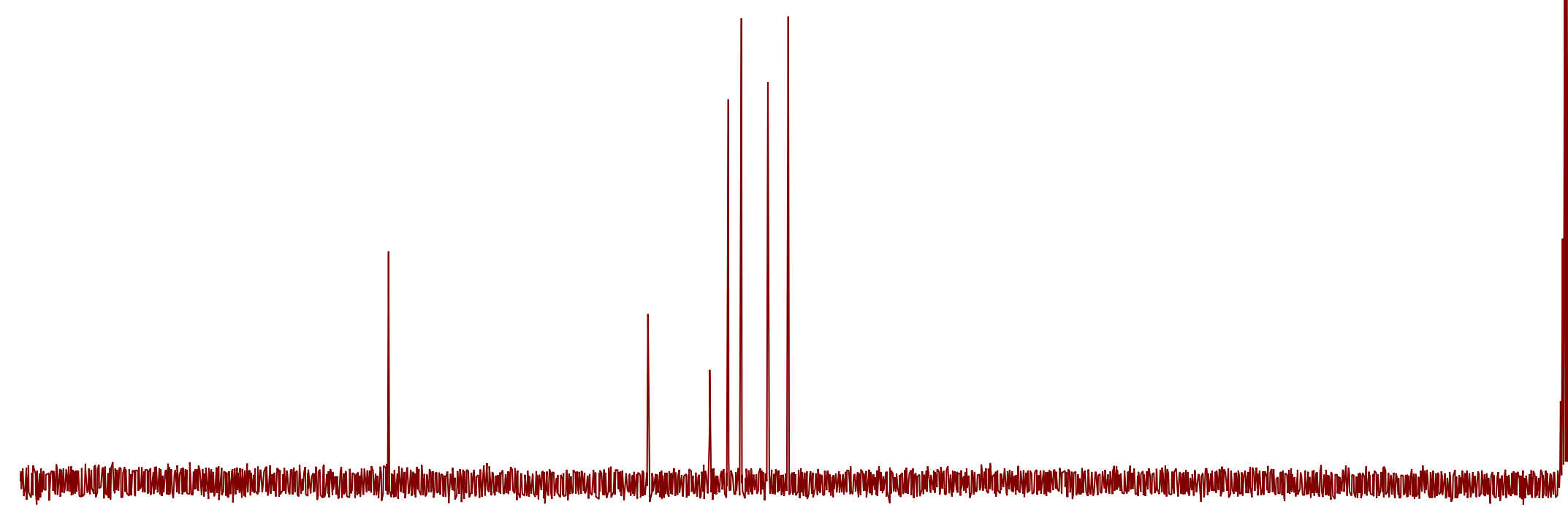

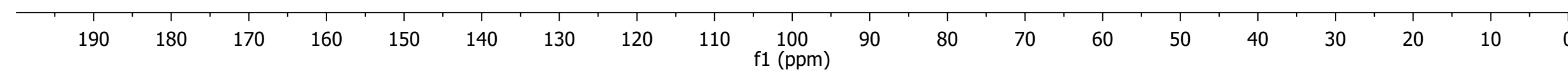


車 o

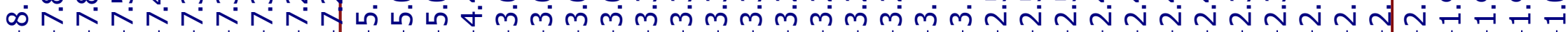

\begin{tabular}{|ll|}
\hline \multicolumn{1}{|c|}{ Parameter } & \multicolumn{1}{c|}{ Value } \\
1 Solvent & cdcl3 \\
2 Experiment & $1 \mathrm{D}$ \\
3 Number of Scans & 8 \\
4 Relaxation Delay & 1.0000 \\
5 Acquisition Time & 2.0447 \\
6 Spectrometer Frequency & 499.81 \\
7 Nucleus & $1 \mathrm{H}$ \\
\hline
\end{tabular}

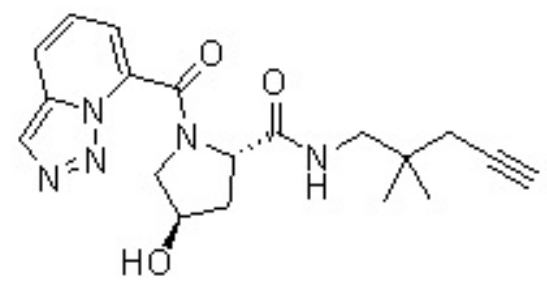

38
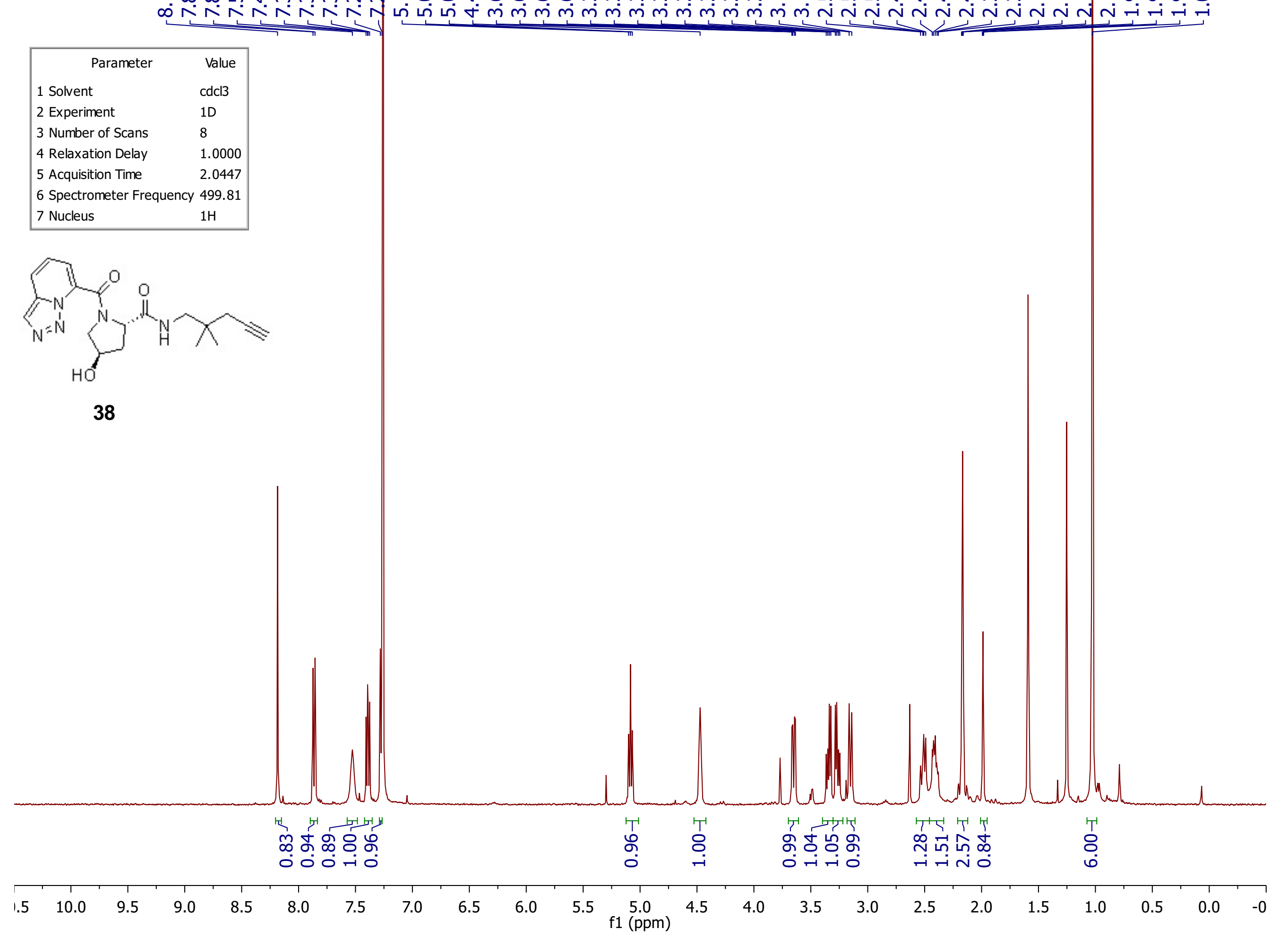


\begin{tabular}{|l|l|}
\hline \multicolumn{1}{|c|}{ Parameter } & Value \\
1 Solvent & cdc13 \\
2 Experiment & $1 \mathrm{D}$ \\
3 Number of Scans & 512 \\
4 Relaxation Delay & 1.0000 \\
5 Acquisition Time & 1.3042 \\
6 Spectrometer Frequency 100.61 \\
7 Nucleus & $13 \mathrm{C}$ \\
\hline
\end{tabular}

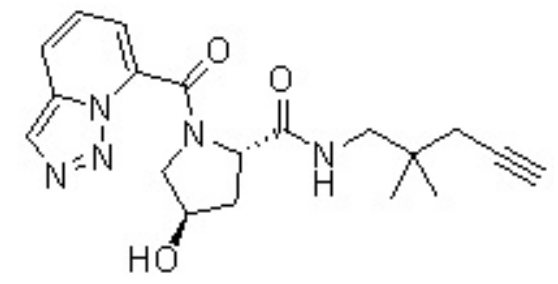

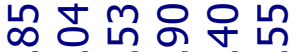

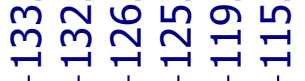

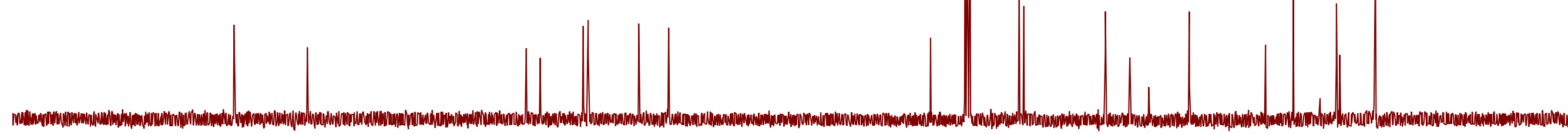

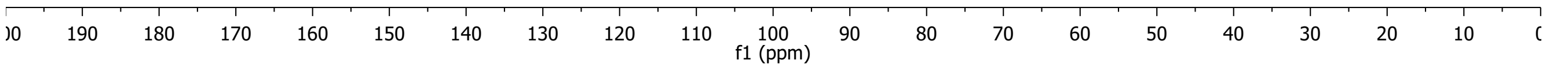


ஜ

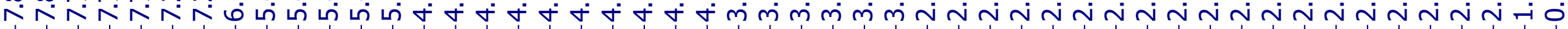

\begin{tabular}{|ll|}
\hline \multicolumn{1}{|c|}{ Parameter } & \multicolumn{1}{c|}{ Value } \\
1 Solvent & cdcl3 \\
2 Experiment & $1 \mathrm{D}$ \\
3 Number of Scans & 8 \\
4 Relaxation Delay & 1.0000 \\
5 Acquisition Time & 2.5559 \\
6 Spectrometer Frequency & 400.01 \\
7 Nucleus & $1 \mathrm{H}$ \\
\hline
\end{tabular}
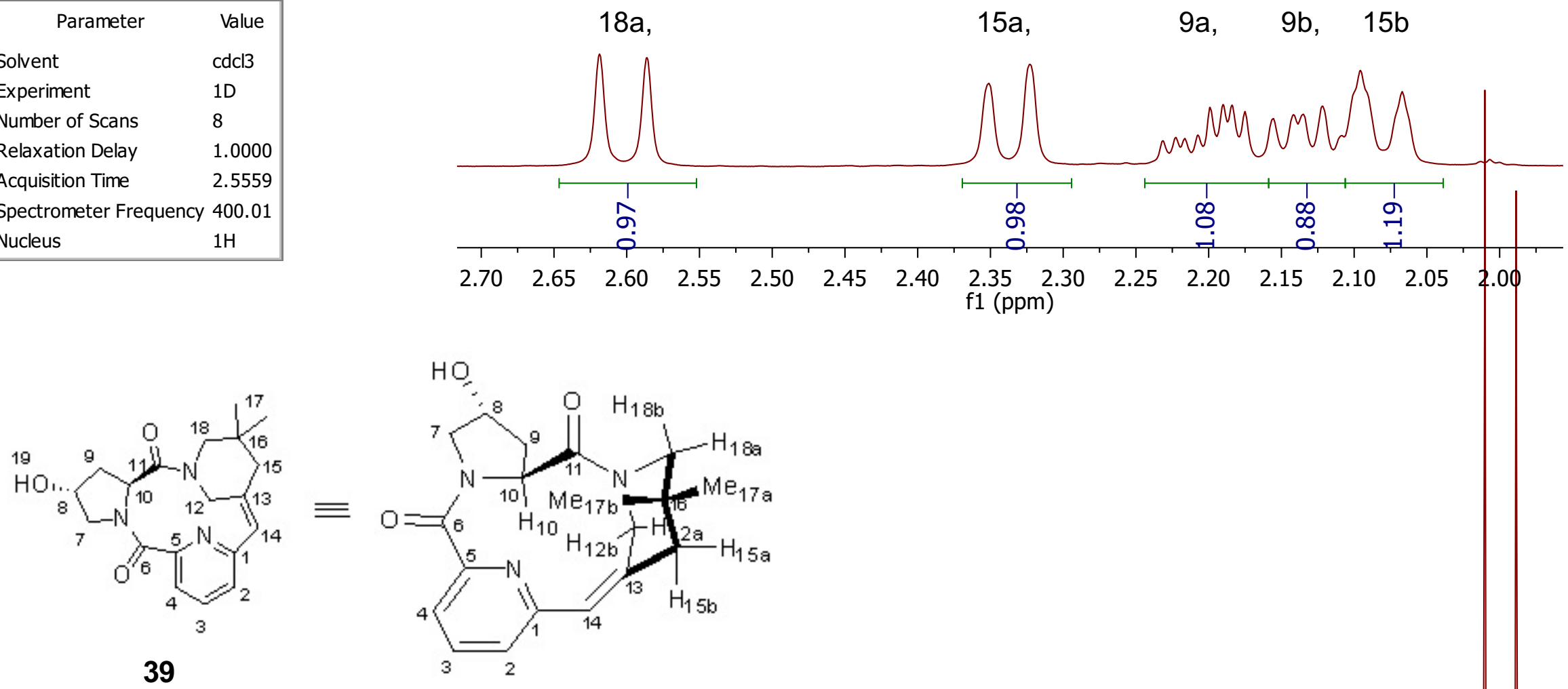

39

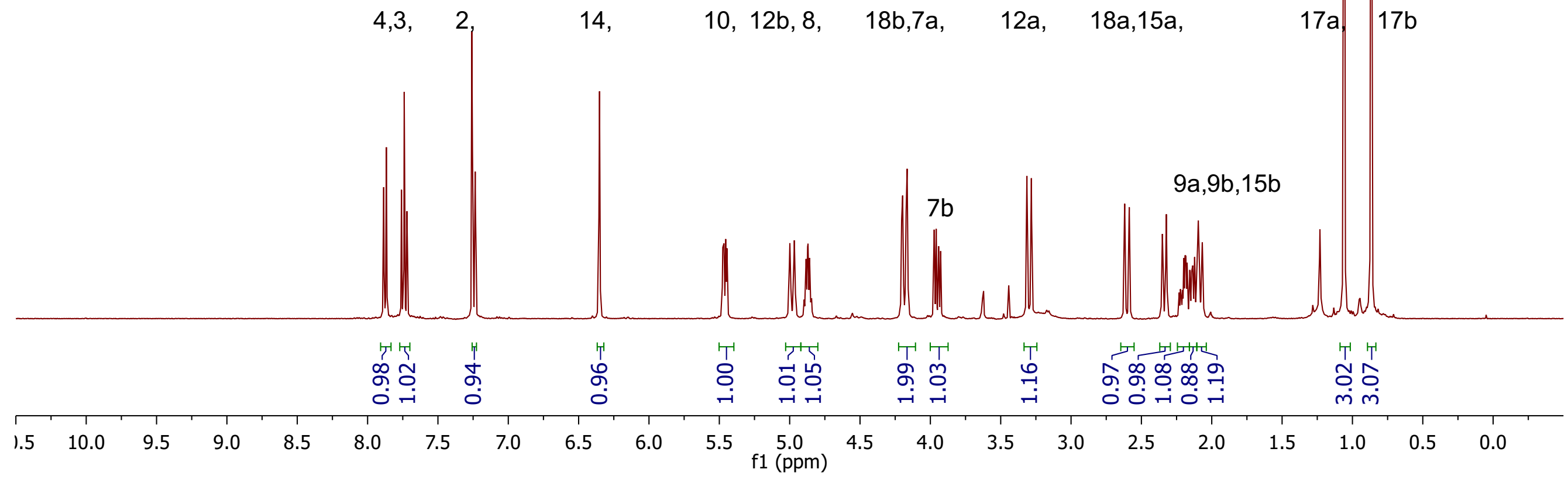

14

$10,12 b, 8, \quad 18 b, 7 a$

12a, $18 a, 15 a$,

17a $17 b$ 


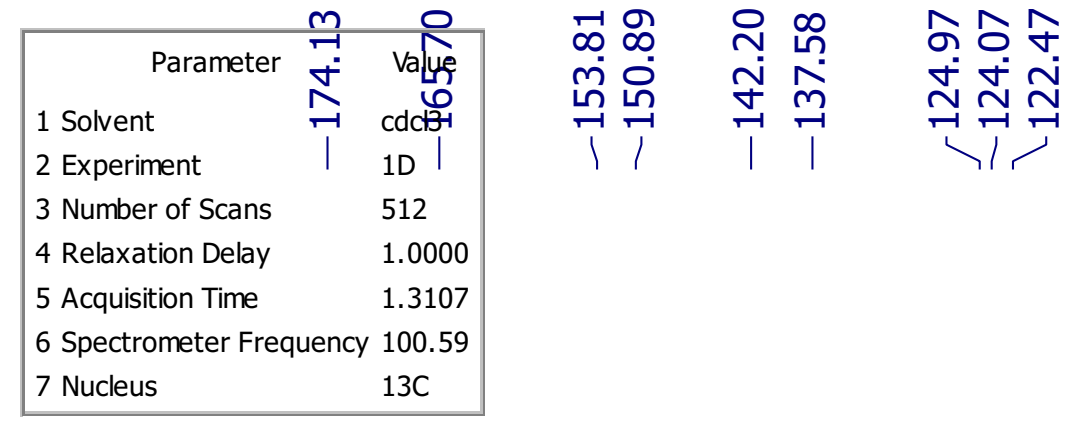

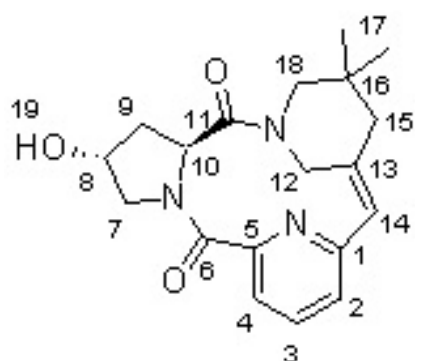

39

\section{సิ

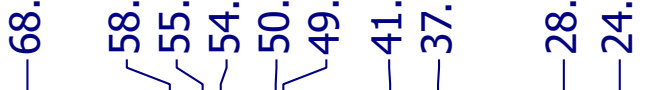

$11, \quad 6, \quad 1,5, \quad 13,3, \quad 2,14,4$ 


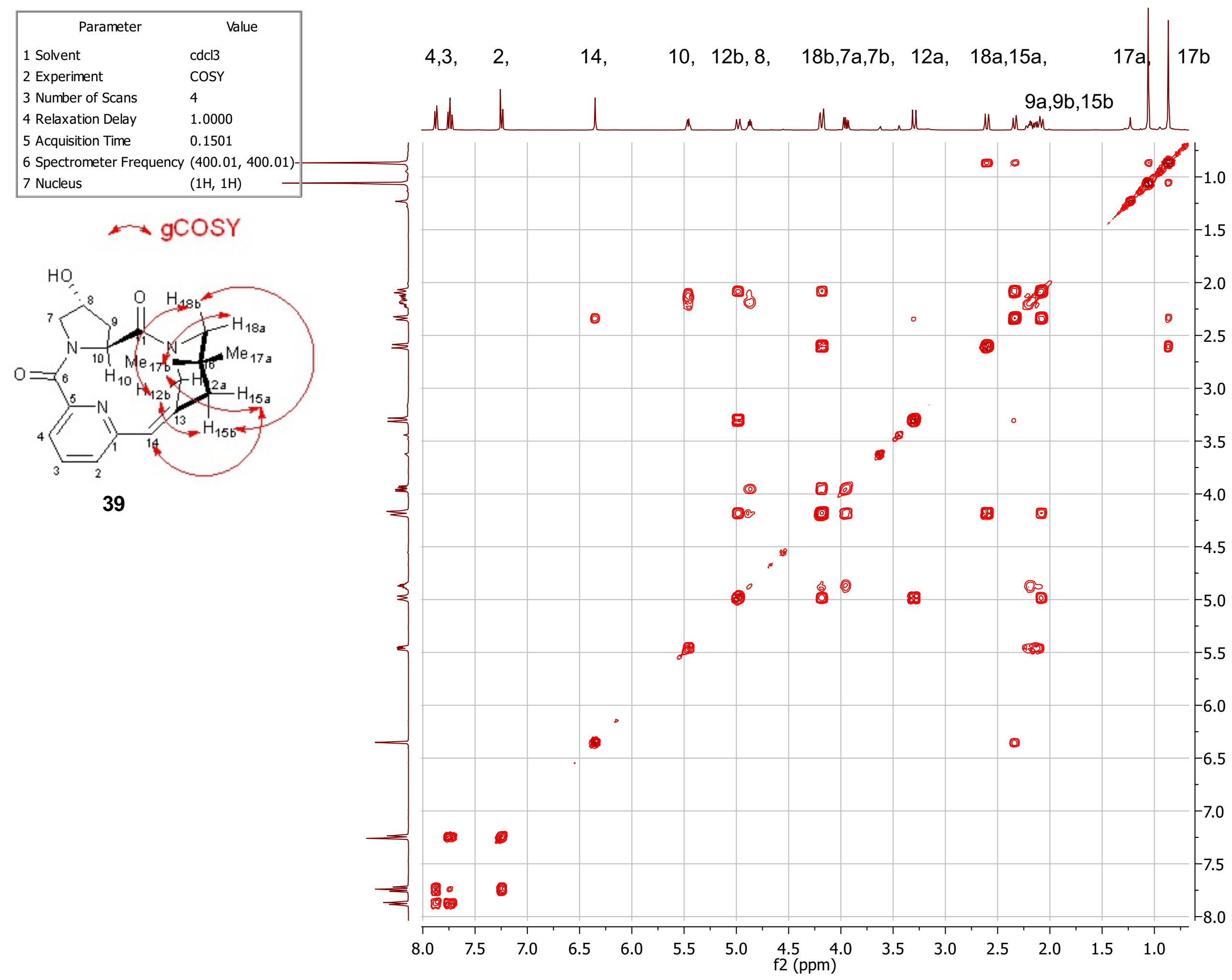




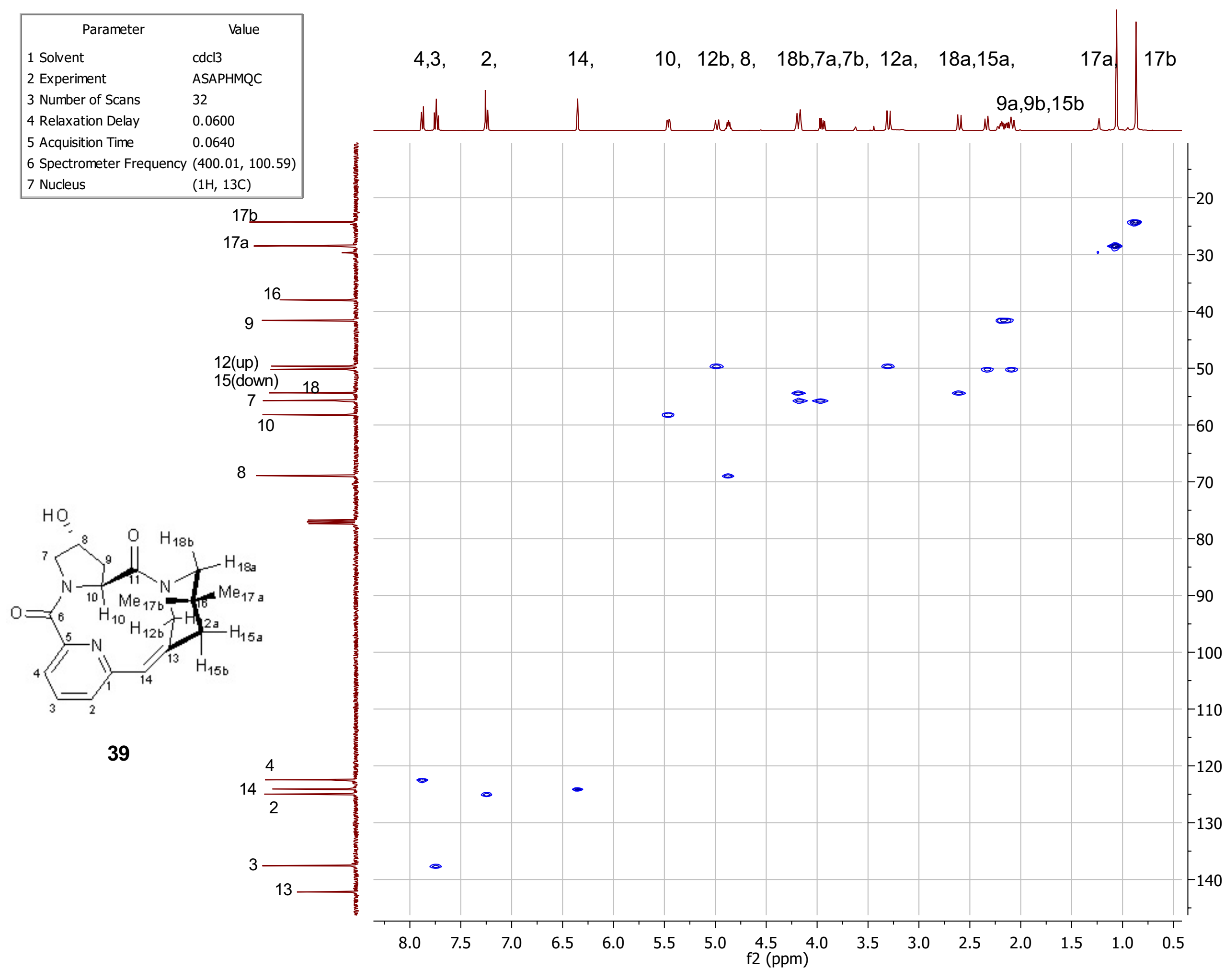

$\hat{\varepsilon}$
$\stackrel{0}{0}$
$\square$ 


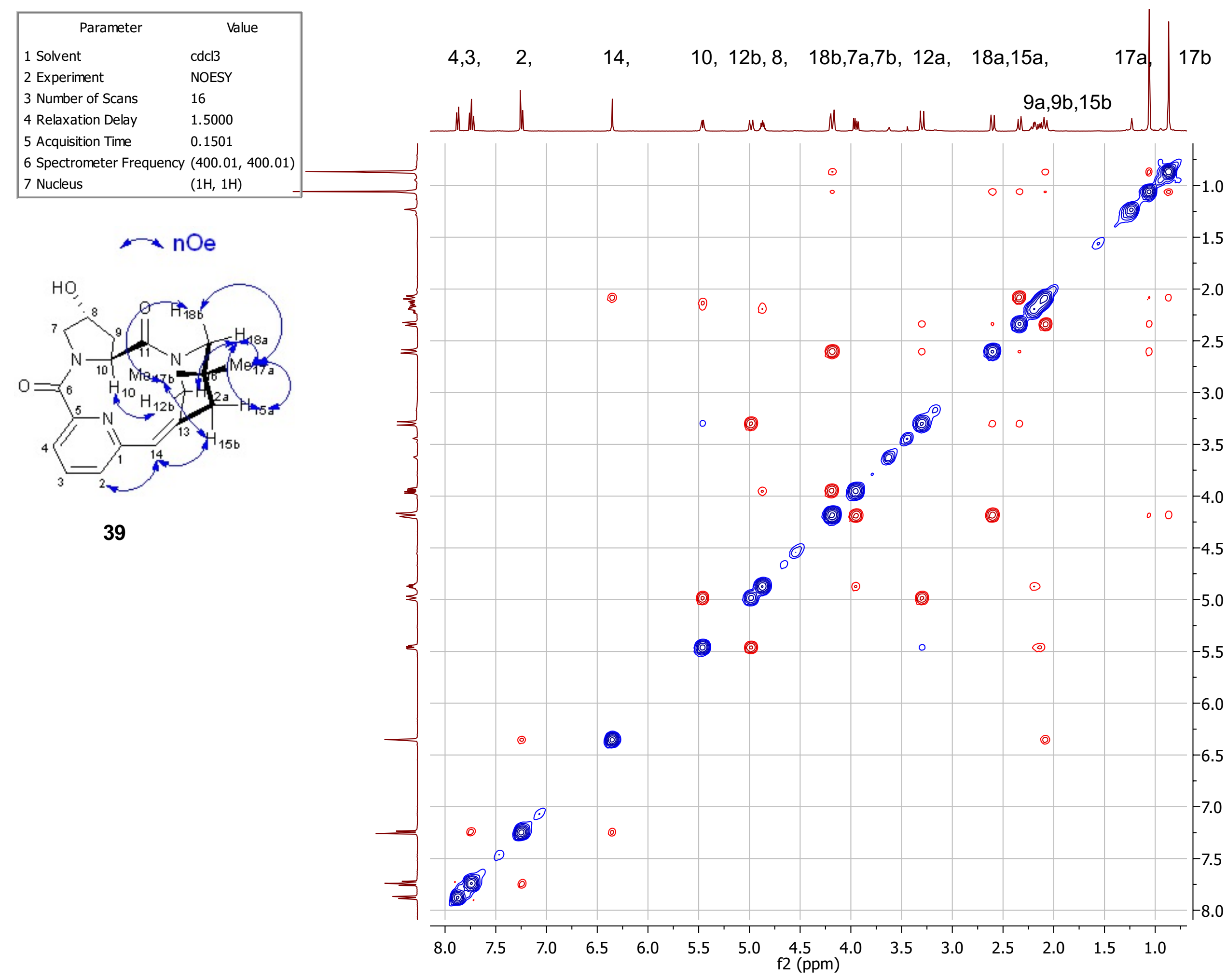

$\widehat{E}$
은
$\bar{L}$ 


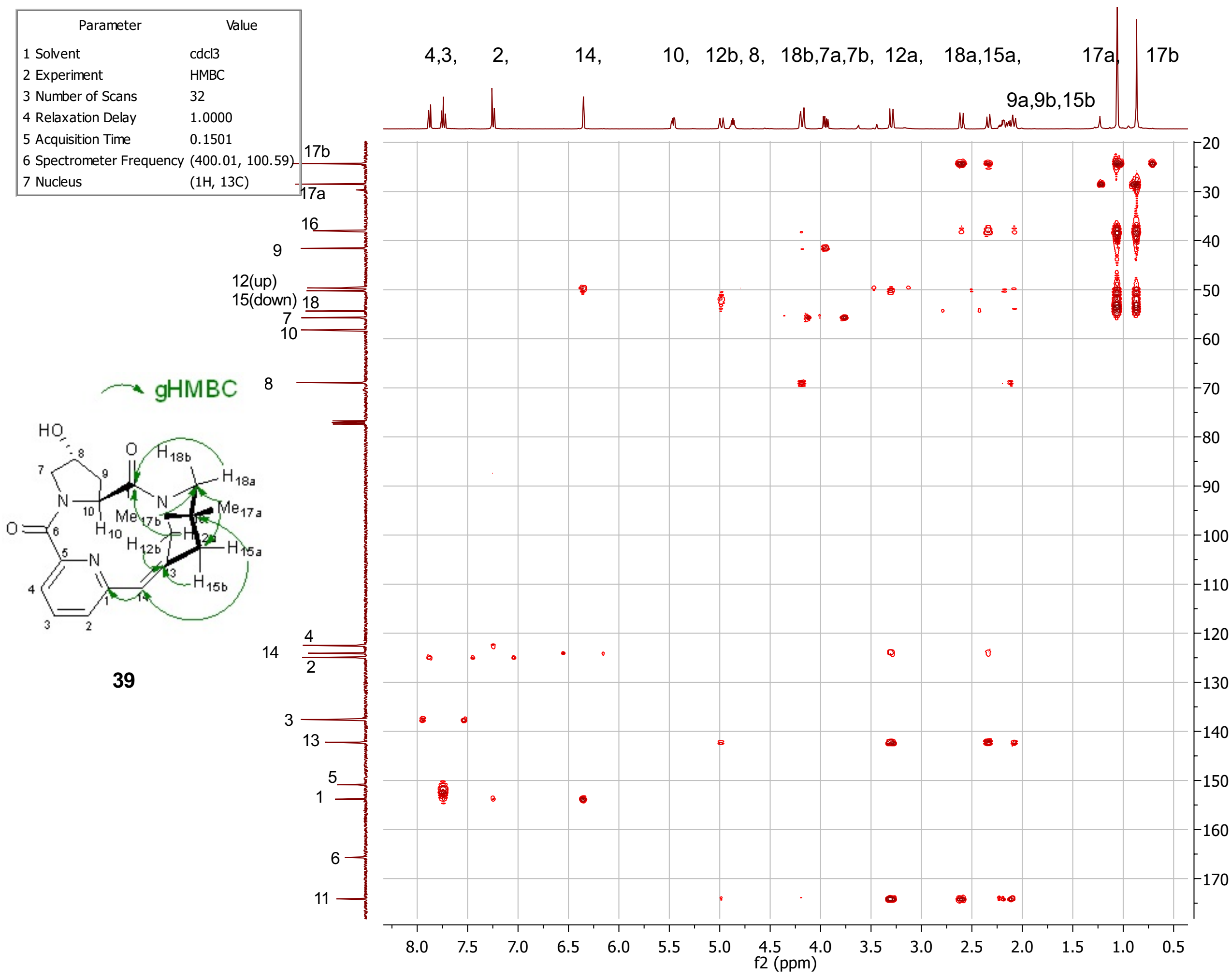

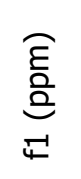




\begin{tabular}{|ll|}
\hline \multicolumn{1}{|c|}{ Parameter } & \multicolumn{1}{c|}{ Value } \\
1 Solvent & cdcl3 \\
2 Experiment & $1 \mathrm{D}$ \\
3 Number of Scans & 8 \\
4 Relaxation Delay & 1.0000 \\
5 Acquisition Time & 2.0447 \\
6 Spectrometer Frequency & 499.81 \\
7 Nucleus & $1 \mathrm{H}$ \\
\hline
\end{tabular}

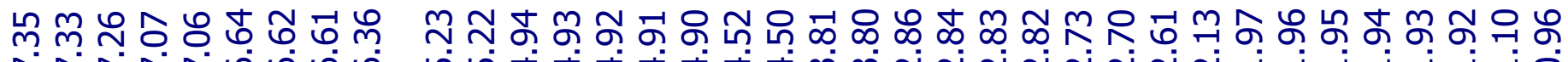
NヘNベ

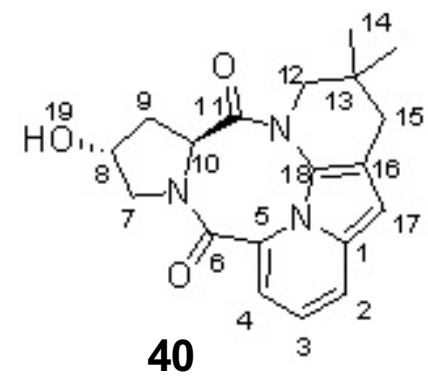

$2, \quad 4,3, \quad 17$,

$10,8, \quad 12$

7

$9,12^{\prime}, 15,19,9^{\prime}$

14

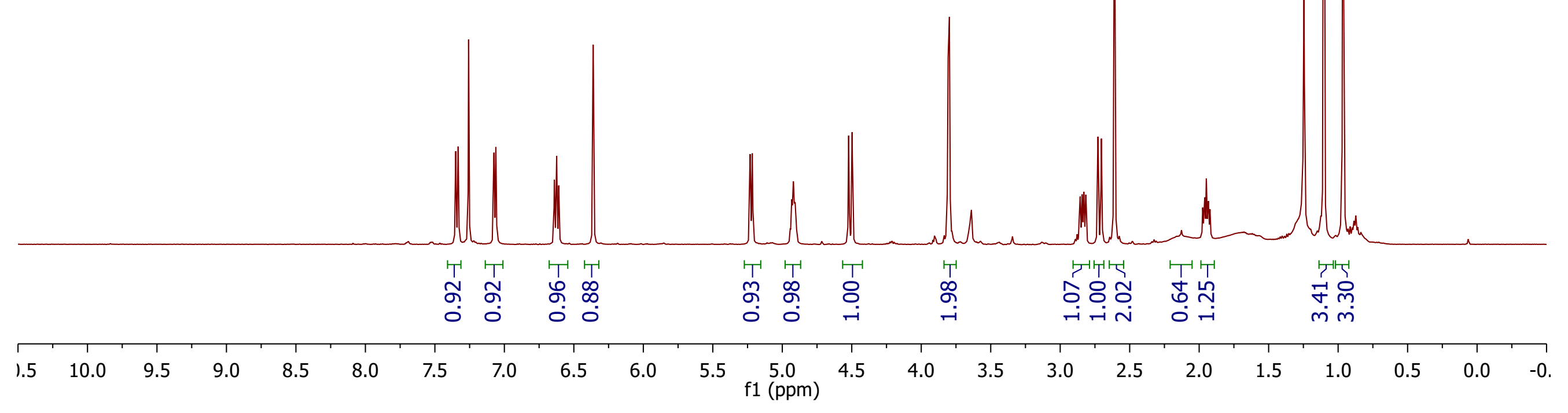




\begin{tabular}{|ll|}
\hline \multicolumn{1}{|c}{ Parameter } & \multicolumn{1}{c|}{ Value } \\
1 Solvent & cdcl3 \\
2 Experiment & $1 \mathrm{D}$ \\
3 Number of Scans & 18700 \\
4 Relaxation Delay & 1.0000 \\
5 Acquisition Time & 1.3042 \\
6 Spectrometer Frequency & 100.61 \\
7 Nucleus & $13 \mathrm{C}$ \\
\hline
\end{tabular}

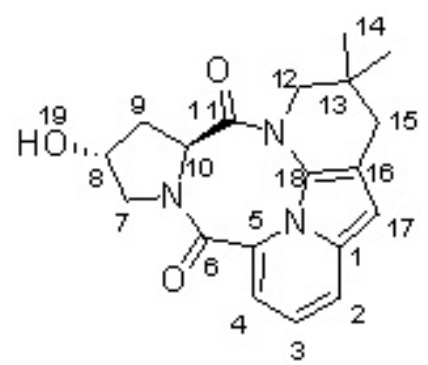

40

11,6

$1,5, \quad 2,18,16,4,3$

17,

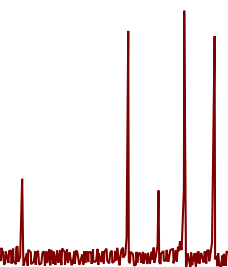

$8, \quad 10,12,7$

$15,9,13,14$ 


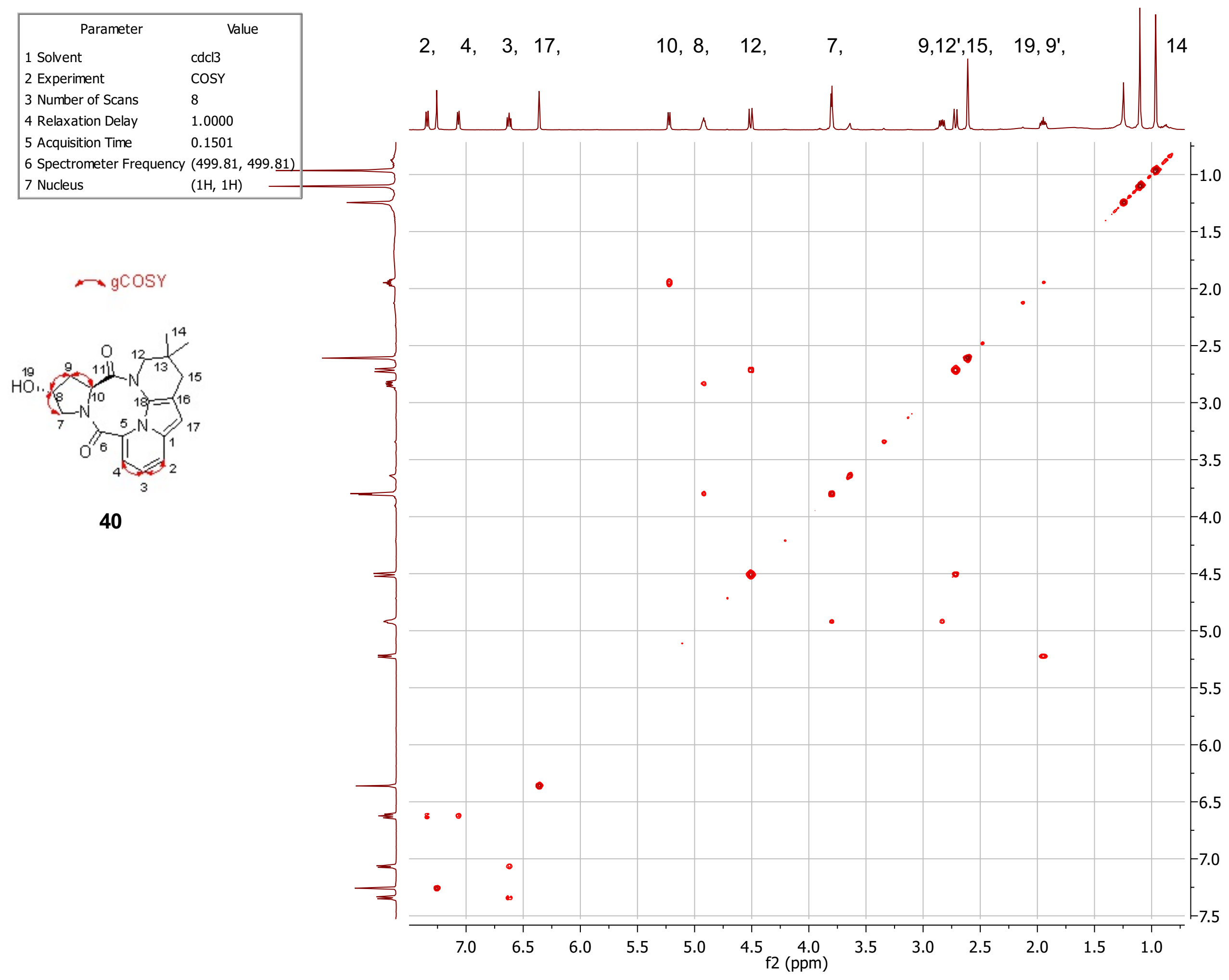




\begin{tabular}{|ll|}
\hline \multicolumn{1}{|c|}{ Parameter } & \multicolumn{1}{c|}{ Value } \\
1 Solvent & cdcl3 \\
2 Experiment & ASAPHMQC \\
3 Number of Scans & 16 \\
4 Relaxation Delay & 0.0600 \\
5 Acquisition Time & 0.0640 \\
6 Spectrometer Frequency & $(499.81,125.69)$ \\
7 Nucleus & $(1 \mathrm{H}, 13 \mathrm{C})$ \\
\hline
\end{tabular}

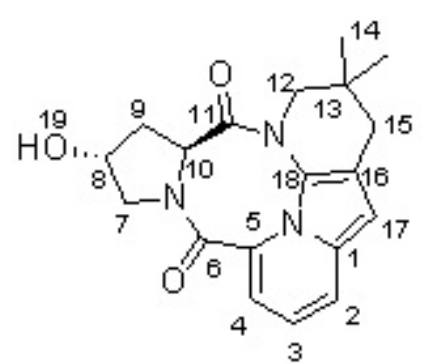

40
$2, \quad 4, \quad 3,17, \quad 10,8, \quad 12, \quad 7, \quad 9,12^{\prime}, 15, \quad 19,9^{\prime}$,

14
7 Nucleus

.

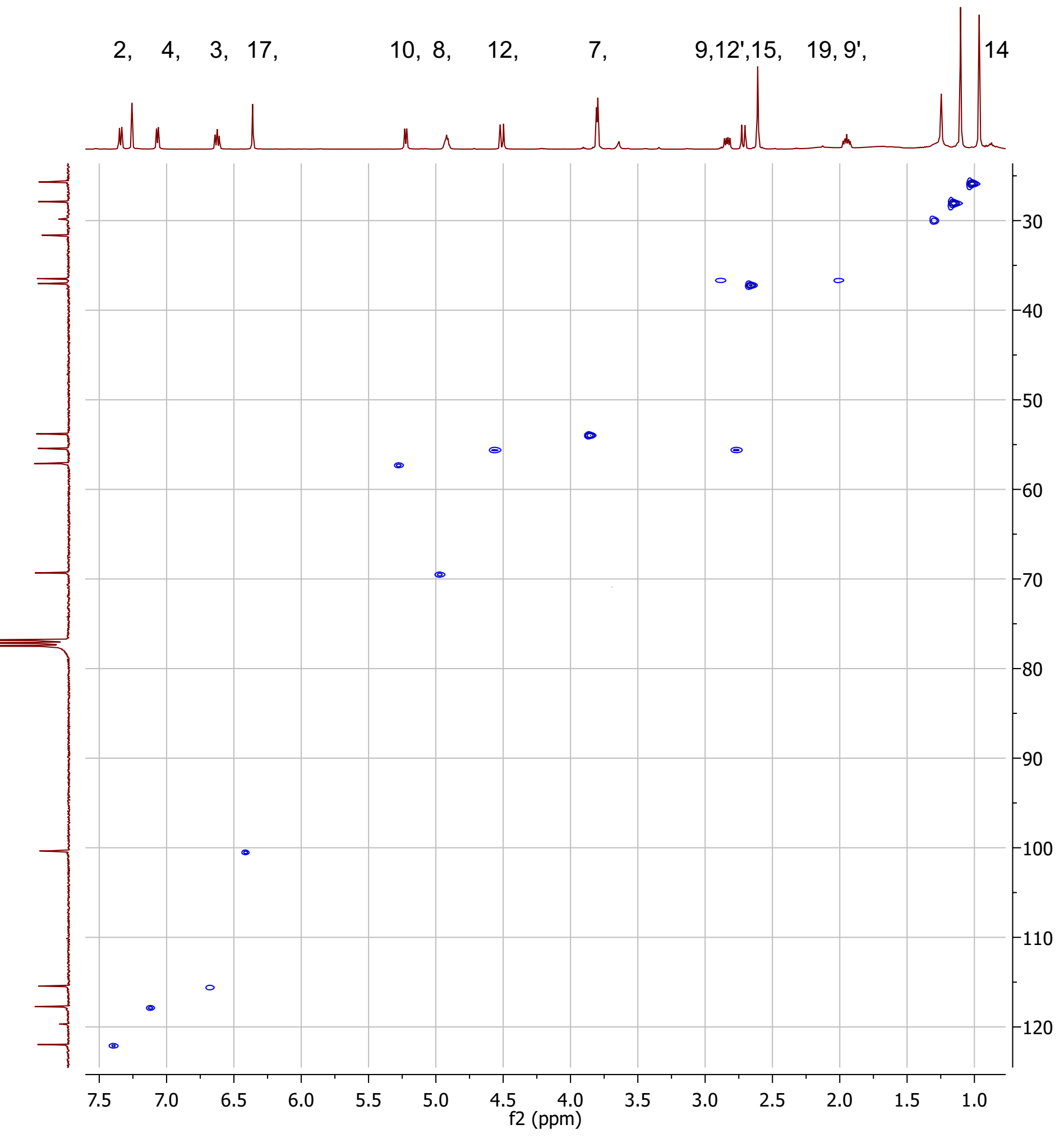




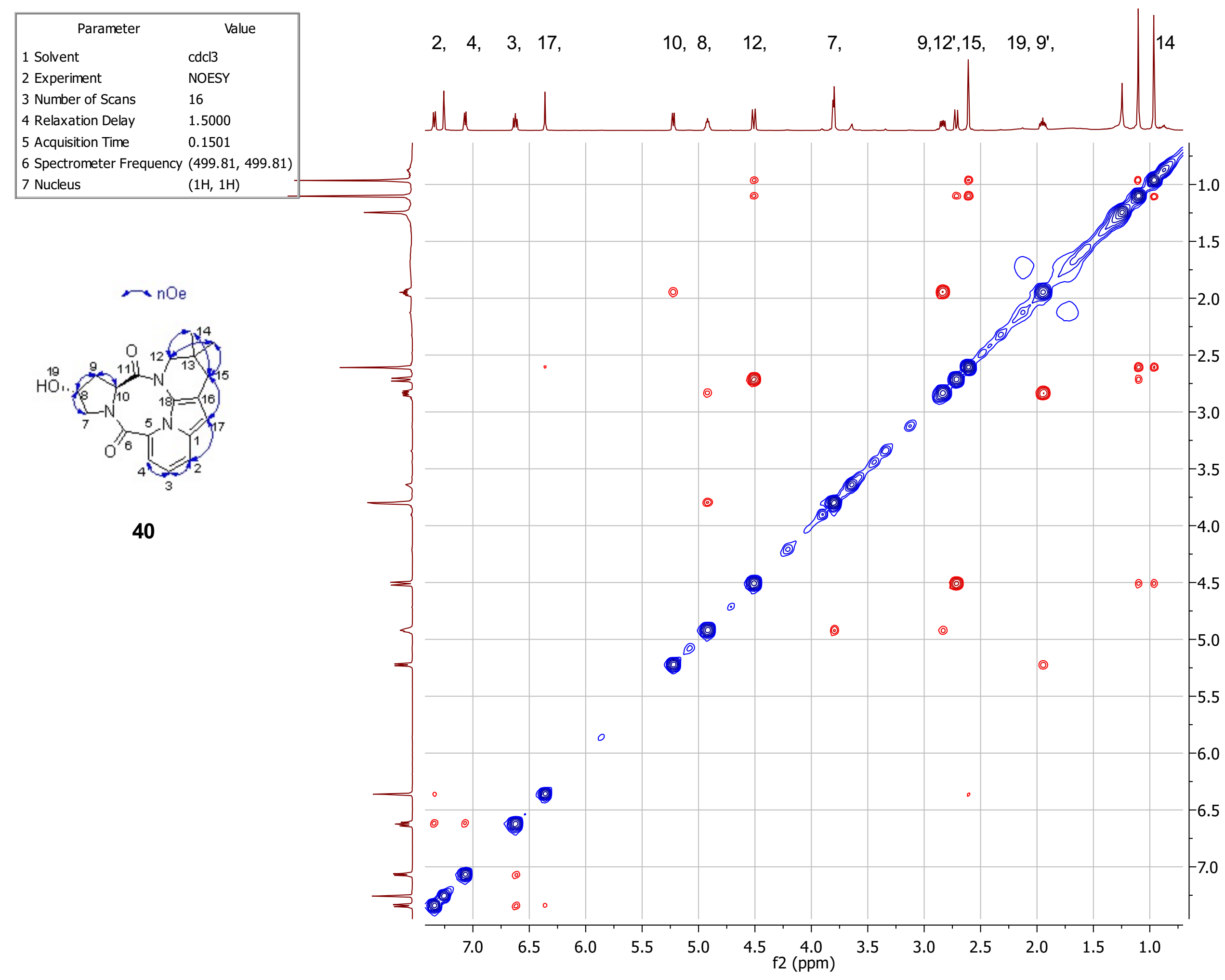

है
든 


\begin{tabular}{|ll|}
\hline \multicolumn{1}{|c|}{ Parameter } & \multicolumn{1}{c|}{ Value } \\
1 Solvent & cdcl3 \\
2 Experiment & HMBC \\
3 Number of Scans & 32 \\
4 Relaxation Delay & 1.0000 \\
5 Acquisition Time & 0.1500 \\
6 Spectrometer Frequency & $(499.81,125.69)$ \\
7 Nucleus & $(1 \mathrm{H}, 13 \mathrm{C})$ \\
\hline
\end{tabular}

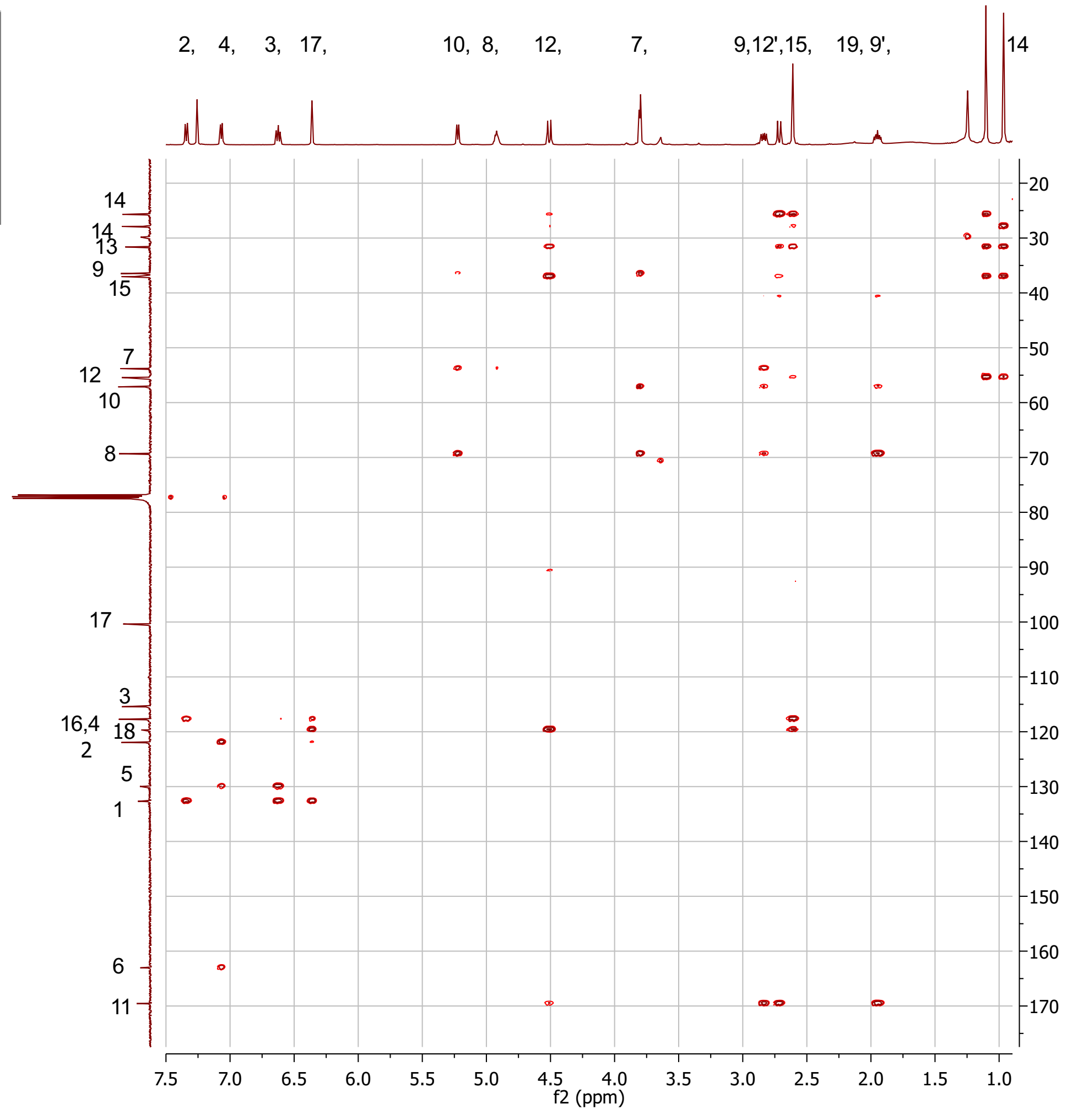

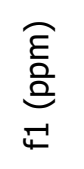

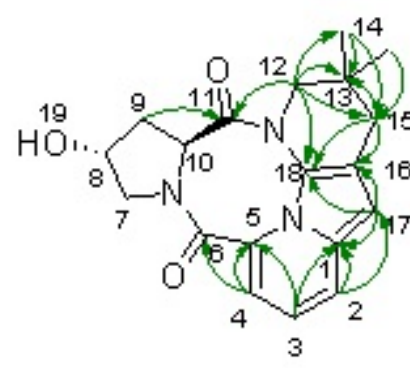

40 


\begin{tabular}{|ll|}
\hline \multicolumn{1}{|c|}{ Parameter } & \multicolumn{1}{c|}{$\begin{array}{c}\text { Value } \\
1 \text { Comment }\end{array}$} \\
& $60^{\circ} \mathrm{C}$ \\
& \\
2 Solvent & $\mathrm{cdcl} 3$ \\
3 Experiment & $1 \mathrm{D}$ \\
4 Number of Scans & 8 \\
5 Relaxation Delay & 1.0000 \\
6 Acquisition Time & 2.5559 \\
7 Spectrometer Frequency & 400.01 \\
8 Nucleus & $1 \mathrm{H}$ \\
\hline
\end{tabular}

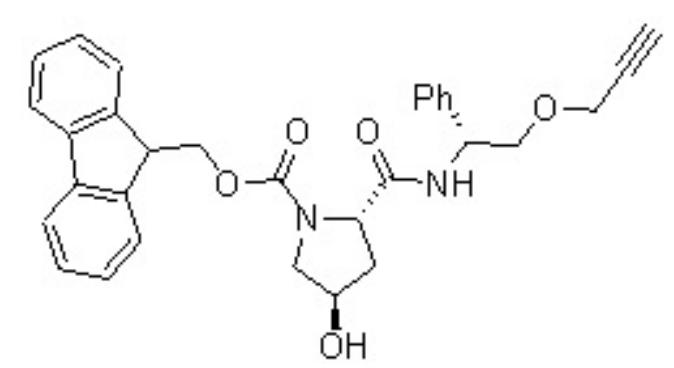

48b

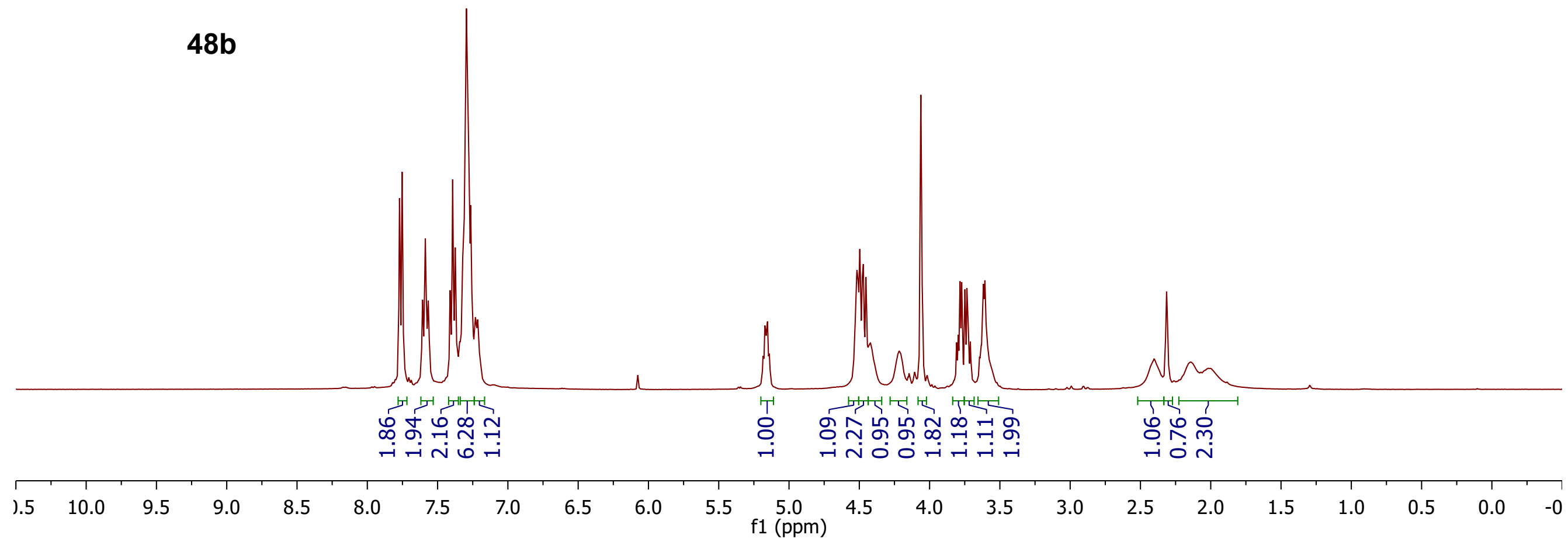



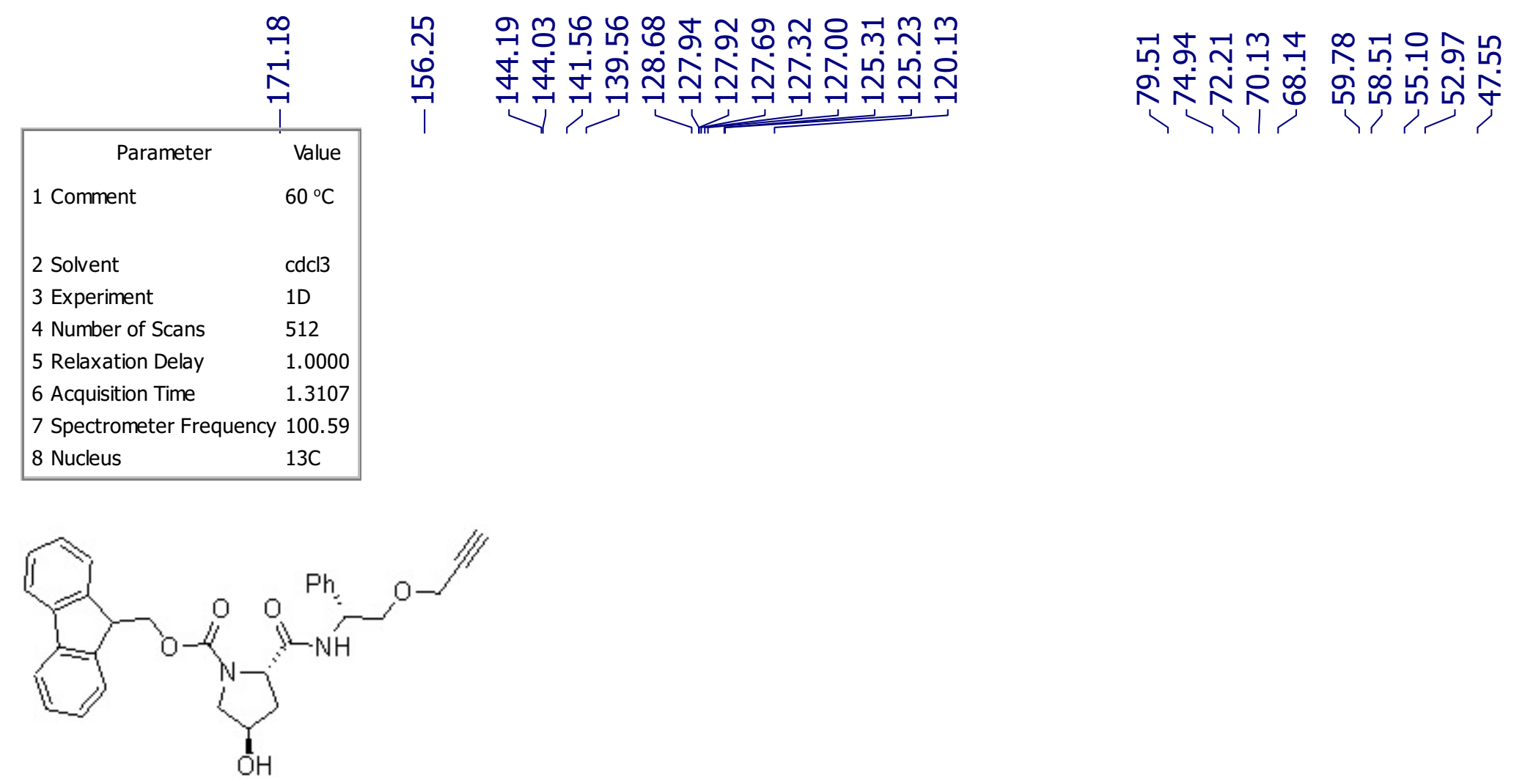

$48 b$

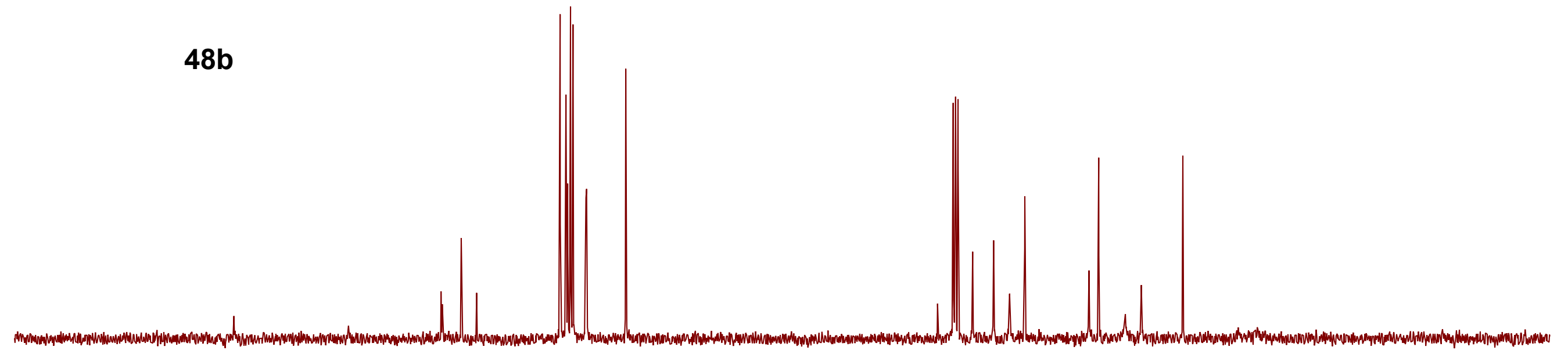

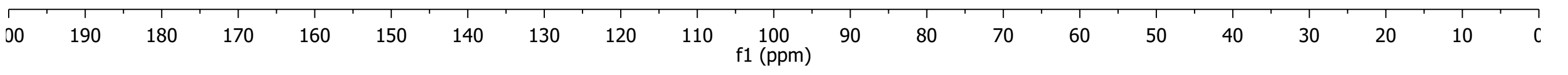




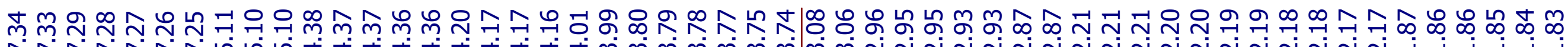

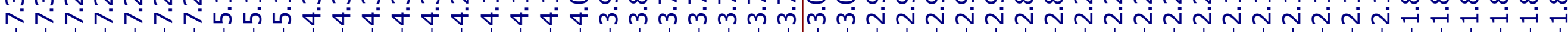

\begin{tabular}{|ll|}
\hline \multicolumn{1}{|c|}{ Parameter } & \multicolumn{1}{c|}{ Value } \\
1 Solvent & cd3od \\
2 Experiment & $1 \mathrm{D}$ \\
3 Number of Scans & 8 \\
4 Relaxation Delay & 1.0000 \\
5 Acquisition Time & 2.0447 \\
6 Spectrometer Frequency & 499.81 \\
7 Nucleus & $1 \mathrm{H}$ \\
\hline
\end{tabular}

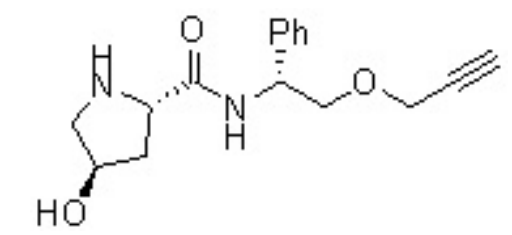

48c

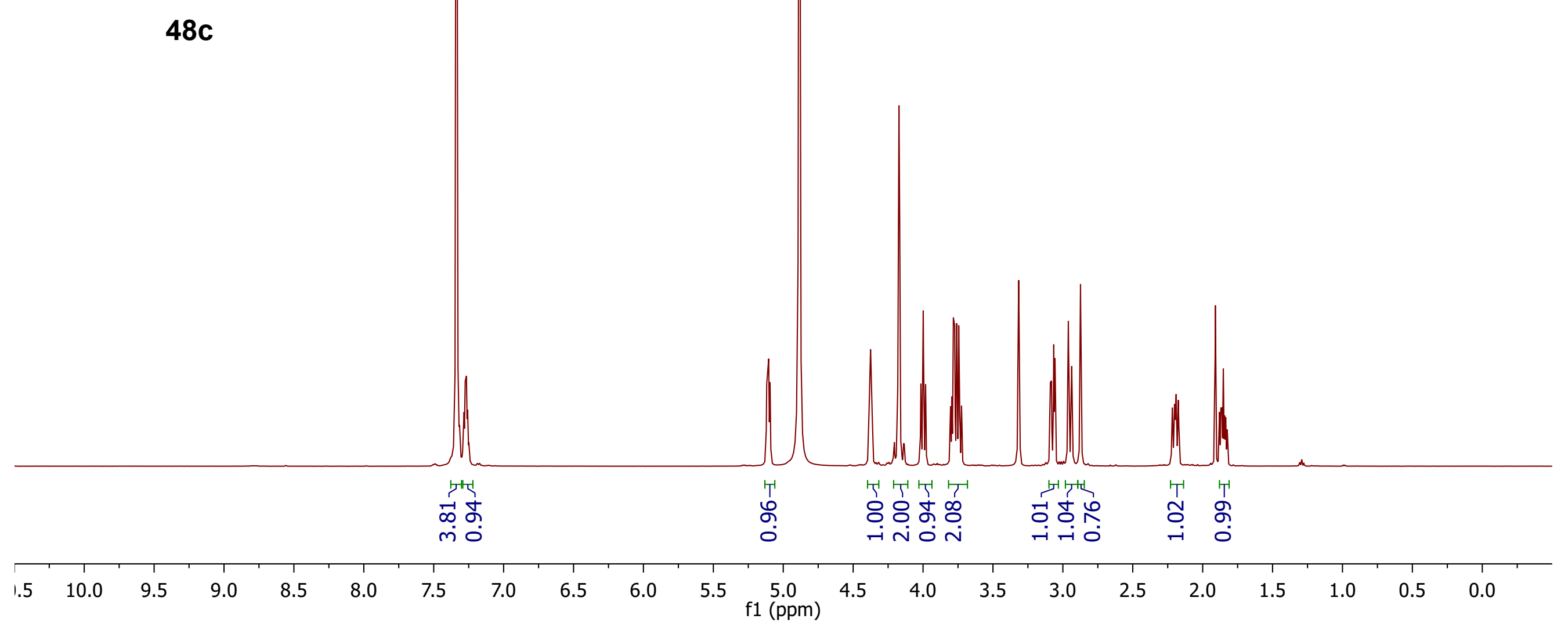




\begin{tabular}{|l|l|}
\hline \multicolumn{1}{|c|}{ Parameter } & Value \\
1 Solvent & cd3od \\
2 Experiment & $1 \mathrm{D}$ \\
3 Number of Scans & 64 \\
4 Relaxation Delay & 1.0000 \\
5 Acquisition Time & 1.3042 \\
6 Spectrometer Frequency 100.61 \\
7 Nucleus & $13 \mathrm{C}$ \\
\hline
\end{tabular}

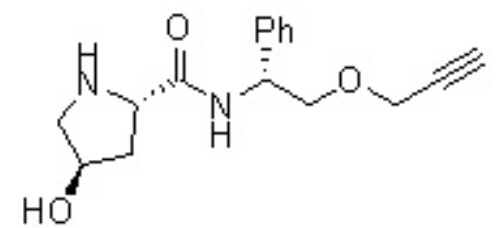

48c

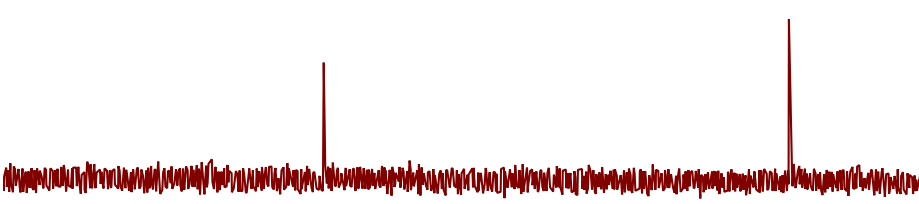

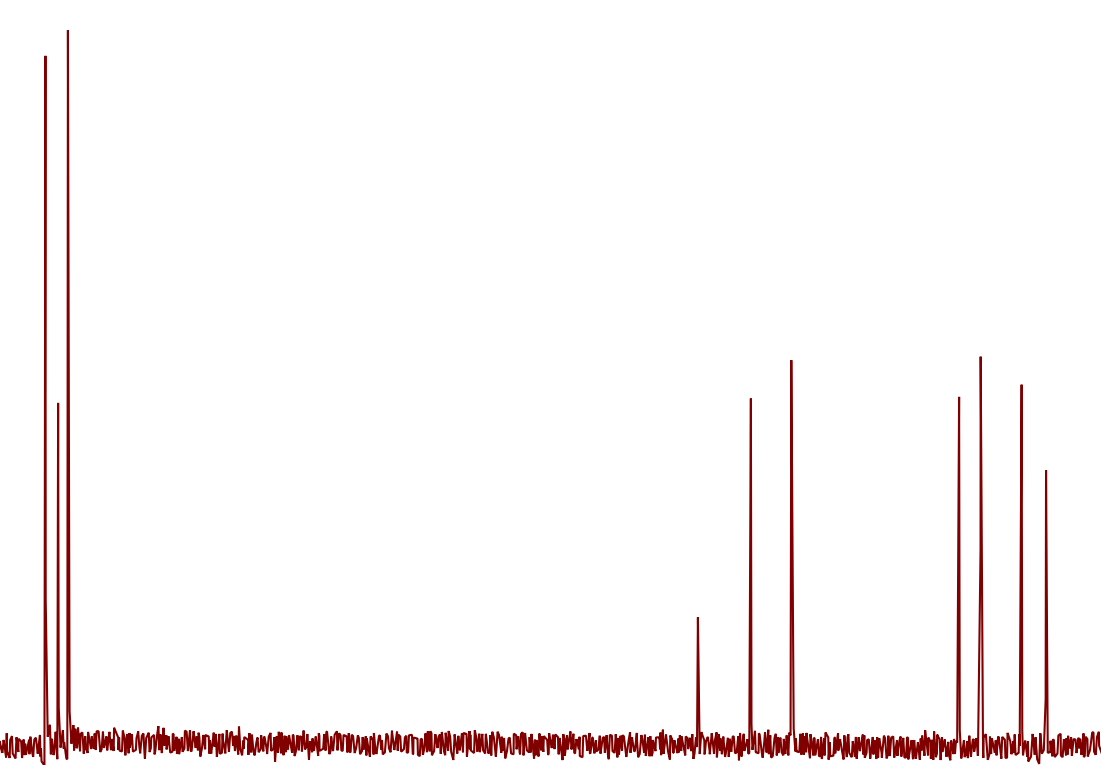

$190 \quad 180$

170

160

150

140

130

120

110

100

f1 (ppm)

$90 \quad 80$

70

60




\begin{tabular}{|l|l|}
\hline \multicolumn{1}{|c|}{ Parameter } & \multicolumn{1}{c|}{ Value } \\
1 Solvent & cdcl3 \\
2 Experiment & $1 \mathrm{D}$ \\
3 Number of Scans & 256 \\
4 Relaxation Delay & 1.0000 \\
5 Acquisition Time & 1.3042 \\
6 Spectrometer Frequency 100.61 \\
7 Nucleus & $13 \mathrm{C}$ \\
\hline
\end{tabular}

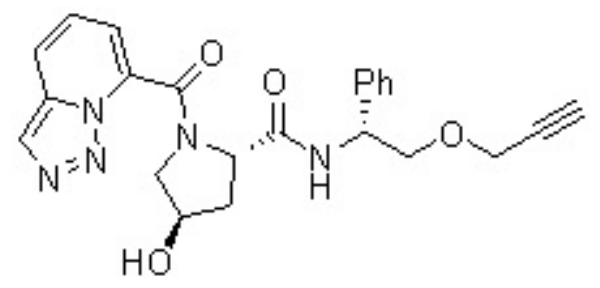

48

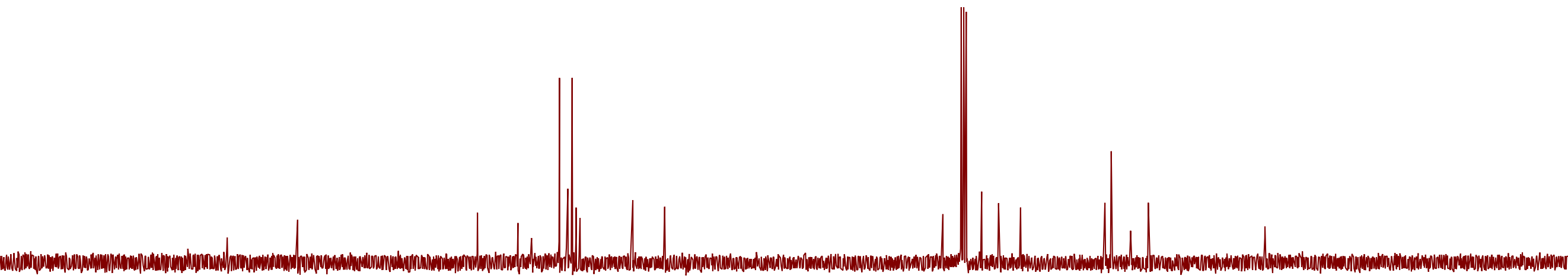

130

120

110

100

f1 (ppm)

$\begin{array}{llll}90 & 80 & 70 & 60\end{array}$




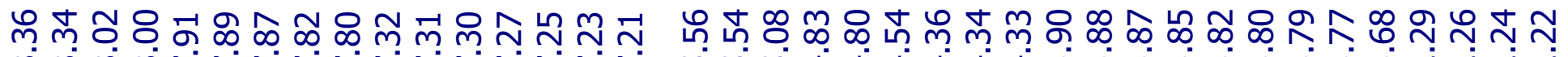

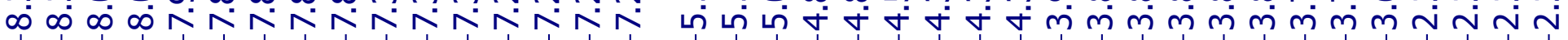

\begin{tabular}{|ll|}
\hline \multicolumn{1}{|c|}{ Parameter } & \multicolumn{1}{c|}{ Value } \\
1 Solvent & CDCl3/CD3OD $=1: 1$ \\
2 Experiment & $1 \mathrm{D}$ \\
3 Number of Scans & 8 \\
4 Relaxation Delay & 1.0000 \\
5 Acquisition Time & 2.5559 \\
6 Spectrometer Frequency & 400.02 \\
7 Nucleus & $1 \mathrm{H}$ \\
\hline
\end{tabular}

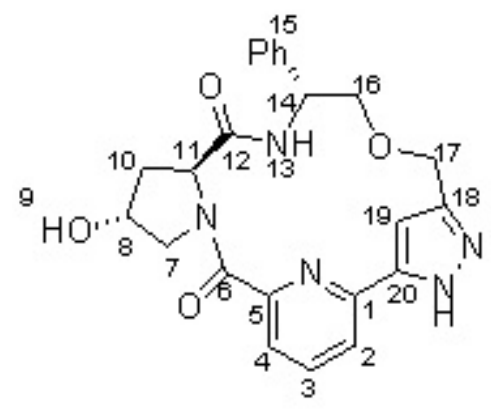

49

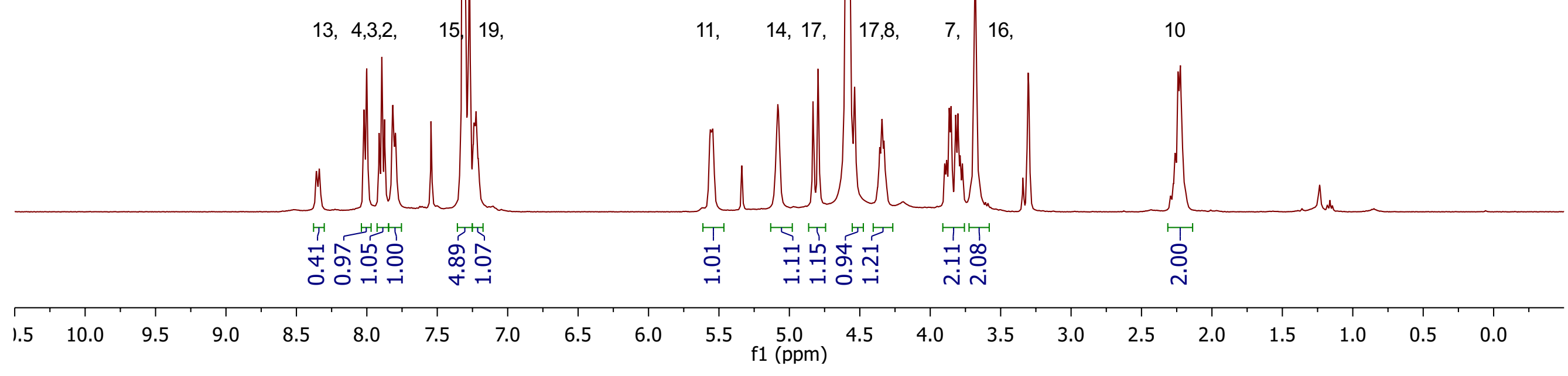




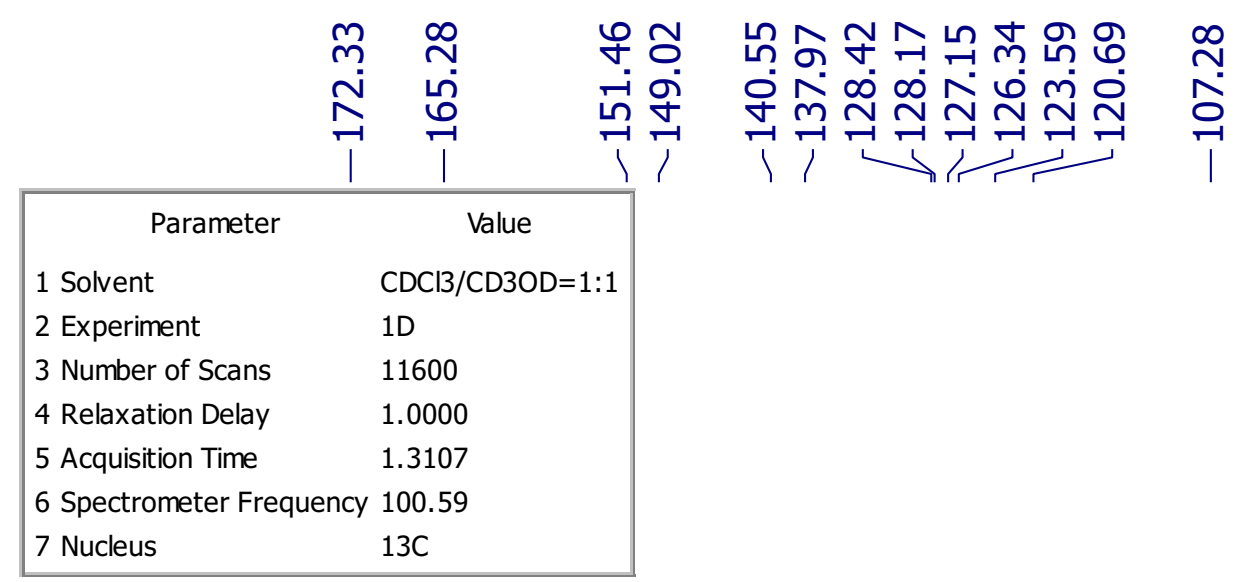

꼿의듬둥

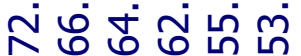

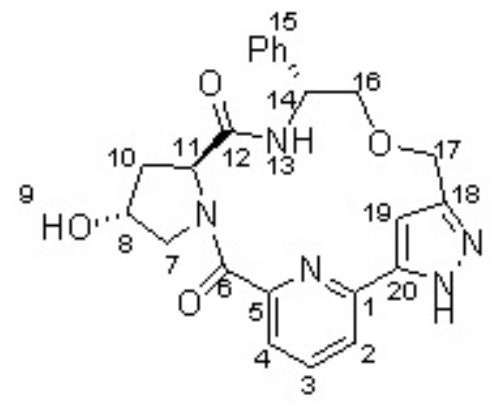

49

12, 6

15,3

4, 2 ,

19 ,

$14,8,17,11, \quad 7,14$

10

1, 5 


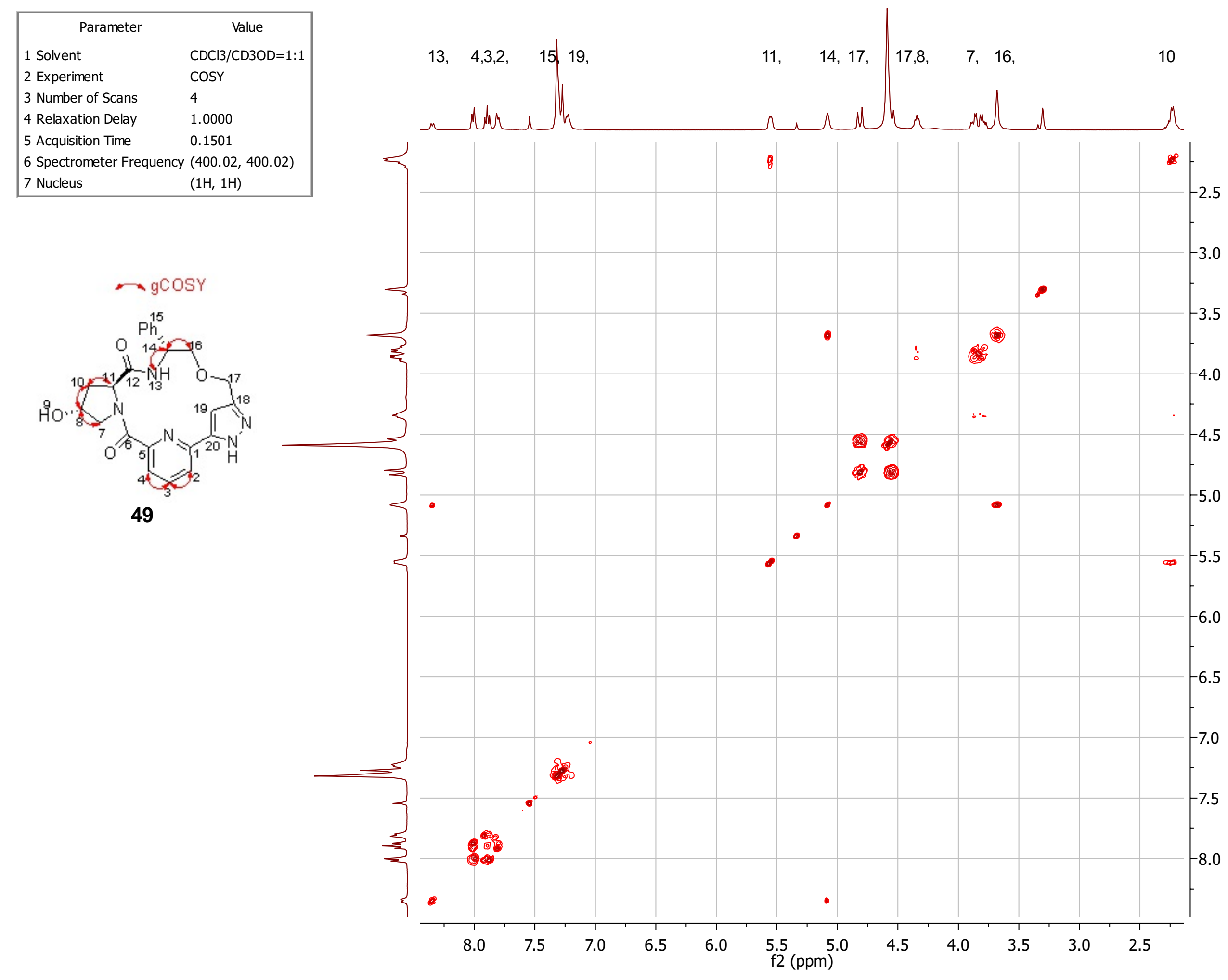

हैํㅡㄹ 


\begin{tabular}{|ll|}
\hline \multicolumn{1}{|c|}{ Parameter } & \multicolumn{1}{c|}{ Value } \\
1 Solvent & CDCl3/CD3OD $=1: 1$ \\
2 Experiment & HMQC \\
3 Number of Scans & 16 \\
4 Relaxation Delay & 1.0000 \\
5 Acquisition Time & 0.1501 \\
6 Spectrometer Frequency & $(400.02,100.59)$ \\
7 Nucleus & $(1 \mathrm{H}, 13 \mathrm{C})$ \\
\hline
\end{tabular}

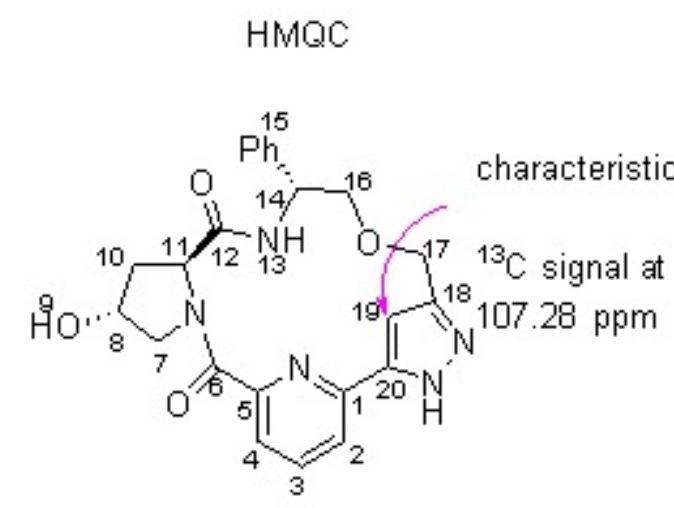

49
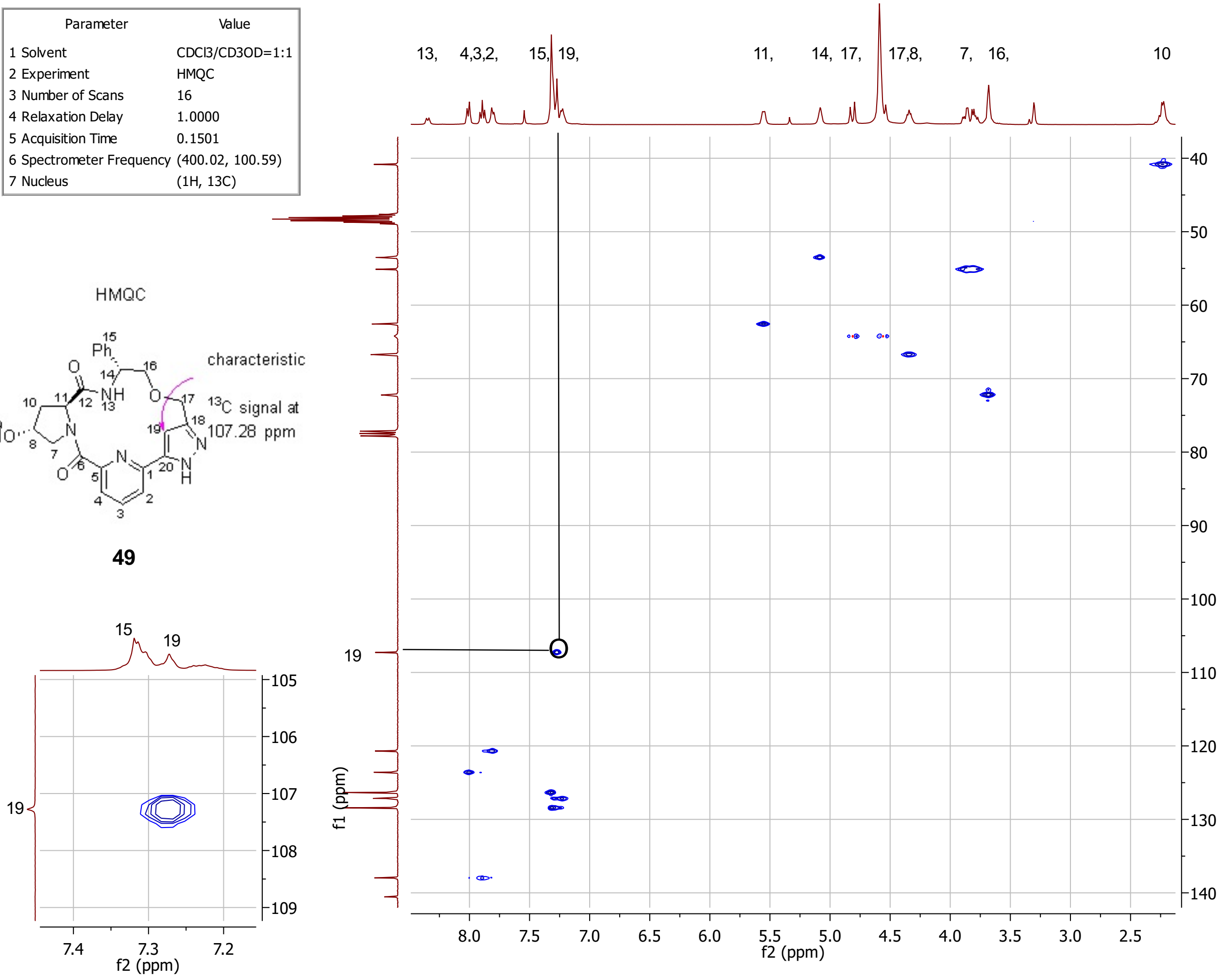


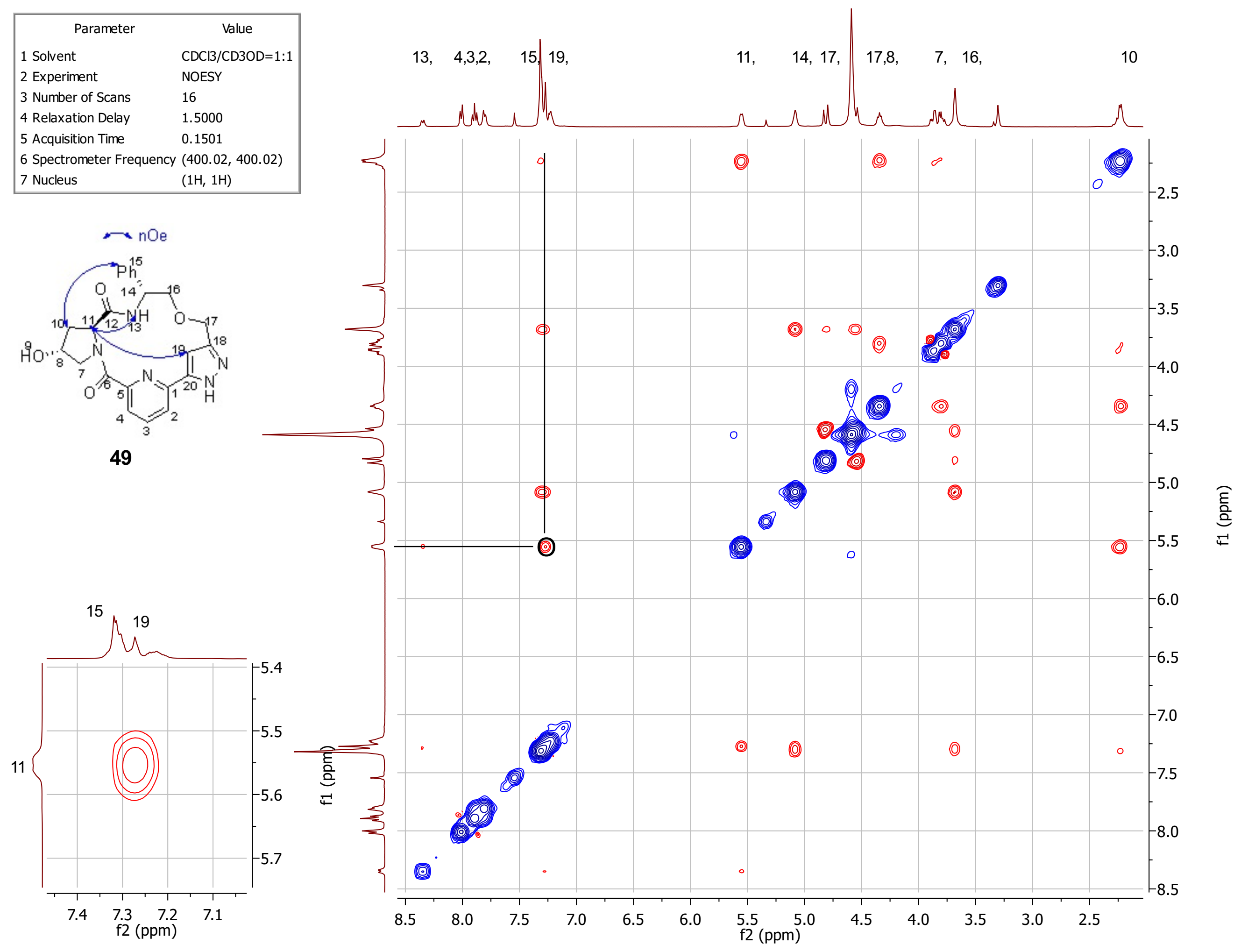




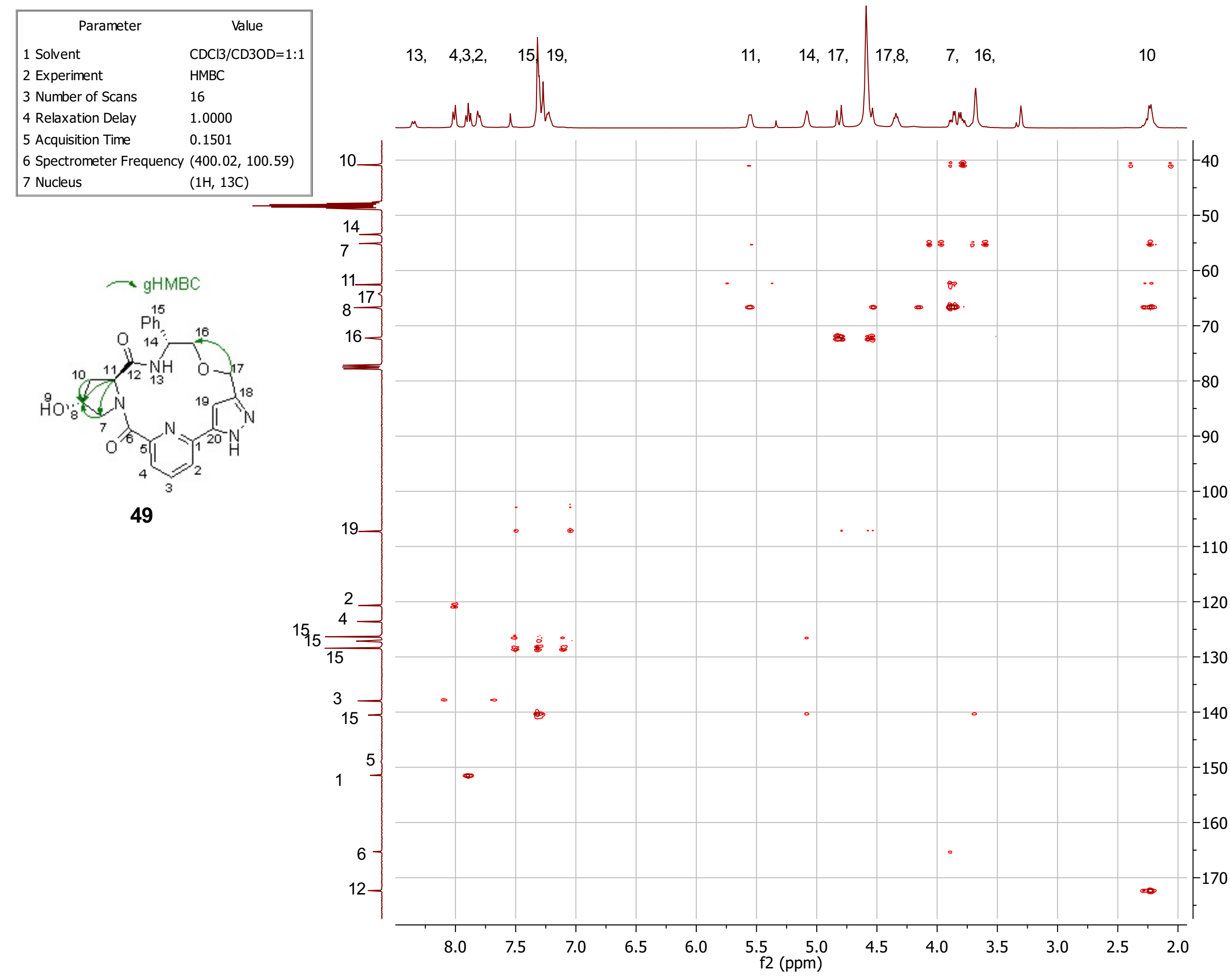

है
든 


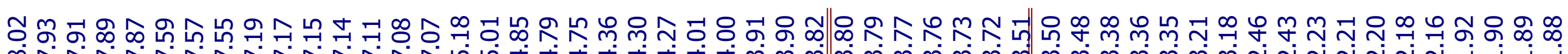

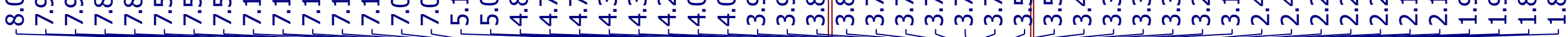

\begin{tabular}{|ll|}
\hline \multicolumn{1}{|c|}{ Parameter } & \multicolumn{1}{c|}{ Value } \\
1 Solvent & CD3OD/CDCl3=3:1 \\
2 Experiment & $1 \mathrm{D}$ \\
3 Number of Scans & 8 \\
4 Relaxation Delay & 1.0000 \\
5 Acquisition Time & 2.5592 \\
6 Spectrometer Frequency & 400.09 \\
7 Nucleus & $1 \mathrm{H}$ \\
\hline
\end{tabular}

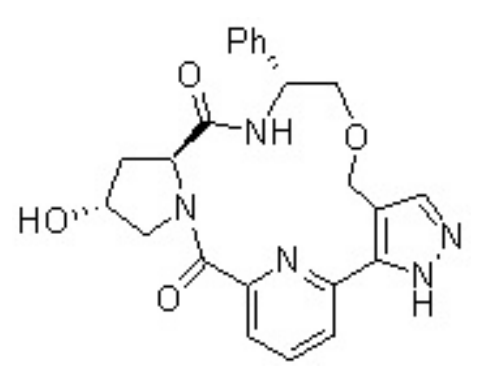

50

\section{0}

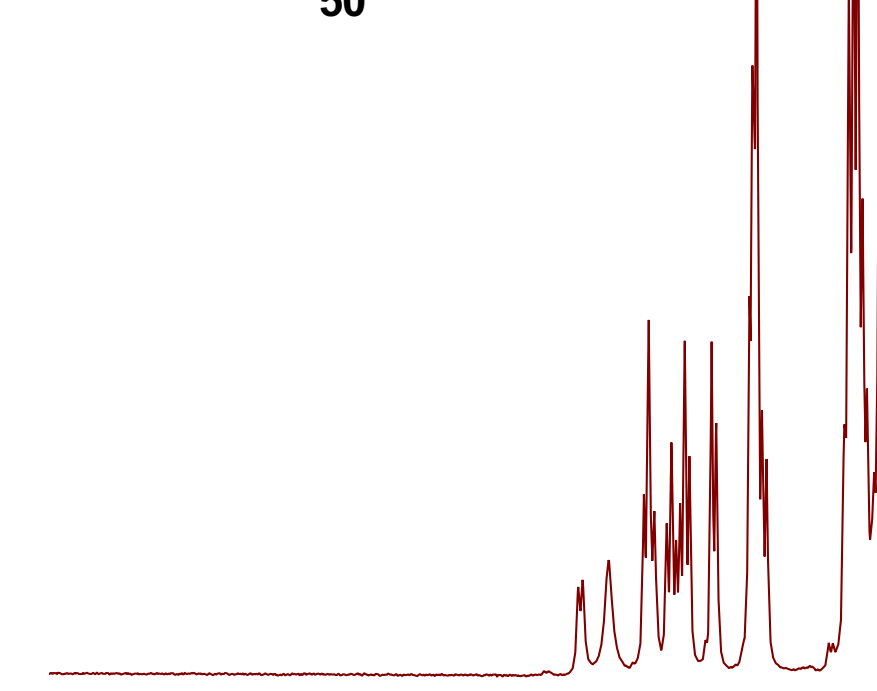

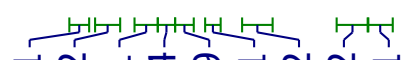
ㄲํำำำำํำ

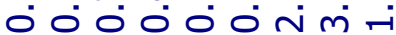

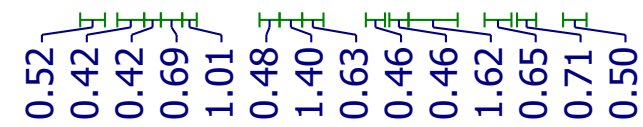

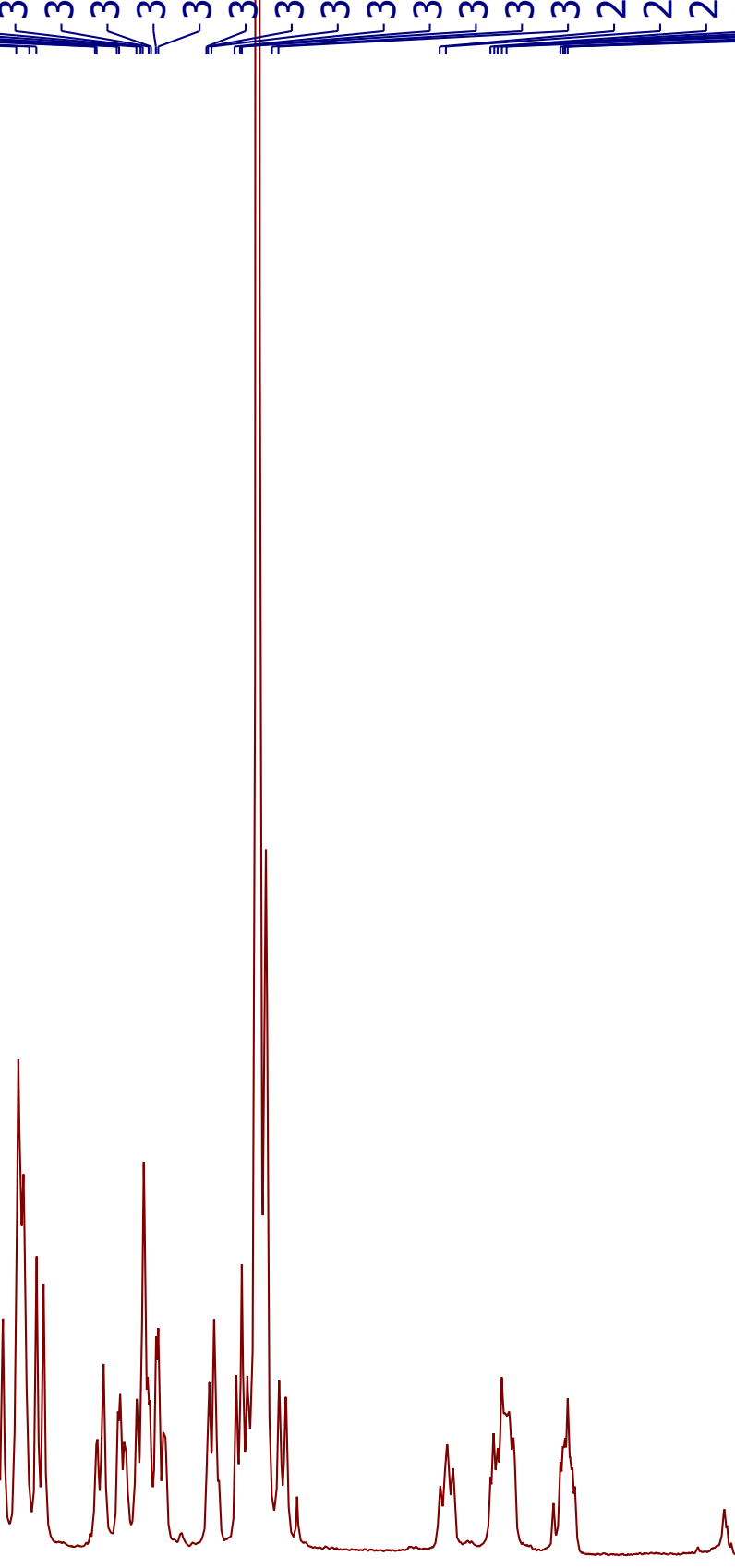

म्ञ m 8 ต

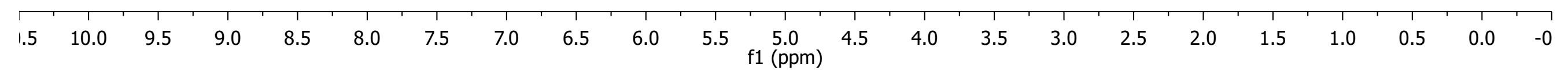




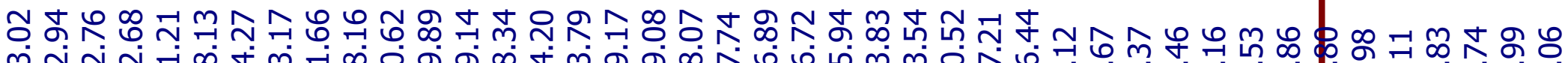

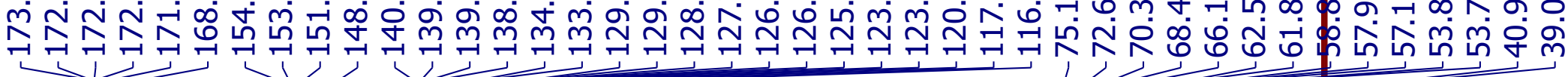

\begin{tabular}{|ll|}
\hline \multicolumn{1}{|c|}{ Parameter } & \multicolumn{1}{c|}{ Value } \\
1 Solvent & CD3OD/CDCl3=3:1 \\
2 Experiment & $1 \mathrm{D}$ \\
3 Number of Scans & 17000 \\
4 Relaxation Delay & 1.0000 \\
5 Acquisition Time & 1.3042 \\
6 Spectrometer Frequency & 100.61 \\
7 Nucleus & $13 \mathrm{C}$ \\
\hline
\end{tabular}

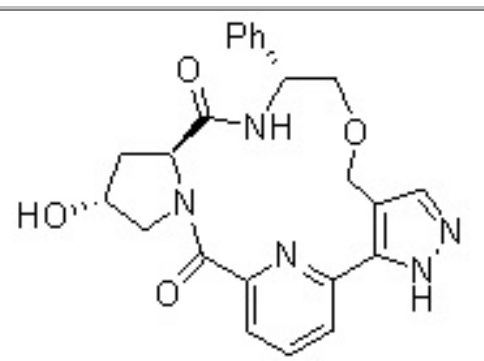

50

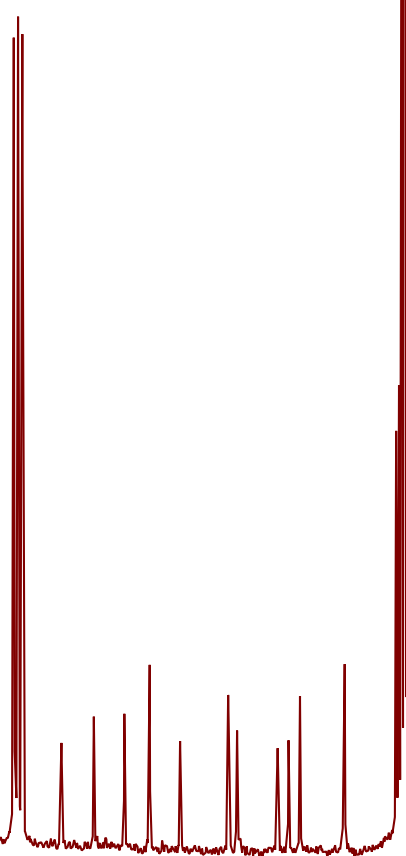

$190 \quad 180$

170

160

150

140

130

120

110

100

f1 (ppm)

$90 \quad 80$

$80 \quad 70$

70

60

50

30

20 
$\left(\mathrm{CD}_{3}\right)_{2} \mathrm{SO}, 90^{\circ} \mathrm{C}, 400 \mathrm{MHz}$

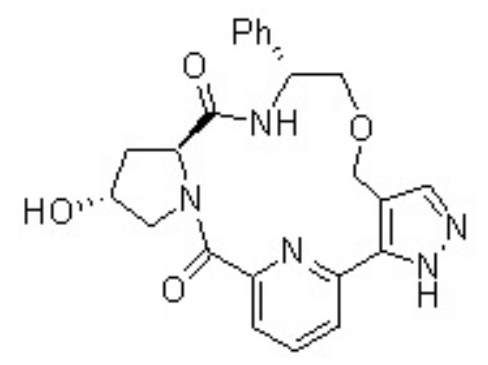

50

$\left(\mathrm{CD}_{3}\right)_{2} \mathrm{SO}, 25^{\circ} \mathrm{C}, 400 \mathrm{MHz}$ chold NMUNAWW Mrar

$\begin{array}{lllllll}13 & 12 & 11 & 10 & 9 & 8 & 7 \\ & & & & & \end{array}$




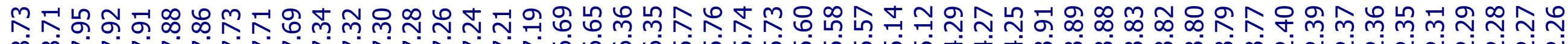

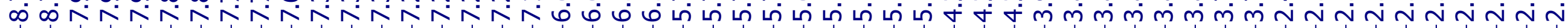

\begin{tabular}{ll}
\hline \multicolumn{1}{|c|}{ Parameter } & Value \\
1 Solvent & CD3OD/CDCl3 $=1: 1$ \\
2 Experiment & $1 \mathrm{D}$ \\
3 Number of Scans & 8 \\
4 Relaxation Delay & 1.0000 \\
5 Acquisition Time & 2.5559 \\
6 Spectrometer Frequency 400.02 \\
7 Nucleus & $1 \mathrm{H}$ \\
\hline
\end{tabular}

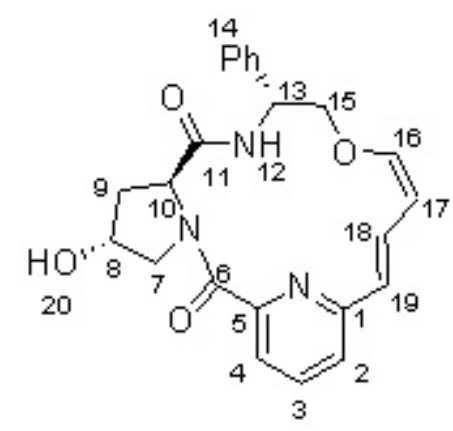

51

12. $18,4,3,14=19,16, \quad 17,10, \quad 13, \quad 8, \quad 15,7, \quad 9$

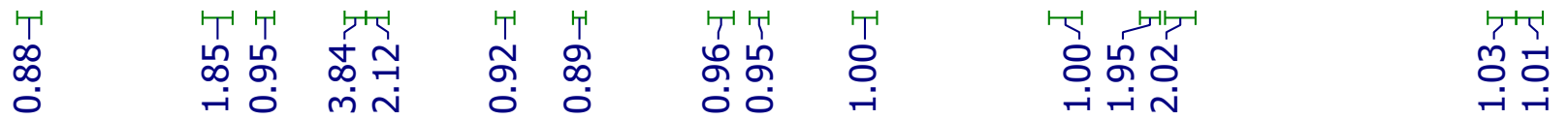

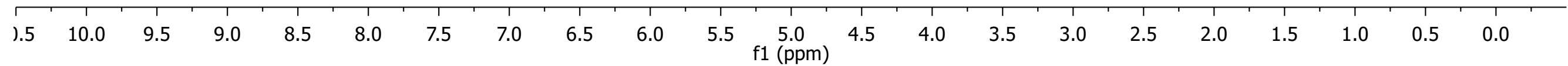




\begin{tabular}{|l|l|}
\hline \multicolumn{1}{|c|}{ Parameter } & CD3OD/CDCl3=1:1 \\
1 Solvent & $1 \mathrm{D}$ \\
2 Experiment & 1800 \\
3 Number of Scans & 1.0000 \\
4 Relaxation Delay & 1.3107 \\
5 Acquisition Time & 100.59 \\
6 Spectrometer Frequency \\
7 Nucleus
\end{tabular}

ลิ효

लं

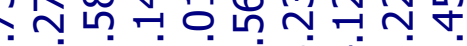

m

j人

ำ

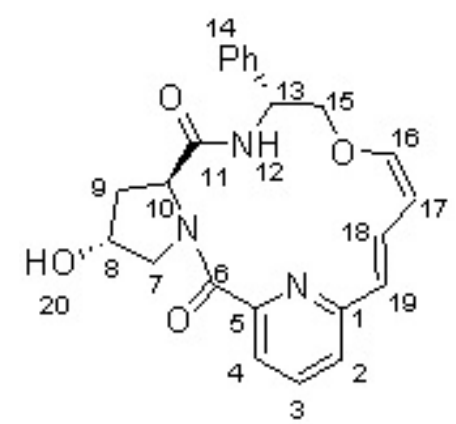

51
$11,6, \quad 1,5,16, \quad 14,3,14,19,18,2,4, \quad 17$
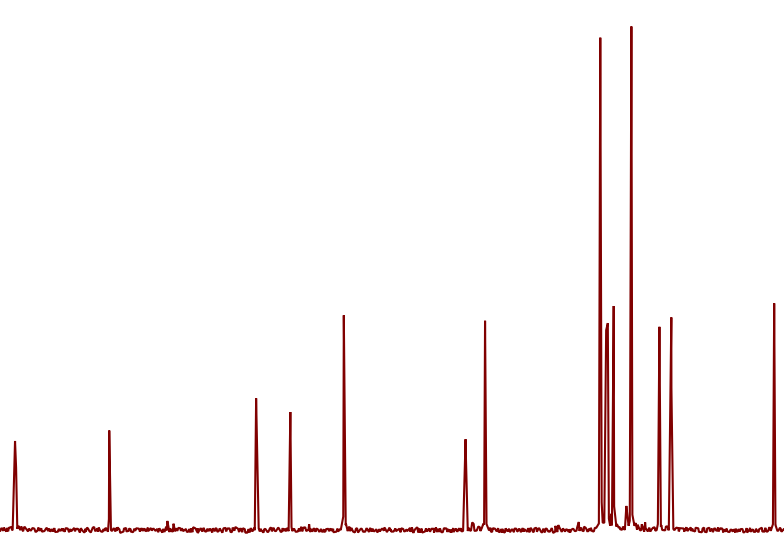

$15,8, \quad 10,7,13, \quad 9$

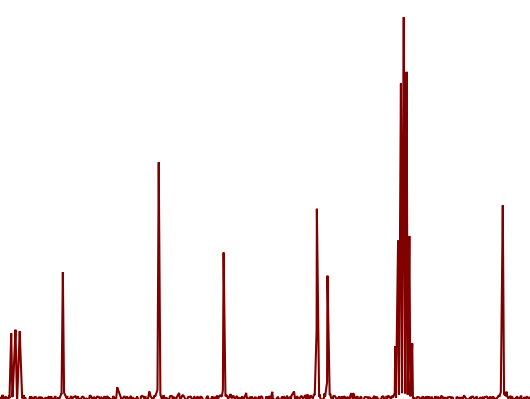

70

60

60

50




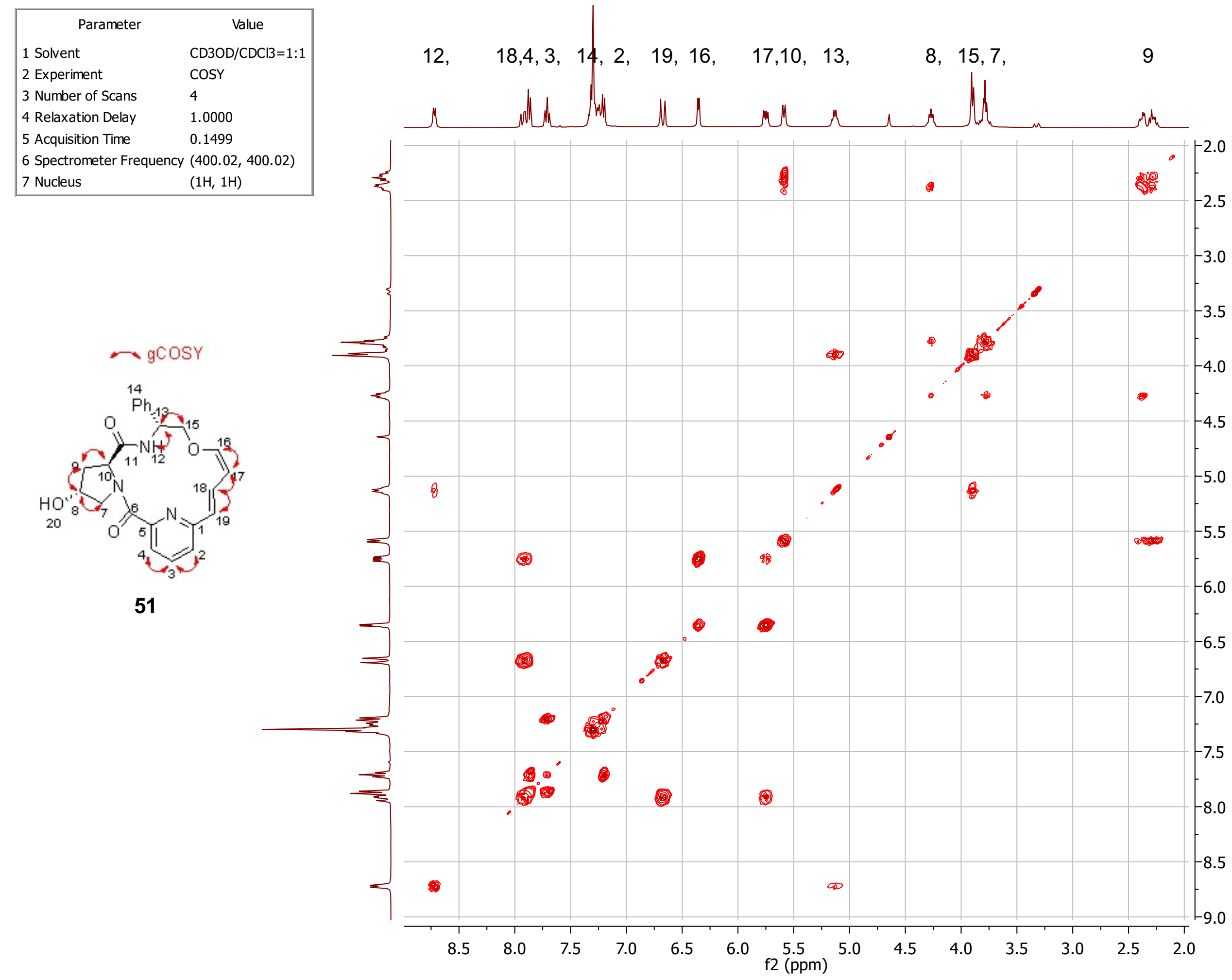

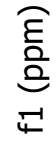




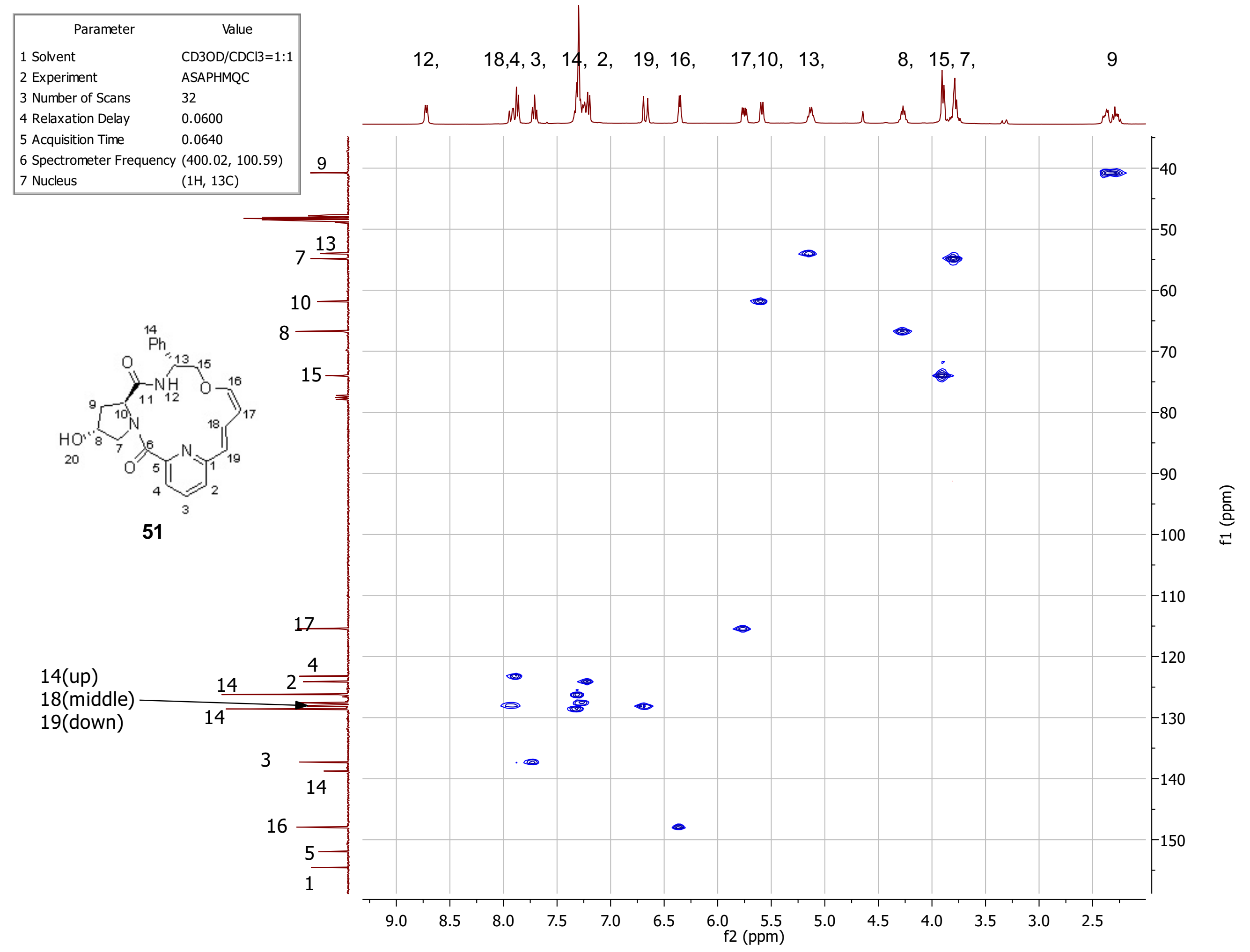




\begin{tabular}{|ll|}
\hline \multicolumn{1}{|c|}{ Parameter } & \multicolumn{1}{c|}{ Value } \\
1 Solvent & CD3OD/CDCl3 $=1: 1$ \\
2 Experiment & NOESY \\
3 Number of Scans & 16 \\
4 Relaxation Delay & 1.0000 \\
5 Acquisition Time & 0.1499 \\
6 Spectrometer Frequency & $(400.02,400.02)$ \\
7 Nucleus & $(1 \mathrm{H}, 1 \mathrm{H})$ \\
\hline
\end{tabular}

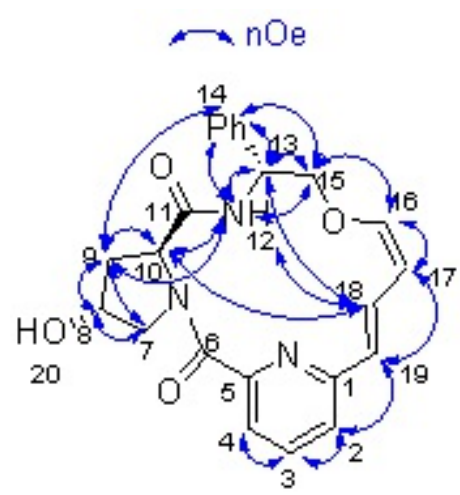

51

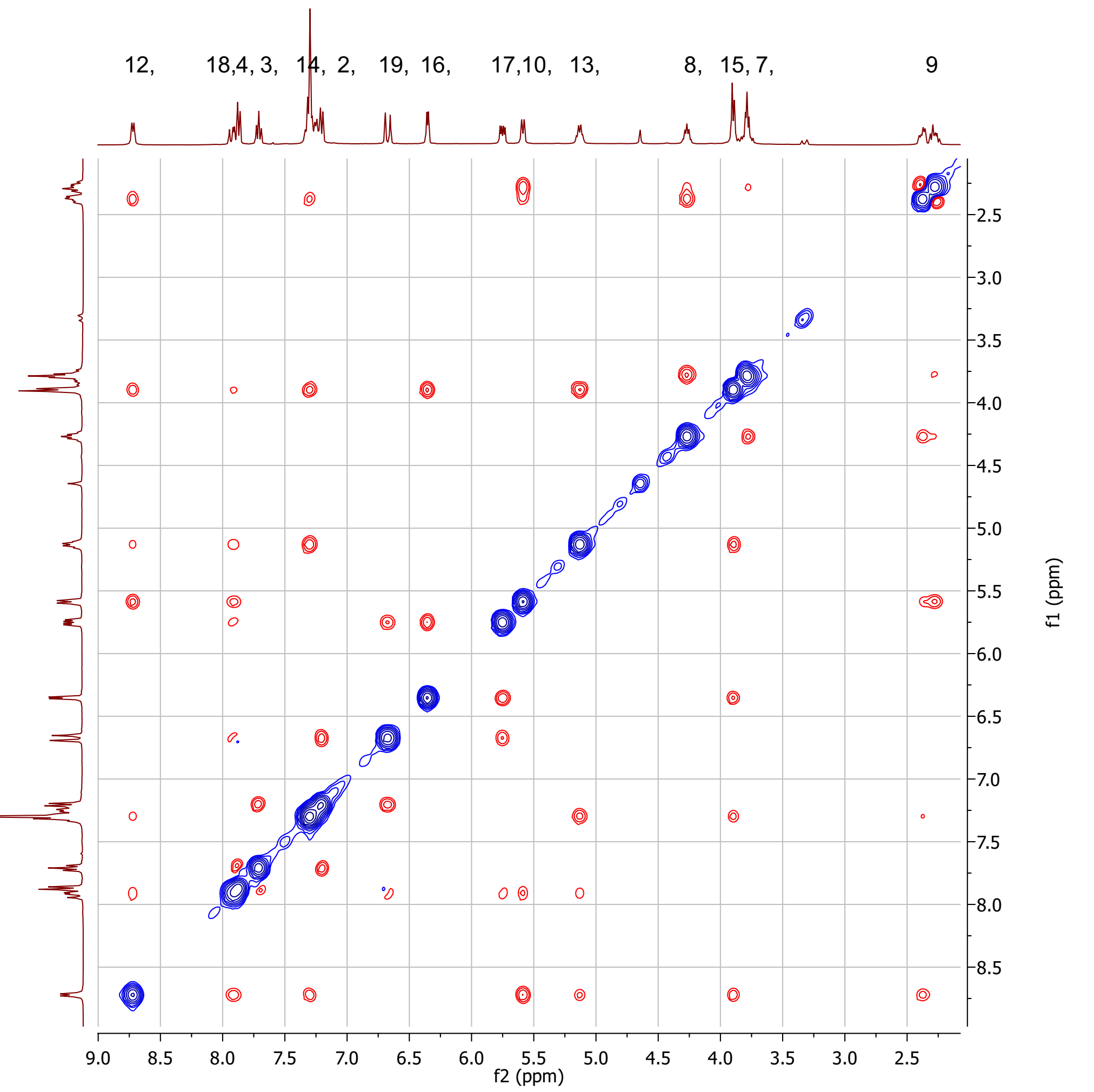




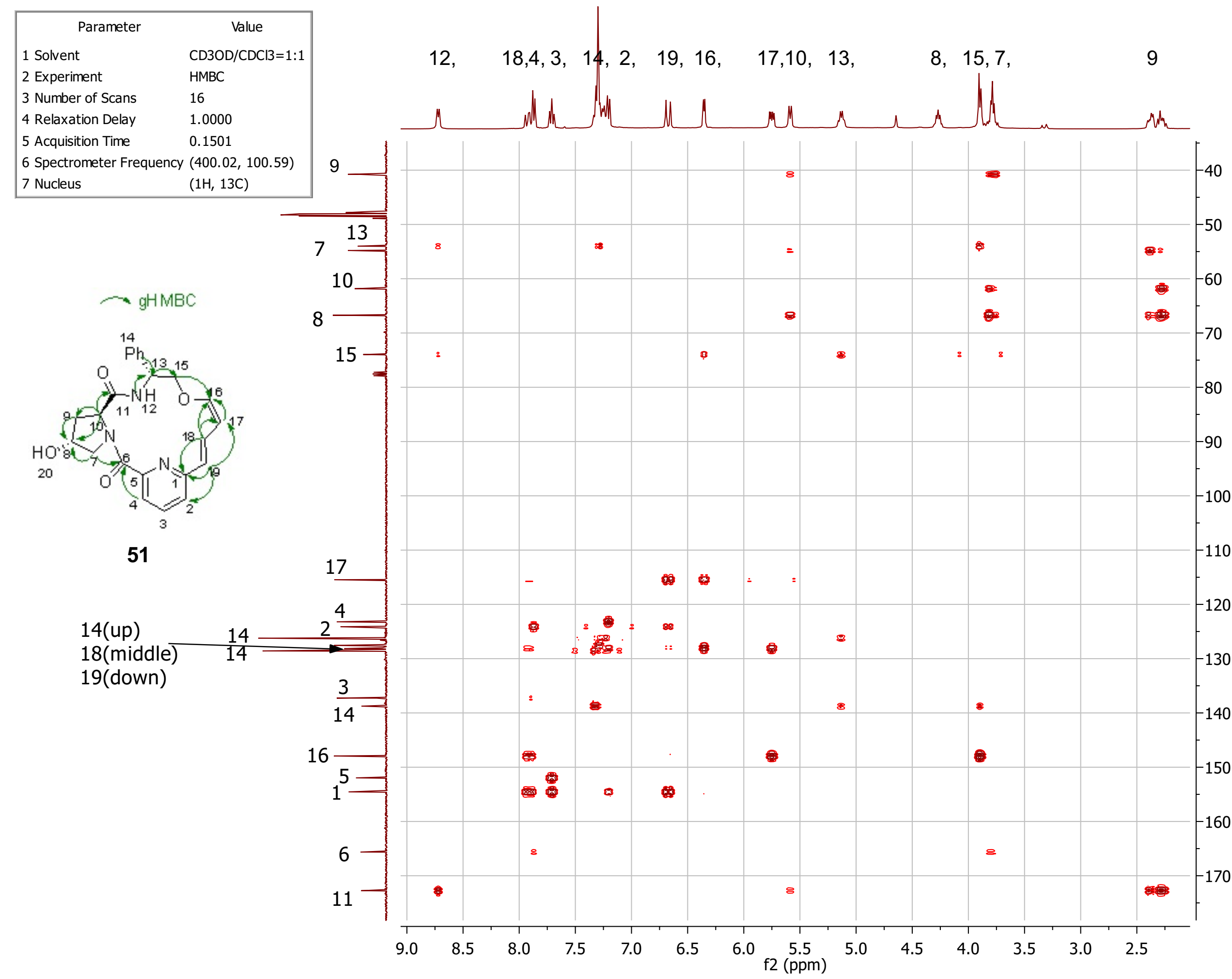

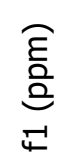




\begin{tabular}{|ll|}
\hline \multicolumn{1}{|c|}{ Parameter } & \multicolumn{1}{c|}{ Value } \\
1 Solvent & cdcl3 \\
2 Experiment & $1 \mathrm{D}$ \\
3 Number of Scans & 8 \\
4 Relaxation Delay & 1.0000 \\
5 Acquisition Time & 2.5559 \\
6 Spectrometer Frequency & 400.01 \\
7 Nucleus & $1 \mathrm{H}$ \\
\hline
\end{tabular}

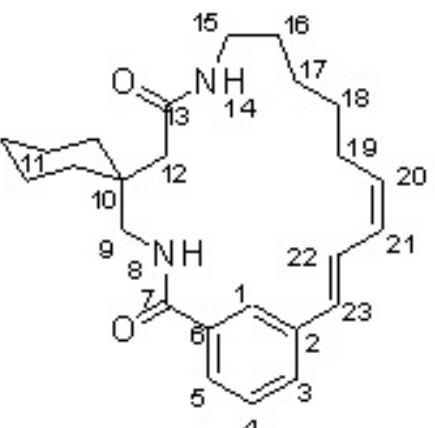

$\nu_{20.2}=10.9 \mathrm{~Hz}, v_{21,22}=10.9 \mathrm{~Hz}, \jmath_{22,23}=15.5 \mathrm{~Hz}$

52

8 ,

$1,522,4,3, \quad 23,21,14,20$,
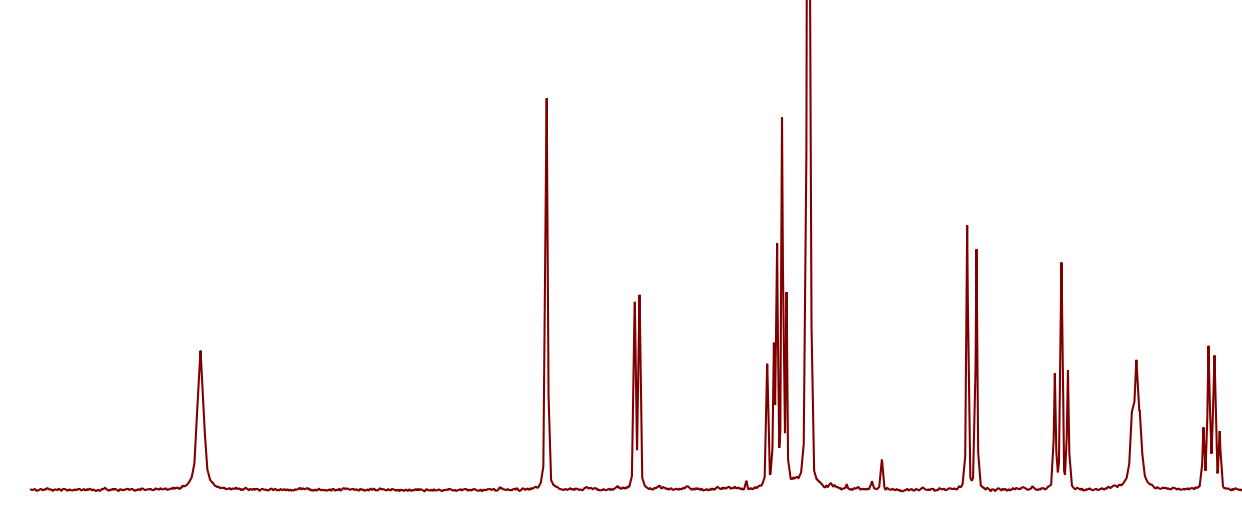

†

'T 'T

上世

म다 †什

ลิ ๙

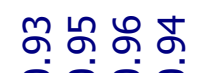

No 0

(T)

ㅇํㅇㅇㅛ

- $~$

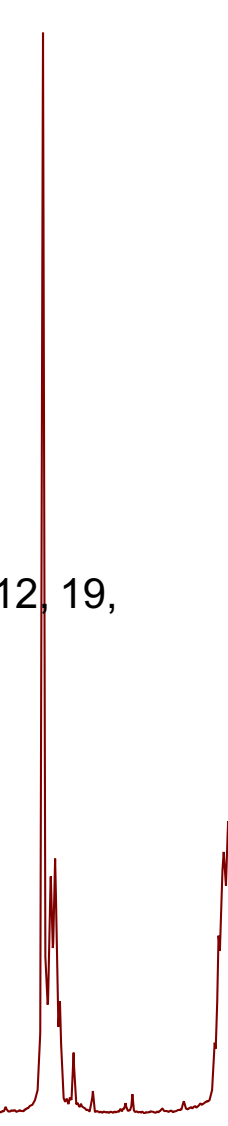

$16,17,18,11$

$8.0 \quad 7.5$

7.0

6.56.

\title{
म्त
}

ติ ำ

ชิ่

\begin{abstract}
The
\end{abstract}
क

กี

1.5




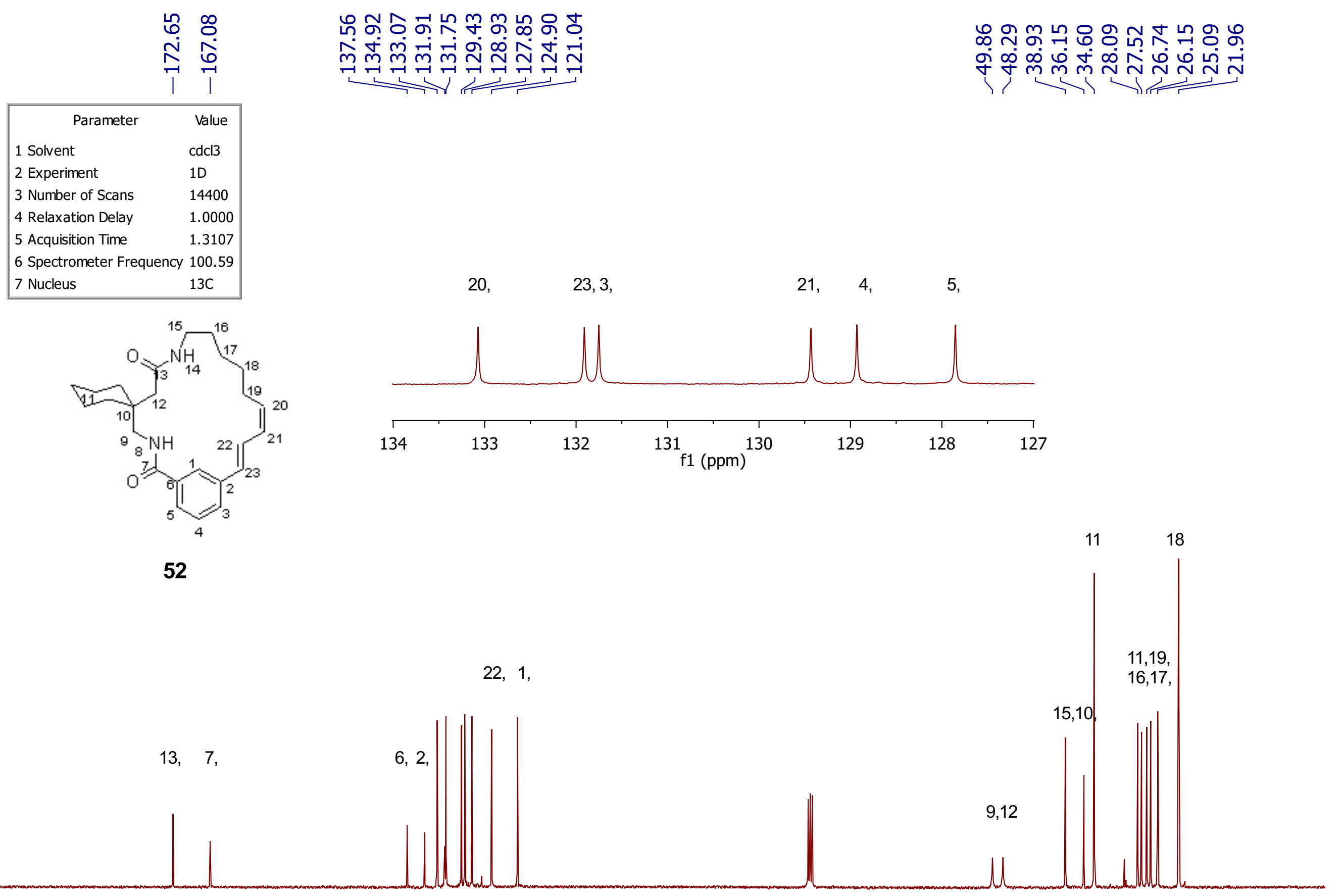

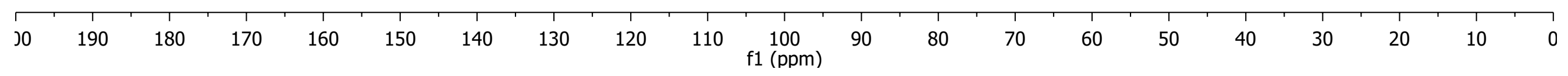




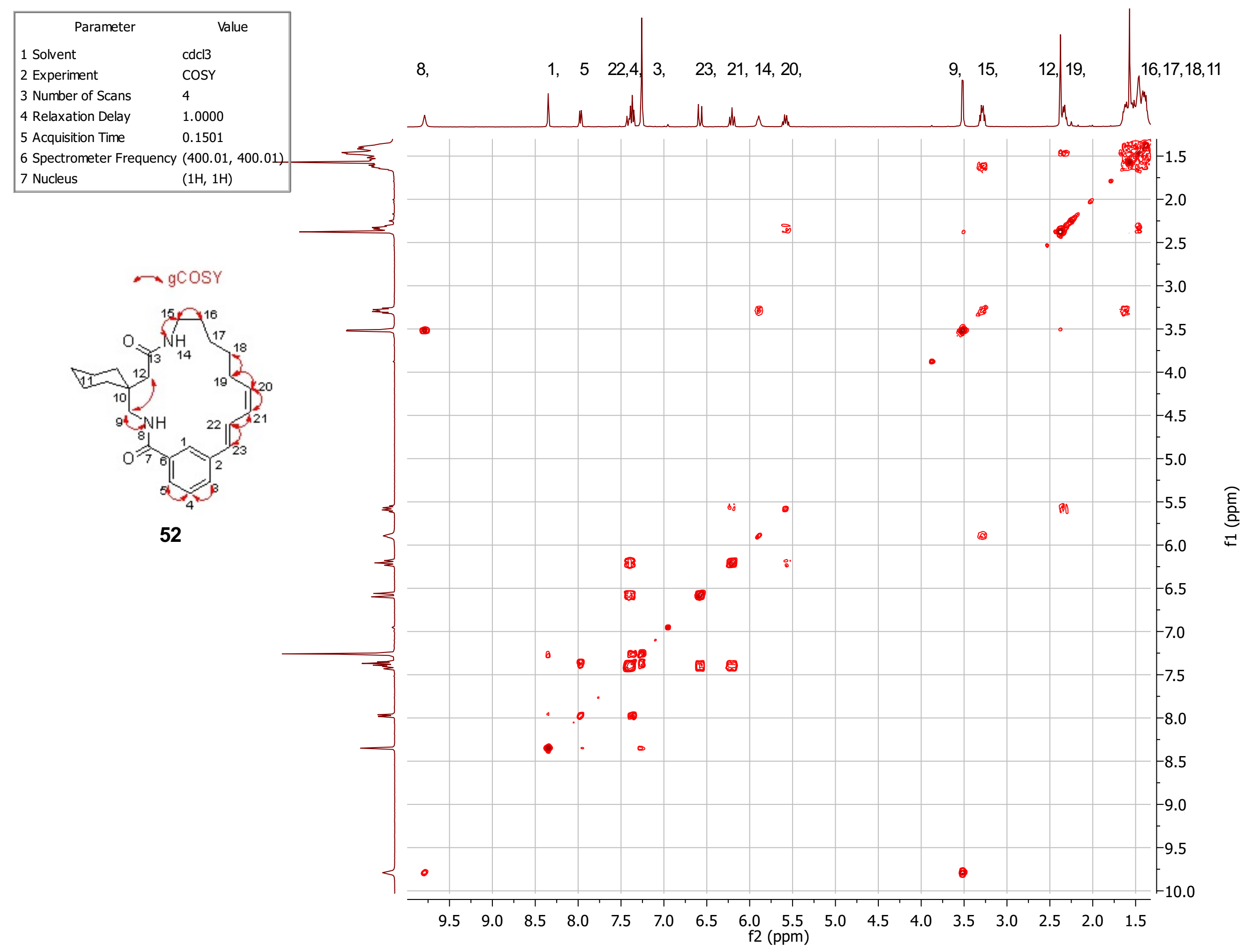




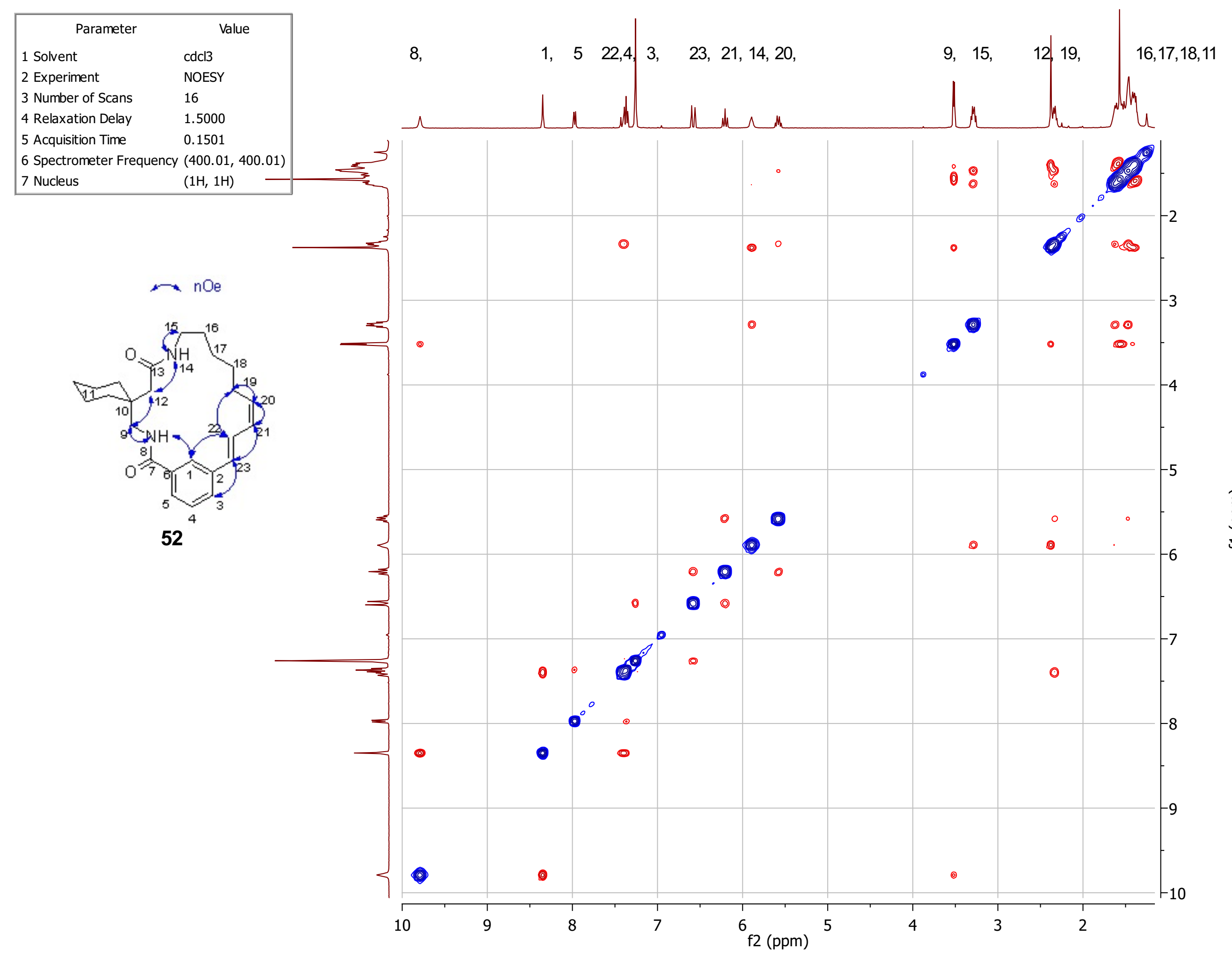

$\widehat{E}$
흔 


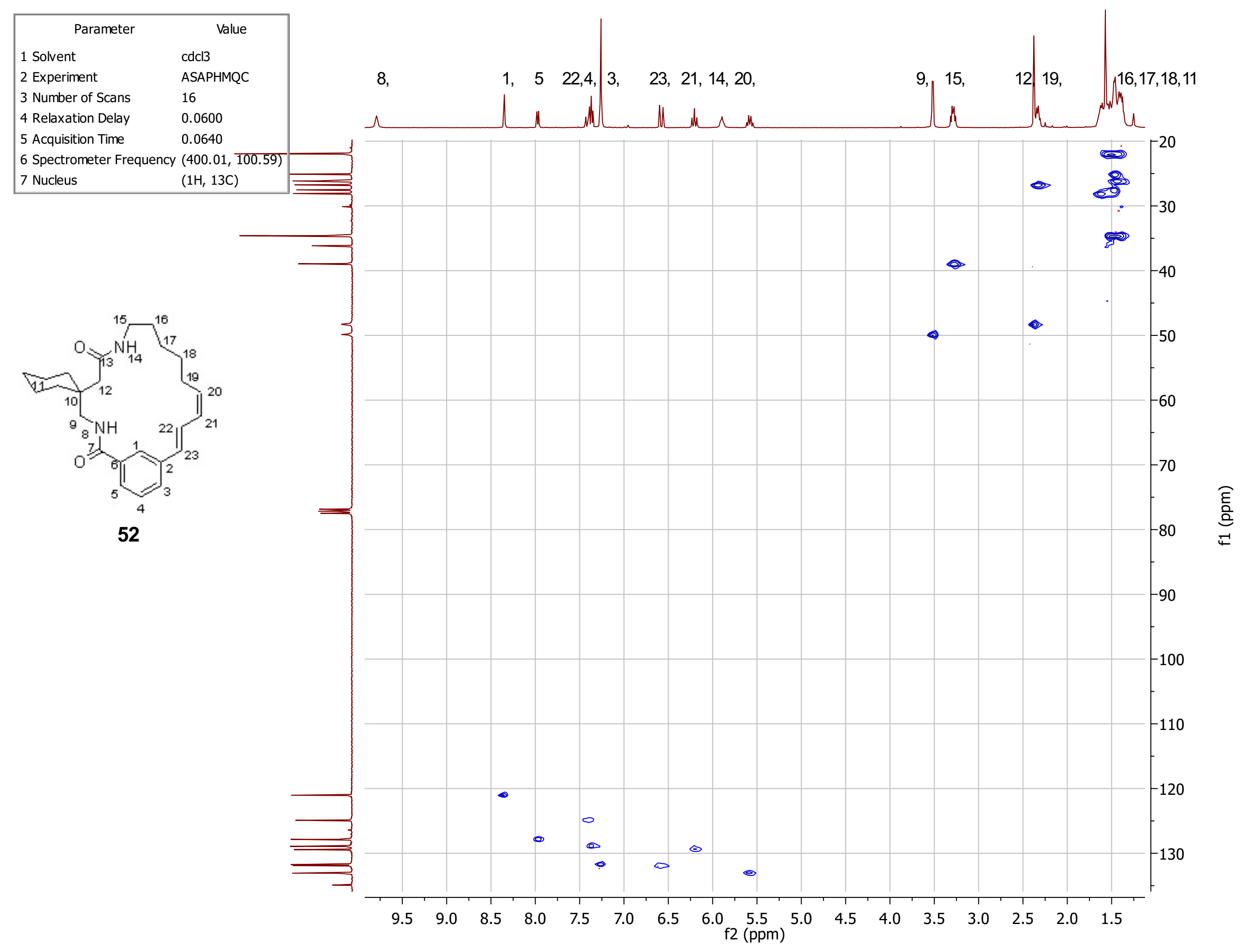




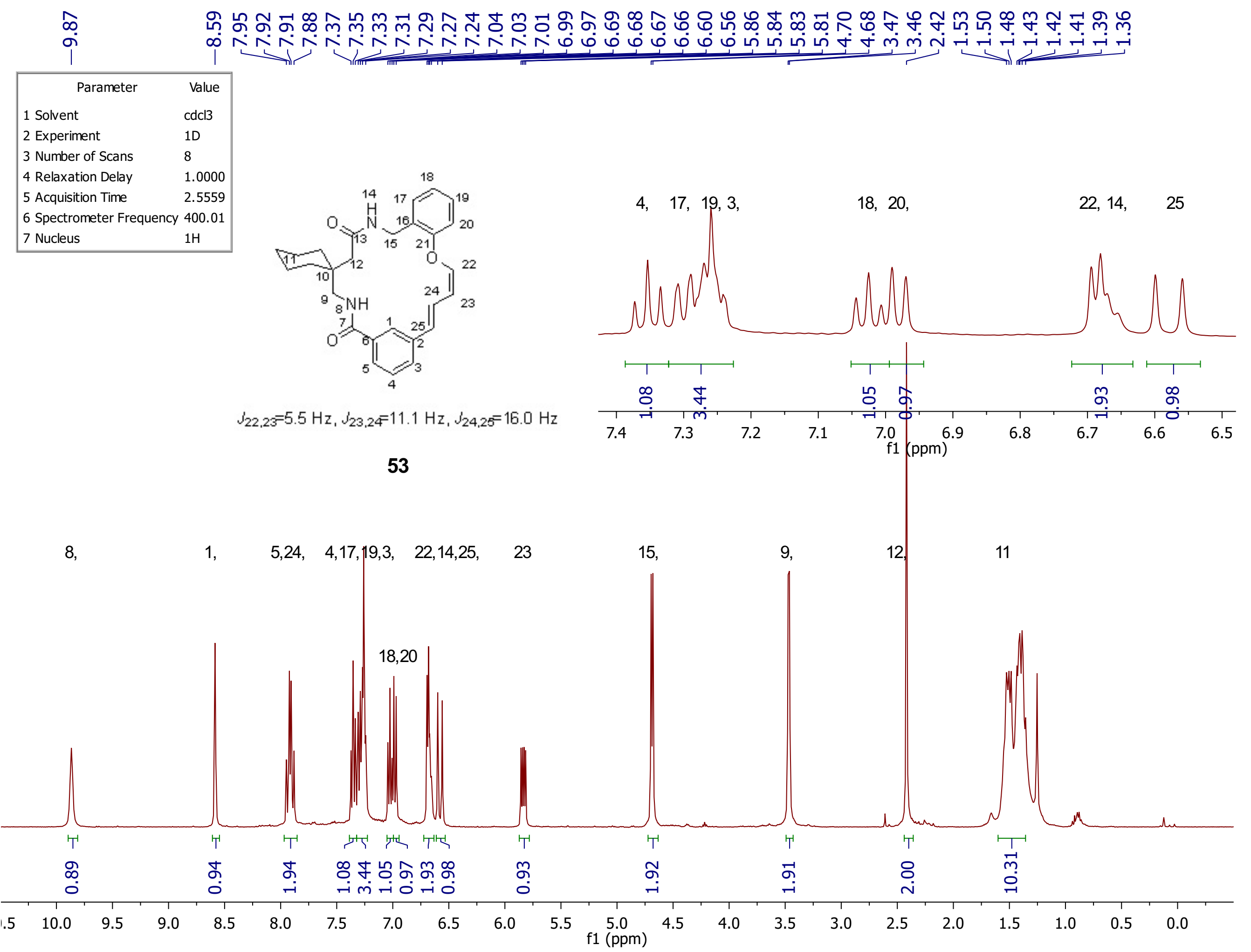




\begin{tabular}{|ll|}
\hline \multicolumn{1}{|c|}{ Parameter } & Value \\
1 Solvent & cdcl3 \\
2 Experiment & $1 \mathrm{D}$ \\
3 Number of Scans & 1700 \\
4 Relaxation Delay & 1.0000 \\
5 Acquisition Time & 1.3107 \\
6 Spectrometer Frequency 100.59 \\
7 Nucleus & $13 \mathrm{C}$ \\
\hline
\end{tabular}

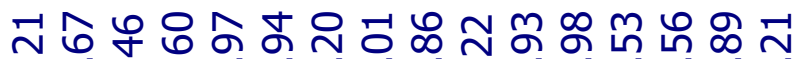
ㄴํำ 득 瓷

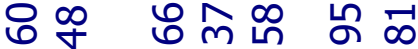

จें
3
17, $\quad 19,25,4$,
5,16
$24, \quad 18, \quad 1$
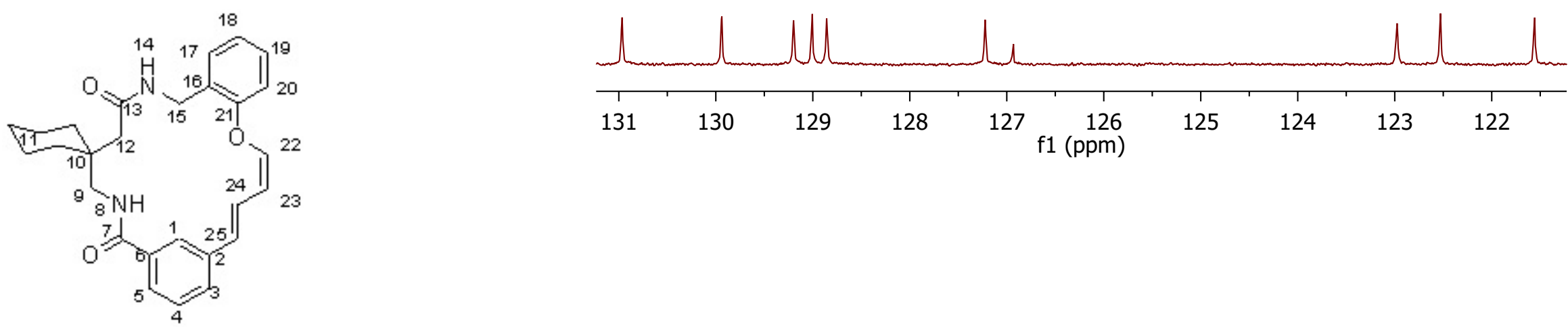

53

13, $7, \quad 21, \quad 22,6,2, \quad 5,16 \quad 23,20$,

$9,12, \quad 15,10,11,11,1$
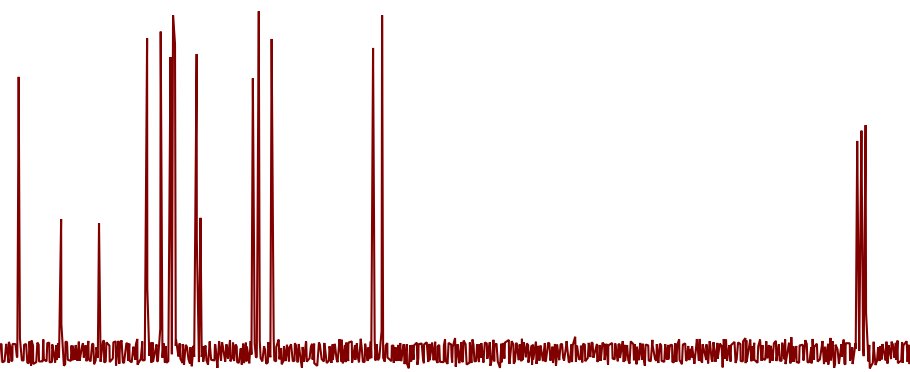

0




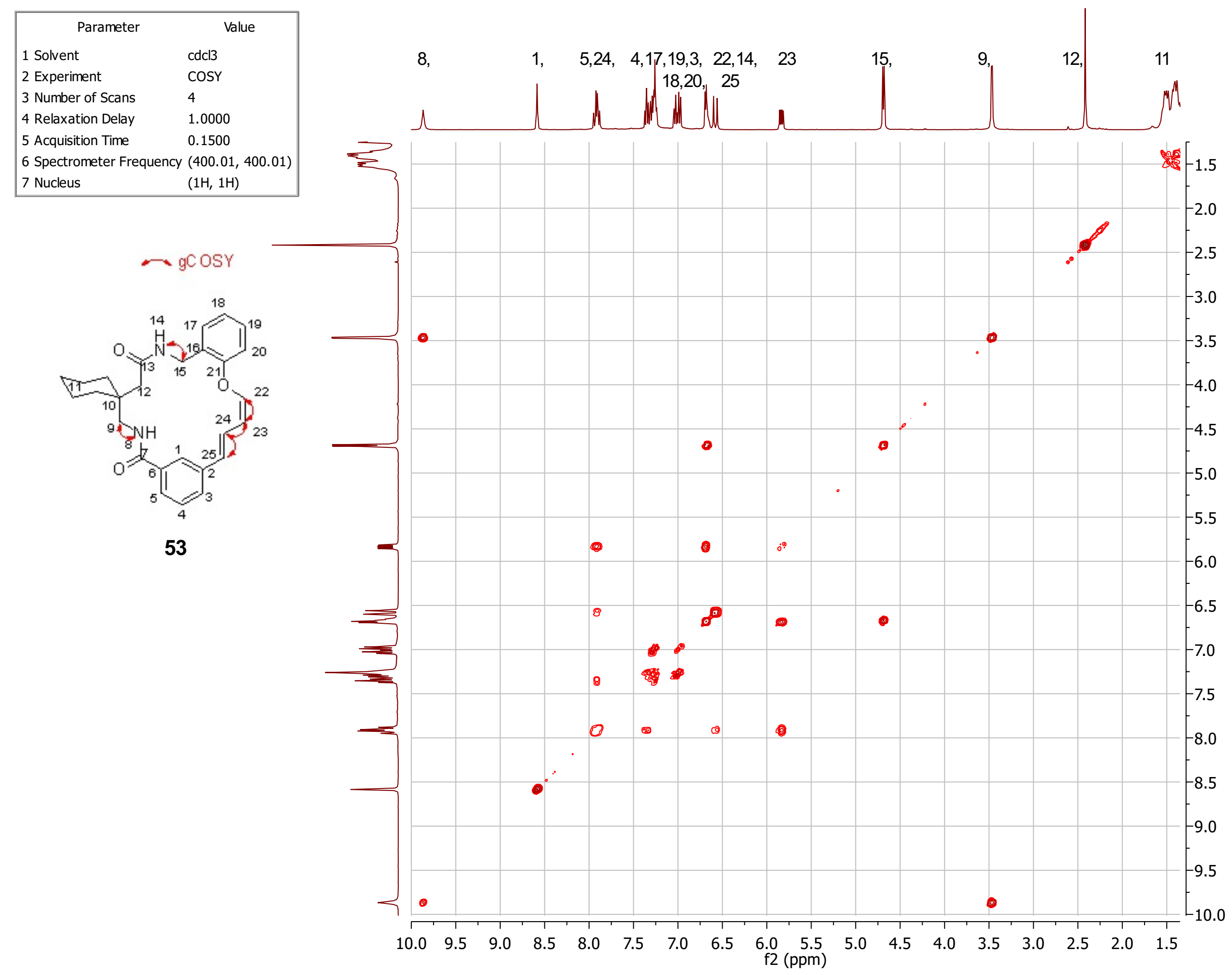

흔 


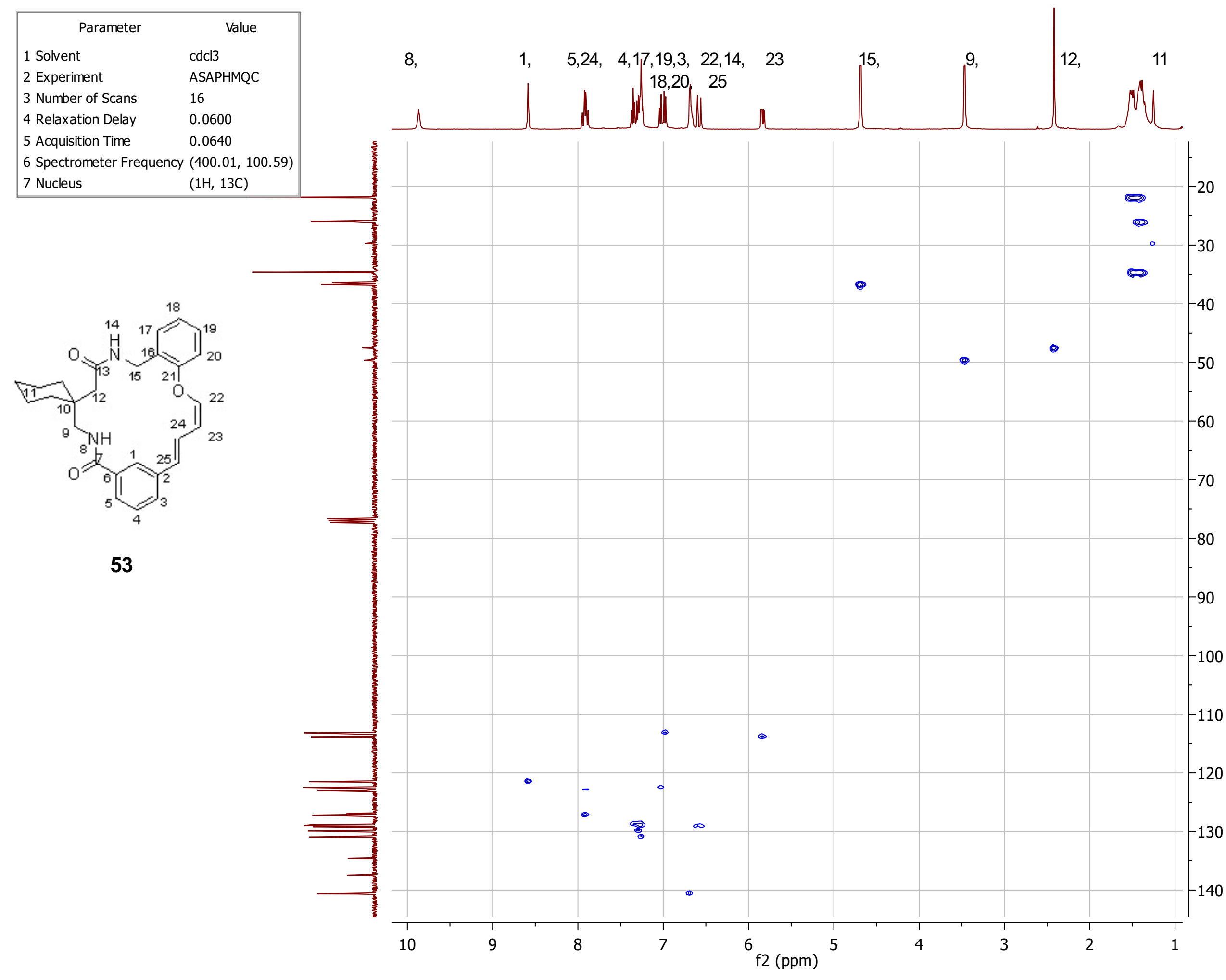




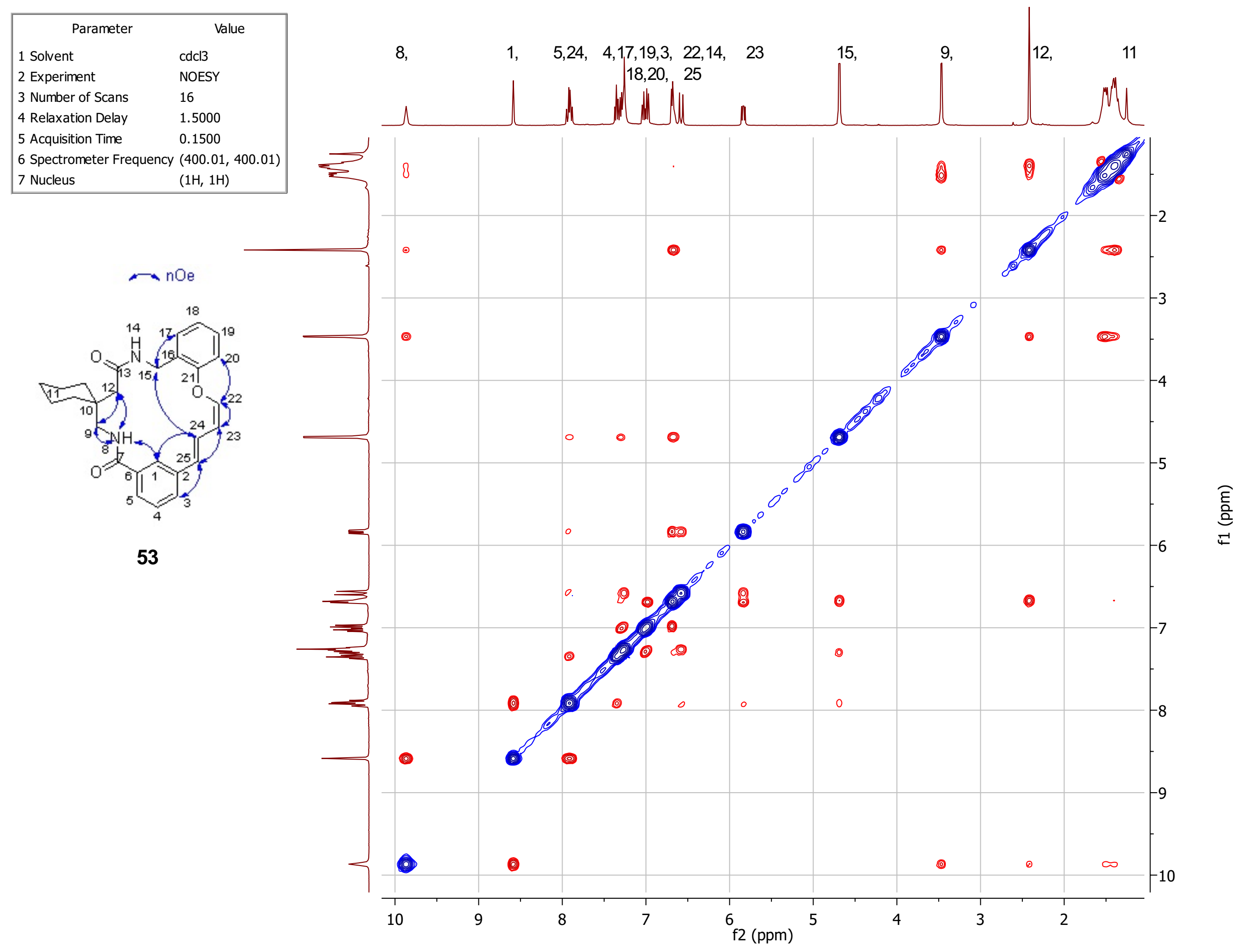




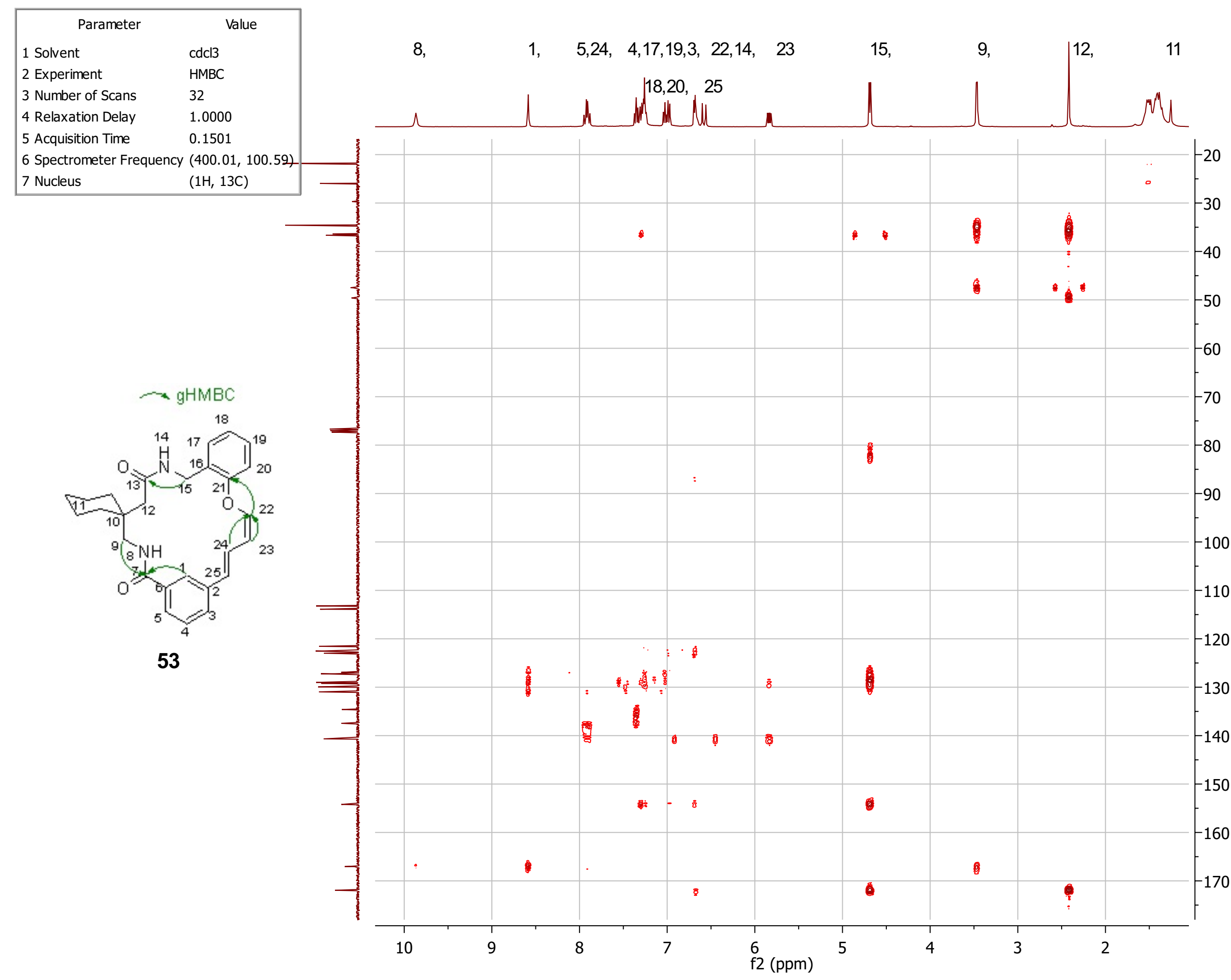




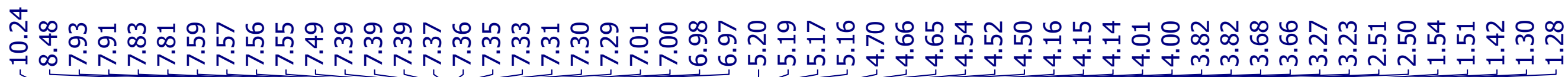

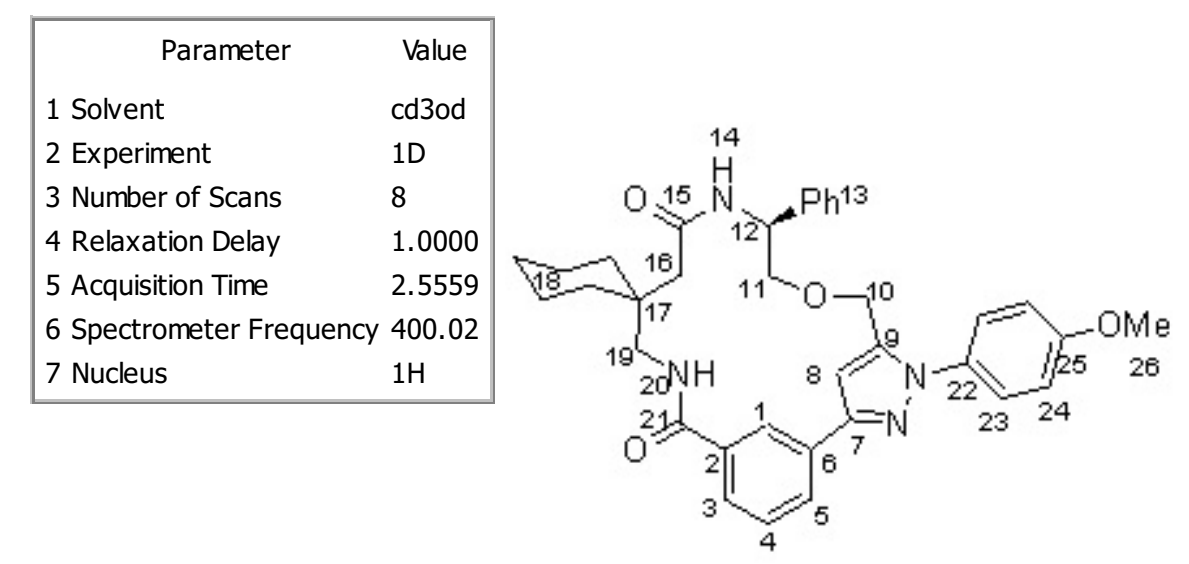

56

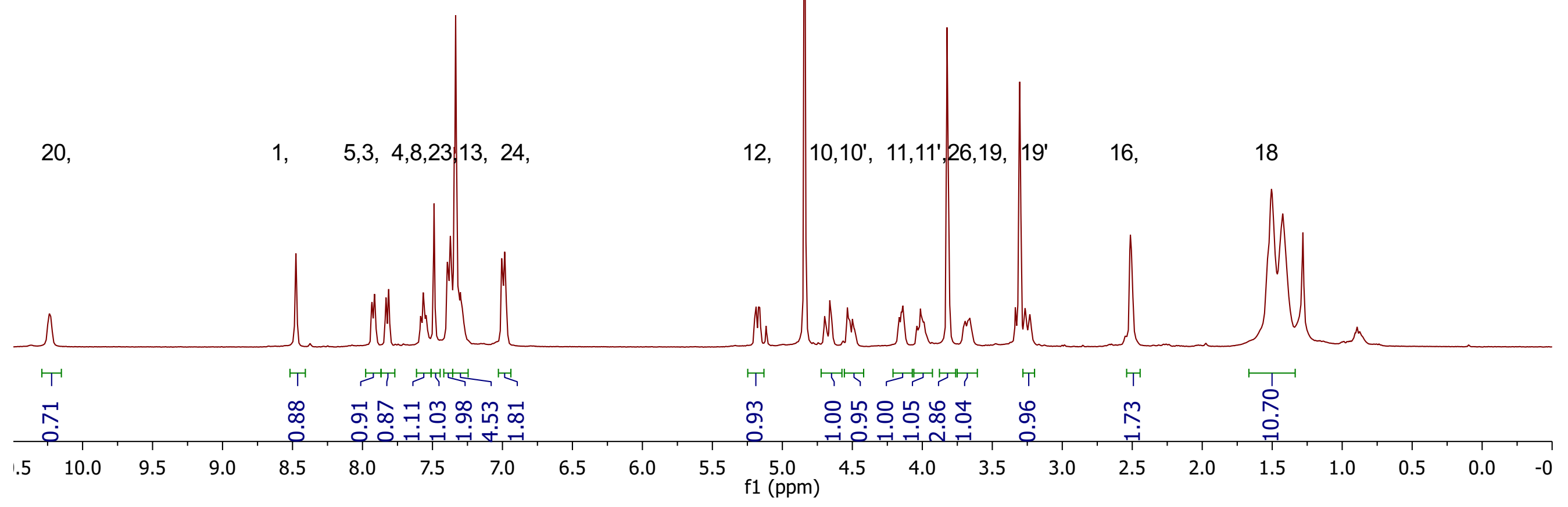




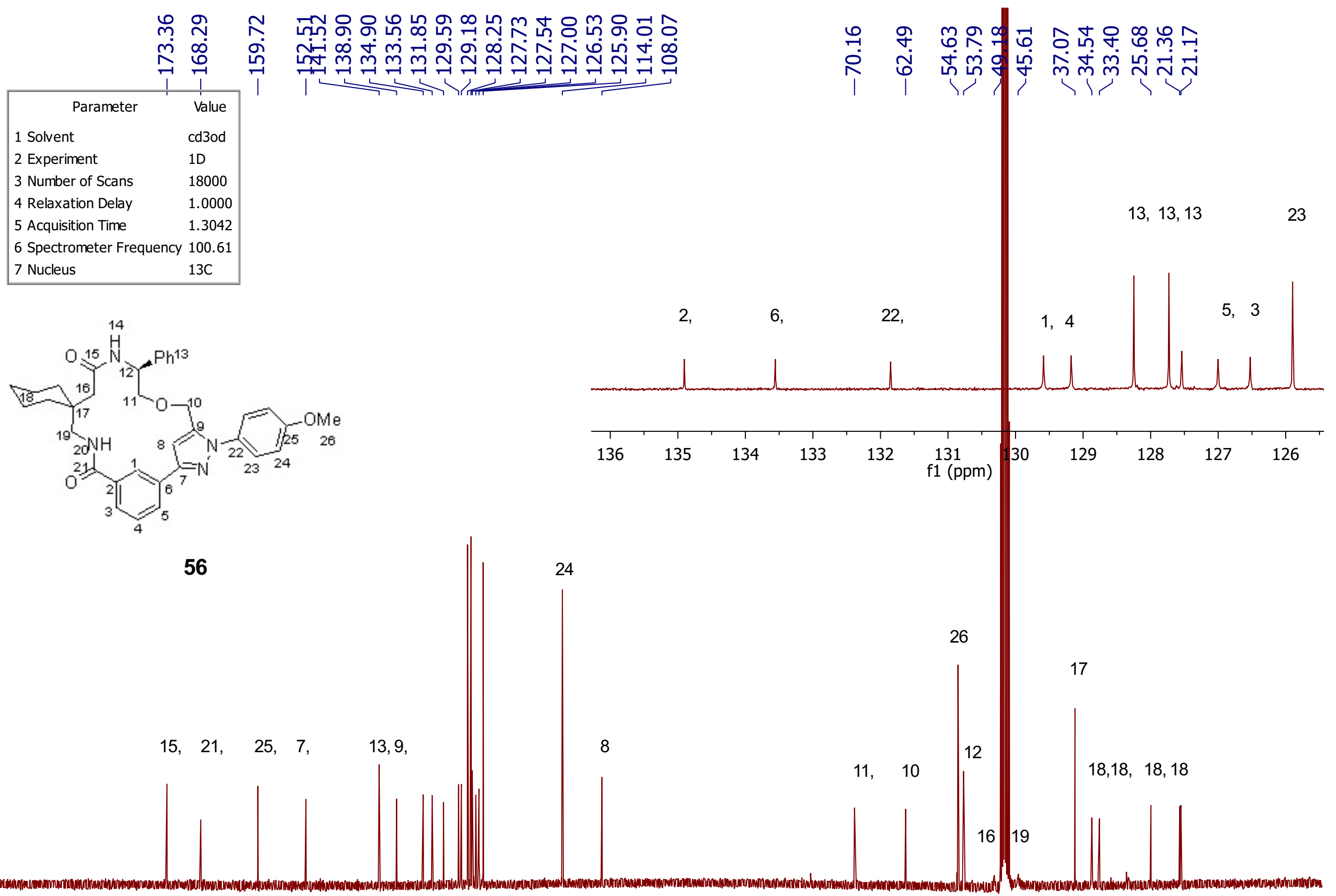

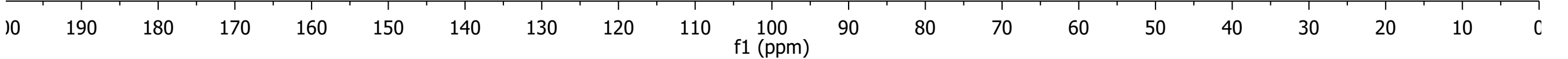




\begin{tabular}{|ll|}
\hline \multicolumn{1}{|c|}{ Parameter } & \multicolumn{1}{c|}{ Value } \\
1 Solvent & cd3od \\
2 Experiment & COSY \\
3 Number of Scans & 8 \\
4 Relaxation Delay & 1.0000 \\
5 Acquisition Time & 0.1500 \\
6 Spectrometer Frequency & $(400.02,400.02)$ \\
7 Nucleus & $(1 \mathrm{H}, 1 \mathrm{H})$ \\
\hline
\end{tabular}

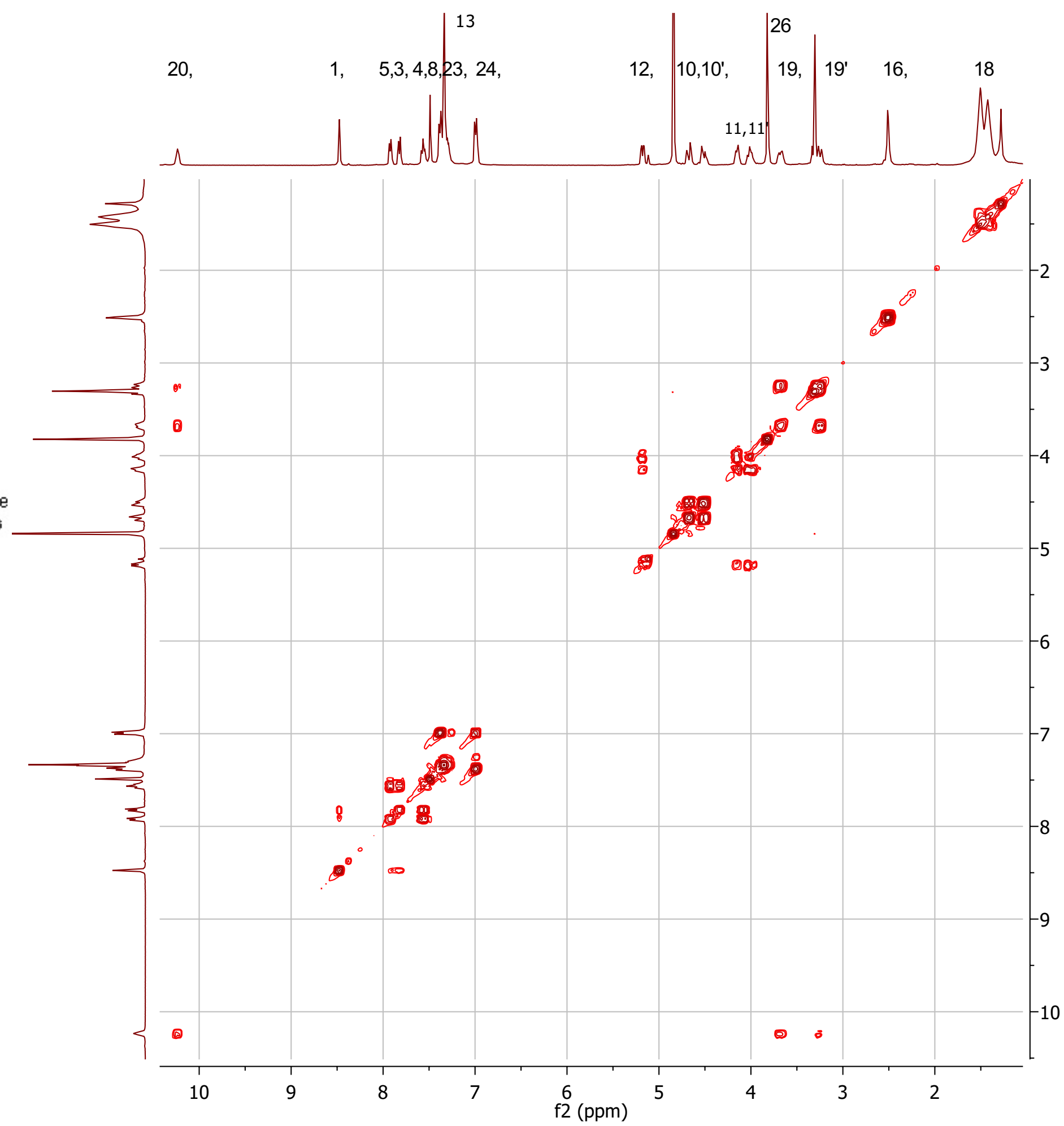

${ }_{\bar{E}}^{\stackrel{0}{0}}$

56

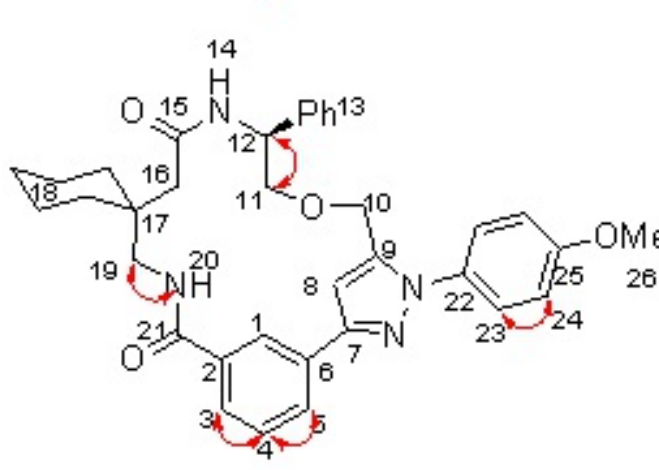




\begin{tabular}{|ll|}
\hline \multicolumn{1}{|c|}{ Parameter } & \multicolumn{1}{c|}{ Value } \\
1 Solvent & cd3od \\
2 Experiment & ASAPHMQC \\
3 Number of Scans & 32 \\
4 Relaxation Delay & 0.0600 \\
5 Acquisition Time & 0.0640 \\
6 Spectrometer Frequency & $(400.02,100.59)$ \\
7 Nucleus & $(1 \mathrm{H}, 13 \mathrm{C})$ \\
\hline
\end{tabular}
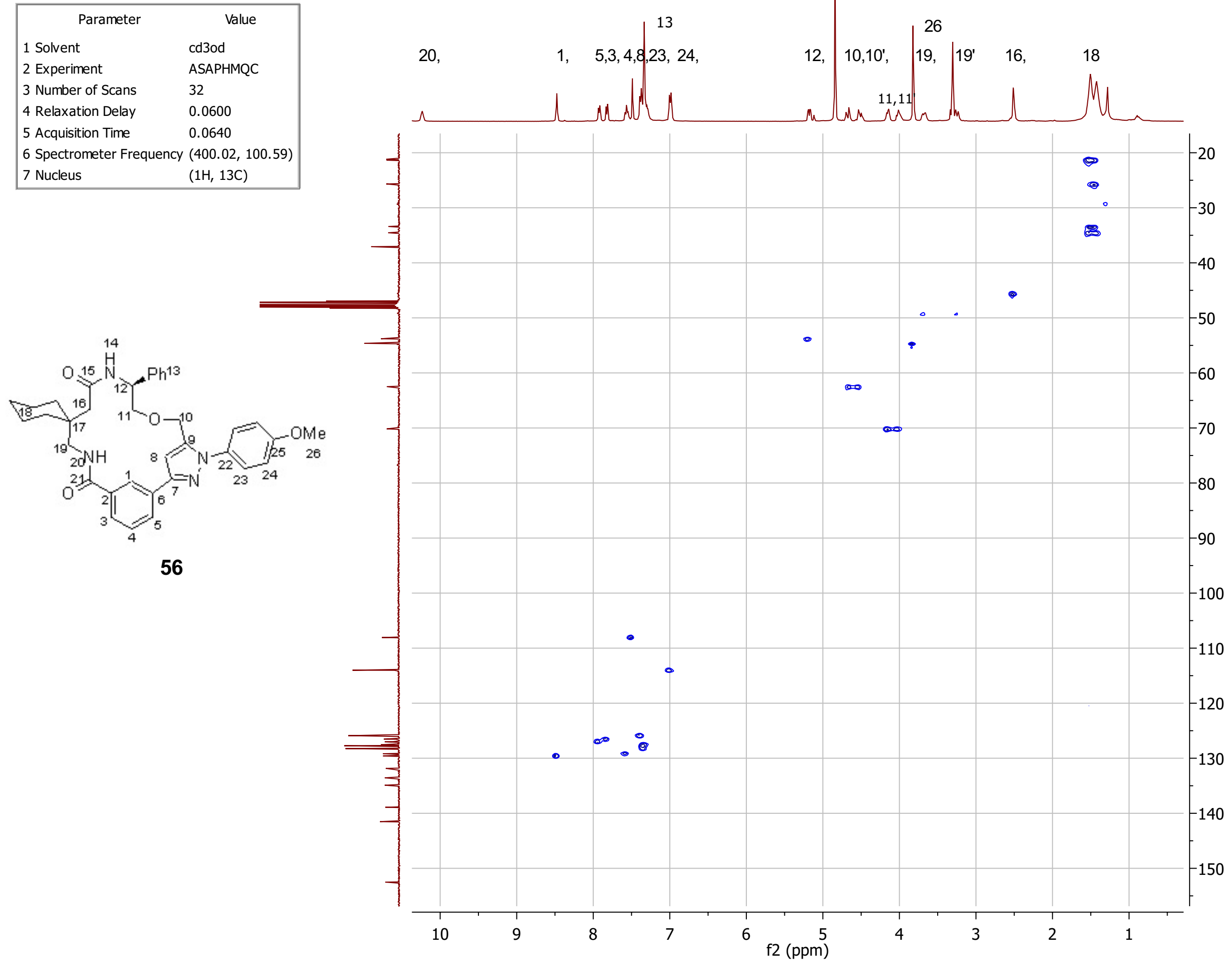


\begin{tabular}{|ll|}
\hline \multicolumn{1}{|c|}{ Parameter } & \multicolumn{1}{c|}{ Value } \\
1 Solvent & cd3od \\
2 Experiment & NOESY \\
3 Number of Scans & 16 \\
4 Relaxation Delay & 1.5000 \\
5 Acquisition Time & 0.1500 \\
6 Spectrometer Frequency & $(400.02,400.02)$ \\
7 Nucleus & $(1 \mathrm{H}, 1 \mathrm{H})$ \\
\hline
\end{tabular}
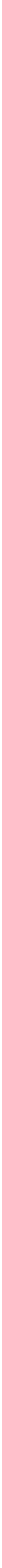
চ চ

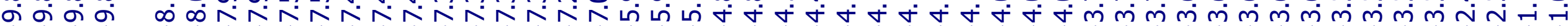

\begin{tabular}{|ll|}
\hline \multicolumn{1}{|c|}{ Parameter } & \multicolumn{1}{c|}{ Value } \\
1 Solvent & cdcl3 \\
2 Experiment & $1 \mathrm{D}$ \\
3 Number of Scans & 8 \\
4 Relaxation Delay & 1.0000 \\
5 Acquisition Time & 2.0447 \\
6 Spectrometer Frequency & 499.81 \\
7 Nucleus & $1 \mathrm{H}$ \\
\hline
\end{tabular}

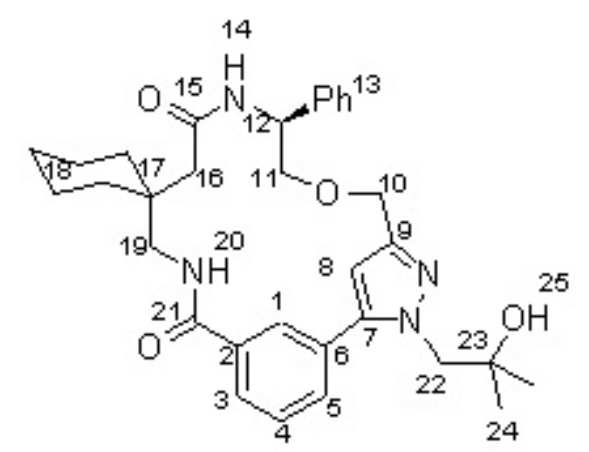

58

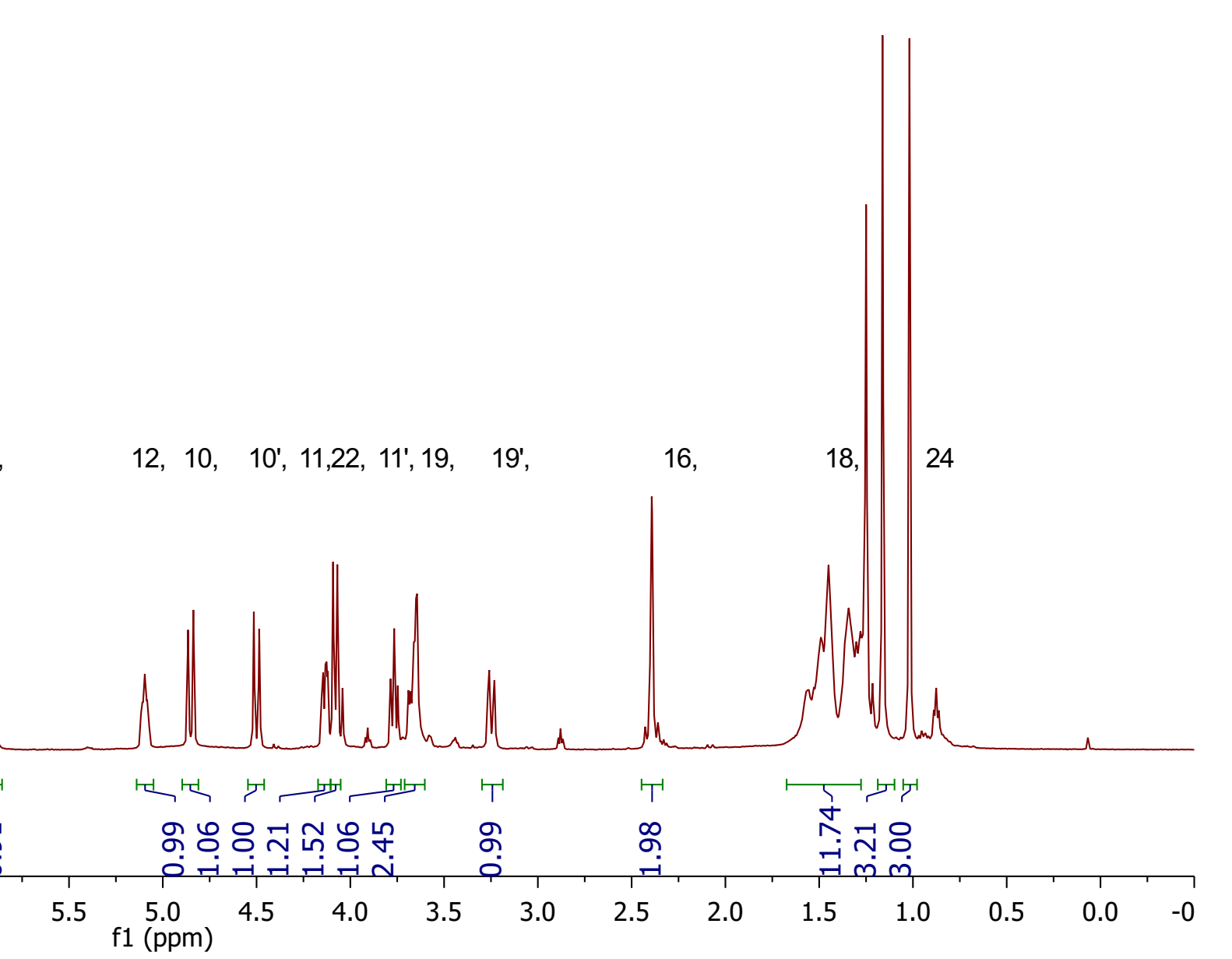




\begin{tabular}{|c|c|}
\hline 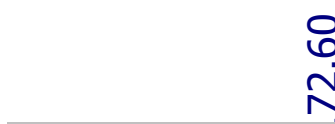 & $\begin{array}{l}\text { N } \\
\widehat{\sigma}\end{array}$ \\
\hline Parameter & Value \\
\hline 1 Solvent & cdcl3 \\
\hline 2 Experiment & $1 \mathrm{D}$ \\
\hline 3 Number of Scans & 18200 \\
\hline 4 Relaxation Delay & 1.0000 \\
\hline 5 Acquisition Time & 1.3107 \\
\hline 6 Spectrometer Frequen & 100.59 \\
\hline 7 Nucleus & $13 \mathrm{C}$ \\
\hline
\end{tabular}

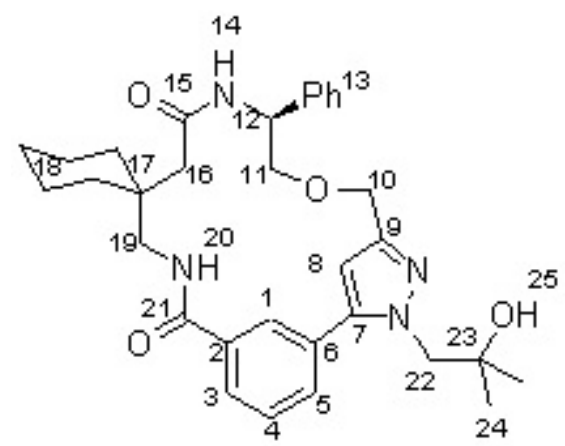

58

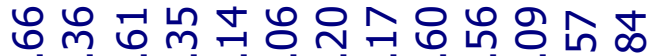
守

开

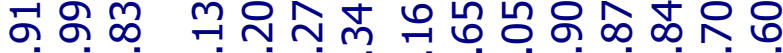

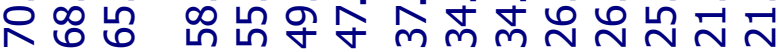

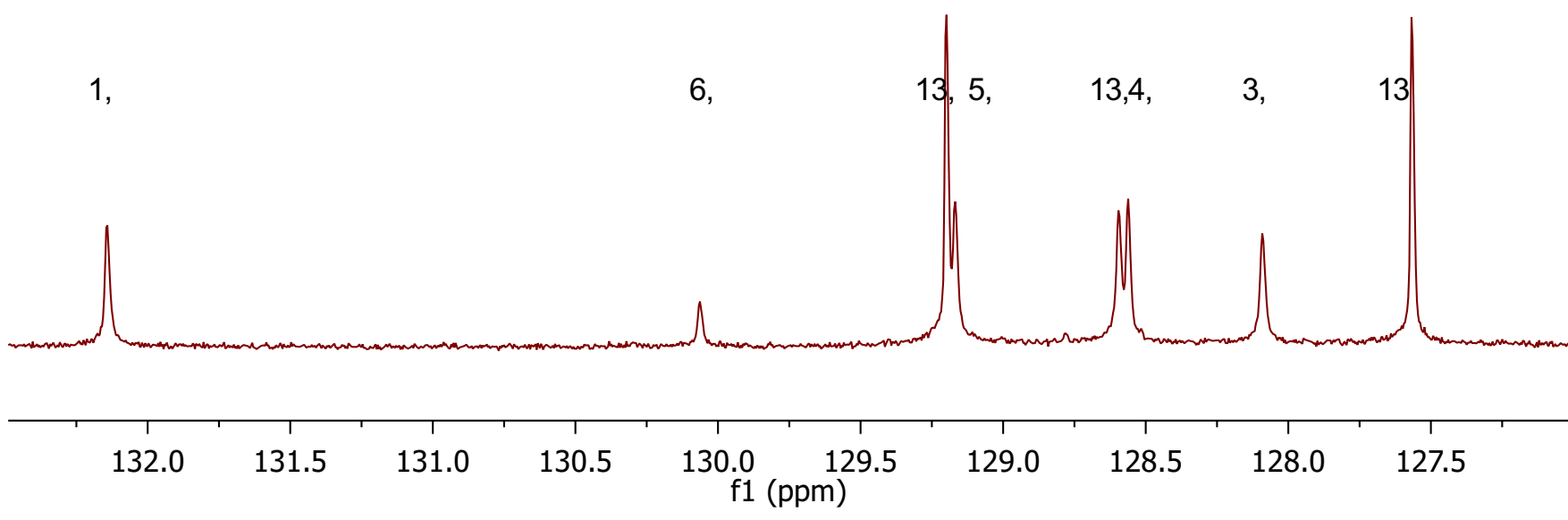

15, 21,

$9,7, \quad 13,2$,

8

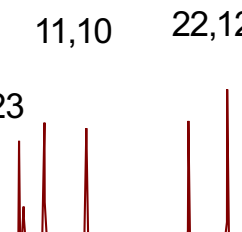

19,16

$80-70$




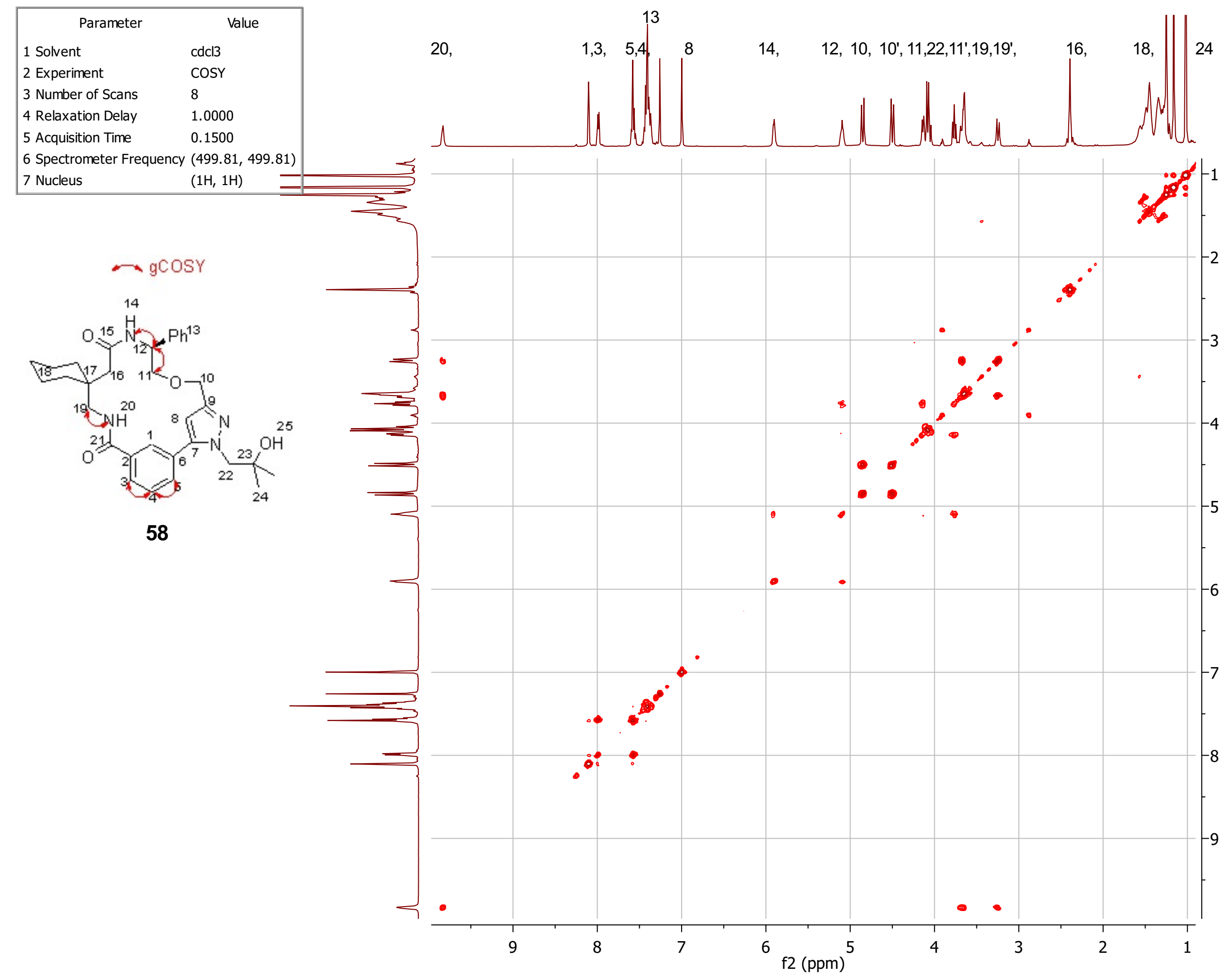




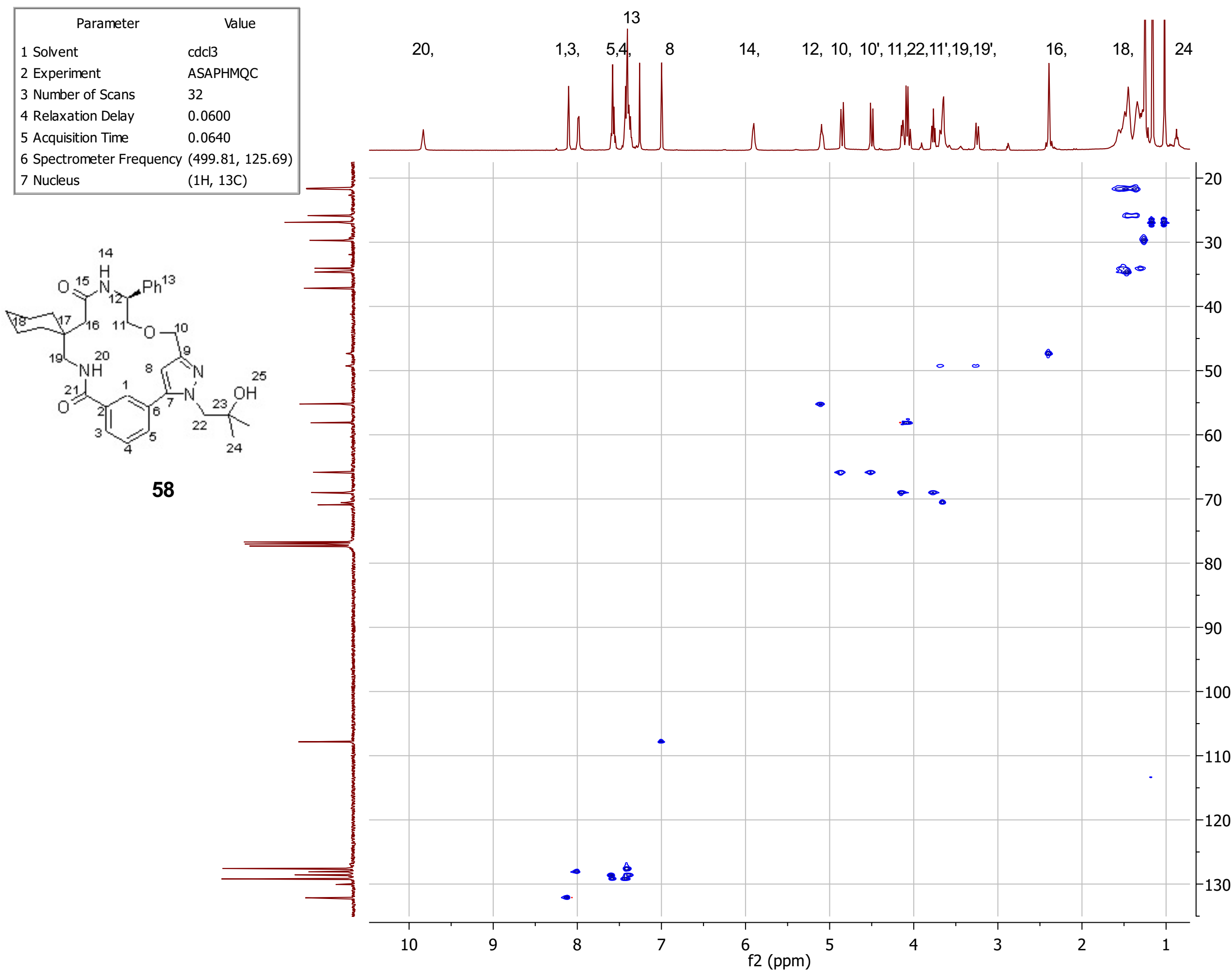

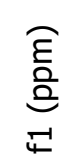




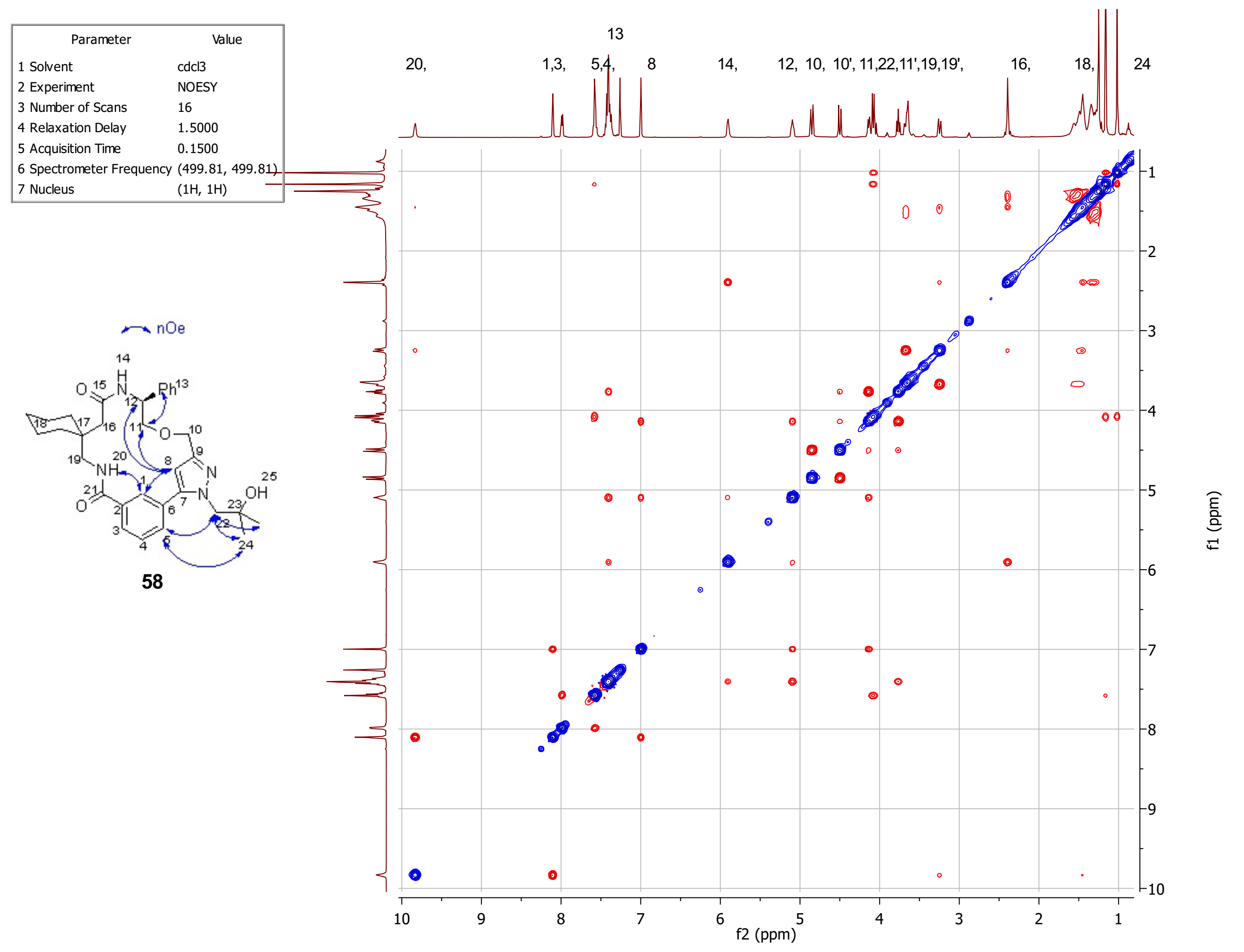


ๆ ₹

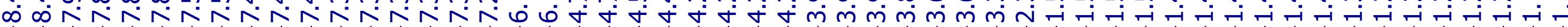

\begin{tabular}{|ll|}
\hline \multicolumn{1}{|c|}{ Parameter } & \multicolumn{1}{c|}{ Value } \\
1 Solvent & cdcl3 \\
2 Experiment & $1 \mathrm{D}$ \\
3 Number of Scans & 8 \\
4 Relaxation Delay & 1.0000 \\
5 Acquisition Time & 2.0447 \\
6 Spectrometer Frequency & 499.81 \\
7 Nucleus & $1 \mathrm{H}$ \\
\hline
\end{tabular}

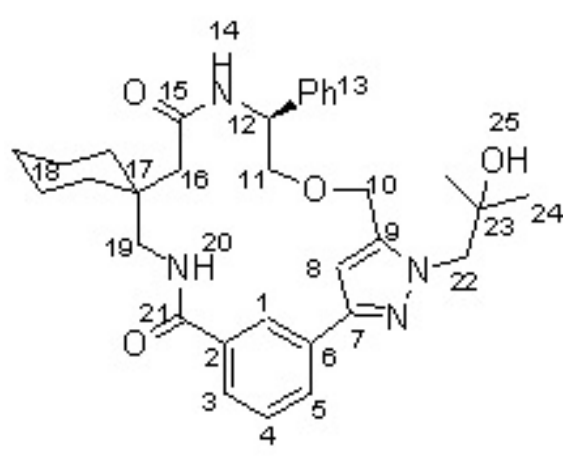

59

8,13

20 ,

1 ,

$5,3, \quad 4$

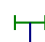

$\infty$

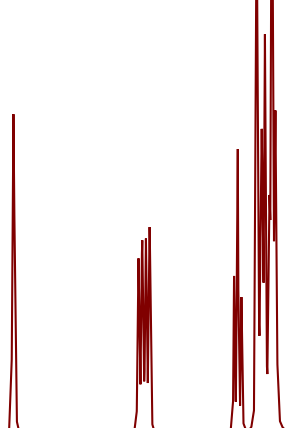

14

12. 10, 10', 11, 22, 11', 19, 19',

18 ,

24

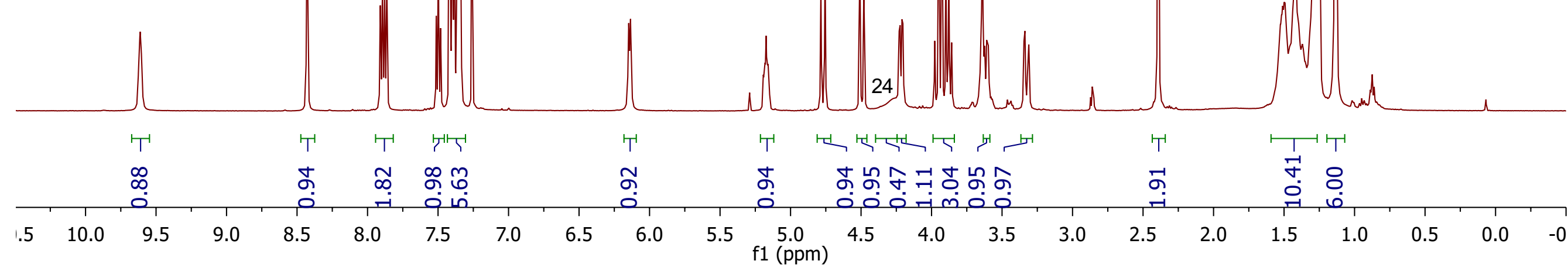




\begin{tabular}{|lc|}
\hline \multicolumn{1}{|c|}{ Parameter } & value \\
1 Solvent & cdcl3 \\
2 Experiment & $1 \mathrm{D}$ \\
3 Number of Scans & 1000 \\
4 Relaxation Delay & 1.0000 \\
5 Acquisition Time & 1.3042 \\
6 Spectrometer Frequency & 100.61 \\
7 Nucleus & $13 \mathrm{C}$ \\
\hline \multicolumn{2}{|c|}{} \\
\hline
\end{tabular}

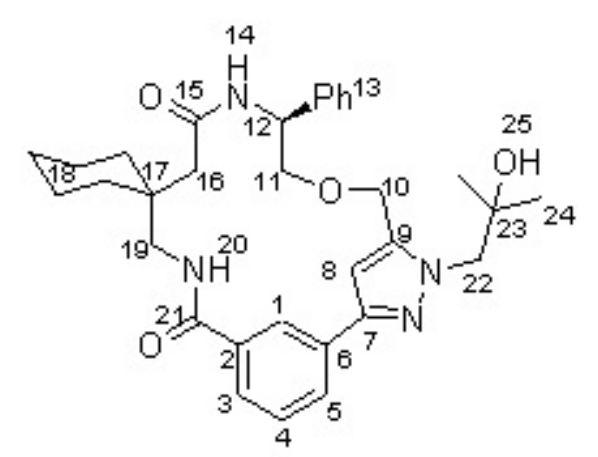

59

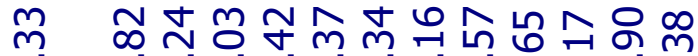

กิ่

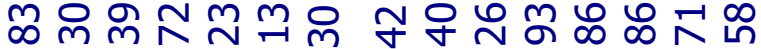

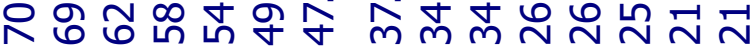

$1,4, \quad 13$,

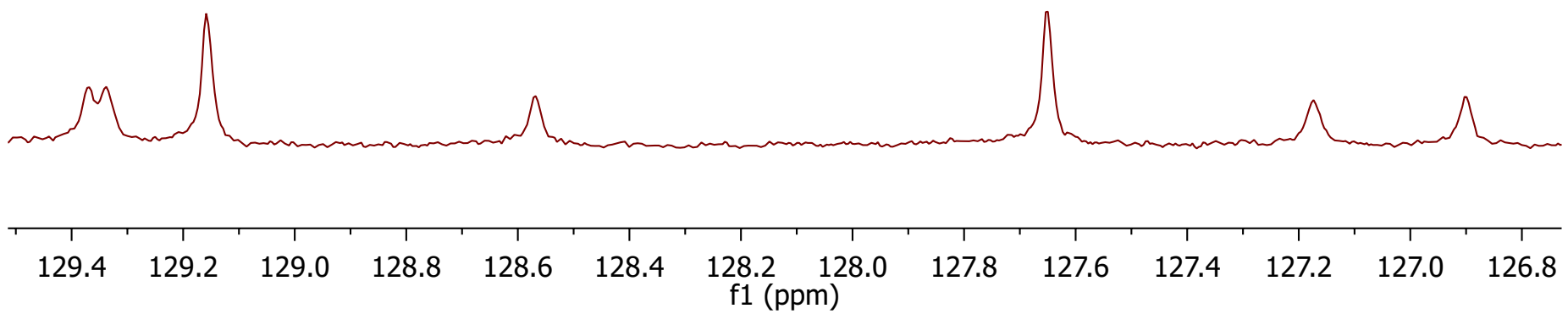

13

5 ,
15, 21,

7

$9,13,2,6$

8
23,11

$10,22,12$

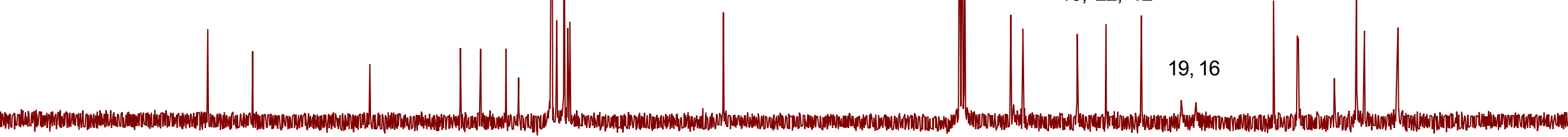

$190 \quad 180$

50




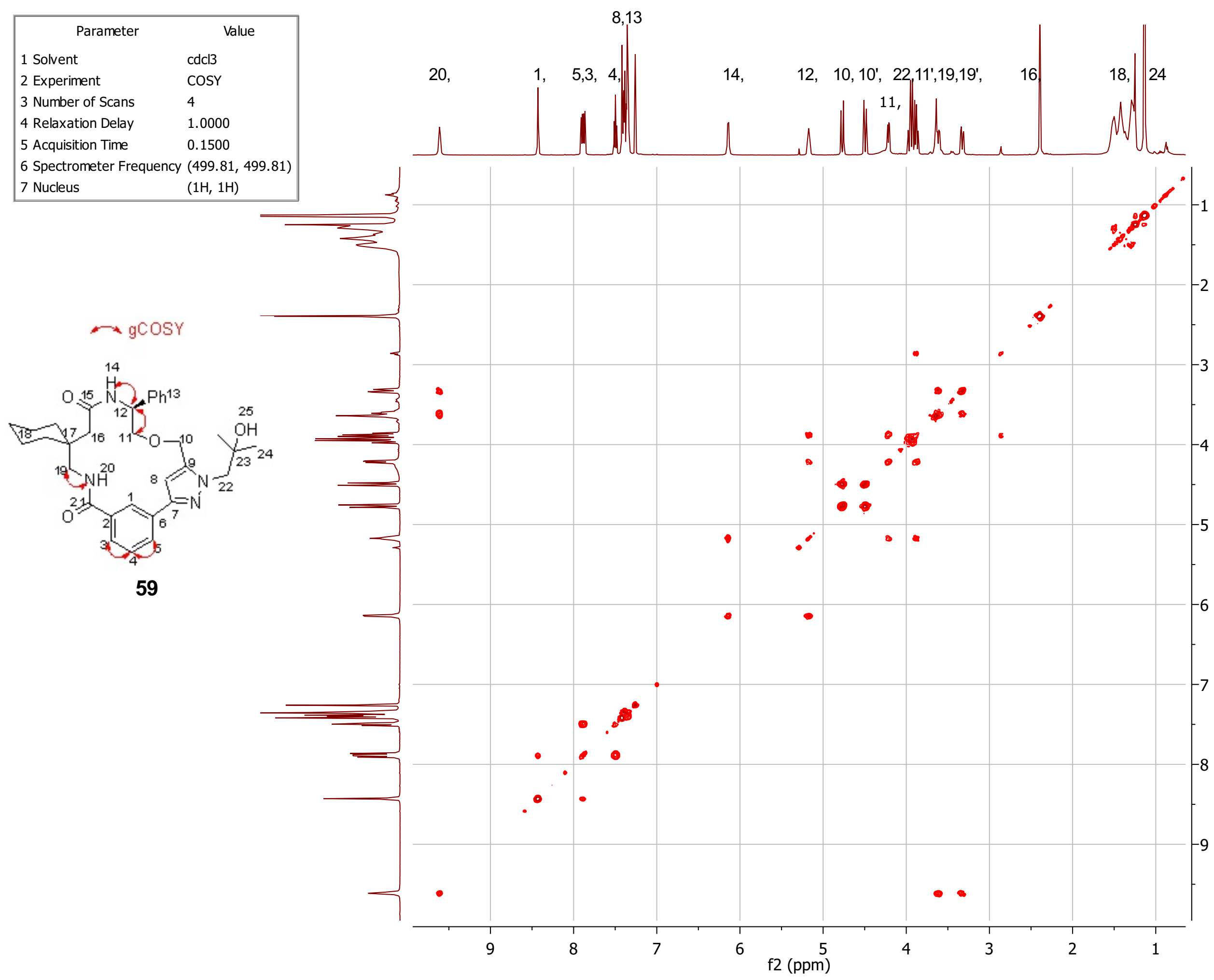

$\underbrace{\widehat{E}}_{\bar{L}}$ 


\begin{tabular}{|ll|}
\hline \multicolumn{1}{|c|}{ Parameter } & \multicolumn{1}{c|}{ Value } \\
1 Solvent & cdcl3 \\
2 Experiment & ASAPHMQC \\
3 Number of Scans & 32 \\
4 Relaxation Delay & 0.0600 \\
5 Acquisition Time & 0.0640 \\
6 Spectrometer Frequency & $(499.81,125.69)$ \\
7 Nucleus & $(1 \mathrm{H}, 13 \mathrm{C})$ \\
\hline
\end{tabular}

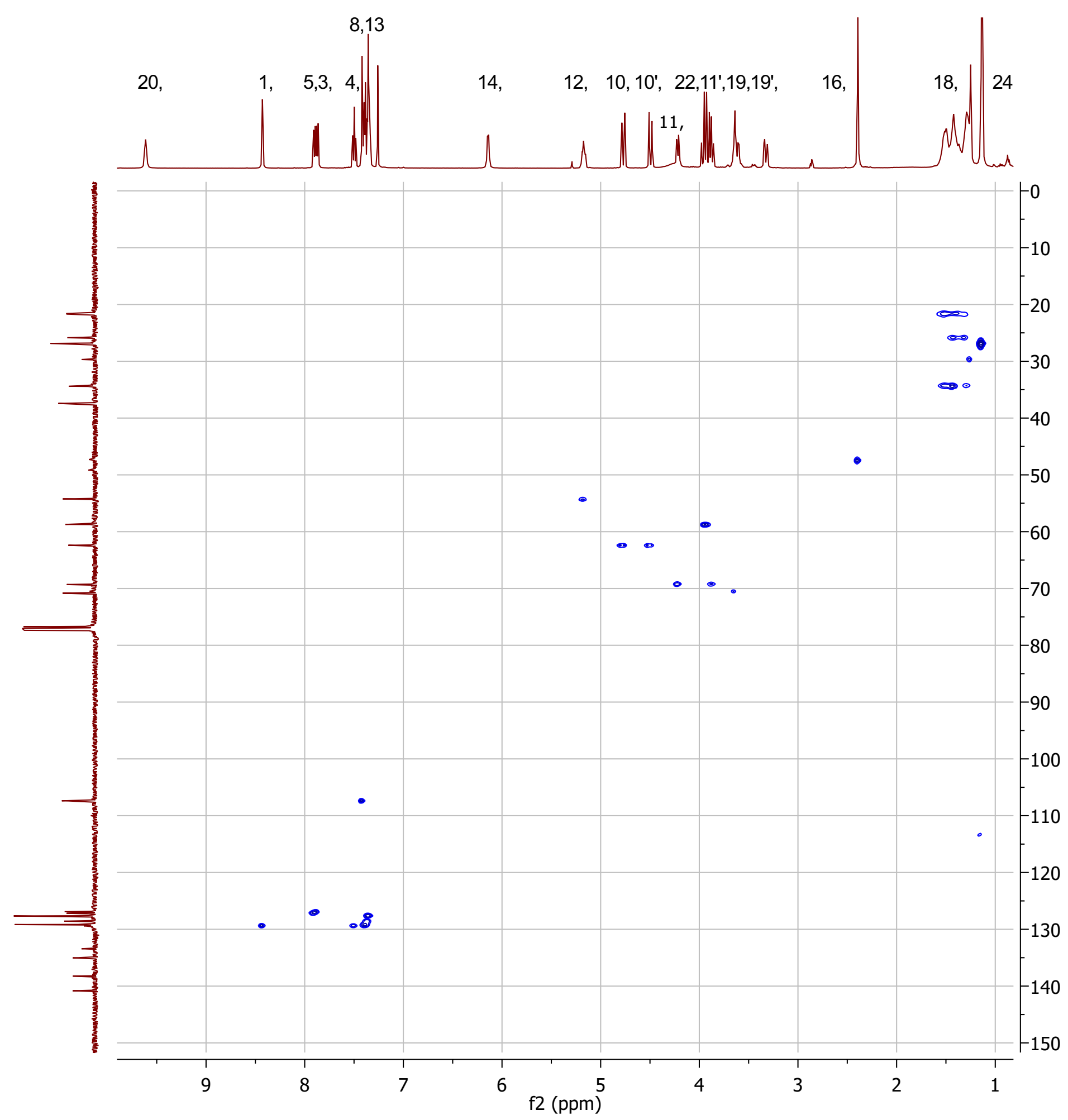

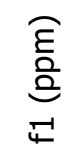

59

$\left.{ }_{19}^{20} \mathrm{NH}_{\mathrm{N}}^{25}\right)_{23}^{24}$

21

4 


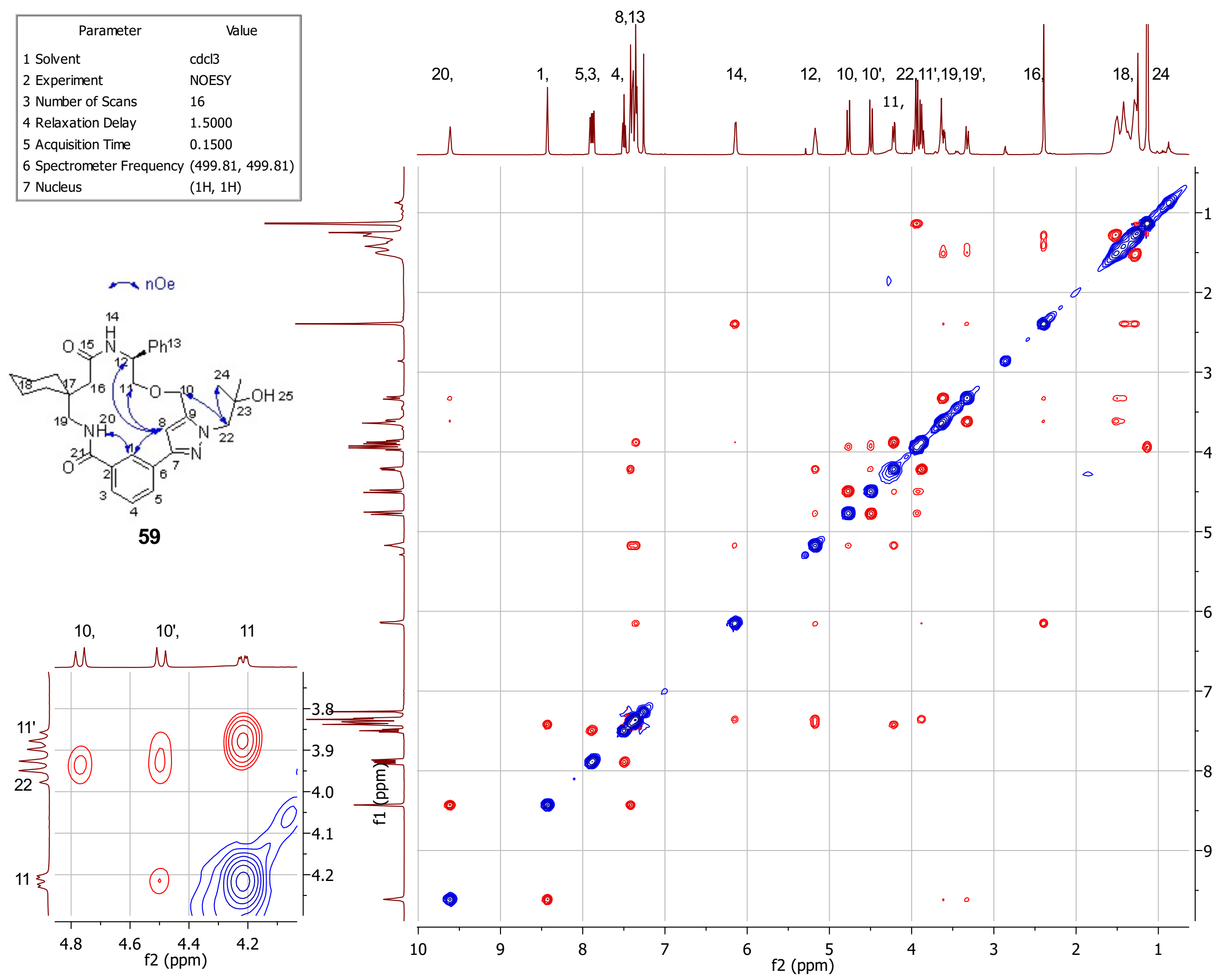


ㄱㅇㅇㅇ

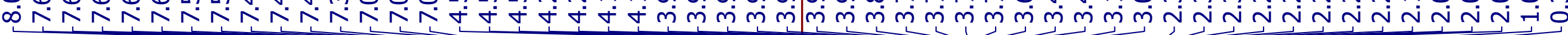

\begin{tabular}{|ll|}
\hline \multicolumn{1}{|c|}{ Parameter } & \multicolumn{1}{c|}{ Value } \\
1 Solvent & cd3od \\
2 Experiment & $1 \mathrm{D}$ \\
3 Number of Scans & 8 \\
4 Relaxation Delay & 1.0000 \\
5 Acquisition Time & 2.5559 \\
6 Spectrometer Frequency & 400.02 \\
7 Nucleus & $1 \mathrm{H}$ \\
\hline
\end{tabular}

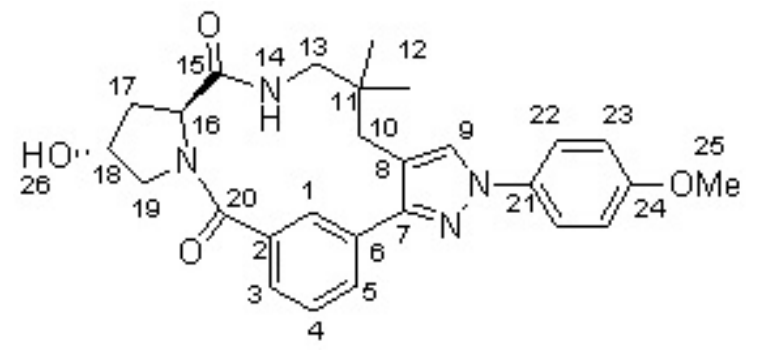

61

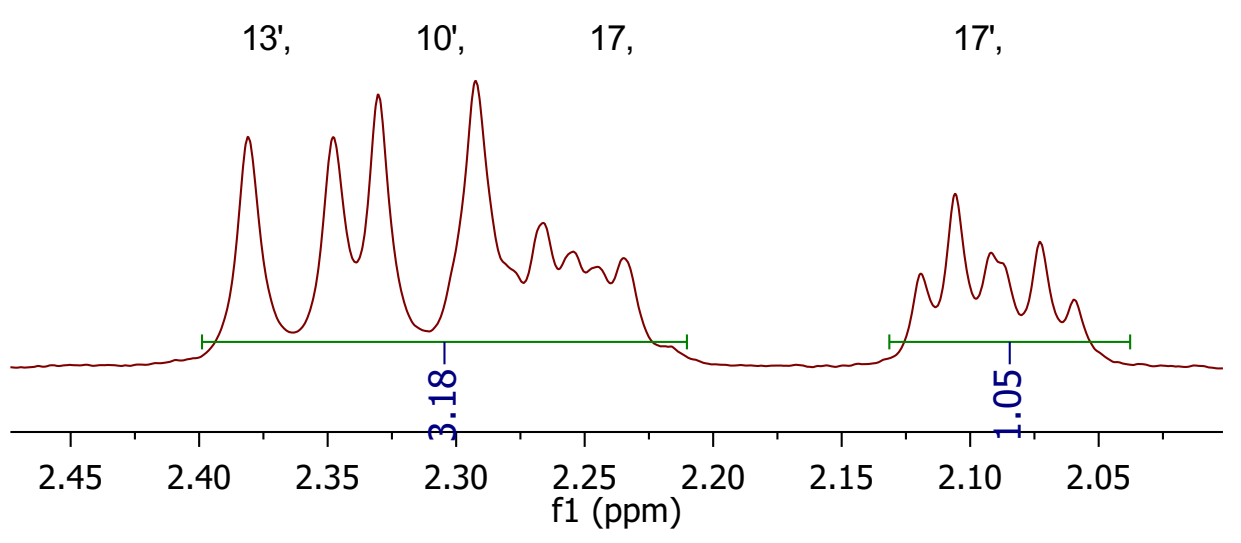

$9,22,3,1,23$,

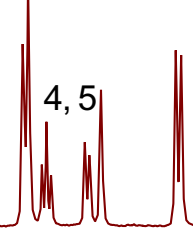

18. 16, 19, 19', 13, 10, 13',10',17,17',

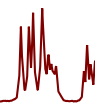

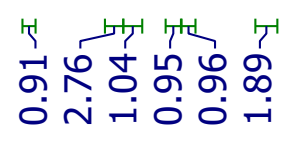

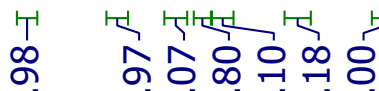

。․

T'T

$\stackrel{\infty}{\rightarrow} \stackrel{1}{\circ}$

mi

$8.0 \quad 7.5 \quad 7.0$

6.5

6.0

5.5

5.0

$\begin{array}{lll}4.5 & 4.0 \quad 3.5\end{array}$

3.0

2.5

$2.0 \quad 1.5$




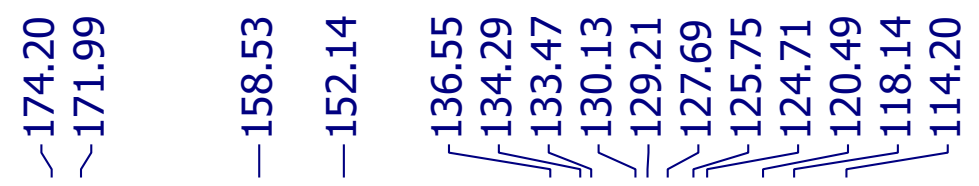

ำ ํํำ

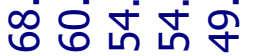

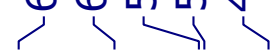

\begin{tabular}{|ll|}
\hline \multicolumn{1}{|c}{ Parameter } & \multicolumn{1}{c|}{ Value } \\
1 Solvent & cd3od \\
2 Experiment & $1 \mathrm{D}$ \\
3 Number of Scans & 17800 \\
4 Relaxation Delay & 1.0000 \\
5 Acquisition Time & 1.3042 \\
6 Spectrometer Frequency & 100.61 \\
7 Nucleus & $13 \mathrm{C}$ \\
\hline
\end{tabular}
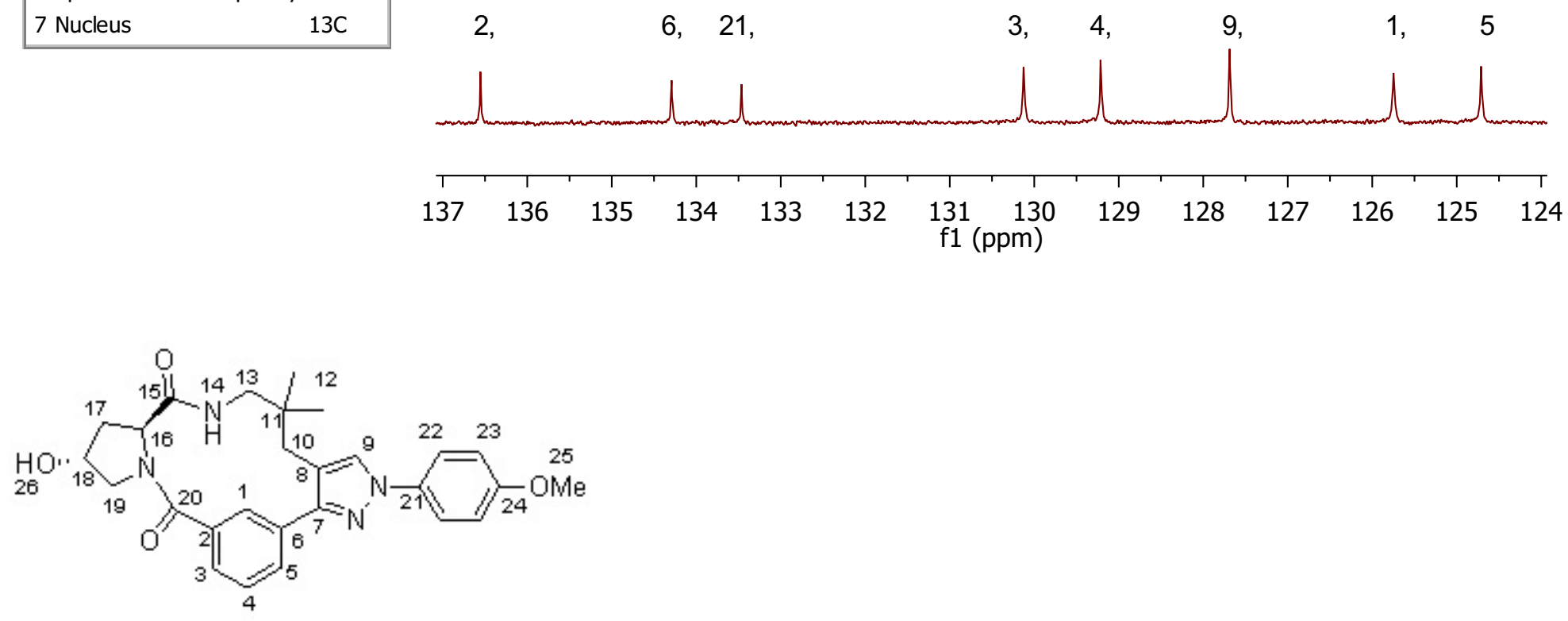

61

15,20,

$24, \quad 7$,

2 ,

22, 8 ,

23,

$18, \quad 16,25,19,13$

$17,11, \quad 10,12,12$

$90 \quad 80$

8070

$50 \quad 40$

ตับ m m $1,1\} 1$ 


\begin{tabular}{|ll|}
\hline \multicolumn{1}{|c|}{ Parameter } & \multicolumn{1}{c|}{ Value } \\
1 Solvent & cd3od \\
2 Experiment & COSY \\
3 Number of Scans & 4 \\
4 Relaxation Delay & 1.0000 \\
5 Acquisition Time & 0.1501 \\
6 Spectrometer Frequency & $(400.02,400.02)$ \\
7 Nucleus & $(1 \mathrm{H}, 1 \mathrm{H})$ \\
\hline
\end{tabular}

$9,22,3,1,23$,

4. 4,5

18, 16,1申,19',13, 10, 13',10',17,17', crombunla ma
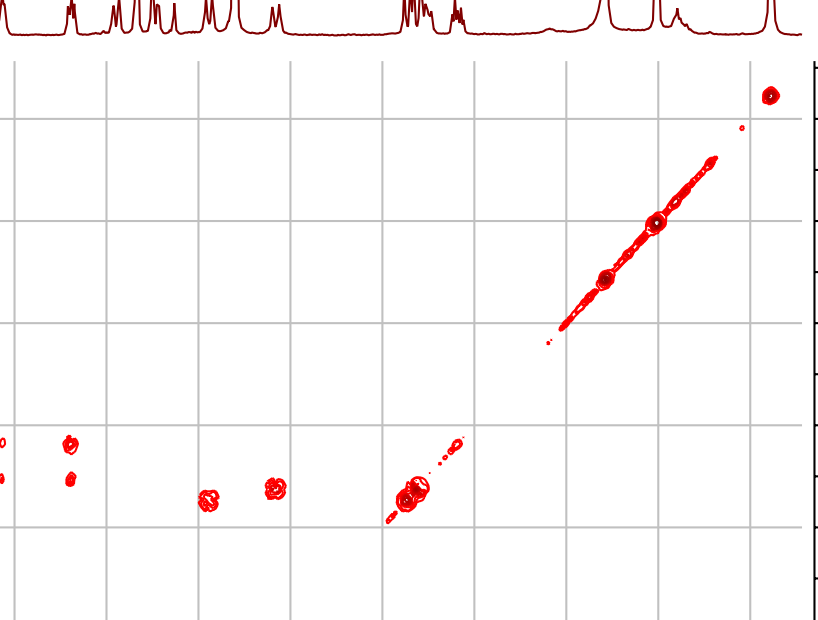

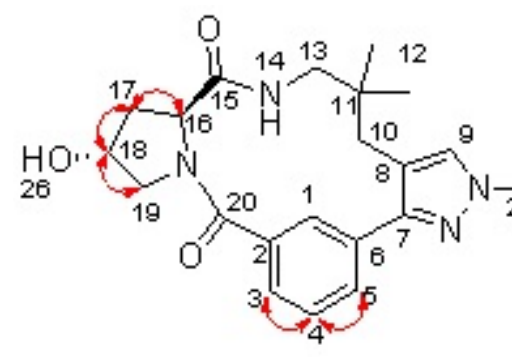

61
$>_{24}^{23}{ }_{-25}^{23}$
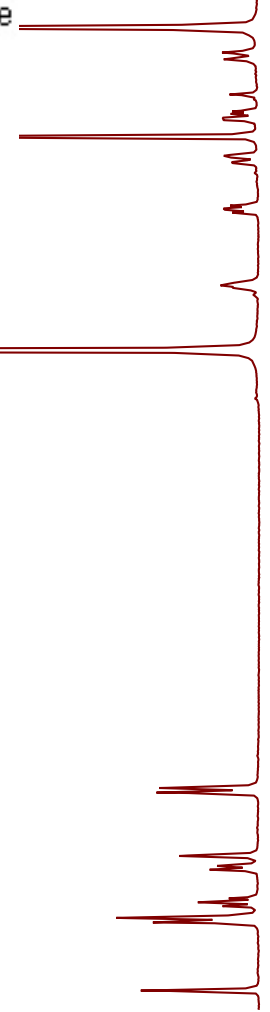

$\begin{array}{llllll}8.0 & 7.5 & 7.0 & 6.5 & 6.0 & 5.5\end{array}$

$\begin{array}{rrr}5.0 & 4.5 & 4.0 \\ & \text { f2 }(\mathrm{ppm})\end{array}$ 


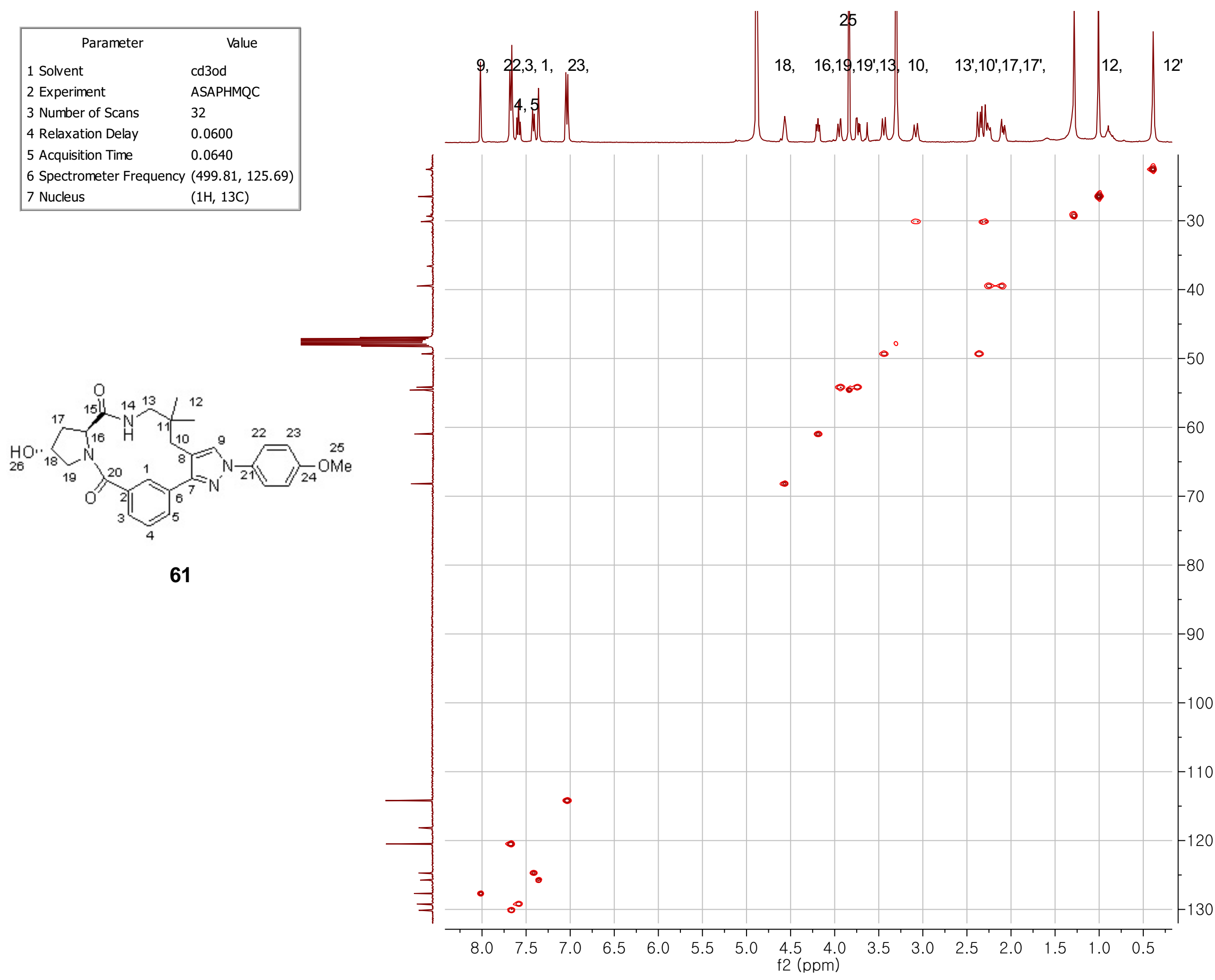




\begin{tabular}{|ll|}
\hline \multicolumn{1}{|c|}{ Parameter } & \multicolumn{1}{c|}{ Value } \\
1 Solvent & cd3od \\
2 Experiment & NOESY \\
3 Number of Scans & 16 \\
4 Relaxation Delay & 1.5000 \\
5 Acquisition Time & 0.1501 \\
6 Spectrometer Frequency & $(400.02,400.02)$ \\
7 Nucleus & $(1 \mathrm{H}, 1 \mathrm{H})$ \\
\hline
\end{tabular}

25

$9,22,3,1,23$,

$\because \mathrm{nOe}$

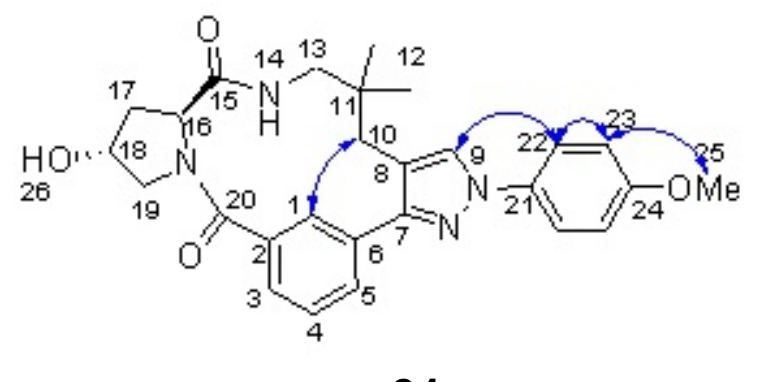

61

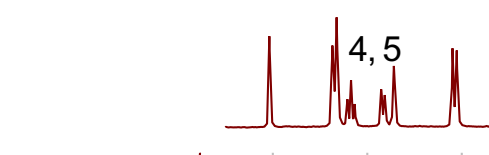

18, 16,19,19',13, 10, 13',10',17,17',

12, $12^{\prime}$
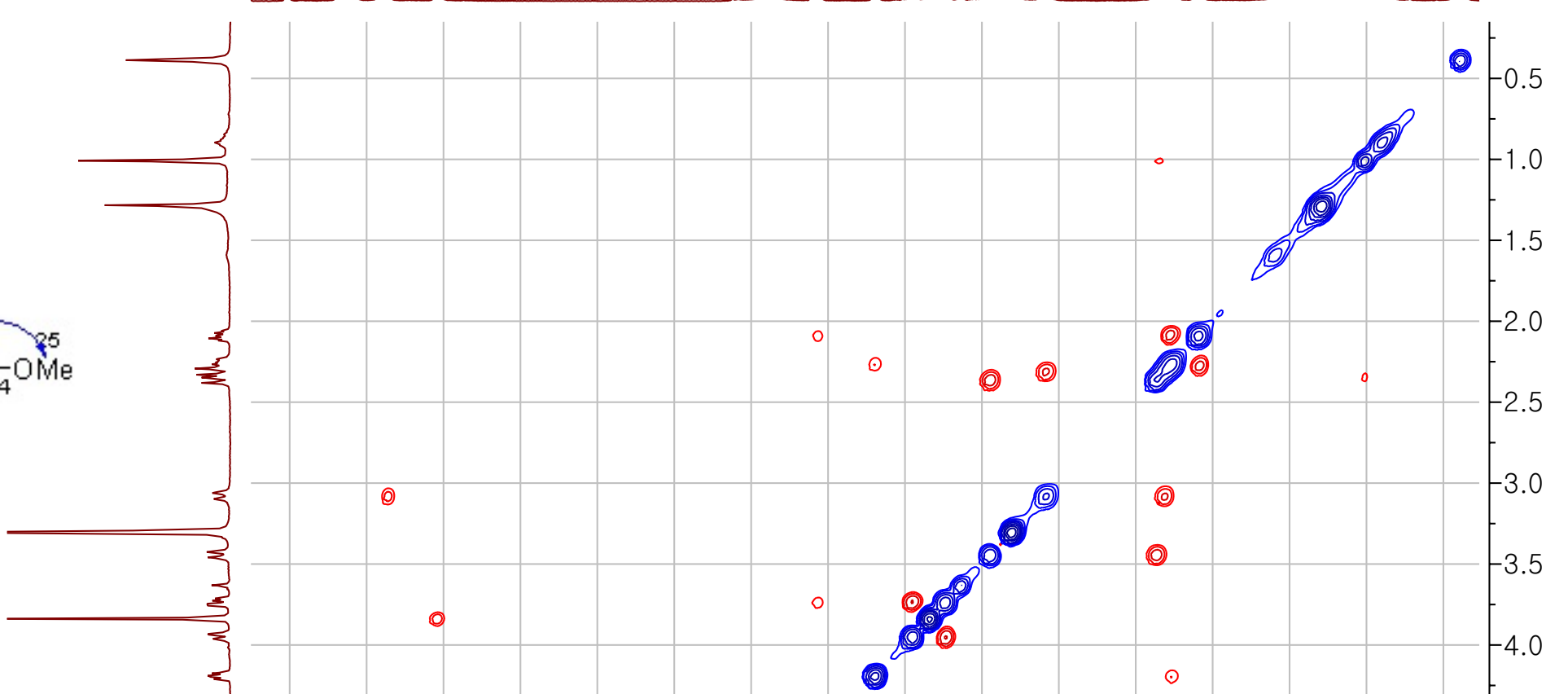

(2)
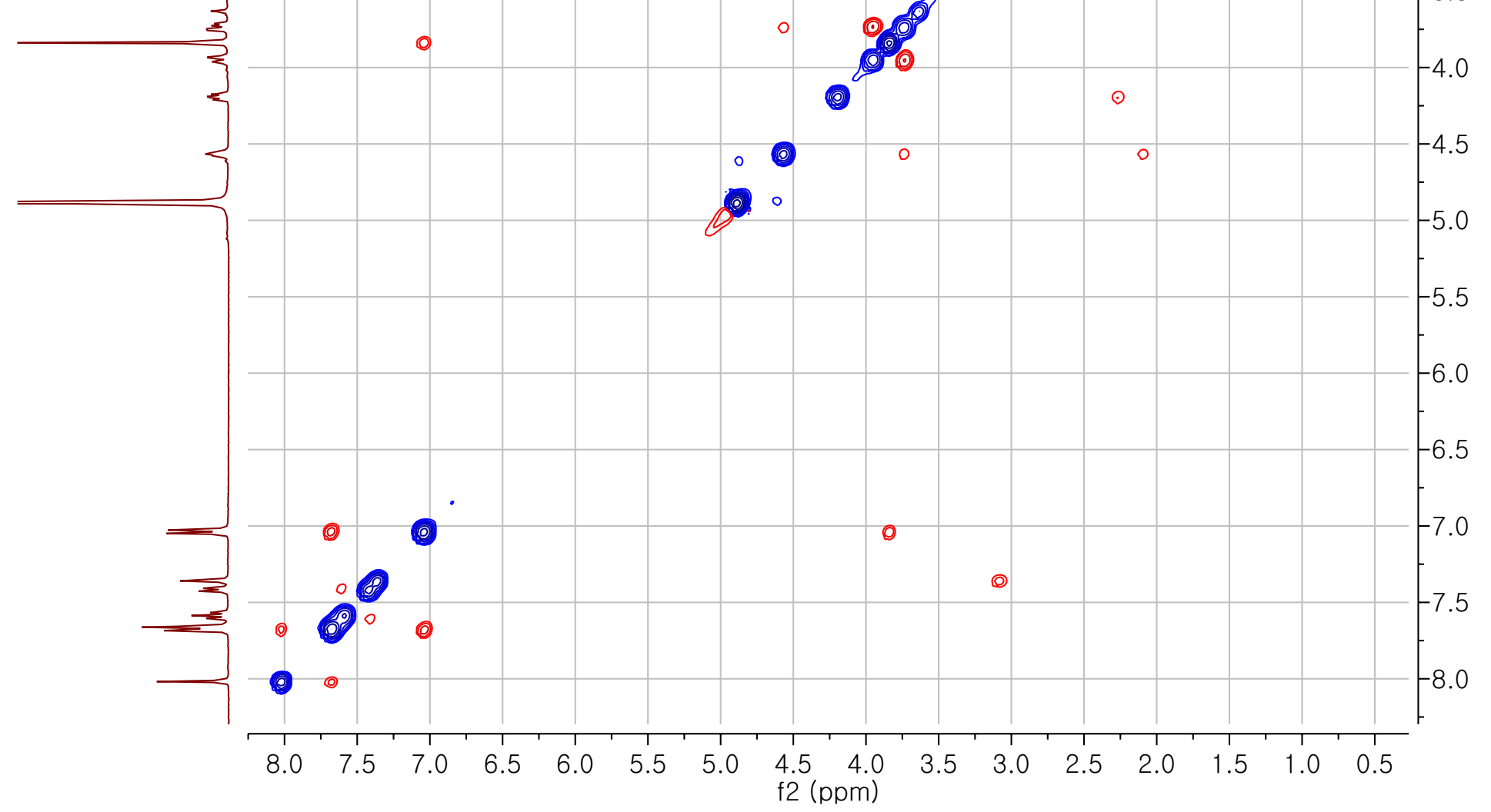


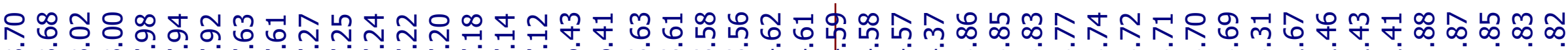

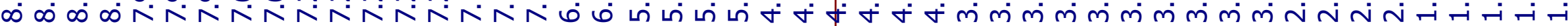

\begin{tabular}{|ll|}
\hline \multicolumn{1}{|c|}{ Parameter } & \multicolumn{1}{c|}{$\begin{array}{r}\text { Value } \\
\text { 1 Solvent }\end{array}$} \\
2 Experiment & 1D \\
3 Number of Scans & 8 \\
4 Relaxation Delay & 1.0000 \\
5 Acquisition Time & 2.5559 \\
6 Spectrometer Frequency & 400.02 \\
7 Nucleus & $1 \mathrm{H}$ \\
\hline
\end{tabular}

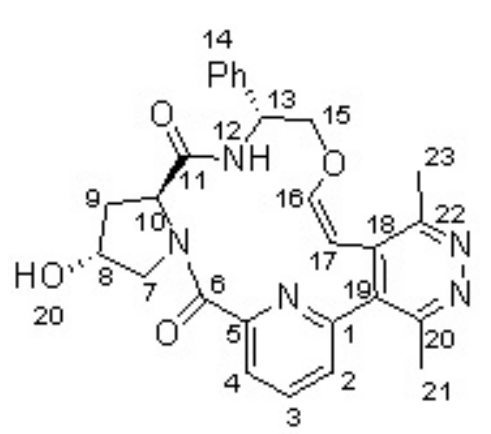

$J_{16,17}=6.8 \mathrm{~Hz}$

63
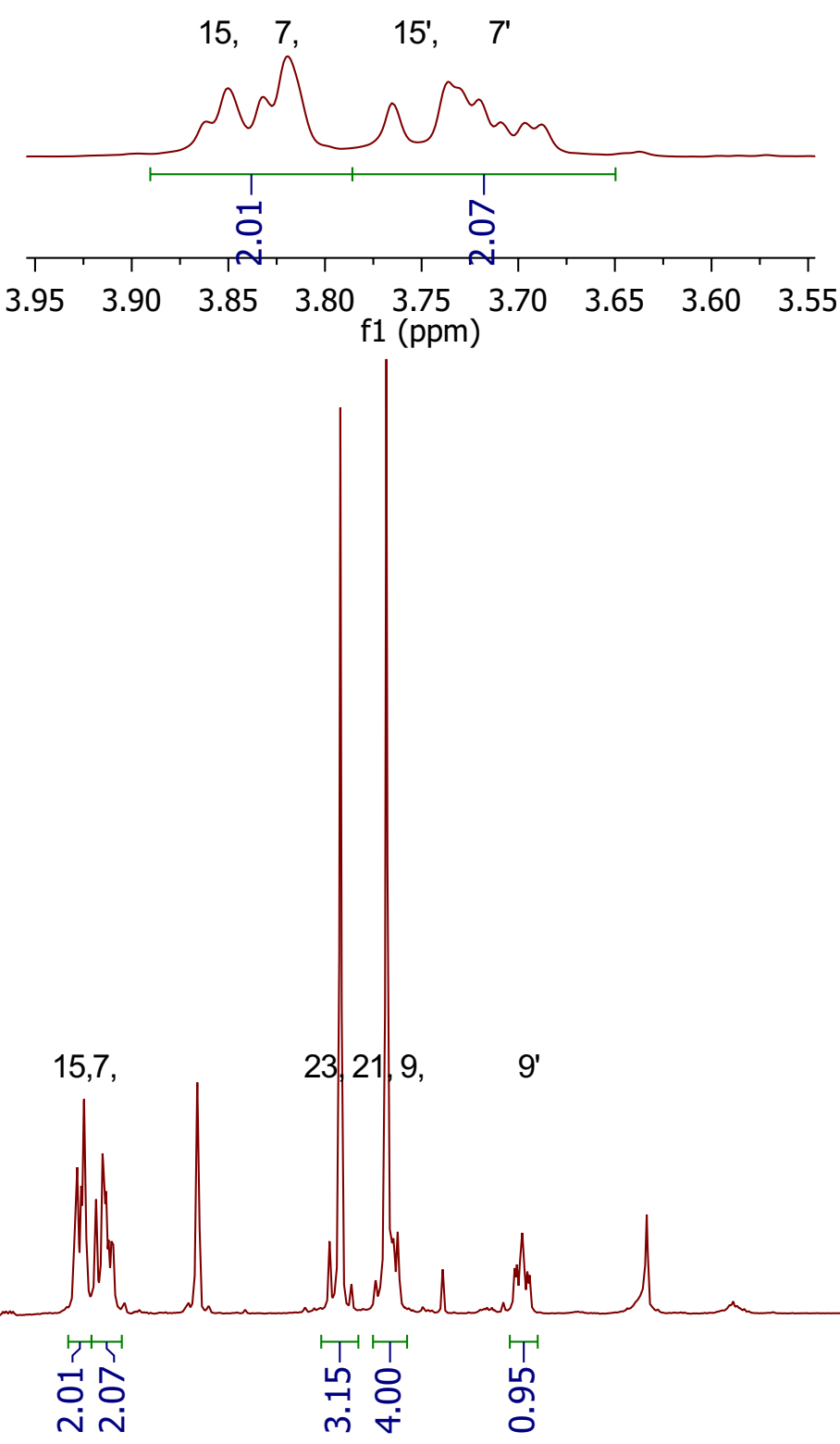

$\begin{array}{lll}1 & 1 \\ 8.0 & 7.5\end{array}$




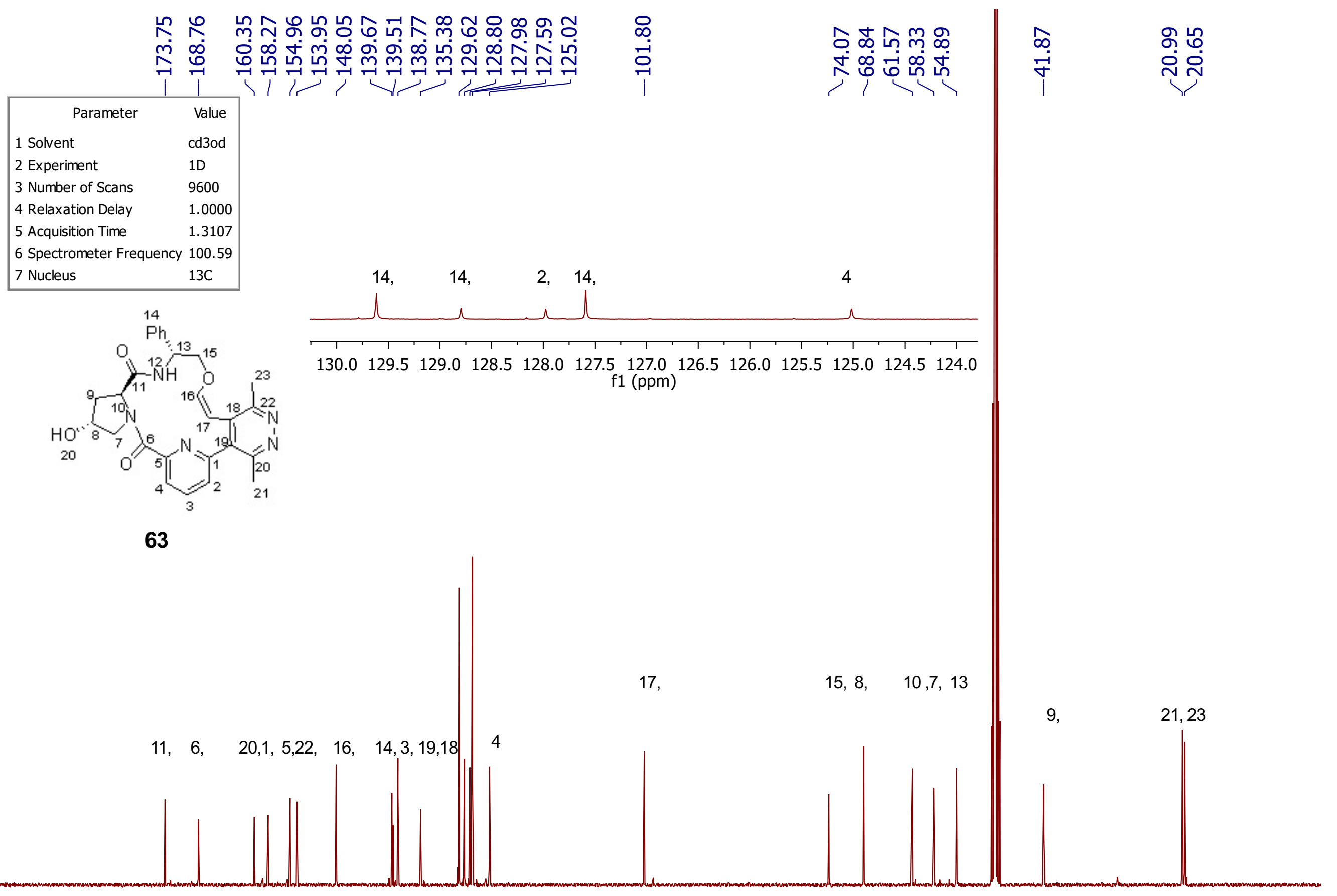

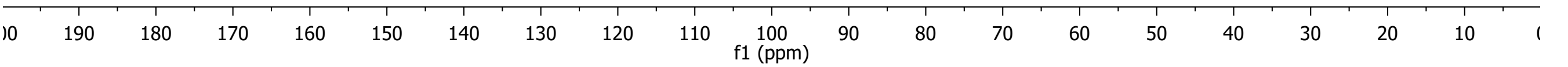




\begin{tabular}{|ll|}
\hline \multicolumn{1}{|c|}{ Parameter } & \multicolumn{1}{c|}{ Value } \\
1 Solvent & cd3od \\
2 Experiment & COSY \\
3 Number of Scans & 4 \\
4 Relaxation Delay & 1.0000 \\
5 Acquisition Time & 0.1500 \\
6 Spectrometer Frequency & $(400.02,400.02)$ \\
7 Nucleus & $(1 \mathrm{H}, 1 \mathrm{H})$ \\
\hline
\end{tabular}

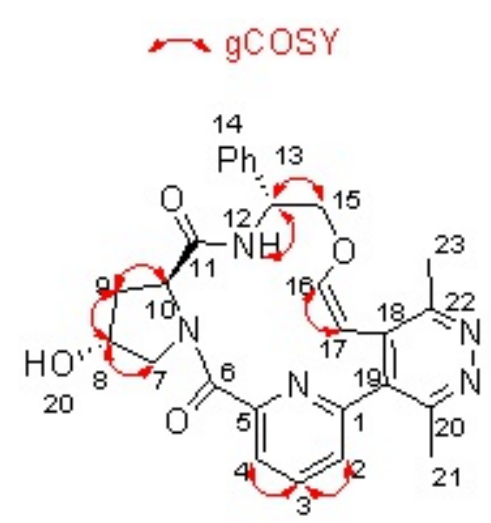

63

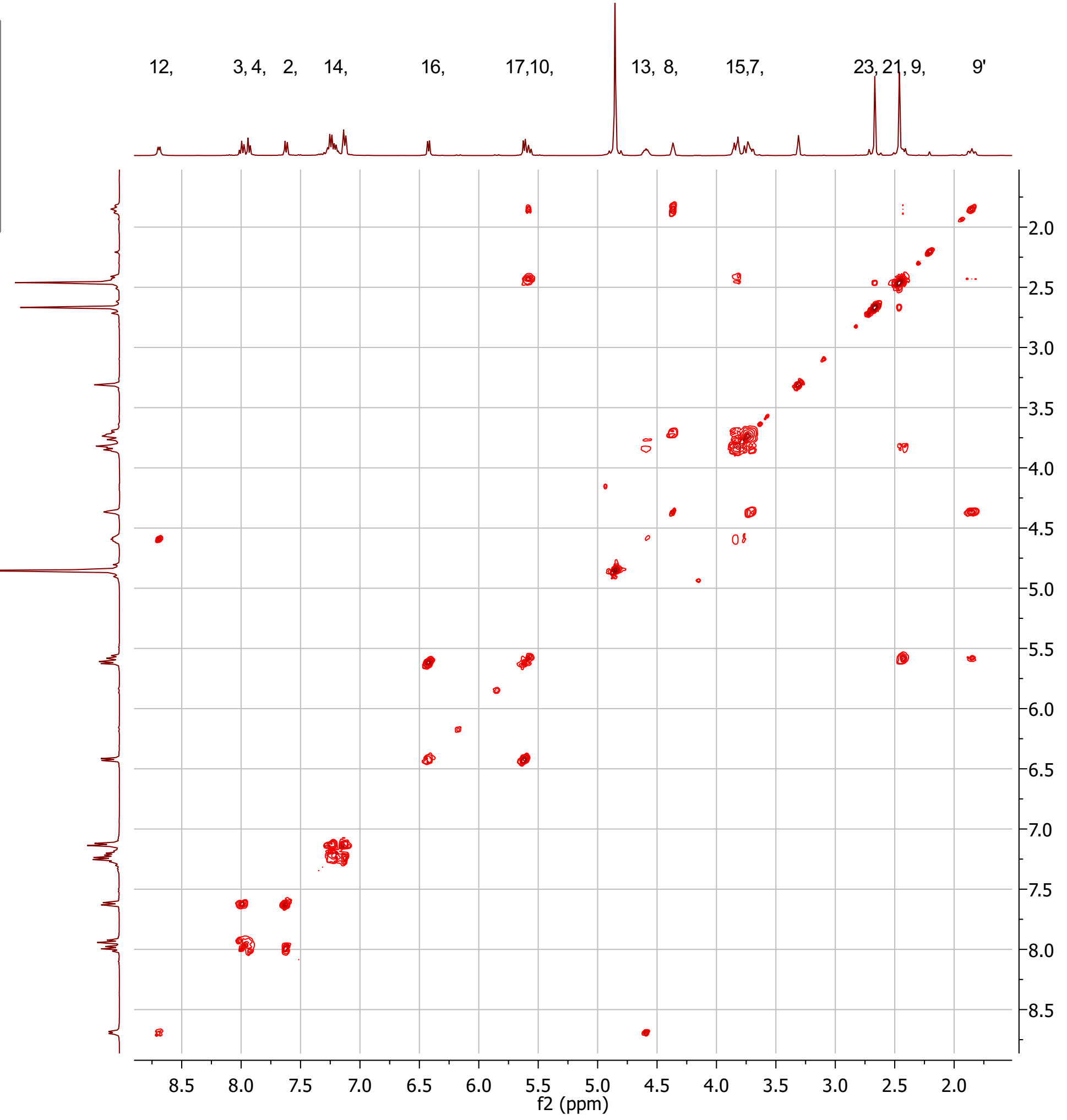

$4,2, \quad 14, \quad 16, \quad 17,10$$$
\text { . }
$$

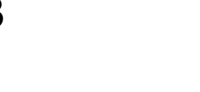




\begin{tabular}{|ll|}
\hline \multicolumn{1}{|c|}{ Parameter } & \multicolumn{1}{c|}{ Value } \\
1 Solvent & cd3od \\
2 Experiment & ASAPHMQC \\
3 Number of Scans & 32 \\
4 Relaxation Delay & 0.0600 \\
5 Acquisition Time & 0.0640 \\
6 Spectrometer Frequency & $(400.02,100.59)$ \\
7 Nucleus & $(1 \mathrm{H}, 13 \mathrm{C})$ \\
\hline
\end{tabular}

12, $3,4,2, \quad 14, \quad 16, \quad 17,10$,

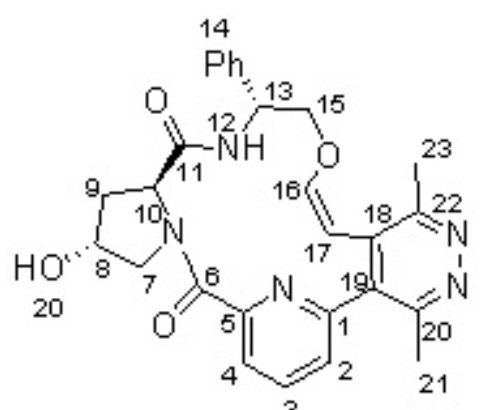

63

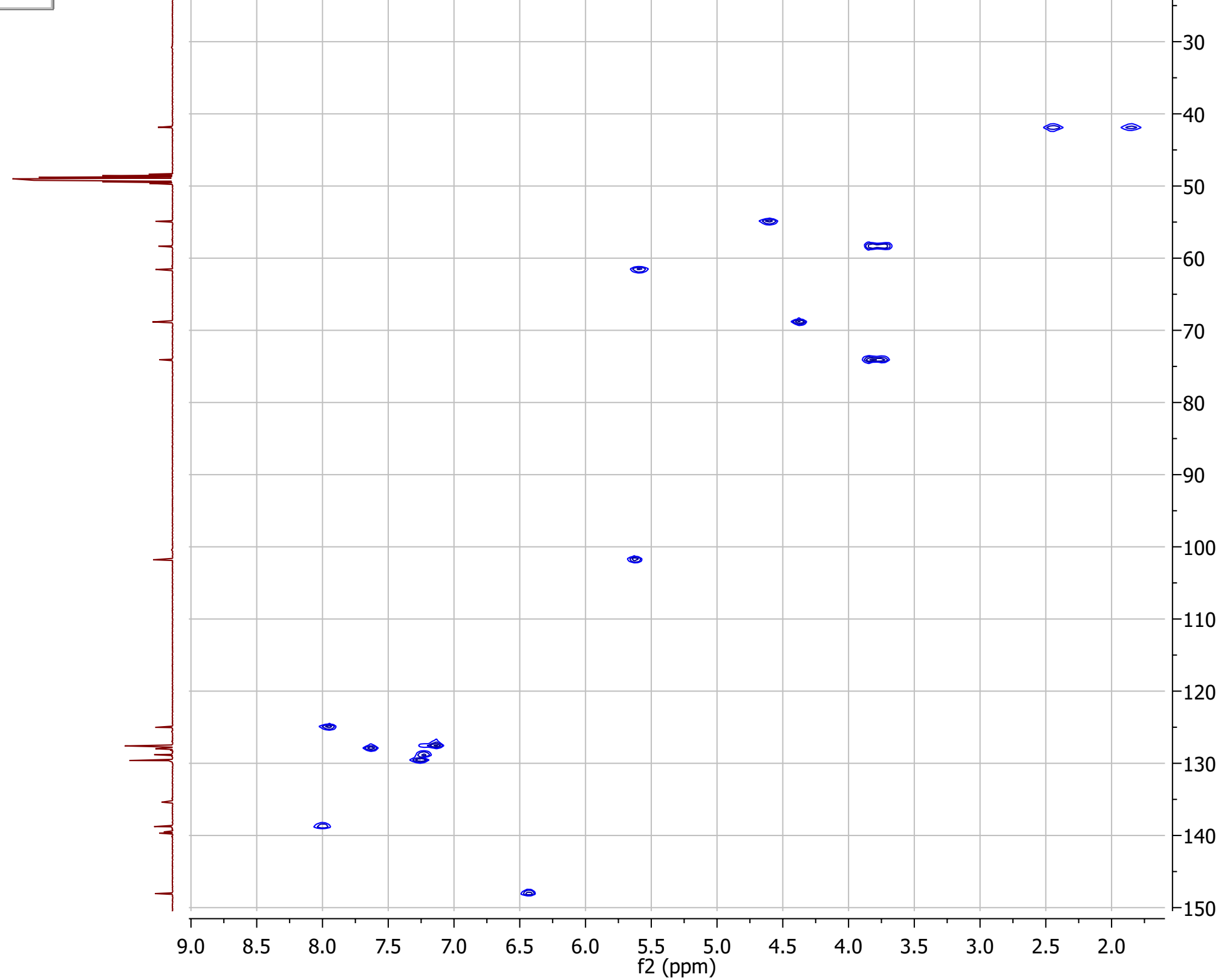




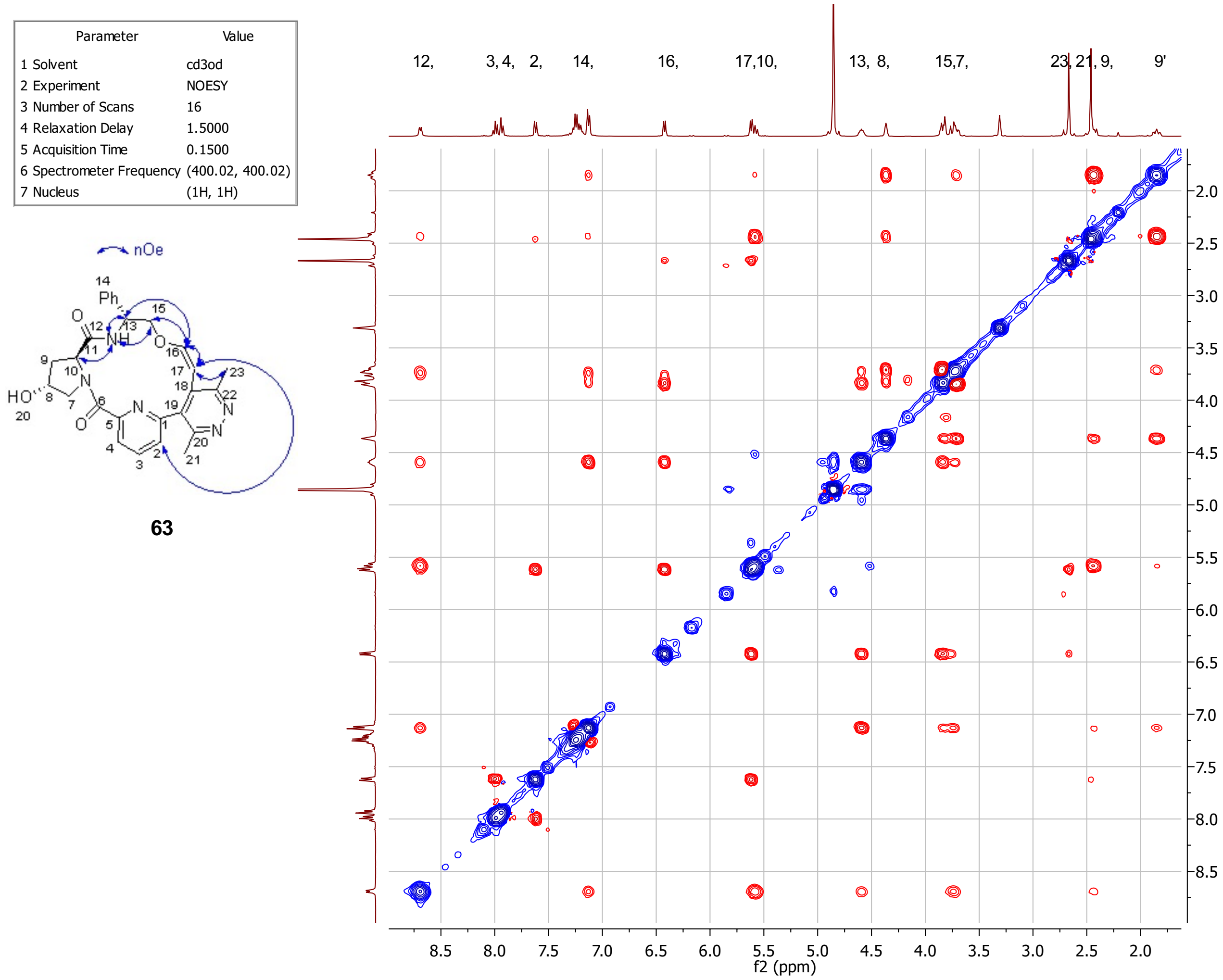

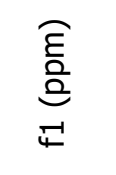


ஜ

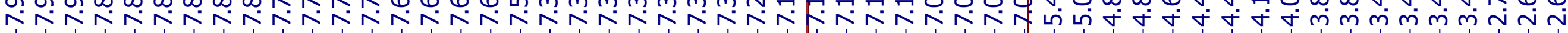

\begin{tabular}{|ll|}
\hline \multicolumn{1}{|c|}{ Parameter } & \multicolumn{1}{c|}{ Value } \\
1 Solvent & cd3od \\
2 Experiment & $1 \mathrm{D}$ \\
3 Number of Scans & 8 \\
4 Relaxation Delay & 1.0000 \\
5 Acquisition Time & 2.0447 \\
6 Spectrometer Frequency & 499.81 \\
7 Nucleus & $1 \mathrm{H}$ \\
\hline
\end{tabular}

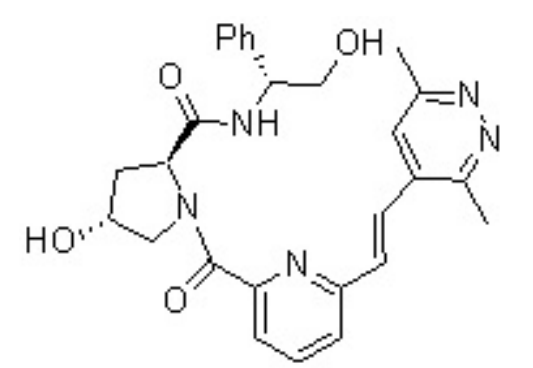

64

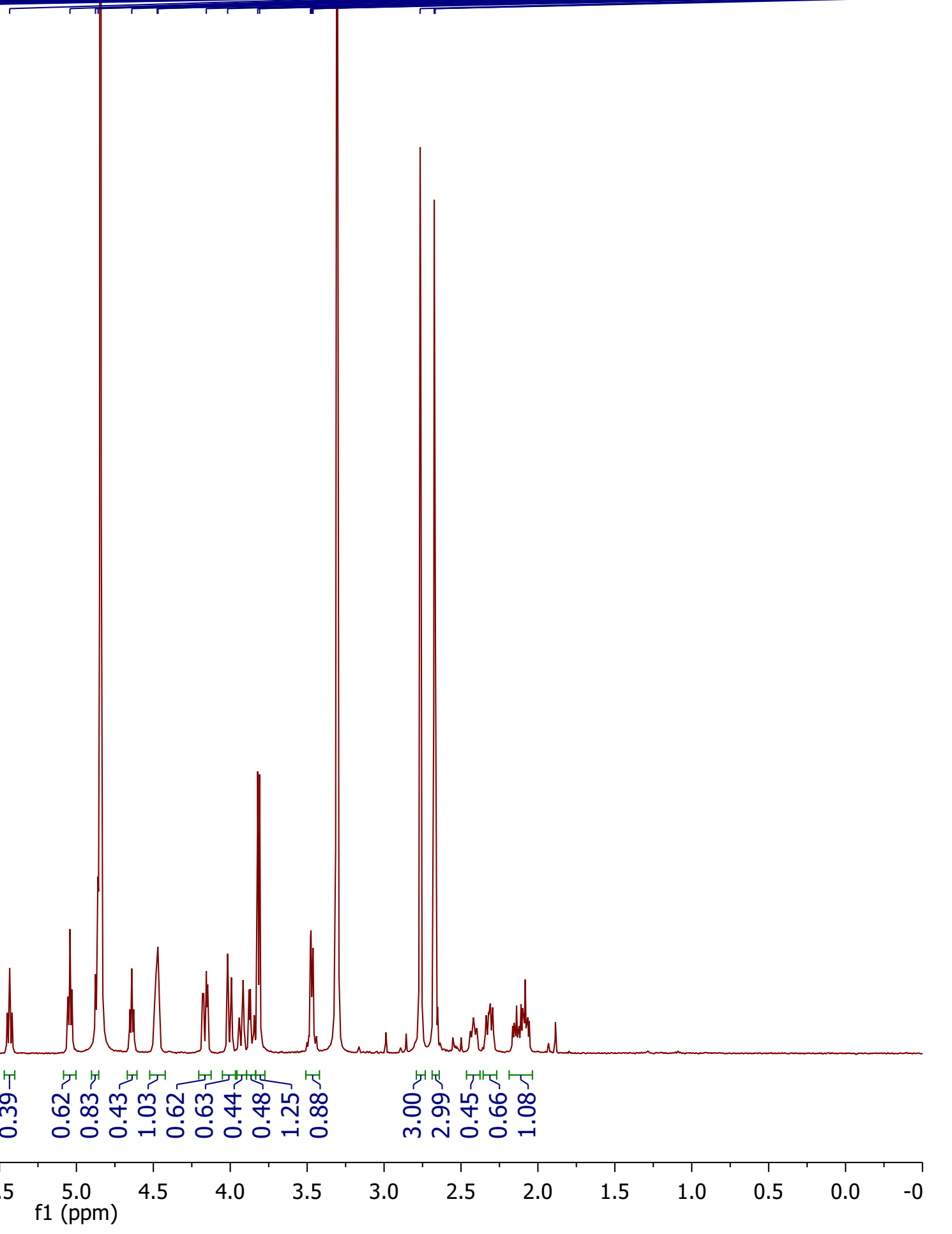




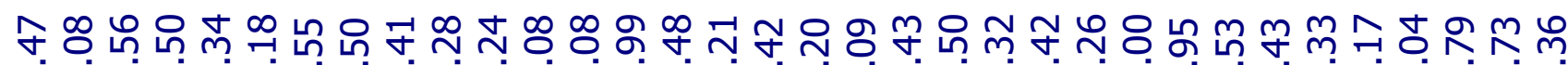
ச்

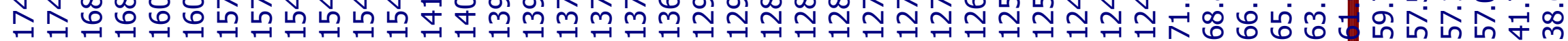

\begin{tabular}{ll} 
& \multicolumn{1}{c}{ cd3od } \\
1 Solvent & $1 \mathrm{D}$ \\
2 Experiment & 18000 \\
3 Number of Scans & 1.0000 \\
4 Relaxation Delay & 1.3107 \\
5 Acquisition Time & 100.59 \\
6 Spectrometer Frequency \\
7 Nucleus & $13 \mathrm{C}$
\end{tabular}

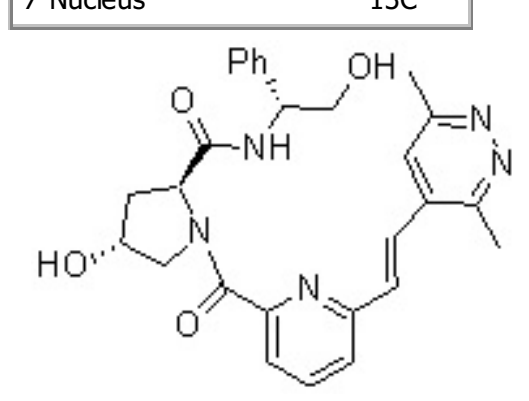

64
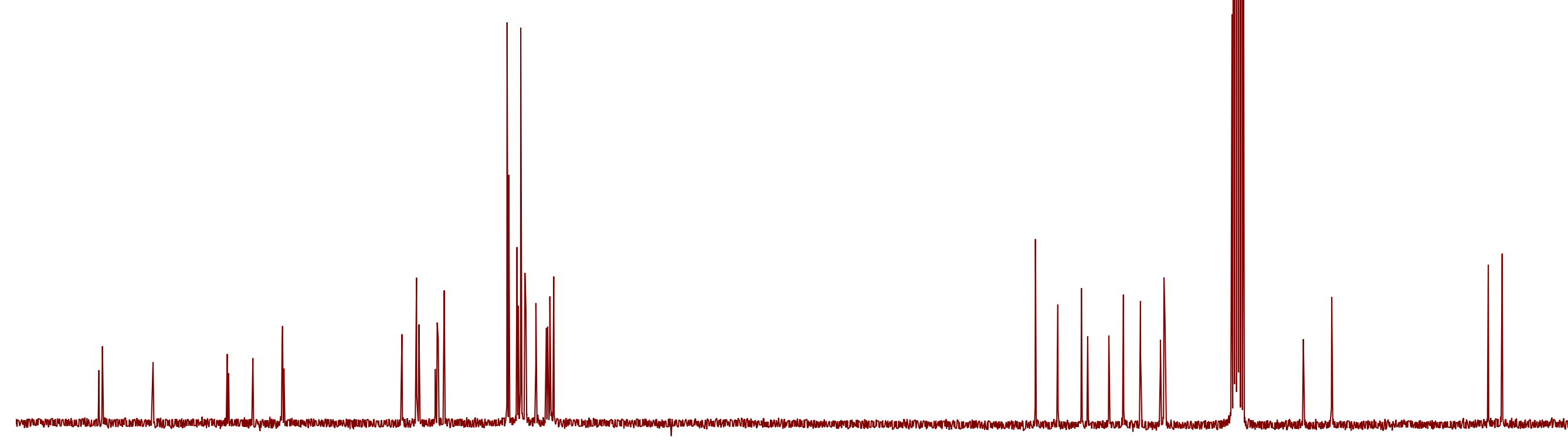

\begin{tabular}{|c|c|c|c|c|c|c|c|c|c|c|c|c|c|c|c|c|}
\hline 180 & 170 & 160 & 150 & 140 & 130 & 120 & 110 & $\begin{array}{c}100 \\
\mathrm{f} 1(\mathrm{ppm})\end{array}$ & 90 & 80 & 70 & 60 & 50 & 40 & 30 & 20 \\
\hline
\end{tabular}




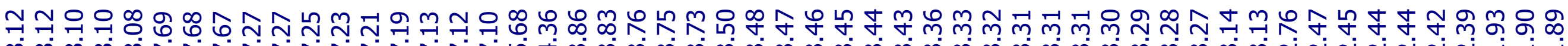

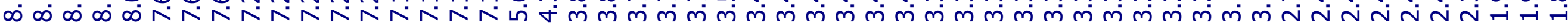

\begin{tabular}{|ll|}
\hline \multicolumn{1}{|c|}{ Parameter } & \multicolumn{1}{c|}{ Value } \\
1 Solvent & cd3od \\
2 Experiment & $1 \mathrm{D}$ \\
3 Number of Scans & 8 \\
4 Relaxation Delay & 1.0000 \\
5 Acquisition Time & 2.5592 \\
6 Spectrometer Frequency & 400.09 \\
7 Nucleus & $1 \mathrm{H}$ \\
\hline
\end{tabular}

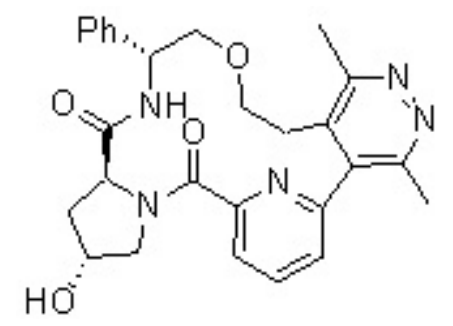

65

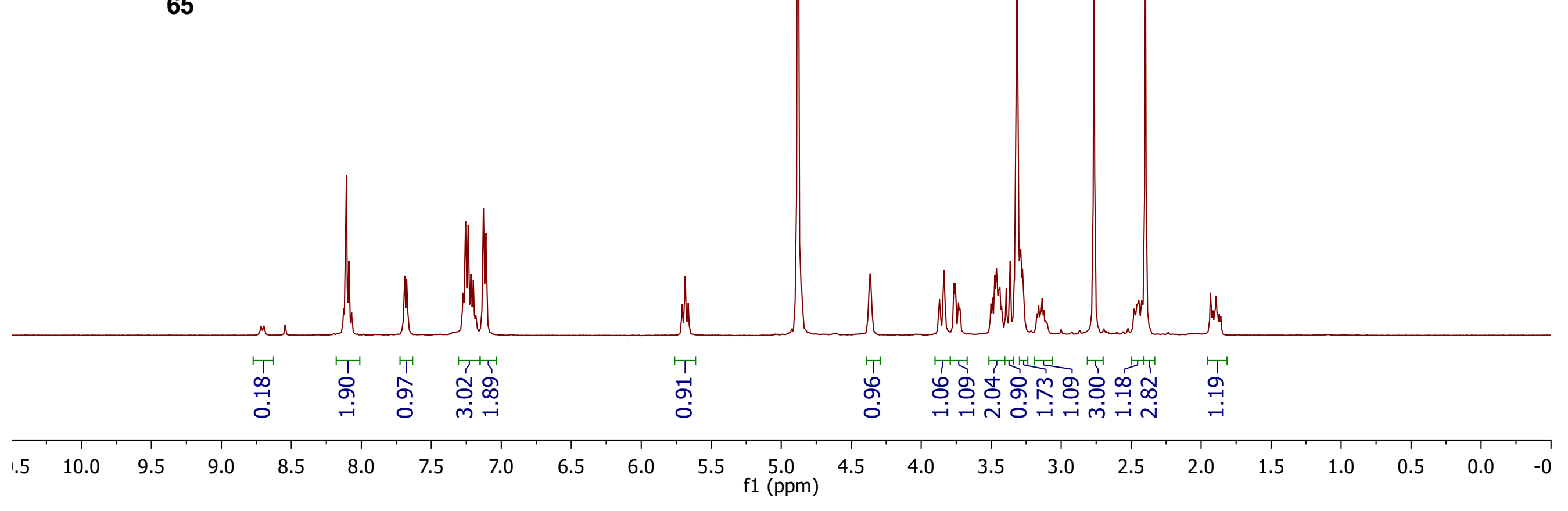




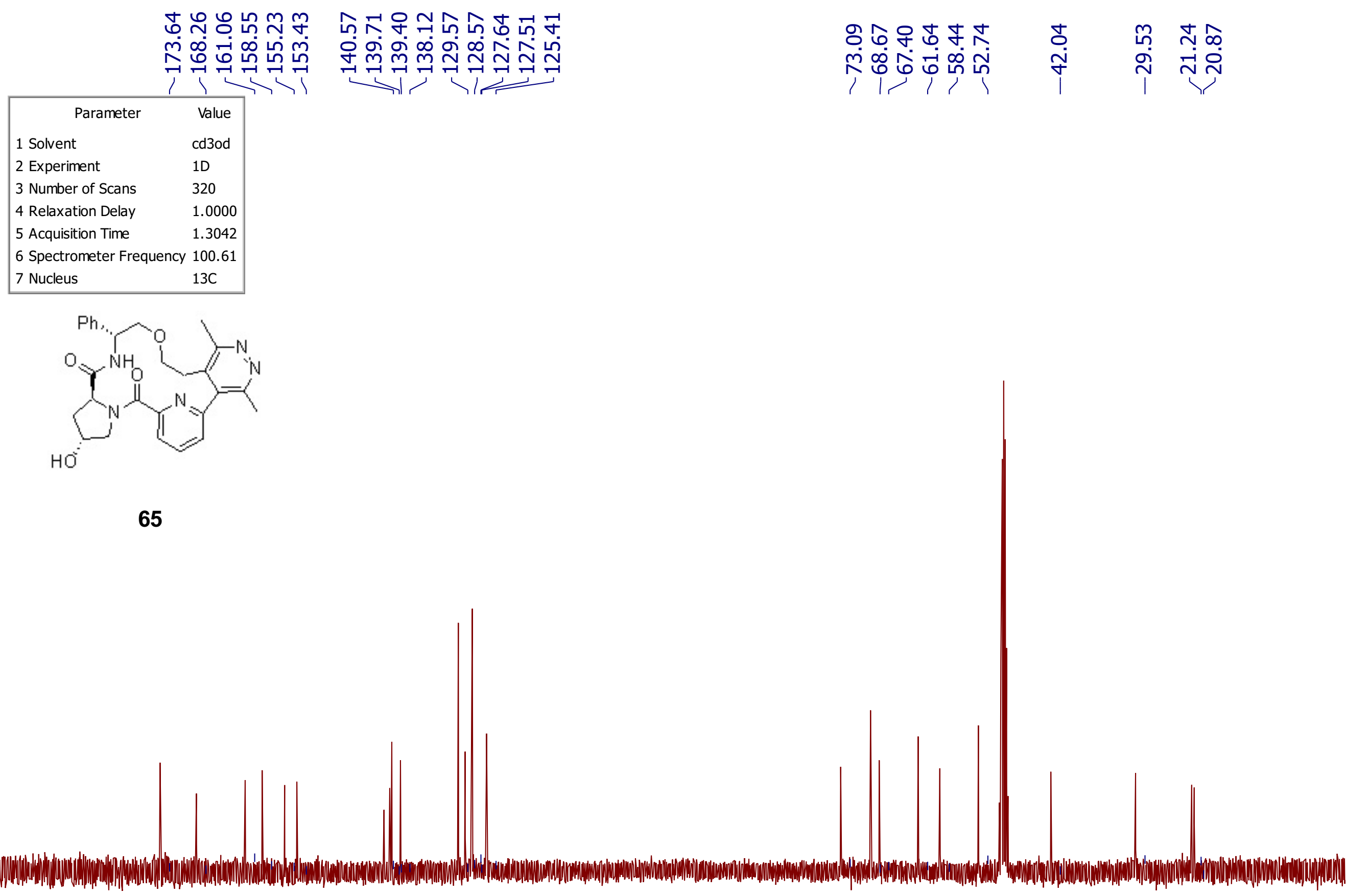

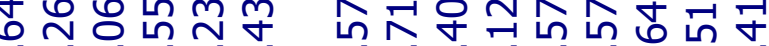

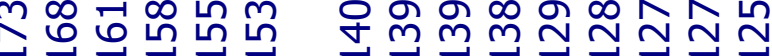

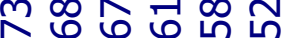
(

$\begin{array}{ll}1 \text { Solvent } & \text { cd3od } \\ 2 \text { Experiment } & 1 \mathrm{D} \\ 3 \text { Number of Scans } & 320 \\ 4 \text { Relaxation Delay } & 1.0000 \\ 5 \text { Acquisition Time } & 1.3042 \\ 6 \text { Spectrometer Frequency } & 100.61 \\ 7 \text { Nucleus } & 13 \mathrm{C}\end{array}$

65

$\begin{array}{llll}1 & 1 & 1 & 1 \\ 90 & 80 & 70 & 60\end{array}$


늑 б

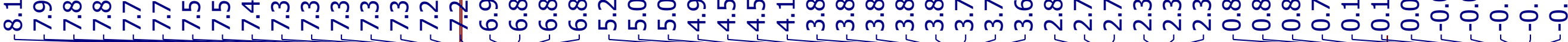

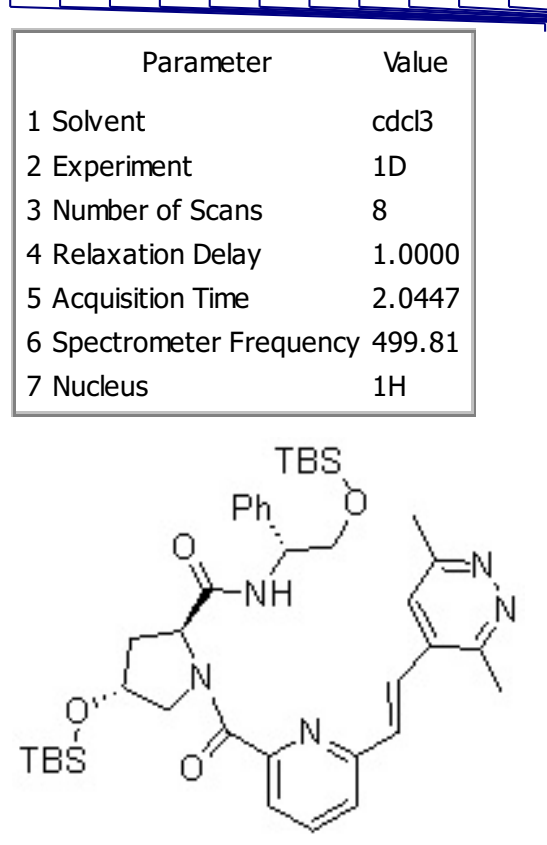

66

\section{6}

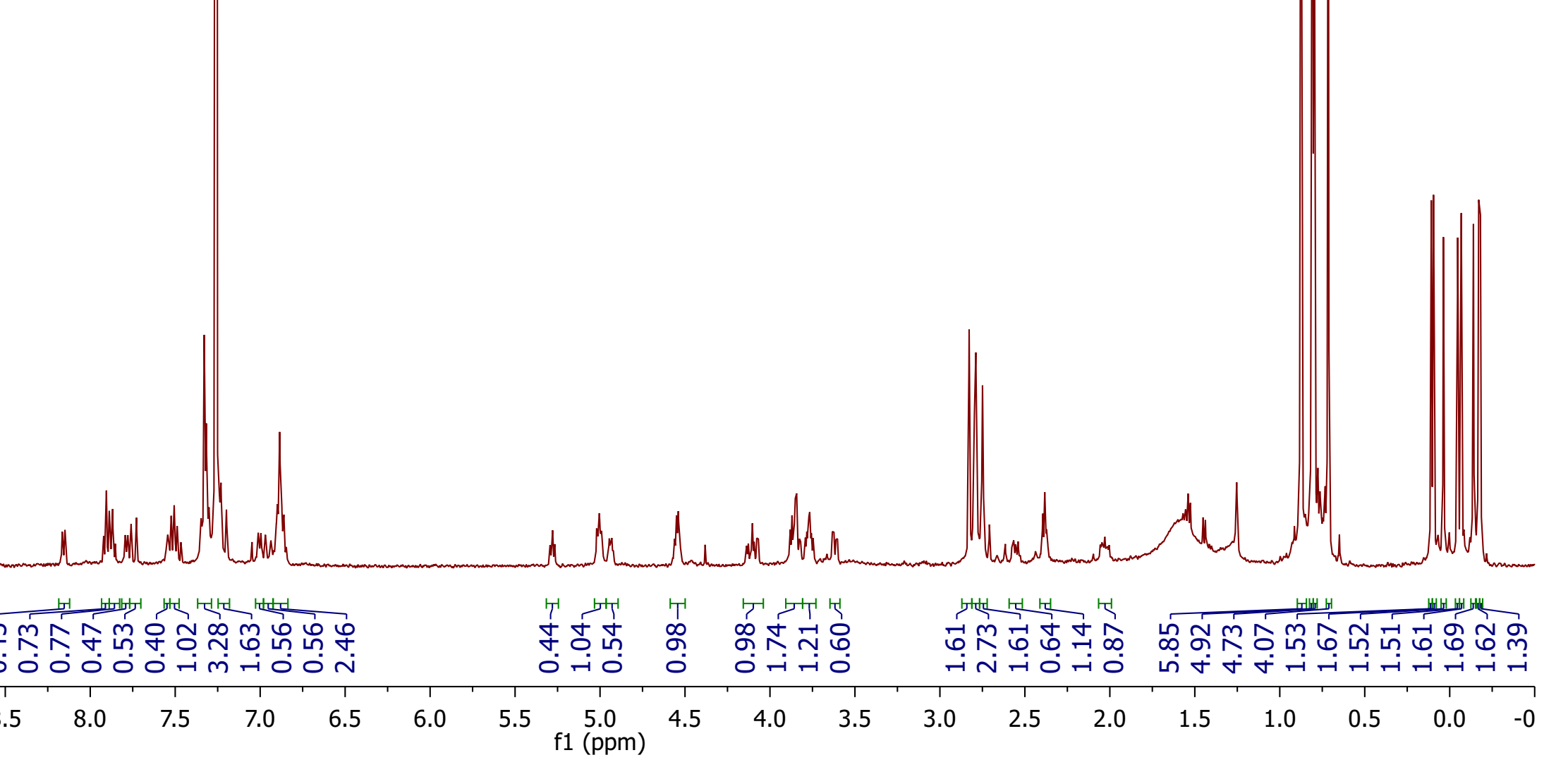




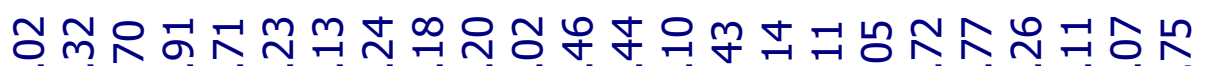
mí

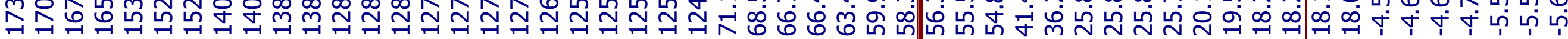

\begin{tabular}{|ll|}
\hline \multicolumn{1}{|c|}{ Parameter } & \multicolumn{1}{c|}{ Value } \\
1 Solvent & cdcl3 \\
2 Experiment & $1 \mathrm{D}$ \\
3 Number of Scans & 25000 \\
4 Relaxation Delay & 1.0000 \\
5 Acquisition Time & 1.0486 \\
6 Spectrometer Frequency 125.69 \\
7 Nucleus & $13 \mathrm{C}$ \\
\hline
\end{tabular}

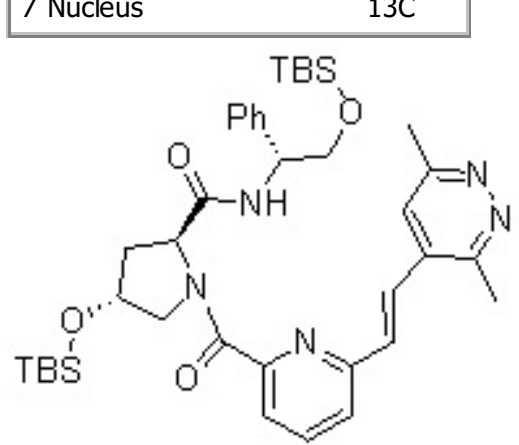

66

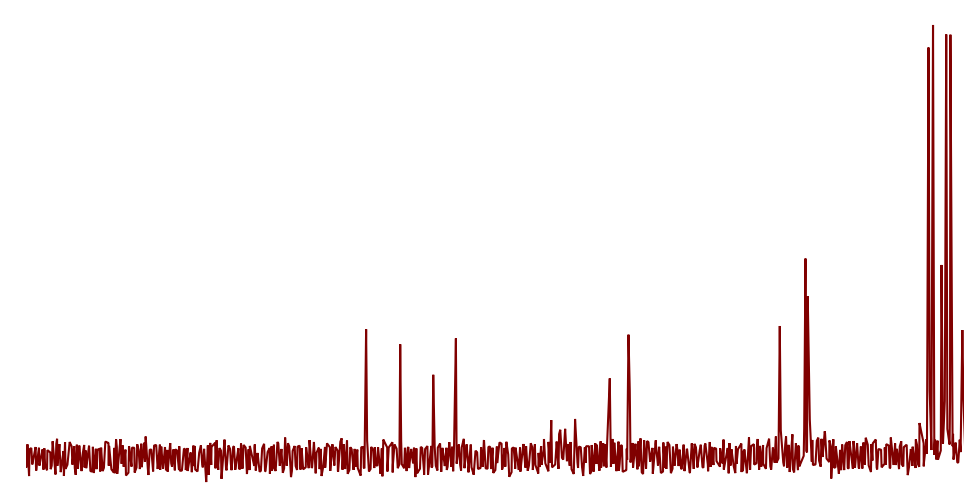

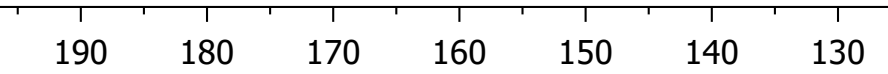

120

110

100

f1 (ppm)

80

70

50

40

30

20 